\title{
A Pivotal Role of Chloride Ion on Nickel-Catalyzed Enantioselective Reductive Cross-Coupling to Perfluoroalkylated Boronate Esters
}

\author{
Dong Wang, and Tao XU
}

Shanghai Key Laboratory of Chemical Assessment and Sustainability, School of Chemical Science and Engineering, Tongji University, 1239 Siping Road, Shanghai, 200092, P. R. of China; Email: taoxu@tongji.edu.cn

\section{Supporting Information}

1. Supplementary Notes.................................................................................2

2. Supplementary Tables............................................................................3

3. Supplementary Methods .........................................................................7

4. Supplementary Discussion .................................................................12

5. The characterization of the compounds ................................................19

6. Single crystal X-ray Diffraction Analysis of compound 3dd ...........86

7. DFT calculation...................................................................................................88

8. Supplementary References...........................................................................103

9. Spectral data of the compounds .......................................................104 


\section{Supplementary Notes}

${ }^{1} \mathrm{H},{ }^{13} \mathrm{C}$ and ${ }^{19} \mathrm{~F}$ NMR spectra were recorded on Bruker 400 (400, 101 and 377 $\mathrm{MHz}$ ) and Bruker $600(600,151$ and $565 \mathrm{MHz})$, and $\mathrm{CDCl}_{3}$ was purchased from J\&K. Chemical shifts are given in ppm with the internal standards as TMS $\left(0 \mathrm{ppm}\right.$ for $\left.{ }^{1} \mathrm{H}\right)$ and $\mathrm{CDCl}_{3}\left(77.0 \mathrm{ppm}\right.$ for $\left.{ }^{13} \mathrm{C}\right)$. Flash column chromatography was performed on silica gel 60 (particle size 200-400 mesh ASTM, purchased from Yantai, China) and eluted with petroleum ether/ethyl acetate. GC spectra were recorded on Agilent Technologies 7890A spectrometer; GC-MS spectra were conducted on Shimadzu GC-MS-QP2010 SE W spectrometer; HPLC analysis was performed on Thermo Fisher Ultimate 3000 spectrometer; Optical rotation was measured using a Rudolph AUTOPOL VI polarimeter; High resolution mass spectra HRMS-ESI were obtained from a Bruker micrOTOF-II instrument. The UV-vis Analysis by Agilent Carry 5000. The Cyclic Voltammetry Analysis by CHI600E (purchased from chinstruments compony).

Unless otherwise noted, all other reagents and starting materials were purchased from commercial sources and used without further purification. Solvents were dried and purified according to the procedure from 'Purification of Laboratory Chemicals book'. 


\section{Supplementary Tables}

General procedure A for Reaction Optimization: An oven-dried 10-mL Schlenk tube containing a Teflon stir bar was charged with Ni-catalyst $(0.02 \mathrm{mmol}, 10 \mathrm{~mol} \%)$, ligand (0.024 mmol, $12 \mathrm{~mol} \%)$, Zn (0.4 mmol, 2 equiv.), TBAC (0.3 mmol, 1.5 equiv.). Then the tube was sealed with a septum and taken out of the $\mathrm{N}_{2}$-filled glovebox. The solvent $(4 \mathrm{~mL})$ was added via syringe under $\mathrm{N}_{2}$ atmosphere and the reaction mixture was stirred for $30 \mathrm{~min}$ at room temperature. Then aryl halide $\mathbf{2 a}(0.2$ $\mathrm{mmol}$ ) and $\mathbf{1 a}(0.4 \mathrm{mmol}, 2.0$ equiv.) were added via micro-syringes. Once added, the tube was closed again, and the reaction mixture was stirred at $1500 \mathrm{rpm}$ for $12 \mathrm{~h}$ at r.t. Upon completed, the mixture was diluted with $\mathrm{H}_{2} \mathrm{O}$ and n-dodecane as a $\mathrm{GC}$ internal standard was added. The yield was determined by GC analysis, and the ee value was obtained by HPLC analysis using the relevant alcohol after purification and oxidation.

Table S1. Screening results on ligands

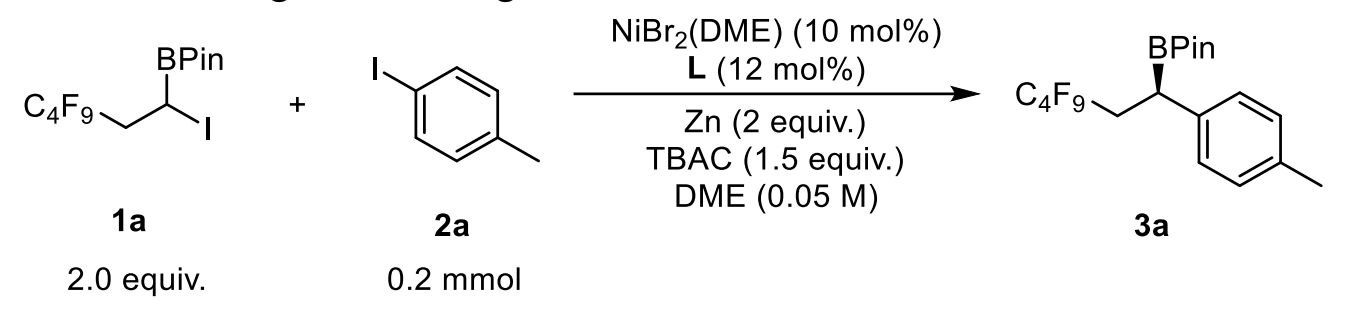

\begin{tabular}{cccc}
\hline Entry & Ligand & Yield & $\boldsymbol{E r}$ \\
\hline $\mathbf{1}$ & $\mathbf{L 1}$ & trace & -- \\
$\mathbf{2}$ & $\mathbf{L 2}$ & $50 \%$ & $78.5: 21.5$ \\
$\mathbf{3}$ & $\mathbf{L 3}$ & $79 \%$. & $91: 9$ \\
$\mathbf{4}$ & $\mathbf{L 4}$ & $82 \%$. & $92.5: 7.5$ \\
$\mathbf{5}$ & $\mathbf{L 5}$ & $75 \%$. & $84.5: 15.5$ \\
$\mathbf{6}$ & $\mathbf{L 6}$ & $81 \%$. & \\
& & &
\end{tabular}

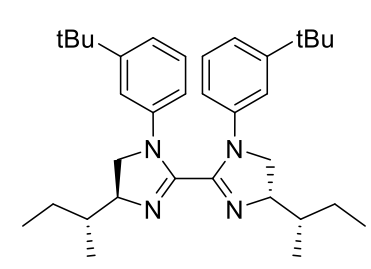

L4

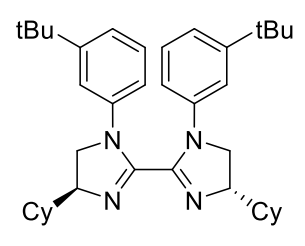

L5

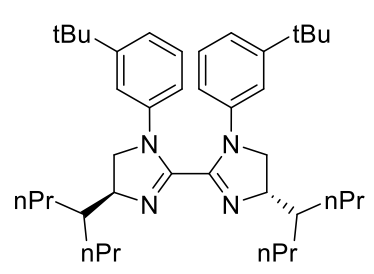

L6 
Table S2. Screening results on Ni-sources

\begin{tabular}{|c|c|c|c|}
\hline & & $\begin{array}{l}\text { Ni-Cat. (10 mol\%) } \\
\text { L4(12 mol\%) }\end{array}$ & \\
\hline 1 & & $\begin{array}{c}\text { Zn (2 equiv.) } \\
\text { TBAC ( } 1.5 \text { equiv. }) \\
\operatorname{DME}(0.05 \mathrm{M})\end{array}$ & \\
\hline 2.0 equiv. & $0.2 \mathrm{mmol}$ & & \\
\hline Entry & Ni-Cat. & Yield & $E r$ \\
\hline 1 & $\mathrm{NiBr}_{2}(\mathrm{DME})$ & $82 \%$. & $92.5: 7.5$ \\
\hline 2 & $\mathrm{Ni}(\mathrm{COD})_{2}$ & $65 \%$ & $91: 9$ \\
\hline 3 & $\mathrm{NiI}_{2}$ & $36 \%$ & $91: 9$ \\
\hline 4 & $\mathrm{Ni}(\mathrm{acac})_{2}$ & N.P. & -- \\
\hline 5 & $\mathrm{NiCl}_{2}(\mathrm{DME})$ & $70 \%$. & $90.5: 9.5$ \\
\hline
\end{tabular}

Table S3. Screening results on reductants

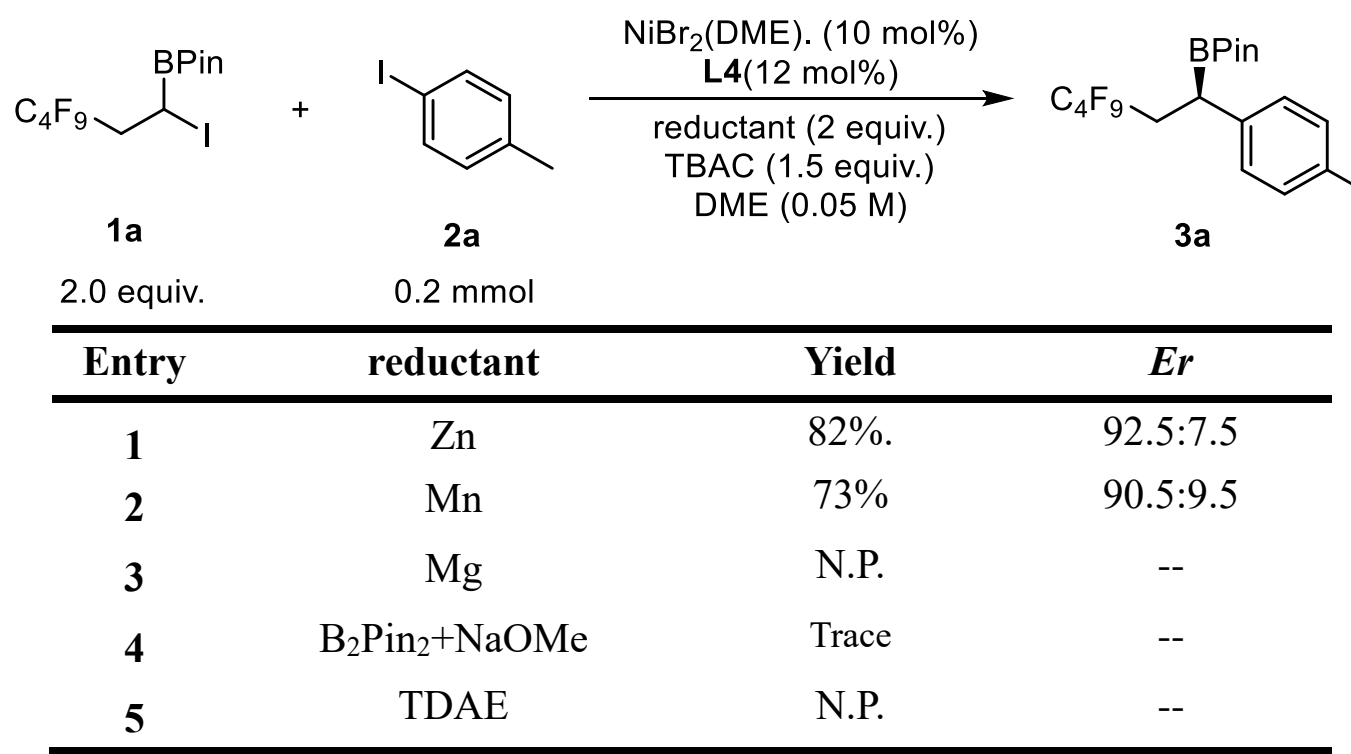

Table S4. Screening results on solvents

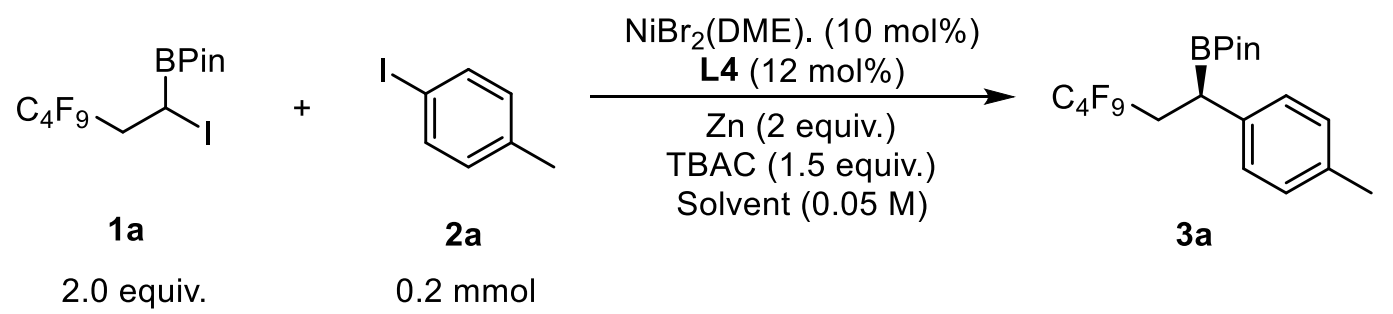




\begin{tabular}{cccc}
\hline Entry & solvent & Yield & $\boldsymbol{E r}$ \\
\hline $\mathbf{1}$ & DME & $82 \%$. & $92.5: 7.5$ \\
$\mathbf{2}$ & THF & $15 \%$ & $91: 9$ \\
$\mathbf{3}$ & Dioxane & $75 \%$ & $84: 16$ \\
$\mathbf{4}$ & MeCN & N.P. & -- \\
$\mathbf{5}$ & DMA & Trace & -- \\
$\mathbf{6}$ & DMF & Trace & -- \\
\hline
\end{tabular}

Table S5. Screening results on the ratio of 1a:2a

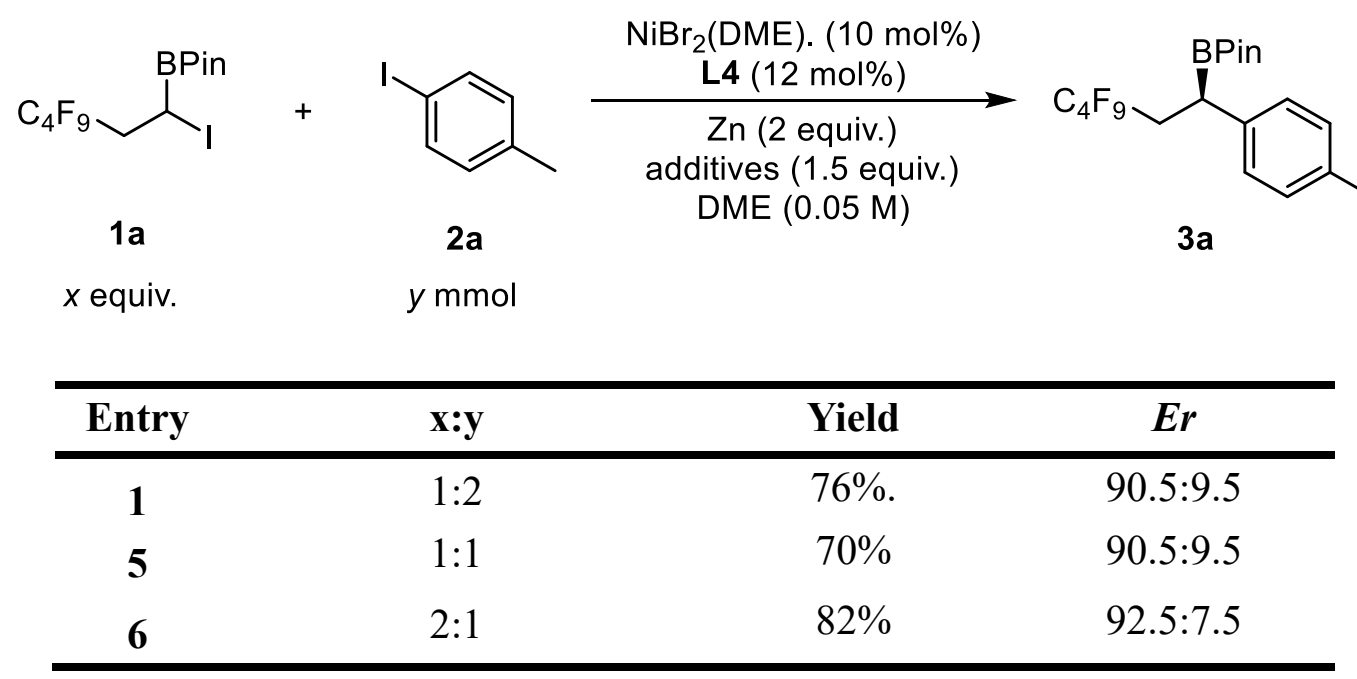

Table S6. Screening results on additives

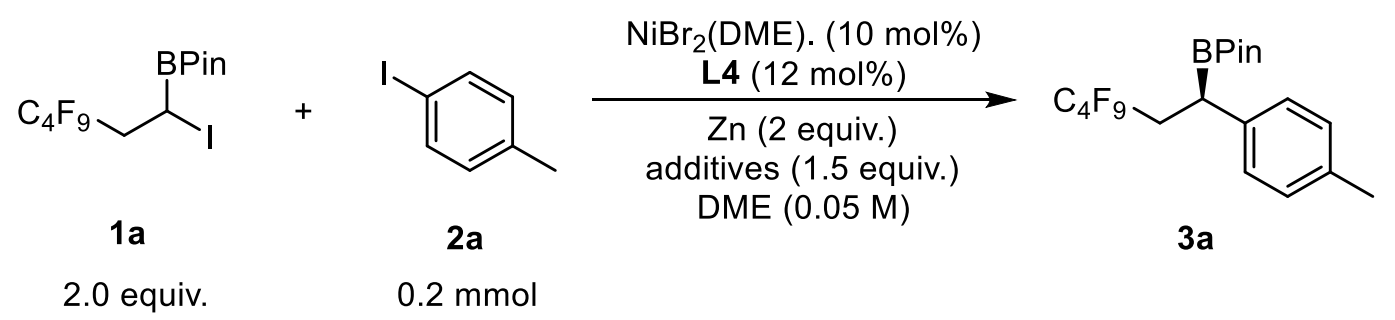

\begin{tabular}{cccc}
\hline Entry & additives & Yield & $\boldsymbol{E r}$ \\
\hline $\mathbf{1}$ & $\mathrm{TBAC}$ & $82 \%$ & $92.5: 7.5$ \\
$\mathbf{2}$ & $\mathrm{LiCl}$ & $10 \%$ & -- \\
$\mathbf{3}$ & $\mathrm{Mg} \mathrm{Cl}$ & $8 \%$ & -- \\
$\mathbf{4}$ & $\mathrm{TMSCl}$ & $2 \%$ & -- \\
$\mathbf{5}$ & $\mathrm{LiBr}$ & $6 \%$ & -- \\
$\mathbf{6}$ & $\mathrm{NaI}$ & $4 \%$ & --
\end{tabular}




\begin{tabular}{lccc}
$\mathbf{7}$ & LiI & $3 \%$ & -- \\
$\mathbf{8}$ & TBAI & $5 \%$ & -- \\
\hline
\end{tabular}




\section{Supplementary Methods}

\section{General procedure B for Product of 3:}

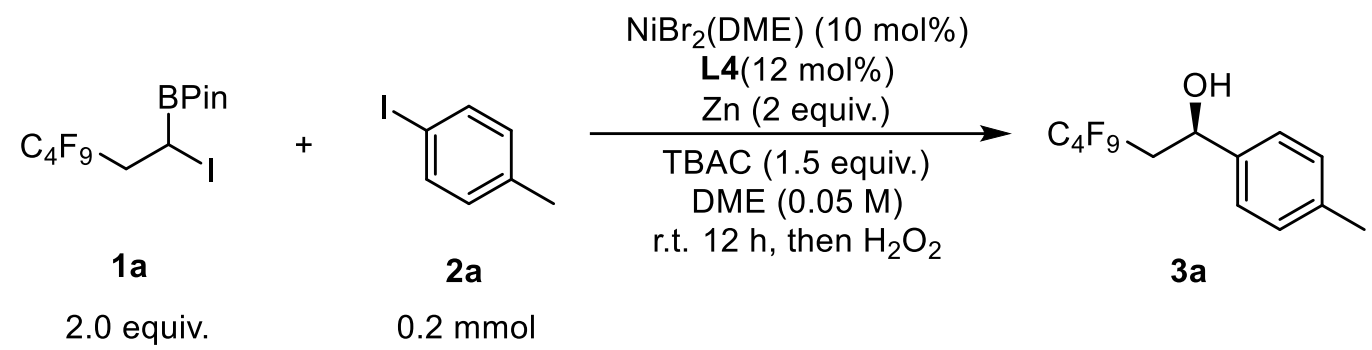

An oven-dried 10-mL Schlenk tube containing a Teflon stir bar was charged with $\mathrm{NiBr}_{2} \cdot \mathrm{DME}$ (0.02 mmol, $\left.10 \mathrm{~mol} \%\right)$, L4 (0.024 mmol, $\left.12 \mathrm{~mol} \%\right)$, Zn (0.4 mmol, 2 equiv.), TBAC ( $0.3 \mathrm{mmol}, 1.5$ equiv.). Then the tube was sealed with a septum and taken out of the $\mathrm{N}_{2}$-filled glovebox. The solvent $(4 \mathrm{~mL})$ was added via syringe under $\mathrm{N}_{2}$ atmosphere and the reaction mixture was stirred for $30 \mathrm{~min}$ at room temperature. Then aryl halide $2 \mathbf{a}(0.2 \mathrm{mmol})$ and $\mathbf{1 a}(0.4 \mathrm{mmol}, 2.0$ equiv. $)$ were added via micro-syringes. Once added, the tube was closed again, and the reaction mixture was stirred at $1500 \mathrm{rpm}$ for $12 \mathrm{~h}$ at r.t. Upon completed, the mixture was diluted with EtOAc and quenched with water. The aqueous solution was extracted with EtOAc three times. The combined organic layers were dried over anhydrous $\mathrm{Na}_{2} \mathrm{SO}_{4}$, filtered through celite, and concentrated in vacuo. Then, the crude product was dissolved in THF $(2 \mathrm{~mL})$ placed in an ice bath. At approximately $0{ }^{\circ} \mathrm{C}$, a $2 \mathrm{~N} \mathrm{NaOH}$ aqueous solution (1.0 mmol, $0.5 \mathrm{~mL}, 5$ equiv.) was added, then $\mathrm{H}_{2} \mathrm{O}_{2}$ (2.0 equiv., $0.4 \mathrm{~mL}, 30 \%$ aqueous solution) was added. The reaction mixture was stirred for 4 6 hours and the temperature were warmed slowly to room temperature. Upon the reaction was completed, the solution was quenched with $2 \mathrm{~mL}$ saturated sodium thiosulfate aqueous. The aqueous solution was extracted with EtOAc three times. The combined organic layers were dried over anhydrous $\mathrm{Na}_{2} \mathrm{SO}_{4}$, filtered and concentrated. The crude material was purified by silica gel column chromatography with a gradient eluent of petroleum ether/ethyl acetate affording 3a. (58.0 mg, 82\% yield, 92.5:7.5 $e r$, ). The $e r$ value was determined by HPLC analysis.

\section{General procedure $\mathbf{C}$ for scale-up reactions:}

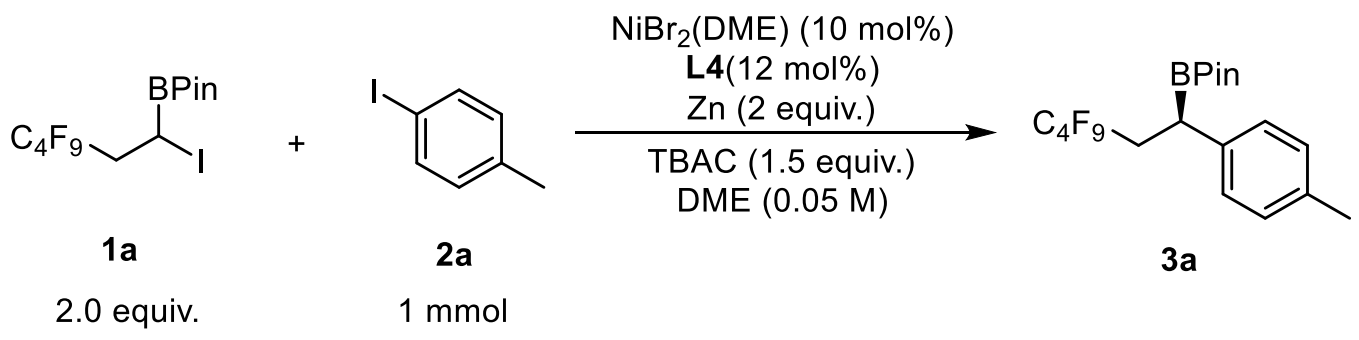

An oven-dried 50-mL Schlenk tube containing a Teflon stir bar was charged with $\mathrm{NiBr}_{2}$. DME (0.02 mmol, $\left.30.8 \mathrm{mg}, 10 \mathrm{~mol} \%\right)$, L4 (0.024 mmol, $\left.61.8 \mathrm{mg}, 12 \mathrm{~mol} \%\right)$, $\mathrm{Zn}(0.4 \mathrm{mmol}, 130 \mathrm{mg}, 2$ equiv.), TBAC (0.3 mmol, $415.5 \mathrm{mg}, 1.5$ equiv.). Then the 
tube was sealed with a septum and taken out of the $\mathrm{N}_{2}$-filled glovebox. The solvent $(20 \mathrm{~mL})$ was added via syringe under $\mathrm{N}_{2}$ atmosphere and the reaction mixture was stirred for $30 \mathrm{~min}$ at room temperature. Then aryl halide $\mathbf{2 a}(1 \mathrm{mmol}, 218 \mathrm{mg})$ and $\mathbf{1 a}$ ( 2 mmol, $1.0 \mathrm{~g}, 2.0$ equiv.) were added via micro-syringes. Once added, the tube was closed again, and the reaction mixture was stirred at $1500 \mathrm{rpm}$ for $12 \mathrm{~h}$ at r.t. Upon completed, the mixture was diluted with EtOAc and quenched with water. The aqueous solution was extracted with EtOAc three times. The combined organic layers were dried over anhydrous $\mathrm{Na}_{2} \mathrm{SO}_{4}$, filtered through celite, and concentrated in vacuo. The crude material was purified by silica gel column chromatography with a gradient eluent of petroleum ether/ethyl acetate affording 3a. (349.6 mg, 75\% yield, 91:9 er). The er value was determined by HPLC analysis using the relative alcohol after oxidation of the product.

\section{General procedure D for synthesis of the starting materials:}

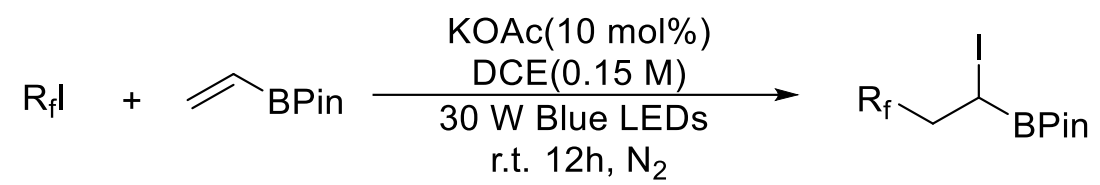

According to the reference ${ }^{\mathrm{S} 1}$, in air, a $200 \mathrm{~mL}$ schlenk tube was charged with KOAc ( $1 \mathrm{mmol}, 99 \mathrm{mg}, 10 \mathrm{~mol} \%)$. The tube was evacuated and filled with nitrogen for three cycles. Then, 1,1-dichloroethane $(65 \mathrm{~mL})$, vinyl borate pinacol ester (10 mmol, $1.7 \mathrm{~mL}, 1.0$ equiv.), perfluoroiodobutane ( $20 \mathrm{mmol}, 2.0$ equiv.) were added to the tube at room temperature. The reaction was stirring under $30 \mathrm{~W}$ blue LEDs irradiation at room temperature. After 12 hours, the crude reaction mixture was concentrated in vacuo and purified by column chromatography (n-pentane: ethyl acetate $=30: 1)$ to afford the desired product.

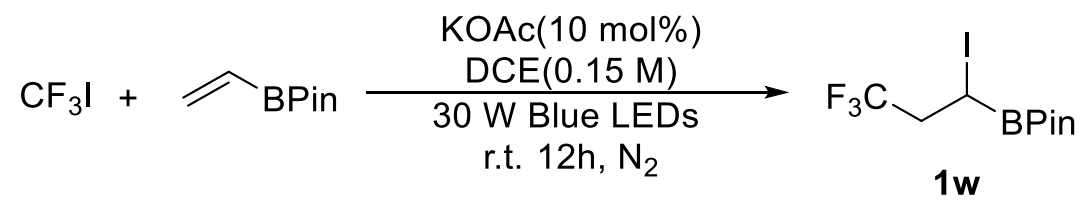

According to the reference ${ }^{\mathrm{S} 1}$, in air, a $200 \mathrm{~mL}$ schlenk tube was charged with KOAc ( $1 \mathrm{mmol}, 99 \mathrm{mg}, 10 \mathrm{~mol} \%)$. The tube was evacuated and filled with nitrogen for three cycles. Then, 1,1-dichloroethane $(65 \mathrm{~mL})$, vinyl borate pinacol ester (10 mmol, $1.7 \mathrm{~mL}, 1.0$ equiv.), $\mathrm{CF}_{3} \mathrm{I}$ (solution of DCE) (20 mmol, 2.0 equiv.) were added to the tube at room temperature. The reaction was stirring under $30 \mathrm{~W}$ blue LEDs irradiation at room temperature. After 12 hours, the crude reaction mixture was concentrated in vacuo and purified by column chromatography (n-pentane: ethyl acetate $=30: 1)$ to afford the desired product $1 \mathbf{w} .{ }^{1} \mathbf{H}$ NMR $\left(600 \mathrm{MHz}, \mathrm{CDCl}_{3}\right) \delta$ 3.29-3.25 (m, 1H), 2.93-2.82 (m, 1H), 2.72-2.64 (m, 1H), $1.28(\mathrm{~s}, 6 \mathrm{H}), 1.26(\mathrm{~s}, 6 \mathrm{H})$; ${ }^{19}$ F NMR $\left(565 \mathrm{MHz}, \mathrm{CDCl}_{3}\right) \delta-66.36(\mathrm{~s}, 3 \mathrm{~F}) ;{ }^{13} \mathbf{C ~ N M R}\left(151 \mathrm{MHz}, \mathrm{CDCl}_{3}\right) \delta 126.0$ (q, $J=275.6 \mathrm{~Hz}), 84.5,39.9$ (t, $J=29.0 \mathrm{~Hz}), 24.2,24.0$. MS: (EI) $[\mathrm{M}]^{+}: 350.00$. 
General procedure $E$ for applications of the products:

Arylation of $3 a$<smiles>Cc1ccc([C@@H](CC(F)(F)F)c2ccccc2)cc1</smiles>

$3 a$

(1.5 equiv.)

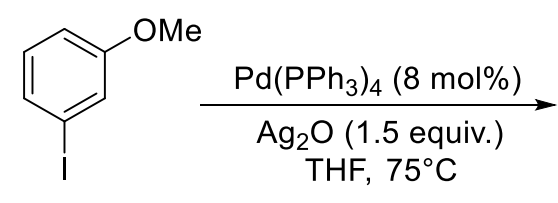

2II

$0.2 \mathrm{mmol}$<smiles>COc1cccc([C@H](CSC(F)(F)F)c2ccc(C)cc2)c1</smiles>

$6 \mathbf{a}$

$51 \%, 91: 9$ er

A $10 \mathrm{~mL}$ oven-dried Schleck tube was charged with $\mathrm{Pd}\left(\mathrm{PPh}_{3}\right)_{4}(0.016 \mathrm{mmol}, 18.5$ $\mathrm{mg}, 8 \mathrm{~mol} \%$ ), $\mathrm{Ag}_{2} \mathrm{O}$ (3.0 mmol, $695.2 \mathrm{mg}, 1.5$ equiv.) in the $\mathrm{N}_{2}$-filled glovebox. Then the tube was sealed with a septum and taken out of the $\mathrm{N}_{2}$-filled glovebox. 3a $(0.3$ mmol, $139.6 \mathrm{mg}, 1.5$ equiv.), $21 \mathrm{l}(0.2 \mathrm{mmol}, 46.8 \mathrm{mg})$ and THF (4 mL) were added via syringe under $\mathrm{N}_{2}$ atmosphere and the reaction mixture was stirred at $75{ }^{\circ} \mathrm{C}$ overnight. The reaction solution was cooled to room temperature and diluted with EtOAc, filtered through celite, and concentrated in vacuo. The residues were purified by silica gel column chromatography with a gradient eluent of petroleum ether/ethyl acetate affording $4 \mathbf{c}$ (45.4 mg, 51\% yield, 91:9 er). The er value was determined by HPLC analysis.

\section{Arylation of $3 e$}<smiles>COc1ccc(C(CC(F)(F)F)C(C)(C)C)cc1</smiles>

(1.5 equiv.)<smiles>N#Cc1ccc(I)cc1</smiles>

$0.2 \mathrm{mmol}$

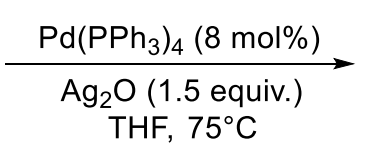

$\mathrm{THF}, 75^{\circ} \mathrm{C}$<smiles>COc1ccc([C@H](CC(F)(F)F)c2ccc(C#N)cc2)cc1</smiles>

$46 \%, 90: 10$ er

A $10 \mathrm{~mL}$ oven-dried Schleck tube was charged with $\mathrm{Pd}\left(\mathrm{PPh}_{3}\right)_{4}(0.016 \mathrm{mmol}, 18.5$ $\mathrm{mg}, 8 \mathrm{~mol} \%$ ), $\mathrm{Ag}_{2} \mathrm{O}$ (3.0 mmol, $695.2 \mathrm{mg}, 1.5$ equiv.) in the $\mathrm{N}_{2}$-filled glovebox. Then the tube was sealed with a septum and taken out of the $\mathrm{N}_{2}$-filled glovebox. 3a $(0.3$ mmol, $139.6 \mathrm{mg}, 1.5$ equiv.), $2 \mathbf{m m}(0.2 \mathrm{mmol}, 45.8 \mathrm{mg})$ and THF (4 mL) were added via syringe under $\mathrm{N}_{2}$ atmosphere and the reaction mixture was stirred at $75{ }^{\circ} \mathrm{C}$ overnight. The reaction solution was cooled to room temperature and diluted with EtOAc, filtered through celite, and concentrated in vacuo. The residues were purified by silica gel column chromatography with a gradient eluent of petroleum ether/ethyl acetate affording $\mathbf{4 c}$ (41.9 $\mathrm{mg}$, 46\% yield, 90:10 er). The er value was determined by HPLC analysis.

\section{Synthesis of $6 b$}


<smiles>Cc1ccc([C@@H](Br)CS(F)(F)(F)(F)F)cc1</smiles>

3a

$(0.2 \mathrm{mmol})$

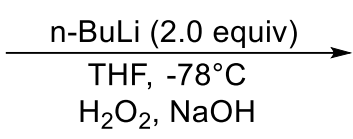

(2.0 equiv.)
$\mathrm{H}_{2} \mathrm{O}_{2}, \mathrm{NaOH}$<smiles>Cc1ccc([C@H](CO)CC(F)(F)F)cc1</smiles>

6b

$54 \%, 91: 9$ er

The title compound was prepared following a known literature procedure with slight modification. ${ }^{\mathrm{S} 2}$ A solution of $\mathbf{3 a}(93.0 \mathrm{mg}, 0.2 \mathrm{mmol}, 1.00$ equiv.) and chloroiodomethane $(30.0 \mu \mathrm{L}, 0.40 \mathrm{mmol}, 2.00$ equiv.) in THF $(1.0 \mathrm{~mL})$ was cooled to $-78^{\circ} \mathrm{C}$. Then $\mathrm{n}$-BuLi (1.6 $\mathrm{M}$ in hexane; $250 \mu \mathrm{L}, 0.40 \mathrm{mmol}, 2.00$ equiv.) was added slowly to it. The resulting reaction mixture was stirred for 30 min at $-78{ }^{\circ} \mathrm{C}$. Then it was allowed to warm to room temperature and stirred for overnight. The reaction flask was then transferred to an ice bath and $\mathrm{NaOH}(2 \mathrm{~mL}, 2.0 \mathrm{M})$ and $\mathrm{H}_{2} \mathrm{O}_{2}(1$ $\mathrm{mL},>30 \% \mathrm{w} / \mathrm{v})$ were added. The reaction mixture was stirred for an additional 2 hours at this temperature and was then diluted with $\mathrm{H}_{2} \mathrm{O}(5.0 \mathrm{~mL})$ and EtOAc $(5.0 \mathrm{~mL})$ and extracted with EtOAc $(3 \times 4.0 \mathrm{~mL})$. The combined organic layers were dried over $\mathrm{Na}_{2} \mathrm{SO}_{4}$, filtered and concentrated. The crude product was purified by flash column chromatography (hexane: EtOAc) to obtain the desired product $6 \mathbf{b}(39.7 \mathrm{mg}, 54 \%$ yield, 91:9 er) as a white solid. The er value was determined by HPLC analysis.

\section{Synthesis of $6 c$}<smiles>Cc1ccc(C([B]C(F)(F)F)CC(F)(F)F)cc1</smiles>

$3 a$

$(0.2 \mathrm{mmol})$<smiles>C=CBr</smiles>

(2.0 equiv.)

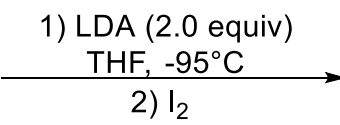

2) $\mathrm{I}_{2}$<smiles>C=C(Br)[C@H](CC(F)(F)F)c1ccc(C)cc1</smiles>

6c

$49 \%, 91: 9$ er

3a (93.0 mg, $0.2 \mathrm{mmol}, 1.0$ equiv.) and vinyl bromide (1.00 $\mathrm{M}$ in THF; $0.40 \mathrm{~mL}$, $0.40 \mathrm{mmol}, 2.0$ equiv.) were dissolved in $\mathrm{Et}_{2} \mathrm{O}(1 \mathrm{~mL})$, and the resulting solution was cooled to $-95^{\circ} \mathrm{C}$. Freshly prepared LDA $(0.86 \mathrm{M} ; 0.47 \mathrm{~mL}, 0.40 \mathrm{mmol}, 2.0$ equiv.) was added dropwise over $10 \mathrm{~min}$, and then the reaction mixture was stirred at $-95{ }^{\circ} \mathrm{C}$ for $1 \mathrm{~h}$. A solution of iodine (111.6 mg, $0.40 \mathrm{mmol}, 2.2$ equiv.) in $\mathrm{MeOH}(1 \mathrm{~mL})$ was added dropwise, and the resulting mixture was stirred at $-95{ }^{\circ} \mathrm{C}$ for $5 \mathrm{~min}$ and then at room temperature for $1 \mathrm{~h} . \mathrm{Na}_{2} \mathrm{SO}_{3}$ (saturated aqueous solution, $10 \mathrm{~mL}$ ) was added, and the reaction mixture was extracted with $\mathrm{Et}_{2} \mathrm{O}(3 \times 15 \mathrm{~mL})$. The combined organic phases were dried over $\mathrm{MgSO}_{4}$ and concentrated. The crude product was purified by flash column chromatography to obtain the desired product 6c (43 mg, 49\% yield, 91:9 er). The er value was determined by HPLC analysis.

\section{Vinylation of $3 e$}




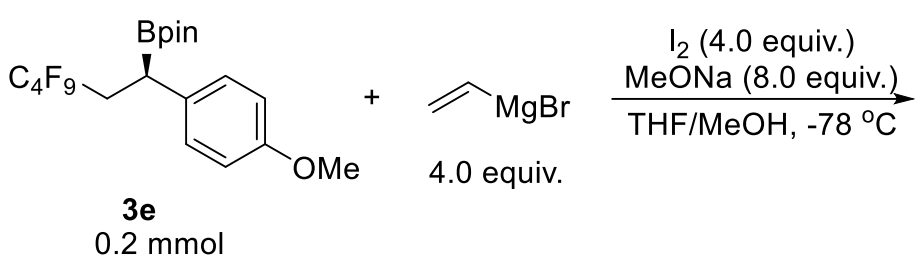

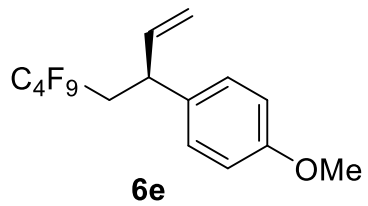

$70 \%, 91: 9$ er

An oven-dried $25 \mathrm{~mL}$ screw-cap Schlenk tube equipped with a Teflon stir bar was added with a solution of $3 \mathbf{e}(99.7 \mathrm{mg}, 0.2 \mathrm{mmol})$ in an hydrous THF $(2 \mathrm{~mL})$ under $\mathrm{N}_{2}$ atmosphere via syringe. Then a solution of vinyl magnesium bromide $(0.8 \mathrm{mmol}, 1.6$ $\mathrm{mL}, 0.5 \mathrm{M}$ in THF) was added dropwise, and the mixture was stirred for $30 \mathrm{~min}$ at room temperature. Then the tube was cooled to $-78{ }^{\circ} \mathrm{C}$ and a solution of $\mathrm{I}_{2}(0.8 \mathrm{mmol}$ in $2 \mathrm{~mL} \mathrm{MeOH}$ ) was added dropwise. The solution was stirred for another $30 \mathrm{~min}$ at this temperature, followed by addition of a solution of NaOMe $(1.6 \mathrm{mmol}$ in $2 \mathrm{~mL}$ $\mathrm{MeOH})$. The reaction mixture was allowed to warm to room temperature and stirred for another 2 hours. Once finished, the reaction was quenched with $2 \mathrm{~mL}$ saturated sodium thiosulfate aqueous. The aqueous solution was extracted with EtOAc three times. The combined organic layers were dried over anhydrous $\mathrm{Na}_{2} \mathrm{SO}_{4}$, filtered and concentrated. The crude material was purified by silica gel column chromatography with a gradient eluent of petroleum ether/ethyl acetate affording 6e (53.4 mg, 70\% yield, 91:9 er). The er value was determined by HPLC analysis.

\section{Synthesis of $6 f$}

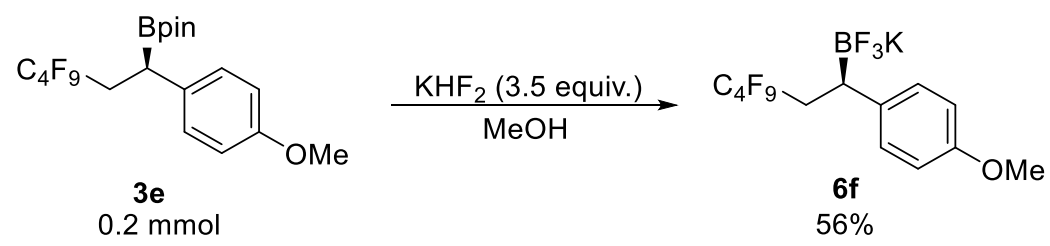

In a $25 \mathrm{~mL}$ round-bottom flask, an aqueous solution of $\mathrm{KHF}_{2}(0.7 \mathrm{mmol}, 54.6 \mathrm{mg}$, 3.5 equiv.) was added to a stirring solution of $3 \mathbf{e}(99.7 \mathrm{mg})$ in $\mathrm{MeOH}(2 \mathrm{~mL})$ at $0{ }^{\circ} \mathrm{C}$. Once added, the ice bath was removed and the reaction mixture was stirred overnight. Upon finished, the resultant solution was concentrated in vacuo, and the residues were washed with hexane/dichloromethane to afford pure $6 \mathbf{f}(55.9 \mathrm{mg}, 59 \%$ yield). 


\section{Supplementary Discussion}

\subsection{Dual Ni/PC regime Reactions}

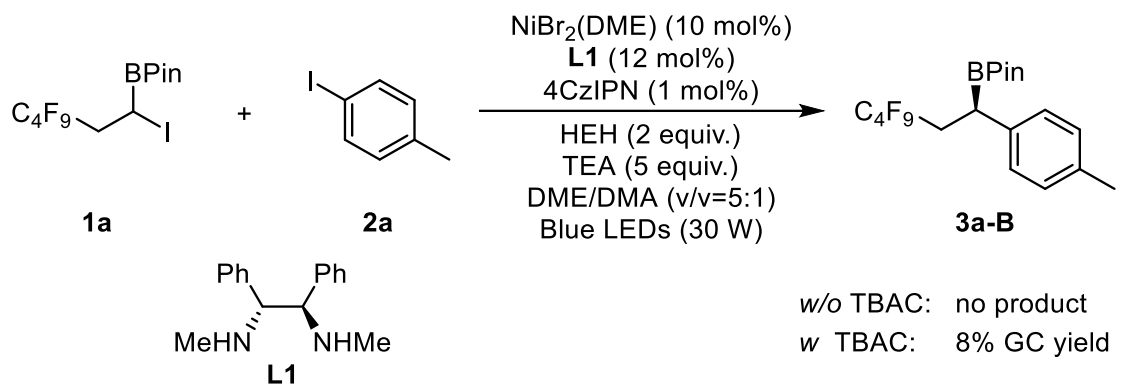

An oven-dried 10-mL Schlenk tube containing a Teflon stir bar was charged with 4CzIPN (0.002 mmol, $1 \mathrm{~mol} \%)$, NiBr $2 \cdot \operatorname{DME}$ (0.02 mmol, $10 \mathrm{~mol} \%)$, L1 (0.024 mmol, $12 \mathrm{~mol} \%)$, HEH (0.4 mmol, 2 equiv.), TEA (1.0 mmol, 5 equiv.), TBAC (0.3 mmol, 1.5 equiv.). Then the tube was sealed with a septum and taken out of the $\mathrm{N}_{2}$-filled glovebox. The solvent $(4 \mathrm{~mL})$ was added via syringe under $\mathrm{N}_{2}$ atmosphere and the reaction mixture was stirred for $30 \mathrm{~min}$ at room temperature. Then aryl halide 1a ( $0.40 \mathrm{mmol}, 2.0$ equiv.) and $\mathbf{2 a}(0.2 \mathrm{mmol})$ were added via micro-syringes. Once added, the tube was closed again, and the reaction mixture was stirred and irradiated under blue light $(\lambda=450-455 \mathrm{~nm})$ for 12 hours, while the temperature was controlled at approximately $20{ }^{\circ} \mathrm{C}$ by cooling with fans and air-conditioner. Upon completed, the mixture was diluted with EtOAc and n-dodecane as a GC internal standard was added. The yield was determined by GC analysis.

\subsection{Comparison Reactions}

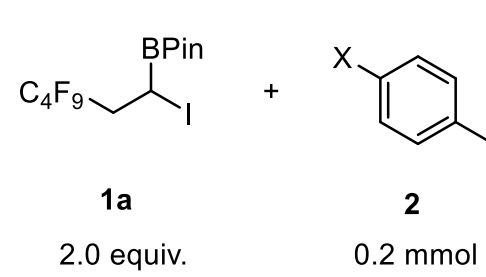

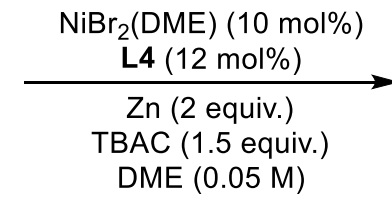

DME $(0.05 \mathrm{M})$

$0.2 \mathrm{mmo}$

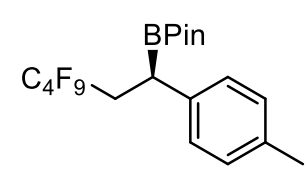

3a-B

$\mathrm{X}=\mathrm{Cl}$ no product

$\mathrm{X}=\mathrm{Br} \quad 12 \%$ GC yield

An oven-dried 10-mL Schlenk tube containing a Teflon stir bar was charged with $\mathrm{NiBr}_{2} \cdot \mathrm{DME}$ (0.02 mmol, $\left.10 \mathrm{~mol} \%\right)$, L4 (0.024 mmol, $\left.12 \mathrm{~mol} \%\right)$, Zn (0.4 mmol, 2 equiv.), TBAC ( 0.3 mmol, 1.5 equiv.). Then the tube was sealed with a septum and taken out of the $\mathrm{N}_{2}$-filled glovebox. The solvent $(4 \mathrm{~mL})$ was added via syringe under $\mathrm{N}_{2}$ atmosphere and the reaction mixture was stirred for $30 \mathrm{~min}$ at room temperature. Then aryl halide $2(0.2 \mathrm{mmol})$ and $1 \mathrm{a}(0.4 \mathrm{mmol}, 2.0$ equiv. $)$ were added via micro-syringes. Once added, the tube was closed again, and the reaction mixture was stirred at $1500 \mathrm{rpm}$ for $12 \mathrm{~h}$ at r.t. Upon completed, the mixture was diluted with $\mathrm{H}_{2} \mathrm{O}$ and n-dodecane as a GC internal standard was added. The yield was determined by GC analysis.

\subsection{Mechanistic Studies Experiments}

\section{Zinc powder activated by $T B A C$}

In $\mathrm{N}_{2}$-filled glovebox, an oven-dried 20-mL Schlenk tube containing a Teflon stir 
bar was charged with $\mathrm{Zn}(5 \mathrm{mmol})$, TBAC (7.5 mmol, 1.5 equiv.) and $20 \mathrm{ml} \mathrm{DME}$ mixture was stirred at $1500 \mathrm{rpm}$ for $24 \mathrm{~h}$ at r.t. Then, under nitrogen atmosphere, it is filtered, washed with DME, and dried under reduced pressure to obtain TBAC-activated zinc powder.
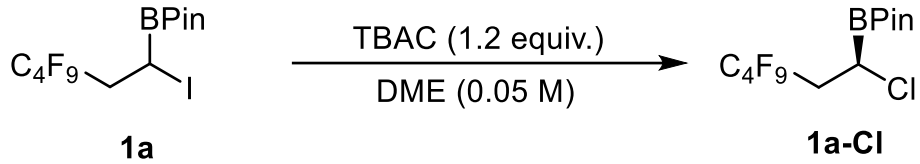

An oven-dried 20-mL Schlenk tube containing a Teflon stir bar was charged with TBAC ( 0.48 mmol, 1.2 equiv.). Then the tube was sealed with a septum and taken out of the $\mathrm{N}_{2}$-filled glovebox. The solvent $(8 \mathrm{~mL})$ was added via syringe under $\mathrm{N}_{2}$ atmosphere Then $1 \mathrm{a}(0.4 \mathrm{mmol}, 1.0$ equiv.) were added via micro-syringes. Once added, the tube was closed again, and the reaction mixture was stirred at $1500 \mathrm{rpm}$ for $12 \mathrm{~h}$ at r.t. Upon completed, the mixture was diluted with EtOAc and quenched with water. The aqueous solution was extracted with EtOAc three times. The combined organic layers were dried over anhydrous $\mathrm{Na}_{2} \mathrm{SO}_{4}$, filtered through celite, and concentrated in vacuo. The crude material was purified by silica gel column chromatography with a gradient eluent of petroleum ether/ethyl acetate affording 1a-Cl. The product was determined by NMR analysis. ${ }^{1} \mathbf{H}$ NMR $\left(600 \mathrm{MHz}, \mathrm{CDCl}_{3}\right) \delta$ $3.65(\mathrm{dd}, J=6.6,9.0 \mathrm{~Hz}, 1 \mathrm{H}), 2.83-2.72(\mathrm{~m}, 1 \mathrm{H}), 2.65-2.54(\mathrm{~m}, 1 \mathrm{H}), 1.29(\mathrm{~s}, 6 \mathrm{H}), 1.28$ $(\mathrm{s}, 6 \mathrm{H}) ;{ }^{19} \mathbf{F}$ NMR $\left(565 \mathrm{MHz}, \mathrm{CDCl}_{3}\right) \delta-80.99--81.03(\mathrm{~m}, 3 \mathrm{~F}),-113.27--113.41(\mathrm{~m}$, 2F), -124.57- -124.63(m, 2F), -125.95- -126.02 (m, 2F); ${ }^{13} \mathbf{C}$ NMR (151 MHz, $\mathrm{CDCl}_{3}$ ) $\delta 119.8-108.3(\mathrm{~m}), 84.5,37.4(\mathrm{t}, J=21.5 \mathrm{~Hz}), 24.2,24.0$. HRMS: $\mathrm{m} / \mathrm{z}$ (ESI) calculated $[\mathrm{M}+\mathrm{H}]^{+}:$409.0783, found: 409.0789 .

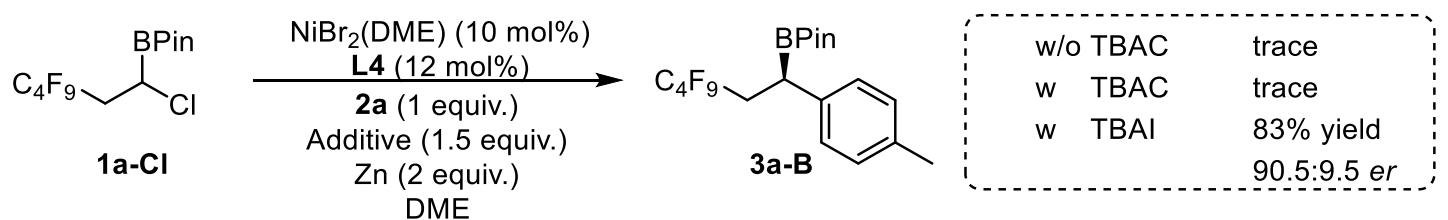

An oven-dried 10-mL Schlenk tube containing a Teflon stir bar was charged with $\mathrm{NiBr}_{2} \cdot \mathrm{DME}$ (0.02 mmol, $\left.10 \mathrm{~mol} \%\right)$, 44 (0.024 mmol, $\left.12 \mathrm{~mol} \%\right)$, Zn (0.4 mmol, 2 equiv.), additives ( $0.3 \mathrm{mmol}, 1.5$ equiv.). Then the tube was sealed with a septum and taken out of the $\mathrm{N}_{2}$-filled glovebox. The solvent $(4 \mathrm{~mL})$ was added via syringe under $\mathrm{N}_{2}$ atmosphere and the reaction mixture was stirred for $30 \mathrm{~min}$ at room temperature. Then aryl halide $\mathbf{2 a}(0.2 \mathrm{mmol})$ and $\mathbf{1 a - C l}(0.4 \mathrm{mmol}, 2.0$ equiv. $)$ were added via micro-syringes. Once added, the tube was closed again, and the reaction mixture was stirred at $1500 \mathrm{rpm}$ for $12 \mathrm{~h}$ at r.t. Upon completed, the mixture was diluted with $\mathrm{H}_{2} \mathrm{O}$ and n-dodecane as a GC internal standard was added. The yield was determined by GC analysis. 

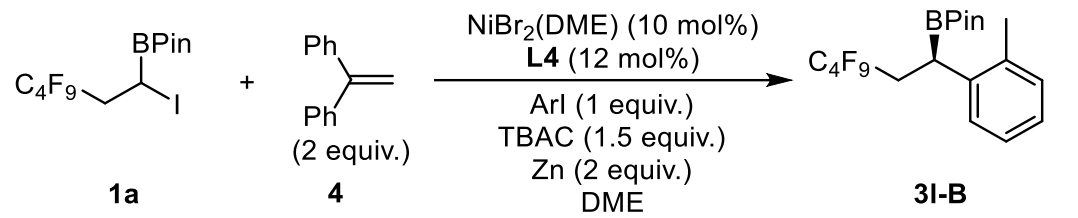

3I-B<smiles>FC(F)(F)CC(CC(c1ccccc1)c1ccccc1)c1ccccc1</smiles>

$$
\begin{array}{lll}
\mathrm{Ar}=p-\mathrm{CH}_{3} \mathrm{C}_{6} \mathrm{H}_{4} \mathrm{I}(\mathbf{2 a}) & 24 \% \text { yield } 91: 9 \text { er } & 17 \% \text { yield } \\
\mathrm{Ar}=0-\mathrm{CH}_{3} \mathrm{C}_{6} \mathrm{H}_{4} \mathrm{I} \text { (2I) } & 7 \% \text { GC yield } & 48 \% \text { yield }
\end{array}
$$

An oven-dried 10-mL Schlenk tube containing a Teflon stir bar was charged with $\mathrm{NiBr}_{2} \cdot \mathrm{DME}$ (0.02 mmol, $\left.10 \mathrm{~mol} \%\right), \mathrm{L} 4$ (0.024 mmol, $\left.12 \mathrm{~mol} \%\right), \mathrm{Zn}(0.4 \mathrm{mmol}, 2$ equiv.), additives ( $0.3 \mathrm{mmol}, 1.5$ equiv.). Then the tube was sealed with a septum and taken out of the $\mathrm{N}_{2}$-filled glovebox. The solvent $(4 \mathrm{~mL})$ was added via syringe under $\mathrm{N}_{2}$ atmosphere and the reaction mixture was stirred for $30 \mathrm{~min}$ at room temperature. Then aryl halide 2 ( $0.2 \mathrm{mmol}), \mathbf{1 a}(0.4 \mathrm{mmol}, 2.0$ equiv.) and 4 (0.4 mmol, 2.0 equiv.) were added via micro-syringes. Once added, the tube was closed again, and the reaction mixture was stirred at $1500 \mathrm{rpm}$ for $12 \mathrm{~h}$ at r.t. Upon completed, the mixture was diluted with $\mathrm{H}_{2} \mathrm{O}$ and n-dodecane as a $\mathrm{GC}$ internal standard was added. The GC yield was determined by GC analysis, and the aqueous solution was extracted with EtOAc three times. The combined organic layers were dried over anhydrous $\mathrm{Na}_{2} \mathrm{SO}_{4}$, filtered and concentrated. The crude material was purified by silica gel column chromatography with a gradient eluent of petroleum ether/ethyl acetate affording $\mathbf{3 l}$ and 5. The product 5 was determined by NMR analysis. ${ }^{1} \mathbf{H}$ NMR $\left(600 \mathrm{MHz}, \mathrm{CDCl}_{3}\right)$ $\delta$ 7.31-7.15 (m, 1H), $4.02(\mathrm{t}, J=8.4 \mathrm{~Hz}, 1 \mathrm{H}), 2.32-2.03(\mathrm{~m}, 5 \mathrm{H}), 1.27(\mathrm{~s}, 12 \mathrm{H}) ;{ }^{19} \mathbf{F}$ NMR $\left(565 \mathrm{MHz}, \mathrm{CDCl}_{3}\right) \delta$-81.27- -81.31 (m, 3F), -112.47- -113.99(m, 2F), -124.78$-124.97(\mathrm{~m}, 2 \mathrm{~F}),-125.95--126.27(\mathrm{~m}, 2 \mathrm{~F}) ;{ }^{13} \mathrm{C}$ NMR $\left(151 \mathrm{MHz}, \mathrm{CDCl}_{3}\right) \delta 144.8$, $143.89,128.6,128.5,128.1,127.7,126.4,126.2,121.4-113.1(\mathrm{~m}), 84.0,83.9,83.6$, $50.1,37.3,32.2(\mathrm{t}, J=21.6 \mathrm{~Hz}), 24.8,24.7,24.3$.

HRMS: m/z (ESI) calculated [M+Na] $]^{+}$555.2116, found: 555.2097.

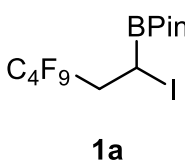

$1 \mathbf{a}$

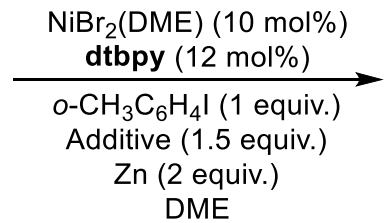

DME<smiles>Cc1ccccc1C(CC(C)(F)F)CC(F)(F)F</smiles>

with TBAC and 4: $\quad 31-B(40 \%$ yield $)+5(47 \%$ yield $)$

An oven-dried 10-mL Schlenk tube containing a Teflon stir bar was charged with $\mathrm{NiBr}_{2} \cdot \operatorname{DME}(0.02 \mathrm{mmol}, 10 \mathrm{~mol} \%)$, dtbpy (0.024 mmol, $\left.12 \mathrm{~mol} \%\right), \mathrm{Zn}(0.4 \mathrm{mmol}, 2$ equiv.), additives ( $0.3 \mathrm{mmol}, 1.5$ equiv.). Then the tube was sealed with a septum and taken out of the $\mathrm{N}_{2}$-filled glovebox. The solvent $(4 \mathrm{~mL})$ was added via syringe under $\mathrm{N}_{2}$ atmosphere and the reaction mixture was stirred for $30 \mathrm{~min}$ at room temperature. Then aryl halide $2 \mathbf{l}(0.2 \mathrm{mmol})$ and $\mathbf{1 a}(0.4 \mathrm{mmol}, 2.0$ equiv. $)$ were added via micro-syringes. Once added, the tube was closed again, and the reaction mixture was stirred at $1500 \mathrm{rpm}$ for $12 \mathrm{~h}$ at r.t. Upon completed, the mixture was diluted with $\mathrm{H}_{2} \mathrm{O}$ and the aqueous solution was extracted with EtOAc three times. The combined organic layers were dried over anhydrous $\mathrm{Na}_{2} \mathrm{SO}_{4}$, filtered and concentrated. The 
crude material was purified by silica gel column chromatography with a gradient eluent of petroleum ether/ethyl acetate affording 31-B and $\mathbf{5}$.

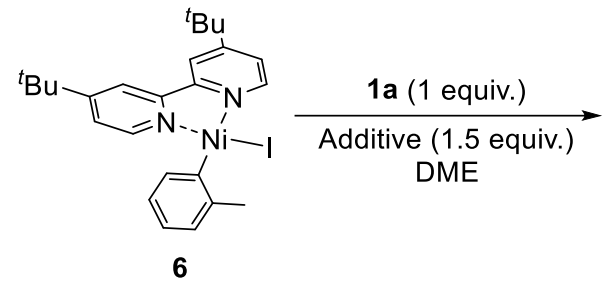

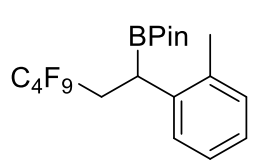

w/o TBAC; with 4 with TBAC and 4: $\quad 3$ I-B $(34 \%$ yield $)+5$ (n.d.)

An oven-dried 10-mL Schlenk tube containing a Teflon stir bar was charged with $6^{\mathrm{S} 3}$ ( $0.1 \mathrm{mmol}, 1.0$ equiv.), TBAC $(0.3 \mathrm{mmol}, 1.5$ equiv. $)$. Then the tube was sealed with a septum and taken out of the $\mathrm{N}_{2}$-filled glovebox. The solvent $(2 \mathrm{~mL})$ was added via syringe under $\mathrm{N}_{2}$ atmosphere. Then $1 \mathrm{a}(0.1 \mathrm{mmol}, 1.0$ equiv.) and $4(0.1 \mathrm{mmol}$, 1.0 equiv.) were added via micro-syringes. Once added, the tube was closed again, and the reaction mixture was stirred at $1500 \mathrm{rpm}$ for $12 \mathrm{~h}$ at r.t. Upon completed, the mixture was diluted with $\mathrm{H}_{2} \mathrm{O}$ and the aqueous solution was extracted with EtOAc three times. The combined organic layers were dried over anhydrous $\mathrm{Na}_{2} \mathrm{SO}_{4}$, filtered and concentrated. The crude material was purified by silica gel column chromatography with a gradient eluent of petroleum ether/ethyl acetate affording $\mathbf{3 I}$ -B and 5.

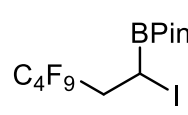

$1 \mathbf{a}$

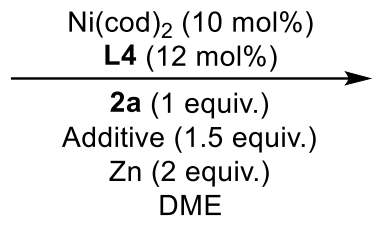

DME<smiles>Cc1ccc(C([18F])CC(F)(F)F)cc1</smiles>

with TBAC and 4 : w/o TBAC $9 \% \quad$ GC yield

w TBAC $67 \%$ yield $91: 9$ er

An oven-dried 10-mL Schlenk tube containing a Teflon stir bar was charged with $\mathrm{Ni}(\text { cod })_{2}(0.02 \mathrm{mmol}, 10 \mathrm{~mol} \%), \mathbf{L} 4$ (0.024 mmol, $\left.12 \mathrm{~mol} \%\right), \mathrm{Zn}(0.4 \mathrm{mmol}, 2$ equiv.), additives $(0.3 \mathrm{mmol}, 1.5$ equiv.). Then the tube was sealed with a septum and taken out of the $\mathrm{N}_{2}$-filled glovebox. The solvent $(4 \mathrm{~mL})$ was added via syringe under $\mathrm{N}_{2}$ atmosphere and the reaction mixture was stirred for $30 \mathrm{~min}$ at room temperature. Then aryl halide $\mathbf{2 a}(0.2 \mathrm{mmol})$, $1 \mathrm{a}(0.4 \mathrm{mmol}, 2.0$ equiv.) and 4 (0.4 mmol, 2.0 equiv.) were added via micro-syringes. Once added, the tube was closed again, and the reaction mixture was stirred at $1500 \mathrm{rpm}$ for $12 \mathrm{~h}$ at r.t. Upon completed, the mixture was diluted with $\mathrm{H}_{2} \mathrm{O}$ and the aqueous solution was extracted with EtOAc three times. The combined organic layers were dried over anhydrous $\mathrm{Na}_{2} \mathrm{SO}_{4}$, filtered and concentrated. The crude material was purified by silica gel column chromatography with a gradient eluent of petroleum ether/ethyl acetate affording $\mathbf{3 a - B}$ and $\mathbf{5}$.

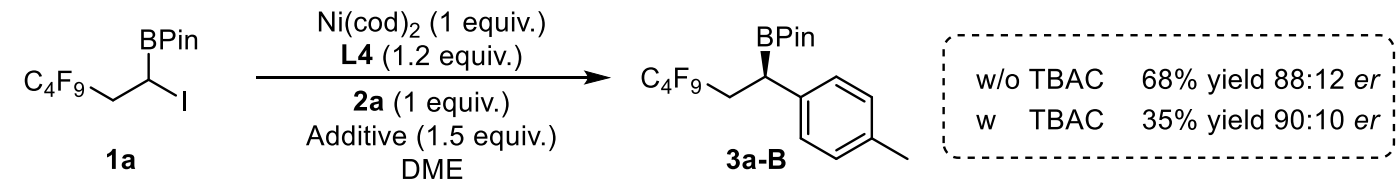


An oven-dried 10-mL Schlenk tube containing a Teflon stir bar was charged with $\mathrm{Ni}(\operatorname{cod})_{2}$ ( $0.2 \mathrm{mmol}, 1.0$ equiv.), $\mathbf{L} 4$ ( $0.24 \mathrm{mmol}, 1.2$ equiv.), additives ( $0.3 \mathrm{mmol}, 1.5$ equiv.). Then the tube was sealed with a septum and taken out of the $\mathrm{N}_{2}$-filled glovebox. The solvent $(4 \mathrm{~mL})$ was added via syringe under $\mathrm{N}_{2}$ atmosphere and the reaction mixture was stirred for $30 \mathrm{~min}$ at room temperature. Then aryl halide $\mathbf{2 a}(0.2$ mmol), 1a ( 0.4 mmol, 2.0 equiv.) and 4 ( 0.4 mmol, 2.0 equiv.) were added via micro-syringes. Once added, the tube was closed again, and the reaction mixture was stirred at $1500 \mathrm{rpm}$ for $12 \mathrm{~h}$ at r.t. Upon completed, the mixture was diluted with $\mathrm{H}_{2} \mathrm{O}$ and the aqueous solution was extracted with EtOAc three times. The combined organic layers were dried over anhydrous $\mathrm{Na}_{2} \mathrm{SO}_{4}$, filtered and concentrated. The crude material was purified by silica gel column chromatography with a gradient eluent of petroleum ether/ethyl acetate affording 3a-B and $\mathbf{5}$.

Table S7. Studies on the substrate 1 nn with different loading of the catalyst.

\begin{tabular}{|c|c|c|c|}
\hline 1nn 2 equiv. & $\begin{array}{c}\mathrm{Ni}(\mathrm{dme}) \mathrm{Br}_{2} \text { (x mol\%) } \\
\mathrm{L4}(1.2 \times \mathrm{mol} \%) \\
\mathbf{2 a}(1 \text { equiv.) } \\
\text { TBAC (1.5 equiv.) } \\
\mathrm{Zn}(2.0 \text { equiv.) } \\
\text { DME }\end{array}$ & $\begin{array}{l}3 \mathrm{nn} \\
\mathrm{Ar}=p-\mathrm{CH}_{3} \mathrm{C}_{6} \mathrm{H}_{4}\end{array}$ & $\sum_{300}^{\mathrm{Ar}}$ \\
\hline Entry & $\mathbf{x}$ & Yield(3nn) & Yield(30o) \\
\hline 1 & 2 & -- & -- \\
\hline 2 & 4 & -- & -- \\
\hline 3 & 6 & -- & -- \\
\hline 4 & 8 & $\sim 1 \%$ & $6 \%$ \\
\hline 5 & 10 & $2 \%$ & $19 \%$ \\
\hline 6 & 20 & $5 \%$ & $46 \%$ \\
\hline
\end{tabular}

An oven-dried 10-mL Schlenk tube containing a Teflon stir bar was charged with $\mathrm{NiBr}_{2} \cdot \mathrm{DME}$ (x mol\%), L4 ( $\left.1.2 \mathrm{x} \mathrm{mol} \%\right)$, Zn (0.4 mmol, 2 equiv.), TBAC ( $0.3 \mathrm{mmol}$, 1.5 equiv.). Then the tube was sealed with a septum and taken out of the $\mathrm{N}_{2}$-filled glovebox. The solvent $(4 \mathrm{~mL})$ was added via syringe under $\mathrm{N}_{2}$ atmosphere and the reaction mixture was stirred for $30 \mathrm{~min}$ at room temperature. Then aryl halide $2 \mathbf{a}(0.2$ mmol) and 1nn ( $0.4 \mathrm{mmol}, 2.0$ equiv.) were added via micro-syringes. Once added, the tube was closed again, and the reaction mixture was stirred at $1500 \mathrm{rpm}$ for $12 \mathrm{~h}$ at r.t. Upon completed, the mixture was diluted with $\mathrm{H}_{2} \mathrm{O}$ and n-dodecane as a GC internal standard was added. The yield was determined by GC analysis. The products are known. ${ }^{\mathrm{S}}$ 


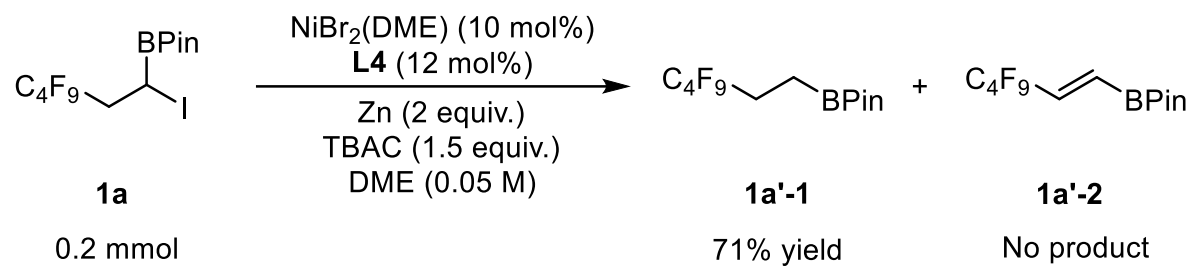

An oven-dried 10-mL Schlenk tube containing a Teflon stir bar was charged with $\mathrm{NiBr}_{2} \cdot \mathrm{DME}$ (0.02 mmol, $\left.10 \mathrm{~mol} \%\right)$, L4 (0.024 mmol, $\left.12 \mathrm{~mol} \%\right)$, Zn (0.4 mmol, 2 equiv.), TBAC ( $0.3 \mathrm{mmol}, 1.5$ equiv.). Then the tube was sealed with a septum and taken out of the $\mathrm{N}_{2}$-filled glovebox. The solvent $(4 \mathrm{~mL})$ was added via syringe under $\mathrm{N}_{2}$ atmosphere and the reaction mixture was stirred for $30 \mathrm{~min}$ at room temperature. Then $1 \mathrm{a}(0.2 \mathrm{mmol})$ were added via micro-syringes. Once added, the tube was closed again, and the reaction mixture was stirred at $1500 \mathrm{rpm}$ for $12 \mathrm{~h}$ at r.t. Upon completed, the mixture was diluted with $\mathrm{H}_{2} \mathrm{O}$ and n-dodecane as a GC internal standard was added. The yield was determined by GC analysis.

\subsection{UV-vis Analysis}

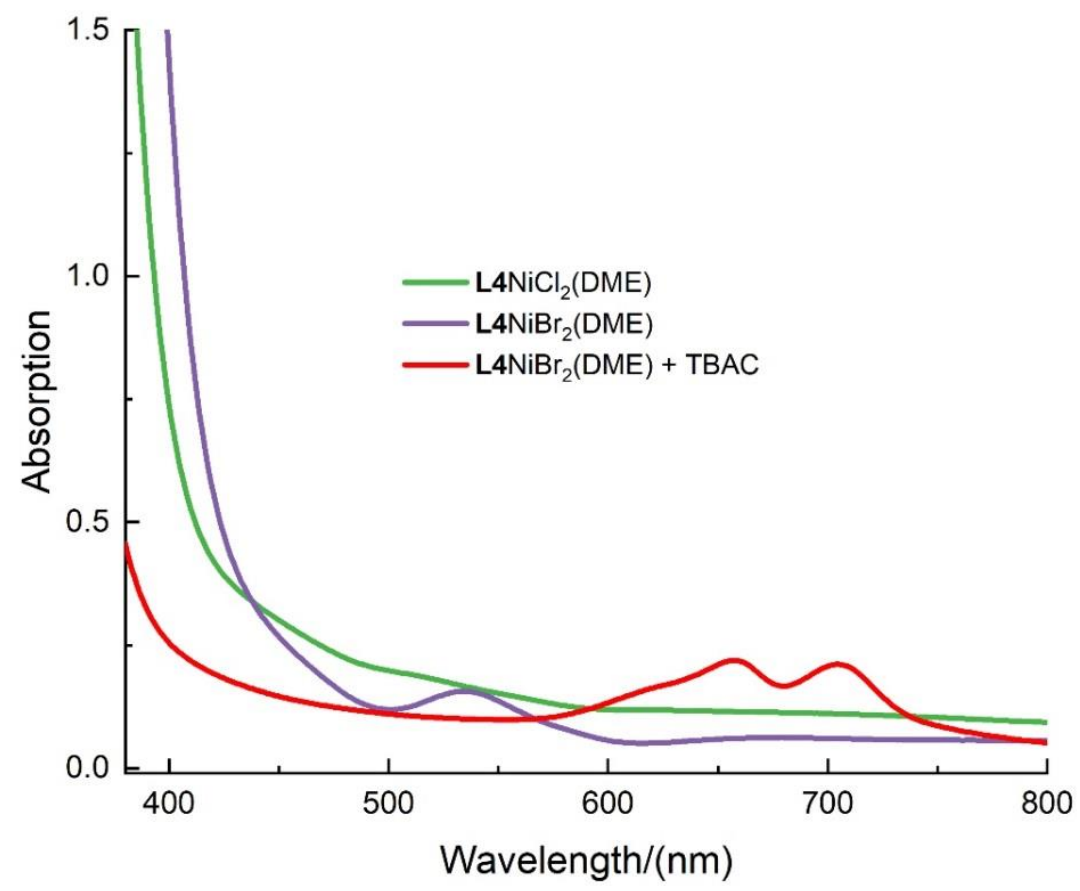

Green line: In a nitrogen filled glovebox, $\mathrm{NiCl}_{2}(\mathrm{DME})(5.5 \mathrm{mg}, 0.025 \mathrm{mmol}, 1$ equiv.) and $\mathbf{L 4}$ (12.8 mg, $0.025 \mathrm{mmol}, 1$ equiv.) were added to a 10-mL Schlenk tube. A solution of DME $(1 \mathrm{~mL})$ was added the reaction was stirred at room temperature for 1 hour. To measure the UV-vis spectrum, a $100 \mathrm{uL}$ aliquot of the solution was diluted to $4 \mathrm{ml}$ total volume with a solution of DME.

Purple line: In a nitrogen filled glovebox, $\mathrm{NiBr}_{2}(\mathrm{DME})(7.7 \mathrm{mg}, 0.025 \mathrm{mmol}, 1$ equiv.) and $\mathbf{L 4}$ (12.8 mg, $0.025 \mathrm{mmol}, 1$ equiv.) were added to a 10-mL Schlenk tube. A solution of DME $(1 \mathrm{~mL})$ was added the reaction was stirred at room temperature for 
1 hour. To measure the UV-vis spectrum, a $100 \mathrm{uL}$ aliquot of the solution was diluted to $4 \mathrm{ml}$ total volume with a solution of DME.

Red line: In a nitrogen filled glovebox, $\mathrm{NiBr}_{2}(\mathrm{DME})(7.7 \mathrm{mg}, 0.025 \mathrm{mmol}, 1$ equiv.), L4 (12.8 mg, $0.025 \mathrm{mmol}$, 1equiv) and TBAC (104.2 mg, $0.375 \mathrm{mmol}, 15$ equiv.) were added to a $10-\mathrm{mL}$ Schlenk tube. A solution of DME (1 mL) was added the reaction was stirred at room temperature for 1 hour. To measure the UV-vis spectrum, a $100 \mathrm{uL}$ aliquot of the solution was diluted to $4 \mathrm{ml}$ total volume with a solution of DME.

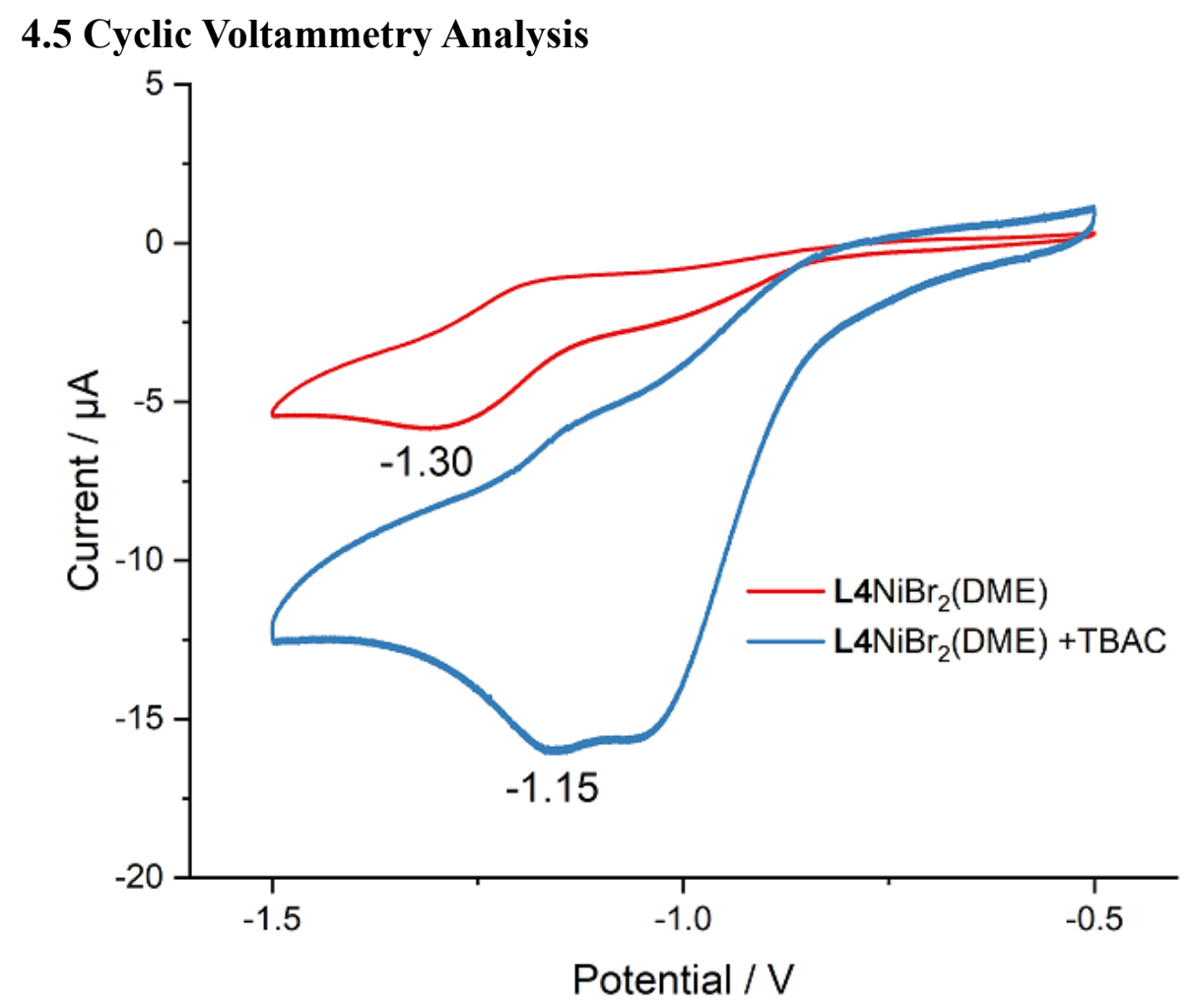

In a nitrogen filled glovebox, $\mathrm{NiBr}_{2}(\mathrm{DME})(6.16 \mathrm{mg}, 0.02 \mathrm{mmol}, 1$ equiv.) and L4 (10.3 mg, $0.02 \mathrm{mmol}, 1$ equiv.) were added to a 10-mL Schlenk tube. A solution of DME $(1 \mathrm{~mL})$ was added the reaction was stirred at room temperature for 2.5 hours. The solvent was removed by vacuum. The samples were dissolved in $20 \mathrm{~mL} 0.1 \mathrm{M}$ tetra-n-butylammonium hexafluorophosphate in dry acetonitrile. (protected with a nitrogen balloon when testing). Measurements were conducted using glassy carbon working electrode, platinum wire counter electrode, and $\mathrm{Ag} / \mathrm{AgCl}$ reference electrode in a scan rate of $0.1 \mathrm{~V} / \mathrm{s}$. 


\section{The characterization of the compounds}<smiles>Cc1ccc([C@@H](O)CC(F)(F)F)cc1</smiles>

The title compound 3a was synthesized according to General Procedure B, and it was purified by column chromatography on silica gel (82\% yield, 92.5:7.5 er, $58.0 \mathrm{mg}$, white solid).

${ }^{1} \mathbf{H}$ NMR $\left(600 \mathrm{MHz}, \mathrm{CDCl}_{3}\right) \delta 7.28(\mathrm{~d}, J=8.4 \mathrm{~Hz}, 2 \mathrm{H}), 7.20(\mathrm{~d}, J=7.8 \mathrm{~Hz}, 2 \mathrm{H}), 5.18$ $(\mathrm{dt}, J=8.4,3.0 \mathrm{~Hz}, 1 \mathrm{H}), 2.66-2.56(\mathrm{~m}, 1 \mathrm{H}), 2.44-2.32(\mathrm{~m}, 4 \mathrm{H}), 2.14(\mathrm{~s}, 1 \mathrm{H}) ;{ }^{19} \mathbf{F}$ NMR (565 MHz, $\left.\mathrm{CDCl}_{3}\right) \delta-81.10--81.15(\mathrm{~m}, 3 \mathrm{~F}),-112.48--114.49$ (m, 2F), -124.65$-124.71(\mathrm{~m}, 2 \mathrm{~F}),-125.95-126.04(\mathrm{~m}, 2 \mathrm{~F}) ;{ }^{13} \mathbf{C} \mathbf{N M R}\left(151 \mathrm{MHz}, \mathrm{CDCl}_{3}\right) \delta 139.7$, 138.3, 129.5, 125.6, 129.8-116.0 (m), 67.8, $39.7(\mathrm{t}, J=20.5 \mathrm{~Hz}), 21.1$.

HRMS: $\mathrm{m} / \mathrm{z}(\mathrm{ESI})$ calculated $[\mathrm{M}+\mathrm{Na}]^{+}: 377.0558$, found: $377.0564 .[\alpha]_{\mathrm{D}}{ }^{25}=12.385(c$ $\left.=0.25, \mathrm{CHCl}_{3}\right)$.

The enantiomeric excess of 3a was determined by chiral HPLC analysis compared to the corresponding racemate alcohol.

Conditions: ChiralPak IB column; hexane $/{ }^{i} \mathrm{PrOH}=98: 2$; flow rate $=1.0 \mathrm{~mL} / \mathrm{min} ; \lambda=$ $220 \mathrm{~nm} ; \mathrm{t}_{\mathrm{R} 1}($ major $)=10.7 \mathrm{~min} ; \mathrm{t}_{\mathrm{R} 2}($ minor $)=9.0 \mathrm{~min}$.

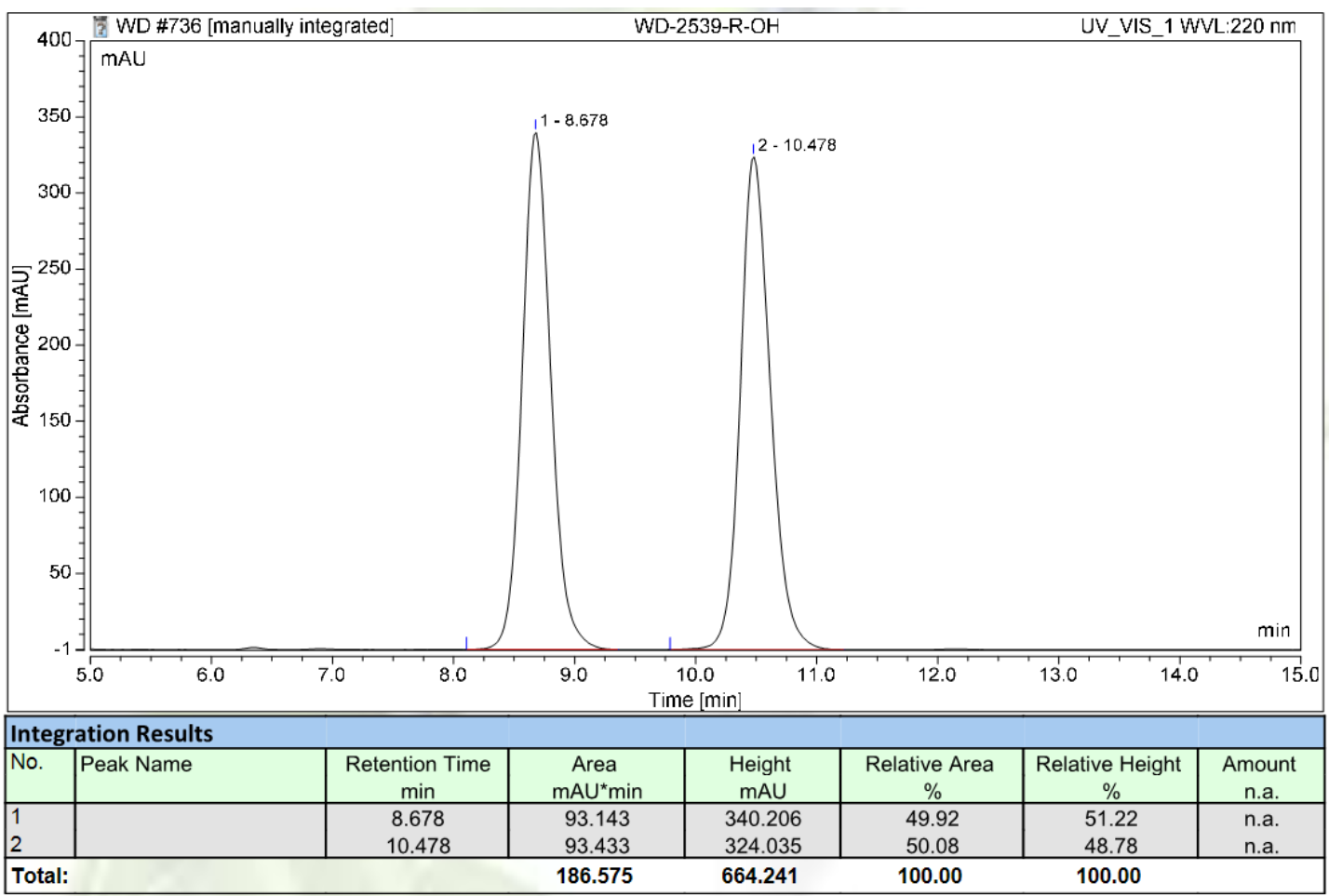




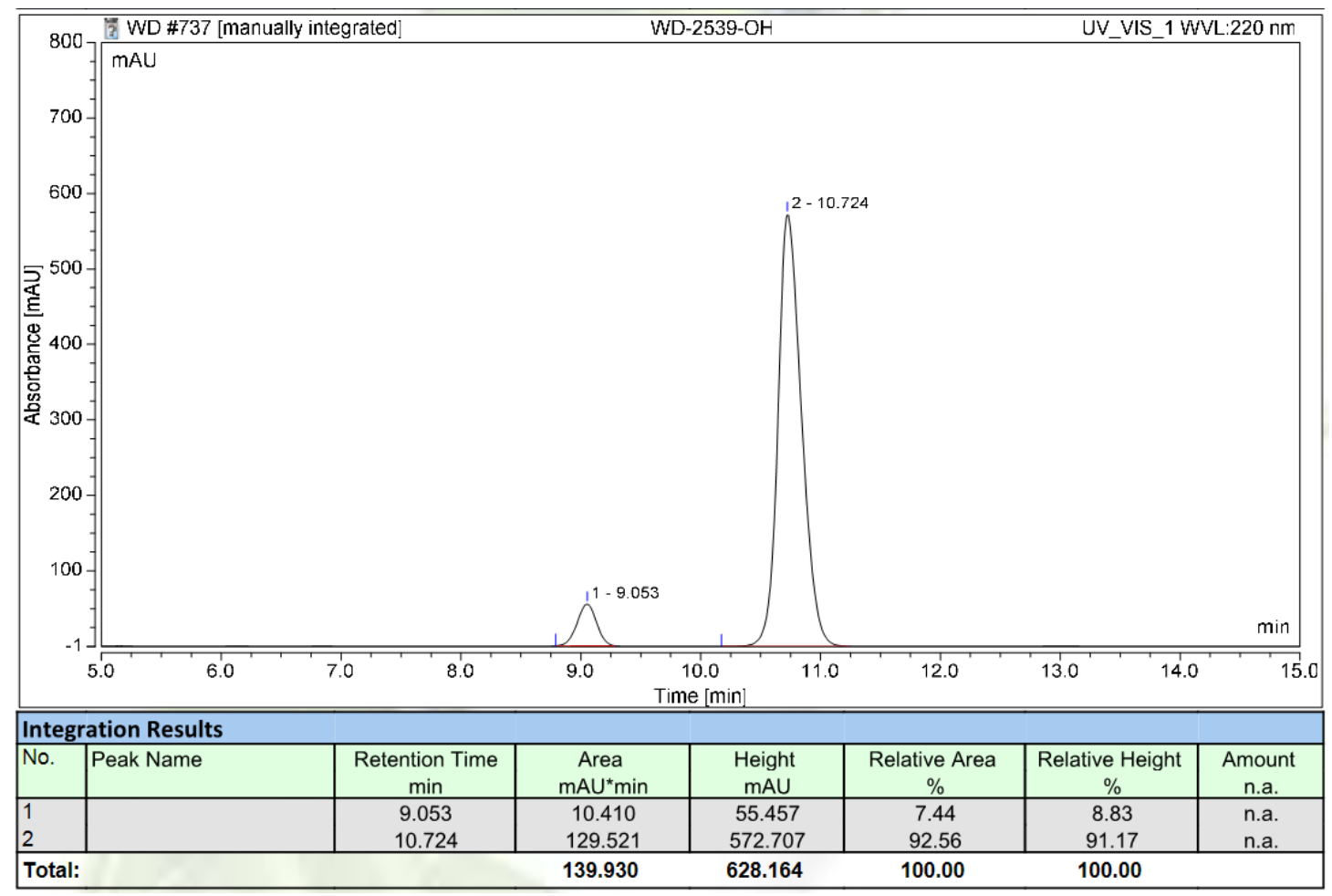<smiles>O[C@H](CC(F)(F)F)c1ccc(Br)cc1</smiles>

The title compound $\mathbf{3 b}$ was synthesized according to General Procedure $\mathbf{B}$, and it was purified by column chromatography on silica gel (59\% yield, 91:9 er, $46.8 \mathrm{mg}$, colourless oil).

${ }^{1}$ H NMR $\left(600 \mathrm{MHz}, \mathrm{CDCl}_{3}\right) \delta 7.41(\mathrm{~d}, J=8.4 \mathrm{~Hz}, 2 \mathrm{H}), 7.31(\mathrm{~d}, J=8.4 \mathrm{~Hz}, 2 \mathrm{H}), 5.20$ $-5.15(\mathrm{~m}, 1 \mathrm{H}), 2.67-2.56(\mathrm{~m}, 1 \mathrm{H}), 2.45-2.36(\mathrm{~m}, 1 \mathrm{H}), 2.18(\mathrm{~d}, J=1.8 \mathrm{~Hz}, 1 \mathrm{H}), 1.32(\mathrm{~s}$, 9H); ${ }^{19}$ F NMR (565 MHz, $\left.\mathrm{CDCl}_{3}\right) \delta-81.00--81.05$ (m, 3F), -112.42- -114.49 (m, 2F), -124.55- -124.60 (m, 2F), -125.86- -125.94. (m, 2F); $\left.{ }^{13} \mathbf{C ~ N M R ~ ( 1 5 1 ~ M H z , ~} \mathrm{CDCl}_{3}\right) \delta$ 151.6, 139.7, 125.8, 125.4, 119.6 - $105.3(\mathrm{~m}), 67.7,39.7$ (t, $J=20.4 \mathrm{~Hz}), 34.6,31.3$.

HRMS: $\mathrm{m} / \mathrm{z}(\mathrm{ESI})$ calculated $[\mathrm{M}+\mathrm{Na}]^{+}: 419.1028$, found: $419.1037 .[\alpha]_{\mathrm{D}}{ }^{25}=20.441(c$ $\left.=0.25, \mathrm{CHCl}_{3}\right)$.

The enantiomeric excess of $\mathbf{3 b}$ was determined by chiral HPLC analysis compared to the corresponding racemate alcohol.

Conditions: ChiralPak IB column; hexane $/ \mathrm{PrOH}=98: 2$; flow rate $=1.0 \mathrm{~mL} / \mathrm{min} ; \lambda=$ $220 \mathrm{~nm} ; \mathrm{tR}_{1}$ (major) $=7.4 \mathrm{~min} ; \mathrm{tR}_{2}$ (minor) $=8.5 \mathrm{~min}$. 

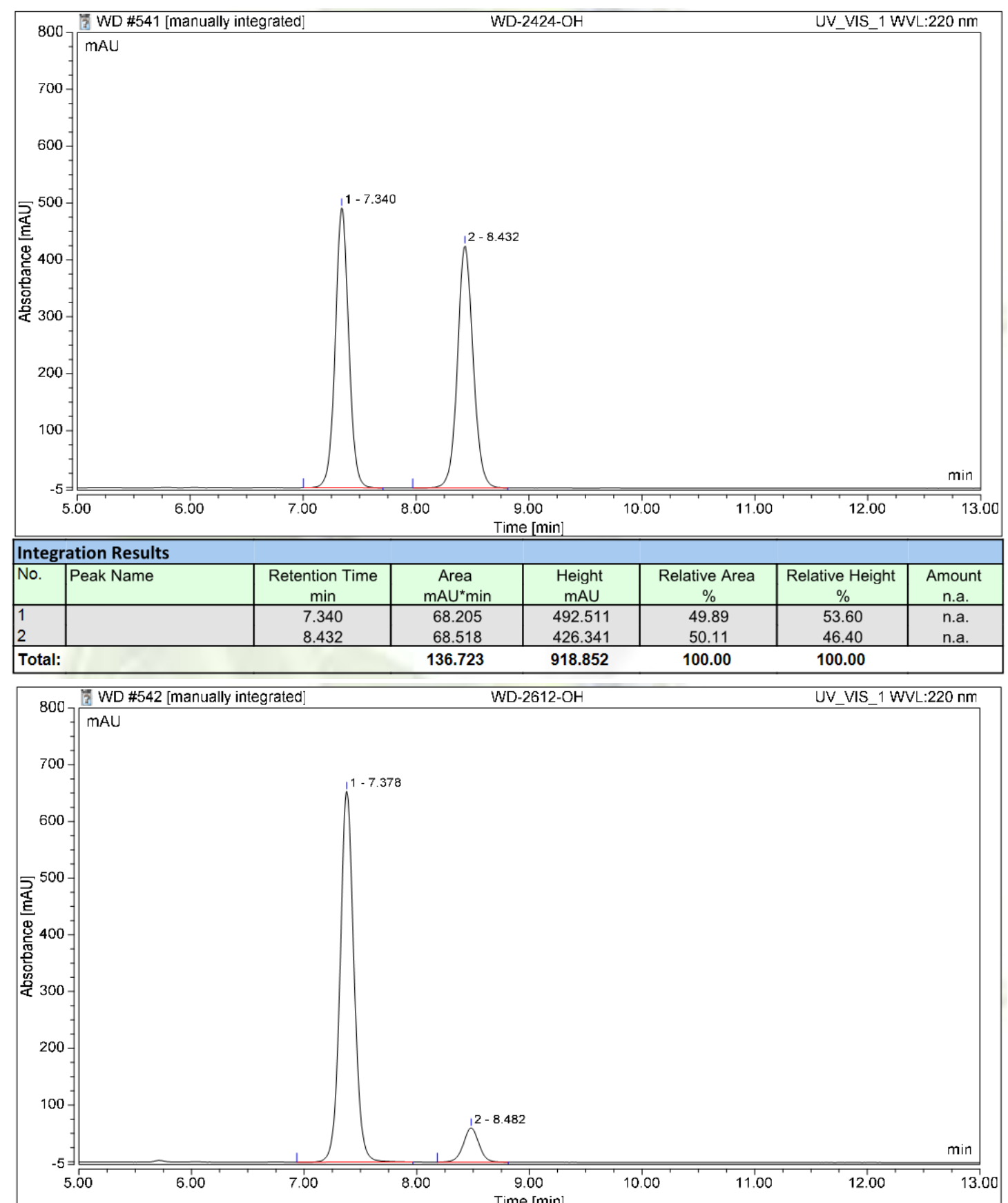

\begin{tabular}{|c|c|c|c|c|c|c|c|}
\hline \multicolumn{8}{|c|}{ Integration Results } \\
\hline No. & Peak Name & $\begin{array}{l}\text { Retention Time } \\
\text { min }\end{array}$ & $\begin{array}{c}\text { Area } \\
\mathrm{mAU}^{*} \min \end{array}$ & $\begin{array}{c}\text { Height } \\
\text { mAU }\end{array}$ & $\begin{array}{c}\text { Relative Area } \\
\%\end{array}$ & $\begin{array}{c}\text { Relative Height } \\
\%\end{array}$ & $\begin{array}{c}\text { Amount } \\
\text { n.a. }\end{array}$ \\
\hline $\begin{array}{l}1 \\
2\end{array}$ & & $\begin{array}{l}7.378 \\
8.482\end{array}$ & $\begin{array}{c}93.734 \\
9.672\end{array}$ & $\begin{array}{c}653.949 \\
60.227\end{array}$ & $\begin{array}{c}90.65 \\
9.35\end{array}$ & $\begin{array}{c}91.57 \\
8.43\end{array}$ & $\begin{array}{l}\text { n.a. } \\
\text { n.a. }\end{array}$ \\
\hline \multicolumn{3}{|c|}{ Total: } & 103.406 & 714.177 & 100.00 & 100.00 & \\
\hline
\end{tabular}<smiles>O[C@H](CC(F)(F)F)c1ccc(Cl)cc1</smiles>

The title compound $\mathbf{3 c}$ was synthesized according to General Procedure B, and it was purified by column chromatography on silica gel (54\% yield, 92:8 er, $40.4 \mathrm{mg}$, colourless oil). 
${ }^{1} \mathbf{H}$ NMR $\left(600 \mathrm{MHz}, \mathrm{CDCl}_{3}\right) \delta 7.36(\mathrm{~d}, J=8.4 \mathrm{~Hz}, 2 \mathrm{H}), 7.33(\mathrm{~d}, J=8.4 \mathrm{~Hz}, 2 \mathrm{H}), 5.20$ $(\mathrm{dt}, J=8.4,3.0 \mathrm{~Hz}, 1 \mathrm{H}), 2.64-2.54(\mathrm{~m}, 1 \mathrm{H}), 2.42-2.33(\mathrm{~m}, 1 \mathrm{H}), 2.28(\mathrm{brs}, 1 \mathrm{H}) ;{ }^{19} \mathbf{F}$ NMR $\left(565 \mathrm{MHz}, \mathrm{CDCl}_{3}\right) \delta$-81.01- -81.06 (m, 3F), -112.46- -114.16 (m, 2F), -124.52$-124.58(\mathrm{~m}, 2 \mathrm{~F}),-125.58--125.96 .(\mathrm{m}, 2 \mathrm{~F}) ;{ }^{13} \mathbf{C ~ N M R}\left(151 \mathrm{MHz}, \mathrm{CDCl}_{3}\right) \delta 141.0$, 134.2, 129.1, 127.0, 121.2-107.3 (m), 67.3, $39.85(\mathrm{t}, J=20.5 \mathrm{~Hz})$.

HRMS: $\mathrm{m} / \mathrm{z}(\mathrm{ESI})$ calculated $[\mathrm{M}-\mathrm{OH}]^{+}: 357.0087$, found: $357.0085 .[\alpha]_{\mathrm{D}}{ }^{25}=19.393(c$ $\left.=0.25, \mathrm{CHCl}_{3}\right)$.

The enantiomeric excess of $\mathbf{3 c}$ was determined by chiral HPLC analysis compared to the corresponding racemate alcohol.

Conditions: ChiralPak IB column; hexane $/ \mathrm{PrOH}=98: 2$; flow rate $=1.0 \mathrm{~mL} / \mathrm{min} ; \lambda=$ $220 \mathrm{~nm} ; \mathrm{tR}_{1}$ (major) $=13.0 \mathrm{~min} ; \mathrm{tR}_{2}$ (minor) $=11.9 \mathrm{~min}$.

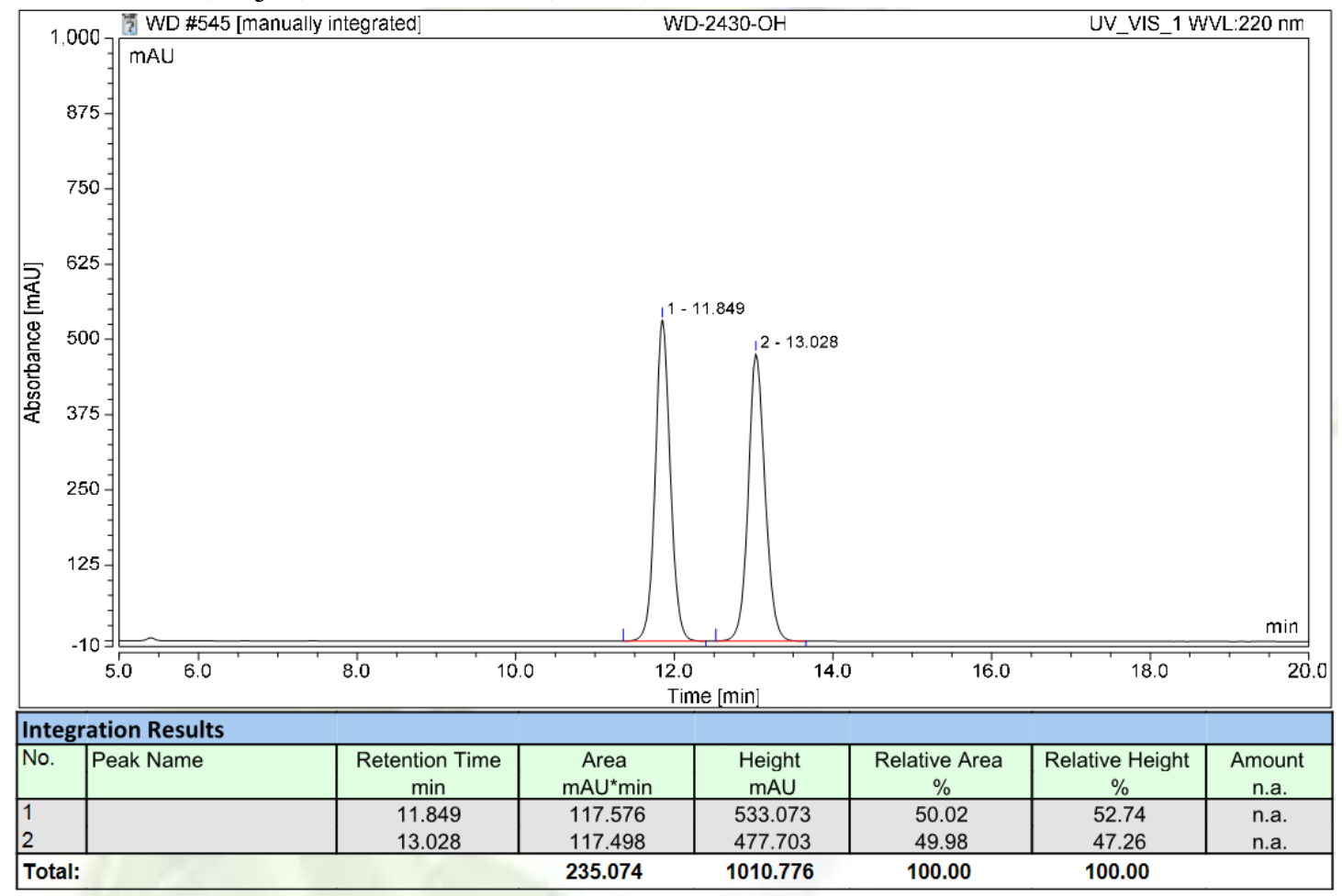




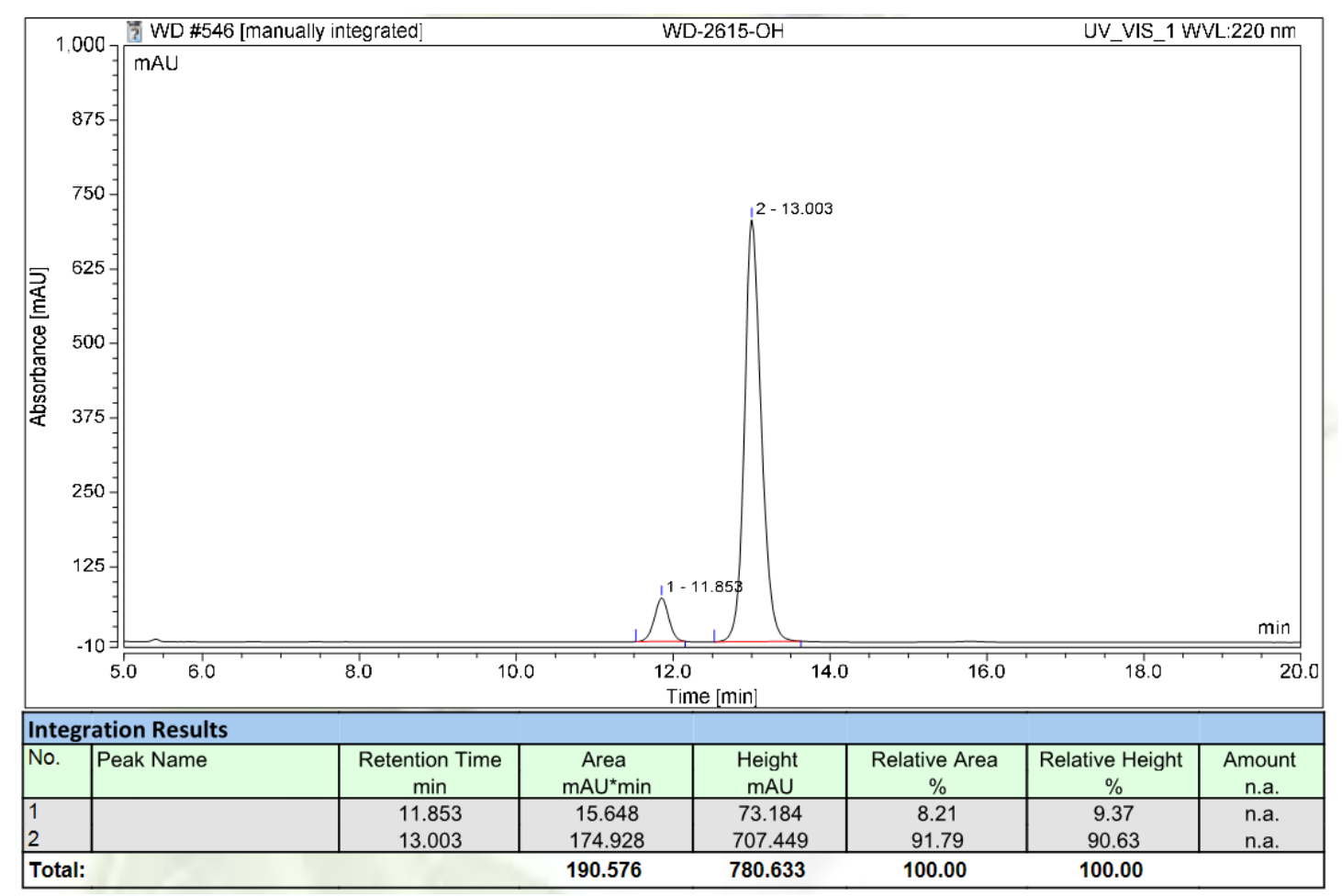<smiles>O[C@H](CC(F)(F)F)c1ccc(F)cc1</smiles>

The title compound 3d was synthesized according to General Procedure B, and it was purified by column chromatography on silica gel (57\% yield, 90.5:9.5 er, $40.9 \mathrm{mg}$, colourless oil).

${ }^{1} \mathbf{H}$ NMR $\left(600 \mathrm{MHz}, \mathrm{CDCl}_{3}\right) \delta$ 7.38-7.36 (m, 2H), $7.07(\mathrm{t}, J=8.4 \mathrm{~Hz}, 2 \mathrm{H}), 5.22(\mathrm{dt}, J$ $=8.4,3.0 \mathrm{~Hz}, 1 \mathrm{H}), 2.66-2.56(\mathrm{~m}, 1 \mathrm{H}), 2.43-2.35(\mathrm{~m}, 1 \mathrm{H}), 2.22(\mathrm{~s}, 1 \mathrm{H}) ;{ }^{19} \mathbf{F} \mathbf{N M R}(565$ $\left.\mathrm{MHz}, \mathrm{CDCl}_{3}\right) \delta-81.34--81.46(\mathrm{~m}, 3 \mathrm{~F}),-112.72--115.01(\mathrm{~m}, 3 \mathrm{~F}),-124.84--124.91(\mathrm{~m}$, 2F), -126.21- -126.30. (m, 2F); ${ }^{13} \mathbf{C}$ NMR (151 MHz, $\left.\mathrm{CDCl}_{3}\right) \delta 162.6(\mathrm{~d}, J=247.2$ $\mathrm{Hz}), 138.4$ (d, $J=3.2 \mathrm{~Hz}), 127.4$ (d, $J=8.2 \mathrm{~Hz}), 119.5-115.9(\mathrm{~m}), 115.8$ (d, $J=21.6$ $\mathrm{Hz}), 67.4,39.9$ (t, $J=20.5 \mathrm{~Hz})$.

HRMS: $\mathrm{m} / \mathrm{z}(\mathrm{ESI})$ calculated $[\mathrm{M}+\mathrm{Na}]^{+}: 381.0308$, found: $381.0321 .[\alpha]_{\mathrm{D}}^{25}=24.983$ $\left(c=0.25, \mathrm{CHCl}_{3}\right)$.

The enantiomeric excess of $\mathbf{3 d}$ was determined by chiral HPLC analysis compared to the corresponding racemate alcohol.

Conditions: ChiralPak IB column; hexane $/ \mathrm{PrOH}=98: 2$; flow rate $=1.0 \mathrm{~mL} / \mathrm{min} ; \lambda=$ $220 \mathrm{~nm} ; \mathrm{tR}_{1}$ (major) $=10.2 \mathrm{~min} ; \mathrm{tR}_{2}$ (minor) $=9.3 \mathrm{~min}$. 


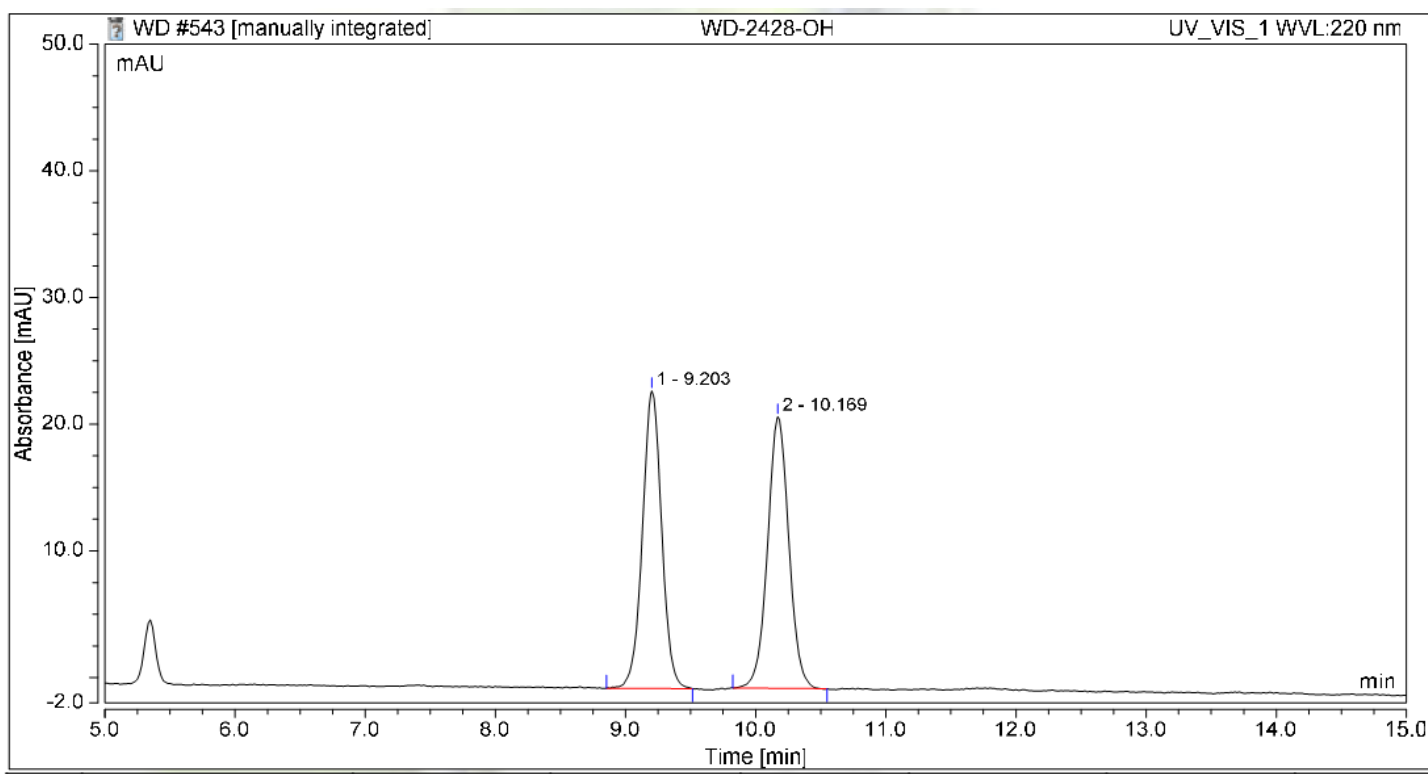

\begin{tabular}{|l|c|c|c|c|c|c|c|}
\hline \multicolumn{2}{|l|}{ Integration Results } \\
\hline No. & Peak Name & $\begin{array}{c}\text { Retention Time } \\
\min \end{array}$ & $\begin{array}{c}\text { Area } \\
\mathrm{mAU}^{*} \text { min }\end{array}$ & $\begin{array}{c}\text { Height } \\
\mathrm{mAU}\end{array}$ & $\begin{array}{c}\text { Relative Area } \\
\%\end{array}$ & $\begin{array}{c}\text { Relative Height } \\
\%\end{array}$ & $\begin{array}{c}\text { Amount } \\
\text { n.a. }\end{array}$ \\
\hline 1 & & 9.203 & 4.025 & 23.497 & 49.85 & 52.30 & n.a. \\
2 & 10.169 & 4.050 & 21.434 & 50.15 & 47.70 & n.a. \\
\hline
\end{tabular}

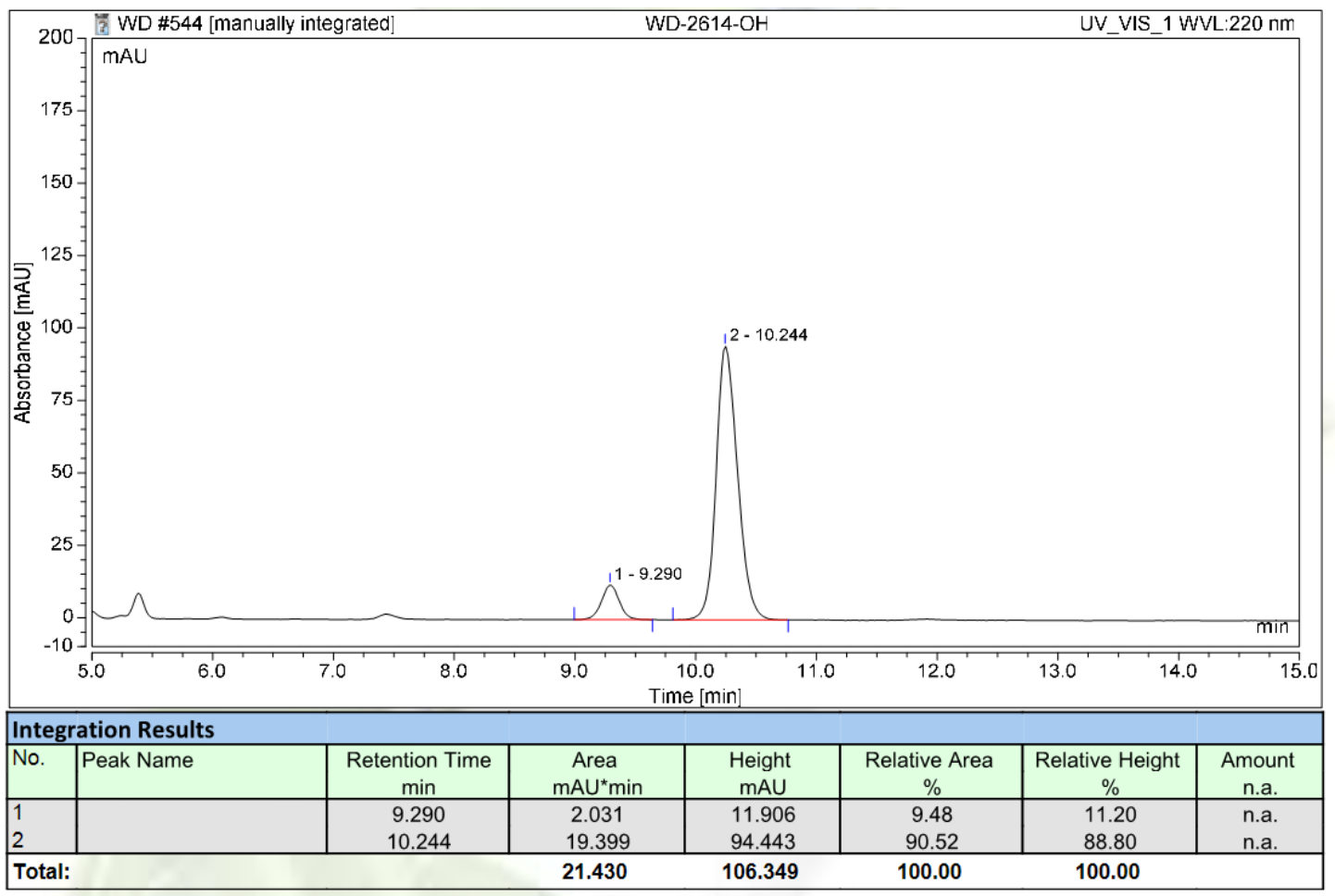<smiles>COc1ccc([C@@H](O)CC(F)(F)F)cc1</smiles>

The title compound 3e was synthesized according to General Procedure B, and it was purified by column chromatography on silica gel (67\% yield, 91:9 er, $49.6 \mathrm{mg}$, colourless oil). 
${ }^{1} \mathbf{H}$ NMR $\left(600 \mathrm{MHz}, \mathrm{CDCl}_{3}\right) \delta 7.30(\mathrm{~d}, J=9.0 \mathrm{~Hz}, 2 \mathrm{H}), 6.90(\mathrm{~d}, J=8.4 \mathrm{~Hz}, 2 \mathrm{H}), 5.15$ $(\mathrm{dt}, J=8.4,3.0 \mathrm{~Hz}, 1 \mathrm{H}), 3.81(\mathrm{~s}, 2 \mathrm{H}), 2.67-2.56(\mathrm{~m}, 1 \mathrm{H}), 2.44-2.34(\mathrm{~m}, 1 \mathrm{H}), 2.20(\mathrm{~s}$, $1 \mathrm{H}) ;{ }^{19} \mathbf{F}$ NMR $\left(565 \mathrm{MHz}, \mathrm{CDCl}_{3}\right) \delta-81.00--81.06(\mathrm{~m}, 3 \mathrm{~F}),-112.50--114.40(\mathrm{~m}, 2 \mathrm{~F})$, -124.54- -124.64 (m, 2F), -125.89- -125.96. (m, 2F); $\left.{ }^{13} \mathrm{C} \mathrm{NMR} \mathrm{(151} \mathrm{MHz,} \mathrm{CDCl}_{3}\right) \delta$ 159.6, 134.8, 126.9, 120.3-108.6 (m), 67.5, 55.3, 39.7 (t, $J=20.5 \mathrm{~Hz})$.

HRMS: $\mathrm{m} / \mathrm{z}(\mathrm{ESI})$ calculated $[\mathrm{M}+\mathrm{Na}]^{+}: 393.0508$, found: $393.0512 .[\alpha]_{\mathrm{D}}{ }^{25}=31.332(c$ $\left.=0.25, \mathrm{CHCl}_{3}\right)$.

The enantiomeric excess of 3e was determined by chiral HPLC analysis compared to the corresponding racemate alcohol.

Conditions: ChiralPak IA column; hexane $/ \mathrm{PrOH}=97: 3$; flow rate $=1.0 \mathrm{~mL} / \mathrm{min} ; \lambda=$ $220 \mathrm{~nm} ; \mathrm{tR}_{1}$ (major) $=14.0 \mathrm{~min} ; \mathrm{tR}_{2}$ (minor) $=13.2 \mathrm{~min}$.

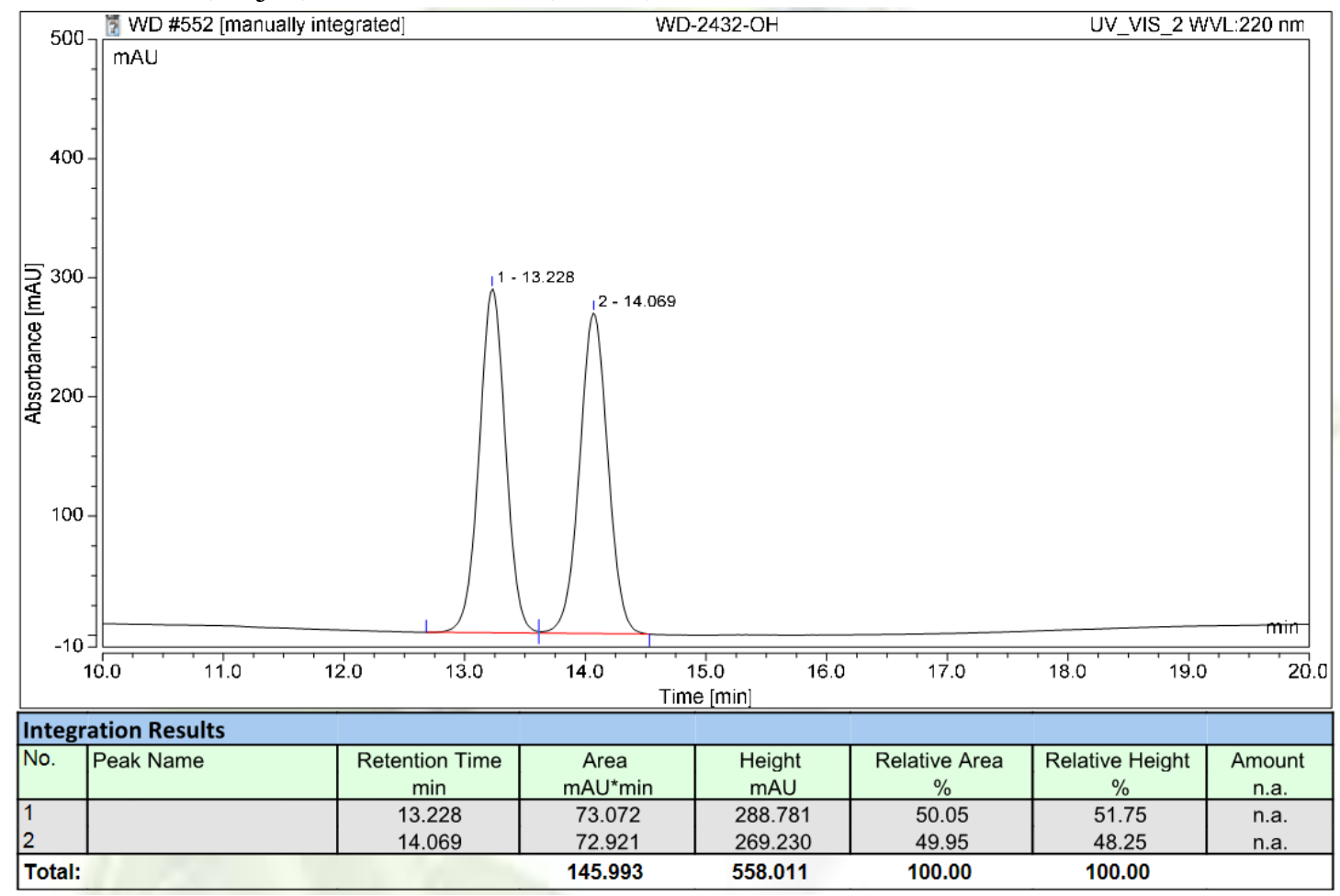




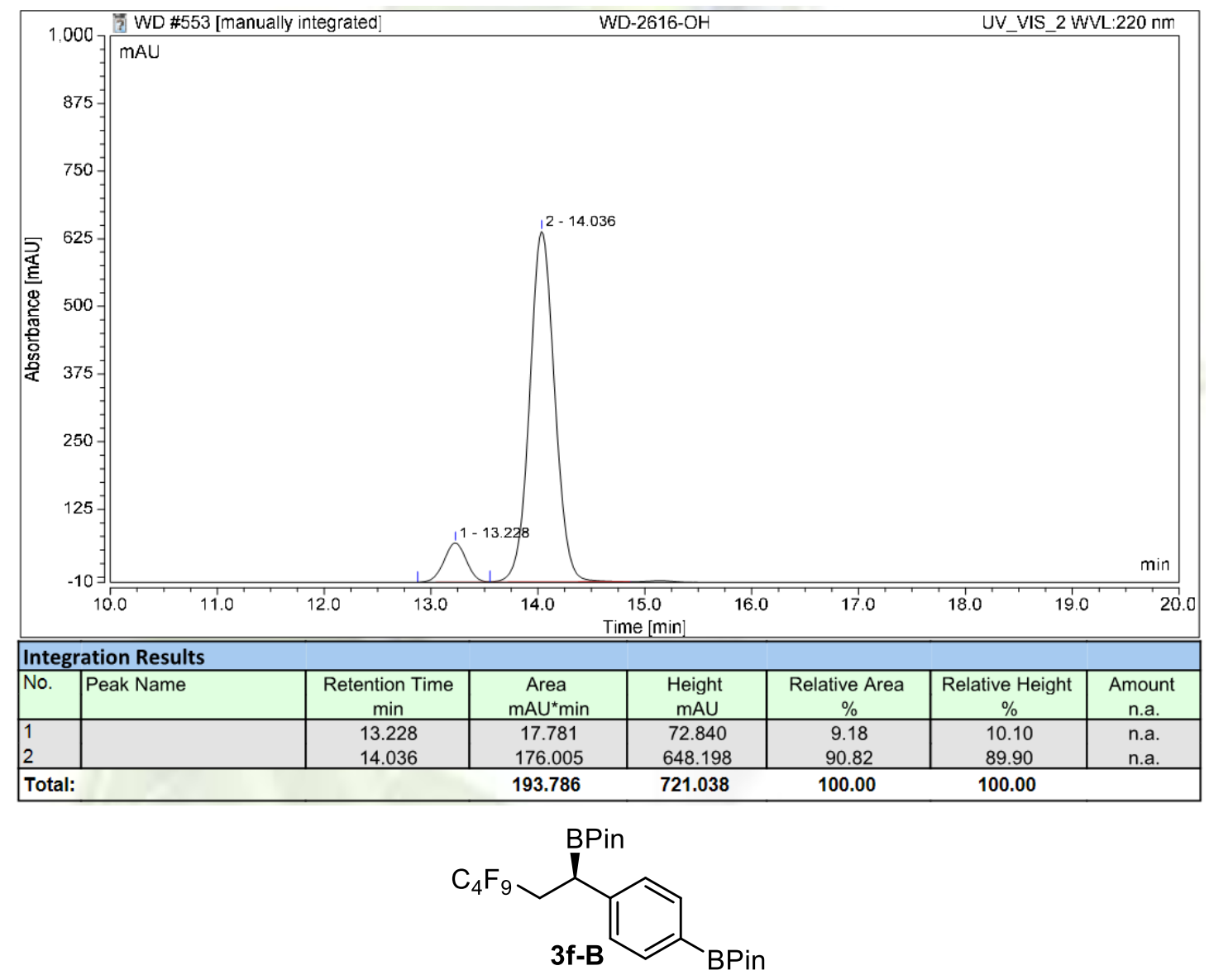

The title compound 3f-B was synthesized according to General Procedure B, and it was purified by column chromatography on silica gel (45\% yield, 92:8 er, $51.9 \mathrm{mg}$, colourless oil).

${ }^{1} \mathbf{H}$ NMR $\left(600 \mathrm{MHz}, \mathrm{CDCl}_{3}\right) \delta 7.73(\mathrm{~d}, J=7.8 \mathrm{~Hz}, 2 \mathrm{H}), 7.24(\mathrm{~d}, J=7.8 \mathrm{~Hz}, 2 \mathrm{H})$, 2.85-2.74 (m, 12H), 2.38-2.27 (m, 1H), $1.34(\mathrm{~s}, 12 \mathrm{H}), 1.18(\mathrm{~s}, 6 \mathrm{H}), 1.14(\mathrm{~s}, 6 \mathrm{H}) ;{ }^{19} \mathbf{F}$ NMR (565 MHz, $\left.\mathrm{CDCl}_{3}\right) \delta$-81.89- -81.04 (m, 3F), -113.55- -113.63 (m, 2F), -124.54-124.59 (m, 2F), -125.94- -126.01. (m, 2F); $\left.{ }^{13} \mathbf{C} \mathbf{~ N M R ~ ( 1 5 1 ~ M H z , ~} \mathrm{CDCl}_{3}\right) \delta$ 143.9, 135.2, 127.4, 120.4-108.1 (m), 84.0, 83.7, 33.3 (t, J = 21.56 Hz), 24.9, 24.8, 24.7, 24.4, 24.3.

HRMS: $\mathrm{m} / \mathrm{z}(\mathrm{ESI})$ calculated $[\mathrm{M}+\mathrm{Na}]^{+}:$577.2345, found: $577.2318 .[\alpha]_{\mathrm{D}}{ }^{25}=-10.878$ $\left(c=0.25, \mathrm{CHCl}_{3}\right)$.

The enantiomeric excess of $\mathbf{3 f}-\mathbf{B}$ was determined by chiral HPLC analysis compared to the corresponding racemate alcohol.

Conditions: ChiralPak IA column; hexane $/ \mathrm{PrOH}=95: 5$; flow rate $=0.7 \mathrm{~mL} / \mathrm{min} ; \lambda=$ $220 \mathrm{~nm} ; \mathrm{tR}_{1}$ (major) $=30.7 \mathrm{~min} ; \mathrm{tR}_{2}$ (minor) $=29.1 \mathrm{~min}$. 

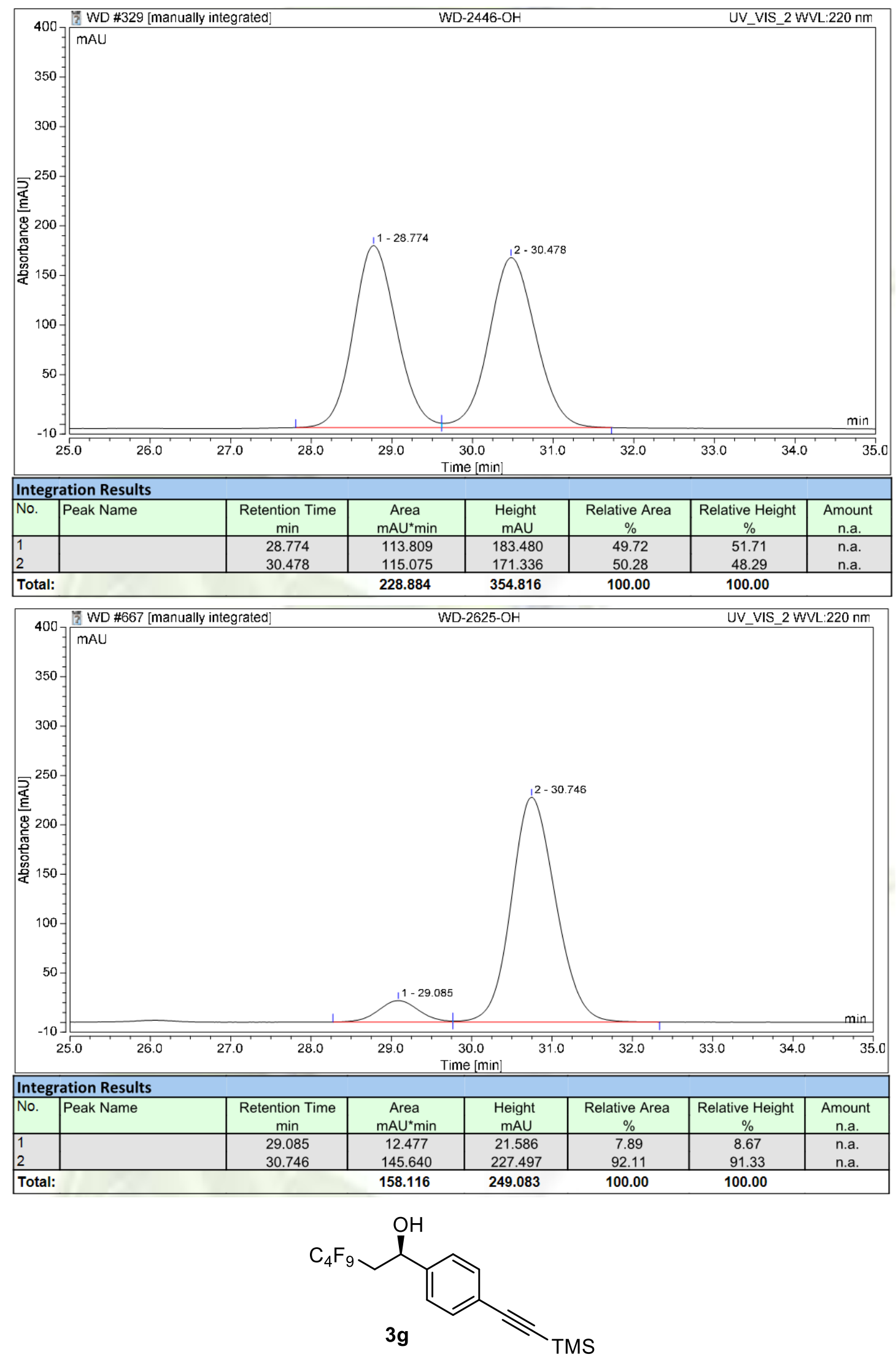

The title compound $\mathbf{3 g}$ was synthesized according to General Procedure B, and it was purified by column chromatography on silica gel (69\% yield, 91:9 er, $60.3 \mathrm{mg}$, colourless oil). 
${ }^{1} \mathbf{H}$ NMR $\left(600 \mathrm{MHz}, \mathrm{CDCl}_{3}\right) \delta 7.48(\mathrm{~d}, J=8.4 \mathrm{~Hz}, 2 \mathrm{H}), 7.32(\mathrm{~d}, J=8.4 \mathrm{~Hz}, 2 \mathrm{H}), 5.23$ -5.20(m, 1H), 2.65-2.54 (m, 1H), 2.43-2.34 (m, 1H), $2.18(\mathrm{~s}, 1 \mathrm{H}), 0.25(\mathrm{~s}, 9 \mathrm{H}) ;{ }^{19} \mathbf{F}$ NMR $\left(565 \mathrm{MHz}, \mathrm{CDCl}_{3}\right) \delta-81.32--81.45(\mathrm{~m}, 3 \mathrm{~F}),-112.64--114.35(\mathrm{~m}, 2 \mathrm{~F})$, -124.79- -124.87 (m, 2F), -126.19- -126.27. (m, 2F); $\left.{ }^{13} \mathbf{C ~ N M R ~ ( 1 5 1 ~ M H z , ~} \mathrm{CDCl}_{3}\right) \delta$ 142.8, 132.5, 125.5, 123.4, 104.4, 94.9, 67.7, 39.8 (t, $J=21.0 \mathrm{~Hz}),-0.1$.

MS: (EI) $[\mathrm{M}]^{+}: 436.15 .[\alpha]_{\mathrm{D}}{ }^{25}=17.750\left(c=0.25, \mathrm{CHCl}_{3}\right)$.

The enantiomeric excess of $\mathbf{3 g}$ was determined by chiral HPLC analysis compared to the corresponding racemate alcohol.

Conditions: ChiralPak IA column; hexane $/{ }^{i} \mathrm{PrOH}=98: 2$; flow rate $=1.0 \mathrm{~mL} / \mathrm{min} ; \lambda=$ $220 \mathrm{~nm} ; \mathrm{t}_{\mathrm{R} 1}($ major $)=14.7 \mathrm{~min} ; \mathrm{t}_{\mathrm{R} 2}($ minor $)=13.7 \mathrm{~min}$. 

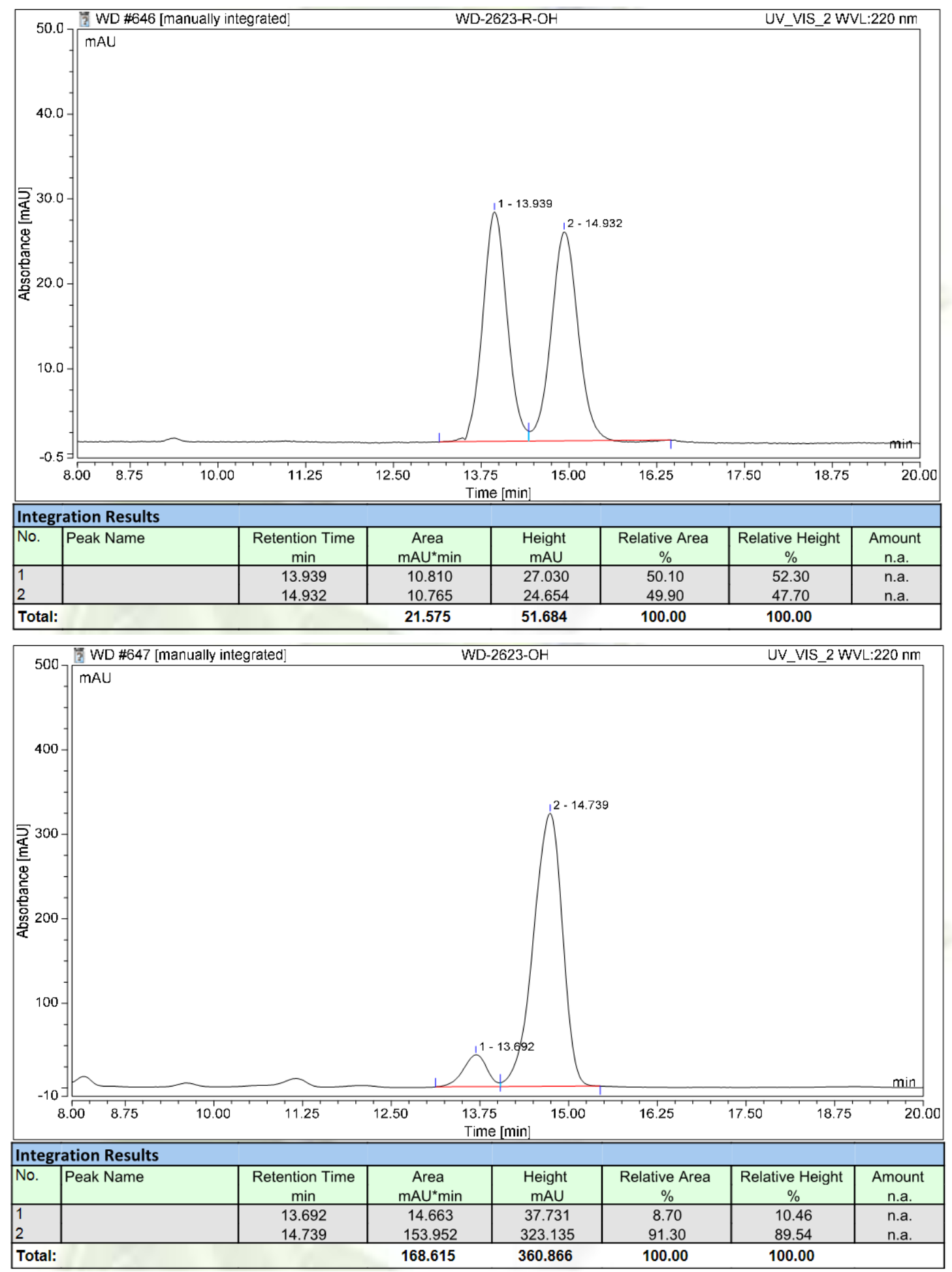<smiles>OC(CC(F)(F)F)c1cccc([Se-])c1</smiles>

$3 \mathrm{~h}$

The title compound $\mathbf{3 h}$ was synthesized according to General Procedure B, and it was purified by column chromatography on silica gel (81\% yield, 92:8 er, $82.7 \mathrm{mg}$, colourless oil). 
${ }^{1} \mathbf{H}$ NMR $\left(600 \mathrm{MHz}, \mathrm{CDCl}_{3}\right) \delta 7.68(\mathrm{~d}, J=7.9 \mathrm{~Hz}, 1 \mathrm{H}), 7.34-7.25(\mathrm{~m}, 2 \mathrm{H}), 6.99(\mathrm{~d}, \mathrm{~J}$ $=7.8 \mathrm{~Hz}, 1 \mathrm{H}), 6.94(\mathrm{~s}, 1 \mathrm{H}), 5.12(\mathrm{dd}, J=8.5,3.1 \mathrm{~Hz}, 1 \mathrm{H}), 2.57-2.39(\mathrm{~m}, 2 \mathrm{H})$, 2.35-2.17 (m, 1H); ${ }^{19} \mathbf{F}$ NMR $\left(565 \mathrm{MHz}, \mathrm{CDCl}_{3}\right) \delta-80.96--81.05(\mathrm{~m}, 3 \mathrm{~F}),-112.47-$ -114.03 (m, 2F), -124.46- -124.55 (m, 2F), -125.87- -125.95. (m, 2F); ${ }^{13}$ C NMR (151 $\left.\mathrm{MHz}, \mathrm{CDCl}_{3}\right) \delta 149.8,145.6,144.6,132.1,130.1,129.7,128.5-106.8(\mathrm{~m}), 124.3$, 122.4, 119.8, 67.1, 39.7 (t, J = 20.64 Hz), 21.6.

HRMS: $\mathrm{m} / \mathrm{z}(\mathrm{ESI})$ calculated $[\mathrm{M}+\mathrm{Na}]^{+}: 528.0620$, found: $528.0610 .[\alpha]_{\mathrm{D}}{ }^{25}=9.432(c$ $\left.=0.25, \mathrm{CHCl}_{3}\right)$.

The enantiomeric excess of $\mathbf{3 h}$ was determined by chiral HPLC analysis compared to the corresponding racemate alcohol.

Conditions: ChiralPak IA column; hexane $/{ }^{i} \mathrm{PrOH}=95: 5$; flow rate $=1.0 \mathrm{~mL} / \mathrm{min} ; \lambda=$ $220 \mathrm{~nm} ; \mathrm{t}_{\mathrm{R} 1}$ (major) $=21.9 \mathrm{~min} ; \mathrm{t}_{\mathrm{R} 2}$ (minor $)=19.7 \mathrm{~min}$.

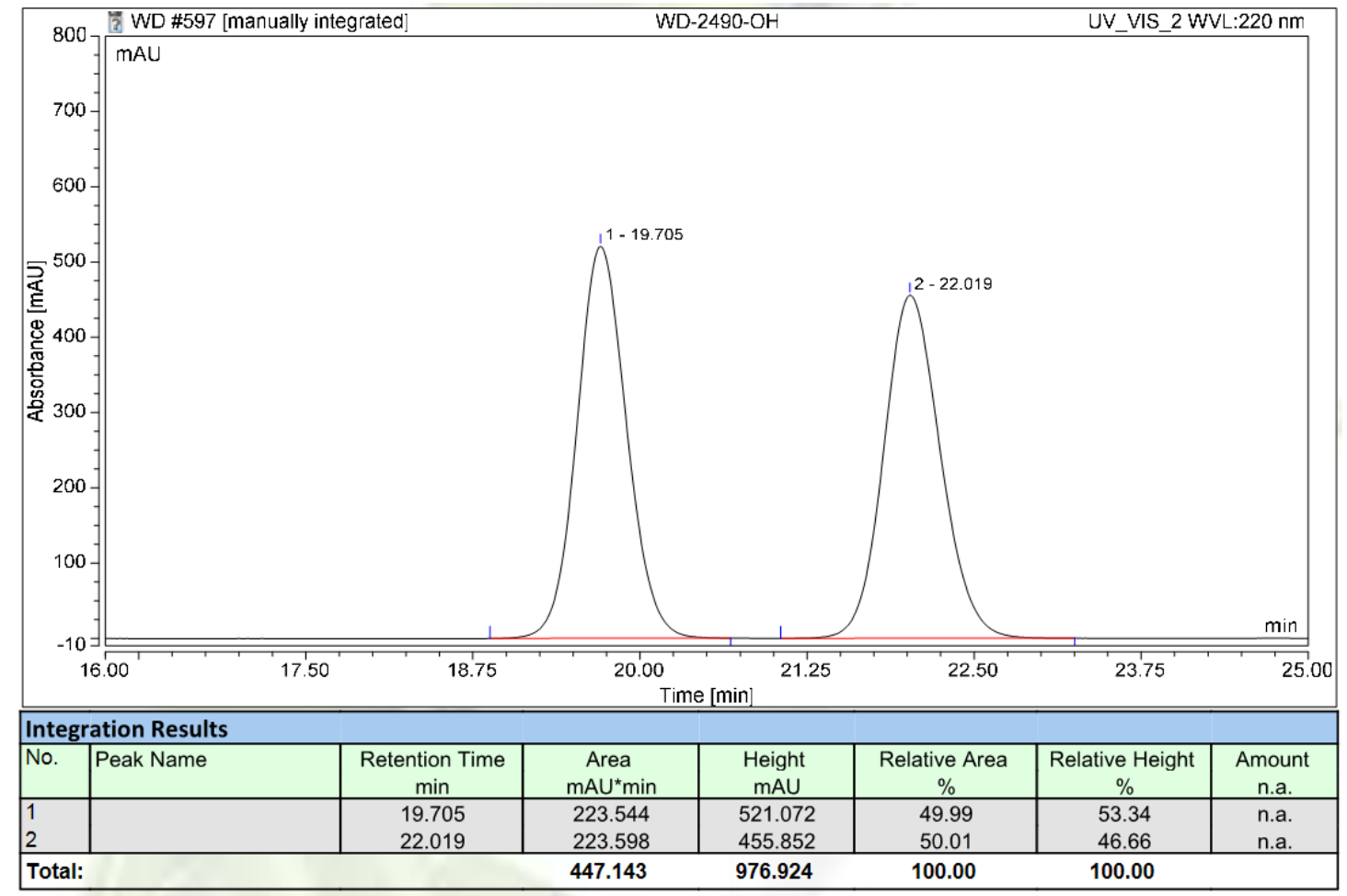




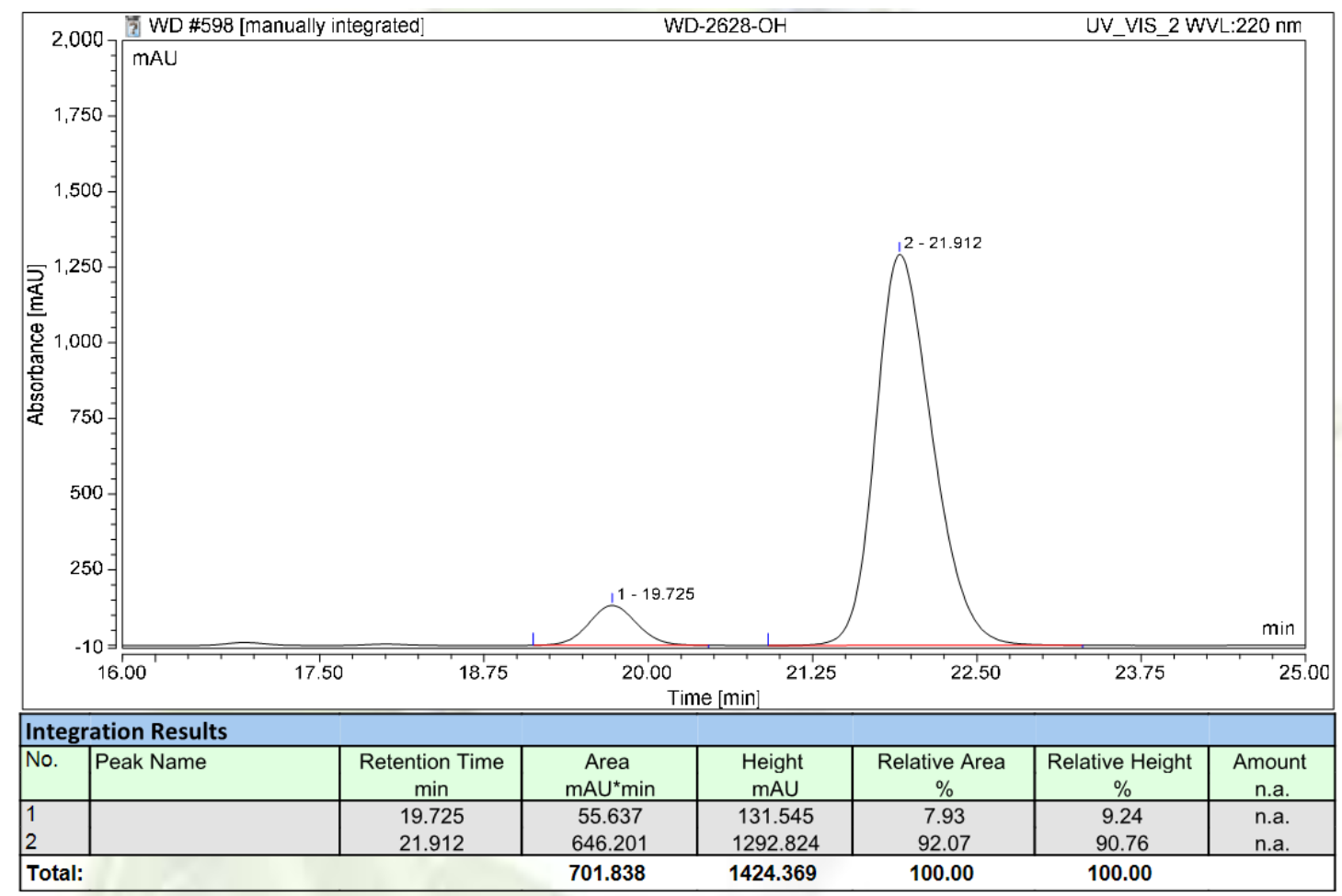<smiles>O=Cc1cccc([C@@H](O)CC(F)(F)F)c1</smiles>

$3 \mathbf{i}$

The title compound $\mathbf{3 i}$ was synthesized according to General Procedure B, and it was purified by column chromatography on silica gel (51\% yield, 90:10 er, $37.6 \mathrm{mg}$, colourless oil).

${ }^{1} \mathbf{H}$ NMR $\left(600 \mathrm{MHz}, \mathrm{CDCl}_{3}\right) \delta 10.0(\mathrm{~s}, 1 \mathrm{H}), 7.94(\mathrm{~s}, 1 \mathrm{H}), 7.87-7.85(\mathrm{~m}, 1 \mathrm{H})$, 7.70-7.69 (m, 1H), $7.58(\mathrm{t}, J=7.2 \mathrm{~Hz}, 1 \mathrm{H}), 5.34(\mathrm{dd}, J=9.0,3.6 \mathrm{~Hz}, 1 \mathrm{H}), 2.71-2.39$ (m, 3H); ${ }^{19}$ F NMR (565 MHz, $\left.\mathrm{CDCl}_{3}\right) \delta$-81.01- -81.09 (m, 3F), -112.38- -114.05 (m, 2F), -124.44- -124.55 (m, 2F), -125.83- -125.97. (m, 2F); ${ }^{13} \mathrm{C}$ NMR (151 MHz, $\left.\mathrm{CDCl}_{3}\right) \delta 191.9,143.7,136.8,131.7,129.9,129.6,126.6,117.6-116.3(\mathrm{~m}), 67.3,39.9$ $(\mathrm{t}, J=20.5 \mathrm{~Hz}), 24.8$.

HRMS: $\mathrm{m} / \mathrm{z}(\mathrm{ESI})$ calculated $[\mathrm{M}+\mathrm{Na}]^{+}: 369.0532$, found: $369.0538 .[\alpha]_{\mathrm{D}}{ }^{25}=12.945(c$ $\left.=0.25, \mathrm{CHCl}_{3}\right)$.

The enantiomeric excess of $\mathbf{3 i}$ was determined by chiral HPLC analysis compared to the corresponding racemate alcohol.

Conditions: ChiralPak IB column; hexane $/{ }^{i} \mathrm{PrOH}=95: 5$; flow rate $=1.0 \mathrm{~mL} / \mathrm{min} ; \lambda=$ $220 \mathrm{~nm} ; \mathrm{t}_{\mathrm{R} 1}$ (major) $=16.0 \mathrm{~min} ; \mathrm{t}_{\mathrm{R} 2}($ minor $)=10.8 \mathrm{~min}$. 

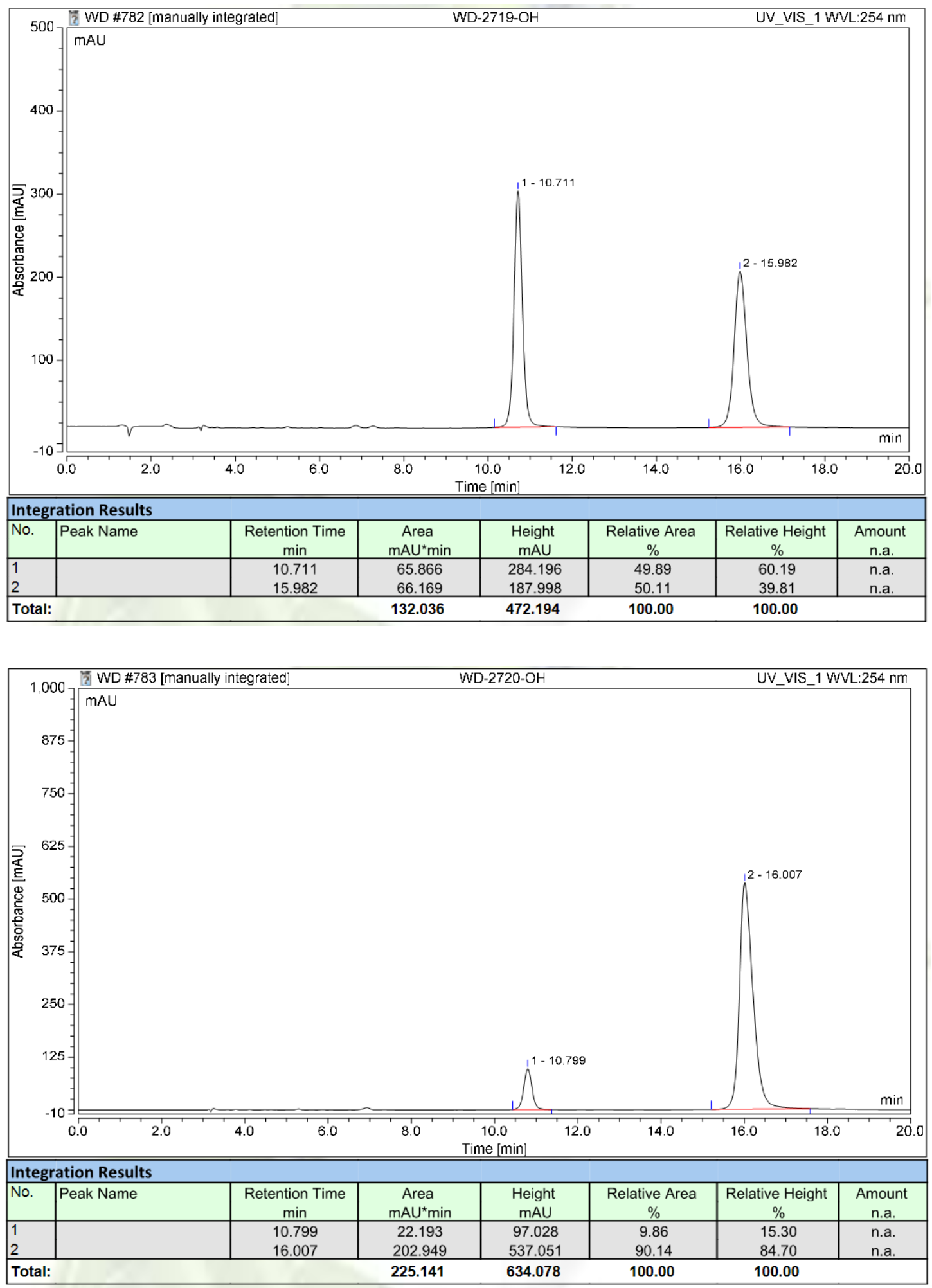<smiles>COC(=O)c1cccc([C@@H](O)CC(F)(F)F)c1</smiles> 
The title compound $\mathbf{3 j}$ was synthesized according to General Procedure B, and it was purified by column chromatography on silica gel (63\% yield, 90:10 er, $50.2 \mathrm{mg}$, colourless oil).

${ }^{1} \mathbf{H}$ NMR $\left(400 \mathrm{MHz}, \mathrm{CDCl}_{3}\right) \delta 8.06(\mathrm{~s}, 1 \mathrm{H}), 8.00(\mathrm{~d}, J=7.6 \mathrm{~Hz}, 1 \mathrm{H}), 7.61(\mathrm{~d}, J=7.6$ $\mathrm{Hz}, 1 \mathrm{H}), 7.47$ (t, $J=7.6 \mathrm{~Hz}, 1 \mathrm{H}), 5.32-5.26(\mathrm{~m}, 1 \mathrm{H}), 3.93$ (s, 3H), 2.75-2.34 (m, 3H); ${ }^{19}$ F NMR $\left(565 \mathrm{MHz}, \mathrm{CDCl}_{3}\right) \delta-81.35--81.42(\mathrm{~m}, 3 \mathrm{~F}),-112.60--114.33(\mathrm{~m}, 2 \mathrm{~F})$, -124.72- -124.82 (m, 2F), -126.21- -126.26. (m, 2F); $\left.{ }^{13} \mathbf{C ~ N M R ~ ( 1 0 1 ~ M H z , ~} \mathrm{CDCl}_{3}\right) \delta$ $166.8,143.1,130.7,130.2,129.5,129.0,126.8,123.0-108.2,67.5,52.3,39.8(\mathrm{t}, \mathrm{J}=$ $20.7 \mathrm{~Hz})$.

HRMS: $\mathrm{m} / \mathrm{z}(\mathrm{ESI})$ calculated $[\mathrm{M}+\mathrm{Na}]^{+}: 416.0903$, found: $416.0902 .[\alpha]_{\mathrm{D}}{ }^{25}=16.670(c$ $\left.=0.25, \mathrm{CHCl}_{3}\right)$.

The enantiomeric excess of $\mathbf{3 j}$ was determined by chiral HPLC analysis compared to the corresponding racemate alcohol.

Conditions: ChiralPak ID column; hexane $/{ }^{i} \mathrm{PrOH}=98: 2$; flow rate $=1.0 \mathrm{~mL} / \mathrm{min} ; \lambda=$ $220 \mathrm{~nm} ; \mathrm{t}_{\mathrm{R} 1}$ (major) $=15.3 \mathrm{~min} ; \mathrm{t}_{\mathrm{R} 2}$ (minor $)=16.3 \mathrm{~min}$.

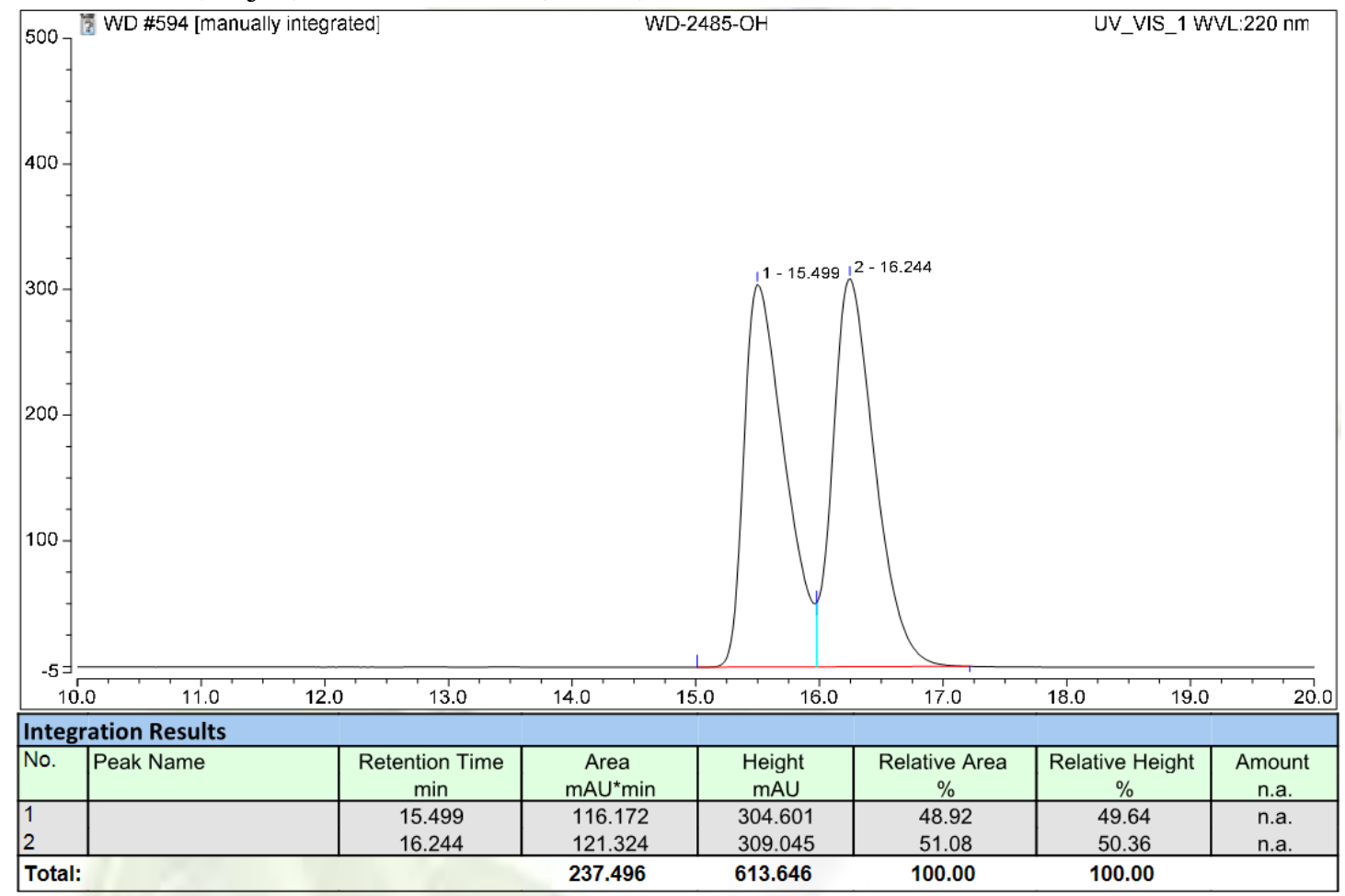




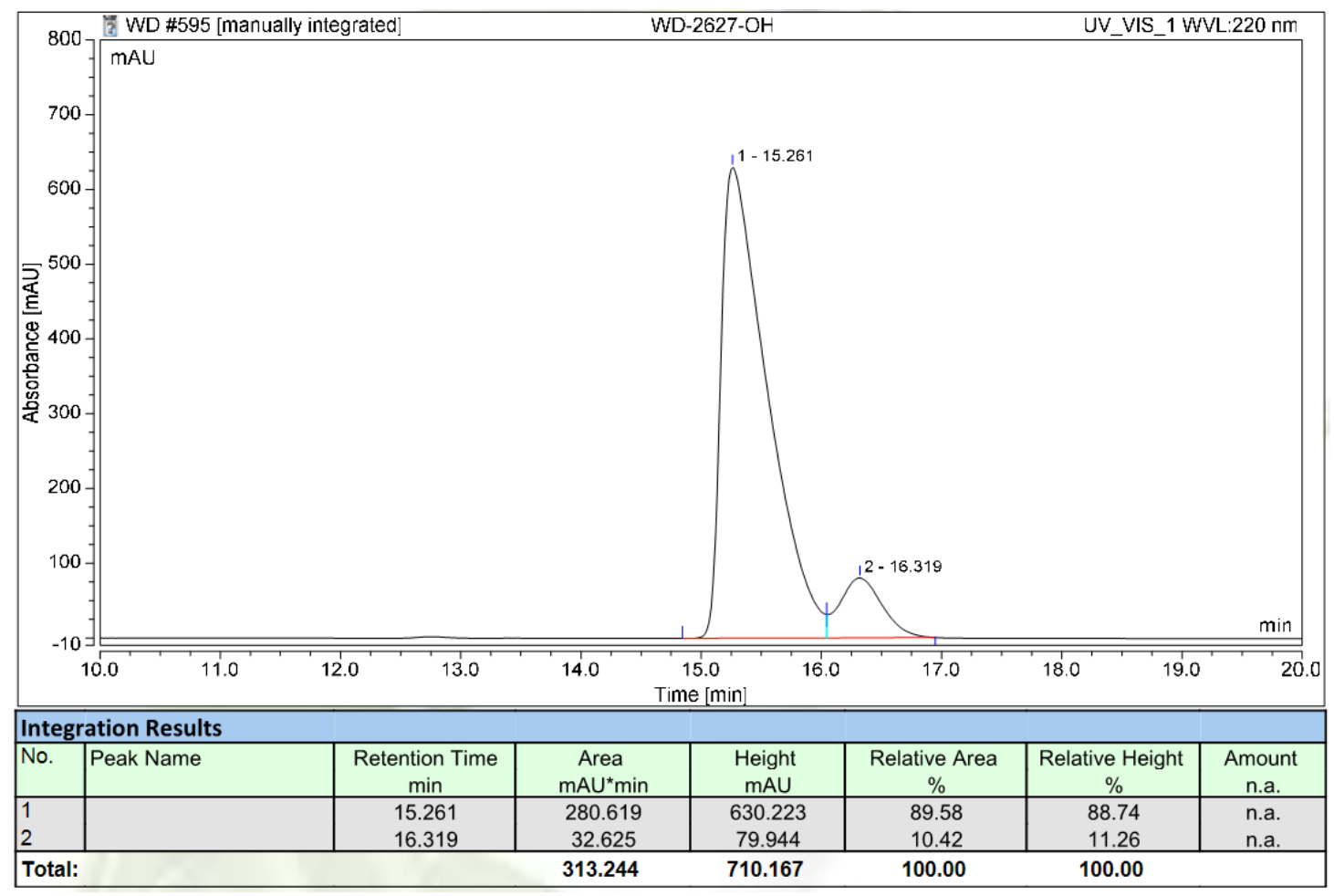<smiles>CC(=O)c1cccc([C@@H](O)CC(F)(F)F)c1</smiles>

The title compound 3k was synthesized according to General Procedure B, and it was purified by column chromatography on silica gel (57\% yield, 88:12 er, $43.6 \mathrm{mg}$, colourless oil).

${ }^{1}$ H NMR $\left(400 \mathrm{MHz}, \mathrm{CDCl}_{3}\right) \delta 8.00(\mathrm{~s}, 1 \mathrm{H}), 7.92(\mathrm{~d}, J=7.6 \mathrm{~Hz}, 1 \mathrm{H}), 7.62(\mathrm{~d}, J=7.6$ $\mathrm{Hz}, 1 \mathrm{H}), 7.51(\mathrm{t}, J=7.7 \mathrm{~Hz}, 1 \mathrm{H}), 5.33-5.30(\mathrm{~m}, 1 \mathrm{H}), 2.73-2.56(\mathrm{~m}, 4 \mathrm{H}), 2.50-2.34(\mathrm{~m}$, $2 \mathrm{H}) ;{ }^{19} \mathbf{F}$ NMR $\left(565 \mathrm{MHz}, \mathrm{CDCl}_{3}\right) \delta-81.55--81.77$ (m, 3F), -112.83- -114.38 (m, 2F), -124.88- -125.05 (m, 2F), -126.32- -126.55. (m, 2F); ${ }^{13} \mathbf{C}$ NMR (101 MHz, $\left.\mathrm{CDCl}_{3}\right) \delta$ 197.8, 143.2, 137.7, 130.3, 129.2, 128.4, 125.4, 70.5, 67.6, 39.9 (t, $J=20.7 \mathrm{~Hz}), 26.7$. HRMS: $\mathrm{m} / \mathrm{z}(\mathrm{ESI})$ calculated $[\mathrm{M}+\mathrm{Na}]^{+}: 400.0954$, found: $400.0952 .[\alpha]_{\mathrm{D}}{ }^{25}=18.176(c$ $\left.=0.25, \mathrm{CHCl}_{3}\right)$.

The enantiomeric excess of $\mathbf{3 k}$ was determined by chiral HPLC analysis compared to the corresponding racemate alcohol.

Conditions: ChiralPak ID column; hexane $/{ }^{i} \mathrm{PrOH}=95: 5$; flow rate $=1.0 \mathrm{~mL} / \mathrm{min} ; \lambda=$ $220 \mathrm{~nm} ; \mathrm{t}_{\mathrm{R} 1}($ major $)=7.9 \mathrm{~min} ; \mathrm{t}_{\mathrm{R} 2}($ minor $)=8.6 \mathrm{~min}$. 

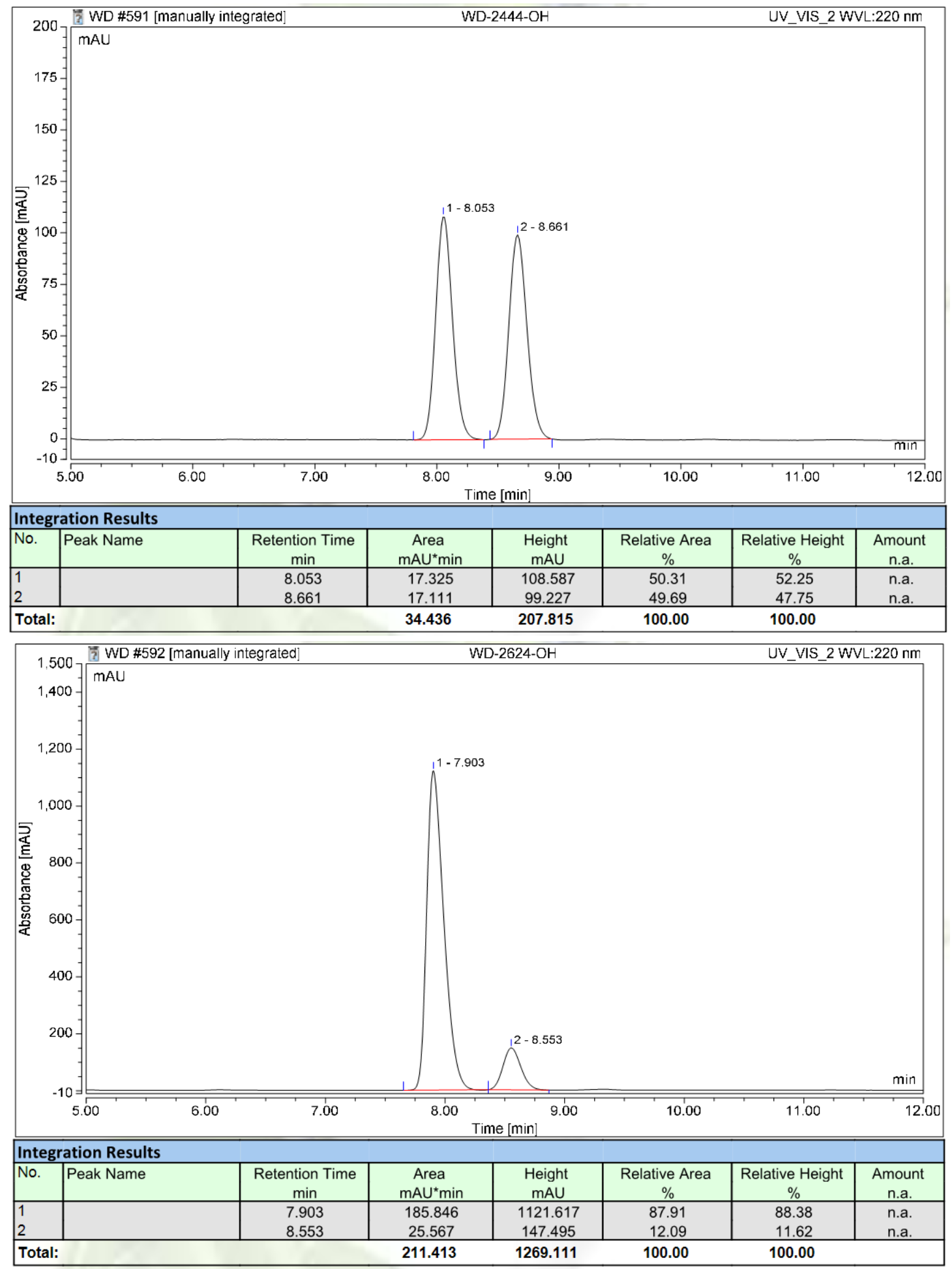<smiles>Cc1ccccc1[C@@H](O)CC(F)(F)F</smiles>

The title compound $\mathbf{3 1}$ was synthesized according to General Procedure B, and it was purified by column chromatography on silica gel (72\% yield, 95:5 er, $51.0 \mathrm{mg}$, colourless oil). 
${ }^{1} \mathbf{H}$ NMR $\left(600 \mathrm{MHz}, \mathrm{CDCl}_{3}\right) \delta 7.53(\mathrm{~d}, J=7.2 \mathrm{~Hz}, 1 \mathrm{H}), 7.27(\mathrm{t}, J=7.2 \mathrm{~Hz}, 1 \mathrm{H}), 7.22$ $(\mathrm{t}, J=7.2 \mathrm{~Hz}, 1 \mathrm{H}), 7.16(\mathrm{~d}, J=7.2 \mathrm{~Hz}, 1 \mathrm{H}), 5.49-5.42(\mathrm{~m}, 1 \mathrm{H}), 2.60-2.50(\mathrm{~m}, 1 \mathrm{H})$, 2.39- $2.29(\mathrm{~m}, 4 \mathrm{H}), 2.18(\mathrm{~m}, 1 \mathrm{H}) ;{ }^{19} \mathbf{F}$ NMR $\left(565 \mathrm{MHz}, \mathrm{CDCl}_{3}\right) \delta$-81.01- -81.06 (m, 3F), -112.86- -114.60 (m, 2F), -124.52- -124.62 (m, 2F), -125.84- -125.95. (m, 2F); ${ }^{13} \mathrm{C}$ NMR $\left(151 \mathrm{MHz}, \mathrm{CDCl}_{3}\right) \delta 140.8,133.9,130.8,128.1,126.7,125.1,120.5-106.6$ (m), 64.4, 38.9 (t, J = 20.7 Hz), 18.7.

HRMS: $\mathrm{m} / \mathrm{z}(\mathrm{ESI})$ calculated $[\mathrm{M}+\mathrm{Na}]^{+}: 377.0558$, found: $377.0564 .[\alpha]_{\mathrm{D}}{ }^{25}=38.247(c$ $\left.=0.25, \mathrm{CHCl}_{3}\right)$.

The enantiomeric excess of $\mathbf{3 l}$ was determined by chiral HPLC analysis compared to the corresponding racemate alcohol.

Conditions: ChiralPak IB column; hexane $/{ }^{i} \mathrm{PrOH}=98: 2$; flow rate $=1.0 \mathrm{~mL} / \mathrm{min} ; \lambda=$ $220 \mathrm{~nm} ; \mathrm{t}_{\mathrm{R} 1}$ (major) $=12.6 \mathrm{~min} ; \mathrm{t}_{\mathrm{R} 2}($ minor $)=10.1 \mathrm{~min}$. 

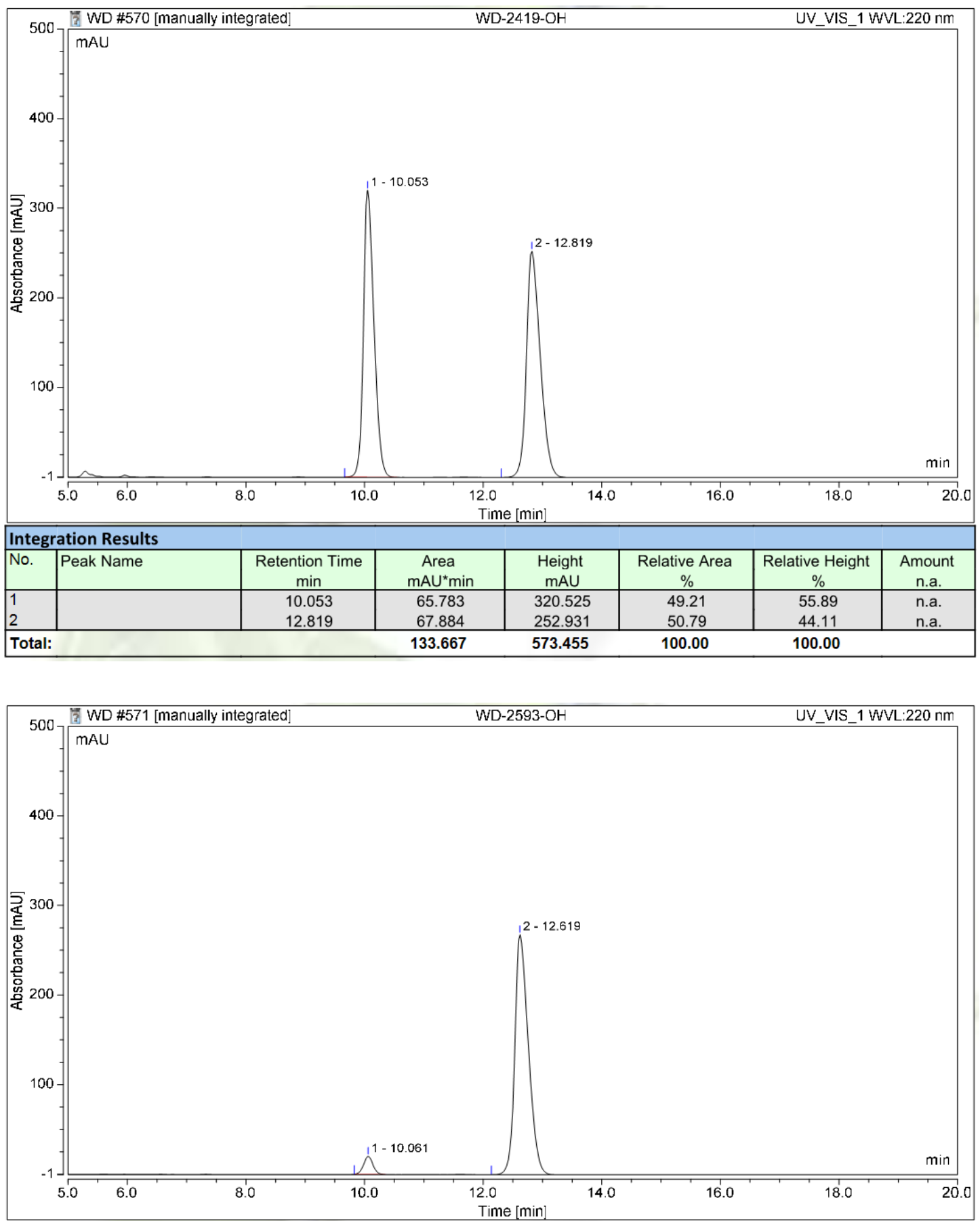

\begin{tabular}{|l|l|c|c|c|c|c|c|}
\hline \multicolumn{2}{|l|}{ Integration Results } \\
\hline No. & Peak Name & $\begin{array}{c}\text { Retention Time } \\
\text { min }\end{array}$ & $\begin{array}{c}\text { Area } \\
\text { mAU*min }\end{array}$ & $\begin{array}{c}\text { Height } \\
\text { mAU }\end{array}$ & $\begin{array}{c}\text { Relative Area } \\
\%\end{array}$ & $\begin{array}{c}\text { Relative Height } \\
\%\end{array}$ & $\begin{array}{c}\text { Amount } \\
\text { n.a. }\end{array}$ \\
\hline 1 & & 10.061 & 3.714 & 20.199 & 4.98 & 7.00 & n.a. \\
2 & 12.619 & 70.836 & 268.184 & 95.02 & 93.00 & n.a. \\
\hline
\end{tabular}<smiles>COc1ccccc1[C@@H](O)CC(F)(F)F</smiles>

The title compound $\mathbf{3 m}$ was synthesized according to General Procedure B, and it was purified by column chromatography on silica gel (64\% yield, 87:13 er, $47.4 \mathrm{mg}$, colourless oil). 
${ }^{1} \mathbf{H}$ NMR $\left(600 \mathrm{MHz}, \mathrm{CDCl}_{3}\right) \delta 7.39(\mathrm{~d}, J=7.2 \mathrm{~Hz}, 1 \mathrm{H}), 7.30(\mathrm{t}, J=7.8 \mathrm{~Hz}, 1 \mathrm{H}), 7.00$ (t, $J=7.2 \mathrm{~Hz}, 1 \mathrm{H}), 6.91(\mathrm{~d}, J=8.4 \mathrm{~Hz}, 1 \mathrm{H}), 5.39-5.35(\mathrm{~m}, 1 \mathrm{H}), 3.88(\mathrm{~s}, 3 \mathrm{H}), 2.75(\mathrm{~d}$, $J=6.6 \mathrm{~Hz}, 1 \mathrm{H}), 2.64-2.50(\mathrm{~m}, 2 \mathrm{H}) ;{ }^{19} \mathbf{F}$ NMR $\left(565 \mathrm{MHz}, \mathrm{CDCl}_{3}\right) \delta-81.04--81.12(\mathrm{~m}$, 3F), -112.20- -115.02 (m, 2F), -124.61- -124.67 (m, 2F), -125.90- -125.99. (m, 2F); ${ }^{13} \mathrm{C}$ NMR $\left(151 \mathrm{MHz}, \mathrm{CDCl}_{3}\right) \delta 156.0,130.3,129.2,126.9,121.0,120.3-107.0(\mathrm{~m})$, 110.6, 64.9, 55.3, $38.0(\mathrm{t}, J=20.5 \mathrm{~Hz})$.

HRMS: $\mathrm{m} / \mathrm{z}(\mathrm{ESI})$ calculated $[\mathrm{M}+\mathrm{Na}]^{+}: 393.0508$, found: $393.0514 .[\alpha]_{\mathrm{D}}{ }^{25}=16.710(c$ $\left.=0.25, \mathrm{CHCl}_{3}\right)$.

The enantiomeric excess of $\mathbf{3 m}$ was determined by chiral HPLC analysis compared to the corresponding racemate alcohol.

Conditions: ChiralPak IB column; hexane $/{ }^{i} \mathrm{PrOH}=98: 2$; flow rate $=1.0 \mathrm{~mL} / \mathrm{min} ; \lambda=$ $220 \mathrm{~nm} ; \mathrm{t}_{\mathrm{R} 1}($ major $)=10.1 \mathrm{~min} ; \mathrm{t}_{\mathrm{R} 2}($ minor $)=9.1 \mathrm{~min}$. 

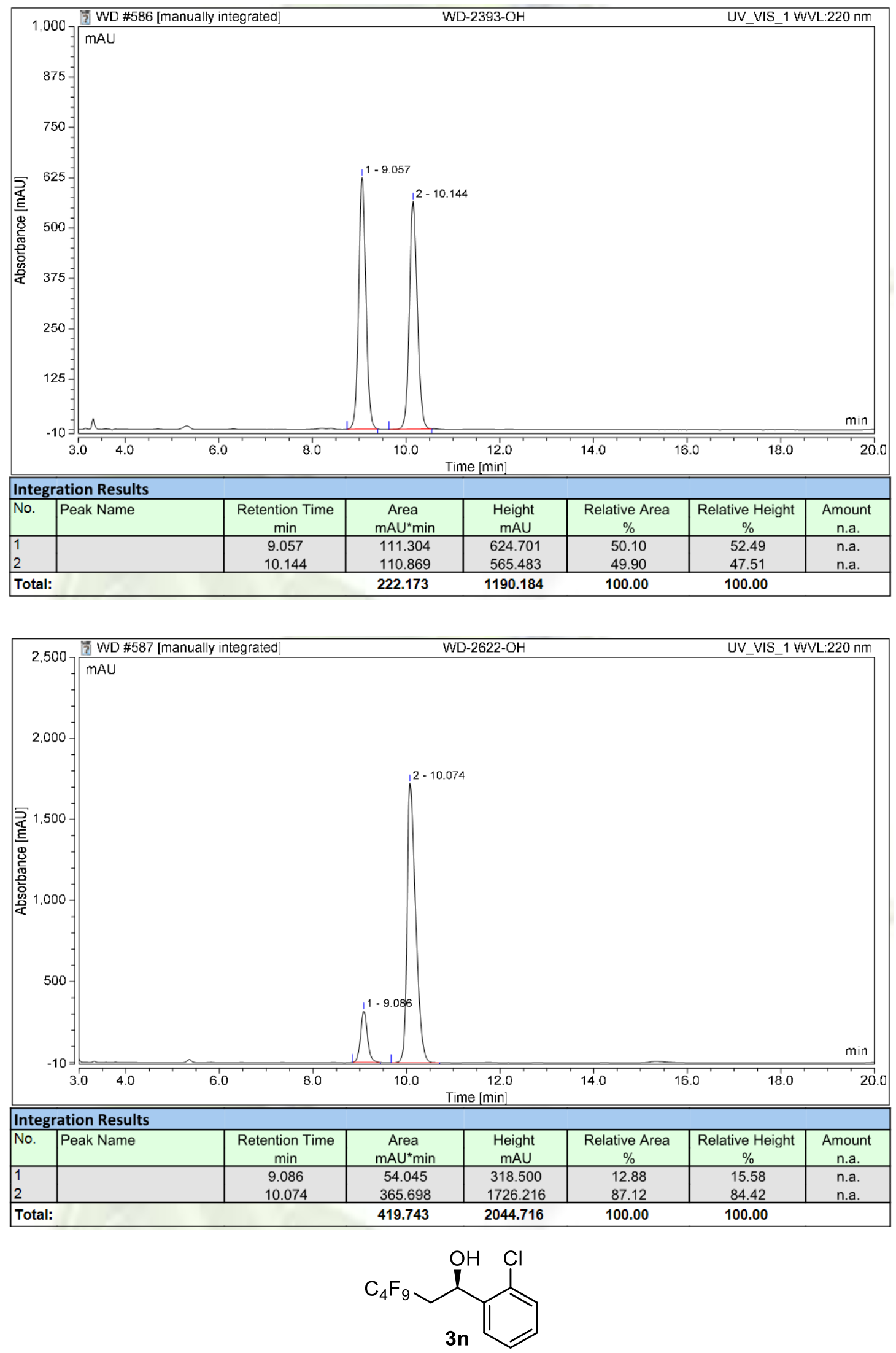

The title compound 3n was synthesized according to General Procedure B, and it was purified by column chromatography on silica gel (34\% yield, 94:6 er, $25.5 \mathrm{mg}$, colourless oil). 
${ }^{1} \mathbf{H}$ NMR $\left(600 \mathrm{MHz}, \mathrm{CDCl}_{3}\right) \delta 7.66(\mathrm{dd}, J=7.8,1.2 \mathrm{~Hz}, 1 \mathrm{H}), 7.38-7.33(\mathrm{~m}, 2 \mathrm{H}), 7.29$ $-7.26(\mathrm{~m}, 1 \mathrm{H}), 5.65(\mathrm{dt}, J=9.0,2.4 \mathrm{~Hz}, 1 \mathrm{H}), 2.58-2.40(\mathrm{~m}, 2 \mathrm{H}), 2.35-2.33(\mathrm{~m}, 1 \mathrm{H})$; ${ }^{19}$ F NMR $\left(565 \mathrm{MHz}, \mathrm{CDCl}_{3}\right) \delta-80.83--81.19(\mathrm{~m}, 3 \mathrm{~F}),-112.03--114.93(\mathrm{~m}, 2 \mathrm{~F})$, -124.54- -124.59 (m, 2F), -125.81- -125.92. (m, 2F); $\left.{ }^{13} \mathbf{C ~ N M R ~ ( 1 5 1 ~ M H z , ~} \mathrm{CDCl}_{3}\right) \delta$ 139.8, 131.2, 129.7, 129.3, 127.5, 127.0, 120.2-108.4, 64.7, $38.3(\mathrm{t}, J=20.5 \mathrm{~Hz})$.

HRMS: $\mathrm{m} / \mathrm{z}(\mathrm{ESI})$ calculated $[\mathrm{M}+\mathrm{Na}]^{+}: 397.0012$, found: $397.0011 .[\alpha]_{\mathrm{D}}{ }^{25}=24.003(c$ $\left.=0.25, \mathrm{CHCl}_{3}\right)$.

The enantiomeric excess of $\mathbf{3 n}$ was determined by chiral HPLC analysis compared to the corresponding racemate alcohol.

Conditions: ChiralPak IB column; hexane $/{ }^{i} \mathrm{PrOH}=98: 52$; flow rate $=1.0 \mathrm{~mL} / \mathrm{min} ; \lambda=$ $220 \mathrm{~nm} ; \mathrm{t}_{\mathrm{R} 1}($ major $)=8.1 \mathrm{~min} ; \mathrm{t}_{\mathrm{R} 2}($ minor $)=7.6 \mathrm{~min}$. 

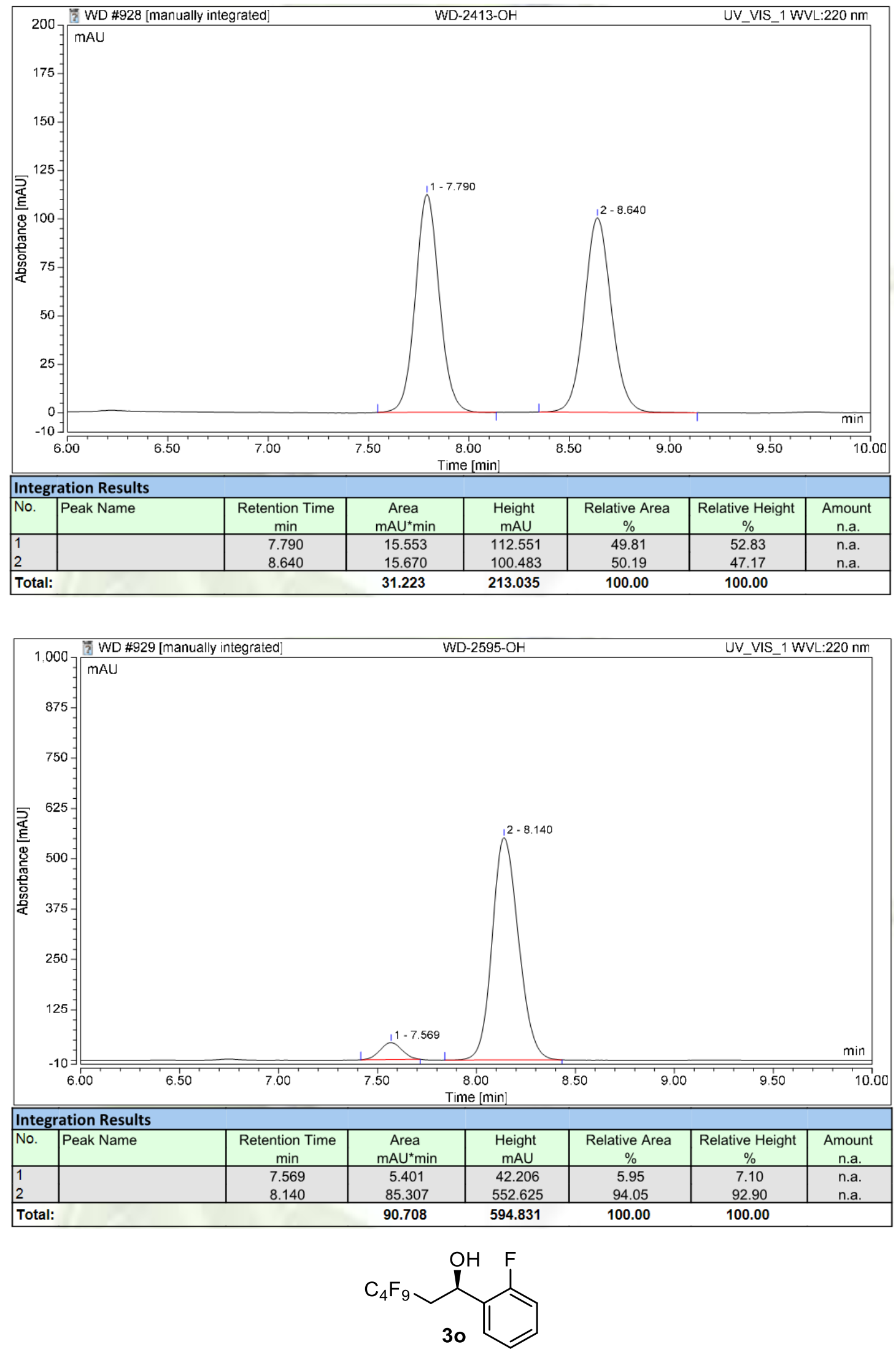
The title compound $\mathbf{3 o}$ was synthesized according to General Procedure B, and it was purified by column chromatography on silica gel (67\% yield, 90:10 er, $47.9 \mathrm{mg}$, colourless oil).

${ }^{1} \mathbf{H}$ NMR $\left(600 \mathrm{MHz}, \mathrm{CDCl}_{3}\right) \delta 7.53(\mathrm{td}, J=7.2,1.2 \mathrm{~Hz}, 1 \mathrm{H}), 7.34-7.30(\mathrm{~m}, 1 \mathrm{H}), 7.20$ (t, $J=7.2 \mathrm{~Hz}, 1 \mathrm{H}), 7.09-7.05(\mathrm{~m}, 1 \mathrm{H}), 5.54-5.51(\mathrm{~m}, 1 \mathrm{H}), 2.67-2.47(\mathrm{~m}, 2 \mathrm{H}), 2.26(\mathrm{~d}$, $J=4.2 \mathrm{~Hz}, 1 \mathrm{H}) ;{ }^{19} \mathbf{F}$ NMR $\left(565 \mathrm{MHz}, \mathrm{CDCl}_{3}\right) \delta-81.43--81.71(\mathrm{~m}, 3 \mathrm{~F}),-112.57-$ -115.49 (m, 2F), -119.80- -120.07(m, 1F), -124.78- -125.14 (m, 2F), -126.25- -126.51. $(\mathrm{m}, 2 \mathrm{~F}) ;{ }^{13} \mathbf{C}$ NMR $\left(151 \mathrm{MHz}, \mathrm{CDCl}_{3}\right) \delta 159.3(\mathrm{~d}, J=246.3 \mathrm{~Hz}), 146.8-103.3(\mathrm{~m})$, 129.9 (d, $J=8.2 \mathrm{~Hz}), 129.46(\mathrm{~d}, \mathrm{~J}=13.0 \mathrm{~Hz}), 127.23$ (d, J = 3.8 Hz), 124.65 (d, J = $3.5 \mathrm{~Hz}), 115.64(\mathrm{~d}, J=21.1 \mathrm{~Hz}), 62.4,38.7(\mathrm{t}, J=20.7 \mathrm{~Hz})$.

HRMS: $\mathrm{m} / \mathrm{z}(\mathrm{ESI})$ calculated $[\mathrm{M}+\mathrm{Na}]^{+}: 381.0808$, found: $381.0330 .[\alpha]_{\mathrm{D}}{ }^{25}=11.029(c$ $\left.=0.25, \mathrm{CHCl}_{3}\right)$.

The enantiomeric excess of 3o was determined by chiral HPLC analysis compared to the corresponding racemate alcohol.

Conditions: ChiralPak IA column; hexane $/{ }^{i} \mathrm{PrOH}=99: 1$; flow rate $=1.0 \mathrm{~mL} / \mathrm{min} ; \lambda=$ $220 \mathrm{~nm} ; \mathrm{t}_{\mathrm{R} 1}$ (major) $=20.8 \mathrm{~min} ; \mathrm{t}_{\mathrm{R} 2}($ minor $)=19.6 \mathrm{~min}$. 

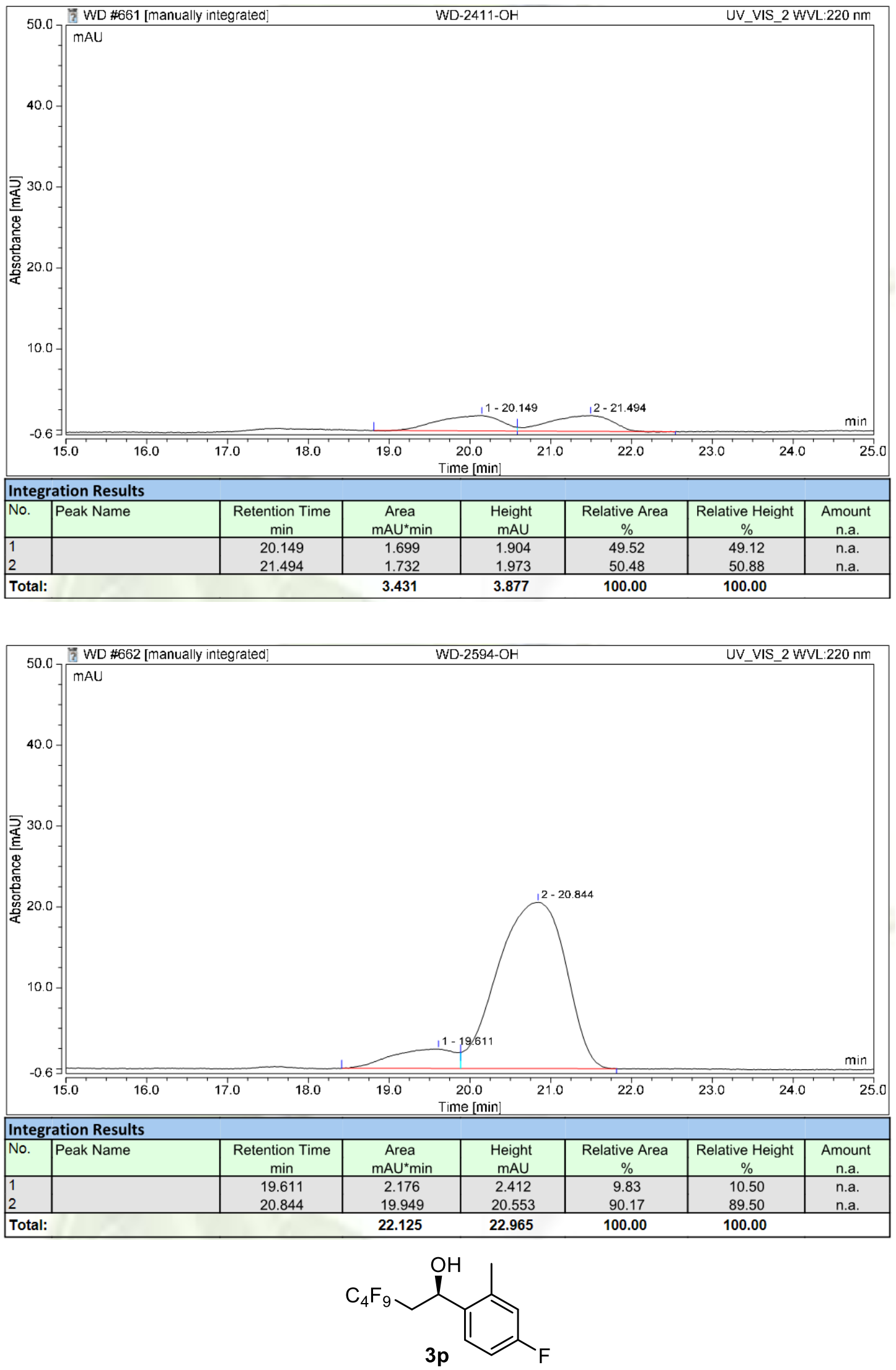

The title compound $\mathbf{3 p}$ was synthesized according to General Procedure B, and it was purified by column chromatography on silica gel (69\% yield, 96:4 er, $51.4 \mathrm{mg}$, colourless oil). 
${ }^{1} \mathbf{H}$ NMR $\left(600 \mathrm{MHz}, \mathrm{CDCl}_{3}\right) \delta 7.50(\mathrm{dd}, J=8.4,5.4 \mathrm{~Hz}, 1 \mathrm{H}), 6.95(\mathrm{td}, J=8.4,2.4 \mathrm{~Hz}$, $1 \mathrm{H}), 6.88$ (dd, $J=9.6,2.4 \mathrm{~Hz}, 1 \mathrm{H}), 5.43$ (dt, $J=9.0,2.4 \mathrm{~Hz}, 1 \mathrm{H}), 2.60-2.50(\mathrm{~m}, 1 \mathrm{H})$, 2.37-2.27 (m, 4H), $2.16(\mathrm{~s}, 1 \mathrm{H}) ;{ }^{19} \mathbf{F}$ NMR $\left(565 \mathrm{MHz}, \mathrm{CDCl}_{3}\right) \delta-81.01--81.06(\mathrm{~m}$, $3 \mathrm{~F}),-112.89--114.53(\mathrm{~m}, 2 \mathrm{~F}),-114.78(\mathrm{~s}, 1 \mathrm{~F}),-124.53--124.59(\mathrm{~m}, 2 \mathrm{~F}),-159.84-$ -125.96. (m, 2F); ${ }^{13} \mathrm{C}$ NMR (151 MHz, CDCl3) $\delta 162.2(\mathrm{~d}, \mathrm{~J}=246.6 \mathrm{~Hz}), 136.5$ (d, $J$ $=3.0 \mathrm{~Hz}), 136.4(\mathrm{~d}, J=7.9 \mathrm{~Hz}), 127.1(\mathrm{~d}, J=8.6 \mathrm{~Hz}), 117.3(\mathrm{~d}, J=21.1 \mathrm{~Hz}), 130.1-$ $108.6(\mathrm{~m}), 113.4(\mathrm{~d}, J=21.1 \mathrm{~Hz}), 63.8,39.1(\mathrm{t}, J=20.4 \mathrm{~Hz}), 35.9-14.0(\mathrm{~m}), 18.8$.

HRMS: $\mathrm{m} / \mathrm{z}(\mathrm{ESI})$ calculated $[\mathrm{M}+\mathrm{Na}]^{+}: 395.0464$, found: $395.0502 .[\alpha]_{\mathrm{D}}{ }^{25}=36.679(c$ $\left.=0.25, \mathrm{CHCl}_{3}\right)$.

The enantiomeric excess of $\mathbf{3 p}$ was determined by chiral HPLC analysis compared to the corresponding racemate alcohol.

Conditions: ChiralPak IB column; hexane $/{ }^{i} \mathrm{PrOH}=98: 2$; flow rate $=1.0 \mathrm{~mL} / \mathrm{min} ; \lambda=$ $220 \mathrm{~nm} ; \mathrm{t}_{\mathrm{R} 1}$ (major) $=11.2 \mathrm{~min} ; \mathrm{t}_{\mathrm{R} 2}$ (minor) $=8.5 \mathrm{~min}$. 

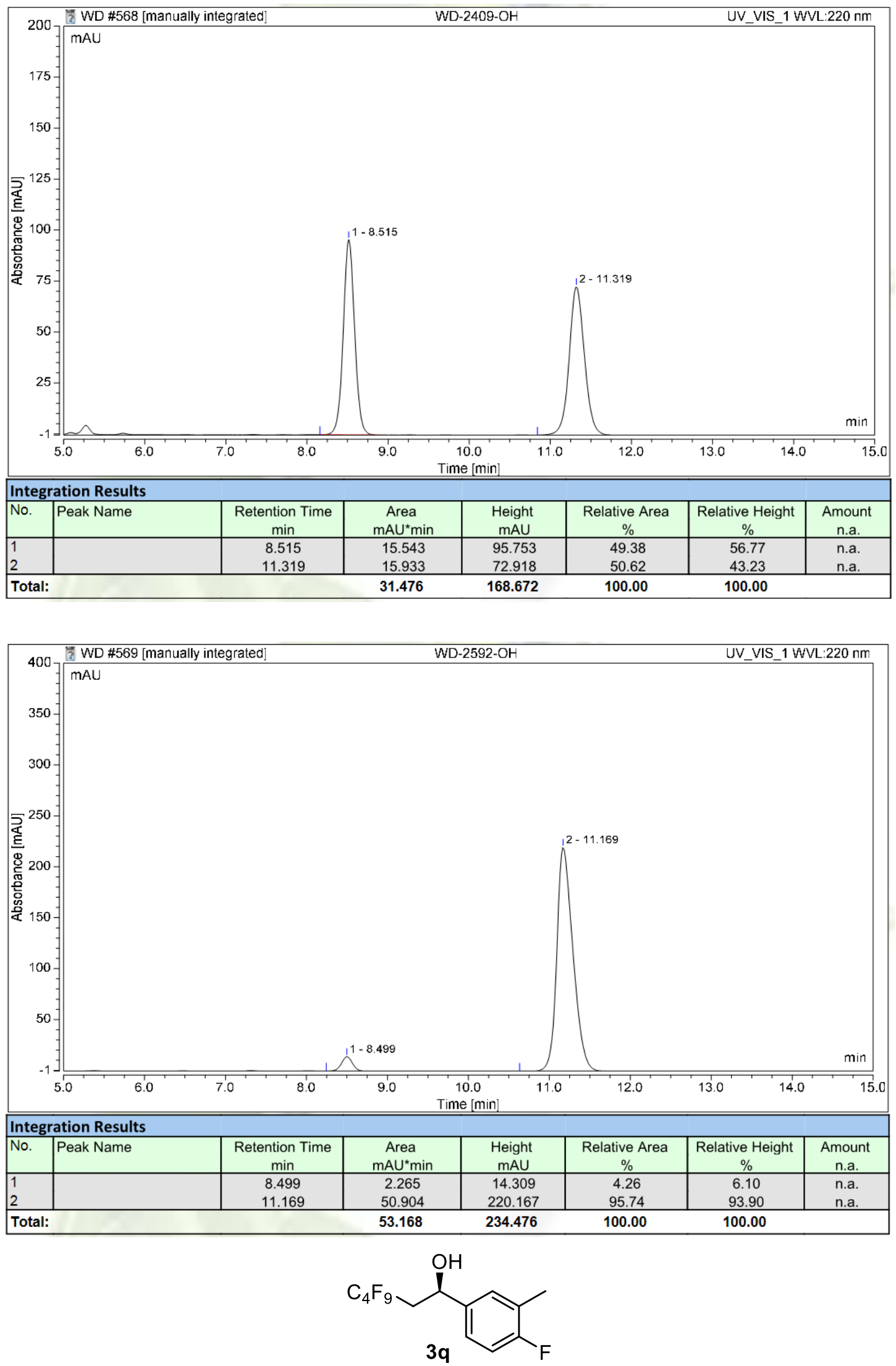

The title compound $\mathbf{3 q}$ was synthesized according to General Procedure B, and it was purified by column chromatography on silica gel (75\% yield, 90:10 er, $55.9 \mathrm{mg}$, colourless oil). 
${ }^{1} \mathbf{H}$ NMR $\left(600 \mathrm{MHz}, \mathrm{CDCl}_{3}\right) \delta 7.20(\mathrm{~d}, J=7.2 \mathrm{~Hz}, 1 \mathrm{H}), 7.17-7.14(\mathrm{~m}, 1 \mathrm{H}), 7.00(\mathrm{t}, J$ $=9.0 \mathrm{~Hz}, 1 \mathrm{H}), 5.15(\mathrm{dt}, J=8.4,3.0 \mathrm{~Hz}, 1 \mathrm{H}), 2.64-2.53(\mathrm{~m}, 1 \mathrm{H}), 2.42-2.32(\mathrm{~m}, 1 \mathrm{H})$, $2.29(\mathrm{~s}, 3 \mathrm{H}), 2.27(\mathrm{~s}, 1 \mathrm{H}) ;{ }^{19} \mathbf{F}$ NMR $\left(565 \mathrm{MHz}, \mathrm{CDCl}_{3}\right) \delta-81.03--81.14(\mathrm{~m}, 3 \mathrm{~F})$, -112.51- -114.34 (m, 2F), -117.87 (s, 1F), -124.55- -124.64 (m, 2F), -125.90- -126.01. $(\mathrm{m}, 2 \mathrm{~F}) ;{ }^{13} \mathbf{C}$ NMR $\left(151 \mathrm{MHz}, \mathrm{CDCl}_{3}\right) \delta 161.1(\mathrm{~d}, J=245.8 \mathrm{~Hz}), 138.1(\mathrm{~d}, J=3.5 \mathrm{~Hz})$, $128.8(\mathrm{~d}, J=5.4 \mathrm{~Hz}), 125.4$ (d, $J=17.7 \mathrm{~Hz}), 124.6$ (d, $J=8.3 \mathrm{~Hz}), 115.3$ (d, $J=22.7$ $\mathrm{Hz}), 119.6-100.0(\mathrm{~m}), 67.4,39.8(\mathrm{t}, J=20.4 \mathrm{~Hz}), 14.5(\mathrm{~d}, J=3.5 \mathrm{~Hz})$.

HRMS: $\mathrm{m} / \mathrm{z}$ (ESI) calculated $[\mathrm{M}+\mathrm{Na}]^{+}: 395.0464$, found: $395.0472 .[\alpha]_{\mathrm{D}}{ }^{25}=13.097(c$ $\left.=0.25, \mathrm{CHCl}_{3}\right)$.

The enantiomeric excess of $\mathbf{3 q}$ was determined by chiral HPLC analysis compared to the corresponding racemate alcohol.

Conditions: ChiralPak IB column; hexane $/ \mathrm{PrOH}=98: 2$; flow rate $=1.0 \mathrm{~mL} / \mathrm{min} ; \lambda=$ $220 \mathrm{~nm} ; \mathrm{t}_{\mathrm{R} 1}($ major $)=9.2 \mathrm{~min} ; \mathrm{t}_{\mathrm{R} 2}($ minor $)=8.4 \mathrm{~min}$.

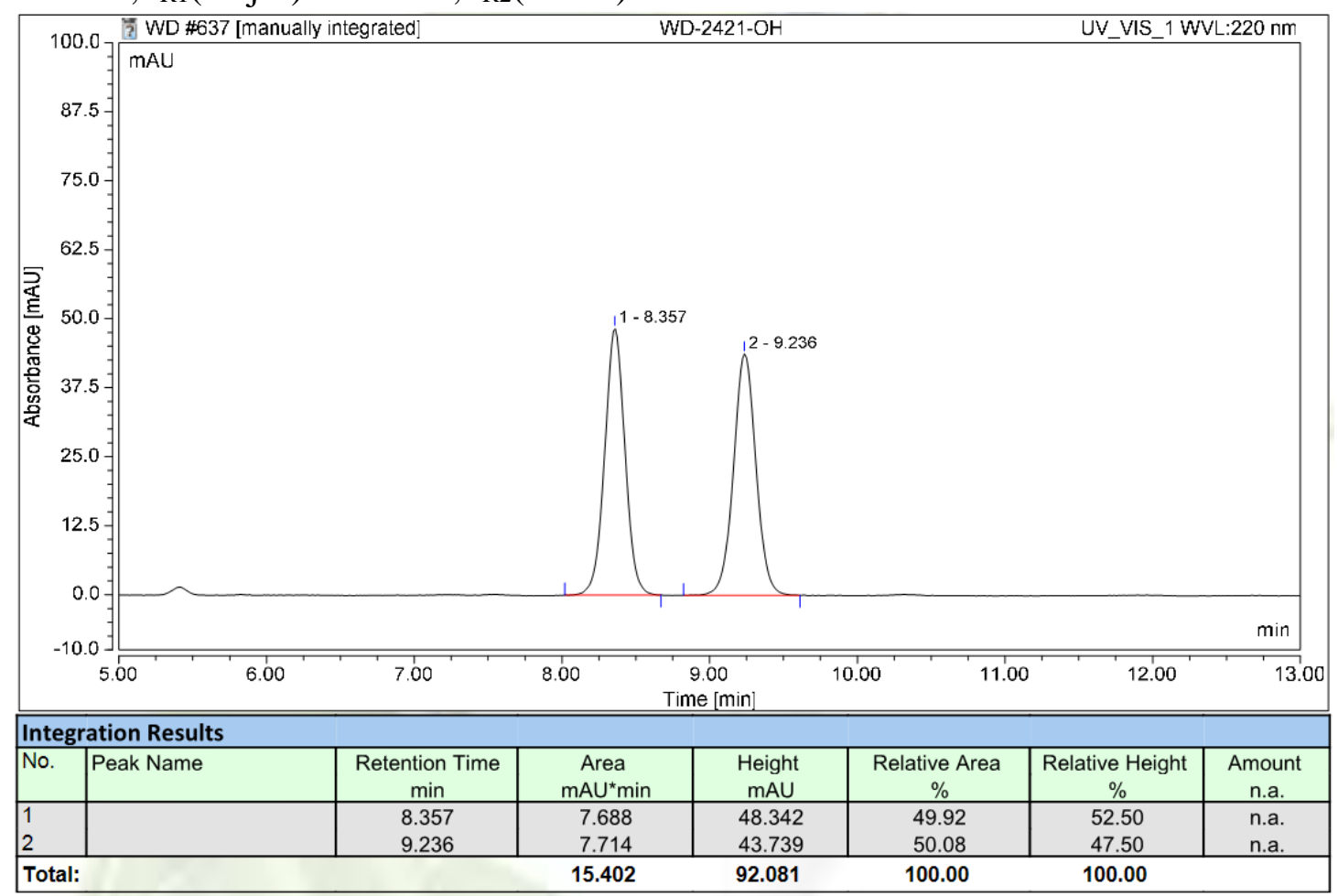




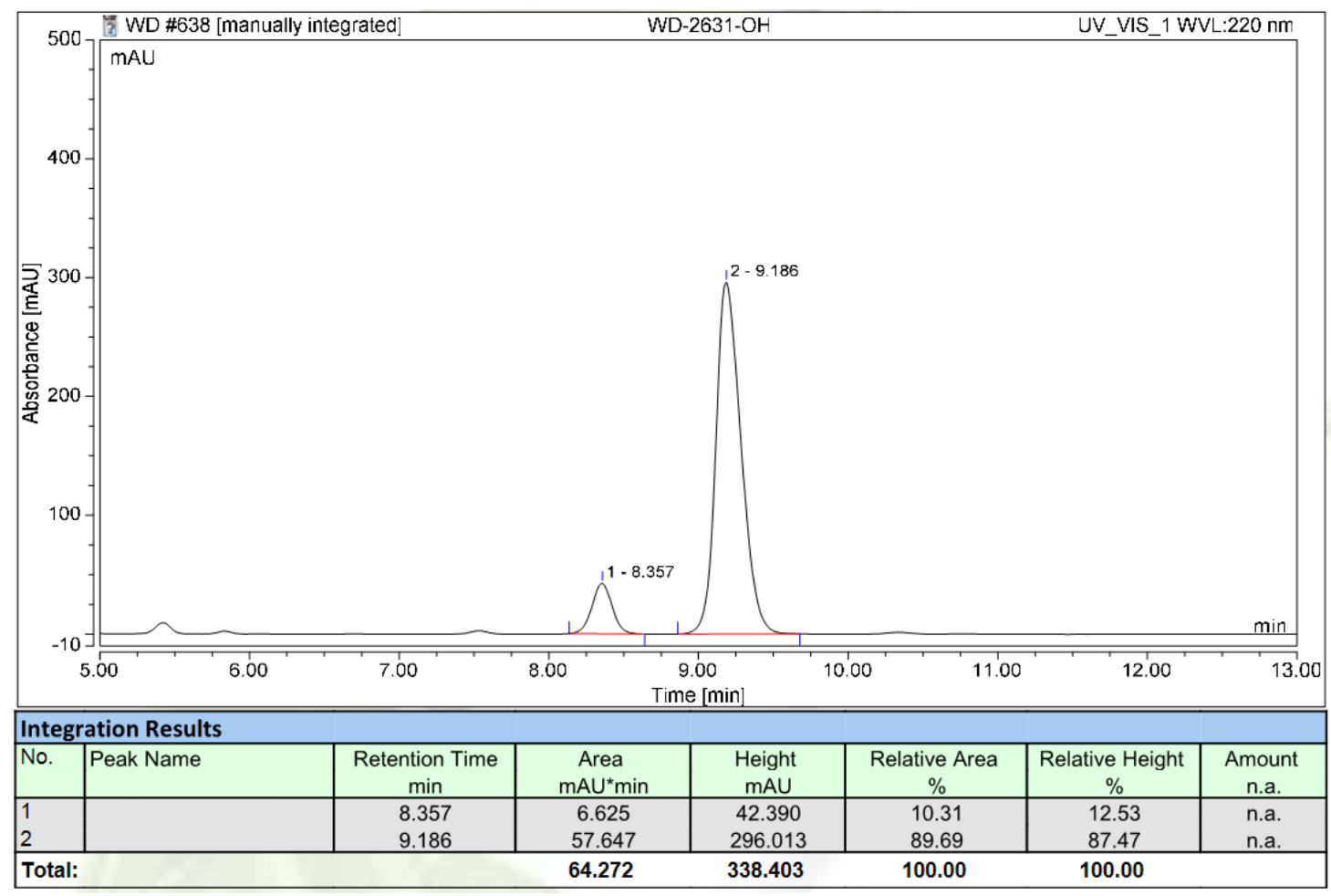<smiles>Cc1ccc([C@@H](O)CC(F)(F)F)cc1C#N</smiles>

The title compound $\mathbf{3 r}$ was synthesized according to General Procedure B, and it was purified by column chromatography on silica gel (34\% yield, 92:8 er, $25.8 \mathrm{mg}$, colourless oil).

${ }^{1} \mathbf{H}$ NMR $\left(600 \mathrm{MHz}, \mathrm{CDCl}_{3}\right) \delta 7.65(\mathrm{~s}, 1 \mathrm{H}), 7.51(\mathrm{dd}, J=7.8,1.2 \mathrm{~Hz}, 1 \mathrm{H}), 7.35(\mathrm{~d}, J$ $=8.4 \mathrm{~Hz}, 1 \mathrm{H}), 5.25-5.22(\mathrm{~m}, 1 \mathrm{H}), 2.66-2.54(\mathrm{~m}, 4 \mathrm{H}), 2.47(\mathrm{~s}, 1 \mathrm{H}), 2.44-2.33(\mathrm{~m}, 1 \mathrm{H})$; ${ }^{19}$ F NMR $\left(565 \mathrm{MHz}, \mathrm{CDCl}_{3}\right) \delta-80.99--81.03(\mathrm{~m}, 3 \mathrm{~F}),-112.43--113.90(\mathrm{~m}, 2 \mathrm{~F})$, -124.42- -124.53 (m, 2F), -125.86- -125.94. (m, 2F); ${ }^{13} \mathbf{C}$ NMR $\left(151 \mathrm{MHz}, \mathrm{CDCl}_{3}\right) \delta$ $142.1,141.1,130.8,130.0,129.7,117.7,120.2-108.2(\mathrm{~m}), 113.2,66.8,39.8(\mathrm{t}, J=$ $20.4 \mathrm{~Hz}), 20.2$.

HRMS: $\mathrm{m} / \mathrm{z}(\mathrm{ESI})$ calculated $[\mathrm{M}+\mathrm{Na}]^{+}: 402.0511$, found: $402.0515 .[\alpha]_{\mathrm{D}}{ }^{25}=10.168(c$ $\left.=0.25, \mathrm{CHCl}_{3}\right)$.

The enantiomeric excess of $\mathbf{3 r}$ was determined by chiral HPLC analysis compared to the corresponding racemate alcohol.

Conditions: ChiralPak IF column; hexane $/ \mathrm{PrOH}=95: 5$; flow rate $=1.0 \mathrm{~mL} / \mathrm{min} ; \lambda=$ $220 \mathrm{~nm} ; \mathrm{t}_{\mathrm{R} 1}($ major $)=7.1 \mathrm{~min} ; \mathrm{t}_{\mathrm{R} 2}($ minor $)=7.7 \mathrm{~min}$. 

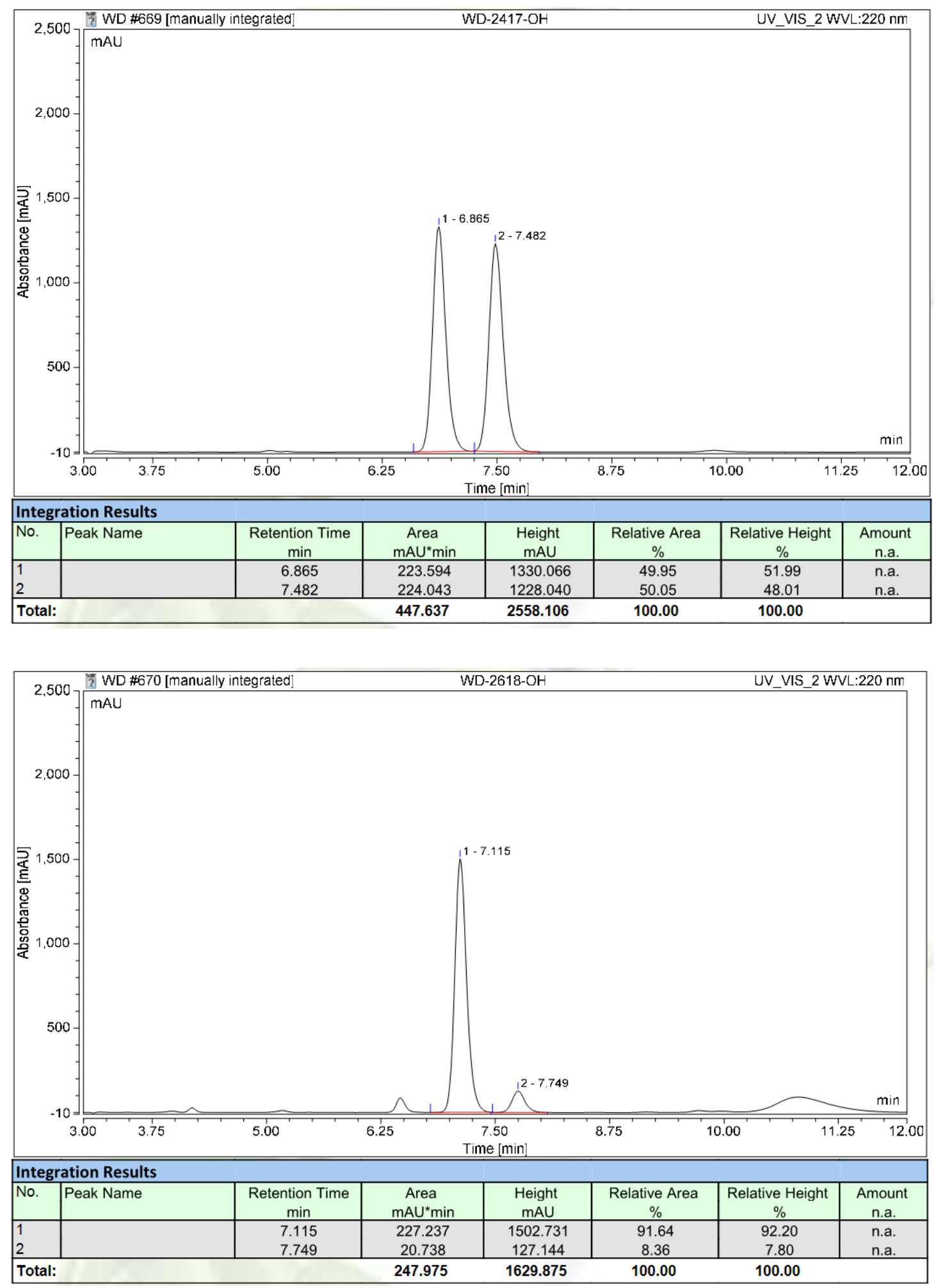<smiles>O[C@H](CC(F)(F)F)c1ccc2c(c1)OCO2</smiles>

The title compound $\mathbf{3 s}$ was synthesized according to General Procedure B, and it was purified by column chromatography on silica gel (45\% yield, 92:8 er, $34.5 \mathrm{mg}$, white solid). 
${ }^{1} \mathbf{H}$ NMR $\left(600 \mathrm{MHz}, \mathrm{CDCl}_{3}\right) \delta 6.89(\mathrm{~s}, 1 \mathrm{H}), 6.83(\mathrm{~d}, J=8.4 \mathrm{~Hz}, 1 \mathrm{H}), 6.79(\mathrm{~d}, J=7.8$ $\mathrm{Hz}, 1 \mathrm{H}), 5.97(\mathrm{~d}, J=1.2 \mathrm{~Hz}, 2 \mathrm{H}), 5.14-5.12(\mathrm{~m}, 1 \mathrm{H}), 2.64-2.54(\mathrm{~m}, 1 \mathrm{H}), 2.42-2.33(\mathrm{~m}$, 1H), 2.18-2.16 (m, 1H); ${ }^{19} \mathbf{F}$ NMR $\left(565 \mathrm{MHz}, \mathrm{CDCl}_{3}\right) \delta-80.99--81.03(\mathrm{~m}, 3 \mathrm{~F})$, -112.55- -114.33 (m, 2F), -124.54- -124.60 (m, 2F), -125.87- -125.94. (m, 2F); ${ }^{13} \mathrm{C}$ NMR $\left(151 \mathrm{MHz}, \mathrm{CDCl}_{3}\right) \delta 148.1,147.6,136.7,119.2,118.5-108.6(\mathrm{~m}), 108.4,106.1$, 101.2, 67.8, $39.8(\mathrm{t}, J=20.4 \mathrm{~Hz})$.

HRMS: $\mathrm{m} / \mathrm{z}(\mathrm{ESI})$ calculated $[\mathrm{M}+\mathrm{Na}]^{+}:$407.0300, found: $407.0296 .[\alpha]_{\mathrm{D}}{ }^{25}=19.075(c$ $\left.=0.25, \mathrm{CHCl}_{3}\right)$.

The enantiomeric excess of 3s was determined by chiral HPLC analysis compared to the corresponding racemate alcohol.

Conditions: ChiralPak IB column; hexane $/ \mathrm{PrOH}=95: 5$; flow rate $=1.0 \mathrm{~mL} / \mathrm{min} ; \lambda=$ $220 \mathrm{~nm} ; \mathrm{t}_{\mathrm{R} 1}$ (major) $=10.8 \mathrm{~min} ; \mathrm{t}_{\mathrm{R} 2}$ (minor $)=9.5 \mathrm{~min}$.

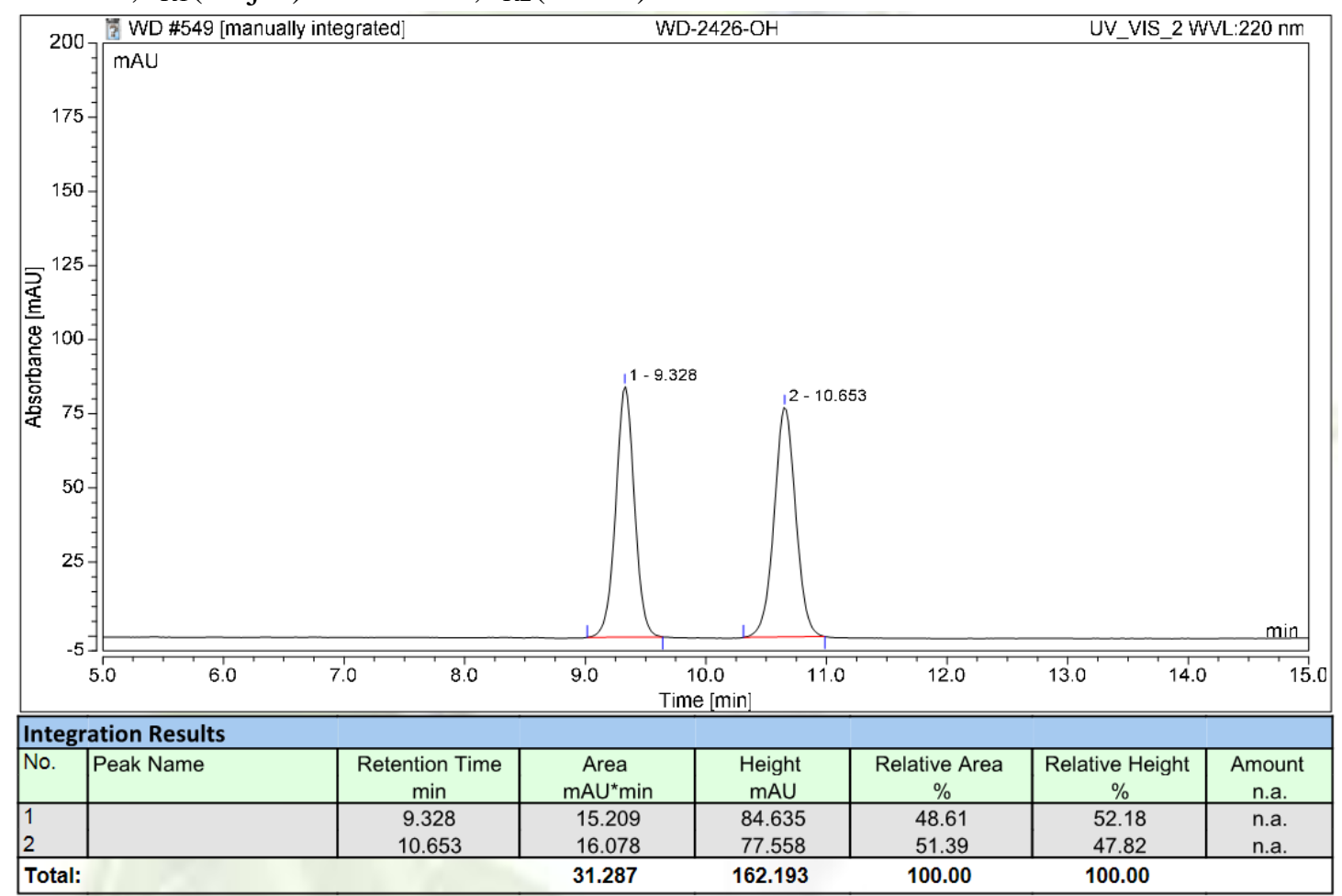




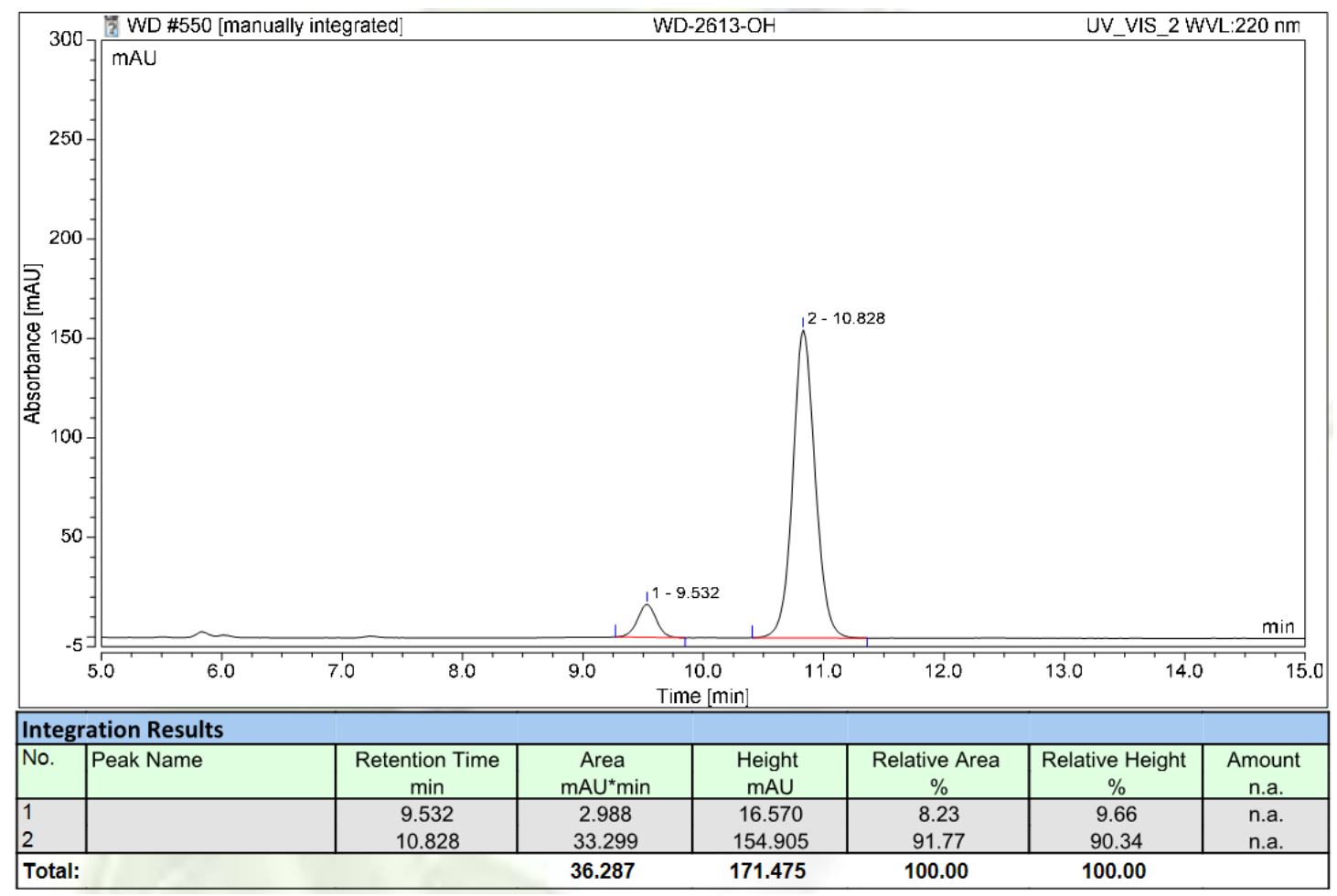

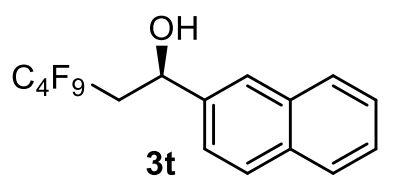

The title compound $\mathbf{3 t}$ was synthesized according to General Procedure B, and it was purified by column chromatography on silica gel (63\% yield, 91:9 er, $49.1 \mathrm{mg}$, white solid).

${ }^{1} \mathbf{H}$ NMR $\left(600 \mathrm{MHz}, \mathrm{CDCl}_{3}\right) \delta$ 7.89-7.63 (m, 4H), 7.53-7.47 (m, 3H), $5.39(\mathrm{dt}, J=8.4$, $3.0 \mathrm{~Hz}, 1 \mathrm{H}), 2.76-2.65(\mathrm{~m}, 1 \mathrm{H}), 2.56-2.46(\mathrm{~m}, 1 \mathrm{H}), 2.29(\mathrm{~s}, 1 \mathrm{H}) ;{ }^{19} \mathbf{F}$ NMR $(565 \mathrm{MHz}$, $\left.\mathrm{CDCl}_{3}\right) \delta-80.98--81.02(\mathrm{~m}, 3 \mathrm{~F}),-112.35--114.17(\mathrm{~m}, 2 \mathrm{~F}),-124.48--124.54(\mathrm{~m}, 2 \mathrm{~F})$, -125.84- -125.92. (m, 2F); ${ }^{13} \mathbf{C}$ NMR (151 MHz, $\left.\mathrm{CDCl}_{3}\right) \delta 148.1,147.6,136.7,119.2$, 118.5-108.6(m), 108.4, 106.1, 101.2, 67.8, 39.8 (t, $J=20.4 \mathrm{~Hz})$.

HRMS: $\mathrm{m} / \mathrm{z}(\mathrm{ESI})$ calculated $[\mathrm{M}+\mathrm{Na}]^{+}: 413.0558$, found: $413.0560 .[\alpha]_{\mathrm{D}}{ }^{25}=19.576(c$ $\left.=0.25, \mathrm{CHCl}_{3}\right)$.

The enantiomeric excess of $\mathbf{3 t}$ was determined by chiral HPLC analysis compared to the corresponding racemate alcohol.

Conditions: ChiralPak IB column; hexane $/ \mathrm{PrOH}=95: 5$; flow rate $=1.0 \mathrm{~mL} / \mathrm{min} ; \lambda=$ $220 \mathrm{~nm} ; \mathrm{t}_{\mathrm{R} 1}($ major $)=16.9 \mathrm{~min} ; \mathrm{t}_{\mathrm{R} 2}($ minor $)=14.8 \mathrm{~min}$. 

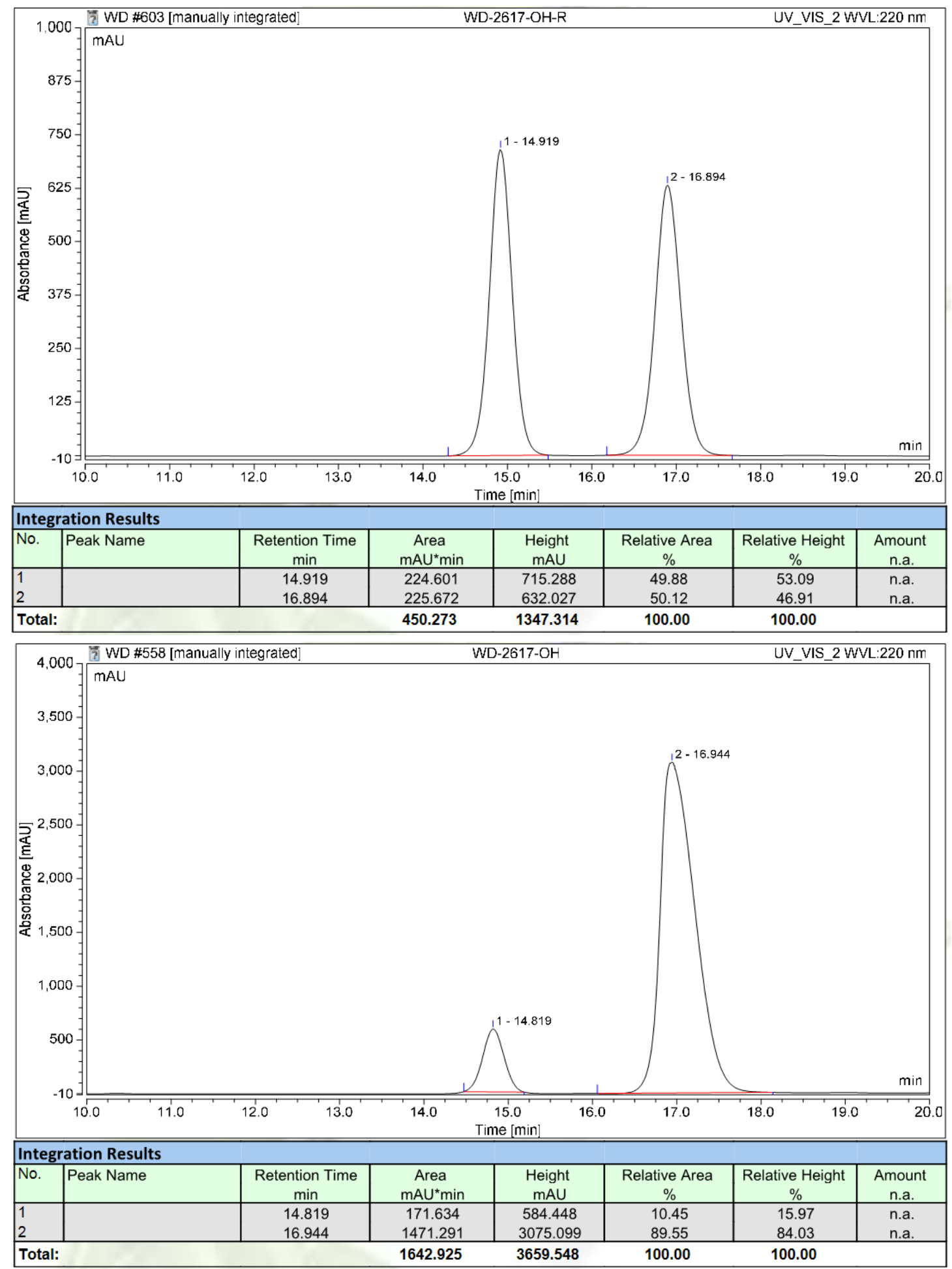<smiles>Cn1ccc2cc([C@@H](O)CC(F)(F)F)ccc21</smiles>

The title compound $\mathbf{3 u}$ was synthesized according to General Procedure B, and it was purified by column chromatography on silica gel (68\% yield, 88:12 er, $53.5 \mathrm{mg}$, white solid). 
${ }^{1}$ H NMR $\left(400 \mathrm{MHz}, \mathrm{CDCl}_{3}\right) \delta 7.64(\mathrm{~s}, 1 \mathrm{H}), 7.34(\mathrm{~d}, J=8.4 \mathrm{~Hz}, 1 \mathrm{H}), 7.24(\mathrm{~d}, J=8.4$ $\mathrm{Hz}, 1 \mathrm{H}), 7.09(\mathrm{~d}, J=2.8 \mathrm{~Hz}, 1 \mathrm{H}), 6.49(\mathrm{~d}, J=2.8 \mathrm{~Hz}, 1 \mathrm{H}), 5.34-5.28(\mathrm{~m}, 1 \mathrm{H}), 3.80(\mathrm{~s}$, $3 \mathrm{H}), 2.79-2.63(\mathrm{~m}, 1 \mathrm{H}), 2.55-2.40(\mathrm{~m}, 1 \mathrm{H}), 2.14(\mathrm{~s}, 1 \mathrm{H}) ;{ }^{19} \mathbf{F} \mathbf{N M R}\left(565 \mathrm{MHz}, \mathrm{CDCl}_{3}\right)$ $\delta$-80.98- -81.03 (m, 3F), -112.48- -114.47 (m, 2F), -124.56- -124.62 (m, 2F), -125.86-125.94. (m, 2F); ${ }^{13} \mathrm{C}$ NMR (101 MHz, $\left.\mathrm{CDCl}_{3}\right) \delta$ 136.6, 133.9, 129.8, 128.5, 119.2, 118.1, 117.8-107.3 (m), 109.7, 101.2, 68.6, $40.0(\mathrm{t}, J=20.7 \mathrm{~Hz}), 32.9$.

HRMS: $\mathrm{m} / \mathrm{z}(\mathrm{ESI})$ calculated $[\mathrm{M}+\mathrm{H}]^{+}: 394.0848$, found: $394.0854 .[\alpha]_{\mathrm{D}}{ }^{25}=17.346(c$ $\left.=0.25, \mathrm{CHCl}_{3}\right)$.

The enantiomeric excess of $\mathbf{3} \mathbf{u}$ was determined by chiral HPLC analysis compared to the corresponding racemate alcohol.

Conditions: ChiralPak IG column; hexane $/ \mathrm{PrOH}=95: 5$; flow rate $=1.0 \mathrm{~mL} / \mathrm{min} ; \lambda=$ $220 \mathrm{~nm} ; \mathrm{t}_{\mathrm{R} 1}$ (major) $=13.9 \mathrm{~min} ; \mathrm{t}_{\mathrm{R} 2}$ (minor) $=15.4 \mathrm{~min}$.

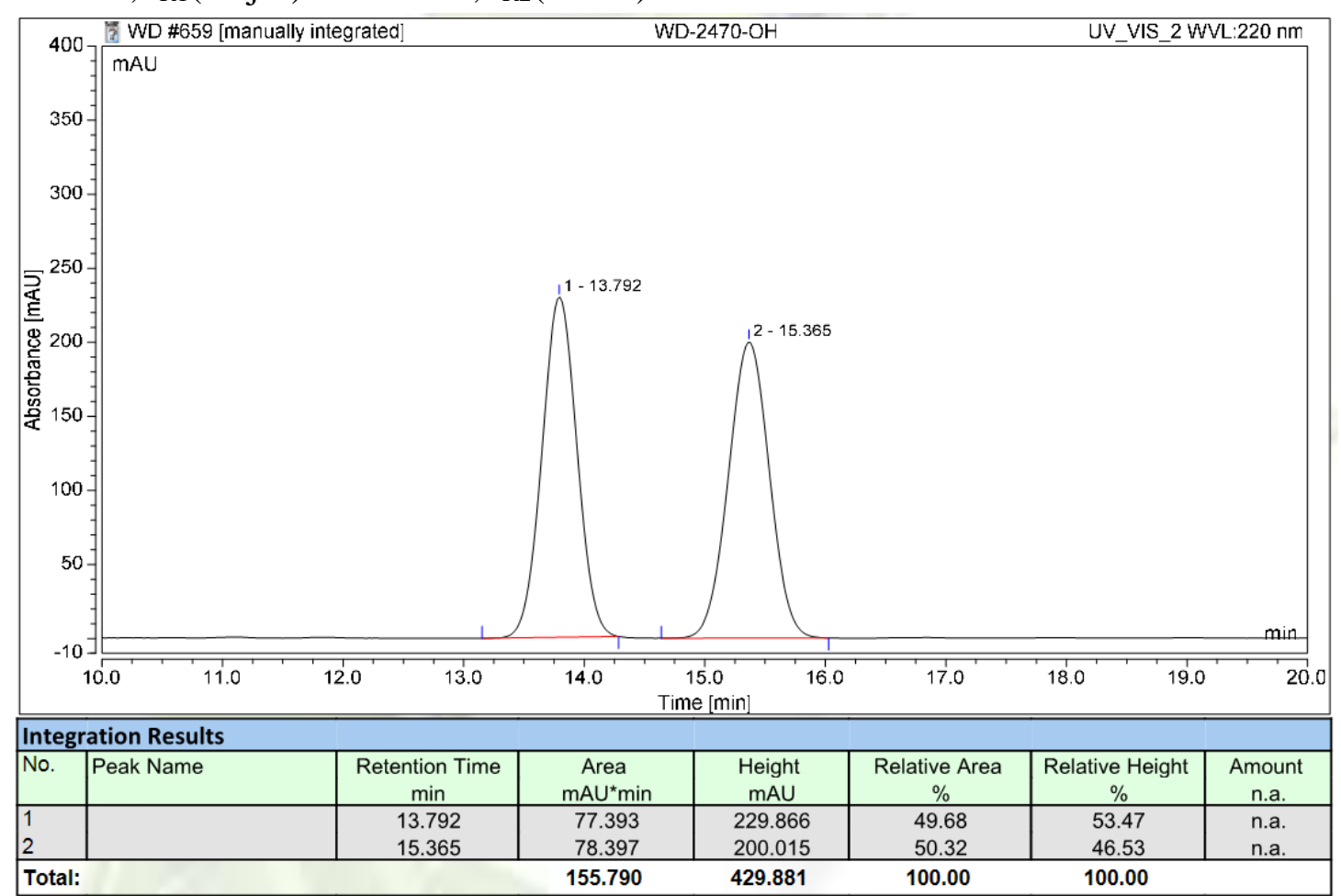




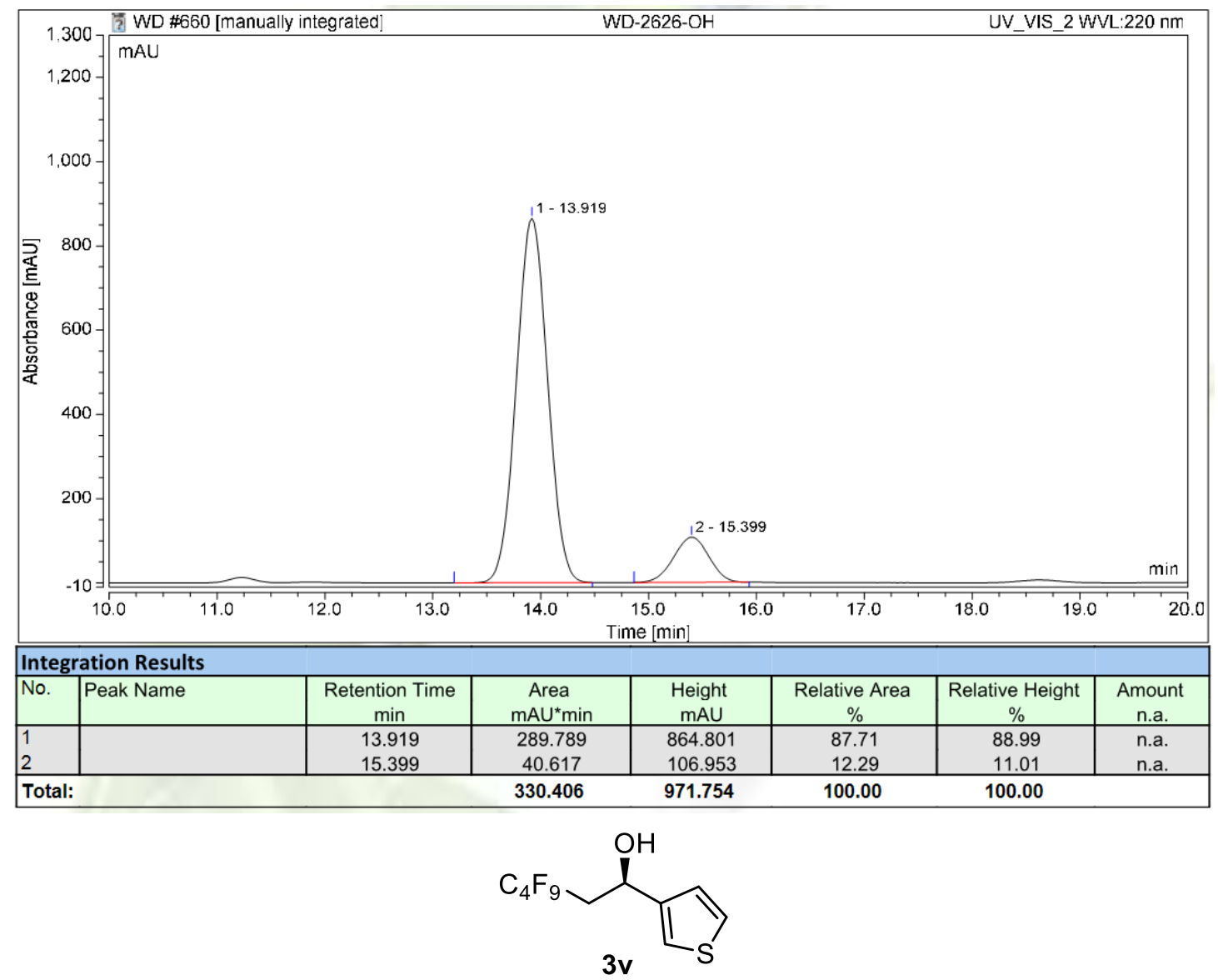

The title compound $\mathbf{3 v}$ was synthesized according to General Procedure B, and it was purified by column chromatography on silica gel (44\% yield, $88: 12 \mathrm{er}, 30.5 \mathrm{mg}$, white solid).

${ }^{1} \mathbf{H}$ NMR $\left(600 \mathrm{MHz}, \mathrm{CDCl}_{3}\right) \delta 7.36(\mathrm{dd}, J=4.8,3.0 \mathrm{~Hz}, 1 \mathrm{H}), 7.29(\mathrm{~d}, J=2.4 \mathrm{~Hz}, 1 \mathrm{H})$, $7.10(\mathrm{dd}, J=4.8,1.2 \mathrm{~Hz}, 1 \mathrm{H}), 5.36-5.31(\mathrm{~m}, 1 \mathrm{H}), 2.69-2.58(\mathrm{~m}, 1 \mathrm{H}), 2.52-2.43(\mathrm{~m}$, $1 \mathrm{H}), 2.28(\mathrm{~s}, 1 \mathrm{H}) ;{ }^{19} \mathbf{F}$ NMR $\left(565 \mathrm{MHz}, \mathrm{CDCl}_{3}\right) \delta-81.01--81.06(\mathrm{~m}, 3 \mathrm{~F}),-112.47-$ -114.48 (m, 2F), -124.54- - 124.60 (m, 2F), -125.88- -125.96. (m, 2F); ${ }^{13}$ C NMR (151 $\left.\mathrm{MHz}, \mathrm{CDCl}_{3}\right) \delta 143.9,127.0,125.0,121.6,119.6-108.5(\mathrm{~m}), 64.1,39.2(\mathrm{t}, J=20.4$ $\mathrm{Hz})$.

HRMS: m/z (ESI) calculated $[\mathrm{M}-\mathrm{OH}]^{+}: 329.0041$, found: $329.0045 .[\alpha]_{\mathrm{D}}{ }^{25}=21.389(c$ $\left.=0.25, \mathrm{CHCl}_{3}\right)$.

The enantiomeric excess of $\mathbf{3 v}$ was determined by chiral HPLC analysis compared to the corresponding racemate alcohol.

Conditions: ChiralPak ID column; hexane $/{ }^{i} \mathrm{PrOH}=98: 2$; flow rate $=1.0 \mathrm{~mL} / \mathrm{min} ; \lambda=$ $220 \mathrm{~nm} ; \mathrm{t}_{\mathrm{R} 1}($ major $)=6.2 \mathrm{~min} ; \mathrm{t}_{\mathrm{R} 2}($ minor $)=5.8 \mathrm{~min}$. 

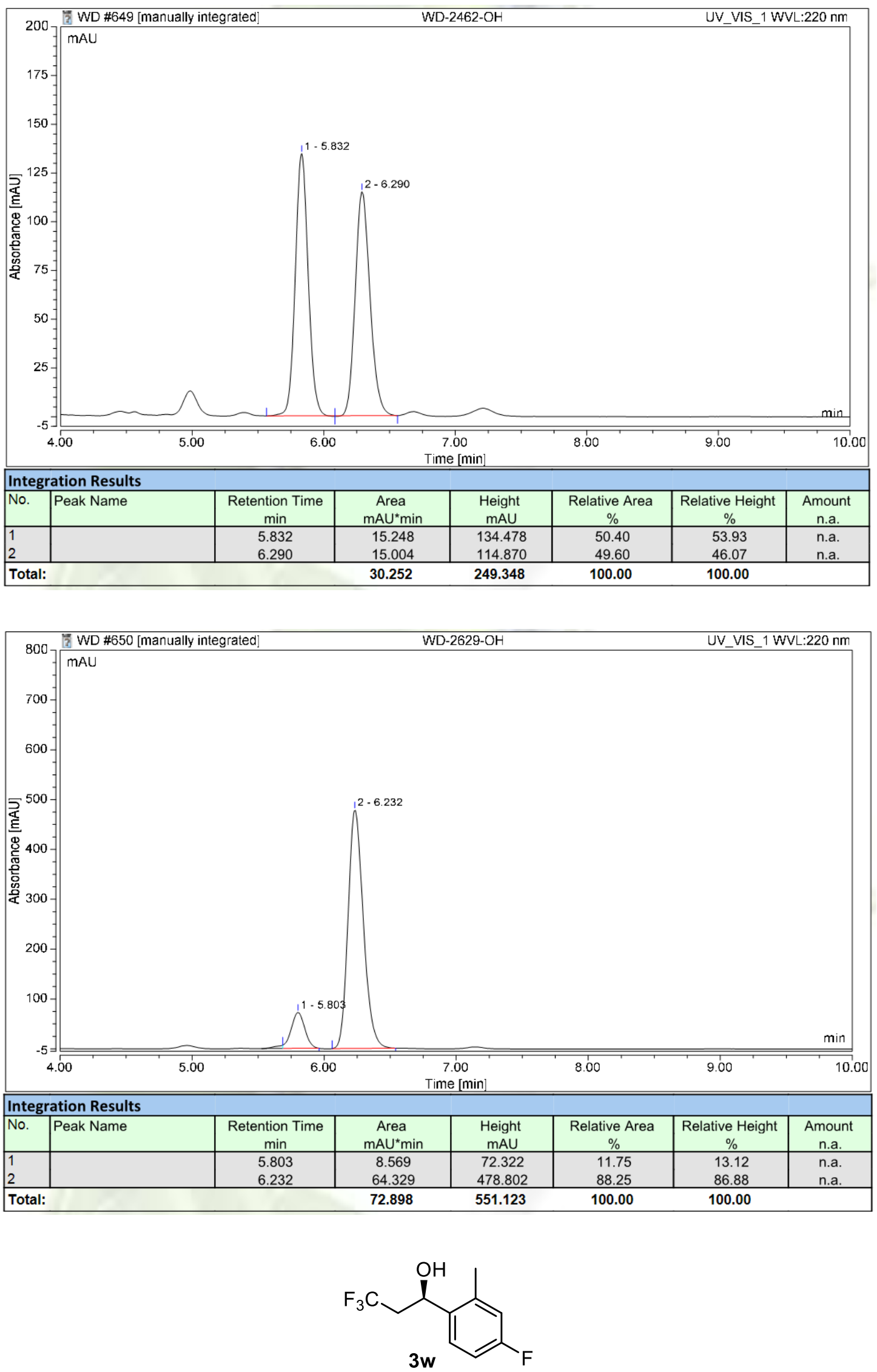
The title compound $\mathbf{3 w}$ was synthesized according to General Procedure B, and it was purified by column chromatography on silica gel (51\% yield, 94:6 er, $22.6 \mathrm{mg}$, colourless oil).

${ }^{1} \mathbf{H}$ NMR $\left(600 \mathrm{MHz} \mathrm{CDCl}_{3}\right) \delta 7.47(\mathrm{dd}, J=9.0,6.0 \mathrm{~Hz}, 1 \mathrm{H}), 6.94(\mathrm{td}, J=8.4,2.4 \mathrm{~Hz}$, $1 \mathrm{H}), 6.87(\mathrm{dd}, J=10.2,3.0 \mathrm{~Hz}, 1 \mathrm{H}), 5.29(\mathrm{dt}, J=9.0,3.0 \mathrm{~Hz}, 1 \mathrm{H}), 2.62-2.52(\mathrm{~m}, 1 \mathrm{H})$, $2.41-2.32(\mathrm{~m}, 4 \mathrm{H}), 2.13(\mathrm{~d}, J=3.6 \mathrm{~Hz}, 1 \mathrm{H}) ;{ }^{19} \mathbf{F}$ NMR $\left(565 \mathrm{MHz} \mathrm{CDCl}_{3}\right) \delta-64.12(\mathrm{~s}$, 3F), -114.81 (s, 1F); ${ }^{13} \mathrm{C}$ NMR $\left(151 \mathrm{MHz}, \mathrm{CDCl}_{3}\right) \delta 162.2(\mathrm{~d}, J=246.3 \mathrm{~Hz}), 136.5(\mathrm{~d}$, $J=7.9 \mathrm{~Hz}), 136.2(\mathrm{~d}, J=3.3 \mathrm{~Hz}), 127.0(\mathrm{~d}, J=8.6 \mathrm{~Hz}), 129.23-121.76(\mathrm{~m}), 117.2(\mathrm{~d}$, $J=21.4 \mathrm{~Hz}), 113.3(\mathrm{~d}, J=21.1 \mathrm{~Hz}), 64.8-64.7(\mathrm{~m}), 42.1(\mathrm{q}, J=27.0 \mathrm{~Hz}), 18.8$.

HRMS: $\mathrm{m} / \mathrm{z}(\mathrm{ESI})$ calculated $[\mathrm{M}+\mathrm{H}]^{+}: 223.0741$, found: $223.0704 .[\alpha]_{\mathrm{D}}{ }^{25}=30.732(c$ $\left.=0.25, \mathrm{CHCl}_{3}\right)$.

The enantiomeric excess of $\mathbf{3 w}$ was determined by chiral HPLC analysis compared to the corresponding racemate alcohol.

Conditions: ChiralPak IB column; hexane $/{ }^{i} \mathrm{PrOH}=98: 2$; flow rate $=1.0 \mathrm{~mL} / \mathrm{min} ; \lambda=$ $220 \mathrm{~nm} ; \mathrm{t}_{\mathrm{R} 1}$ (major) $=11.9 \mathrm{~min} ; \mathrm{t}_{\mathrm{R} 2}($ minor $)=10.7 \mathrm{~min}$.

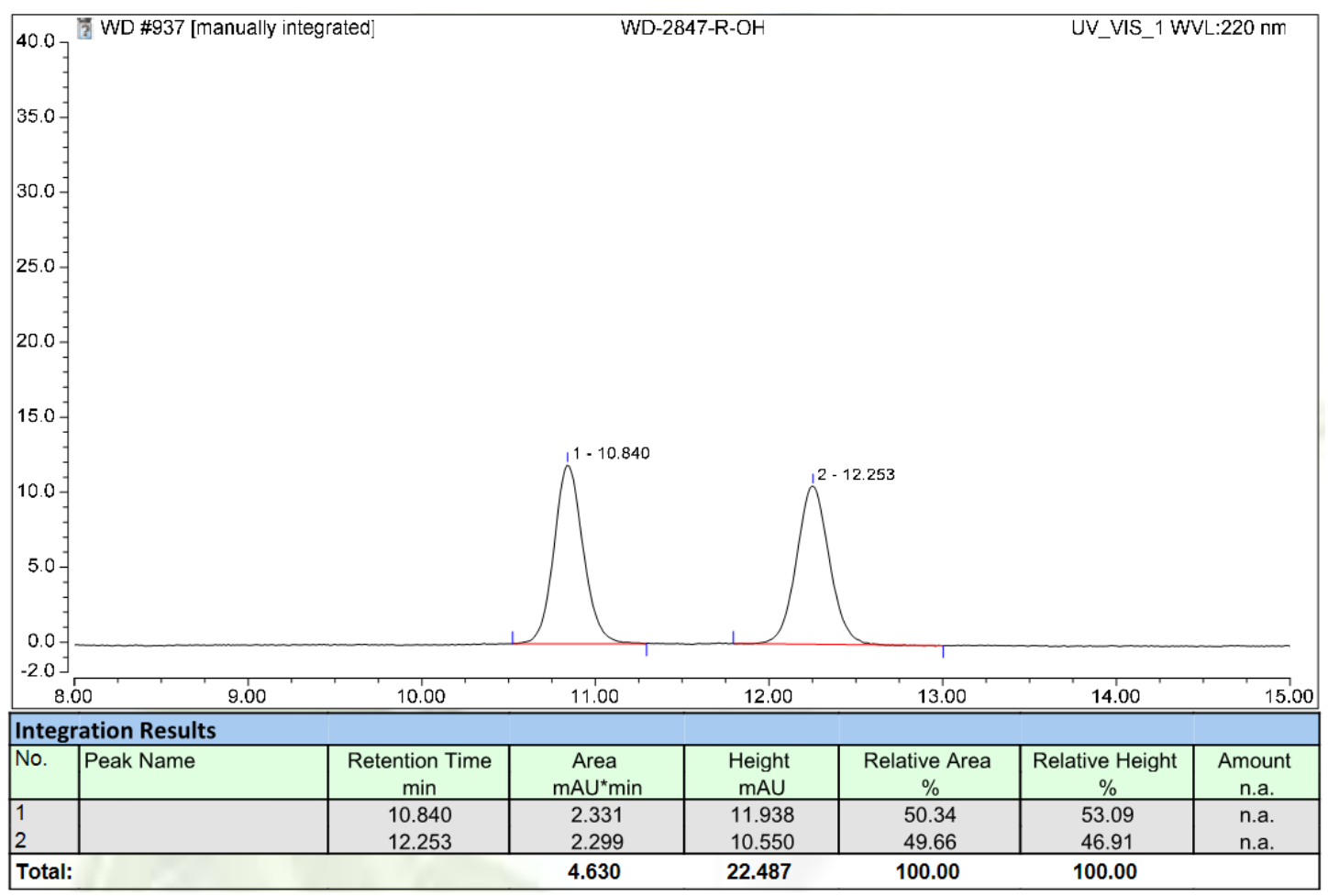




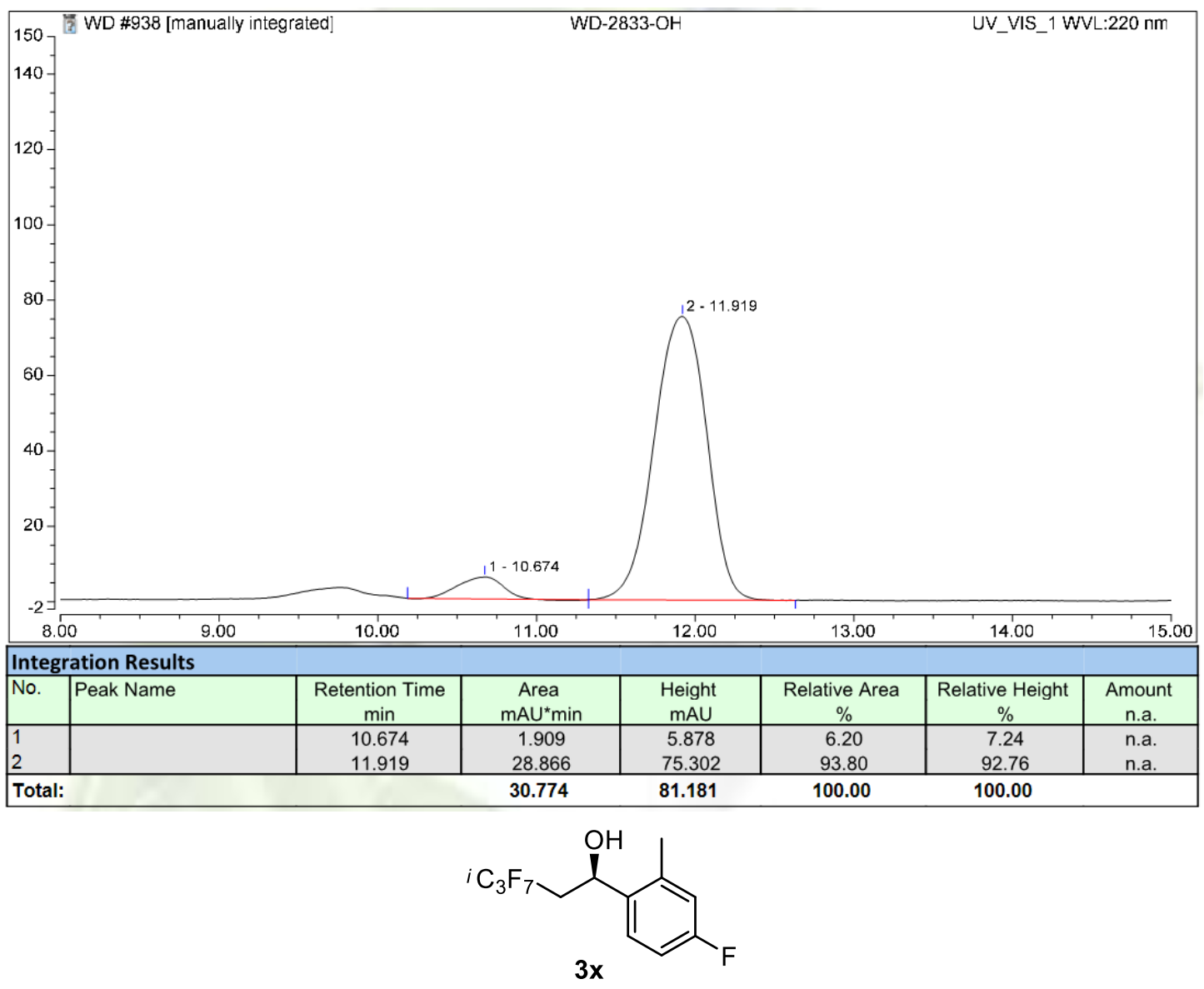

The title compound $\mathbf{3 x}$ was synthesized according to General Procedure B, and it was purified by column chromatography on silica gel (30\% yield, 99:1 er, $19.4 \mathrm{mg}$, colourless oil).

${ }^{1} \mathbf{H}$ NMR $\left(600 \mathrm{MHz}, \mathrm{CDCl}_{3}\right) \delta 7.52(\mathrm{dd}, J=8.4,6.0 \mathrm{~Hz}, 1 \mathrm{H}), 6.95(\mathrm{td}, J=8.4,3.0 \mathrm{~Hz}$, $1 \mathrm{H}), 6.87$ (dd, $J=9.6,2.4 \mathrm{~Hz}, 1 \mathrm{H}), 5.38$ (d, $J=9.6 \mathrm{~Hz}, 1 \mathrm{H}), 2.53(\mathrm{td}, J=15.6,9.6 \mathrm{~Hz}$, 1H), 2.33-2.30 (m, 4H), 2.14-2.12(m, 1H); ${ }^{19} \mathbf{F}$ NMR (565 MHz, $\left.\mathrm{CDCl}_{3}\right) \delta-76.18-$ -76.24 (m, 3F), -77.07- -77.14 (m, 3F), -114.81 (s, 1F), -185.72- -185.77. (m, 2F); ${ }^{13} \mathrm{C}$ NMR $\left(151 \mathrm{MHz}, \mathrm{CDCl}_{3}\right) \delta 162.1(\mathrm{~d}, J=246.4 \mathrm{~Hz}), 136.8(\mathrm{~d}, J=3.2 \mathrm{~Hz}), 136.2(\mathrm{~d}, J$ $=7.9 \mathrm{~Hz}), 127.0(\mathrm{~d}, J=8.6 \mathrm{~Hz}), 122.1-119.7(\mathrm{~m}), 117.3(\mathrm{~d}, J=21.1 \mathrm{~Hz}), 113.4(\mathrm{~d}, J=$ $21.1 \mathrm{~Hz}), 91.4(\mathrm{dt}, J=204.2 \mathrm{~Hz}, J=32.3 \mathrm{~Hz}), 64.5(\mathrm{~d}, J=3.8 \mathrm{~Hz}), 37.2(\mathrm{~d}, J=18.7$ $\mathrm{Hz}), 18.7$.

HRMS: $\mathrm{m} / \mathrm{z}(\mathrm{ESI})$ calculated $[\mathrm{M}-\mathrm{OH}]^{+}: 305.0571$, found: $305.0567 .[\alpha]_{\mathrm{D}}{ }^{25}=52.235(c$ $\left.=0.25, \mathrm{CHCl}_{3}\right)$.

The enantiomeric excess of $\mathbf{3} \mathbf{x}$ was determined by chiral HPLC analysis compared to the corresponding racemate alcohol.

Conditions: ChiralPak IB column; hexane $/{ }^{i} \mathrm{PrOH}=98: 2$; flow rate $=1.0 \mathrm{~mL} / \mathrm{min} ; \lambda=$ $220 \mathrm{~nm} ; \mathrm{t}_{\mathrm{R} 1}($ major $)=12.1 \mathrm{~min} ; \mathrm{t}_{\mathrm{R} 2}($ minor $)=9.9 \mathrm{~min}$. 

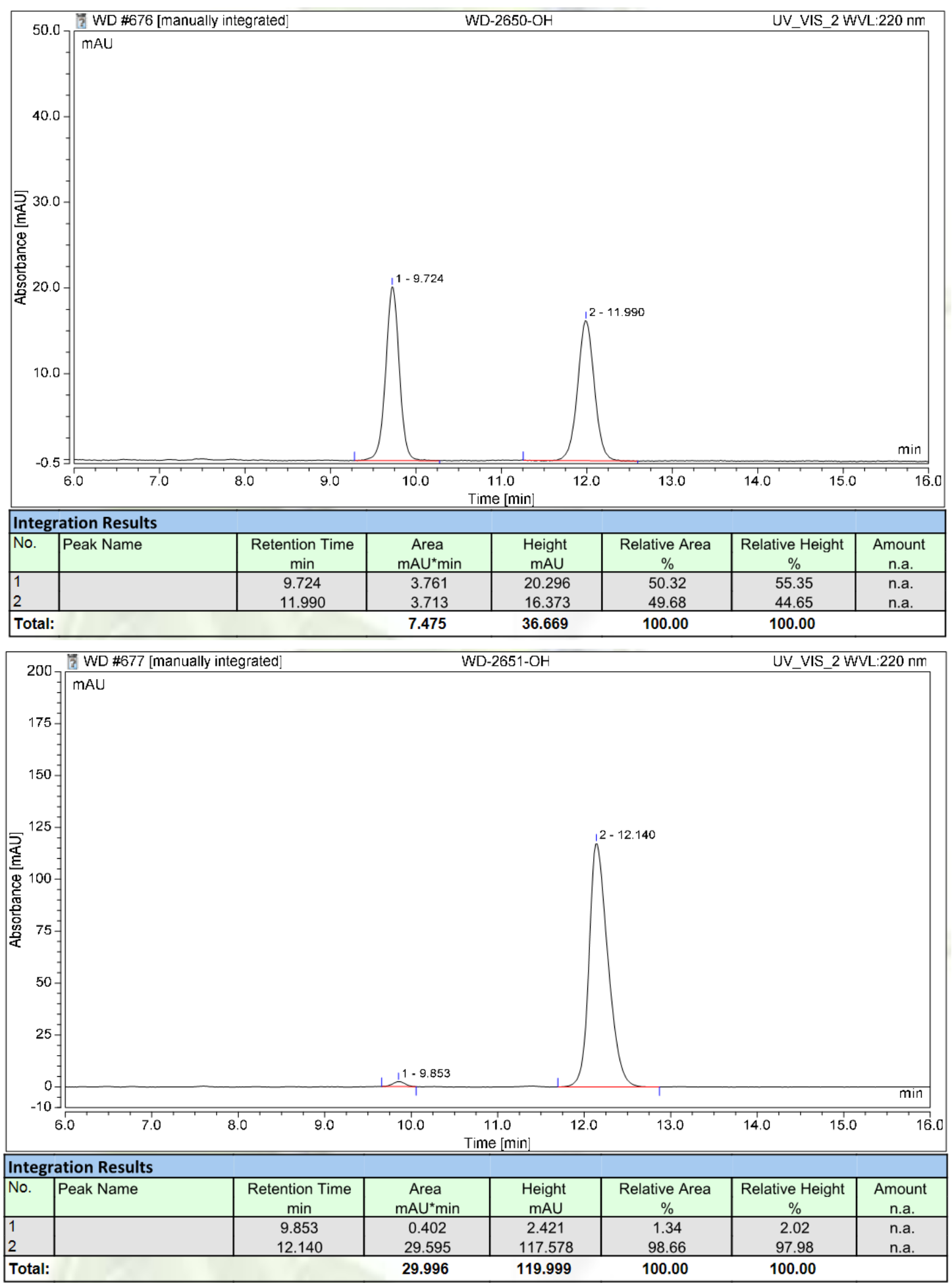<smiles></smiles>

The title compound $\mathbf{3 y}$ was synthesized according to General Procedure B, and it was purified by column chromatography on silica gel (56\% yield, 96:4 er, $47.3 \mathrm{mg}$, colourless oil). 
${ }^{1} \mathbf{H}$ NMR $\left(600 \mathrm{MHz}, \mathrm{CDCl}_{3}\right) \delta 7.51(\mathrm{dd}, J=9.0,6.0 \mathrm{~Hz}, 1 \mathrm{H}), 6.95(\mathrm{td}, J=8.4,2.4 \mathrm{~Hz}$, $1 \mathrm{H}), 6.88(\mathrm{dd}, J=9.6,3.0 \mathrm{~Hz}, 1 \mathrm{H}), 5.44(\mathrm{dt}, J=9.6,3.0 \mathrm{~Hz}, 1 \mathrm{H}), 2.61-2.50(\mathrm{~m}, 1 \mathrm{H})$, 2.37-2.18 (m, 4H), $2.18(\mathrm{~s}, 1 \mathrm{H}) ;{ }^{19} \mathbf{F}$ NMR $\left(565 \mathrm{MHz}, \mathrm{CDCl}_{3}\right) \delta-80.75--80.82(\mathrm{~m}$, $3 \mathrm{~F}),-112.76--114.28(\mathrm{~m}, 2 \mathrm{~F}),-114.78(\mathrm{~s}, 1 \mathrm{~F}),-122.52--122.60$. (m, 2F), -123.81-123.86. (m, 2F), -126.26- -126.33. (m, 2F); ${ }^{13} \mathrm{C}$ NMR (151 MHz, $\left.\mathrm{CDCl}_{3}\right) \delta 162.2(\mathrm{~d}$, $J=246.6 \mathrm{~Hz}), 136.5(\mathrm{~d}, J=2.9 \mathrm{~Hz}), 136.4(\mathrm{~d}, J=7.7 \mathrm{~Hz}), 127.1(\mathrm{~d}, J=8.3 \mathrm{~Hz})$, $117.3(\mathrm{~d}, J=21.1 \mathrm{~Hz}), 113.4(\mathrm{~d}, J=21.3 \mathrm{~Hz}), 63.8(\mathrm{~d}, J=3.9 \mathrm{~Hz}), 39.1(\mathrm{t}, J=20.8$ $\mathrm{Hz}), 18.8$.

HRMS: $\mathrm{m} / \mathrm{z}(\mathrm{ESI})$ calculated $[\mathrm{M}-\mathrm{OH}]^{+}: 405.0507$, found: $405.0527 .[\alpha]_{\mathrm{D}}^{25}=10.233(c$ $\left.=0.25, \mathrm{CHCl}_{3}\right)$.

The enantiomeric excess of $\mathbf{3 y}$ was determined by chiral HPLC analysis compared to the corresponding racemate alcohol.

Conditions: ChiralPak IB column; hexane $/{ }^{i} \mathrm{PrOH}=98: 2$; flow rate $=1.0 \mathrm{~mL} / \mathrm{min} ; \lambda=$ $220 \mathrm{~nm} ; \mathrm{t}_{\mathrm{R} 1}($ major $)=8.8 \mathrm{~min} ; \mathrm{t}_{\mathrm{R} 2}($ minor $)=7.2 \mathrm{~min}$. 

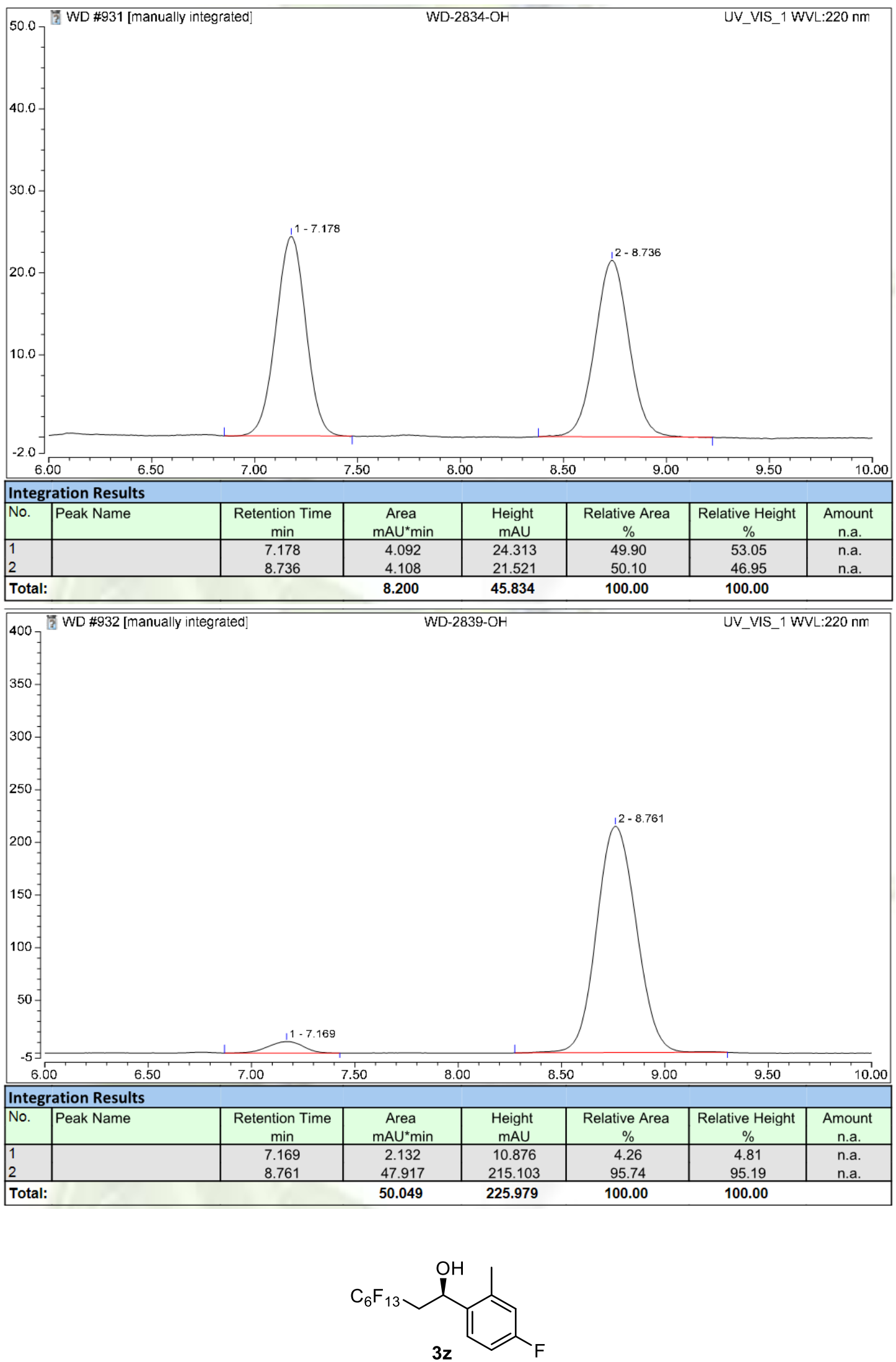

The title compound $\mathbf{3 z}$ was synthesized according to General Procedure B, and it was purified by column chromatography on silica gel (75\% yield, 95.5:4.5 er, $70.8 \mathrm{mg}$, colourless oil). 
${ }^{1}$ H NMR $\left(600 \mathrm{MHz}, \mathrm{CDCl}_{3}\right) \delta$ 7.51-7.48 (m, 1H), $6.94(\mathrm{t}, J=8.4 \mathrm{~Hz}, 1 \mathrm{H}), 6.87(\mathrm{~d}, J$ $=9.6 \mathrm{~Hz}, 1 \mathrm{H}), 5.45-5.40(\mathrm{~m}, 1 \mathrm{H}), 2.60-2.49(\mathrm{~m}, 1 \mathrm{H}), 2.36-2.24(\mathrm{~m}, 5 \mathrm{H}) ;{ }^{19} \mathbf{F}$ NMR $\left(565 \mathrm{MHz}, \mathrm{CDCl}_{3}\right) \delta-80.86--80.92(\mathrm{~m}, 3 \mathrm{~F}),-112.75--114.33(\mathrm{~m}, 2 \mathrm{~F}),-114.84(\mathrm{~s}, 1 \mathrm{~F})$, $-121.78--121.87(\mathrm{~m}, 2 \mathrm{~F}),-122.88--123.00(\mathrm{~m}, 2 \mathrm{~F}),-123.65--123.73(\mathrm{~m}, 2 \mathrm{~F})$, -126.20- -126.27 (m, 2F); ${ }^{13} \mathbf{C}$ NMR (151 MHz, $\left.\mathrm{CDCl}_{3}\right) \delta 162.2(\mathrm{~d}, J=246.6 \mathrm{~Hz})$, $136.5(\mathrm{~d}, J=3.0 \mathrm{~Hz}), 136.4(\mathrm{~d}, J=7.7 \mathrm{~Hz}), 127.1(\mathrm{~d}, J=8.6 \mathrm{~Hz}), 117.3(\mathrm{~d}, J=21.3$ $\mathrm{Hz}), 120.1-108.3(\mathrm{~m}), 113.4(\mathrm{~d}, J=21.1 \mathrm{~Hz}), 63.8,39.1$ (t, $J=20.5 \mathrm{~Hz}), 18.7$.

HRMS: $\mathrm{m} / \mathrm{z}(\mathrm{ESI})$ calculated $[\mathrm{M}-\mathrm{OH}]^{+}: 455.0475$, found: $455.0467 .[\alpha]_{\mathrm{D}}{ }^{25}=30.732(c$ $\left.=0.25, \mathrm{CHCl}_{3}\right)$.

The enantiomeric excess of $\mathbf{3 z}$ was determined by chiral HPLC analysis compared to the corresponding racemate alcohol.

Conditions: ChiralPak IB column; hexane $/{ }^{i} \mathrm{PrOH}=98: 2$; flow rate $=1.0 \mathrm{~mL} / \mathrm{min} ; \lambda=$ $220 \mathrm{~nm} ; \mathrm{t}_{\mathrm{R} 1}($ major $)=9.8 \mathrm{~min} ; \mathrm{t}_{\mathrm{R} 2}($ minor $)=7.7 \mathrm{~min}$.

\begin{tabular}{|l|l|l|l|l|l|l|}
\hline & UV_VIS_2 WVL:220 nm \\
\hline
\end{tabular}




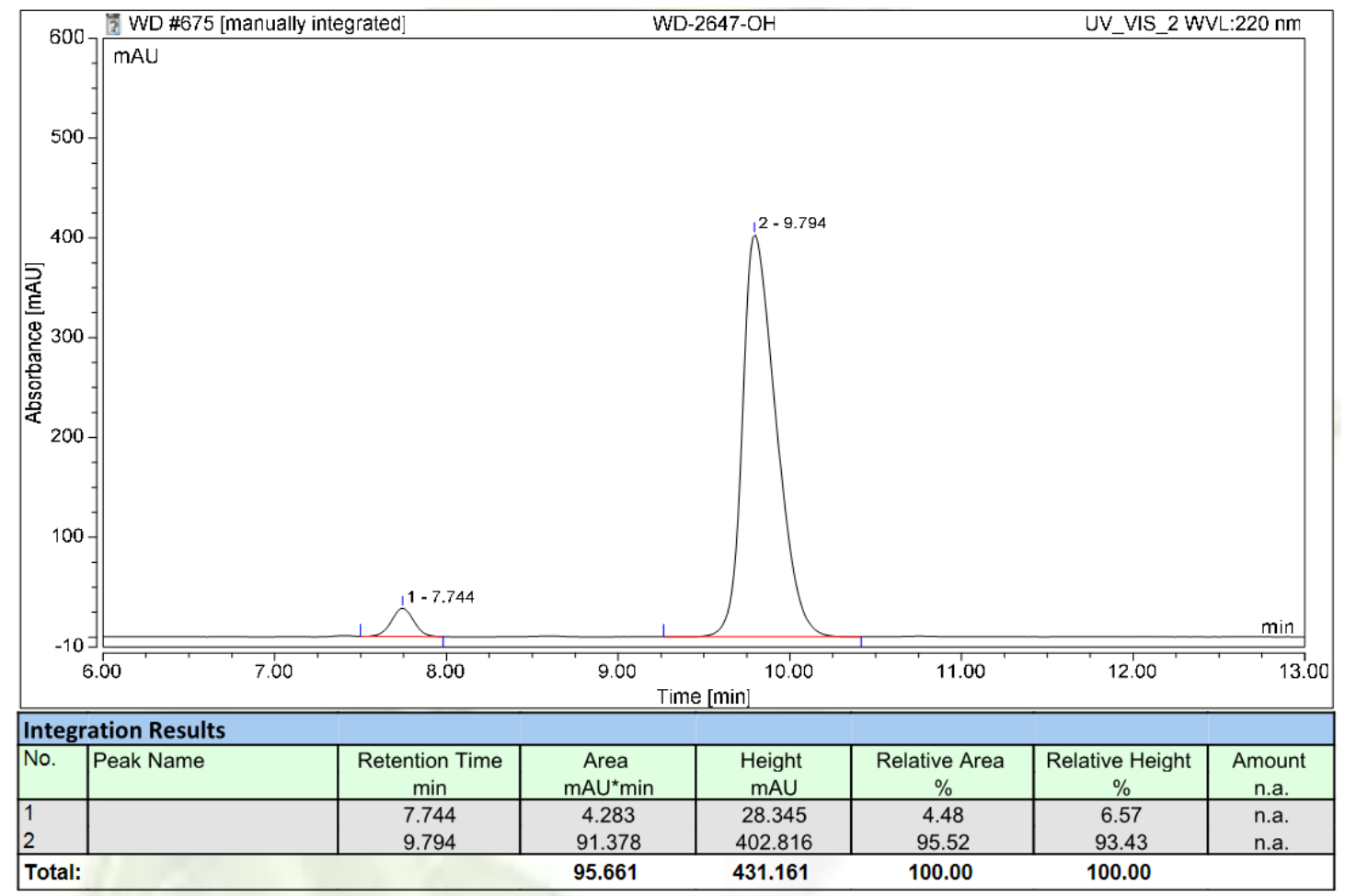<smiles>Cc1cc(F)ccc1[C@@H](O)CC(F)(F)F</smiles>

The title compound 3aa was synthesized according to General Procedure B, and it was purified by column chromatography on silica gel (75\% yield, 95:5 er, $85.8 \mathrm{mg}$, colourless oil).

${ }^{1} \mathbf{H}$ NMR $\left(600 \mathrm{MHz}, \mathrm{CDCl}_{3}\right) \delta 7.49(\mathrm{dd}, J=9.0,6.0 \mathrm{~Hz}, 1 \mathrm{H}), 6.94(\mathrm{td}, J=8.4,2.4 \mathrm{~Hz}$, $1 \mathrm{H}), 6.86(\mathrm{dd}, J=9.6,2.4 \mathrm{~Hz}, 1 \mathrm{H}), 5.42(\mathrm{dt}, J=8.4,3.0 \mathrm{~Hz}, 1 \mathrm{H}), 2.59-2.48(\mathrm{~m}, 1 \mathrm{H})$, 2.36- $2.26(\mathrm{~m}, 5 \mathrm{H}) ;{ }^{19} \mathbf{F}$ NMR $\left(565 \mathrm{MHz}, \mathrm{CDCl}_{3}\right) \delta-80.88--80.98(\mathrm{~m}, 3 \mathrm{~F}),-112.76-$ -114.35 (m, 2F), -114.87 (s, 1F), -121.60- -121.71 (m, 2F), -121.91- -122.08 (m, 4F), -122.80- -122.90 (m, 2F), -123.64- -123.70 (m, 2F), -126.24- -126.29. (m, 2F); ${ }^{13} \mathrm{C}$ NMR $\left(151 \mathrm{MHz}, \mathrm{CDCl}_{3}\right) \delta 162.2(\mathrm{~d}, J=246.4 \mathrm{~Hz}), 136.5(\mathrm{~d}, J=3.2 \mathrm{~Hz}), 136.4(\mathrm{~d}, J$ $=7.9 \mathrm{~Hz}), 127.1(\mathrm{~d}, J=8.6 \mathrm{~Hz}), 120.0-108.2(\mathrm{~m}), 117.3(\mathrm{~d}, J=21.1 \mathrm{~Hz}), 113.4(\mathrm{~d}, J$ $=21.1 \mathrm{~Hz}), 63.8,39.1(\mathrm{t}, J=20.7 \mathrm{~Hz}), 18.7$.

HRMS: $\mathrm{m} / \mathrm{z}(\mathrm{ESI})$ calculated $[\mathrm{M}-\mathrm{OH}]^{+}: 555.0411$, found: $555.0406 .[\alpha]_{\mathrm{D}}{ }^{25}=18.730(c$ $\left.=0.25, \mathrm{CHCl}_{3}\right)$.

The enantiomeric excess of 3aa was determined by chiral HPLC analysis compared to the corresponding racemate alcohol.

Conditions: ChiralPak IB column; hexane $/{ }^{i} \mathrm{PrOH}=98: 2$; flow rate $=1.0 \mathrm{~mL} / \mathrm{min} ; \lambda=$ $220 \mathrm{~nm} ; \mathrm{t}_{\mathrm{R} 1}($ major $)=9.9 \mathrm{~min} ; \mathrm{t}_{\mathrm{R} 2}($ minor $)=7.6 \mathrm{~min}$. 

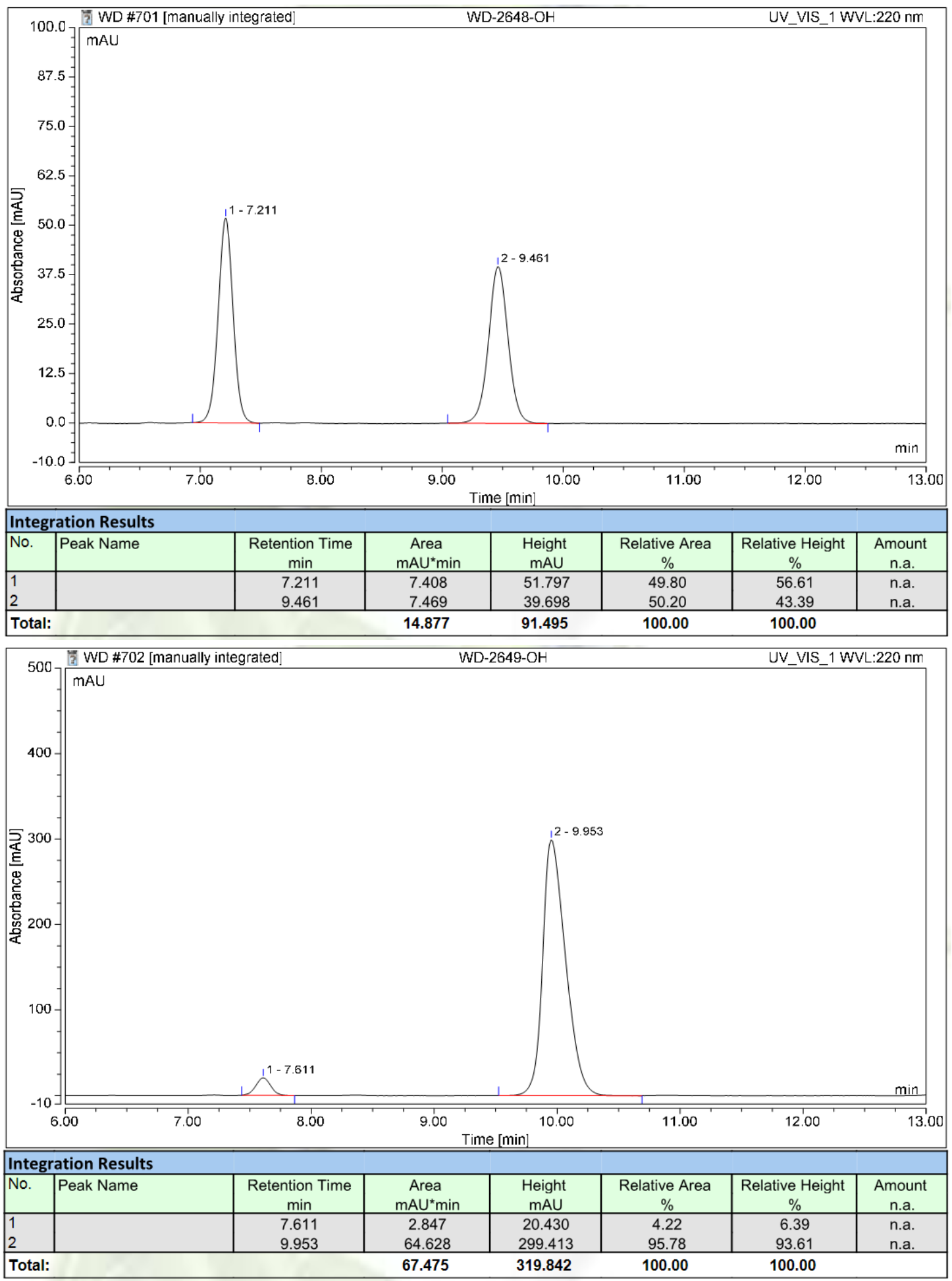<smiles>Cc1ccc([C@@H](O)CC(F)(F)F)cc1</smiles>

The title compound $\mathbf{3 b b}$ was synthesized according to General Procedure B, and it was purified by column chromatography on silica gel (51\% yield, 92:8 er, $31 \mathrm{mg}$, colourless oil). 
${ }^{1} \mathbf{H}$ NMR $\left(600 \mathrm{MHz}, \mathrm{CDCl}_{3}\right) \delta 7.25(\mathrm{~d}, J=8.4 \mathrm{~Hz}, 2 \mathrm{H}), 7.18(\mathrm{~d}, J=7.8 \mathrm{~Hz}, 2 \mathrm{H}), 5.11$ $(\mathrm{d}, J=9.0 \mathrm{~Hz}, 1 \mathrm{H}), 2.64-2.57(\mathrm{~m}, 1 \mathrm{H}), 2.41-2.34(\mathrm{~m}, 1 \mathrm{H}), 2.35(\mathrm{~s}, 3 \mathrm{H}), 2.17(\mathrm{~s}, 1 \mathrm{H})$; ${ }^{19} \mathbf{F}$ NMR $\left(565 \mathrm{MHz}, \mathrm{CDCl}_{3}\right) \delta$ 77.10- -77.30 (m, 6F), -185.53- -185.70 (m, 1F); ${ }^{13} \mathrm{C}$ NMR (151 MHz, $\left.\mathrm{CDCl}_{3}\right) . \delta 140.1,138.3,129.5,125.4,124.0-117.9(\mathrm{~m}), 91.4$ (dp, $J=$ 204.8, 32.3 Hz), 68.4 (d, $J=3.6 \mathrm{~Hz}), 38.1$ (d, $J=18.7 \mathrm{~Hz}), 21.1$.

HRMS: $\mathrm{m} / \mathrm{z}(\mathrm{ESI})$ calculated $[\mathrm{M}-\mathrm{OH}]^{+}: 287.0665$, found: $287.0681 .[\alpha]_{\mathrm{D}}{ }^{25}=51.919(c$ $\left.=0.25, \mathrm{CHCl}_{3}\right)$.

The enantiomeric excess of $\mathbf{3 b b}$ was determined by chiral HPLC analysis compared to the corresponding racemate alcohol.

Conditions: ChiralPak IB column; hexane $/{ }^{i} \mathrm{PrOH}=98: 2$; flow rate $=1.0 \mathrm{~mL} / \mathrm{min} ; \lambda=$ $220 \mathrm{~nm} ; \mathrm{t}_{\mathrm{R} 1}($ major $)=12.0 \mathrm{~min} ; \mathrm{t}_{\mathrm{R} 2}($ minor $)=9.2 \mathrm{~min}$.

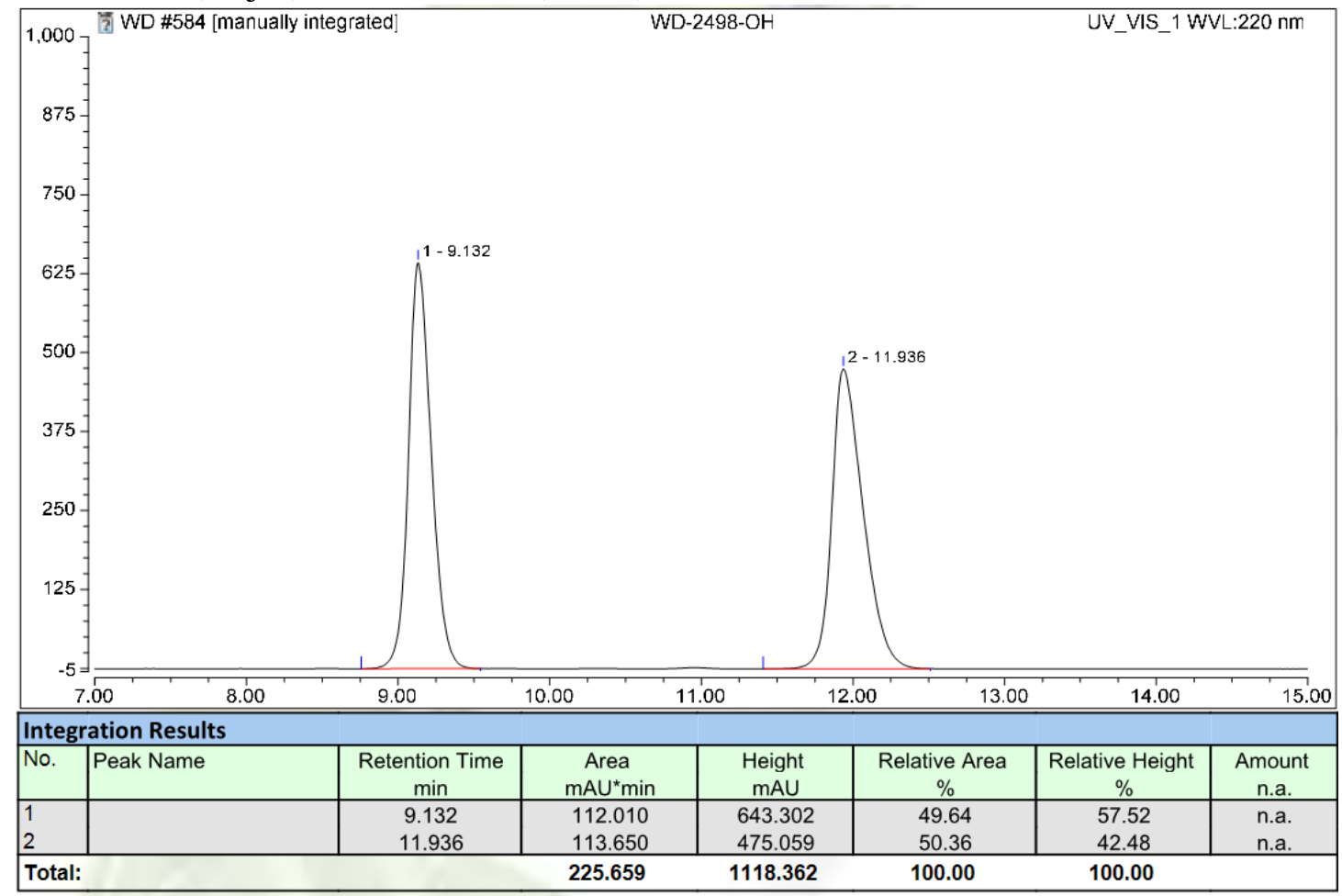




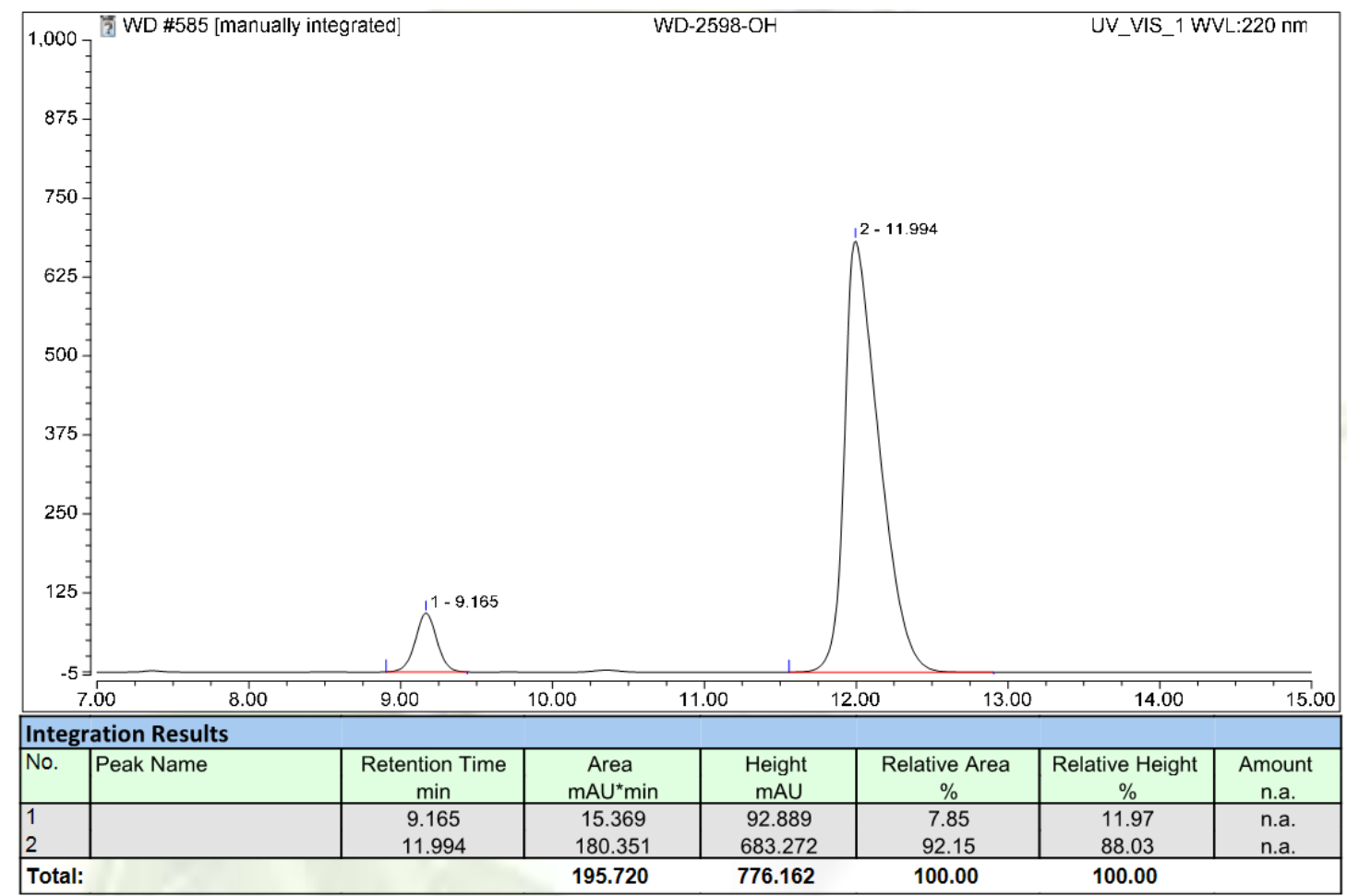<smiles>O[C@H](CC(F)(F)F)c1ccc(-c2ccccc2)cc1</smiles>

The title compound 3cc was synthesized according to General Procedure B, and it was purified by column chromatography on silica gel (43\% yield, 90:10 er, $53.1 \mathrm{mg}$, white solid).

${ }^{1} \mathbf{H}$ NMR $\left(600 \mathrm{MHz}, \mathrm{CDCl}_{3}\right) \delta$ 7.62-7.57 (m, 4H), 7.47-7.43 (m, 4H), 7.38-7.34 (m, 1H), 5.29-5.25 (m, 1H), 2.72-2.61 (m, 1H), 2.51-2.41 (m, 1H), $2.31(\mathrm{~s}, 1 \mathrm{H}) ;{ }^{19}$ F NMR $\left(565 \mathrm{MHz}, \mathrm{CDCl}_{3}\right) \delta-80.67--80.92(\mathrm{~m}, 3 \mathrm{~F}),-112.18--113.99(\mathrm{~m}, 2 \mathrm{~F}),-121.52-$ -121.62 (m, 2F), -121.88- -122.00 (m, 4F), -122.71- -122.81 (m, 2F), -123.55- -123.60 (m, 2F), -126.14- -126.19. (m, 2F); ${ }^{13}$ C NMR (151 MHz, CDCl3) $\delta$ 141.6, 141.4, $140.5,128.8,127.6,127.5,127.1,126.1,119.8-110.8(\mathrm{~m}), 67.7,39.9(\mathrm{t}, J=20.5 \mathrm{~Hz})$.

HRMS: $\mathrm{m} / \mathrm{z}(\mathrm{ESI})$ calculated $[\mathrm{M}-\mathrm{OH}]^{+}:$599.0662, found: $599.0657 .[\alpha]_{\mathrm{D}}{ }^{25}=8.717(c$ $\left.=0.25, \mathrm{CHCl}_{3}\right)$.

The enantiomeric excess of 3cc was determined by chiral HPLC analysis compared to the corresponding racemate alcohol.

Conditions: ChiralPak IG column; hexane $/{ }^{i} \mathrm{PrOH}=98: 2$; flow rate $=1.0 \mathrm{~mL} / \mathrm{min} ; \lambda=$ $220 \mathrm{~nm} ; \mathrm{t}_{\mathrm{R} 1}($ major $)=5.7 \mathrm{~min} ; \mathrm{t}_{\mathrm{R} 2}($ minor $)=5.2 \mathrm{~min}$. 

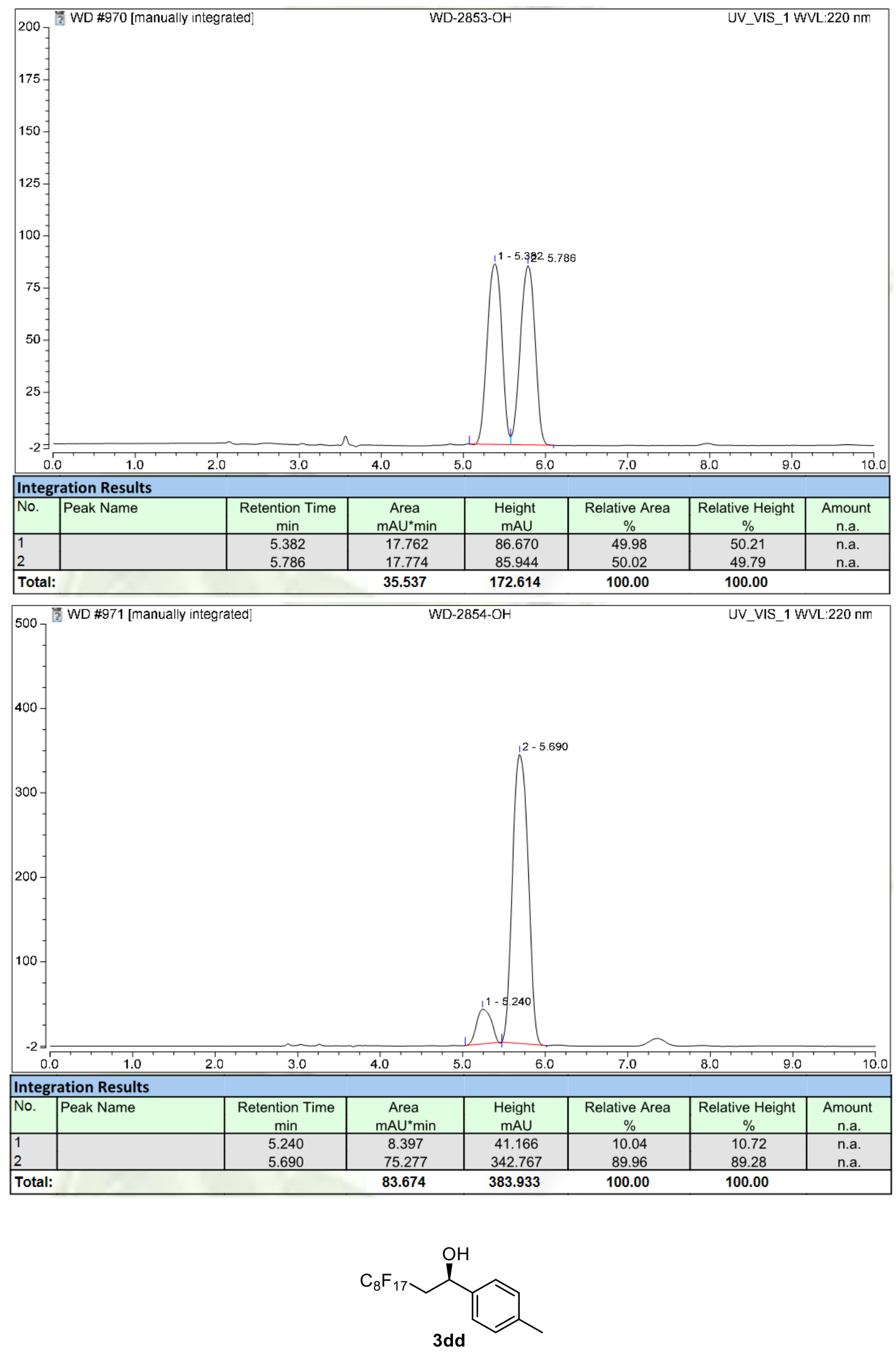

The title compound 3dd was synthesized according to General Procedure B, and it was purified by column chromatography on silica gel (79\% yield, 90:10 er, $87.1 \mathrm{mg}$, white solid). 
${ }^{1} \mathbf{H}$ NMR $\left(600 \mathrm{MHz}, \mathrm{CDCl}_{3}\right) \delta 7.27(\mathrm{~d}, J=7.8 \mathrm{~Hz}, 2 \mathrm{H}), 7.19(\mathrm{~d}, J=7.8 \mathrm{~Hz}, 2 \mathrm{H}), 5.17$ $(\mathrm{d}, J=8.4 \mathrm{~Hz}, 1 \mathrm{H}), 2.66-2.56(\mathrm{~m}, 1 \mathrm{H}), 2.46-2.35(\mathrm{~m}, 2 \mathrm{H}), 2.35(\mathrm{~s}, 3 \mathrm{H}), 2.20(\mathrm{~s}, 1 \mathrm{H})$; ${ }^{19} \mathbf{F}$ NMR $\left(565 \mathrm{MHz}, \mathrm{CDCl}_{3}\right) \delta-80.72--80.78(\mathrm{~m}, 3 \mathrm{~F}),-112.20--114.08(\mathrm{~m}, 2 \mathrm{~F})$, -121.51- -121.59 (m, 2F), -121.86- -122.00 (m, 4F), -122.67- -122.77 (m, 2F), -123.58- -123.63 (m, 2F), -126.09- -126.15 (m, 2F); $\left.{ }^{13} \mathbf{C ~ N M R ~ ( 1 5 1 ~ M H z , ~} \mathrm{CDCl}_{3}\right) \delta$ 139.8, 138.3, 129.5, 125.6, 120.0-106.2 (m), 67.8, $39.8(\mathrm{t}, J=20.4 \mathrm{~Hz}), 21.1$.

MS: (EI) $[\mathrm{M}]^{+}: 554.10 .[\alpha]_{\mathrm{D}}{ }^{25}=15.058\left(c=0.25, \mathrm{CHCl}_{3}\right)$.

The enantiomeric excess of 3dd was determined by chiral HPLC analysis compared to the corresponding racemate alcohol.

Conditions: ChiralPak IB column; hexane $/{ }^{i} \mathrm{PrOH}=98: 2$; flow rate $=1.0 \mathrm{~mL} / \mathrm{min} ; \lambda=$ $220 \mathrm{~nm} ; \mathrm{t}_{\mathrm{R} 1}$ (major) $=10.0 \mathrm{~min} ; \mathrm{t}_{\mathrm{R} 2}($ minor $)=8.5 \mathrm{~min}$.

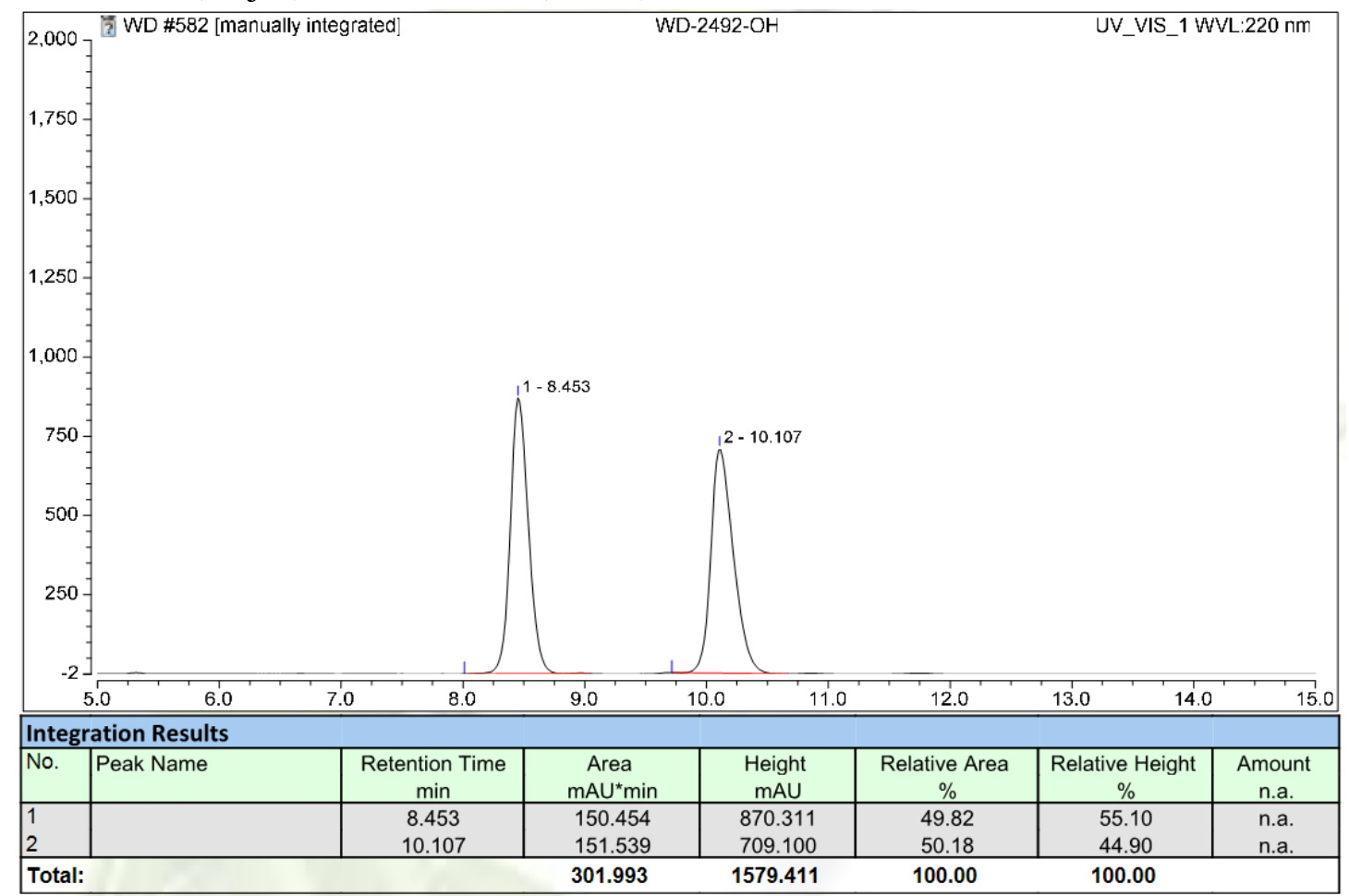




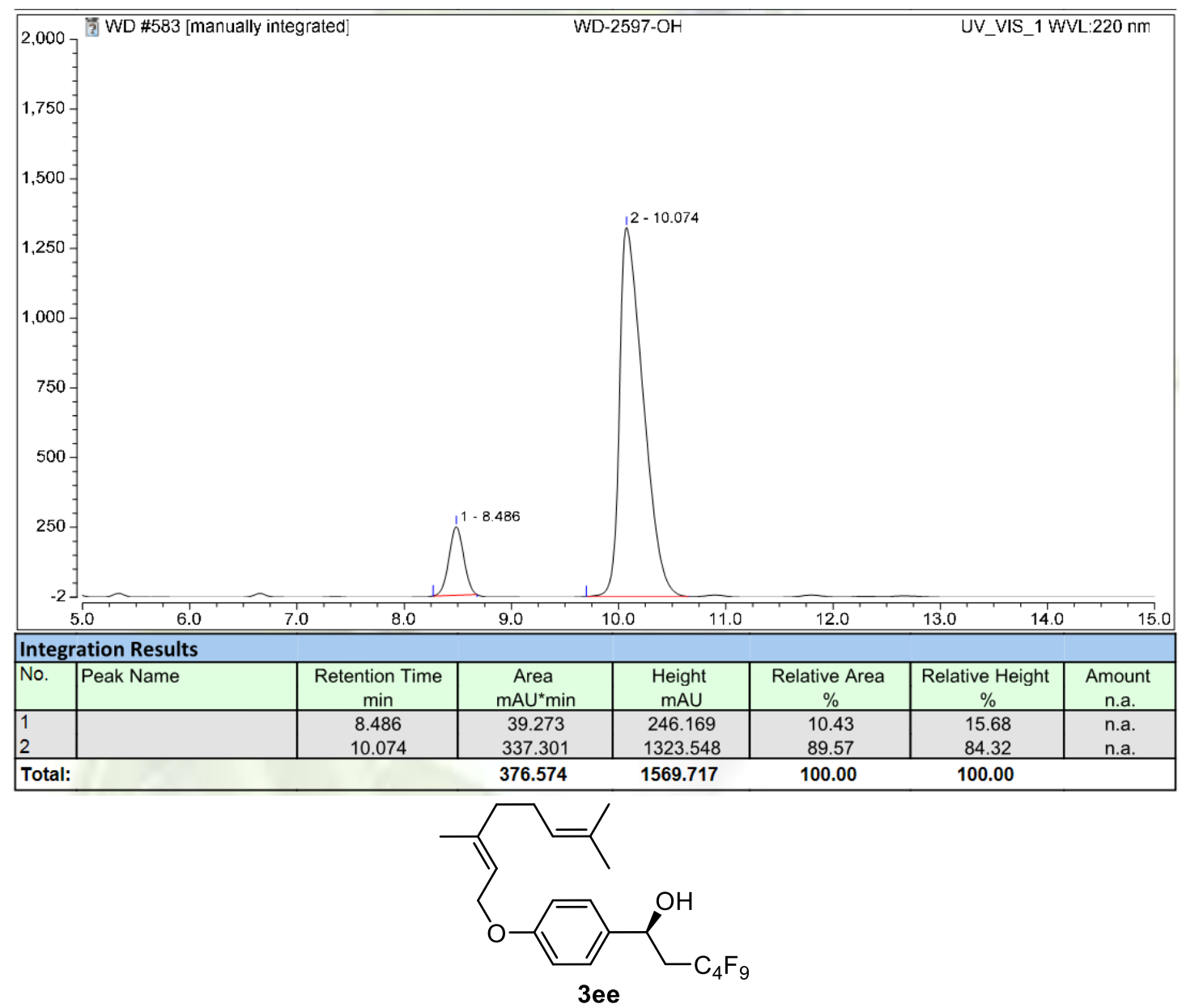

The title compound 3ee was synthesized according to General Procedure B, and it was purified by column chromatography on silica gel (39\% yield, 90:10 er, $38.6 \mathrm{mg}$, colourless oil).

${ }^{1} \mathbf{H}$ NMR $\left(600 \mathrm{MHz}, \mathrm{CDCl}_{3}\right) \delta 7.30(\mathrm{~d}, J=9.0 \mathrm{~Hz}, 2 \mathrm{H}), 6.92(\mathrm{~d}, J=8.4 \mathrm{~Hz}, 2 \mathrm{H}), 5.48$ $(\mathrm{t}, J=6.6 \mathrm{~Hz}, 1 \mathrm{H}), 5.16(\mathrm{dt}, J=8.4,3.0 \mathrm{~Hz}, 1 \mathrm{H}), 5.09(\mathrm{t}, J=6.0 \mathrm{~Hz}, 1 \mathrm{H}), 4.54(\mathrm{~d}, J=$ $6.6 \mathrm{~Hz}, 2 \mathrm{H}), 2.67-2.56(\mathrm{~m}, 1 \mathrm{H}), 2.44-2.35(\mathrm{~m}, 1 \mathrm{H}), 2.16-2.05(\mathrm{~m}, 5 \mathrm{H}), 1.74(\mathrm{~s}, 3 \mathrm{H})$, $1.68(\mathrm{~s}, 3 \mathrm{H}), 1.61(\mathrm{~s}, 3 \mathrm{H}) ;{ }^{19} \mathbf{F}$ NMR $\left(565 \mathrm{MHz}, \mathrm{CDCl}_{3}\right) \delta-81.00--81.06(\mathrm{~m}, 3 \mathrm{~F})$, -112.48- -114.41 (m, 2F), -124.55- -124.61 (m, 2F), -125.87- -125.95 (m, 2F); ${ }^{13} \mathrm{C}$ NMR $\left(151 \mathrm{MHz}, \mathrm{CDCl}_{3}\right) \delta 158.9,141.4,134.7$ 131.8, 126.9, 123.7, 119.3, 118.3-116.0 (m), 115.0, 110.0, 67.6, 64.9, 39.8-39.5 (m), 26.3, 25.7, 17.7, 16.6.

HRMS: $\mathrm{m} / \mathrm{z}(\mathrm{ESI})$ calculated $[\mathrm{M}+\mathrm{Na}]^{+}: 515.1603$, found: $515.1598 .[\alpha]_{\mathrm{D}}{ }^{25}=11.650(c$ $\left.=0.25, \mathrm{CHCl}_{3}\right)$.

The enantiomeric excess of 3ee was determined by chiral HPLC analysis compared to the corresponding racemate alcohol.

Conditions: ChiralPak IG column; hexane $/{ }^{i} \mathrm{PrOH}=99: 1$; flow rate $=1.0 \mathrm{~mL} / \mathrm{min} ; \lambda=$ $220 \mathrm{~nm} ; \mathrm{t}_{\mathrm{R} 1}$ (major) $=18.2 \mathrm{~min} ; \mathrm{t}_{\mathrm{R} 2}$ (minor) $=14.2 \mathrm{~min}$. 

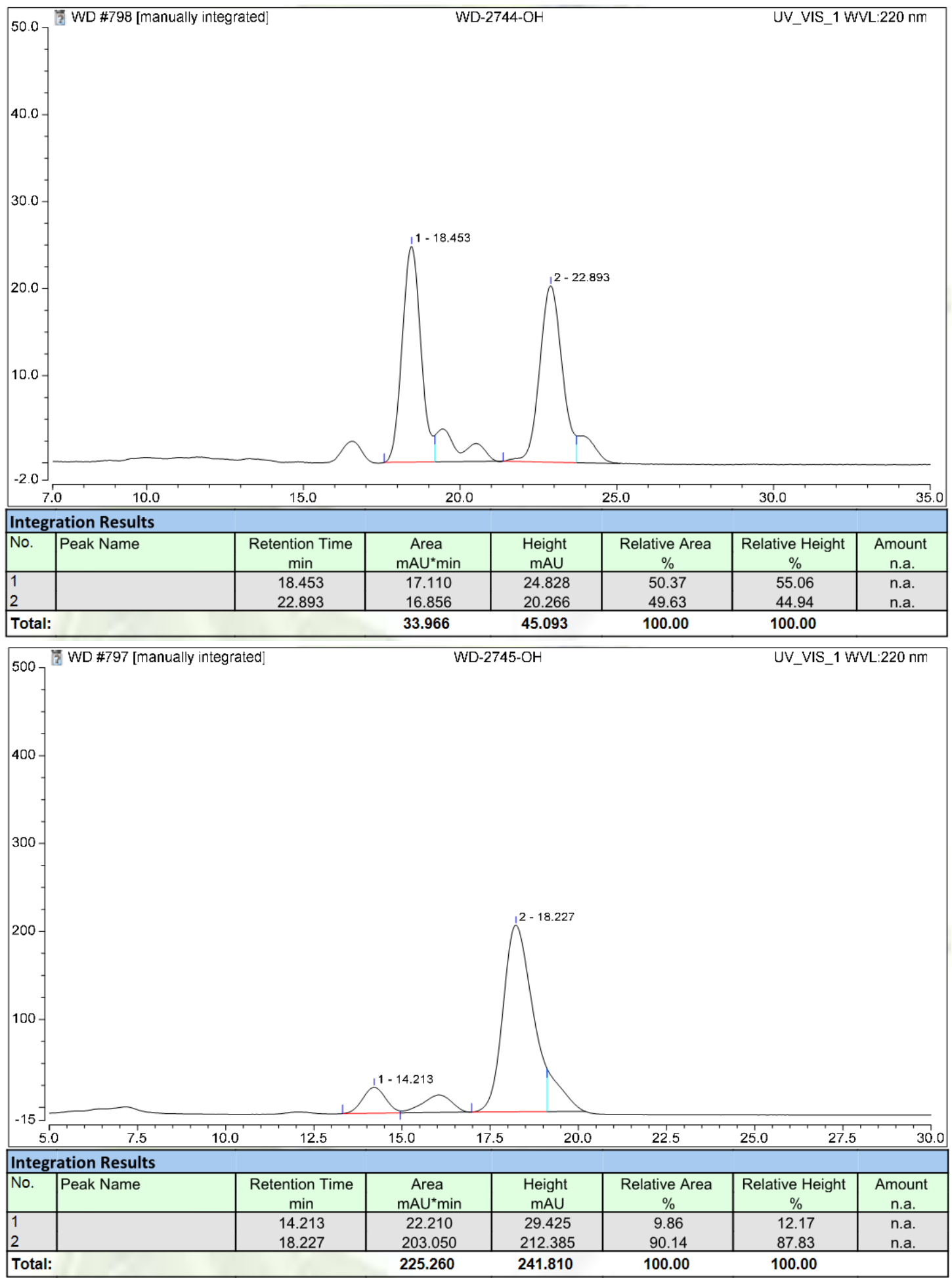<smiles>CCOC(=O)C(C)(C)Oc1ccc([C@@H](O)CC(F)(F)F)cc1</smiles>

The title compound $\mathbf{3 f f}$ was synthesized according to General Procedure B, and it was purified by column chromatography on silica gel (61\% yield, 91:9 er, $57.5 \mathrm{mg}$, colourless oil). 
${ }^{1} \mathbf{H}$ NMR $\left(400 \mathrm{MHz}, \mathrm{CDCl}_{3}\right) \delta 7.26(\mathrm{~d}, J=8.4 \mathrm{~Hz}, 2 \mathrm{H}), 6.84(\mathrm{~d}, J=8.4 \mathrm{~Hz}, 2 \mathrm{H}), 5.21$ $-5.10(\mathrm{~m}, 1 \mathrm{H}), 4.23(\mathrm{q}, J=7.2 \mathrm{~Hz}, 2 \mathrm{H}), 2.68-2.52(\mathrm{~m}, 1 \mathrm{H}), 2.45-2.30(\mathrm{~m}, 1 \mathrm{H}) 1.60(\mathrm{~s}$, $6 \mathrm{H}), 1.25(\mathrm{t}, J=7.2 \mathrm{~Hz}, 3 \mathrm{H}) ;{ }^{19} \mathbf{F}$ NMR $\left(565 \mathrm{MHz}, \mathrm{CDCl}_{3}\right) \delta-81.42--81.50(\mathrm{~m}, 3 \mathrm{~F})$, -112.72- -114.62 (m, 2F), -124.90 (s, 2F), -126.28 (s, 2F); ${ }^{13} \mathrm{C}$ NMR (101 MHz, $\left.\mathrm{CDCl}_{3}\right) \delta 174.2,155.5,136.2,126.6,119.2,118.8-101.2(\mathrm{~m}), 79.2,67.5,61.5,39.7(\mathrm{t}$, $J=20.8 \mathrm{~Hz}$ ), 25.3, 14.0 .

HRMS: $\mathrm{m} / \mathrm{z}(\mathrm{ESI})$ calculated $\left[\mathrm{M}+\mathrm{NH}_{4}\right]^{+}: 488.1478$, found: 488.1480 . $[\alpha]_{\mathrm{D}}^{25}=10.382$ $\left(c=0.25, \mathrm{CHCl}_{3}\right)$.

The enantiomeric excess of $\mathbf{3 f f}$ was determined by chiral HPLC analysis compared to the corresponding racemate alcohol.

Conditions: ChiralPak IG column; hexane $/{ }^{i} \mathrm{PrOH}=98: 2$; flow rate $=1.0 \mathrm{~mL} / \mathrm{min} ; \lambda=$ $220 \mathrm{~nm} ; \mathrm{t}_{\mathrm{R} 1}$ (major) $=19.3 \mathrm{~min} ; \mathrm{t}_{\mathrm{R} 2}($ minor $)=16.8 \mathrm{~min}$. 

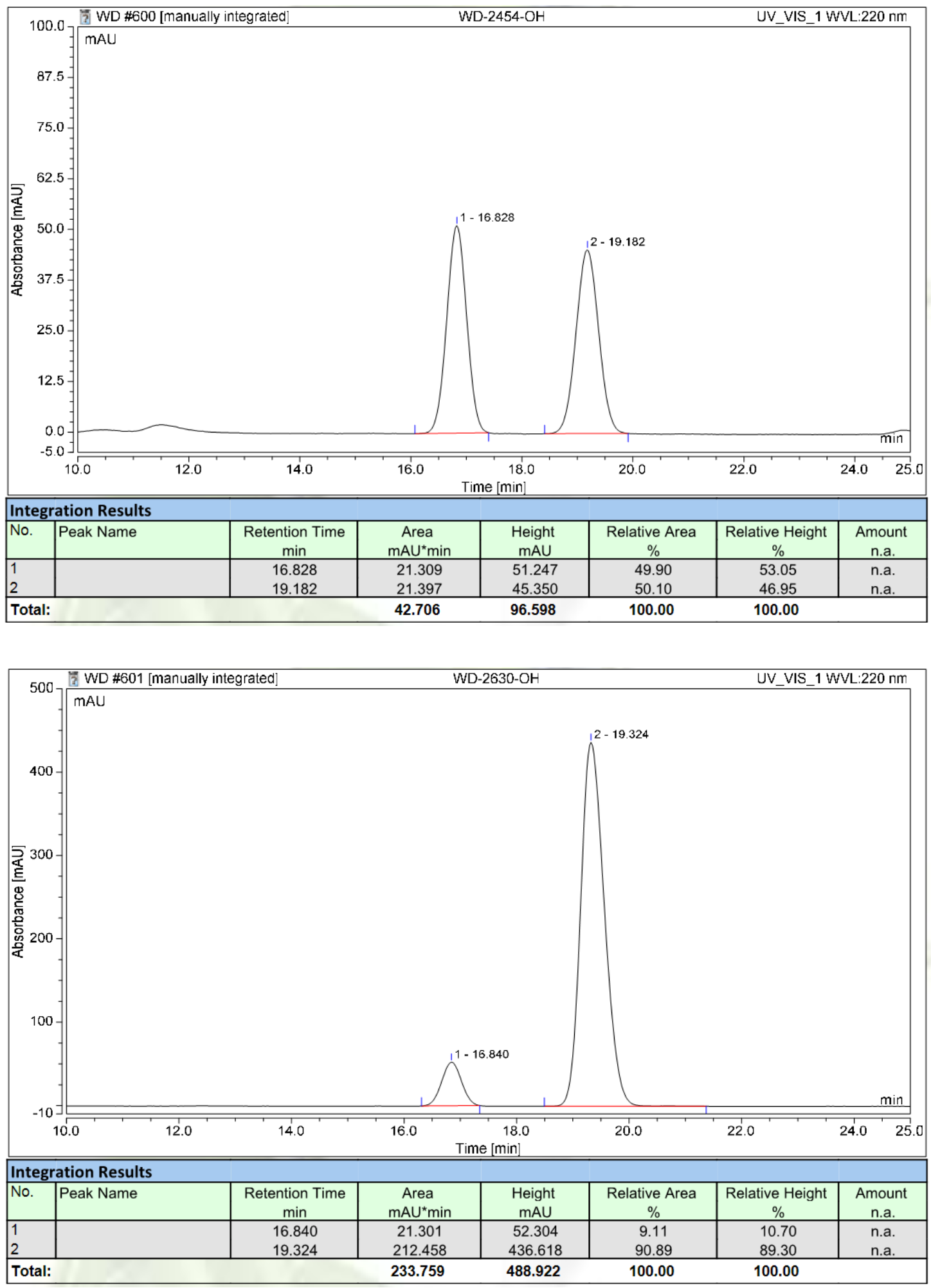<smiles>CN1CN=C2C(=O)N(CCCOc3cccc(C(CC(F)(F)F)CC(F)(F)F)c3)C(=O)N(C)C21</smiles>

3gg-B 
The title compound 3gg-B was synthesized according to General Procedure B, and it was purified by column chromatography on silica gel (36\% yield, 91:9 er, $49.8 \mathrm{mg}$, white solid).

${ }^{1} \mathbf{H}$ NMR $\left(600 \mathrm{MHz}, \mathrm{CDCl}_{3}\right) \delta 7.51(\mathrm{~s}, 1 \mathrm{H}), 7.16(\mathrm{t}, J=7.8 \mathrm{~Hz}, 1 \mathrm{H}), 6.80(\mathrm{~d}, J=7.8$ $\mathrm{Hz}, 1 \mathrm{H}), 6.72-6.70(\mathrm{~m}, 1 \mathrm{H}), 6.65(\mathrm{dd}, J=7.8,1.8 \mathrm{~Hz}, 1 \mathrm{H}), 4.24(\mathrm{t}, J=7.2 \mathrm{~Hz}, 2 \mathrm{H})$, 4.07-4.03 (m, 2H), $3.95(\mathrm{~s}, 3 \mathrm{H}), 3.57(\mathrm{~s}, 3 \mathrm{H}), 2.82-2.71(\mathrm{~m}, 1 \mathrm{H}), 2.67(\mathrm{dd}, J=10.8$, $4.2 \mathrm{~Hz}, 1 \mathrm{H}), 2.35-2.25(\mathrm{~m}, 1 \mathrm{H}), 2.19-2.12(\mathrm{~m}, 2 \mathrm{H}), 1.20(\mathrm{~s}, 6 \mathrm{H}), 1.16(\mathrm{~s}, 6 \mathrm{H}) ;{ }^{19}$ F NMR $\left(565 \mathrm{MHz}, \mathrm{CDCl}_{3}\right) \delta-80.97--81.06(\mathrm{~m}, 3 \mathrm{~F}),-113.55--113.64(\mathrm{~m}, 2 \mathrm{~F}),-124.52-$ $-124.57(\mathrm{~m}, 2 \mathrm{~F}),-125.91--125.96(\mathrm{~m}, 2 \mathrm{~F}) ;{ }^{13} \mathrm{C}$ NMR $\left(151 \mathrm{MHz}, \mathrm{CDCl}_{3}\right) \delta 159.1$, 155.3, 151.5, 148.8, 142.0, 141.4, 129.5, 120.3, 114.3, 112.1, 107.7, 83.9, 66.0, 39.0, $33.5,29.7,27.9,24.8,24.6,24.4(\mathrm{~d}, J=6.6 \mathrm{~Hz})$.

HRMS: $\mathrm{m} / \mathrm{z}($ ESI $)$ calculated $[\mathrm{M}+\mathrm{H}]^{+}: 689.2551$, found: $689.2464 .[\alpha]_{\mathrm{D}}{ }^{25}=8.678(c=$ $\left.0.25, \mathrm{CHCl}_{3}\right)$.

The enantiomeric excess of 3gg-B was determined by chiral HPLC analysis compared to the corresponding racemate alcohol.

Conditions: ChiralPak IC column; hexane $/{ }^{i} \mathrm{PrOH}=85: 15$; flow rate $=1.0 \mathrm{~mL} / \mathrm{min} ; \lambda=$ $220 \mathrm{~nm} ; \mathrm{t}_{\mathrm{R} 1}$ (major) $=67.0 \mathrm{~min} ; \mathrm{t}_{\mathrm{R} 2}$ (minor) $=63.1 \mathrm{~min}$.

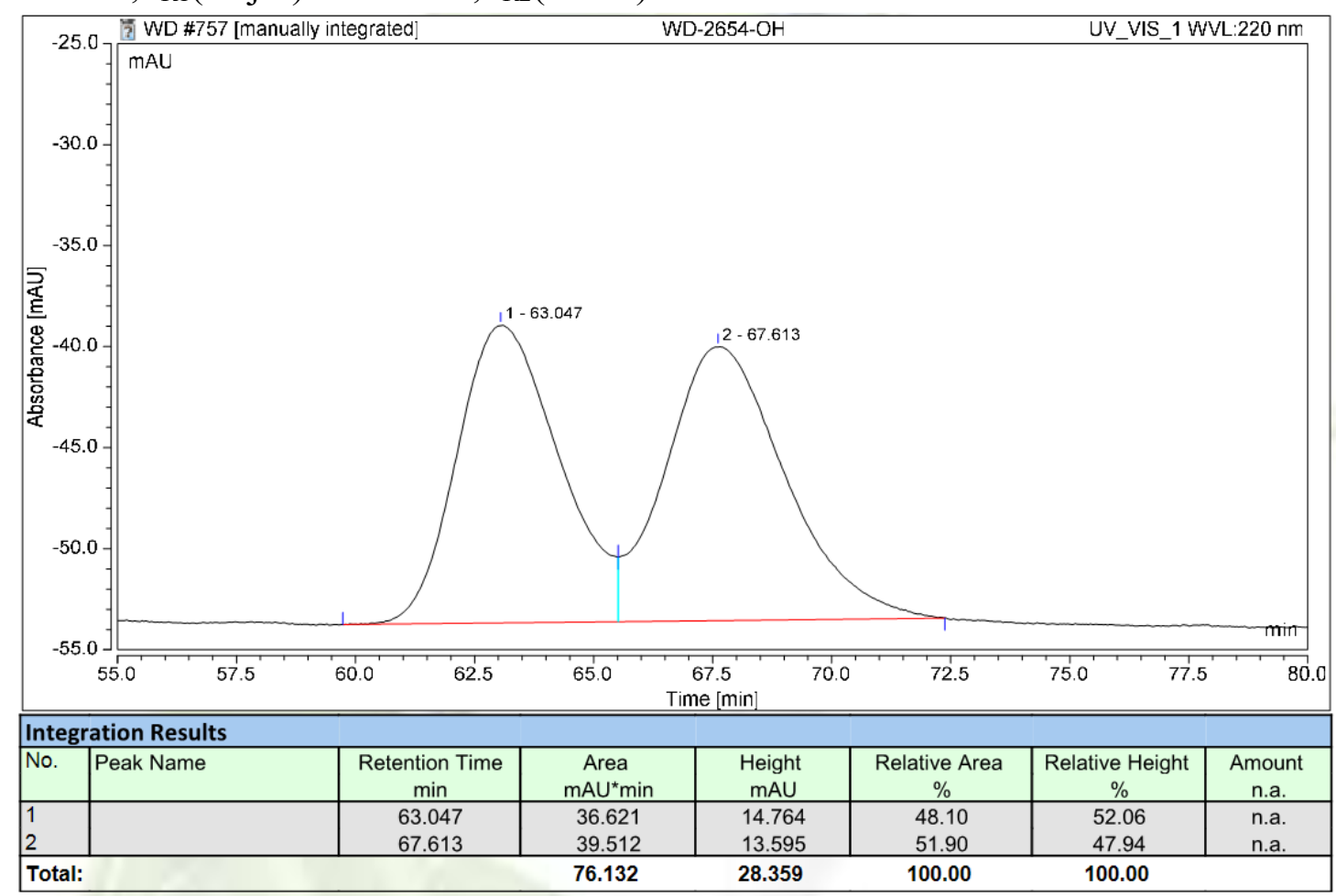




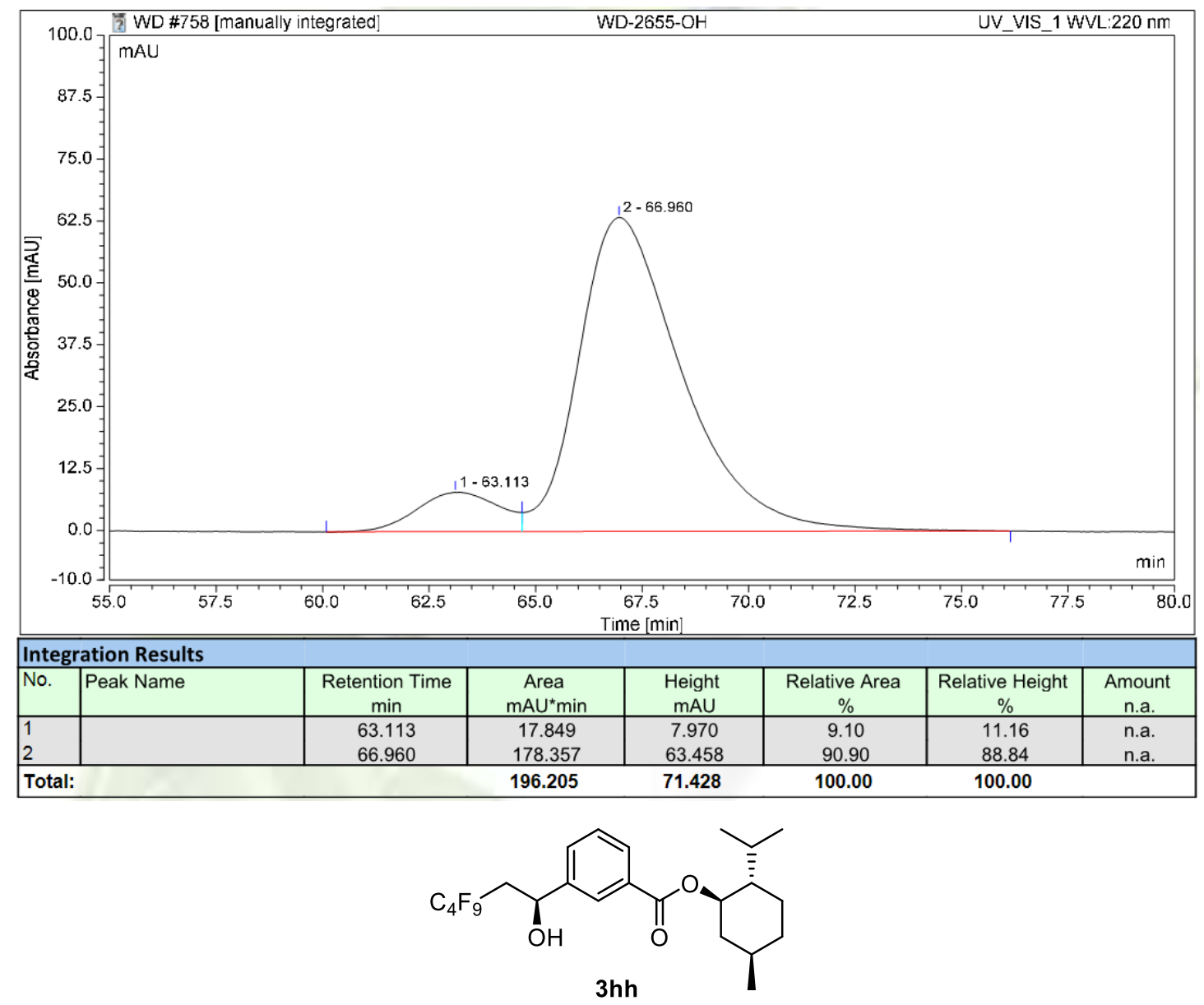

The title compound 3hh was synthesized according to General Procedure B, and it was purified by column chromatography on silica gel (69\% yield, 86:14 $d r, 72.3 \mathrm{mg}$, white solid).

${ }^{1} \mathbf{H}$ NMR $\left(400 \mathrm{MHz}, \mathrm{CDCl}_{3}\right) \delta 8.06(\mathrm{~s}, 1 \mathrm{H}), 7.99(\mathrm{~d}, J=7.6 \mathrm{~Hz}, 1 \mathrm{H}), 7.60(\mathrm{~d}, J=7.6$ $\mathrm{Hz}, 1 \mathrm{H}), 7.46(\mathrm{t}, J=7.6 \mathrm{~Hz}, 1 \mathrm{H}), 5.34-5.24(\mathrm{~m}, 1 \mathrm{H}), 4.95(\mathrm{td}, J=10.8,4.0 \mathrm{~Hz}, 1 \mathrm{H})$, 2.72-2.56 (m, 2H), 2.49-2.35 (m, $1 \mathrm{H}), 2.11(\mathrm{~d}, J=11.6 \mathrm{~Hz}, 1 \mathrm{H}), 1.98-1.88(\mathrm{~m}, 1 \mathrm{H})$, $1.73(\mathrm{~d}, J=11.2 \mathrm{~Hz}, 2 \mathrm{H}), 1.64-1.51(\mathrm{~m}, 2 \mathrm{H}), 1.30-1.21(\mathrm{~m}, 1 \mathrm{H}), 1.19-1.07(\mathrm{~m}, 2 \mathrm{H})$, $0.93(\mathrm{t}, J=6.4 \mathrm{~Hz}, 6 \mathrm{H}), 0.79(\mathrm{~d}, J=6.8 \mathrm{~Hz}, 3 \mathrm{H}) ;{ }^{19} \mathbf{F}$ NMR $\left(565 \mathrm{MHz}, \mathrm{CDCl}_{3}\right) \delta$ -81.02- -81.10 (m, 3F), -112.33- -114.22 (m, 2F), -124.51- -124.57 (m, 2F), -125.90-125.98 (m, 2F); ${ }^{13} \mathrm{C}$ NMR $\left(101 \mathrm{MHz}, \mathrm{CDCl}_{3}\right) \delta 165.8,143.1,131.5,129.9,129.5$, 128.9, 126.8, 122.6-108.5 (m), 75.2, 67.5, 47.2, 40.9, 39.8 (t, $J=20.6$ Hz), 34.3, 31.4, 26.6, 23.7, 22.0, 20.7, 16.5.

HRMS: $\mathrm{m} / \mathrm{z}(\mathrm{ESI})$ calculated $[\mathrm{M}+\mathrm{Na}]^{+}: 545.1709$, found: $545.1712 .[\alpha]_{\mathrm{D}}{ }^{25}=-25.625$ $\left(c=0.25, \mathrm{CHCl}_{3}\right)$.

The enantiomeric excess of $\mathbf{3 h h}$ was determined by chiral HPLC analysis compared to the corresponding racemate alcohol.

Conditions: ChiralPak IB column; hexane $/{ }^{i} \mathrm{PrOH}=98: 2$; flow rate $=1.0 \mathrm{~mL} / \mathrm{min} ; \lambda=$ $220 \mathrm{~nm} ; \mathrm{t}_{\mathrm{R} 1}$ (major) $=10.5 \mathrm{~min} ; \mathrm{t}_{\mathrm{R} 2}($ minor $)=6.7 \mathrm{~min}$. 

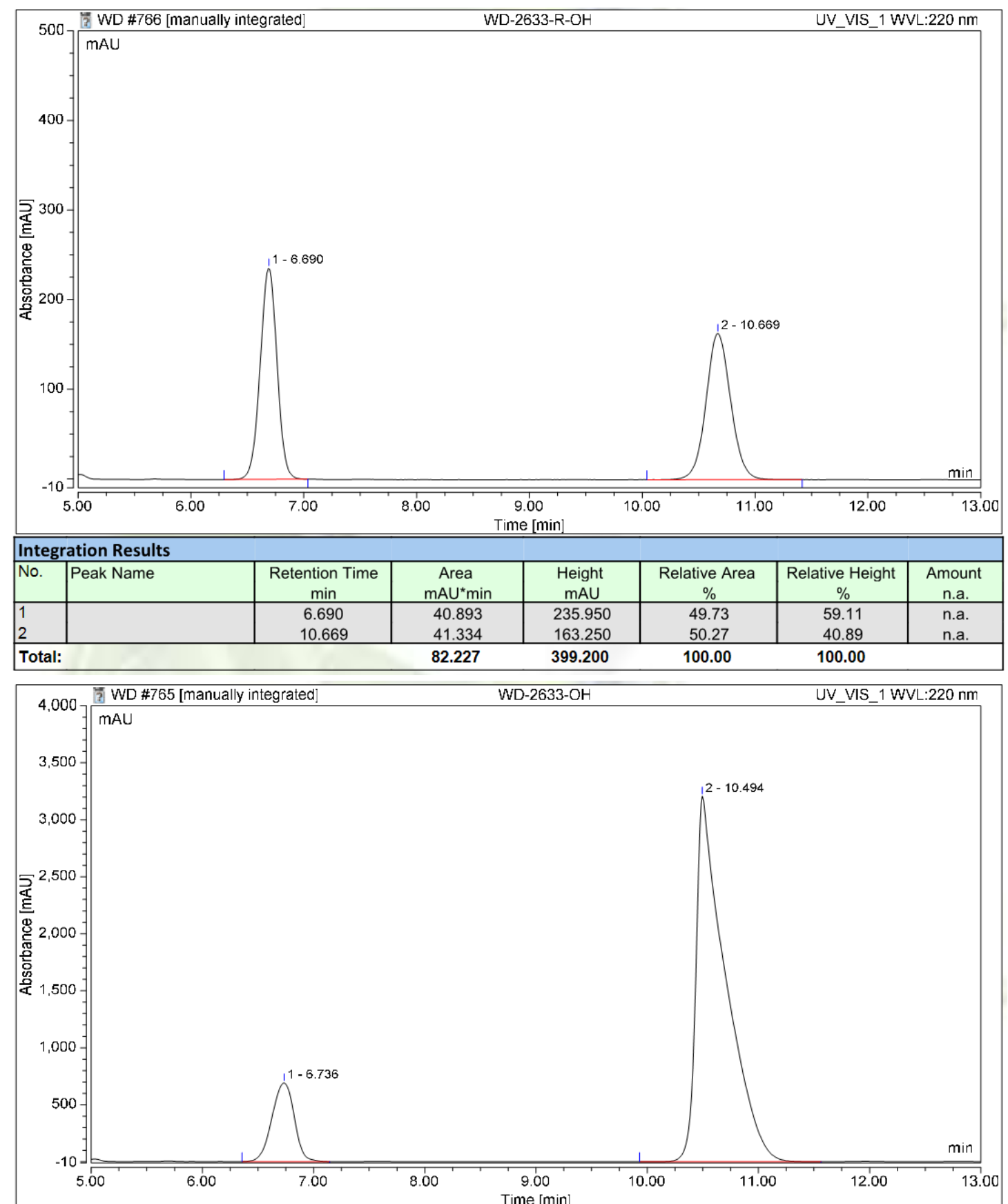

\begin{tabular}{|c|c|c|c|c|c|c|c|}
\hline \multicolumn{8}{|c|}{ Integration Results } \\
\hline No. & Peak Name & $\begin{array}{l}\text { Retention Time } \\
\text { min }\end{array}$ & $\begin{array}{c}\text { Area } \\
\mathrm{mAU}^{*} \min \end{array}$ & $\begin{array}{c}\text { Height } \\
\text { mAU }\end{array}$ & $\begin{array}{c}\text { Relative Area } \\
\% \\
\end{array}$ & $\begin{array}{c}\text { Relative Height } \\
\%\end{array}$ & $\begin{array}{c}\text { Amount } \\
\text { n.a. }\end{array}$ \\
\hline $\begin{array}{l}1 \\
2 \\
\end{array}$ & & $\begin{array}{c}6.736 \\
10.494 \\
\end{array}$ & $\begin{array}{l}160.486 \\
987.126 \\
\end{array}$ & \begin{tabular}{|c|}
690.015 \\
3205.668 \\
\end{tabular} & $\begin{array}{l}13.98 \\
86.02 \\
\end{array}$ & $\begin{array}{l}17.71 \\
82.29 \\
\end{array}$ & $\begin{array}{l}\text { n.a. } \\
\text { n.a. }\end{array}$ \\
\hline \multicolumn{3}{|c|}{ Total: } & 1147.612 & 3895.684 & 100.00 & 100.00 & \\
\hline
\end{tabular}<smiles>COc1ccc2c(CC(=O)Oc3cccc(C(Cc4ccccc4)CC(F)(F)F)c3)c(C)n(C(=O)c3ccc(Cl)cc3)c2c1</smiles> 
The title compound 3ii-B was synthesized according to General Procedure B, and it was purified by column chromatography on silica gel (54\% yield, 90:10 er, $87.2 \mathrm{mg}$, white solid).

${ }^{1} \mathbf{H}$ NMR $\left(600 \mathrm{MHz}, \mathrm{CDCl}_{3}\right) \delta 7.68(\mathrm{~d}, J=9.0 \mathrm{~Hz}, 2 \mathrm{H}), 7.48(\mathrm{~d}, J=7.8 \mathrm{~Hz}, 1 \mathrm{H}), 7.29$ -7.25 (m, 1H), 7.11-7.05 (m, 2H), 6.97-6.89 (m, 3H), $6.70(\mathrm{dd}, J=9.0,2.4 \mathrm{~Hz}, 1 \mathrm{H})$, $3.90(\mathrm{~s}, 2 \mathrm{H}), 3.84(\mathrm{~s}, 3 \mathrm{H}), 2.80-2.71(\mathrm{~m}, 2 \mathrm{H}), 2.46(\mathrm{~s}, 3 \mathrm{H}), 2.34-2.27(\mathrm{~m}, 1 \mathrm{H}), 1.16(\mathrm{~s}$, 6H), $1.14(\mathrm{~s}, 6 \mathrm{H}) ;{ }^{19} \mathbf{F}$ NMR $\left(565 \mathrm{MHz}, \mathrm{CDCl}_{3}\right) \delta-81.07--81.15(\mathrm{~m}, 3 \mathrm{~F}),-112.94-$ -114.40 (m, 2F), -124.20- -124.90 (m, 2F), -125.73- -126.39 (m, 2F); ${ }^{13}$ C NMR (151 $\left.\mathrm{MHz}, \mathrm{CDCl}_{3}\right) \delta 169.1,168.2,156.1,150.9,142.2,139.3,136.2,133.8,131.2,130.8$, $130.5,129.6,125.6,121.0,119.2,115.0,112.0,111.8,101.2,84.1,55.6,33.3(\mathrm{t}, J=$ 21.6 Hz), 30.5, 24.7, 24.3 (d, J = 7.7 Hz), 13.4.

HRMS: $\mathrm{m} / \mathrm{z}(\mathrm{ESI})$ calculated $[\mathrm{M}+2 \mathrm{H}]^{+}:$807.2170, found: $807.2184 .[\alpha]_{\mathrm{D}}{ }^{25}=10.897(\mathrm{c}$ $\left.=0.25, \mathrm{CHCl}_{3}\right)$.

The enantiomeric excess of 3ii-B was determined by chiral HPLC analysis compared to the corresponding racemate alcohol.

Conditions: ChiralPak IG column; hexane $/{ }^{i} \mathrm{PrOH}=97: 3$; flow rate $=1.0 \mathrm{~mL} / \mathrm{min} ; \lambda=$ $220 \mathrm{~nm} ; \mathrm{t}_{\mathrm{R} 1}$ (major) $=19.6 \mathrm{~min} ; \mathrm{t}_{\mathrm{R} 2}$ (minor) $=13.7 \mathrm{~min}$.

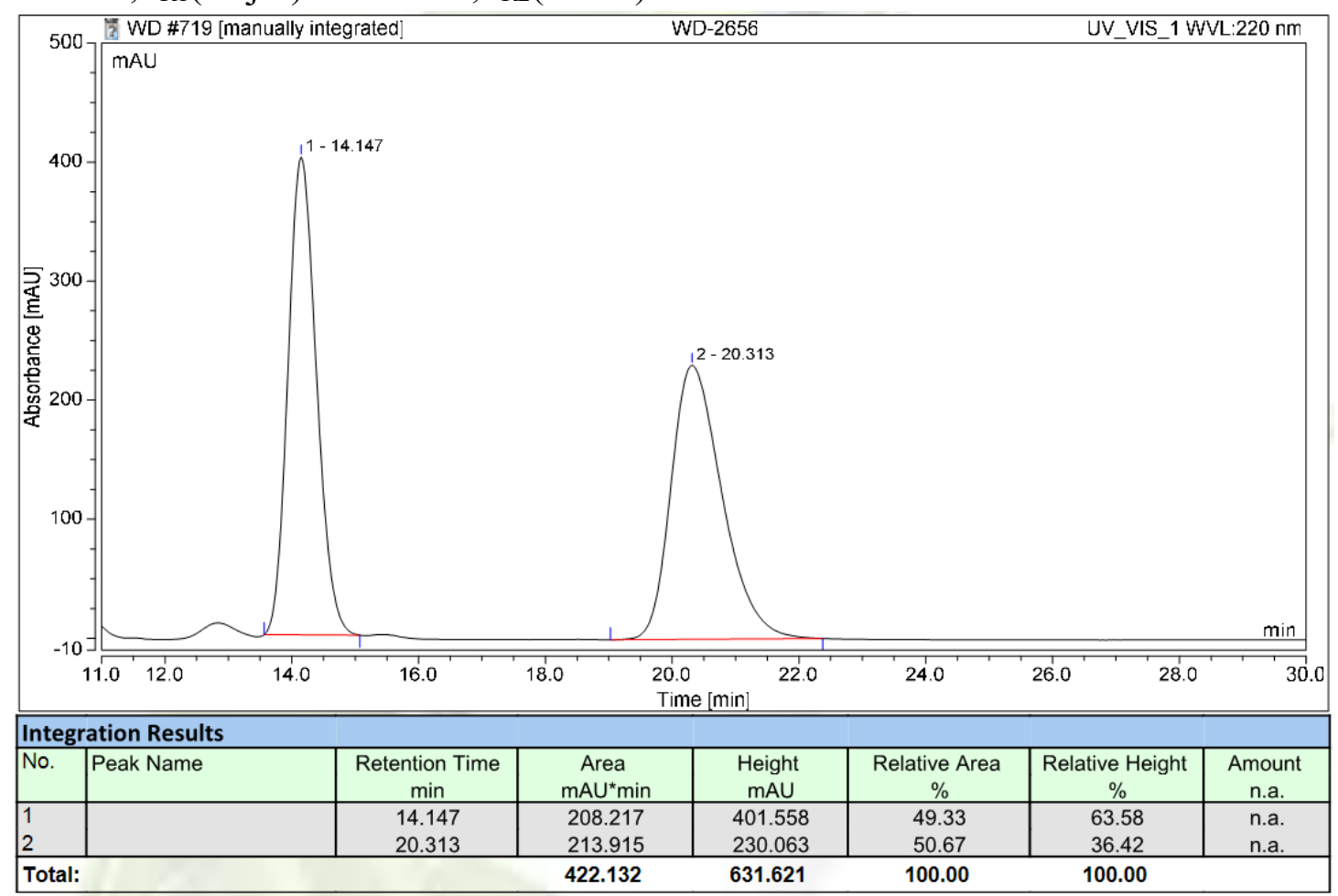




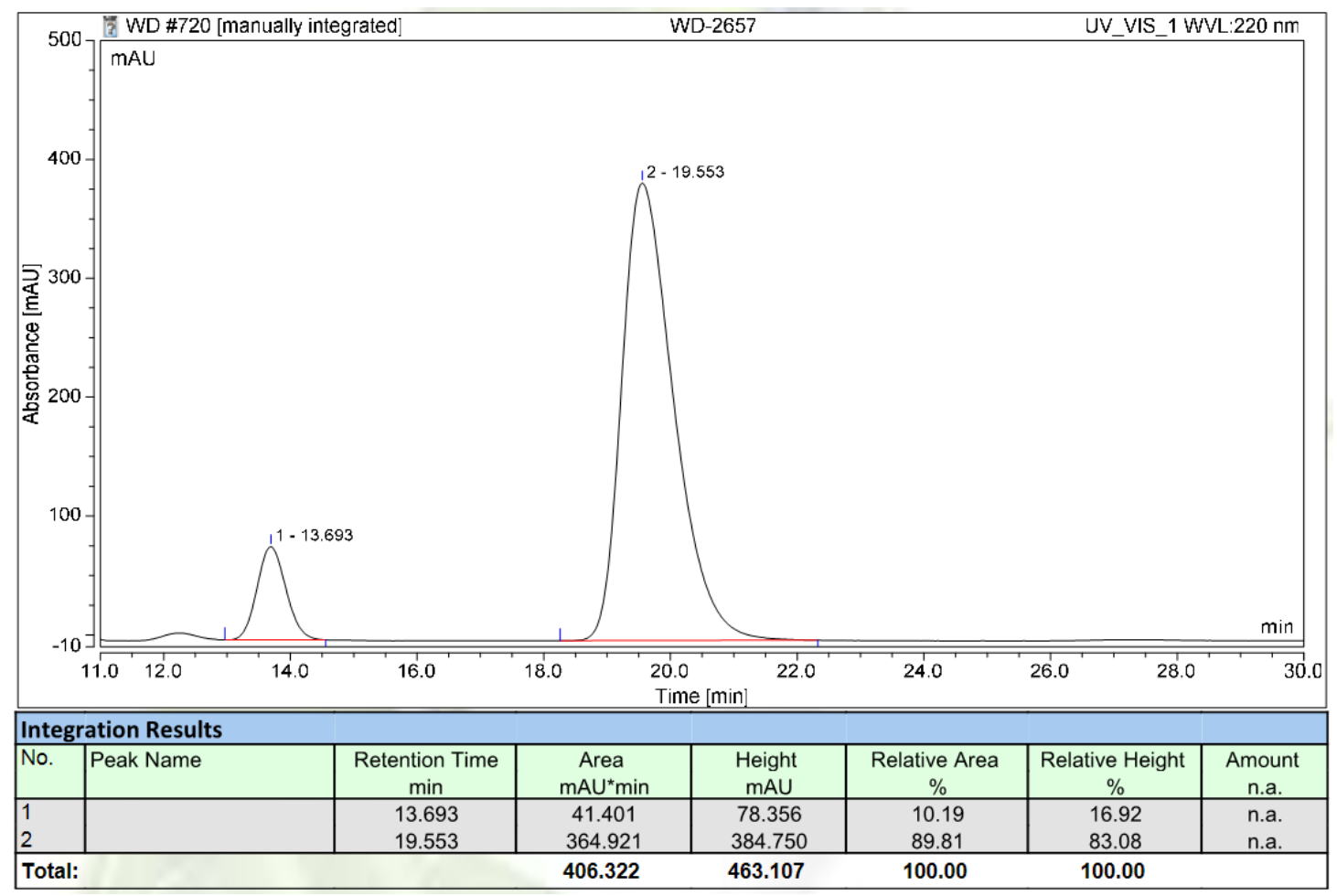<smiles>C[C@]12CCC3c4ccc(OC(=O)c5cccc([C@@H](CC(F)(F)F)c6ccccc6)c5)cc4CC[C@H]3C1CCC2=O</smiles>

The title compound 3jj-B was synthesized according to General Procedure B, and it was purified by column chromatography on silica gel (68\% yield, 91:9 $d r, 101.5 \mathrm{mg}$, white solid).

${ }^{1} \mathbf{H}$ NMR $\left(400 \mathrm{MHz}, \mathrm{CDCl}_{3}\right) \delta 8.22(\mathrm{~s}, 1 \mathrm{H}), 8.08-8.06(\mathrm{~m}, 1 \mathrm{H}), 8.04-8.02(\mathrm{~m}, 1 \mathrm{H})$, 7.53-7.51 (m, 1H), $7.44(\mathrm{t}, J=7.8 \mathrm{~Hz}, 1 \mathrm{H}), 7.34(\mathrm{~d}, J=8.4 \mathrm{~Hz}, 1 \mathrm{H}), 6.99-6.98(\mathrm{~m}$, $2 \mathrm{H}), 6.96-6.94(\mathrm{~m}, 1 \mathrm{H}), 2.96-2.94(\mathrm{~m}, 2 \mathrm{H}), 2.86-2.79(\mathrm{~m}, 2 \mathrm{H}), 2.54-2.49(\mathrm{~m}, 1 \mathrm{H})$, 2.46-2.30 (m, 3H), 2.18-2.13 (m, 1H), 2.09-1.97 (m, 4H), 1.67-1.47 (m, 7H), $1.21(\mathrm{~s}$, $6 \mathrm{H}), 1.18(\mathrm{~s}, 6 \mathrm{H}), 0.93(\mathrm{~s}, 3 \mathrm{H}) ;{ }^{19} \mathbf{F}$ NMR $\left(565 \mathrm{MHz}, \mathrm{CDCl}_{3}\right) \delta-80.89--81.06(\mathrm{~m}, 3 \mathrm{~F})$, -112.82- -114.05 (m, 2F), -124.45- -124.51 (m, 2F), -125.86- -125.98 (m, 2F); ${ }^{13} \mathrm{C}$ NMR $\left(101 \mathrm{MHz}, \mathrm{CDCl}_{3}\right)$ 165.3, 148.8, 141.3, 138.0, 137.4, 133.3, 133.3, 130.1, 130.1, 129.6, 128.9, 128.5, 128.0, 126.4, 121.7, 118.8, 118.7-108.4 (m), 50.4, 47.9, 44.1, 38.0, 35.8, 33.4 (t, J = 21.6 Hz), 31.5, 29.4, 26.3, 25.7, 24.8, 24.4, 21.5, 13.8.

HRMS: $\mathrm{m} / \mathrm{z}(\mathrm{ESI})$ calculated $[\mathrm{M}+\mathrm{H}]^{+}:$747.2898, found: $747.2928 .[\alpha]_{\mathrm{D}}{ }^{25}=16.642(c$ $\left.=0.25, \mathrm{CHCl}_{3}\right)$.<smiles>CC(C)C(C)CC[C@H](C)C1CCC2[C@H]3CC=C4CC(OC(=O)c5cccc(C(CC(F)(F)F)C(F)(F)F)c5)CC[C@]4(C)[C@H]3CC[C@]12C</smiles> 
The title compound 3kk-B was synthesized according to General Procedure B, and it was purified by column chromatography on silica gel (44\% yield, 87:13 dr, $77.2 \mathrm{mg}$, white solid).

${ }^{1} \mathbf{H}$ NMR $\left(600 \mathrm{MHz}, \mathrm{CDCl}_{3}\right){ }^{1} \mathrm{H}$ NMR $\left(600 \mathrm{MHz}, \mathrm{CDCl}_{3}\right) \delta 7.93(\mathrm{~s}, 1 \mathrm{H}), 7.88(\mathrm{~d}, J=$ $7.2 \mathrm{~Hz}, 1 \mathrm{H}), 7.43(\mathrm{~d}, J=7.8 \mathrm{~Hz}, 1 \mathrm{H}), 7.38-7.34(\mathrm{~m}, 1 \mathrm{H}), 5.43(\mathrm{~d}, J=3.6 \mathrm{~Hz}, 1 \mathrm{H})$, 4.89-4.83 (m, 1H), 2.87-2.75 (m, 2H), 2.47 (d, $J=7.2 \mathrm{~Hz}, 1 \mathrm{H}), 2.39-2.30(\mathrm{~m}, 1 \mathrm{H})$, $2.24-1.47(\mathrm{~m}, 15 \mathrm{H}), 1.37-1.07(\mathrm{~m}, 30 \mathrm{H}), 1.08(\mathrm{~s}, 3 \mathrm{H}), 0.94(\mathrm{~d}, J=6.6 \mathrm{~Hz}, 3 \mathrm{H})$, 0.87-0.81 (m, 9H), $0.70(\mathrm{~s}, 3 \mathrm{H}) ;{ }^{19} \mathbf{F}$ NMR $\left(565 \mathrm{MHz}, \mathrm{CDCl}_{3}\right) \delta-80.95--81.06(\mathrm{~m}$, 3F), -112.91- -114.04 (m, 2F), -124.46- -124.52 (m, 2F), -125.92- -125.99 (m, 2F); ${ }^{13}$ C NMR $\left(151 \mathrm{MHz}, \mathrm{CDCl}_{3}\right) \delta 165.9,140.9,139.6,132.6,131.3,129.1,128.7,127.5$, 122.8, 118.9-108.4 (m), 84.1, 74.6, 56.7, 56.0, 50.0, 45.8, 42.3, 39.7, 38.2, 37.0, 36.7, $36.2,33.9,31.9(\mathrm{~d}, J=8.2 \mathrm{~Hz}), 29.1,28.3,27.9,26.0,24.7,24.4,23.0,21.0,19.8$, 19.4, 19.0, 18.8, 12.0, 11.8 .

$[\alpha]_{\mathrm{D}}^{25}=-15.914\left(c=0.25, \mathrm{CHCl}_{3}\right)$.<smiles>Cc1ccc([C@@H](CC(F)(F)F)c2ccccc2)cc1</smiles>

The title compound 3a-B was synthesized according to General Procedure C, and it was purified by column chromatography on silica gel (75\% yield, 91:9 er, $349.6 \mathrm{mg}$, white solid).

${ }^{1} \mathbf{H}$ NMR $\left(400 \mathrm{MHz}, \mathrm{CDCl}_{3}\right) \delta 7.05(\mathrm{~d}, J=7.8 \mathrm{~Hz}, 2 \mathrm{H}), 7.01(\mathrm{~d}, J=7.8 \mathrm{~Hz}, 2 \mathrm{H})$, 2.75-2.60 (m, 2H), 2.28-2.17 (m, 4H), $1.12(\mathrm{~s}, 6 \mathrm{H}), 1.08(\mathrm{~s}, 6 \mathrm{H}) ;{ }^{19}$ F NMR $(565 \mathrm{MHz}$, $\left.\mathrm{CDCl}_{3}\right) \delta-81.08--81.22(\mathrm{~m}, 3 \mathrm{~F}),-113.52--113.71(\mathrm{~m}, 2 \mathrm{~F}),-124.57--124.68(\mathrm{~m}, 2 \mathrm{~F})$, -125.97- -126.10 (m, 2F); ${ }^{13} \mathbf{C}$ NMR (151 MHz, $\left.\mathrm{CDCl}_{3}\right) \delta 137.4,135.6,129.4,127.9$, 120.3-108.5 (m), 83.9, $33.7(\mathrm{t}, J=21.4 \mathrm{~Hz}), 24.4,24.3$.

HRMS: $\mathrm{m} / \mathrm{z}(\mathrm{ESI})$ calculated $[\mathrm{M}+\mathrm{H}]^{+}:$465.1642, found: $465.1636 .[\alpha]_{\mathrm{D}}{ }^{25}=-7.259(c$ $\left.=0.25, \mathrm{CHCl}_{3}\right)$.<smiles>COc1ccc([C@H](CC(F)(F)F)c2ccccc2)cc1</smiles>

The title compound 3e-B was synthesized according to General Procedure C, and it was purified by column chromatography on silica gel (59\% yield, 91:9 er, $283.6 \mathrm{mg}$, white solid).

${ }^{1} \mathbf{H}$ NMR $\left(600 \mathrm{MHz}, \mathrm{CDCl}_{3}\right) \delta 7.15(\mathrm{~d}, J=9.0 \mathrm{~Hz}, 2 \mathrm{H}), 6.83(\mathrm{~d}, J=9.0 \mathrm{~Hz}, 2 \mathrm{H}), 3.78$ $(\mathrm{s}, 3 \mathrm{H}), 2.80-2.66(\mathrm{~m}, 2 \mathrm{H}), 2.33-2.21(\mathrm{~m}, 1 \mathrm{H}), 1.20(\mathrm{~s}, 6 \mathrm{H}), 1.16(\mathrm{~s}, 6 \mathrm{H}) ;{ }^{19} \mathbf{F}$ NMR $(565$ $\mathrm{MHz}, \mathrm{CDCl} 3) \delta-80.96--81.13(\mathrm{~m}, 3 \mathrm{~F}),-113.53--113.66(\mathrm{~m}, 2 \mathrm{~F}),-124.55--124.61$ $(\mathrm{m}, 2 \mathrm{~F}),-125.94--126.01(\mathrm{~m}, 2 \mathrm{~F}) ;{ }^{13} \mathrm{C}$ NMR (151 MHz, $\left.\mathrm{CDCl}_{3}\right) \delta$ 157.9, 132.4, 129.0, 114.1, 83.9, 55.2, $33.8(\mathrm{t}, J=20.2 \mathrm{~Hz}), 24.4(\mathrm{~d}, J=11.8 \mathrm{~Hz})$.

HRMS: $\mathrm{m} / \mathrm{z}(\mathrm{ESI})$ calculated $\left[\mathrm{M}+\mathrm{NH}_{4}\right]^{+}:$498.1860, found: $498.1857 .[\alpha]_{\mathrm{D}}{ }^{25}=-4.160$ $\left(c=0.25, \mathrm{CHCl}_{3}\right)$. 


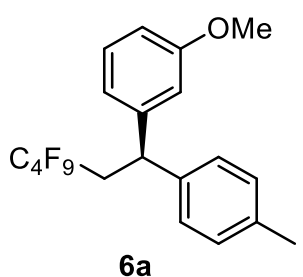

The title compound 6a was synthesized according to General Procedure E, and it was purified by column chromatography on silica gel (51\% yield, 91:9 er, $45.4 \mathrm{mg}$, colourless oil).

${ }^{1} \mathbf{H}$ NMR $\left(600 \mathrm{MHz}, \mathrm{CDCl}_{3}\right) \delta 7.22(\mathrm{t}, J=7.8 \mathrm{~Hz}, 1 \mathrm{H}), 7.15(\mathrm{~d}, J=8.4 \mathrm{~Hz}, 2 \mathrm{H}), 7.11$ $(\mathrm{d}, J=7.8 \mathrm{~Hz}, 2 \mathrm{H}), 6.85(\mathrm{~d}, J=7.2 \mathrm{~Hz}, 1 \mathrm{H}), 6.79(\mathrm{~s}, 1 \mathrm{H}), 6.73(\mathrm{dd}, J=8.4,2.4 \mathrm{~Hz}$, $1 \mathrm{H}), 4.38$ (t, $J=7.2 \mathrm{~Hz}, 1 \mathrm{H}), 3.77(\mathrm{~s}, 3 \mathrm{H}), 2.85(\mathrm{td}, J=18.6,6.6 \mathrm{~Hz}, 2 \mathrm{H}), 2.30(\mathrm{~s}, 3 \mathrm{H})$; ${ }^{19}$ F NMR $\left(565 \mathrm{MHz}, \mathrm{CDCl}_{3}\right) \delta-81.01--81.09(\mathrm{~m}, 3 \mathrm{~F}),-113.16--113.22(\mathrm{~m}, 2 \mathrm{~F})$, -124.43- -125.48 (m, 2F), -125.86- -125.94 (m, 2F); $\left.{ }^{13} \mathbf{C ~ N M R ~ ( 1 5 1 ~ M H z , ~} \mathrm{CDCl}_{3}\right) \delta$ $159.7,144.9,139.9,136.5,129.7,129.4,127.2$, 119.7, 120.2-108.6 (m), 113.8, 111.4, $55.1,43.2,36.0(\mathrm{t}, J=20.7 \mathrm{~Hz}), 29.7,21.0$.

HRMS: $\mathrm{m} / \mathrm{z}(\mathrm{ESI})$ calculated $[\mathrm{M}+\mathrm{H}]^{+}: 445.1208$, found: $445.1197 \cdot[\alpha]_{\mathrm{D}}^{25}=5.350(c=$ $\left.0.25, \mathrm{CHCl}_{3}\right)$.

The enantiomeric excess of 6a was determined by chiral HPLC analysis compared to the corresponding racemate alcohol.

Conditions: ChiralPak OJ-H column; hexane $/{ }^{i} \mathrm{PrOH}=95: 5$; flow rate $=1.0 \mathrm{~mL} / \mathrm{min}$; $\lambda=220 \mathrm{~nm} ; \mathrm{t}_{\mathrm{R} 1}$ (major) $=3.9 \mathrm{~min} ; \mathrm{t}_{\mathrm{R} 2}($ minor $)=4.5 \mathrm{~min}$. 

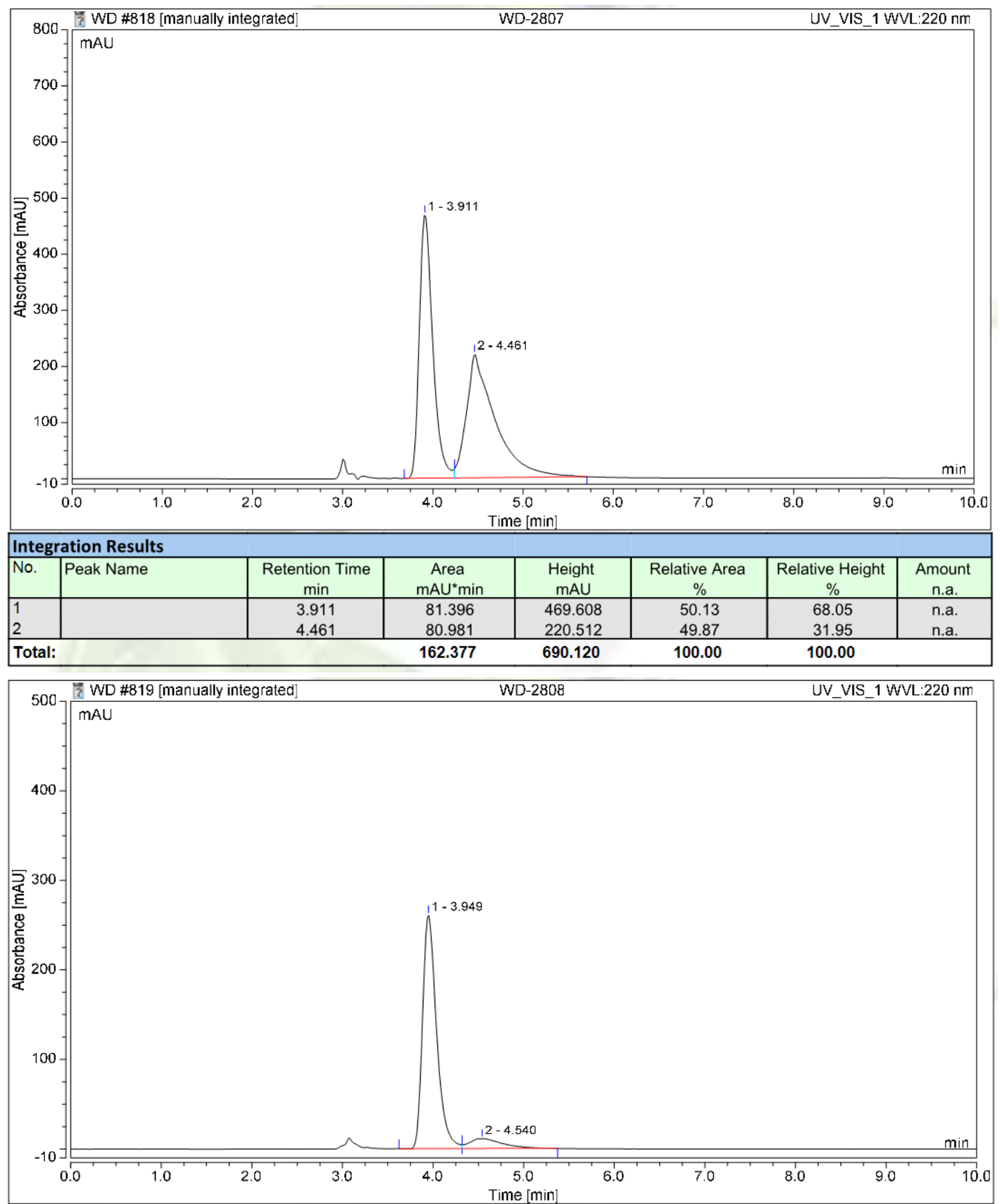

\begin{tabular}{|l|l|c|c|c|c|c|c|}
\hline \multicolumn{2}{|l|}{ Integration Results } \\
\hline No. & Peak Name & $\begin{array}{c}\text { Retention Time } \\
\text { min }\end{array}$ & $\begin{array}{c}\text { Area } \\
\text { mAU*min }\end{array}$ & $\begin{array}{c}\text { Height } \\
\mathrm{mAU}\end{array}$ & $\begin{array}{c}\text { Relative Area } \\
\%\end{array}$ & $\begin{array}{c}\text { Relative Height } \\
\%\end{array}$ & $\begin{array}{c}\text { Amount } \\
\text { n.a. }\end{array}$ \\
\hline 1 & 3.949 & 46.682 & 260.779 & 90.88 & 95.94 & n.a. \\
2 & & 4.540 & 4.683 & 11.031 & 9.12 & n.a. \\
\hline
\end{tabular}<smiles>Cc1ccc(C(CO)CC(F)(F)F)cc1</smiles>

The title compound $\mathbf{6 b}$ was synthesized according to General Procedure E, and it was purified by column chromatography on silica gel (54\% yield, 91:9 er, $39.8 \mathrm{mg}$, white solid). 
${ }^{1} \mathbf{H}$ NMR $\left(600 \mathrm{MHz}, \mathrm{CDCl}_{3}\right) \delta 7.17(\mathrm{~d}, J=8.4 \mathrm{~Hz}, 2 \mathrm{H}), 7.13(\mathrm{~d}, J=8.4 \mathrm{~Hz}, 2 \mathrm{H})$, 3.81-3.75 (m, 2H), 3.29-3.23 (m, 1H), 2.66-2.55 (m, 1H), 2.48-2.37 (m, 1H), $2.34(\mathrm{~s}$, $3 \mathrm{H}) ;{ }^{19} \mathbf{F}$ NMR $\left(565 \mathrm{MHz}, \mathrm{CDCl}_{3}\right) \delta-81.01--81.10(\mathrm{~m}, 3 \mathrm{~F}),-112.32--113.52(\mathrm{~m}, 2 \mathrm{~F})$, -124.39- -125.45 (m, 2F), -125.90- -125.97 (m, 2F); $\left.{ }^{13} \mathbf{C ~ N M R ~ ( 1 5 1 ~ M H z , ~} \mathrm{CDCl}_{3}\right) \delta$ 137.4, 137.1, 129.6, 127.5, 66.6, 40.5, 32.5 (t, $J=20.8 \mathrm{~Hz}$ ), 21.0.

HRMS: $\mathrm{m} / \mathrm{z}(\mathrm{ESI})$ calculated $[2 \mathrm{M}+\mathrm{H}]^{+}:$737.1718, found: $737.1736 .[\alpha]_{\mathrm{D}}{ }^{25}=-10.332$ $\left(c=0.25, \mathrm{CHCl}_{3}\right)$.

The enantiomeric excess of $\mathbf{6 b}$ was determined by chiral HPLC analysis compared to the corresponding racemate alcohol.

Conditions: ChiralPak OD-H column; hexane $/{ }^{i} \mathrm{PrOH}=99: 1$; flow rate $=1.0 \mathrm{~mL} / \mathrm{min}$; $\lambda=220 \mathrm{~nm} ; t_{\mathrm{R} 1}$ (major) $=17.5 \mathrm{~min} ; \mathrm{t}_{\mathrm{R} 2}($ minor $)=16.0 \mathrm{~min}$.

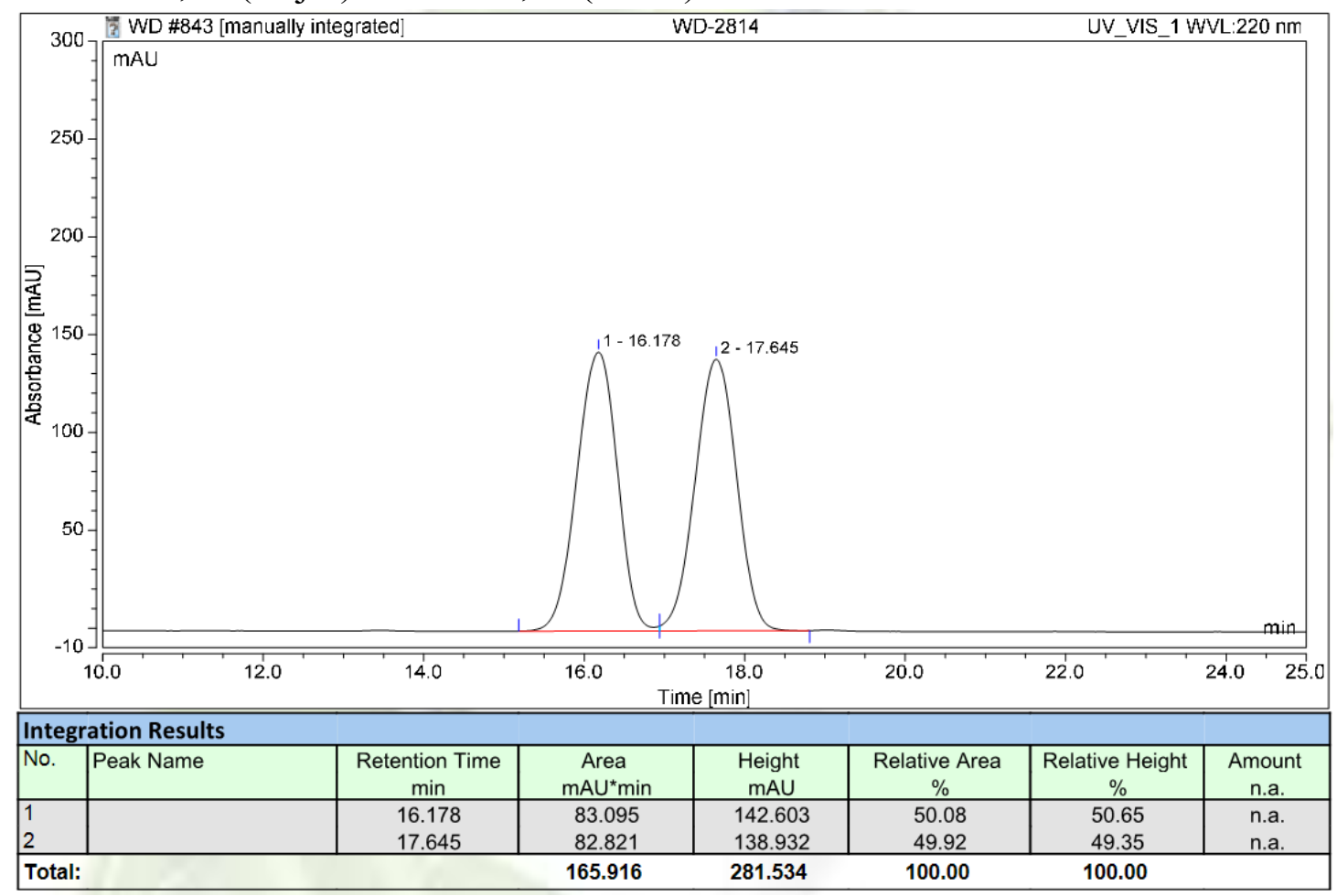




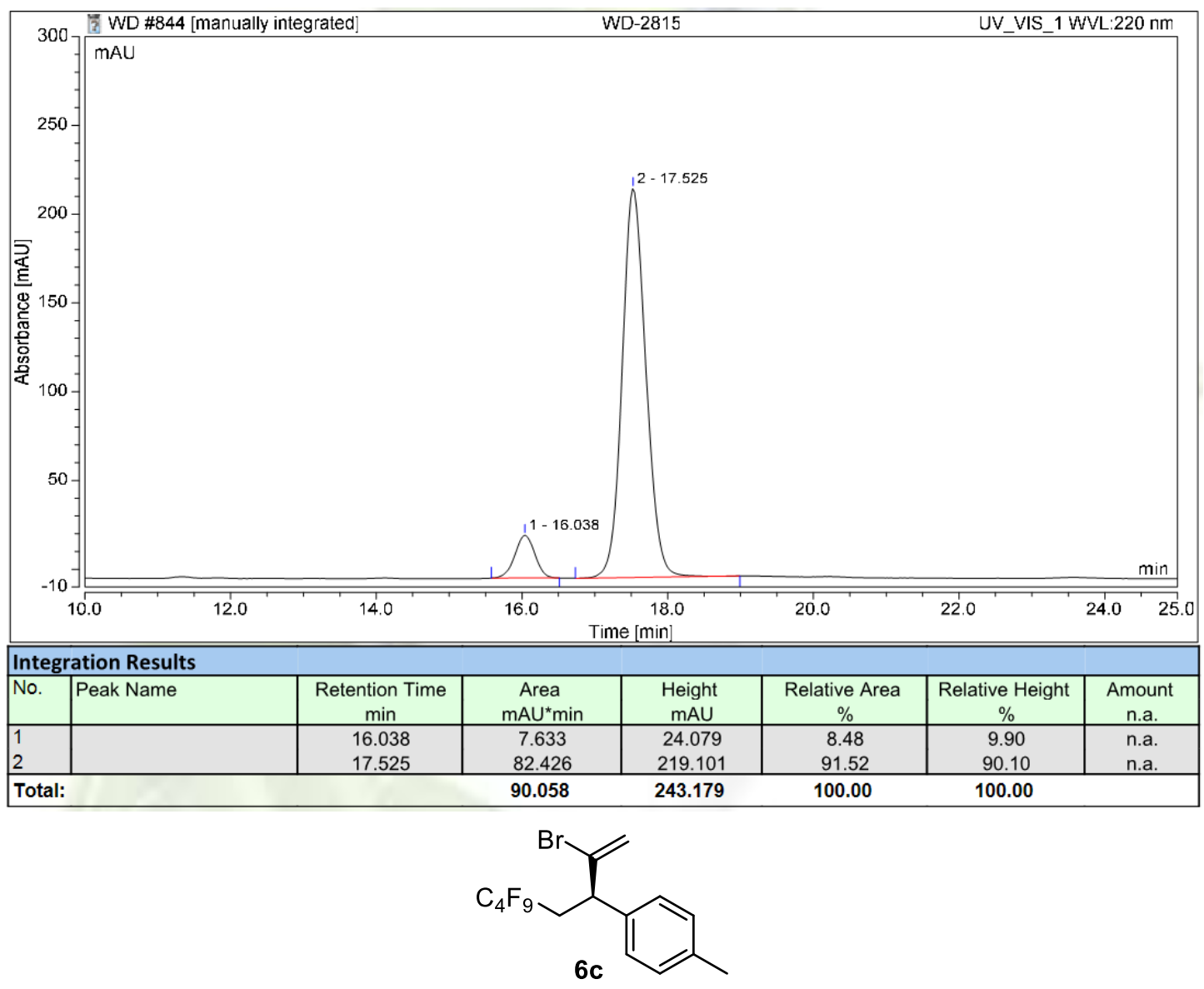

The title compound $\mathbf{6 c}$ was synthesized according to General Procedure E, and it was purified by column chromatography on silica gel (49\% yield, 91:9 er, $43.2 \mathrm{mg}$, colourless oil).

${ }^{1} \mathbf{H}$ NMR $\left(400 \mathrm{MHz}, \mathrm{CDCl}_{3}\right) \delta 7.17(\mathrm{~s}, 4 \mathrm{H}), 5.80(\mathrm{~s}, 1 \mathrm{H}), 5.53(\mathrm{~d}, J=1.2 \mathrm{~Hz}, 1 \mathrm{H})$, $4.08(\mathrm{t}, J=6.8 \mathrm{~Hz}, 1 \mathrm{H}), 2.90(\mathrm{ddd}, J=34.4,16.0,7.2 \mathrm{~Hz}, 1 \mathrm{H}), 2.65-2.49(\mathrm{~m}, 1 \mathrm{H})$, $2.34(\mathrm{~s}, 3 \mathrm{H}) ;{ }^{19} \mathbf{F}$ NMR $\left(565 \mathrm{MHz}, \mathrm{CDCl}_{3}\right) \delta-81.00--81.08(\mathrm{~m}, 3 \mathrm{~F}),-113.49(\mathrm{t}, J=$ 13.0 Hz, 2F), -124.39- -124.50 (m, 2F), -125.83- -125.93 (m, 2F); ${ }^{13}$ C NMR (151 $\left.\mathrm{MHz} \mathrm{CDCl}_{3}\right) \delta 137.5,136.2,136.1,129.4,127.4,118.1,47.3,33.9(\mathrm{t}, J=20.7 \mathrm{~Hz})$, 21.1.

MS: $(\mathrm{EI})[\mathrm{M}]^{+}:$443.00. $[\alpha]_{\mathrm{D}}{ }^{25}=3.342\left(c=0.25, \mathrm{CHCl}_{3}\right)$.

The enantiomeric excess of $\mathbf{6 c}$ was determined by chiral HPLC analysis compared to the corresponding racemate alcohol.

Conditions: ChiralPak OJ-H column; hexane $/{ }^{i} \mathrm{PrOH}=100: 0$; flow rate $=0.5 \mathrm{~mL} / \mathrm{min}$; $\lambda=220 \mathrm{~nm} ; \mathrm{t}_{\mathrm{R} 1}($ major $)=10.2 \mathrm{~min} ; \mathrm{t}_{\mathrm{R} 2}$ (minor $)=9.2 \mathrm{~min}$. 

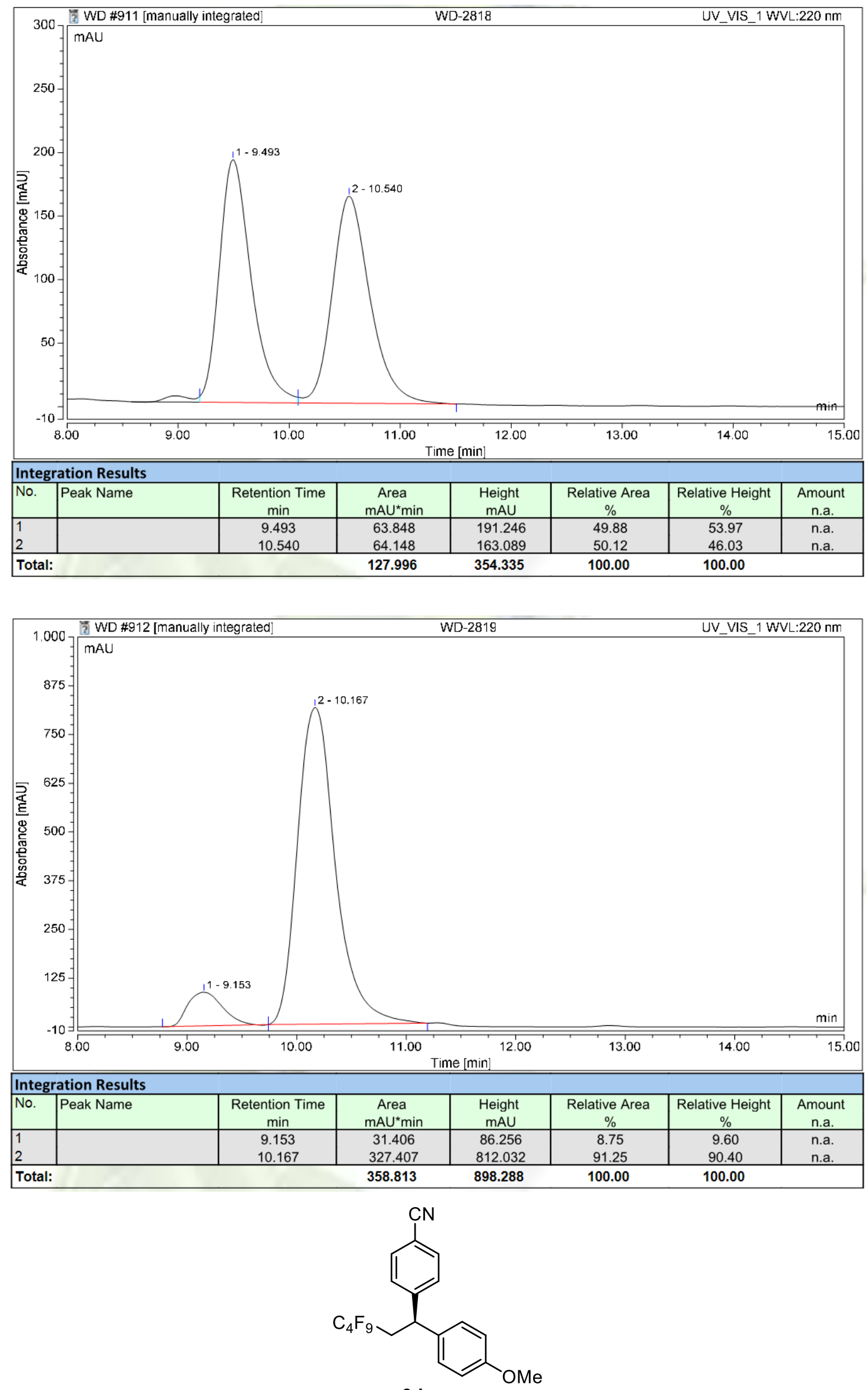

6d 
The title compound $\mathbf{6 d}$ was synthesized according to General Procedure E, and it was purified by column chromatography on silica gel (46\% yield, 90:10 er, $41.9 \mathrm{mg}$, colourless oil).

${ }^{1} \mathbf{H}$ NMR $\left(600 \mathrm{MHz}, \mathrm{CDCl}_{3}\right) \delta 7.60(\mathrm{~d}, J=8.4 \mathrm{~Hz}, 2 \mathrm{H}), 7.37$ (d, $\left.J=8.4 \mathrm{~Hz}, 2 \mathrm{H}\right), 7.14$ $(\mathrm{d}, J=8.4 \mathrm{~Hz}, 1 \mathrm{H}), 6.86(\mathrm{~d}, J=8.4 \mathrm{~Hz}, 2 \mathrm{H}), 4.46(\mathrm{t}, J=6.6 \mathrm{~Hz}, 1 \mathrm{H}), 3.78(\mathrm{~s}, 3 \mathrm{H})$, 2.90-2.81 (m, 2H); ${ }^{19} \mathbf{F}$ NMR (565 MHz, $\left.\mathrm{CDCl}_{3}\right) \delta-80.96--81.07(\mathrm{~m}, 3 \mathrm{~F}),-112.20-$ -113.59 (m, 2F), -124.36- -124.41 (m, 2F), -125.86- -125.93 (m, 2F); ${ }^{13}$ C NMR (151 $\left.\mathrm{MHz}, \mathrm{CDCl}_{3}\right) \delta 158.8,148.6,133.6,132.6,128.3,128.2,118.6,114.4,110.8,55.3$, 42.9, $35.8(\mathrm{t}, J=20.8 \mathrm{~Hz})$.

HRMS: m/z (ESI) calculated $[\mathrm{M}+\mathrm{H}]^{+}: 456.1004$, found: $456.1011 .[\alpha]_{\mathrm{D}}{ }^{25}=5.576(c=$ $\left.0.25, \mathrm{CHCl}_{3}\right)$.

The enantiomeric excess of $\mathbf{6 d}$ was determined by chiral HPLC analysis compared to the corresponding racemate alcohol.

Conditions: ChiralPak IB column; hexane $/{ }^{i} \mathrm{PrOH}=98: 2$; flow rate $=1.0 \mathrm{~mL} / \mathrm{min} ; \lambda=$ $220 \mathrm{~nm} ; \mathrm{t}_{\mathrm{R} 1}$ (major) $=9.8 \mathrm{~min} ; \mathrm{t}_{\mathrm{R} 2}$ (minor) $=9.5 \mathrm{~min}$.

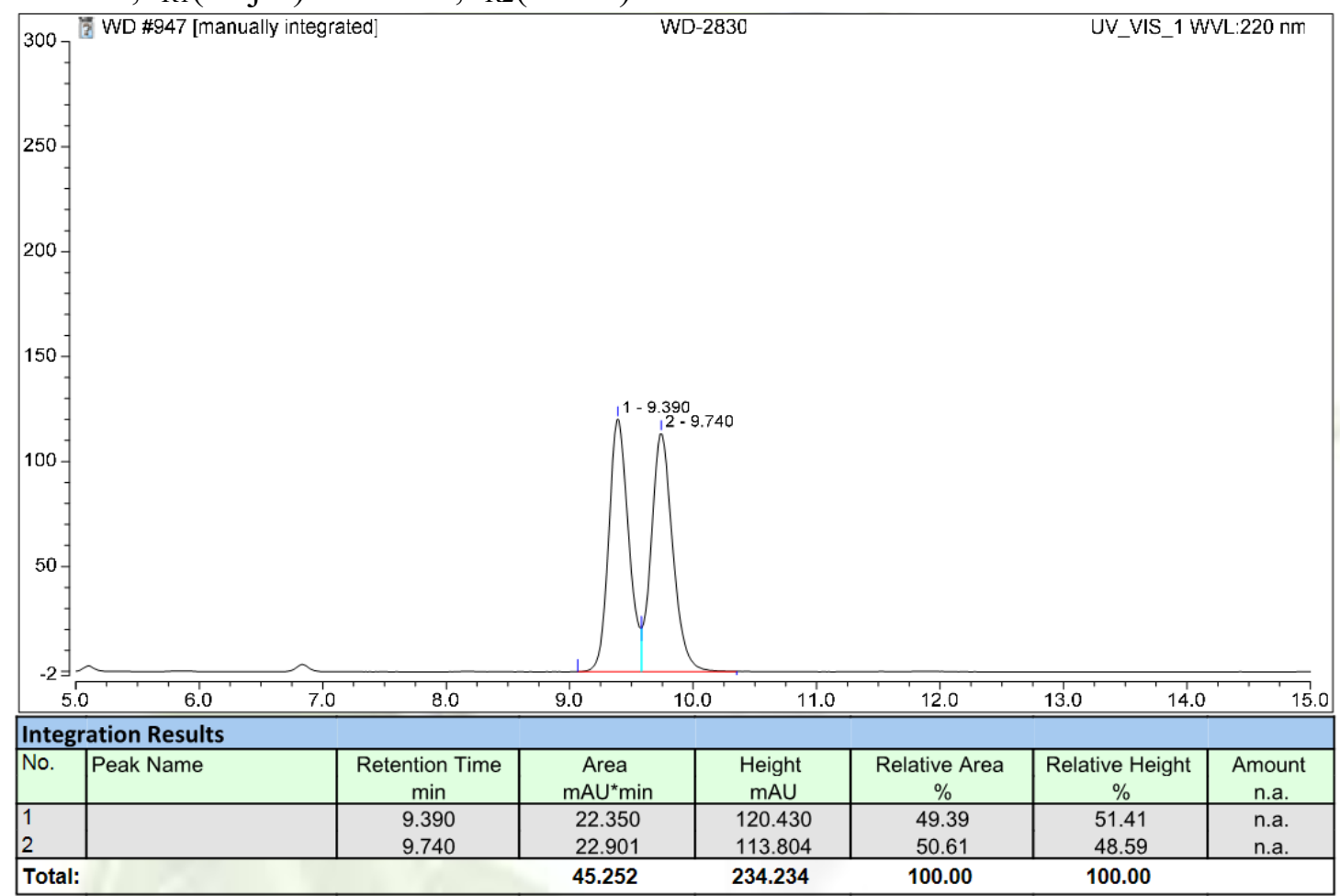




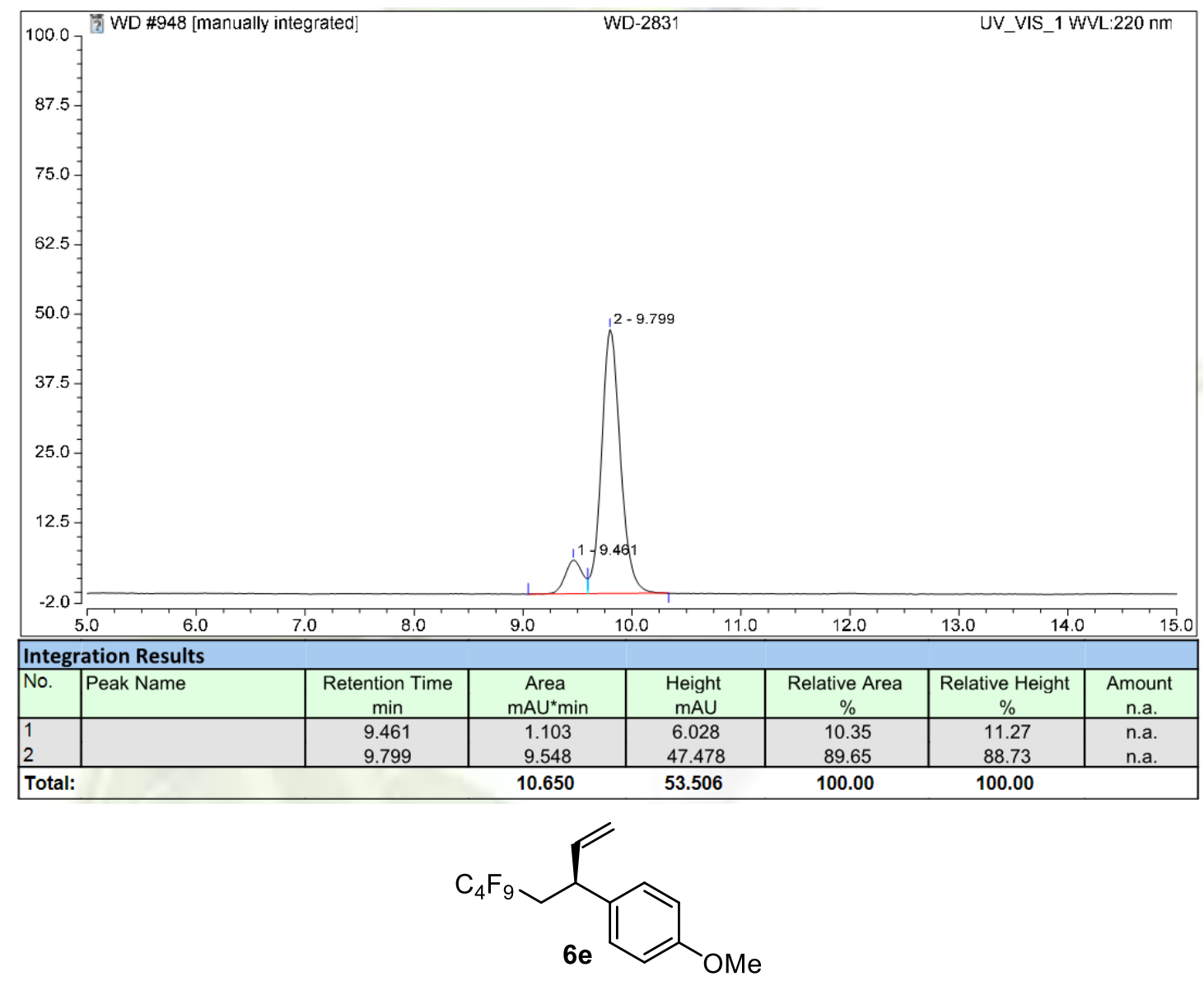

The title compound 6e was synthesized according to General Procedure E, and it was purified by column chromatography on silica gel (70\% yield, 91:9 er, $53.4 \mathrm{mg}$, colourless oil).

${ }^{1} \mathbf{H}$ NMR $\left(600 \mathrm{MHz}, \mathrm{CDCl}_{3}\right) \delta 7.13(\mathrm{~d}, J=9.0 \mathrm{~Hz}, 2 \mathrm{H}), 6.87(\mathrm{~d}, J=8.4 \mathrm{~Hz}, 2 \mathrm{H})$, 6.02-5.96 (m, 1H), 5.10-5.04 (m, 2H), 3.80-3.75 (m, 4H), 2.58-2.40 (m, 2H); ${ }^{19} \mathbf{F}$ NMR $(565 \mathrm{MHz}, \mathrm{CDCl3}) \delta$-81.04- $-81.08(\mathrm{~m}, 3 \mathrm{~F}),-112.26--113.62(\mathrm{~m}, 2 \mathrm{~F})$, -124.51- -124.56 (m, 2F), -125.90- -125.98 (m, 2F); $\left.{ }^{13} \mathbf{C ~ N M R ~ ( 1 5 1 ~ M H z , ~} \mathrm{CDCl}_{3}\right) \delta$ $158.5,140.3,134.3,128.3,115.0,114.2,55.2,41.3,36.0$ (t, $J=20.7 \mathrm{~Hz})$.

HRMS: $\mathrm{m} / \mathrm{z}(\mathrm{ESI})$ calculated $[\mathrm{M}+\mathrm{H}]^{+}: 381.0895$, found: $381.0910 .[\alpha]_{\mathrm{D}}{ }^{25}=12.372(c$ $\left.=0.25, \mathrm{CHCl}_{3}\right)$.

The enantiomeric excess of $\mathbf{6 e}$ was determined by chiral HPLC analysis compared to the corresponding racemate alcohol.

Conditions: ChiralPak OJ-H column; hexane $/{ }^{i} \mathrm{PrOH}=100: 0$; flow rate $=0.2 \mathrm{~mL} / \mathrm{min}$; $\lambda=220 \mathrm{~nm} ; \mathrm{t}_{\mathrm{R} 1}$ (major) $=27.8 \mathrm{~min} ; \mathrm{t}_{2}($ minor $)=26.9 \mathrm{~min}$. 

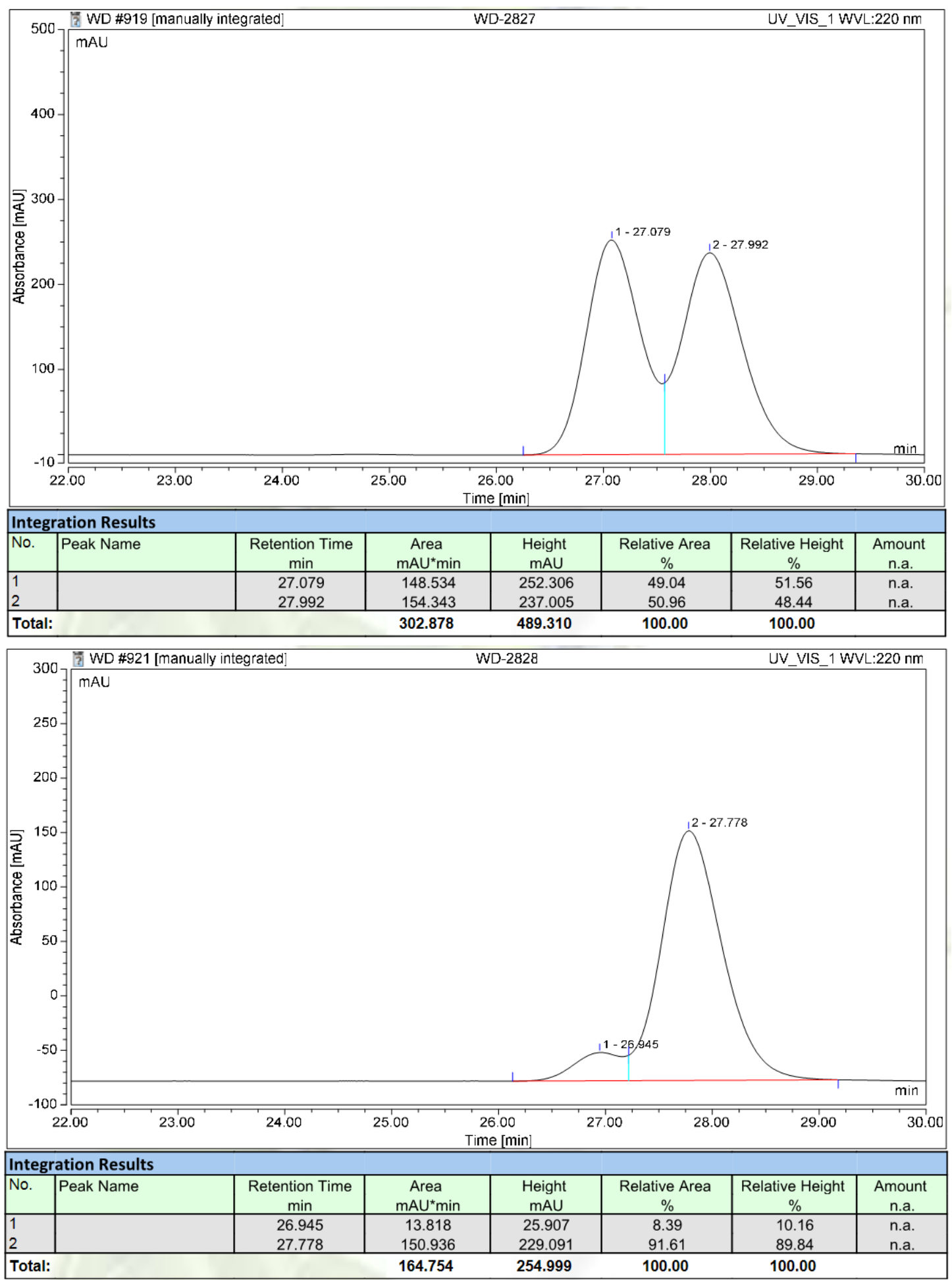<smiles>COc1ccc([C@@H](Br)CSC(F)(F)F)cc1</smiles>

The title compound $\mathbf{6 f}$ was synthesized according to General Procedure E, and it was purified by column chromatography on silica gel (56\% yield, $51.4 \mathrm{mg}$, white solid).

${ }^{1}$ H NMR (600 MHz, Acetone-d $\left.{ }_{6}\right) \delta$ 7.11-7.08 (m, 2H), 6.71-6.68 (m, 2H), 3.70 (s, 
3H), $2.97(\mathrm{~m}, 1 \mathrm{H}), 2.58-2.41(\mathrm{~m}, 2 \mathrm{H}) ;{ }^{19} \mathbf{F}$ NMR $(565 \mathrm{MHz}$, Acetone-d 6$) \delta-82.05(\mathrm{~s}$, 3F), -111.70- -115.58 (m, 2F), -125.14- -125.20 (m, 2F), -126.57- -126.72 (m, 2F), $-147.27(\mathrm{~s}, 3 \mathrm{~F}) ;{ }^{13} \mathrm{C}$ NMR $\left(151 \mathrm{MHz}\right.$, Acetone- $\left.\mathrm{d}_{6}\right) \delta 157.2,129.5,129.4,134.7-113.9$ (m), 113.6, 113.5, 55.2, 25.2.

HRMS: m/z (ESI) calculated [M+K] $]^{+}: 498.9904$, found: 498.9906. 


\section{Single crystal X-ray Diffraction Analysis of compound}

3dd

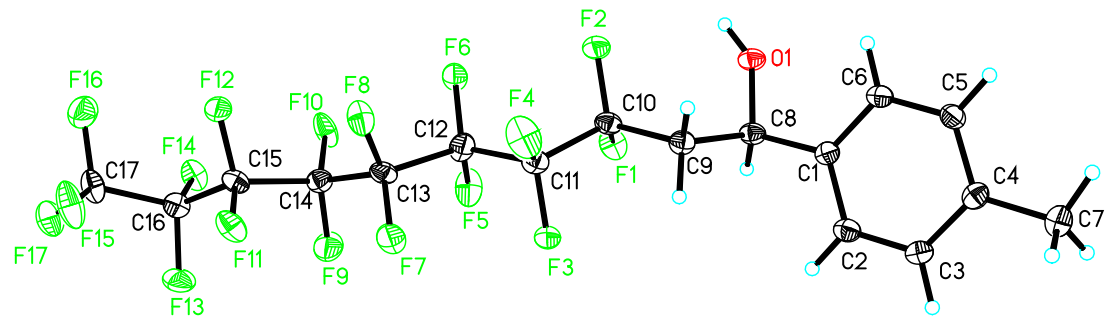

Table S8 Crystal data and structure refinement for cu_210701aa_a.

Identification code

cu_210701aa_a

Empirical formula

$\mathrm{C}_{17} \mathrm{H}_{11} \mathrm{~F}_{17} \mathrm{O}$

Formula weight

554.26

Temperature/K

124.0

Crystal system

monoclinic

Space group

$\mathrm{P} 2{ }_{1}$

$\mathrm{a} / \AA$

9.6770(3)

$\mathrm{b} / \AA$

37.3779(13)

$\mathrm{c} / \AA$

11.1712(4)

$\alpha /{ }^{\circ}$

90

$\beta /{ }^{\circ}$

89.9537(18)

$\gamma /{ }^{\circ}$

90

Volume/ $\AA^{3}$

4040.7(2)

Z

8

$\rho_{\text {calc } g / \mathrm{cm}^{3}}$

1.822

$\mu / \mathrm{mm}^{-1}$

2.040

$\mathrm{F}(000)$

2192.0

Crystal size/ $/ \mathrm{mm}^{3}$

$0.3 \times 0.26 \times 0.12$

Radiation

$\operatorname{CuK} \alpha(\lambda=1.54178)$

$2 \Theta$ range for data collection/ ${ }^{\circ}$

7.914 to 149.544

Index ranges

$-12 \leq \mathrm{h} \leq 12,-46 \leq \mathrm{k} \leq 46,-13 \leq 1 \leq 13$

Reflections collected

44336

Independent reflections

$15518\left[\mathrm{R}_{\text {int }}=0.0516, \mathrm{R}_{\text {sigma }}=0.0431\right]$

Data/restraints/parameters

$15518 / 826 / 1428$

Goodness-of-fit on $\mathrm{F}^{2}$

1.030

Final $\mathrm{R}$ indexes $[\mathrm{I}>=2 \sigma(\mathrm{I})]$

$\mathrm{R}_{1}=0.0507, \mathrm{wR}_{2}=0.1291$ 
Final $\mathrm{R}$ indexes [all data]

Largest diff. peak/hole / e $\AA^{-3}$

Flack parameter
$\mathrm{R}_{1}=0.0547, \mathrm{wR}_{2}=0.1340$

$0.64 /-0.47$

$0.12(6)$ 


\section{DFT calculation.}

Computational Methods: All of the density functional theory (DFT) calculations were performed with the Gaussian $09^{\mathrm{S} 5}$ series of programs. The B3-LYP functional ${ }^{\mathrm{S} 6}$ including Grimme empirical dispersion correction $(\mathrm{GD} 3 \mathrm{BJ})^{\mathrm{S} 7}$ with the standard 6-31G(d) basis set (SDD basis set for Ni and I ) was used for the geometry optimizations in the gas phase. Harmonic vibrational frequency calculations were performed for all of the stationary points to determine whether they are local minima or transition structures and to derive the thermochemical corrections for free energies. The M06 functional ${ }^{\mathrm{S} 8}$ proposed by Truhlar et al. with the 6-311+G(d,p) basis set (SDD basis set for Ni and I) was used to calculate the single-point energies in 1,4-dioxane solvent to provide more accurate energy information. The solvent effect was considered by single-point calculations based on the gas-phase stationary points with the $\mathrm{SMD}^{S 9}$ continuum solvation model. The Gibbs free energies of the stationary points calculated using the M06 functional are used to discuss the energies. The 3D images of the calculated structures were prepared using CYLview. ${ }^{\text {S10 }}$.

TS-L4Ni-I+Cl

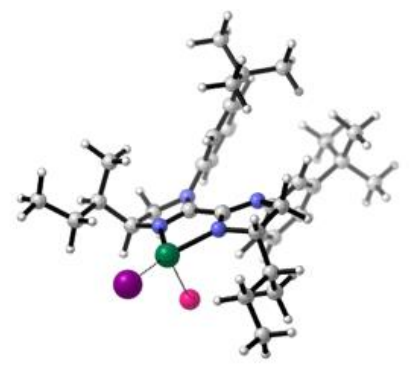

TS-L4Ni-I

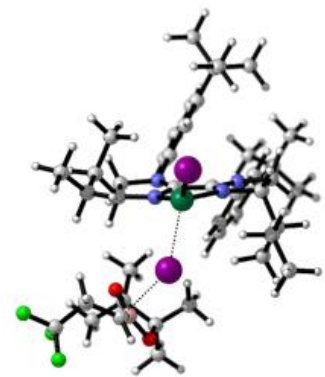

TS-L4Ni-Cl

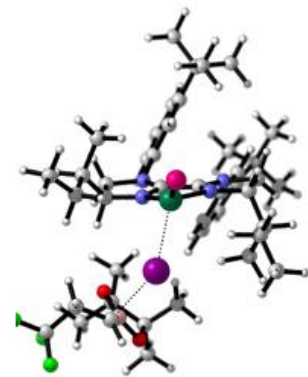

Cartesian coordinates of the calculated transition states.

TS-L4Ni-Cl

Thermal Correction to Free Energy: 0.932146 Hartree

SCF energy: -3013.405844 Hartree

Gibbs free energy: -3012.473698 Hartree

Imaginary frequency: -144.17

Coordinates:

$\begin{array}{llll}\text { C } & -1.32732800 & -1.77791200 & 2.82682100 \\ \text { C } & -1.27552900 & -0.87847000 & 0.75750000\end{array}$




\begin{tabular}{|c|c|c|c|}
\hline $\mathrm{C}$ & -2.66758500 & -1.02856000 & 2.57106200 \\
\hline $\mathrm{H}$ & -1.51189600 & -2.83284900 & 3.04810200 \\
\hline $\mathrm{H}$ & -2.88463600 & -0.27332800 & 3.33042200 \\
\hline $\mathrm{H}$ & -3.51996400 & -1.71298500 & 2.50274500 \\
\hline $\mathrm{N}$ & -0.63299800 & -1.70184600 & 1.52614600 \\
\hline $\mathrm{C}$ & -0.68630200 & -0.64865500 & -0.58465100 \\
\hline $\mathrm{C}$ & -0.25721600 & -0.04023000 & -2.75399800 \\
\hline $\mathrm{C}$ & 0.94784100 & -0.80599600 & -2.13152500 \\
\hline $\mathrm{H}$ & -0.71864800 & -0.58767900 & -3.58209300 \\
\hline $\mathrm{H}$ & 0.01956700 & 0.95559000 & -3.10376900 \\
\hline $\mathrm{H}$ & 1.79971200 & -0.12703900 & -2.01343600 \\
\hline $\mathrm{N}$ & 0.48909400 & -1.15025100 & -0.77572400 \\
\hline $\mathrm{C}$ & -0.47538800 & -1.17253600 & 3.96457300 \\
\hline $\mathrm{H}$ & -1.14333400 & -1.14140500 & 4.84056000 \\
\hline $\mathrm{C}$ & -0.03706900 & 0.26252900 & 3.64123800 \\
\hline $\mathrm{H}$ & 0.71217200 & 0.26814600 & 2.84438100 \\
\hline $\mathrm{H}$ & -0.87925400 & 0.89175000 & 3.32786000 \\
\hline $\mathrm{H}$ & 0.41068000 & 0.73710000 & 4.51848300 \\
\hline $\mathrm{C}$ & 0.70522400 & -2.09893100 & 4.30564500 \\
\hline $\mathrm{H}$ & 0.30613100 & -3.09346300 & 4.54557600 \\
\hline $\mathrm{H}$ & 1.33399800 & -2.23493200 & 3.42011400 \\
\hline $\mathrm{C}$ & 1.57251200 & -1.60415900 & 5.46457600 \\
\hline $\mathrm{H}$ & 2.32294500 & -2.35728800 & 5.72574900 \\
\hline $\mathrm{H}$ & 2.10910300 & -0.68665700 & 5.20202500 \\
\hline $\mathrm{H}$ & 0.97119000 & -1.40218200 & 6.36094900 \\
\hline $\mathrm{C}$ & 1.38658400 & -2.05277900 & -2.92775700 \\
\hline $\mathrm{H}$ & 1.50024900 & -1.71449500 & -3.96989200 \\
\hline $\mathrm{C}$ & 0.31571600 & -3.15266400 & -2.88731700 \\
\hline $\mathrm{H}$ & -0.68190400 & -2.76625600 & -3.13143000 \\
\hline $\mathrm{H}$ & 0.54190000 & -3.94477900 & -3.60624900 \\
\hline $\mathrm{H}$ & 0.26768300 & -3.60948700 & -1.89235800 \\
\hline $\mathrm{C}$ & 2.75927600 & -2.55283700 & -2.44636100 \\
\hline $\mathrm{H}$ & 3.45975900 & -1.70810100 & -2.46332000 \\
\hline $\mathrm{H}$ & 2.68993400 & -2.86858300 & -1.39870600 \\
\hline $\mathrm{C}$ & 3.33156900 & -3.70268200 & -3.27787000 \\
\hline $\mathrm{H}$ & 4.34509300 & -3.94823500 & -2.94450800 \\
\hline $\mathrm{H}$ & 2.72746800 & -4.61038200 & -3.18041200 \\
\hline $\mathrm{H}$ & 3.38139900 & -3.44010300 & -4.34268500 \\
\hline $\mathrm{N}$ & -1.22898700 & 0.05736600 & -1.63186200 \\
\hline $\mathrm{N}$ & -2.44248300 & -0.37018200 & 1.26144000 \\
\hline $\mathrm{C}$ & -2.62594600 & 0.04028600 & -1.91975800 \\
\hline $\mathrm{C}$ & -3.36940200 & -1.12180200 & -1.68588500 \\
\hline $\mathrm{C}$ & -3.23002100 & 1.17177400 & -2.46210300 \\
\hline $\mathrm{C}$ & -4.74053900 & -1.16921500 & -1.94657800 \\
\hline
\end{tabular}




\begin{tabular}{|c|c|c|c|}
\hline $\mathrm{H}$ & -2.85402500 & -1.99107600 & -1.29649600 \\
\hline $\mathrm{C}$ & -4.59224600 & 1.12879500 & -2.75241700 \\
\hline $\mathrm{H}$ & -2.64692600 & 2.07218300 & -2.62027600 \\
\hline $\mathrm{C}$ & -5.33774000 & -0.01934600 & -2.48926700 \\
\hline $\mathrm{H}$ & -5.07989300 & 2.00390700 & -3.17085500 \\
\hline $\mathrm{H}$ & -6.39910400 & -0.01966000 & -2.71763000 \\
\hline $\mathrm{C}$ & -2.96697800 & 0.91680700 & 0.97680100 \\
\hline $\mathrm{C}$ & -4.35218800 & 1.10941000 & 1.01192600 \\
\hline $\mathrm{C}$ & -2.11023800 & 1.98226000 & 0.70185500 \\
\hline $\mathrm{C}$ & -4.91031500 & 2.36455100 & 0.75814500 \\
\hline $\mathrm{H}$ & -4.98269700 & 0.25465200 & 1.22103400 \\
\hline $\mathrm{C}$ & -2.65722500 & 3.22999200 & 0.41216000 \\
\hline $\mathrm{H}$ & -1.03866600 & 1.83527700 & 0.72306000 \\
\hline $\mathrm{C}$ & -4.03604400 & 3.41864500 & 0.44099700 \\
\hline $\mathrm{H}$ & -1.99933000 & 4.06335100 & 0.18233100 \\
\hline $\mathrm{H}$ & -4.43681300 & 4.40421600 & 0.22674800 \\
\hline $\mathrm{C}$ & -5.57481100 & -2.43105400 & -1.68957100 \\
\hline $\mathrm{C}$ & -4.74405400 & -3.55785200 & -1.05257700 \\
\hline $\mathrm{H}$ & -3.92846700 & -3.88647500 & -1.70582600 \\
\hline $\mathrm{H}$ & -4.31197700 & -3.24907400 & -0.09356900 \\
\hline $\mathrm{H}$ & -5.38416900 & -4.42604200 & -0.86443200 \\
\hline $\mathrm{C}$ & -6.14423900 & -2.94043400 & -3.03146700 \\
\hline $\mathrm{H}$ & -6.74754900 & -3.84165900 & -2.87190100 \\
\hline $\mathrm{H}$ & -6.78063300 & -2.18960900 & -3.51077600 \\
\hline $\mathrm{H}$ & -5.33517000 & -3.18907000 & -3.72685100 \\
\hline $\mathrm{C}$ & -6.73781200 & -2.09554000 & -0.73241300 \\
\hline $\mathrm{H}$ & -7.38751900 & -1.31266400 & -1.13622300 \\
\hline $\mathrm{H}$ & -7.35483900 & -2.98459000 & -0.55934200 \\
\hline $\mathrm{H}$ & -6.35848300 & -1.75289600 & 0.23683600 \\
\hline $\mathrm{C}$ & -6.42167000 & 2.62304200 & 0.82107500 \\
\hline $\mathrm{C}$ & -6.70179300 & 3.68720800 & 1.90511100 \\
\hline $\mathrm{H}$ & -7.77775100 & 3.88538400 & 1.97352800 \\
\hline $\mathrm{H}$ & -6.35364900 & 3.34488100 & 2.88587700 \\
\hline $\mathrm{H}$ & -6.19984900 & 4.63436800 & 1.68329900 \\
\hline $\mathrm{C}$ & -7.21568500 & 1.35465100 & 1.17073600 \\
\hline $\mathrm{H}$ & -7.07198800 & 0.57063800 & 0.42122700 \\
\hline $\mathrm{H}$ & -6.93043800 & 0.95401700 & 2.14992500 \\
\hline $\mathrm{H}$ & -8.28490400 & 1.58869100 & 1.20826100 \\
\hline $\mathrm{C}$ & -6.91654600 & 3.13860000 & -0.54724200 \\
\hline $\mathrm{H}$ & -7.99148300 & 3.34961700 & -0.50672900 \\
\hline $\mathrm{H}$ & -6.40589900 & 4.06019600 & -0.84362000 \\
\hline $\mathrm{H}$ & -6.74084300 & 2.38978400 & -1.32552000 \\
\hline $\mathrm{Ni}$ & 1.08544900 & -2.42390400 & 0.70110000 \\
\hline $\mathrm{Cl}$ & 1.76409900 & -4.43912100 & 1.19127800 \\
\hline
\end{tabular}




\begin{tabular}{lrrr} 
C & 2.41154300 & 3.75418300 & 0.53333400 \\
C & 2.32852200 & 3.37145400 & -0.98616200 \\
B & 3.82777200 & 2.03654200 & 0.08276100 \\
O & 3.62971900 & 3.08867300 & 0.94701500 \\
O & 2.97149900 & 2.07134200 & -1.00699400 \\
C & 2.54538300 & 5.24551000 & 0.81632000 \\
H & 1.66971700 & 5.79127000 & 0.44631900 \\
H & 2.61802400 & 5.40963500 & 1.89586000 \\
H & 3.44067900 & 5.66134900 & 0.34965300 \\
C & 1.27716100 & 3.14832900 & 1.36501200 \\
H & 1.51902100 & 3.25314700 & 2.42643500 \\
H & 0.32412400 & 3.65333700 & 1.17838700 \\
H & 1.16851200 & 2.07988400 & 1.15454700 \\
C & 3.16032300 & 4.29313000 & -1.88433200 \\
H & 3.23941700 & 3.84037900 & -2.87714800 \\
H & 2.70048600 & 5.28153200 & -1.98653200 \\
H & 4.17334400 & 4.41344600 & -1.49081800 \\
C & 0.91230200 & 3.23602900 & -1.53390300 \\
H & 0.36245600 & 4.17767600 & -1.42247100 \\
H & 0.95215800 & 2.99806100 & -2.60208100 \\
H & 0.35534000 & 2.44471300 & -1.02886200 \\
C & 5.46949700 & 0.22715600 & -0.88326400 \\
H & 4.69909500 & -0.01310400 & -1.62129800 \\
H & 5.97258600 & -0.70580600 & -0.60976400 \\
C & 6.49322000 & 1.09906300 & -1.58677700 \\
F & 7.53828400 & 1.39916500 & -0.78515200 \\
F & 5.95959300 & 2.27747400 & -1.99943500 \\
I & 3.21183000 & -0.80009100 & 1.29087600 \\
F & 6.99058200 & 0.48652300 & -2.68521200 \\
C & 4.87025800 & 0.92993400 & 0.30791600 \\
H & 5.53225700 & 1.02595700 & 1.16520500 \\
& & & \\
\hline
\end{tabular}

Cartesian coordinates of the calculated transition states.

TS-L4Ni-I

Thermal Correction to Free Energy: 0.930530 Hartree

SCF energy: -2564.606045 Hartree

Gibbs free energy: -2563.675515 Hartree

Imaginary frequency: -151.12

Coordinates:

$\begin{array}{llll}\mathrm{C} & -1.30402500 & -1.88387700 & 2.51490600 \\ \mathrm{C} & -1.32912600 & -0.70247400 & 0.59189200 \\ \mathrm{C} & -2.70664000 & -1.23125900 & 2.34266200 \\ \mathrm{H} & -1.39131500 & -2.97157900 & 2.58573200\end{array}$




\begin{tabular}{|c|c|c|c|}
\hline $\mathrm{H}$ & -3.00314800 & -0.62225900 & 3.20002400 \\
\hline $\mathrm{H}$ & -3.48829400 & -1.97432300 & 2.15096800 \\
\hline $\mathrm{N}$ & -0.61739000 & -1.56678100 & 1.24624100 \\
\hline $\mathrm{C}$ & -0.77528100 & -0.24867500 & -0.70706900 \\
\hline $\mathrm{C}$ & -0.43778900 & 0.65872800 & -2.78631900 \\
\hline $\mathrm{C}$ & 0.83933000 & -0.07006800 & -2.27247500 \\
\hline $\mathrm{H}$ & -0.86147200 & 0.18510800 & -3.67754500 \\
\hline $\mathrm{H}$ & -0.25557700 & 1.71222600 & -3.00371100 \\
\hline $\mathrm{H}$ & 1.63609500 & 0.65826000 & -2.08587400 \\
\hline $\mathrm{N}$ & 0.44008000 & -0.60847700 & -0.96139500 \\
\hline $\mathrm{C}$ & -0.51677400 & -1.36544500 & 3.73873200 \\
\hline $\mathrm{H}$ & -1.19128400 & -1.51136400 & 4.59790800 \\
\hline $\mathrm{C}$ & -0.20732300 & 0.13300500 & 3.61898900 \\
\hline $\mathrm{H}$ & 0.52571800 & 0.31548800 & 2.82780300 \\
\hline $\mathrm{H}$ & -1.10528700 & 0.72372400 & 3.40016900 \\
\hline $\mathrm{H}$ & 0.21201000 & 0.51756900 & 4.55245800 \\
\hline $\mathrm{C}$ & 0.73740900 & -2.22373300 & 3.97822100 \\
\hline $\mathrm{H}$ & 0.42746400 & -3.27365600 & 4.06158300 \\
\hline $\mathrm{H}$ & 1.38965200 & -2.17613500 & 3.10039200 \\
\hline $\mathrm{C}$ & 1.53814800 & -1.82775100 & 5.21987700 \\
\hline $\mathrm{H}$ & 2.34759300 & -2.54406000 & 5.39394600 \\
\hline $\mathrm{H}$ & 1.99562700 & -0.83951400 & 5.10676000 \\
\hline $\mathrm{H}$ & 0.90604500 & -1.80898300 & 6.11753500 \\
\hline $\mathrm{C}$ & 1.35564100 & -1.17634100 & -3.21522800 \\
\hline $\mathrm{H}$ & 1.43075300 & -0.70764800 & -4.20928200 \\
\hline $\mathrm{C}$ & 0.36825700 & -2.34914700 & -3.30318900 \\
\hline $\mathrm{H}$ & -0.65879600 & -2.00923600 & -3.48687600 \\
\hline $\mathrm{H}$ & 0.63749800 & -3.02469100 & -4.11955200 \\
\hline $\mathrm{H}$ & 0.37218100 & -2.93175800 & -2.37519300 \\
\hline $\mathrm{C}$ & 2.76728100 & -1.62537500 & -2.80213400 \\
\hline $\mathrm{H}$ & 3.40733700 & -0.73592700 & -2.74116600 \\
\hline $\mathrm{H}$ & 2.73690900 & -2.04748700 & -1.79017300 \\
\hline $\mathrm{C}$ & 3.40350100 & -2.64442000 & -3.74865000 \\
\hline $\mathrm{H}$ & 4.43669800 & -2.85026000 & -3.45073900 \\
\hline $\mathrm{H}$ & 2.86453600 & -3.59685100 & -3.73574700 \\
\hline $\mathrm{H}$ & 3.41782400 & -2.27474900 & -4.78223800 \\
\hline $\mathrm{N}$ & -1.39744400 & 0.52204000 & -1.65892900 \\
\hline $\mathrm{N}$ & -2.53232400 & -0.36657400 & 1.15117100 \\
\hline $\mathrm{C}$ & -2.79290000 & 0.42303800 & -1.93610000 \\
\hline $\mathrm{C}$ & -3.43339300 & -0.81726300 & -1.83998600 \\
\hline $\mathrm{C}$ & -3.49735400 & 1.55687900 & -2.33264500 \\
\hline $\mathrm{C}$ & -4.80074600 & -0.94760300 & -2.09148300 \\
\hline $\mathrm{H}$ & -2.84049400 & -1.68110200 & -1.56613700 \\
\hline $\mathrm{C}$ & -4.85639100 & 1.43469800 & -2.61633100 \\
\hline
\end{tabular}




\begin{tabular}{|c|c|c|c|}
\hline $\mathrm{H}$ & -2.99310800 & 2.51535400 & -2.38570900 \\
\hline $\mathrm{C}$ & -5.50027300 & 0.20503300 & -2.48617900 \\
\hline $\mathrm{H}$ & -5.42133800 & 2.30915900 & -2.92432200 \\
\hline $\mathrm{H}$ & -6.56233600 & 0.14301500 & -2.70254200 \\
\hline $\mathrm{C}$ & -3.15321500 & 0.90422100 & 1.05003800 \\
\hline $\mathrm{C}$ & -4.54852300 & 0.98478100 & 1.10953500 \\
\hline $\mathrm{C}$ & -2.38096300 & 2.05918100 & 0.92446500 \\
\hline $\mathrm{C}$ & -5.20163900 & 2.21730200 & 1.03315300 \\
\hline $\mathrm{H}$ & -5.10999700 & 0.06306100 & 1.19254000 \\
\hline $\mathrm{C}$ & -3.02324900 & 3.28984200 & 0.81080700 \\
\hline $\mathrm{H}$ & -1.30077800 & 1.99436300 & 0.92766700 \\
\hline $\mathrm{C}$ & -4.41188200 & 3.36836000 & 0.86703100 \\
\hline $\mathrm{H}$ & -2.43224200 & 4.19466300 & 0.70024200 \\
\hline $\mathrm{H}$ & -4.88690500 & 4.34144700 & 0.79405100 \\
\hline $\mathrm{C}$ & -5.52440700 & -2.29538000 & -1.97540900 \\
\hline $\mathrm{C}$ & -4.58718200 & -3.41987100 & -1.50337600 \\
\hline $\mathrm{H}$ & -3.76649000 & -3.59130900 & -2.20817200 \\
\hline $\mathrm{H}$ & -4.15442700 & -3.20144400 & -0.52007700 \\
\hline $\mathrm{H}$ & -5.14866600 & -4.35590200 & -1.41780500 \\
\hline $\mathrm{C}$ & -6.09784200 & -2.68267800 & -3.35593400 \\
\hline $\mathrm{H}$ & -6.62220500 & -3.64323200 & -3.29434300 \\
\hline $\mathrm{H}$ & -6.80830200 & -1.93468800 & -3.72242800 \\
\hline $\mathrm{H}$ & -5.29665900 & -2.77686700 & -4.09692400 \\
\hline $\mathrm{C}$ & -6.67742500 & -2.17239400 & -0.95710300 \\
\hline $\mathrm{H}$ & -7.40142400 & -1.40601900 & -1.25109300 \\
\hline $\mathrm{H}$ & -7.21390900 & -3.12447300 & -0.87336400 \\
\hline $\mathrm{H}$ & -6.29381000 & -1.91097900 & 0.03554100 \\
\hline $\mathrm{C}$ & -6.72727700 & 2.34907600 & 1.13095100 \\
\hline $\mathrm{C}$ & -7.07632200 & 3.21500600 & 2.36160300 \\
\hline $\mathrm{H}$ & -8.16349300 & 3.32101000 & 2.45439000 \\
\hline $\mathrm{H}$ & -6.69749800 & 2.75558600 & 3.28124700 \\
\hline $\mathrm{H}$ & -6.64618400 & 4.21876500 & 2.28643600 \\
\hline $\mathrm{C}$ & -7.42080600 & 0.98658000 & 1.28368300 \\
\hline $\mathrm{H}$ & -7.22241300 & 0.33753000 & 0.42569900 \\
\hline $\mathrm{H}$ & -7.09952800 & 0.46591300 & 2.19272200 \\
\hline $\mathrm{H}$ & -8.50442400 & 1.13031600 & 1.35089000 \\
\hline $\mathrm{C}$ & -7.27290500 & 3.02484000 & -0.14519300 \\
\hline $\mathrm{H}$ & -8.36063600 & 3.14171600 & -0.07581100 \\
\hline $\mathrm{H}$ & -6.83959000 & 4.01821800 & -0.29821500 \\
\hline $\mathrm{H}$ & -7.04703500 & 2.41823800 & -1.02741500 \\
\hline $\mathrm{Ni}$ & 1.15156100 & -2.00967800 & 0.34884700 \\
\hline $\mathrm{C}$ & 2.03648000 & 4.17082300 & 0.912557 \\
\hline $\mathrm{C}$ & 1.95339500 & 3.94560600 & -0.63821600 \\
\hline B & 3.54986600 & 2.60481700 & 0.267926 \\
\hline
\end{tabular}




$\begin{array}{lrrr}\mathrm{O} & 3.30080400 & 3.54438900 & 1.24124000 \\ \mathrm{O} & 2.67563900 & 2.69749200 & -0.80317900 \\ \mathrm{C} & 2.08040000 & 5.62981600 & 1.34831500 \\ \mathrm{H} & 1.17135700 & 6.15538200 & 1.03405100 \\ \mathrm{H} & 2.14762400 & 5.68727100 & 2.43910300 \\ \mathrm{H} & 2.94641300 & 6.14512000 & 0.92799300 \\ \mathrm{C} & 0.95561800 & 3.40855300 & 1.68512000 \\ \mathrm{H} & 1.20138100 & 3.42480800 & 2.75071600 \\ \mathrm{H} & -0.03195200 & 3.86206500 & 1.55317400 \\ \mathrm{H} & 0.91917300 & 2.36144900 & 1.36925800 \\ \mathrm{C} & 2.71399400 & 5.00556900 & -1.44158500 \\ \mathrm{H} & 2.79897800 & 4.66801000 & -2.47877300 \\ \mathrm{H} & 2.19778100 & 5.97099800 & -1.42949200 \\ \mathrm{H} & 3.72630200 & 5.14065400 & -1.05098400 \\ \mathrm{C} & 0.53944000 & 3.78029000 & -1.18276000 \\ \mathrm{H} & -0.06868000 & 4.66411000 & -0.95818000 \\ \mathrm{H} & 0.57519100 & 3.66953000 & -2.27149600 \\ \mathrm{H} & 0.04432200 & 2.90131600 & -0.76568400 \\ \mathrm{C} & 5.29467000 & 1.00890900 & -0.89188100 \\ \mathrm{H} & 4.53368700 & 0.79600300 & -1.64759100 \\ \mathrm{H} & 5.85596400 & 0.08623600 & -0.71333600 \\ \mathrm{C} & 6.25797500 & 2.00587700 & -1.51018200 \\ \mathrm{~F} & 7.28747100 & 2.29268900 & -0.68426200 \\ \mathrm{~F} & 5.64900900 & 3.18215100 & -1.80813800 \\ \mathrm{I} & 3.12898800 & -0.38015700 & 1.20862100 \\ \mathrm{~F} & 6.78430100 & 1.53168400 & -2.66174900 \\ \mathrm{C} & 4.66917100 & 1.55520200 & 0.36500000 \\ \mathrm{H} & 5.33111900 & 1.59866900 & 1.22661200 \\ \mathrm{I} & 1.90233400 & -4.42208700 & 0.51869200\end{array}$

Cartesian coordinates of the calculated transition states.

TS-L4Ni-I+Cl

Thermal Correction to Free Energy: 0.706571 Hartree SCF energy: -2186.782101 Hartree

Gibbs free energy: -2186.075530 Hartree Imaginary frequency: -85.31

Coordinates:

$\begin{array}{lrrc}\mathrm{C} & -0.80837500 & -2.08664500 & 1.77419000 \\ \mathrm{C} & -0.13603700 & -0.45464600 & 0.37341500 \\ \mathrm{C} & 0.74813200 & -2.00847000 & 1.80164800 \\ \mathrm{H} & -1.20899100 & -1.93240200 & 2.78203400 \\ \mathrm{H} & 1.22977900 & -2.96966900 & 1.60827100 \\ \mathrm{H} & 1.11469300 & -1.61987600 & 2.75976300\end{array}$




\begin{tabular}{|c|c|c|c|}
\hline $\mathrm{N}$ & -1.20562300 & -0.93191500 & 0.94574400 \\
\hline $\mathrm{C}$ & -0.32300700 & 0.65636200 & -0.54625700 \\
\hline $\mathrm{C}$ & -0.08891300 & 1.93678400 & -2.43201500 \\
\hline $\mathrm{C}$ & -1.55330100 & 1.96532300 & -1.90679300 \\
\hline $\mathrm{H}$ & 0.33271600 & 2.93061500 & -2.60984500 \\
\hline $\mathrm{H}$ & -0.02391200 & 1.32902000 & -3.33862400 \\
\hline $\mathrm{H}$ & -2.21856300 & 1.55741600 & -2.67128800 \\
\hline $\mathrm{N}$ & -1.55084000 & 0.99521700 & -0.80026700 \\
\hline $\mathrm{C}$ & -1.36282300 & -3.42805400 & 1.24170300 \\
\hline $\mathrm{H}$ & -0.98361900 & -4.18846100 & 1.94742900 \\
\hline $\mathrm{C}$ & -0.84957200 & -3.75936200 & -0.16504900 \\
\hline $\mathrm{H}$ & -1.22794600 & -3.04827600 & -0.90942100 \\
\hline $\mathrm{H}$ & 0.24567000 & -3.75046100 & -0.21744800 \\
\hline $\mathrm{H}$ & -1.17232700 & -4.76233400 & -0.46334500 \\
\hline $\mathrm{C}$ & -2.89898600 & -3.44549700 & 1.31425200 \\
\hline $\mathrm{H}$ & -3.21911100 & -3.06216800 & 2.29275400 \\
\hline $\mathrm{H}$ & -3.30382500 & -2.74717200 & 0.57434200 \\
\hline $\mathrm{C}$ & -3.51820100 & -4.82666500 & 1.08846200 \\
\hline $\mathrm{H}$ & -4.60616600 & -4.78143000 & 1.20956700 \\
\hline $\mathrm{H}$ & -3.31485300 & -5.19825300 & 0.07886200 \\
\hline $\mathrm{H}$ & -3.12739100 & -5.56393200 & 1.80400300 \\
\hline $\mathrm{C}$ & -2.05693500 & 3.34951800 & -1.44779600 \\
\hline $\mathrm{H}$ & -1.92772700 & 4.02177600 & -2.31213200 \\
\hline $\mathrm{C}$ & -1.22626800 & 3.89930800 & -0.27947600 \\
\hline $\mathrm{H}$ & -0.15346000 & 3.90739200 & -0.50531800 \\
\hline $\mathrm{H}$ & -1.51776100 & 4.92533800 & -0.03270100 \\
\hline $\mathrm{H}$ & -1.37589700 & 3.28005300 & 0.61182100 \\
\hline $\mathrm{C}$ & -3.55821100 & 3.28429400 & -1.11769500 \\
\hline $\mathrm{H}$ & -4.07985500 & 2.81708100 & -1.96289200 \\
\hline $\mathrm{H}$ & -3.71455900 & 2.61054000 & -0.26761700 \\
\hline $\mathrm{C}$ & -4.19950600 & 4.64270000 & -0.82307000 \\
\hline $\mathrm{H}$ & -5.27986600 & 4.53311200 & -0.67749000 \\
\hline $\mathrm{H}$ & -3.79205600 & 5.09967200 & 0.08608200 \\
\hline $\mathrm{H}$ & -4.04098600 & 5.34872900 & -1.65006100 \\
\hline $\mathrm{N}$ & 0.63935200 & 1.26824800 & -1.33431900 \\
\hline $\mathrm{N}$ & 1.06729100 & -1.05371500 & 0.71565800 \\
\hline $\mathrm{C}$ & 1.89836300 & 1.66556900 & -0.87527200 \\
\hline $\mathrm{C}$ & 2.11548200 & 1.87047200 & 0.49761900 \\
\hline $\mathrm{C}$ & 2.95319500 & 1.85553800 & -1.77530700 \\
\hline $\mathrm{C}$ & 3.36839700 & 2.21936800 & 0.99734000 \\
\hline $\mathrm{H}$ & 1.27157100 & 1.75349300 & 1.16298600 \\
\hline $\mathrm{C}$ & 4.20421800 & 2.22311000 & -1.28646100 \\
\hline $\mathrm{H}$ & 2.79613200 & 1.67446000 & -2.83312700 \\
\hline $\mathrm{C}$ & 4.41975300 & 2.39298000 & 0.08113200 \\
\hline
\end{tabular}




\begin{tabular}{|c|c|c|c|}
\hline $\mathrm{H}$ & 5.02937700 & 2.35835300 & -1.98084500 \\
\hline $\mathrm{H}$ & 5.40977200 & 2.67049800 & 0.43136100 \\
\hline $\mathrm{C}$ & 2.05433100 & -1.35752800 & -0.26527400 \\
\hline $\mathrm{C}$ & 3.39322500 & -1.44457200 & 0.13706900 \\
\hline $\mathrm{C}$ & 1.69877900 & -1.55328600 & -1.60343000 \\
\hline $\mathrm{C}$ & 4.40880600 & -1.71887400 & -0.78244200 \\
\hline $\mathrm{H}$ & 3.62052900 & -1.26276100 & 1.18057300 \\
\hline $\mathrm{C}$ & 2.71273700 & -1.79569300 & -2.52832100 \\
\hline $\mathrm{H}$ & 0.65764800 & -1.51372100 & -1.92753200 \\
\hline $\mathrm{C}$ & 4.04468100 & -1.87820200 & -2.12978600 \\
\hline $\mathrm{H}$ & 2.44580900 & -1.93957100 & -3.57186500 \\
\hline $\mathrm{H}$ & 4.80923000 & -2.07408900 & -2.87663200 \\
\hline $\mathrm{C}$ & 3.60032600 & 2.45123800 & 2.49727100 \\
\hline $\mathrm{C}$ & 2.33626100 & 2.18185700 & 3.33126400 \\
\hline $\mathrm{H}$ & 1.51939600 & 2.85964400 & 3.06168400 \\
\hline $\mathrm{H}$ & 1.98157700 & 1.15351500 & 3.20075400 \\
\hline $\mathrm{H}$ & 2.55601400 & 2.33093000 & 4.39458100 \\
\hline $\mathrm{C}$ & 4.03037500 & 3.91662400 & 2.72334000 \\
\hline $\mathrm{H}$ & 4.20100200 & 4.10758900 & 3.79040100 \\
\hline $\mathrm{H}$ & 4.95484200 & 4.15220300 & 2.18589200 \\
\hline $\mathrm{H}$ & 3.25357000 & 4.60436000 & 2.37134800 \\
\hline $\mathrm{C}$ & 4.71087400 & 1.50767600 & 3.00286500 \\
\hline $\mathrm{H}$ & 5.64771900 & 1.65012900 & 2.45491500 \\
\hline $\mathrm{H}$ & 4.91150200 & 1.68667300 & 4.06662500 \\
\hline $\mathrm{H}$ & 4.40843500 & 0.46190000 & 2.88522900 \\
\hline $\mathrm{C}$ & 5.88654900 & -1.82176700 & -0.37893400 \\
\hline $\mathrm{C}$ & 6.44510300 & -3.19350300 & -0.81480600 \\
\hline $\mathrm{H}$ & 7.50357100 & -3.28258400 & -0.53833700 \\
\hline $\mathrm{H}$ & 5.89377800 & -4.00731400 & -0.33037300 \\
\hline $\mathrm{H}$ & 6.36732900 & -3.33733100 & -1.89688600 \\
\hline $\mathrm{C}$ & 6.09105900 & -1.68484100 & 1.13774700 \\
\hline $\mathrm{H}$ & 5.76873300 & -0.70435400 & 1.49846100 \\
\hline $\mathrm{H}$ & 5.53898900 & -2.45368200 & 1.69015400 \\
\hline $\mathrm{H}$ & 7.15447000 & -1.79628500 & 1.38044900 \\
\hline $\mathrm{C}$ & 6.68142100 & -0.69541600 & -1.07416300 \\
\hline $\mathrm{H}$ & 7.74542600 & -0.75330000 & -0.80992500 \\
\hline $\mathrm{H}$ & 6.59905500 & -0.76147000 & -2.16399700 \\
\hline $\mathrm{H}$ & 6.29656900 & 0.28275900 & -0.76950700 \\
\hline $\mathrm{Ni}$ & -2.88383800 & -0.19289600 & 0.04736800 \\
\hline I & -5.37995200 & -0.18133200 & 0.69554600 \\
\hline $\mathrm{Cl}$ & -1.71023500 & -1.33668800 & -2.80429100 \\
\hline
\end{tabular}

Cartesian coordinates of the calculated transition states.

L4Ni-Cl 
Thermal Correction to Free Energy: 0.710270 Hartree

SCF energy: -2175.254896 Hartree

Gibbs free energy: -2174.544626 Hartree

Coordinates:

\begin{tabular}{|c|c|c|c|}
\hline C & 1.24110800 & 2.27886200 & 1.50565000 \\
\hline $\mathrm{C}$ & 0.70704300 & 0.66712700 & 0.02621500 \\
\hline $\mathrm{C}$ & -0.31103700 & 2.19143400 & 1.39883700 \\
\hline $\mathrm{H}$ & 1.56071700 & 2.12062700 & 2.53979200 \\
\hline $\mathrm{H}$ & -0.77538200 & 3.14921300 & 1.14869000 \\
\hline $\mathrm{H}$ & -0.76652700 & 1.81207300 & 2.32103000 \\
\hline $\mathrm{N}$ & 1.71968800 & 1.13868800 & 0.69518100 \\
\hline $\mathrm{C}$ & 1.00644500 & -0.43758500 & -0.88664200 \\
\hline $\mathrm{C}$ & 0.89822300 & -2.17239600 & -2.37791200 \\
\hline $\mathrm{C}$ & 2.37254700 & -1.85260900 & -1.98201500 \\
\hline $\mathrm{H}$ & 0.62442400 & -3.21357200 & -2.18634100 \\
\hline $\mathrm{H}$ & 0.69590500 & -1.94617100 & -3.43023000 \\
\hline $\mathrm{H}$ & 2.95665400 & -1.60001700 & -2.87212600 \\
\hline $\mathrm{N}$ & 2.26594200 & -0.64016800 & -1.14227600 \\
\hline $\mathrm{C}$ & 1.82538300 & 3.62267800 & 1.01255800 \\
\hline $\mathrm{H}$ & 1.27450800 & 4.39964500 & 1.56711900 \\
\hline $\mathrm{C}$ & 1.57307100 & 3.83189200 & -0.48686400 \\
\hline $\mathrm{H}$ & 2.17100000 & 3.12896100 & -1.07731900 \\
\hline $\mathrm{H}$ & 0.51876100 & 3.68878000 & -0.75298300 \\
\hline $\mathrm{H}$ & 1.84888200 & 4.84540300 & -0.79079200 \\
\hline $\mathrm{C}$ & 3.31255500 & 3.74590000 & 1.38556600 \\
\hline $\mathrm{H}$ & 3.41833400 & 3.54296400 & 2.46031900 \\
\hline $\mathrm{H}$ & 3.88882700 & 2.96651100 & 0.87443300 \\
\hline $\mathrm{C}$ & 3.92025600 & 5.11597400 & 1.07404900 \\
\hline $\mathrm{H}$ & 4.95146200 & 5.16781100 & 1.43818200 \\
\hline $\mathrm{H}$ & 3.94441200 & 5.31384400 & -0.00292700 \\
\hline $\mathrm{H}$ & 3.35254700 & 5.92573400 & 1.55153700 \\
\hline $\mathrm{C}$ & 3.08320500 & -3.00857300 & -1.24081600 \\
\hline $\mathrm{H}$ & 2.92918200 & -3.89677700 & -1.87482800 \\
\hline $\mathrm{C}$ & 2.44308500 & -3.27912800 & 0.12782000 \\
\hline $\mathrm{H}$ & 1.35598700 & -3.40612200 & 0.05592600 \\
\hline $\mathrm{H}$ & 2.84732000 & -4.19216900 & 0.57350100 \\
\hline $\mathrm{H}$ & 2.64391900 & -2.45021600 & 0.81564600 \\
\hline $\mathrm{C}$ & 4.59814700 & -2.75779400 & -1.15000200 \\
\hline $\mathrm{H}$ & 4.97202700 & -2.53800600 & -2.15975200 \\
\hline $\mathrm{H}$ & 4.79130000 & -1.86001800 & -0.55238300 \\
\hline C & 5.38909500 & -3.93300400 & -0.57012100 \\
\hline $\mathrm{H}$ & 6.46402800 & -3.73141400 & -0.62079500 \\
\hline & 5.14113200 & 095 & 0.481853 \\
\hline
\end{tabular}




\begin{tabular}{|c|c|c|c|}
\hline $\mathrm{H}$ & 5.19346000 & -4.86171500 & -1.12264700 \\
\hline $\mathrm{N}$ & 0.10817000 & -1.27784900 & -1.50158900 \\
\hline $\mathrm{N}$ & -0.51550800 & 1.22983500 & 0.29527000 \\
\hline $\mathrm{C}$ & -1.10310900 & -1.69804800 & -0.89379400 \\
\hline $\mathrm{C}$ & -1.17760600 & -1.81859100 & 0.49961900 \\
\hline $\mathrm{C}$ & -2.21307600 & -1.99074000 & -1.68541100 \\
\hline $\mathrm{C}$ & -2.36341600 & -2.18860100 & 1.13541200 \\
\hline $\mathrm{H}$ & -0.28416300 & -1.62010200 & 1.07640100 \\
\hline $\mathrm{C}$ & -3.39500500 & -2.38948100 & -1.06374100 \\
\hline $\mathrm{H}$ & -2.15631700 & -1.87457100 & -2.76222700 \\
\hline $\mathrm{C}$ & -3.47513900 & -2.47441800 & 0.32556400 \\
\hline $\mathrm{H}$ & -4.26750000 & -2.61826900 & -1.66843900 \\
\hline $\mathrm{H}$ & -4.41323700 & -2.77582600 & 0.78155400 \\
\hline $\mathrm{C}$ & -1.58554200 & 1.33486300 & -0.62456300 \\
\hline $\mathrm{C}$ & -2.89916300 & 1.31846400 & -0.13963700 \\
\hline $\mathrm{C}$ & -1.34106000 & 1.45768500 & -1.99454000 \\
\hline $\mathrm{C}$ & -3.99084500 & 1.40558700 & -1.00583700 \\
\hline $\mathrm{H}$ & -3.04610800 & 1.20580900 & 0.92689400 \\
\hline $\mathrm{C}$ & -2.42390000 & 1.52102200 & -2.86915500 \\
\hline $\mathrm{H}$ & -0.32258700 & 1.50482300 & -2.36435500 \\
\hline $\mathrm{C}$ & -3.72825200 & 1.49606900 & -2.38348400 \\
\hline $\mathrm{H}$ & -2.24625000 & 1.61003100 & -3.93719600 \\
\hline $\mathrm{H}$ & -4.55275300 & 1.55712300 & -3.08664400 \\
\hline $\mathrm{C}$ & -2.45890400 & -2.31494500 & 2.66173100 \\
\hline $\mathrm{C}$ & -1.13994200 & -1.94293600 & 3.36043400 \\
\hline $\mathrm{H}$ & -0.31919400 & -2.60547700 & 3.06520600 \\
\hline $\mathrm{H}$ & -0.84230800 & -0.91107100 & 3.14049000 \\
\hline $\mathrm{H}$ & -1.26100800 & -2.03026700 & 4.44532600 \\
\hline $\mathrm{C}$ & -2.81298100 & -3.77231600 & 3.02856100 \\
\hline $\mathrm{H}$ & -2.88566200 & -3.88500900 & 4.11661300 \\
\hline $\mathrm{H}$ & -3.77074400 & -4.07605100 & 2.59392900 \\
\hline $\mathrm{H}$ & -2.04442000 & -4.46211400 & 2.66329000 \\
\hline $\mathrm{C}$ & -3.56072300 & -1.37093200 & 3.18715700 \\
\hline $\mathrm{H}$ & -4.53733300 & -1.60071500 & 2.74988800 \\
\hline $\mathrm{H}$ & -3.65209800 & -1.46046900 & 4.27586300 \\
\hline $\mathrm{H}$ & -3.32323100 & -0.32756500 & 2.95139200 \\
\hline $\mathrm{C}$ & -5.44201800 & 1.39994000 & -0.50714900 \\
\hline $\mathrm{C}$ & -6.12322000 & 2.72243200 & -0.92170600 \\
\hline $\mathrm{H}$ & -7.16416400 & 2.73742500 & -0.57765200 \\
\hline $\mathrm{H}$ & -5.60492800 & 3.58181900 & -0.48216200 \\
\hline $\mathrm{H}$ & -6.12690200 & 2.85323500 & -2.00848300 \\
\hline $\mathrm{C}$ & -5.53355200 & 1.26596300 & 1.02101000 \\
\hline $\mathrm{H}$ & -5.09114000 & 0.32784100 & 1.36901000 \\
\hline $\mathrm{H}$ & -5.03266700 & 2.09519100 & 1.53317600 \\
\hline
\end{tabular}




$\begin{array}{lrrr}\mathrm{H} & -6.58476600 & 1.27481300 & 1.32862900 \\ \mathrm{C} & -6.19779800 & 0.21092600 & -1.13847300 \\ \mathrm{H} & -7.23880500 & 0.19748500 & -0.79463200 \\ \mathrm{H} & -6.20762400 & 0.26892400 & -2.23153500 \\ \mathrm{H} & -5.72700000 & -0.73549100 & -0.85536100 \\ \mathrm{Ni} & 3.49131000 & 0.42820000 & 0.06023800 \\ \mathrm{Cl} & 5.60776900 & 0.66242000 & 0.48726000\end{array}$

Cartesian coordinates of the calculated transition states.

L4Ni-I

Thermal Correction to Free Energy: 0.707759 Hartree

SCF energy: -1726.454530 Hartree

Gibbs free energy: -1725.746771 Hartree

Coordinates:

$\begin{array}{lrrr}\mathrm{C} & 0.76579800 & 2.25824000 & 1.30774800 \\ \mathrm{C} & 0.13082000 & 0.63289600 & -0.11396900 \\ \mathrm{C} & -0.78861800 & 2.17281400 & 1.30685400 \\ \mathrm{H} & 1.15709700 & 2.12578100 & 2.32020600 \\ \mathrm{H} & -1.27002200 & 3.13143200 & 1.09732000 \\ \mathrm{H} & -1.18026300 & 1.78208600 & 2.25350800 \\ \mathrm{~N} & 1.18234300 & 1.09407300 & 0.49578000 \\ \mathrm{C} & 0.35841000 & -0.49271300 & -1.02903700 \\ \mathrm{C} & 0.10909600 & -2.23762400 & -2.49016600 \\ \mathrm{C} & 1.61384400 & -1.95318900 & -2.19484100 \\ \mathrm{H} & -0.17855200 & -3.26808100 & -2.26453300 \\ \mathrm{H} & -0.15616400 & -2.02072100 & -3.53023500 \\ \mathrm{H} & 2.14163200 & -1.71383100 & -3.12299100 \\ \mathrm{~N} & 1.59442000 & -0.73511600 & -1.35287500 \\ \mathrm{C} & 1.31662600 & 3.58519300 & 0.73546300 \\ \mathrm{H} & 0.83259100 & 4.37814200 & 1.32831400 \\ \mathrm{C} & 0.92449900 & 3.77631900 & -0.73592200 \\ \mathrm{H} & 1.43691000 & 3.04120000 & -1.36649100 \\ \mathrm{H} & -0.15476500 & 3.66931100 & -0.89571200 \\ \mathrm{H} & 1.20611700 & 4.77263500 & -1.08740600 \\ \mathrm{C} & 2.83463000 & 3.69060000 & 0.95610200 \\ \mathrm{H} & 3.05412000 & 3.45751000 & 2.00644500 \\ \mathrm{H} & 3.34418600 & 2.91778700 & 0.37015800 \\ \mathrm{C} & 3.42254000 & 5.06176600 & 0.61455400 \\ \mathrm{H} & 4.48829500 & 5.09073100 & 0.86377600 \\ \mathrm{H} & 3.33074300 & 5.29177000 & -0.45243700 \\ \mathrm{H} & 2.92323300 & 5.86341500 & 1.17458800 \\ \mathrm{C} & 2.34202300 & -3.13011000 & -1.50575700 \\ \mathrm{H} & 2.09871500 & -4.01614600 & -2.11424400\end{array}$




\begin{tabular}{|c|c|c|c|}
\hline $\mathrm{C}$ & 1.81208400 & -3.36516700 & -0.08462100 \\
\hline $\mathrm{H}$ & 0.71795200 & -3.44238900 & -0.06305000 \\
\hline $\mathrm{H}$ & 2.21090800 & -4.29425700 & 0.33158700 \\
\hline $\mathrm{H}$ & 2.10950000 & -2.54415800 & 0.57724400 \\
\hline $\mathrm{C}$ & 3.86656600 & -2.93813900 & -1.55328000 \\
\hline $\mathrm{H}$ & 4.15944800 & -2.77164700 & -2.59950100 \\
\hline $\mathrm{H}$ & 4.14079900 & -2.02817300 & -1.00870300 \\
\hline $\mathrm{C}$ & 4.66340300 & -4.11657200 & -0.98971100 \\
\hline $\mathrm{H}$ & 5.73578100 & -3.96096000 & -1.14557900 \\
\hline $\mathrm{H}$ & 4.50593700 & -4.23175600 & 0.08763100 \\
\hline $\mathrm{H}$ & 4.38446300 & -5.06078100 & -1.47621500 \\
\hline $\mathrm{N}$ & -0.59874600 & -1.31114600 & -1.57826800 \\
\hline $\mathrm{N}$ & -1.06524400 & 1.21947200 & 0.21127900 \\
\hline $\mathrm{C}$ & -1.77903200 & -1.69634600 & -0.88943900 \\
\hline $\mathrm{C}$ & -1.76454700 & -1.80713700 & 0.50643800 \\
\hline $\mathrm{C}$ & -2.94349200 & -1.96919900 & -1.60573000 \\
\hline $\mathrm{C}$ & -2.91433800 & -2.14648200 & 1.22092000 \\
\hline $\mathrm{H}$ & -0.83173600 & -1.62554000 & 1.02340500 \\
\hline $\mathrm{C}$ & -4.08977500 & -2.34157100 & -0.90586500 \\
\hline $\mathrm{H}$ & -2.95415000 & -1.85985600 & -2.68467900 \\
\hline $\mathrm{C}$ & -4.08109900 & -2.41599600 & 0.48636600 \\
\hline $\mathrm{H}$ & -5.00349800 & -2.55832800 & -1.45106100 \\
\hline $\mathrm{H}$ & -4.99320500 & -2.69654200 & 1.00385800 \\
\hline $\mathrm{C}$ & -2.18517600 & 1.33631100 & -0.64528300 \\
\hline $\mathrm{C}$ & -3.46843600 & 1.33946500 & -0.08508000 \\
\hline $\mathrm{C}$ & -2.01727700 & 1.45622200 & -2.02675600 \\
\hline $\mathrm{C}$ & -4.60662700 & 1.44661200 & -0.88693700 \\
\hline $\mathrm{H}$ & -3.55593100 & 1.22651000 & 0.98805500 \\
\hline $\mathrm{C}$ & -3.14744400 & 1.54028700 & -2.83747700 \\
\hline $\mathrm{H}$ & -1.02093900 & 1.48906100 & -2.45404700 \\
\hline $\mathrm{C}$ & -4.42180100 & 1.53786900 & -2.27707800 \\
\hline $\mathrm{H}$ & -3.03024200 & 1.62918700 & -3.91380200 \\
\hline $\mathrm{H}$ & -5.28387500 & 1.61849900 & -2.93152700 \\
\hline $\mathrm{C}$ & -2.91347700 & -2.25684000 & 2.75129700 \\
\hline $\mathrm{C}$ & -1.54928600 & -1.88929700 & 3.35998100 \\
\hline $\mathrm{H}$ & -0.75487800 & -2.56393500 & 3.02308600 \\
\hline $\mathrm{H}$ & -1.25595900 & -0.86342200 & 3.10762700 \\
\hline $\mathrm{H}$ & -1.60185700 & -1.96068300 & 4.45139500 \\
\hline $\mathrm{C}$ & -3.25517600 & -3.70724100 & 3.15537400 \\
\hline $\mathrm{H}$ & -3.25969300 & -3.80785700 & 4.24695700 \\
\hline $\mathrm{H}$ & -4.24101400 & -4.00764700 & 2.78574000 \\
\hline $\mathrm{H}$ & -2.51649600 & -4.40723100 & 2.75009200 \\
\hline C & -3.97125800 & -1.29734300 & 3.33591300 \\
\hline & -4.97768600 & -1.52762600 & 2.97251000 \\
\hline
\end{tabular}




$\begin{array}{lrrr}\mathrm{H} & -3.98666100 & -1.36883400 & 4.42956200 \\ \mathrm{H} & -3.74499800 & -0.25952600 & 3.06684900 \\ \mathrm{C} & -6.02646300 & 1.46610800 & -0.30605600 \\ \mathrm{C} & -6.69319400 & 2.81451200 & -0.65582000 \\ \mathrm{H} & -7.71290500 & 2.84988900 & -0.25465100 \\ \mathrm{H} & -6.12820300 & 3.65056900 & -0.22906100 \\ \mathrm{H} & -6.75210500 & 2.96769900 & -1.73810800 \\ \mathrm{C} & -6.03376600 & 1.30077600 & 1.22196200 \\ \mathrm{H} & -5.58894800 & 0.34772600 & 1.52356600 \\ \mathrm{H} & -5.49006500 & 2.10973800 & 1.72264400 \\ \mathrm{H} & -7.06541000 & 1.32020000 & 1.58919700 \\ \mathrm{C} & -6.84985800 & 0.31093400 & -0.91496500 \\ \mathrm{H} & -7.86878400 & 0.31802700 & -0.51042300 \\ \mathrm{H} & -6.92285200 & 0.39093500 & -2.00412700 \\ \mathrm{H} & -6.39021600 & -0.65323600 & -0.67720400 \\ \mathrm{Ni} & 2.89707600 & 0.25864800 & -0.14767400 \\ \mathrm{I} & 5.30235700 & 0.36159300 & 0.58929900\end{array}$

Cartesian coordinates of the calculated transition states.

CF3-I-Bpin

Thermal Correction to Free Energy: 0.196872 Hartree

SCF energy: -838.141921 Hartree

Gibbs free energy: -837.945049 Hartree

Coordinates:

$\begin{array}{lrrr}\text { C } & -3.02987600 & -0.11869700 & 0.56938700 \\ \text { C } & -2.84230200 & 0.92636200 & -0.59182800 \\ \text { B } & -0.83407000 & 0.36602000 & 0.30925600 \\ \text { O } & -1.73192400 & -0.07442200 & 1.23759400 \\ \text { O } & -1.40181200 & 0.87362900 & -0.82606300 \\ \text { C } & -4.10915600 & 0.23135700 & 1.58555400 \\ \mathrm{H} & -5.09220700 & 0.27333800 & 1.10360500 \\ \mathrm{H} & -4.14396000 & -0.53804900 & 2.36263200 \\ \mathrm{H} & -3.91402800 & 1.19153200 & 2.06759200 \\ \mathrm{C} & -3.20983900 & -1.55310500 & 0.06605100 \\ \mathrm{H} & -3.13345100 & -2.23898500 & 0.91449900 \\ \mathrm{H} & -4.18775200 & -1.69069200 & -0.40568100 \\ \mathrm{H} & -2.43138400 & -1.82082500 & -0.65459600 \\ \mathrm{C} & -3.16076700 & 2.36296000 & -0.17032400 \\ \mathrm{H} & -2.81424600 & 3.04775400 & -0.94989800 \\ \mathrm{H} & -4.23642000 & 2.51100300 & -0.03286500 \\ \mathrm{H} & -2.65275700 & 2.62310300 & 0.76352500 \\ \mathrm{C} & -3.55491500 & 0.58052500 & -1.89315400\end{array}$




$\begin{array}{lrrc}\mathrm{H} & -4.63912500 & 0.54047300 & -1.74036900 \\ \mathrm{H} & -3.34534900 & 1.34966900 & -2.64278300 \\ \mathrm{H} & -3.22115800 & -0.37999300 & -2.29052800 \\ \mathrm{C} & 0.72360300 & 0.31448400 & 0.51388900 \\ \mathrm{H} & 1.00735500 & 0.29927300 & 1.56550700 \\ \mathrm{C} & 1.48506000 & 1.37882600 & -0.25994400 \\ \mathrm{H} & 1.02641600 & 2.34679800 & -0.01850600 \\ \mathrm{H} & 1.38319100 & 1.24452000 & -1.33927800 \\ \mathrm{C} & 2.95526800 & 1.52515400 & 0.07938900 \\ \mathrm{~F} & 3.70919400 & 0.50158500 & -0.35971100 \\ \mathrm{~F} & 3.14089600 & 1.62077300 & 1.41723600 \\ \mathrm{I} & 1.23429700 & -1.77104000 & -0.15372100 \\ \mathrm{~F} & 3.44712400 & 2.65489100 & -0.47844700\end{array}$

Cartesian coordinates of the calculated transition states.

Chloride ion

Thermal Correction to Free Energy: -0.015023 Hartree

SCF energy: -460.3216787 Hartree

Gibbs free energy: -460.3367017 Hartree 


\section{Supplementary References}

1. Vedrenne, E.; Wallner, O. A.; Vitale, M.; Schmidt, F.; Aggarwal, V. K. Homologation of Boronic Esters with Lithiated Epoxides for the Stereocontrolled Synthesis of 1,2-and 1,3-Diols and 1,2,4-Triols. Org. Lett. 2009, 11, 165-168.

2. Fang, T.; Qiu, J.; Yang, K.; Song, Q. Photo-induced weak base-catalyzed synthesis of $\alpha$-haloboronates from vinyl boronates and polyfluoroalkyl halides. Org. Chem. Front. 2021, 8, 1991-1996.

3. Huihui, K. M.; Caputo, J. A.; Melchor, Z.; Olivares, A. M.; Spiewak, A. M.; Johnson, K. A.; DiBenedetto, T. A.; Kim, S.; Ackerman, L. K. G.; Weix, D. J. J. Am. Chem. Soc. 2016, 138, 5016-5019.

4. Joung, S.; Bergmann, A. M.; Brown, M. K. Chem. Sci., 2019, 10, 10944-10947.

5. Frisch, M. J.; Trucks, G. W.; Schlegel, H. B.; Scuseria, G.E.; Robb, M. A.; Cheeseman, J. R.; Scalmani, G.; Barone, V.; Mennucci, B.; Petersson, G. A.; Nakatsuji, H.; Caricato, M.; Li, X.; Hratchian, H. P.; Izmaylov, A. F.; Bloino, J.; Zheng, G.; Sonnenberg, J. L.; Hada, M.; Ehara, M.; Toyota, K.; Fukuda, R.; Hasegawa, J.; Ishida, M.; Nakajima, T.; Honda, Y.; Kitao, O.; Nakai, H.; Vreven, T.; Montgomery, J. A.; Jr.; Peralta, J. E.; Ogliaro, F.; Bearpark, M.; Heyd, J. J.; Brothers, E.; Kudin, K. N.; Staroverov, V. N.; Keith, T.; Kobayashi, R.; Normand, J.; Raghavachari, K.; Rendell, A.; Burant, J. C.; Iyengar, S. S.; Tomasi, J.; Cossi, M. ; Rega, N.; Millam, J. M.; Klene, M. ; Knox, J. E.; Cross, J. B.; Bakken, V.; Adamo, C.; Jaramillo, J.; Gomperts, R.; Stratmann, R. E.; Yazyev, O.; Austin, A. J.; Cammi, R.; Pomelli, C.; Ochterski, J. W.; Martin, R. L.; Morokuma, K.; Zakrzewski, V. G.; Voth, G. A.; Salvador, P.; Dannenberg, J. J.; Dapprich, S.; Daniels, A. D.; Farkas, O.; Foresman, J. B.; Ortiz, J. V.; Cioslowski, J.; Fox, D. J. Gaussian 09, revision E.01; Gaussian, Inc., Wallingford CT, 2013.

6. (a) Lee, C.; Yang, W.; Parr, R. G., Development of the Colle-Salvetti correlation-energy formula into a functional of the electron density. Phys. Rev. B. 1988, 37, 785; (b) Becke, A. D., Density-functional thermochemistry. III. the role of exact exchange. J. Chem. Phys. 1993, 98, 5648.

7. Grimme, S.; Antony, J.; Ehrlich, S.; Krieg, H., A consistent and accurate ab initio parametrization of density functional dispersion correction (DFT-D) for the 94 elements H-Pu. J. Chem. Phys. 2010, 132, 154104.

8. Zhao, Y.; Truhlar, D. G. The M06 suite of density functionals for main group thermochemistry, thermochemical kinetics, noncovalent interactions, excited states, and transition elements: two new functionals and systematic testing of four M06-class functionals and 12 other functionals. Theor. Chem. Acc. 2008, 120, 215.

9. (a) Cossi, M.; Barone, V.; Cammi, R.; Tomasi, J. Ab initio study of solvated molecules: a new implementation of the polarizable continuum model. Chem. Phys. Lett. 1996, 255 (327-335); (b) Cancès, E.; Mennucci, B.; Tomasi, J., A new integral equation formalism for the polarizable continuum model: theoretical background and applications to isotropic and anisotropic dielectrics. J. Chem. Phys. 1997, 107, 3032.

10. Legault, C. Y., CYLview, 1.0b; Universitéde Sherbrooke:. 2009. 
9. Spectra data of the compounds

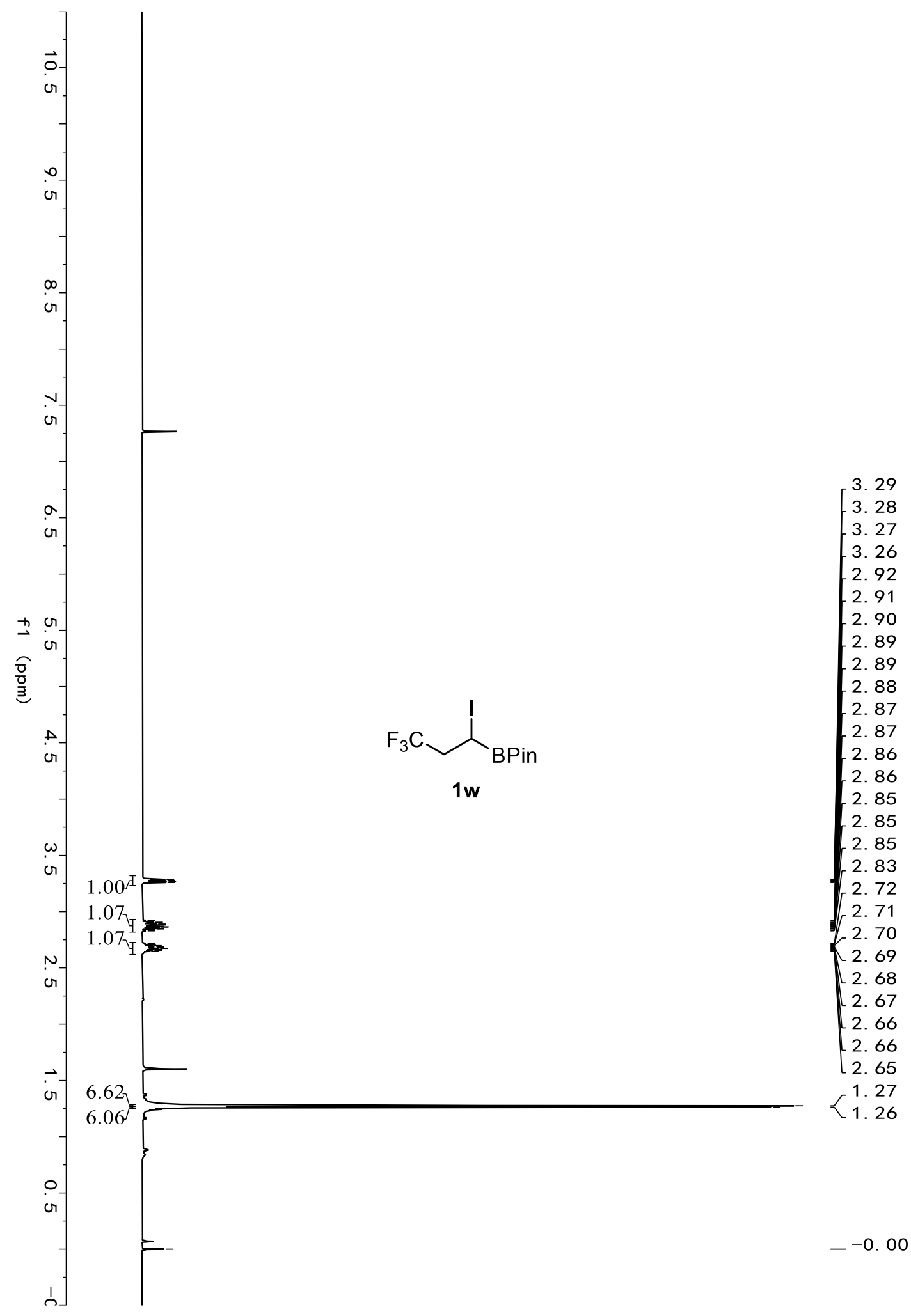




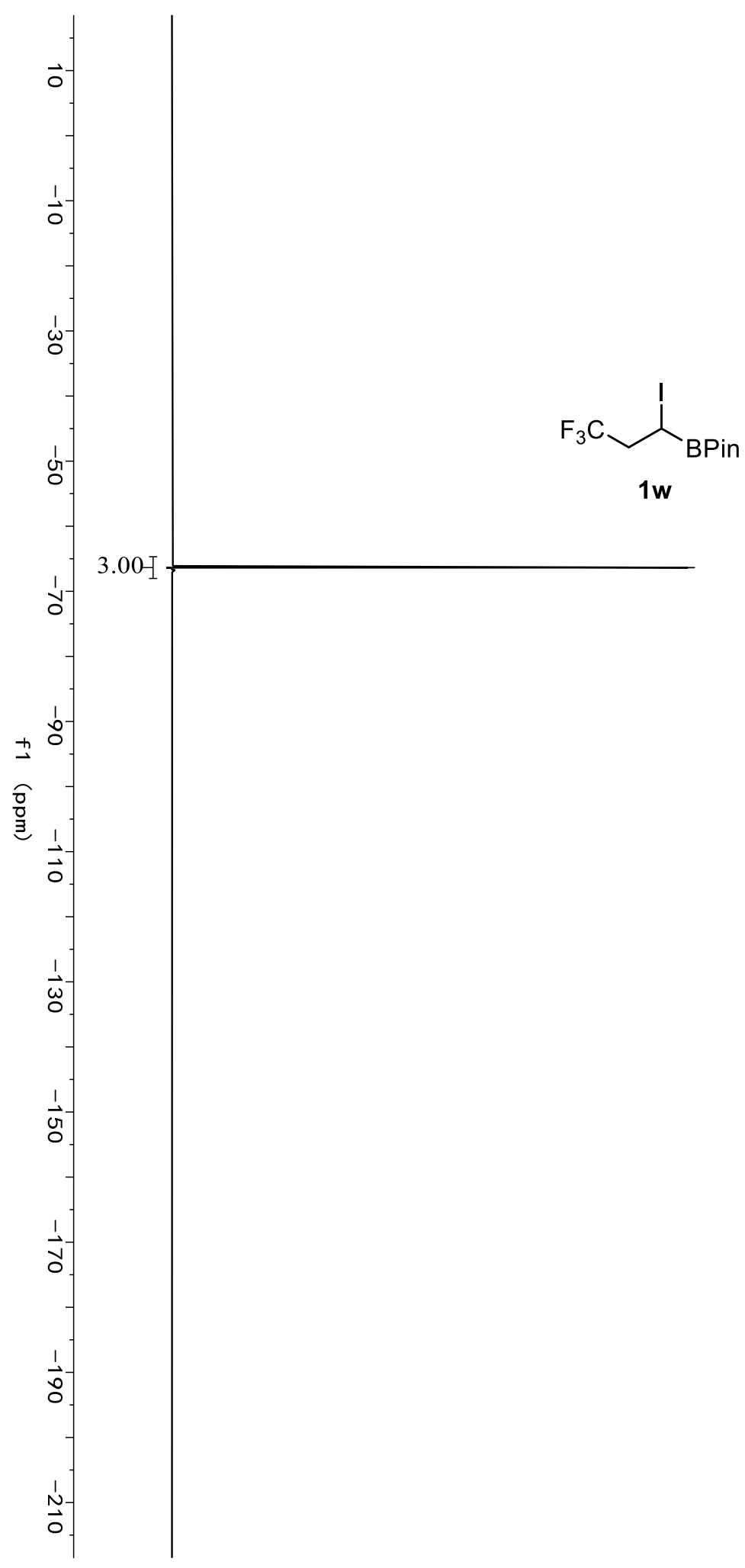

$--66.36$ 


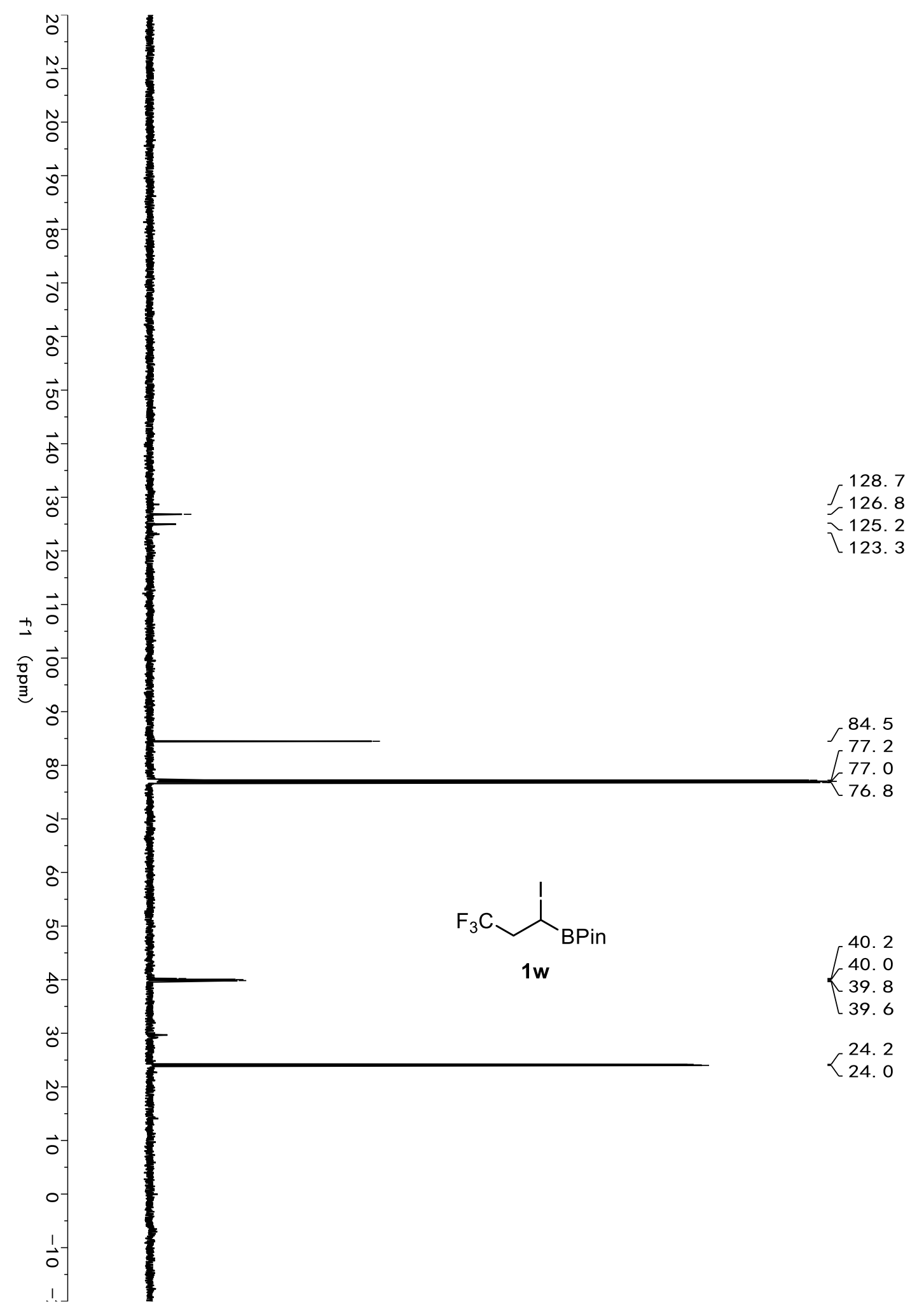



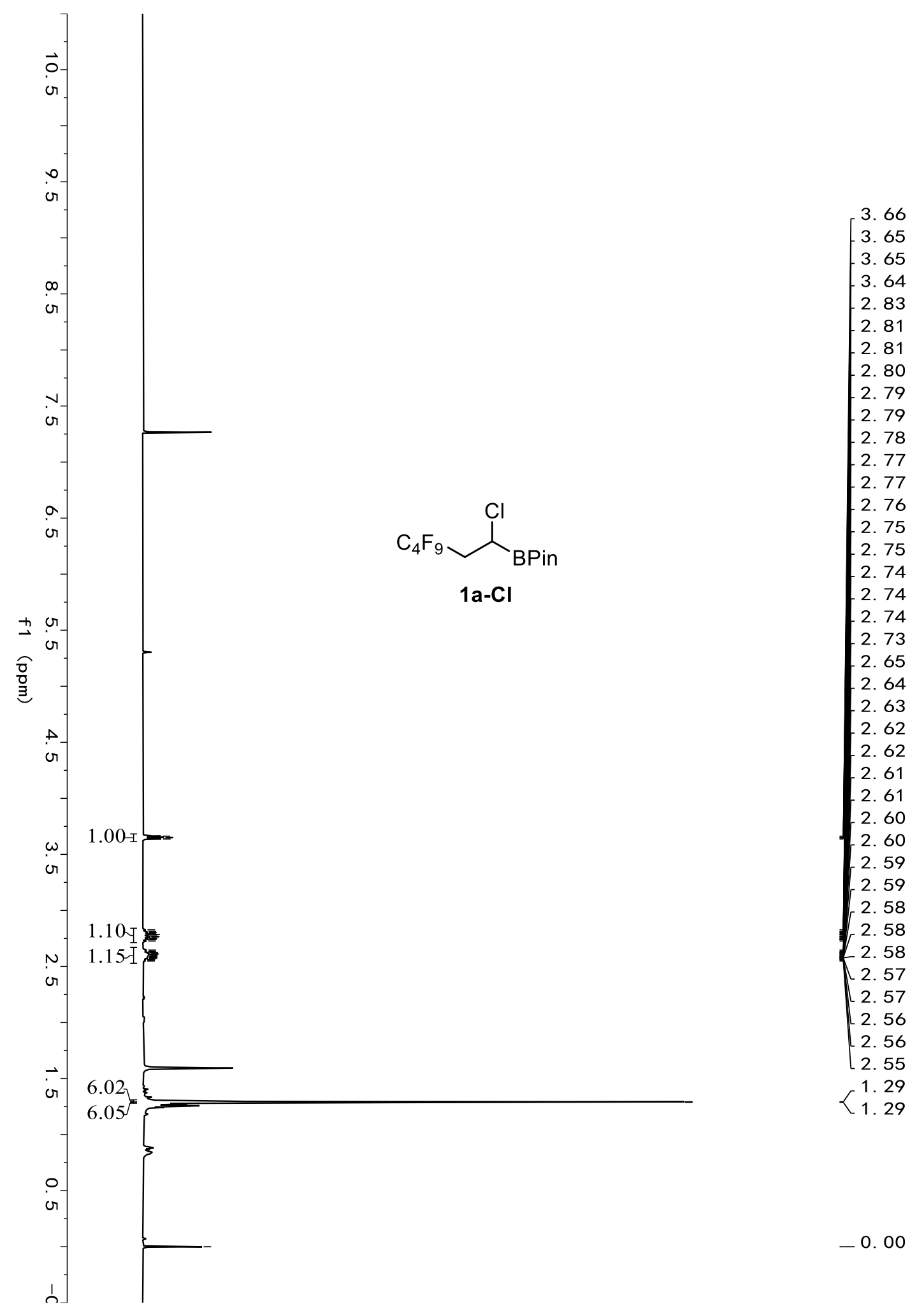

$-0.00$ 


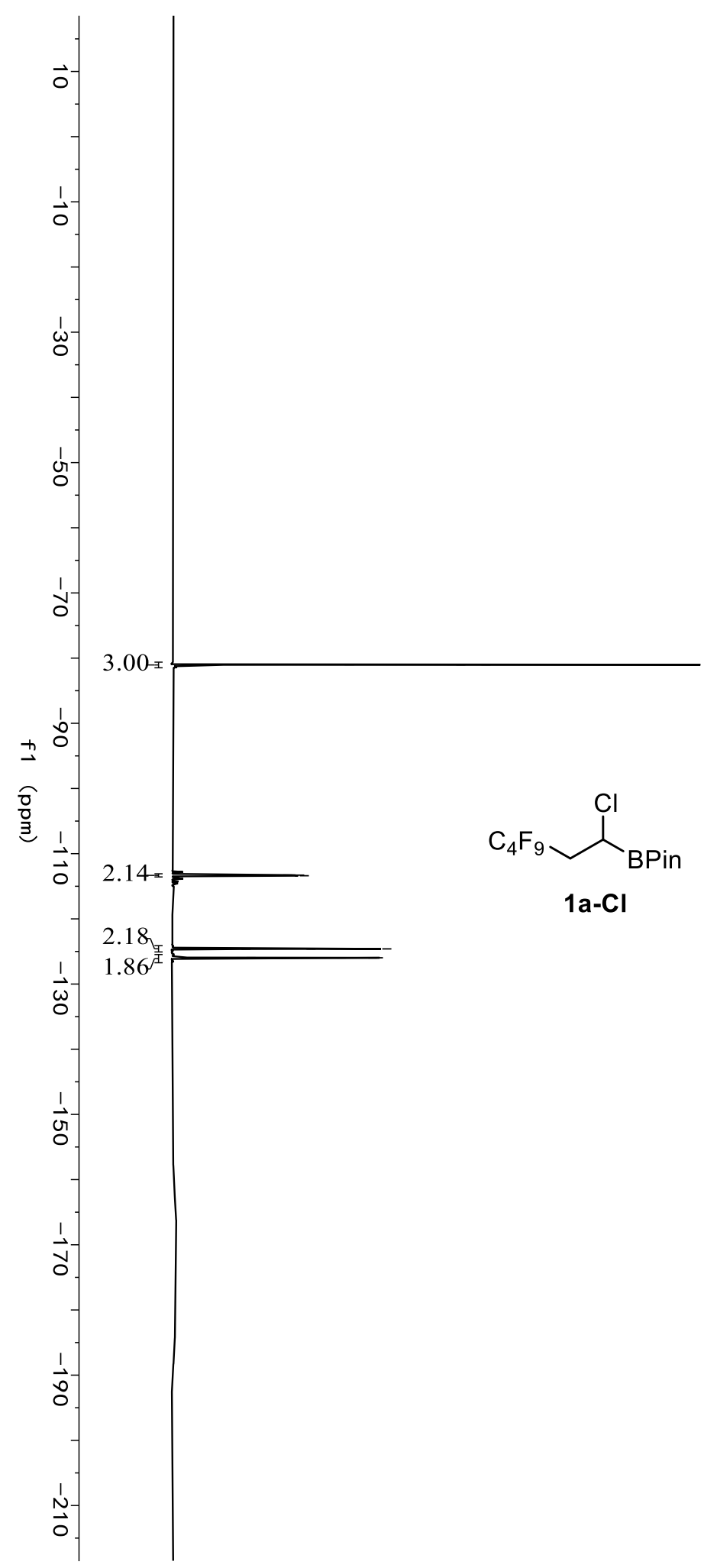

$-80.99$

$-80.99$

$-81.00$

$-81.01$

$-81.02$

$-81.03$

$-113.27$

$-113.29$

$-113.29$

$-113.31$

$-113.36$

$-113.38$

$-113.41$

$-124.57$

$-124.58$

$-124.59$

$-124.60$

$-124.61$

$-124.62$

$-124.62$

$-124.63$

$-125.95$

$-125.95$

$-125.97$

$-125.98$

$-125.99$

$-126.00$

$-126.01$

-126. 02 


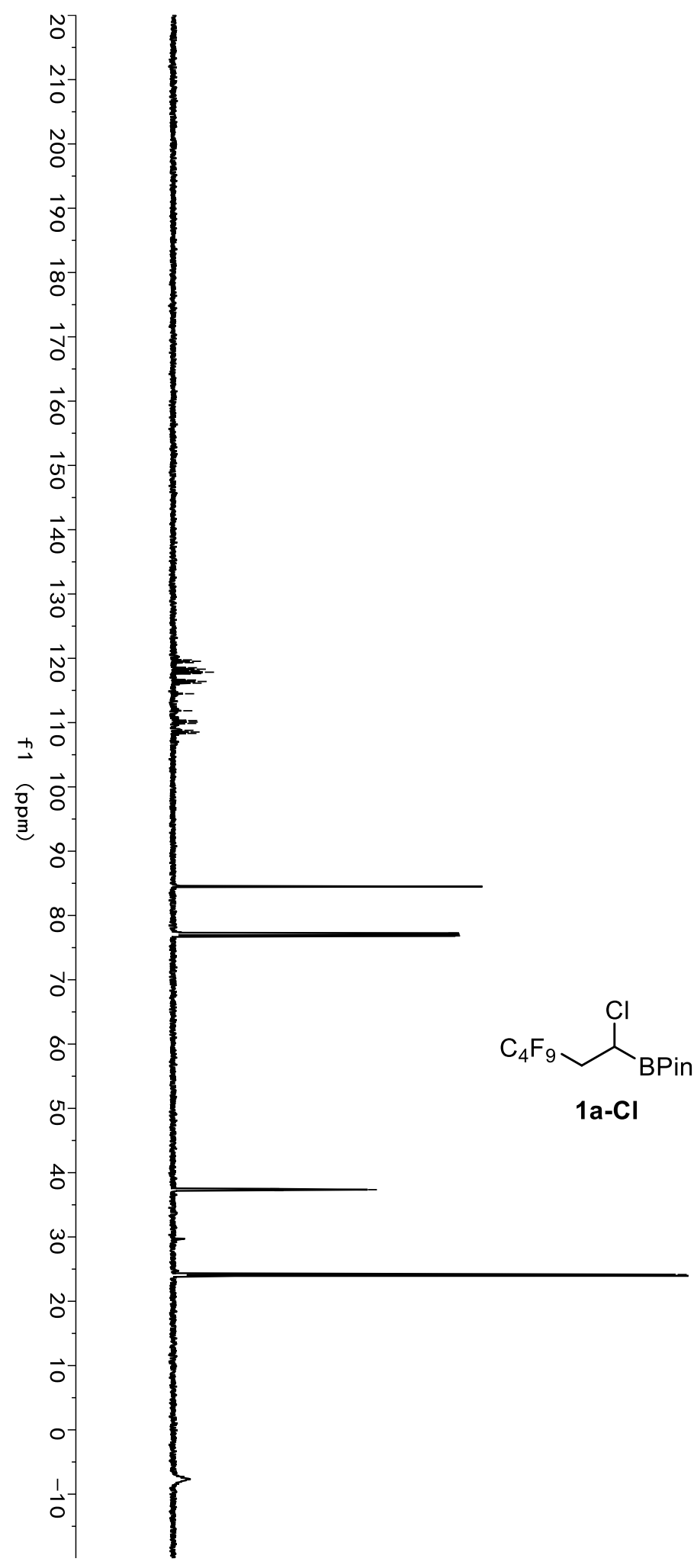

119. 7

119. 5

119. 3

118. 5

118. 3

118. 1

118. 0

117.8

117.6

116. 6

116. 4

116. 1

114. 5

111.8

110. 3

110. 1

109.9

108. 8

108. 5

108. 3

84.5

77.3

77.0

र6.8

37.5

$-37.4$

37. 2

24. 2

<24. 0 

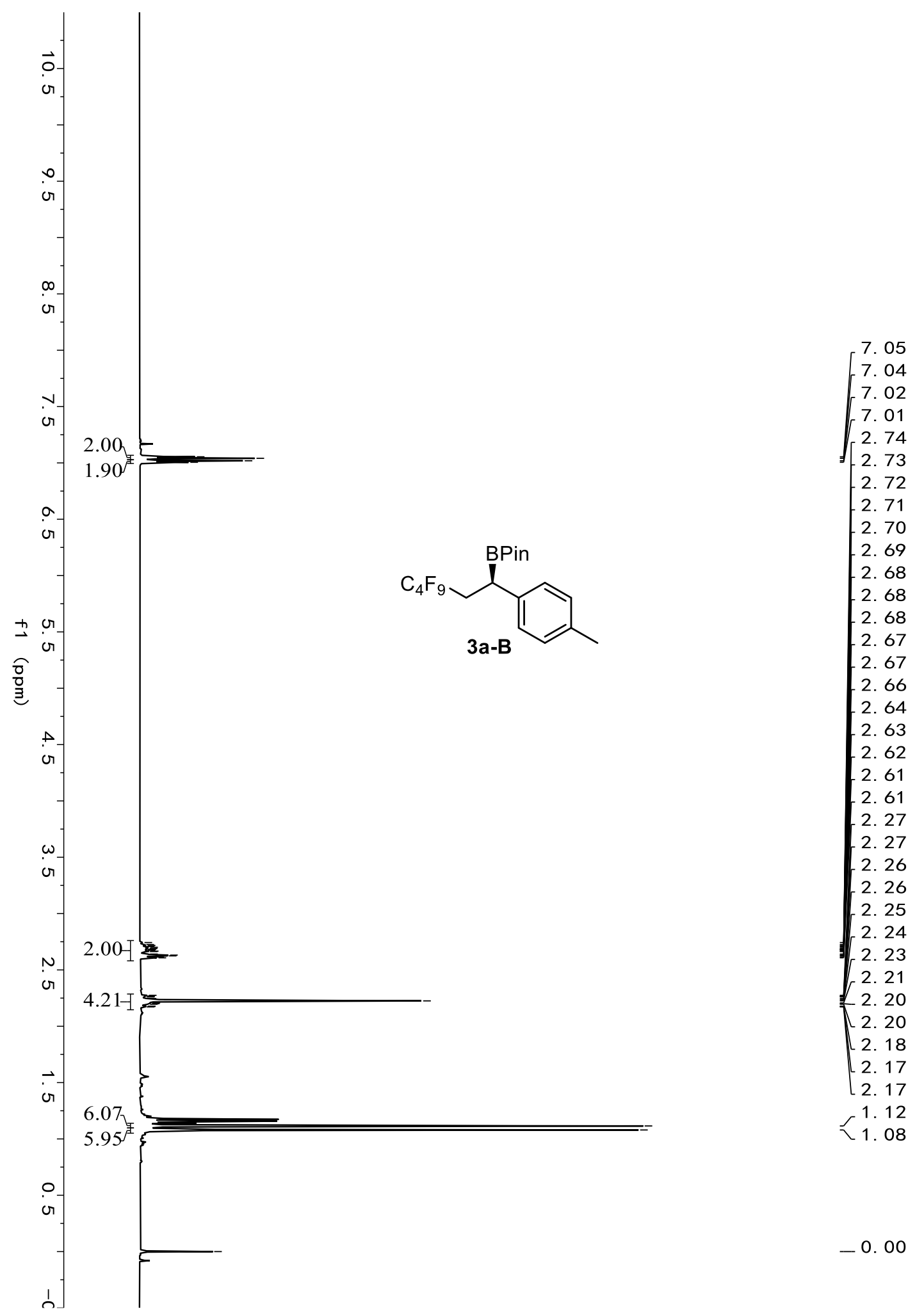


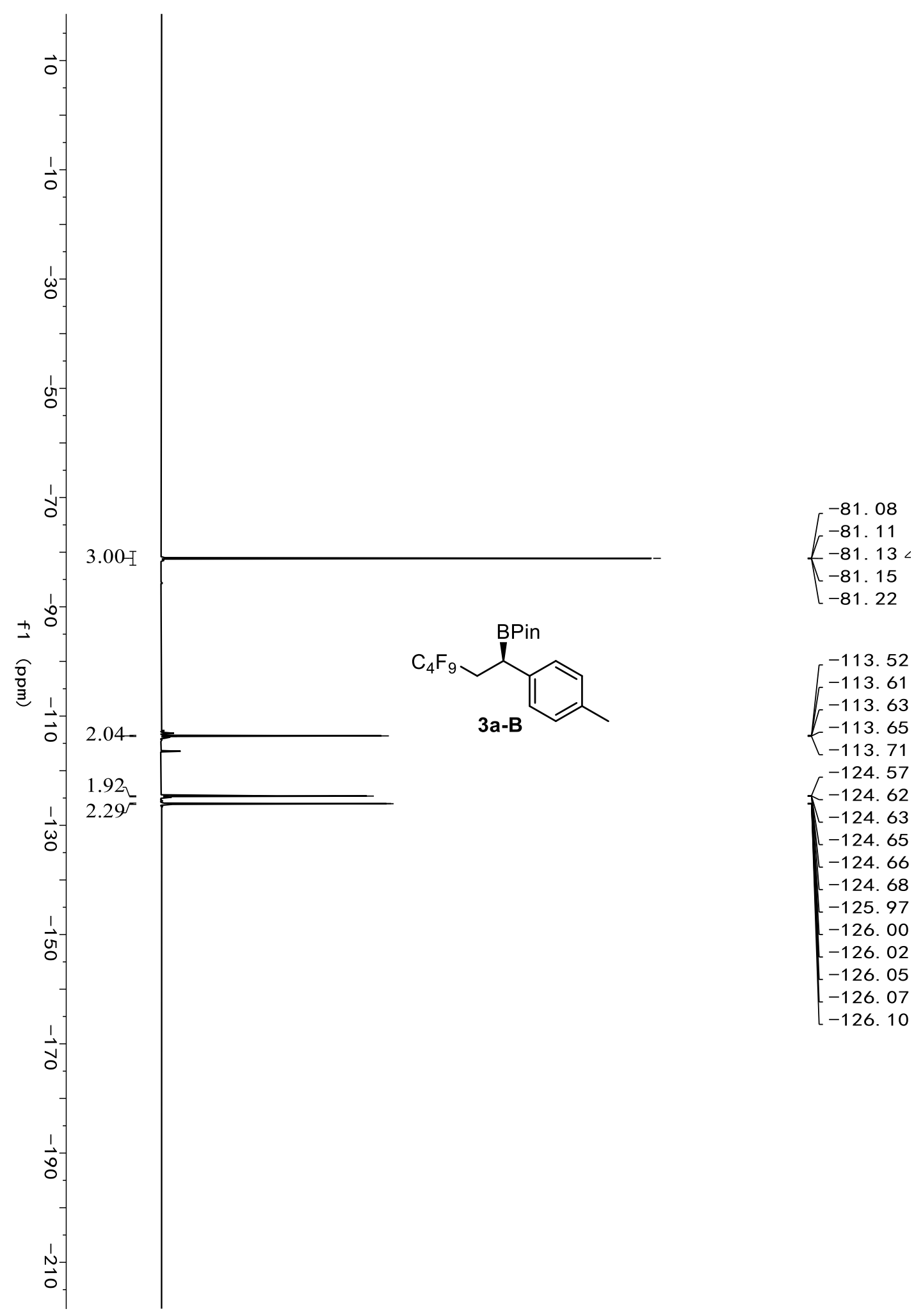




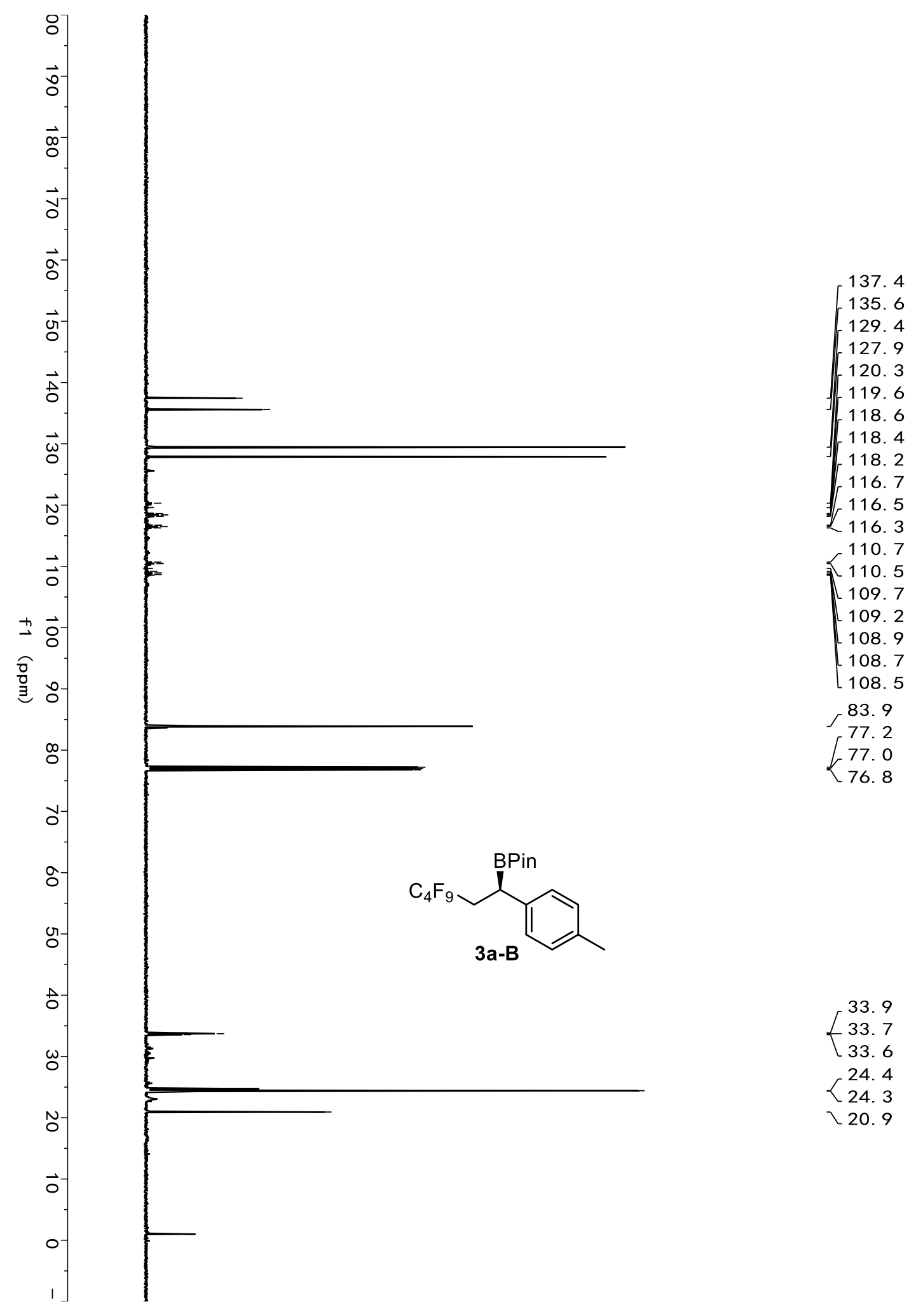




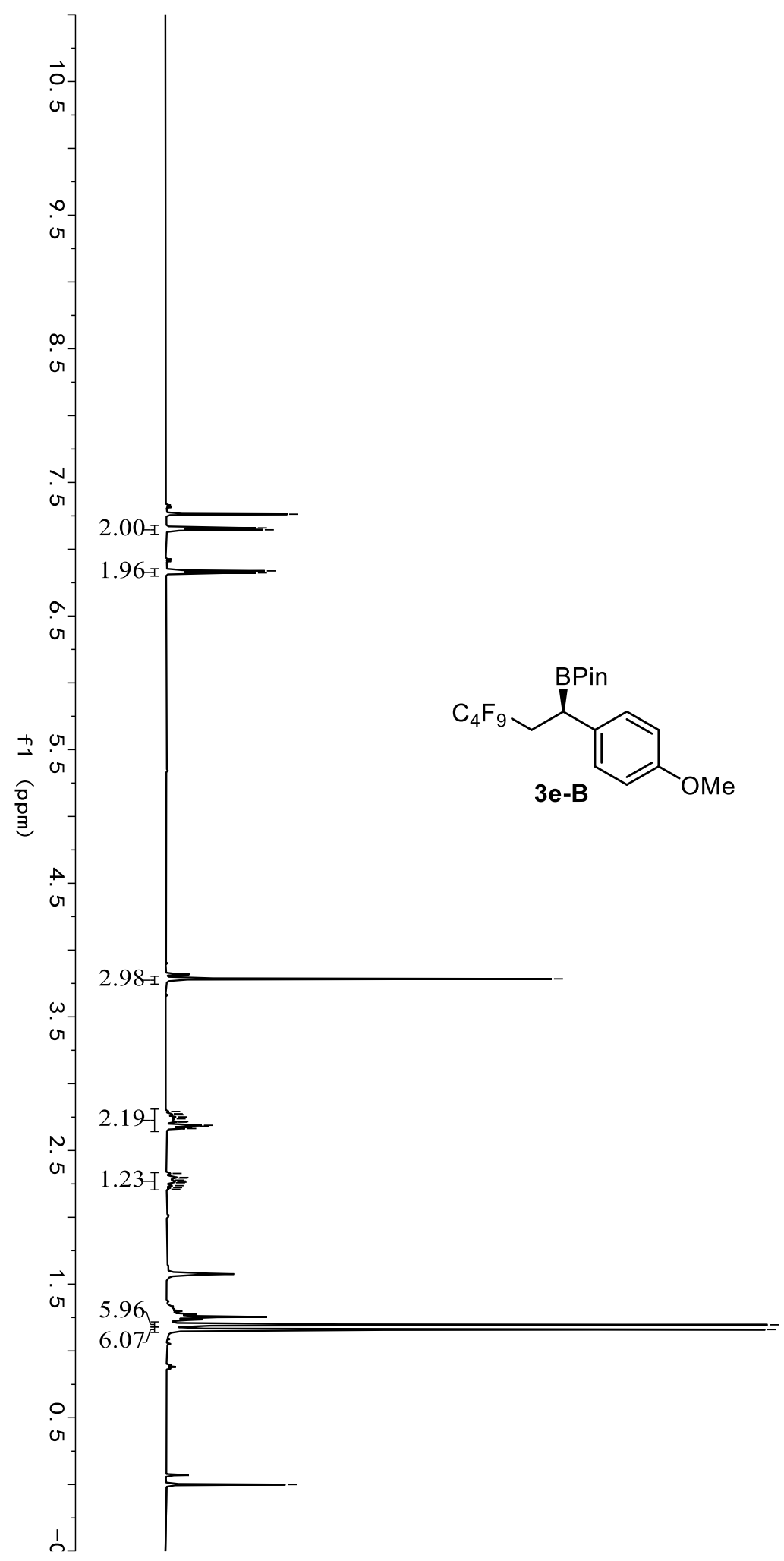

7. 26

7.16

7.15

$-6.84$

6. 82

3. 78

2. 79

2. 77

2. 77

2. 75

2. 74

2. 72

2. 71

2. 69

2. 68

2. 67

2. 66

2. 33

2. 30

2. 29

2. 28

2. 27

-2. 26

2. 26

2. 24

2. 22

2. 21

1. 20

$\Upsilon 1.16$

$-0.00$ 


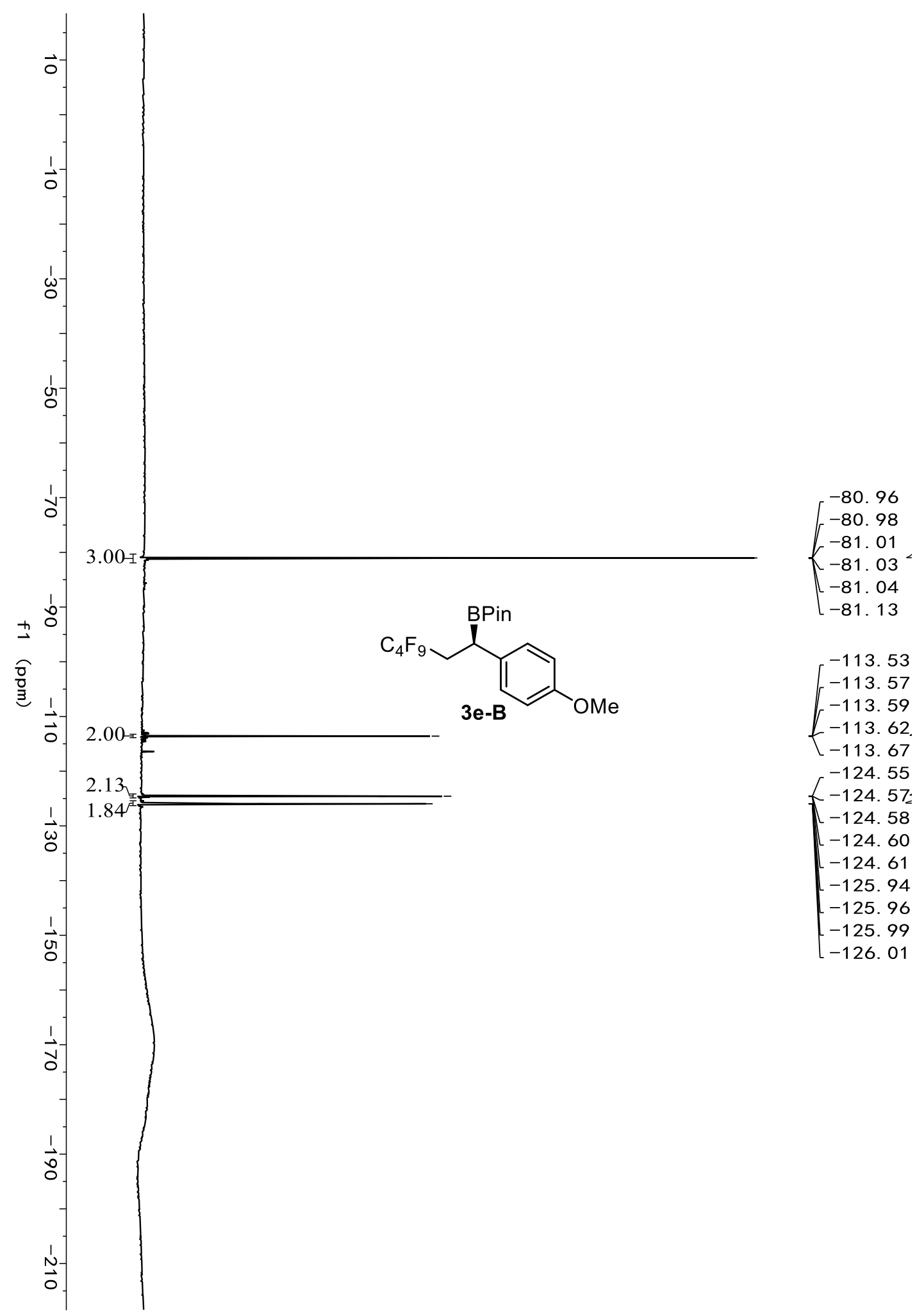




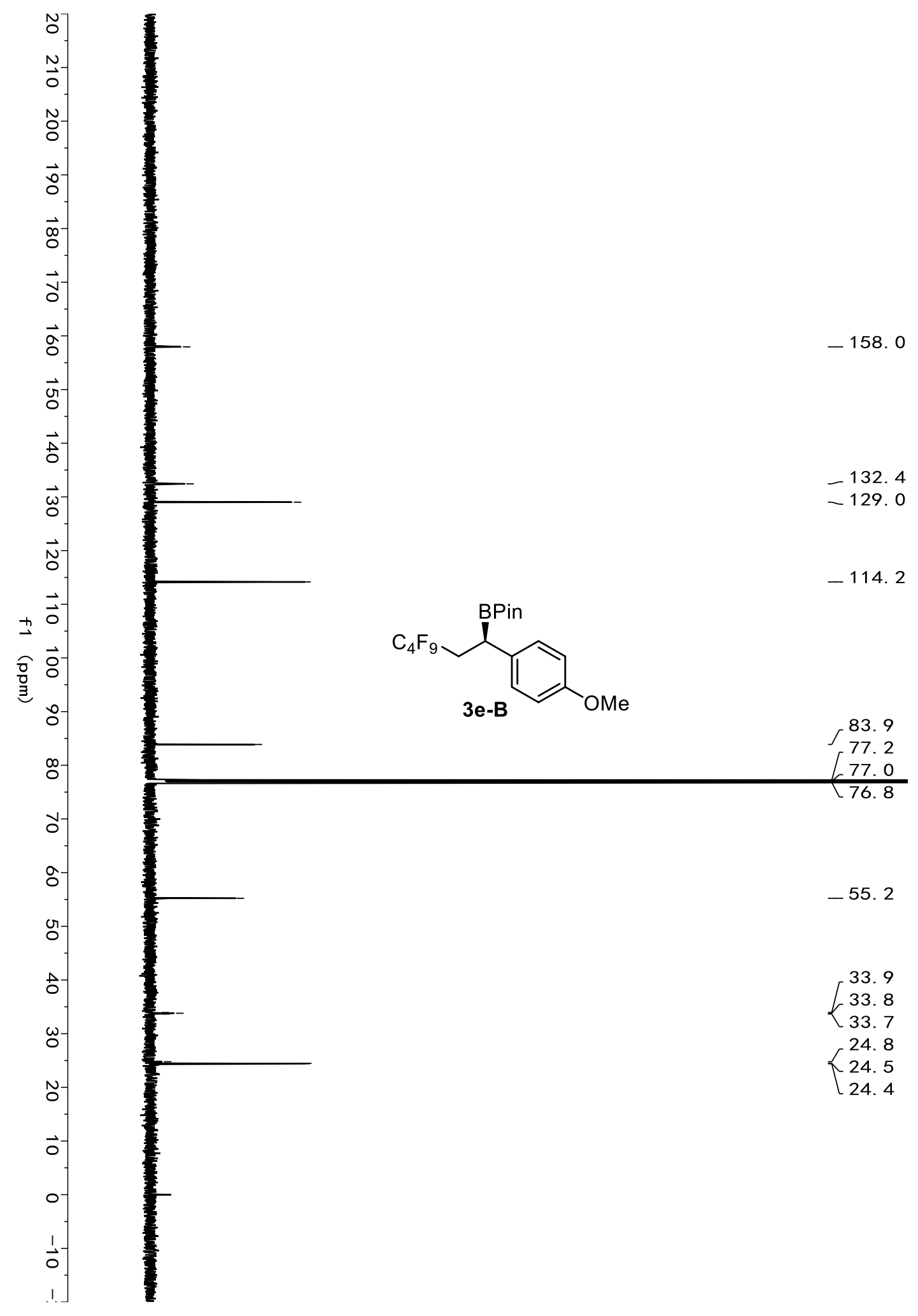




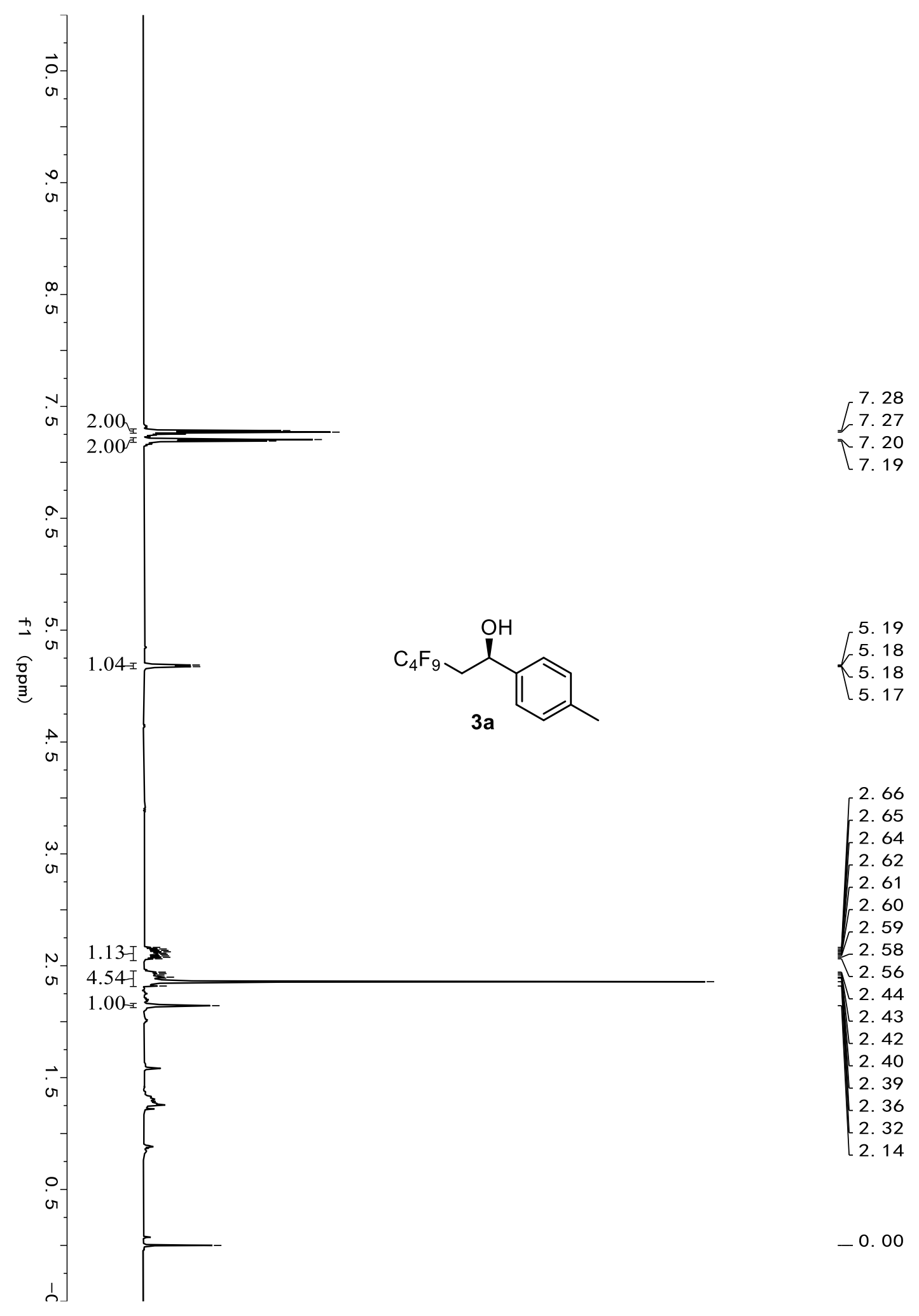




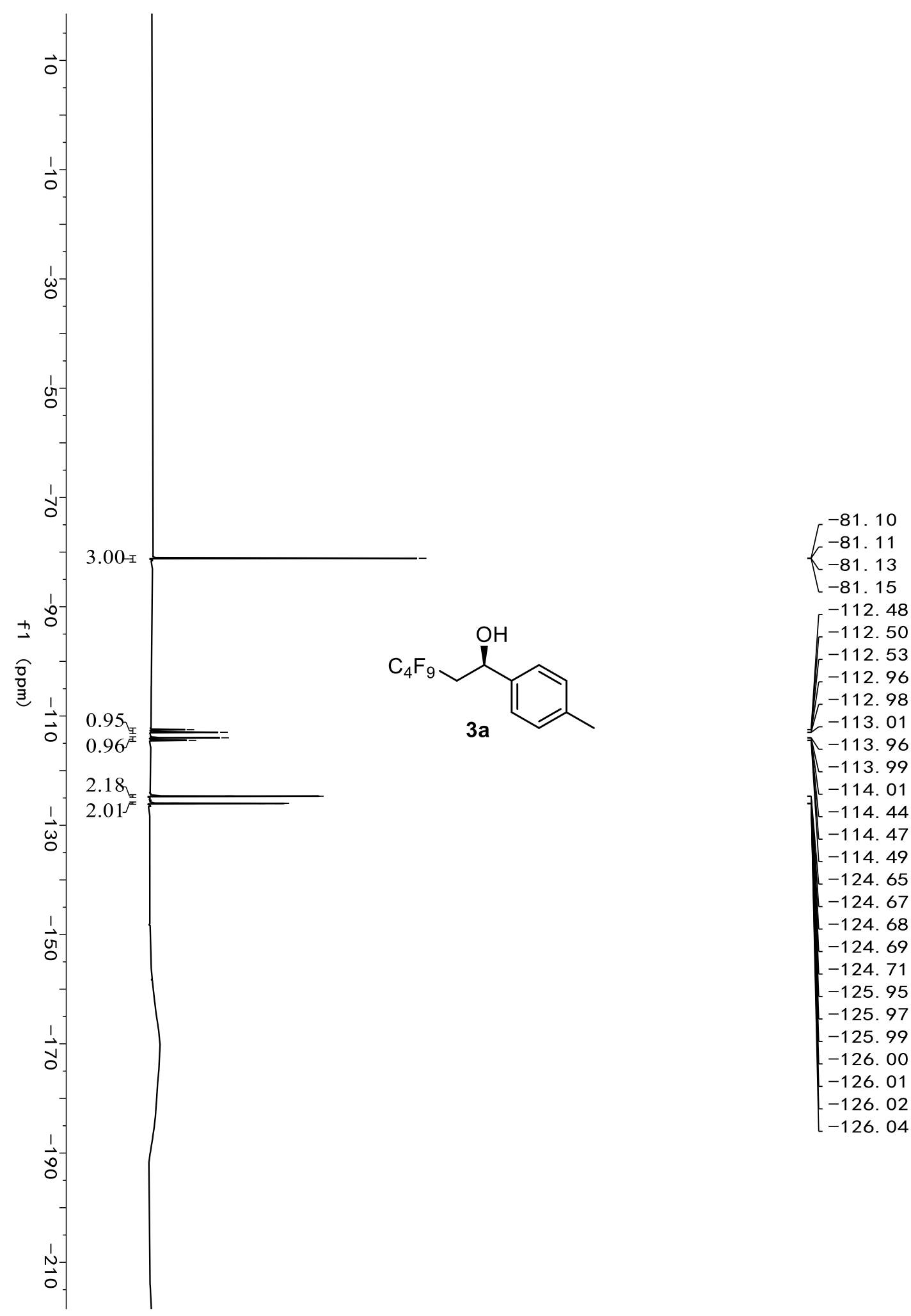



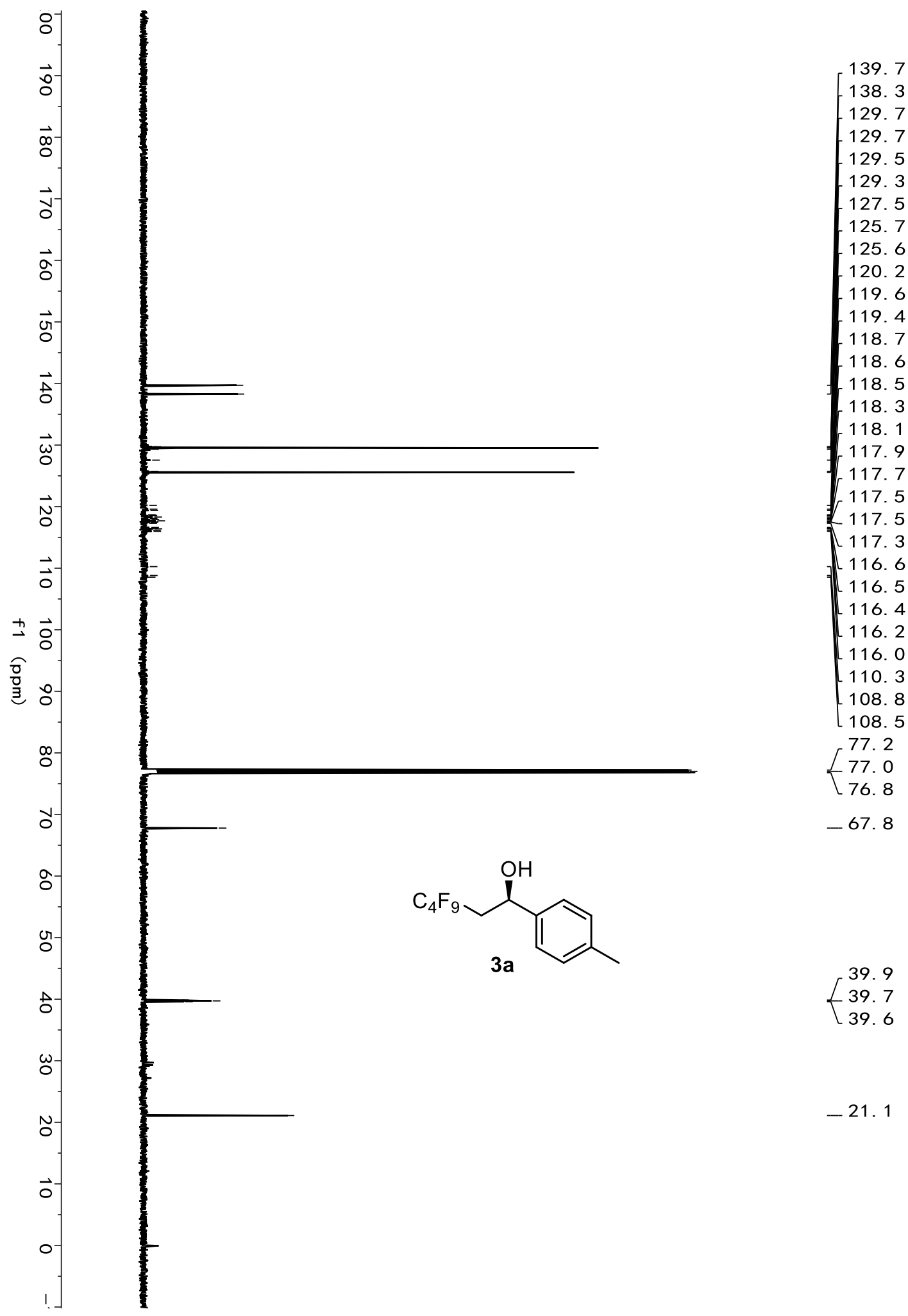

$$
\begin{array}{r}
39.9 \\
-39.7 \\
39.6
\end{array}
$$

$-21.1$

118 


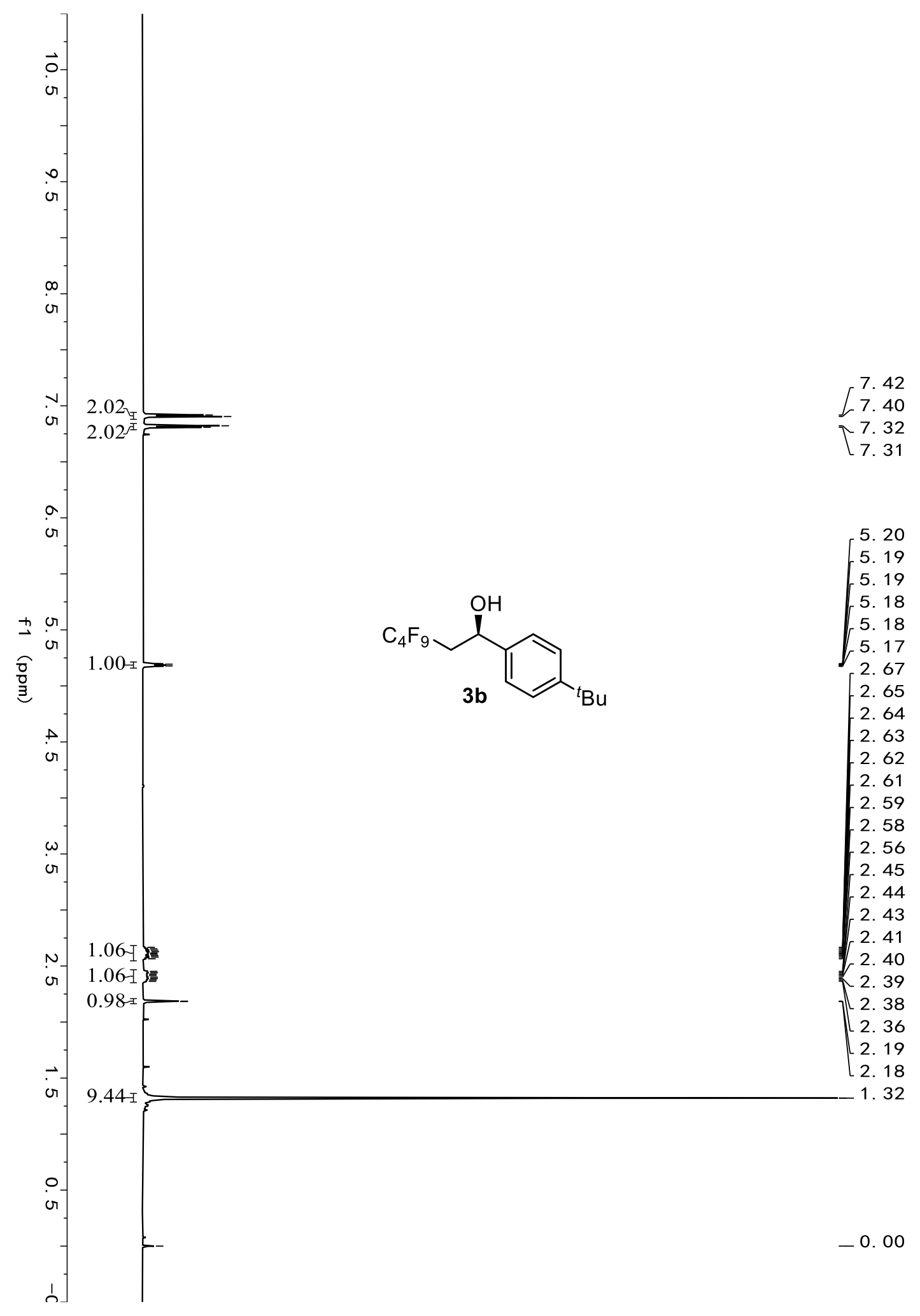




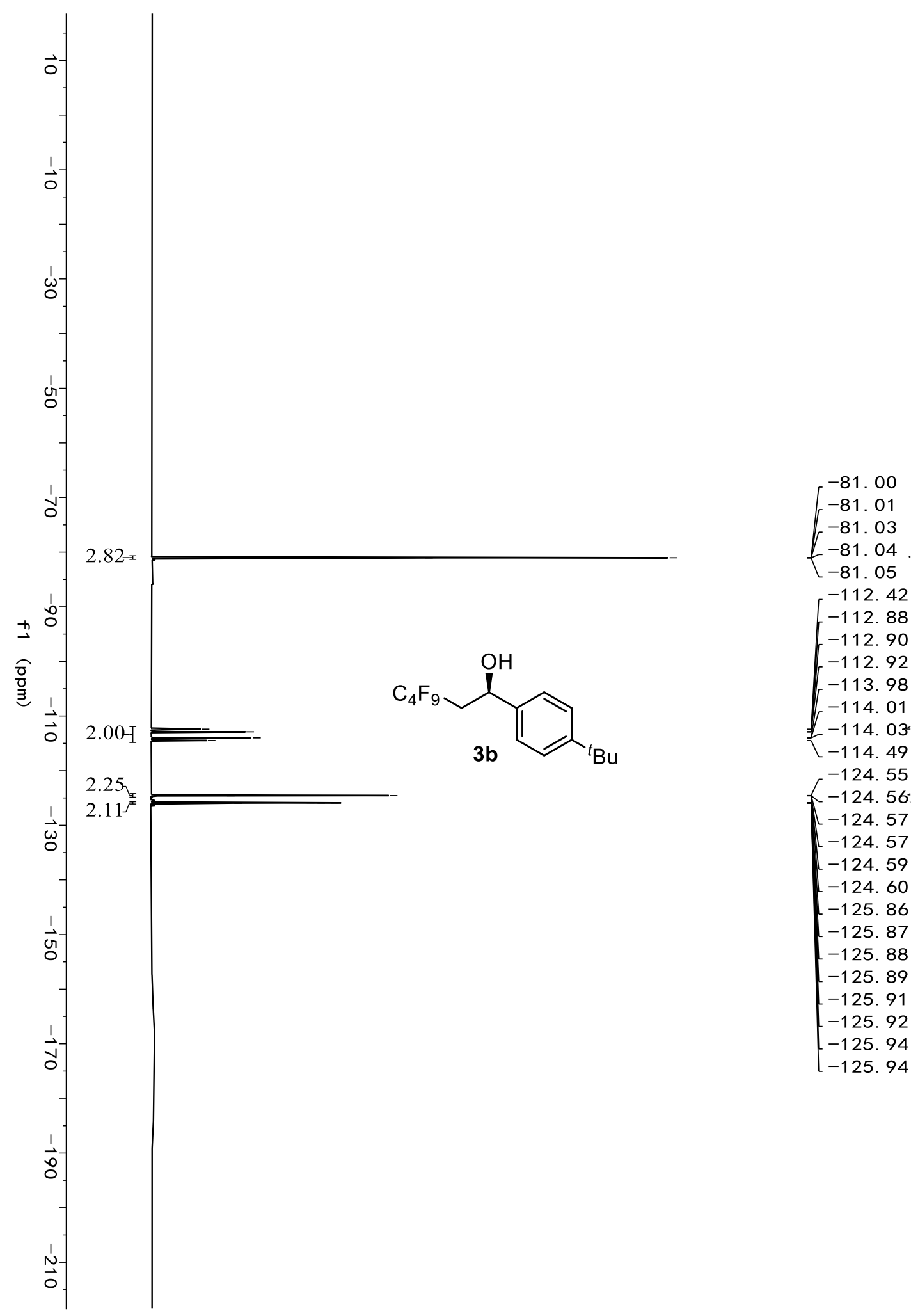




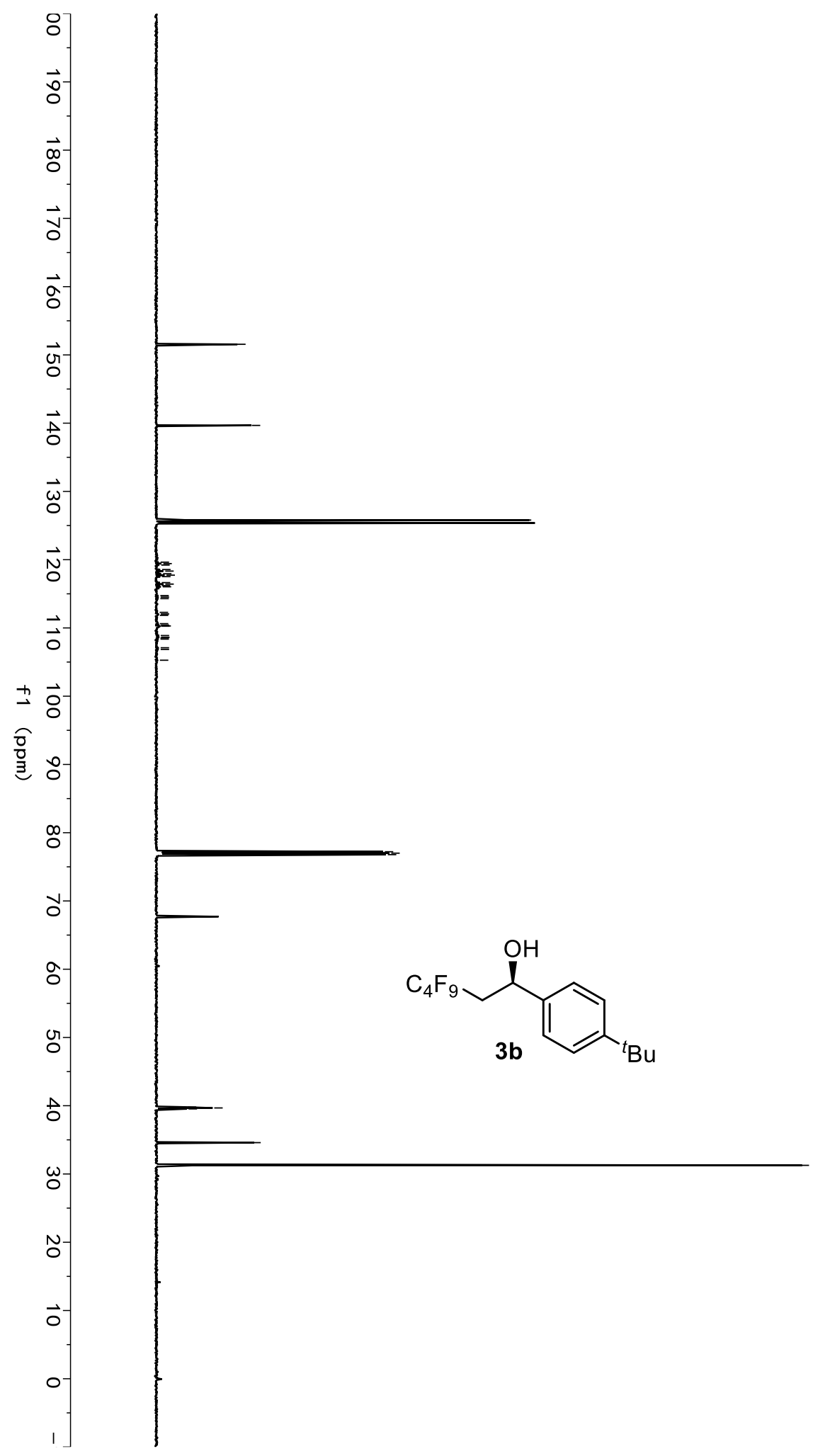

125. 37

119.63 119.42 119. 21 118.55 118. 33 117.94 117. 73

117.52

116. 64

116. 42

116. 20

116. 03

114. 73

114. 51

114. 29

112. 28

112. 05

111.85

110. 63

110. 40

110. 29

110. 26

108. 88

108. 62

108. 53

108. 37

107. 10

106. 84

105. 27

77. 21

77. 00

76. 79

39. 81

39.67

39. 54

34. 59

[31. 27 


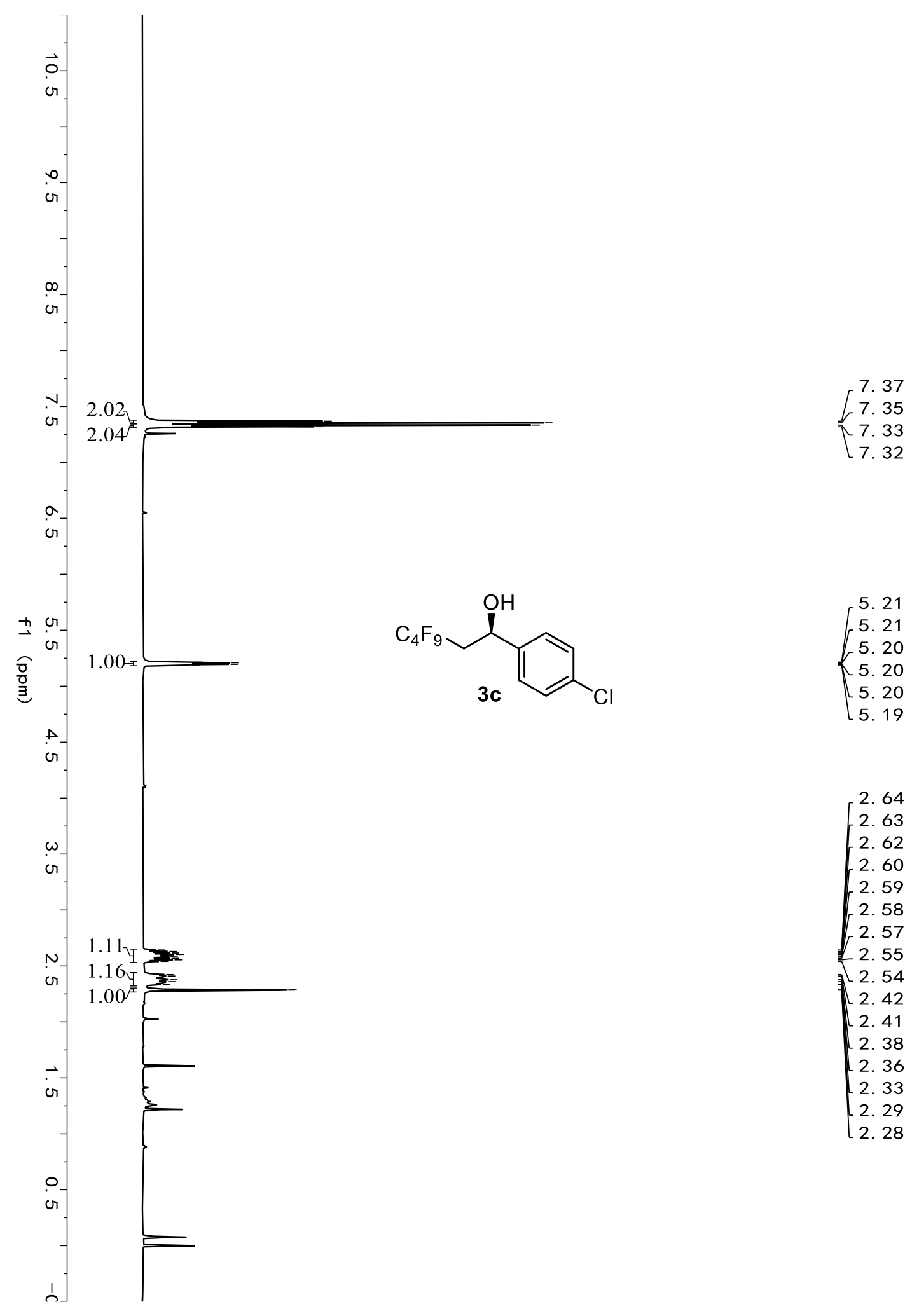




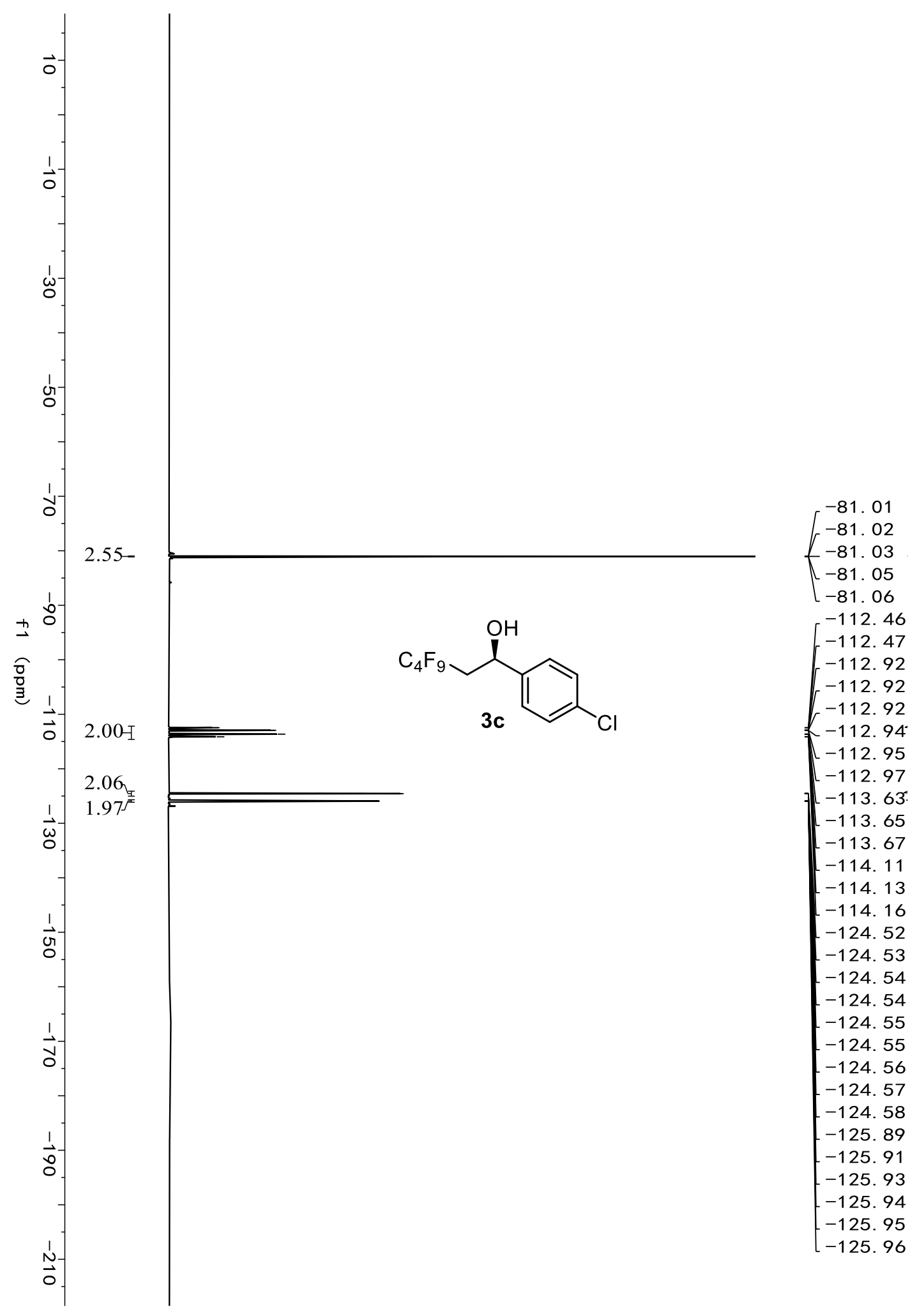




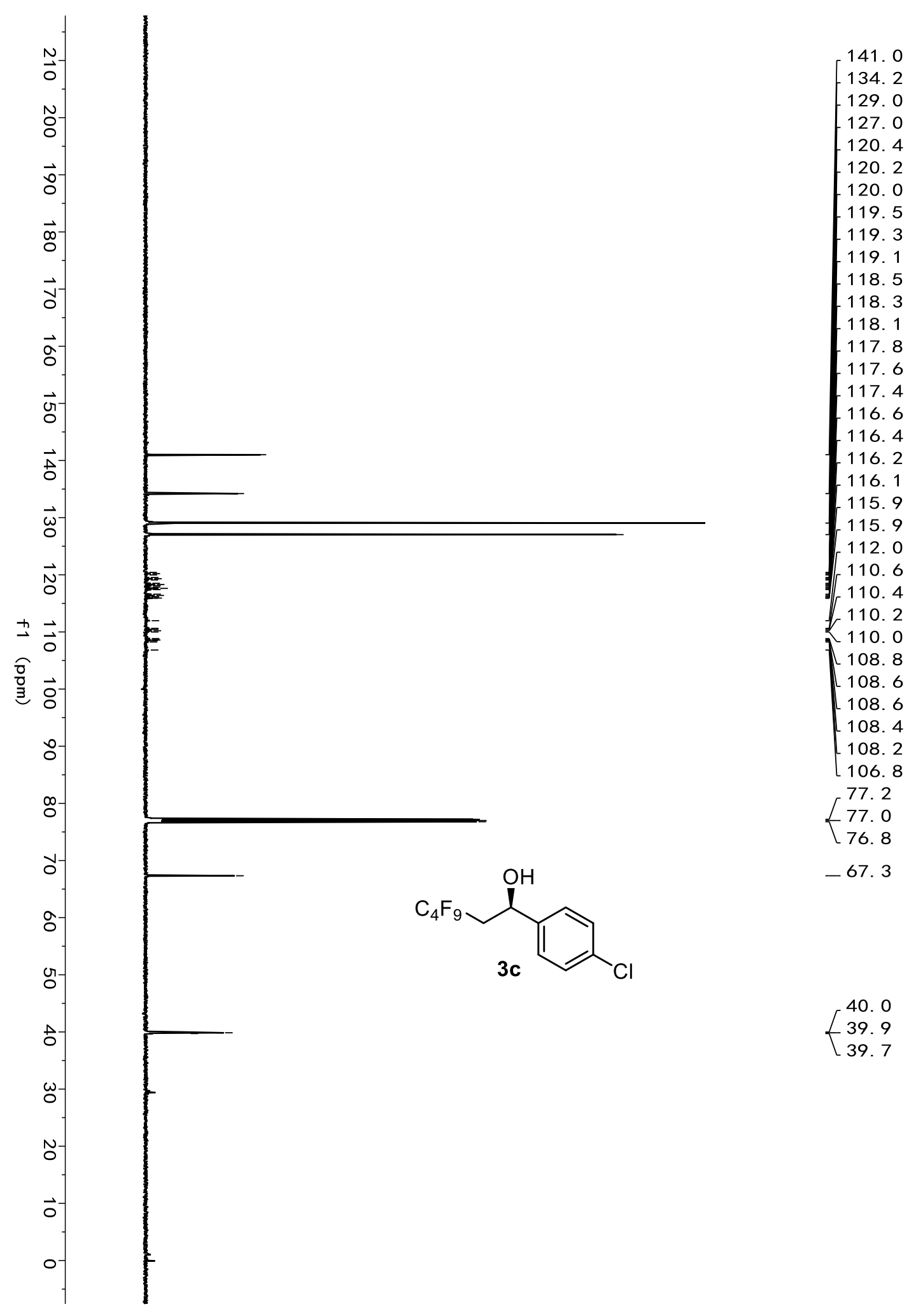




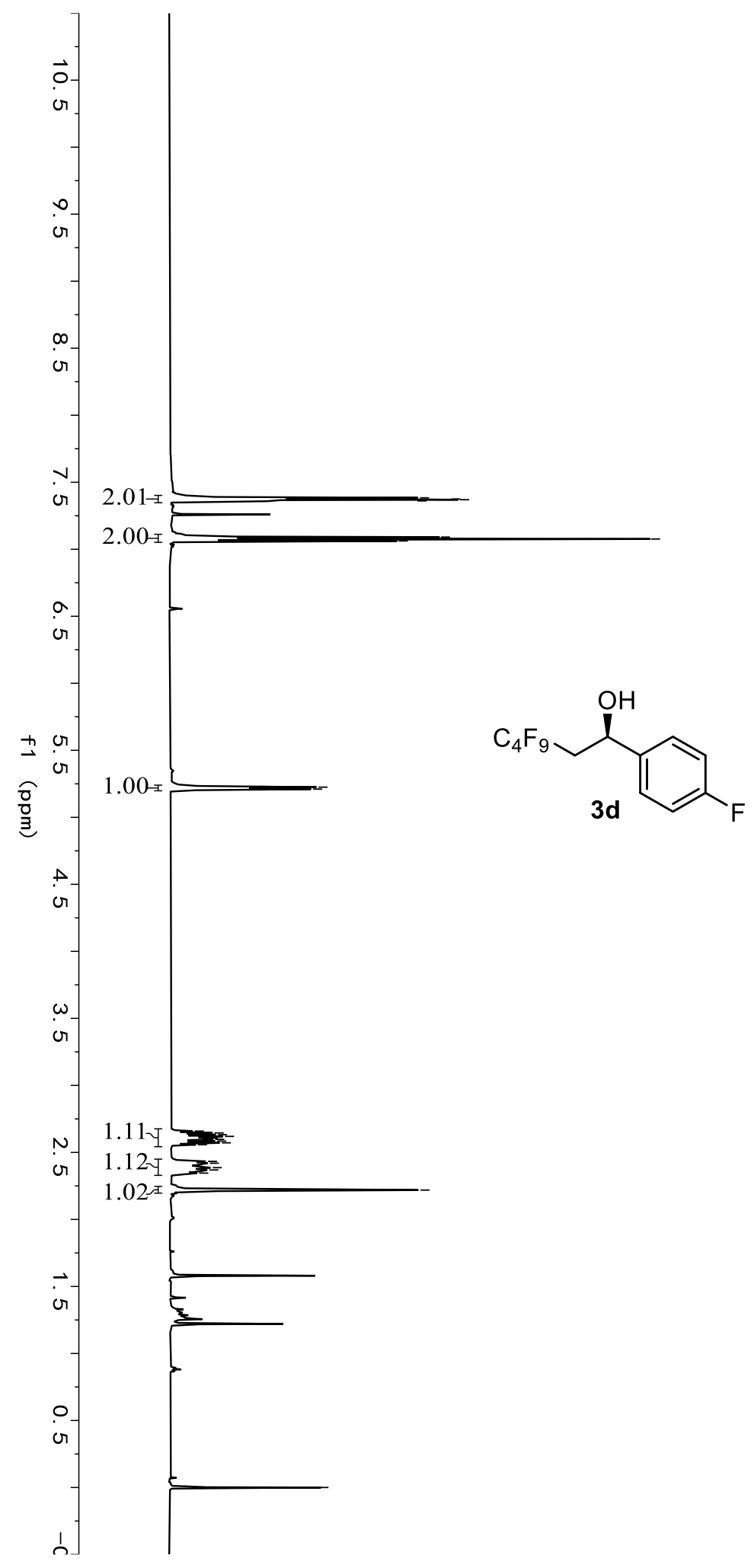

7.38
$\left\{\begin{array}{l}7.37 \\ 7.37 \\ 7.36\end{array}\right.$
$\left\{\begin{array}{l}7.09 \\ 7.08 \\ 7.06\end{array}\right.$

5. 23

5. 23

5. 22

5. 22

5. 21

5. 21

2. 66

2. 65

2. 63

2. 62

2. 61

2. 60

2. 58

2. 57

2. 56

2. 43

2. 42

2. 39

2. 37

2. 35

2. 22

$-0.00$ 


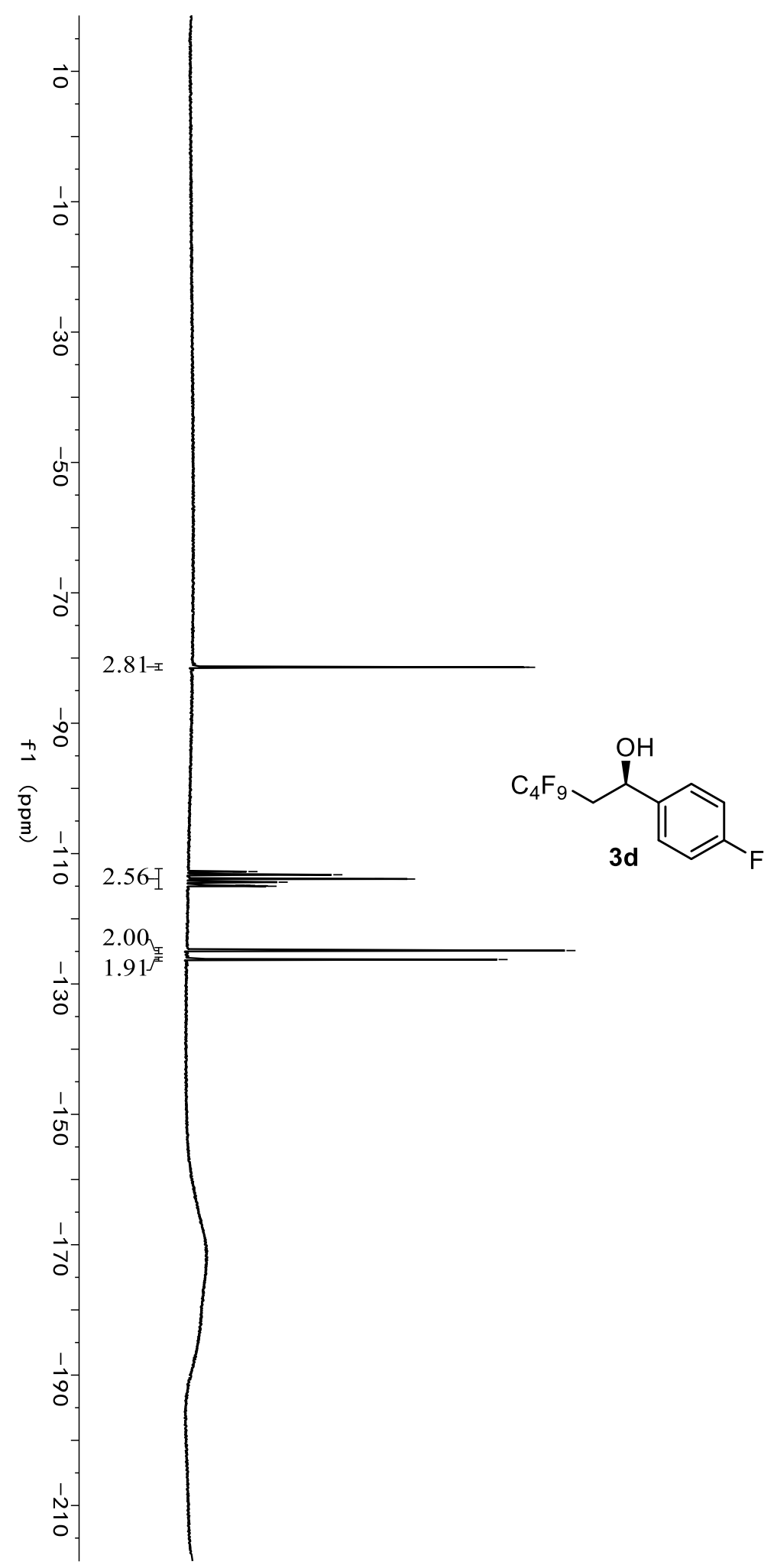

$-81.34$

$-81.36$

$-81.38$

$-81.40$

$-81.42$

$-81.44$

$-81.46$

$-112.72$

$-112.76$

$-112.79$

$-113.21$

$-113.25$

$-113.28$

$-113.88$

$-113.90$

$-113.92$

$-114.36$

$-114.38$

$-114.41$

$-114.84$

$-114.87$

$-114.89$

$-114.93$

$-114.96$

$-114.98$

$-115.01$

$-124.84$

$-124.86$

$-124.88$

$-124.90$

$-124.91$

$-126.21$

$-126.23$

$-126.25$

$-126.28$

$-126.30$ 


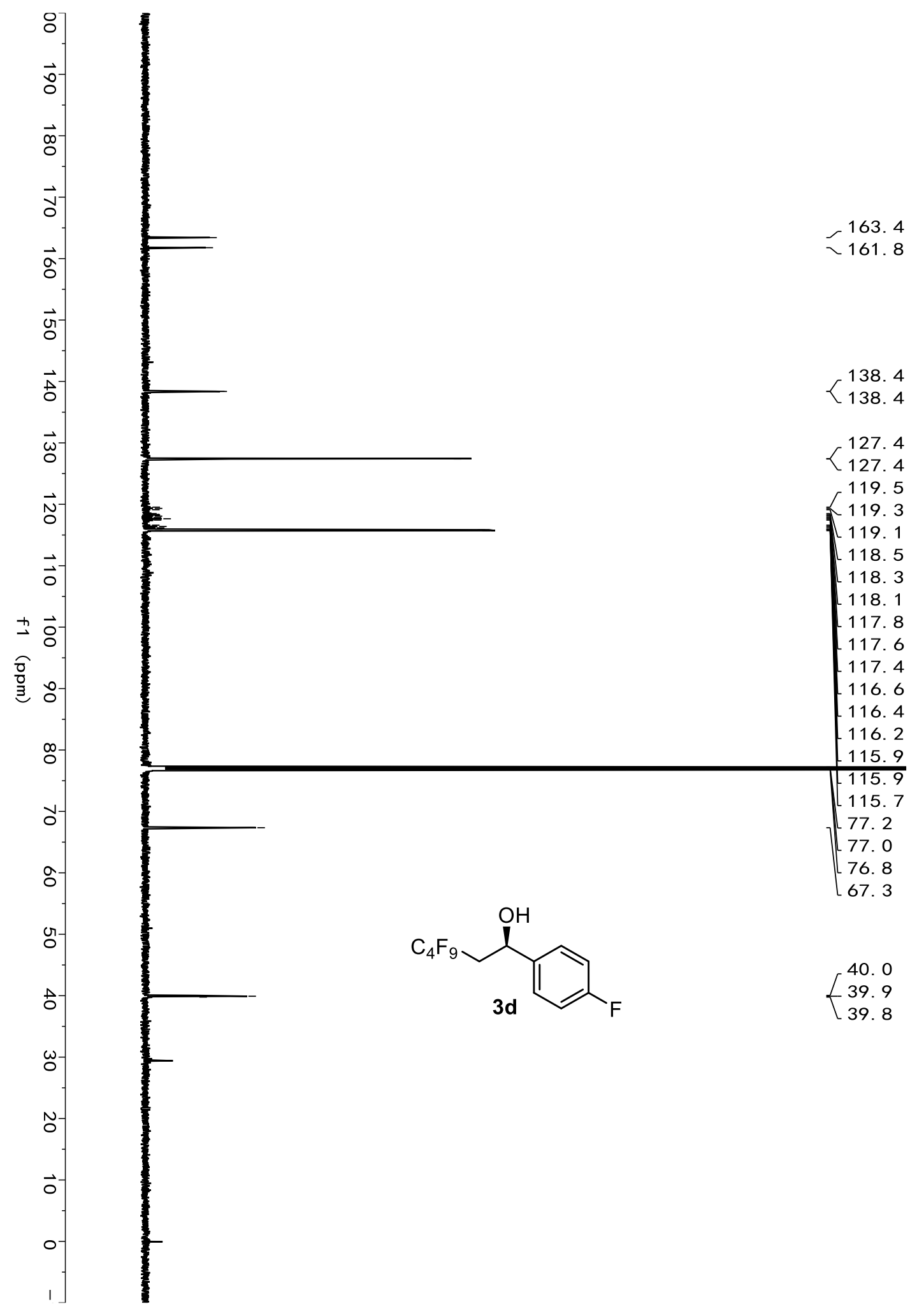




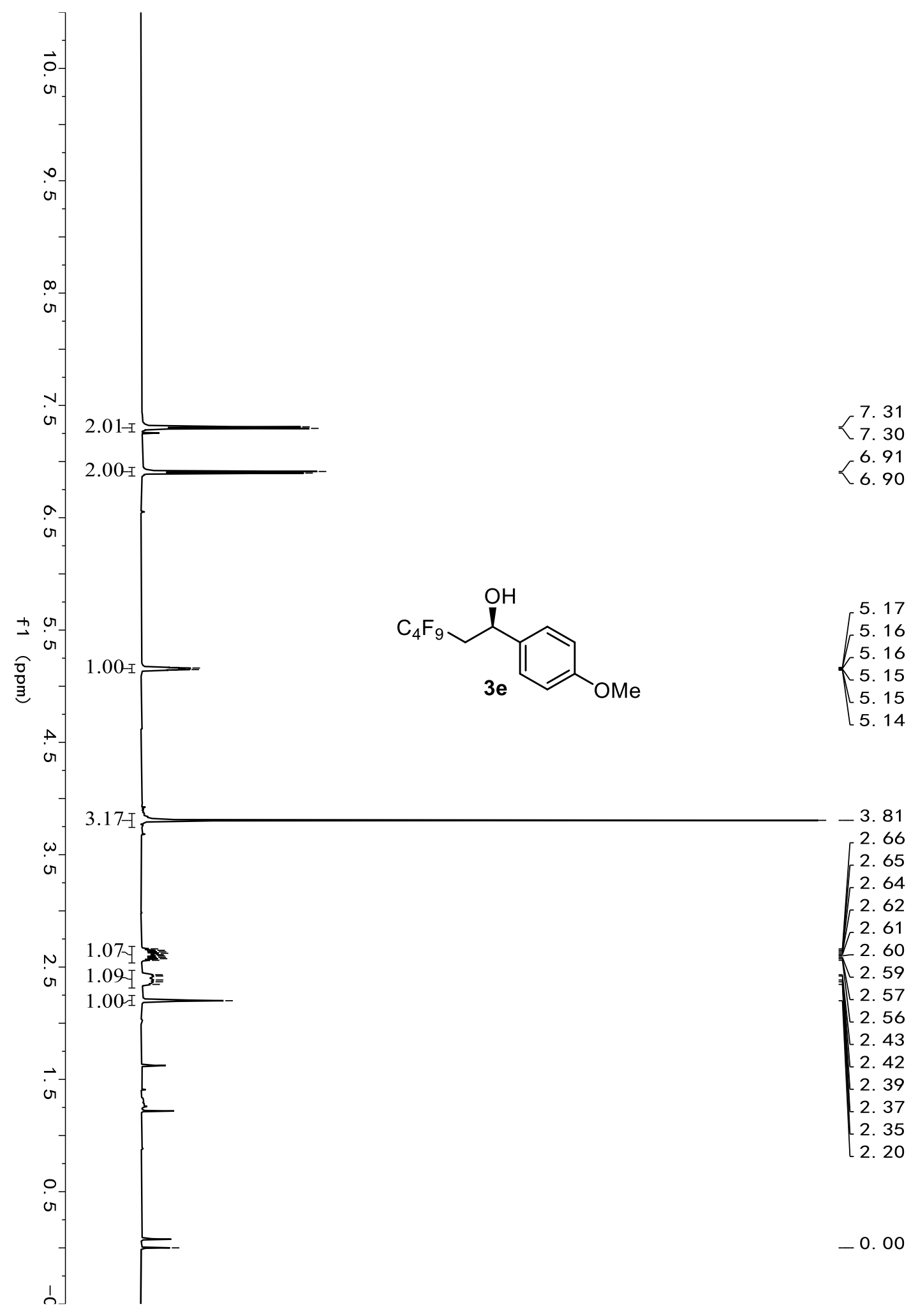




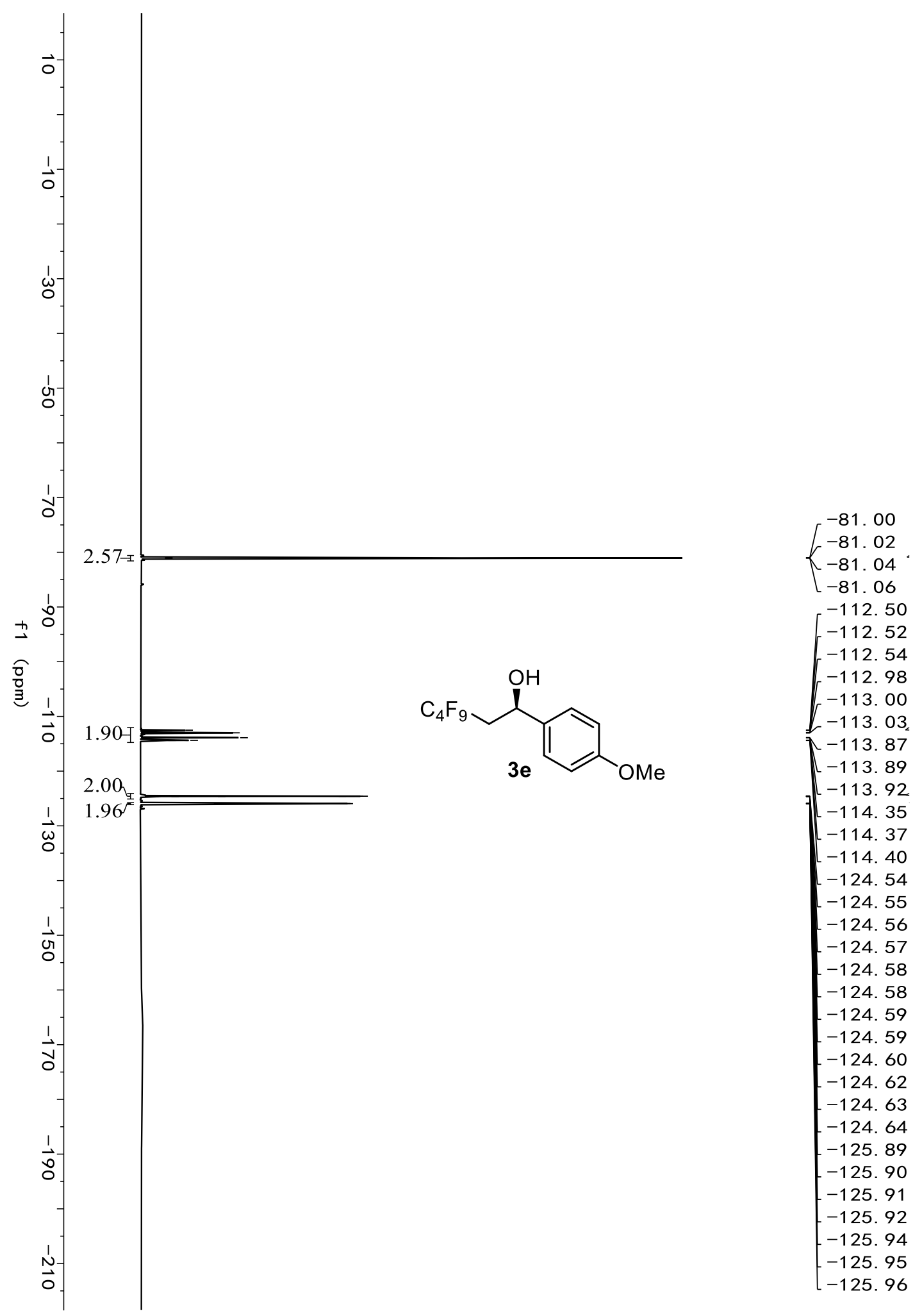




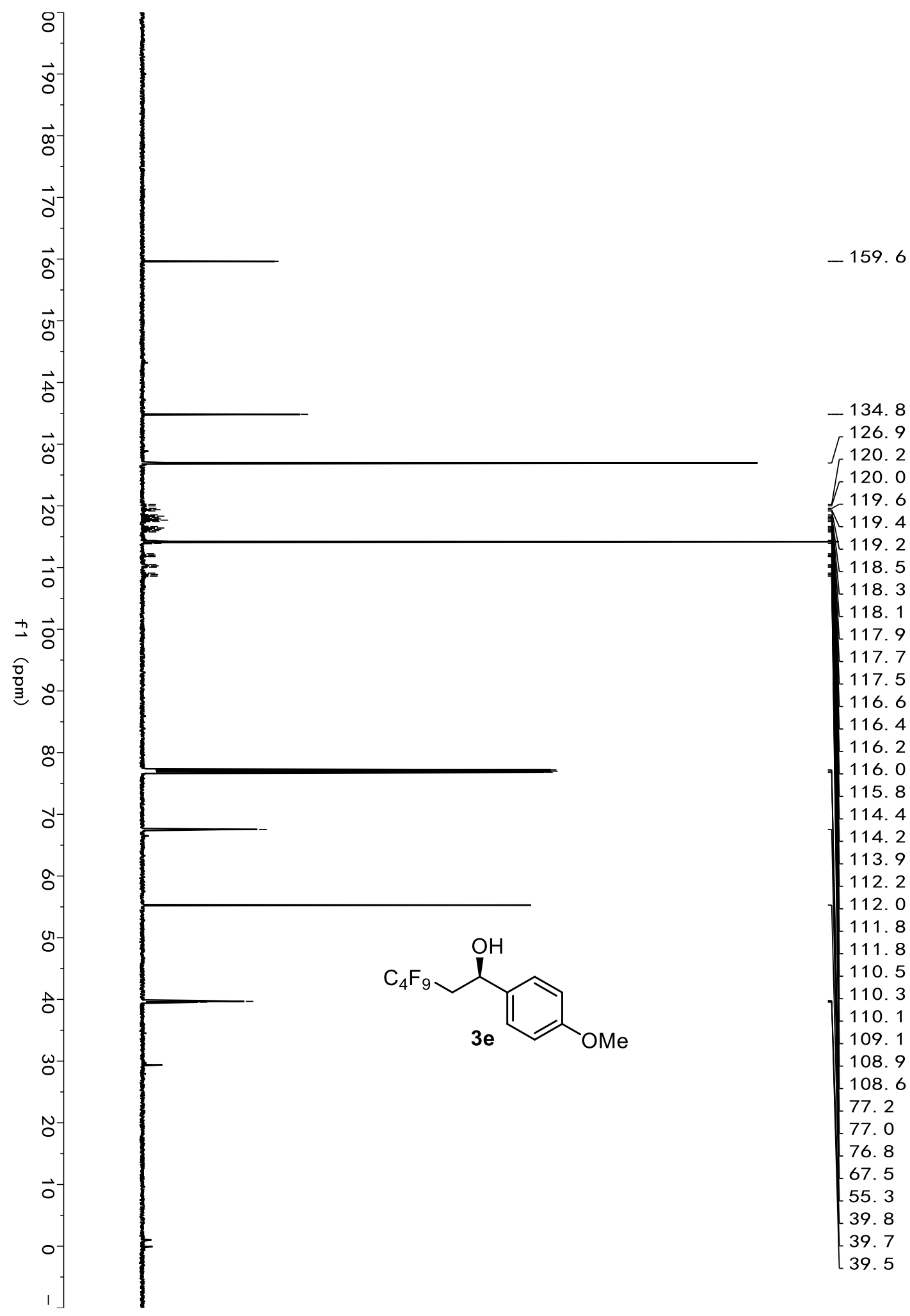




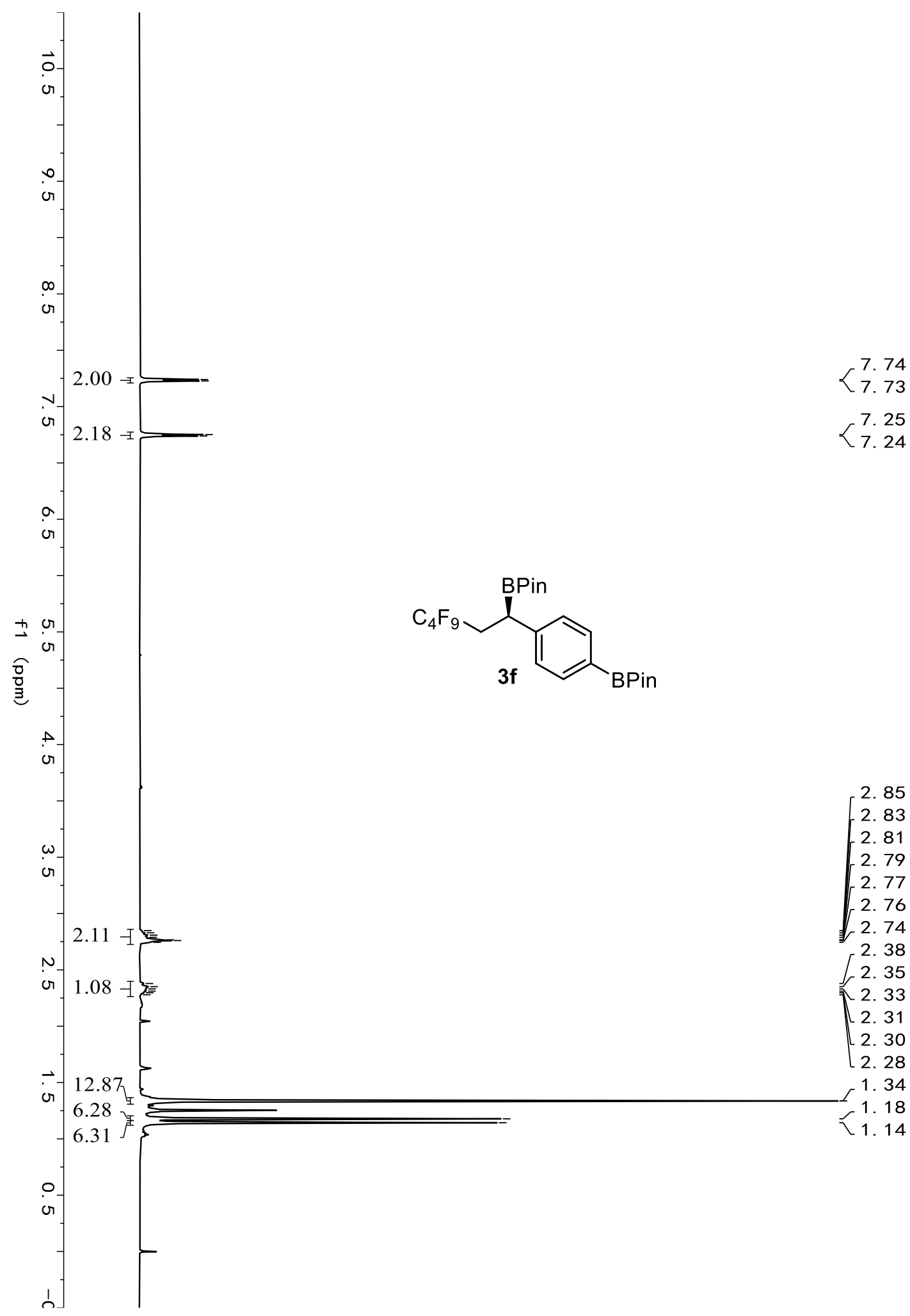




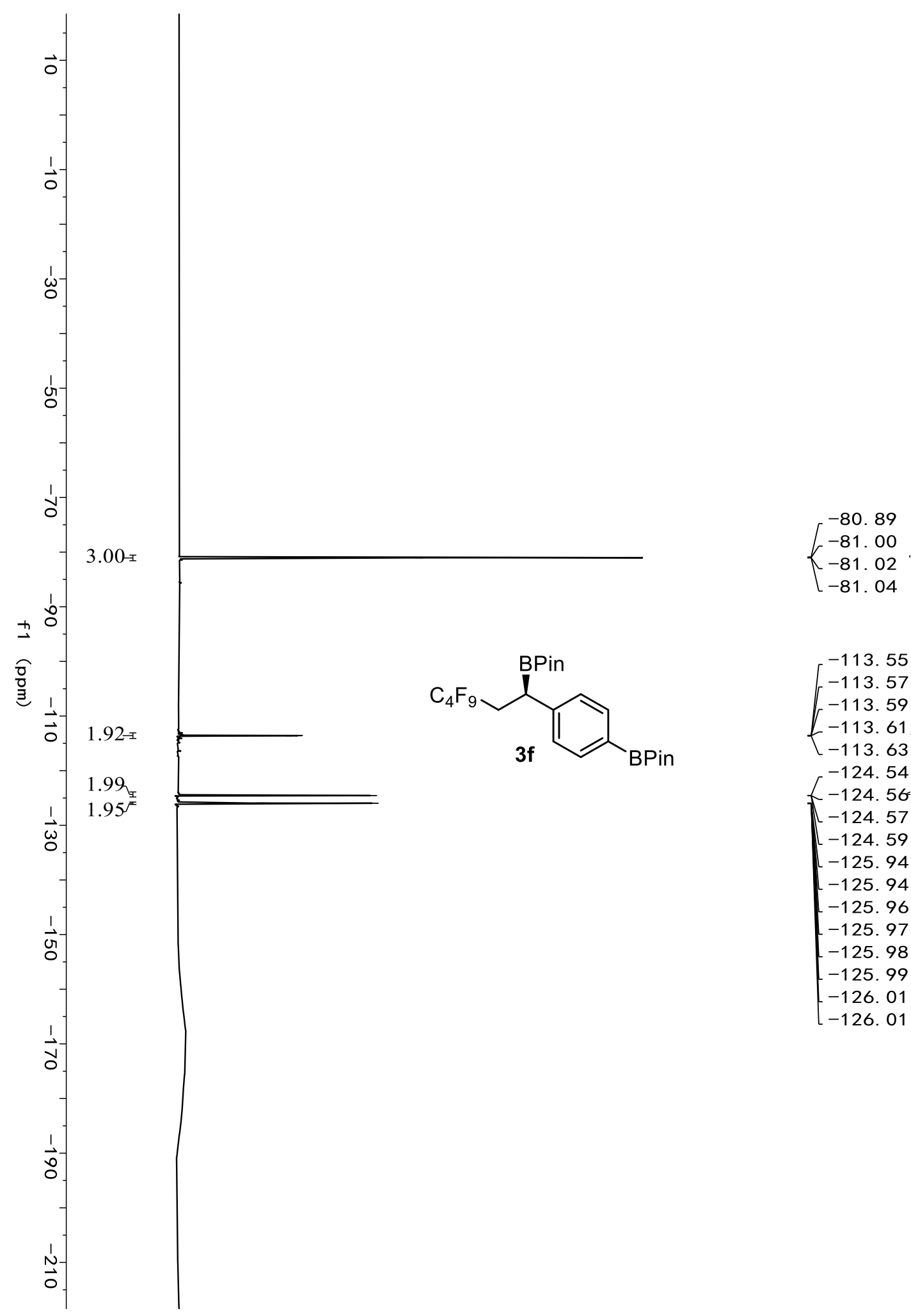




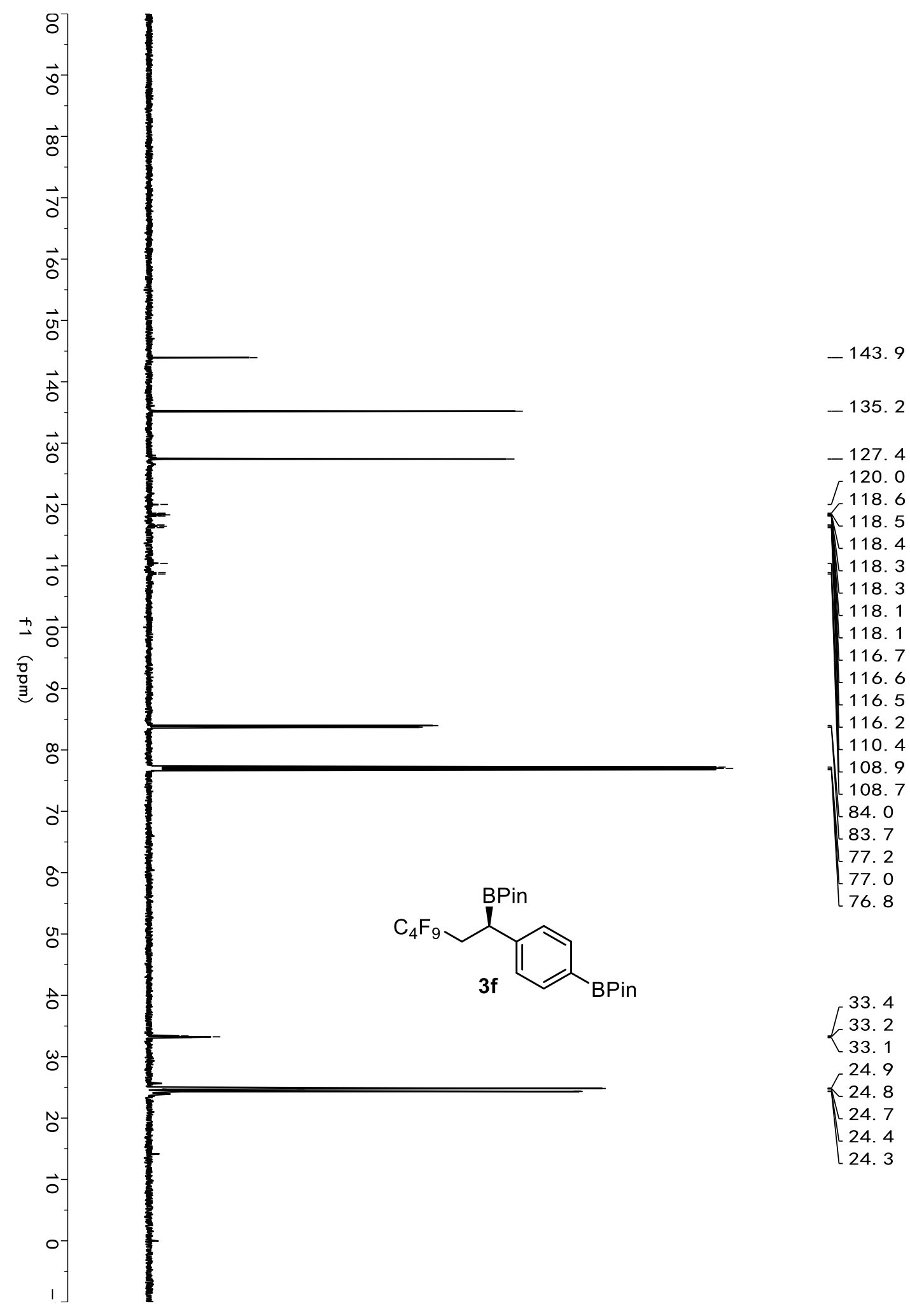




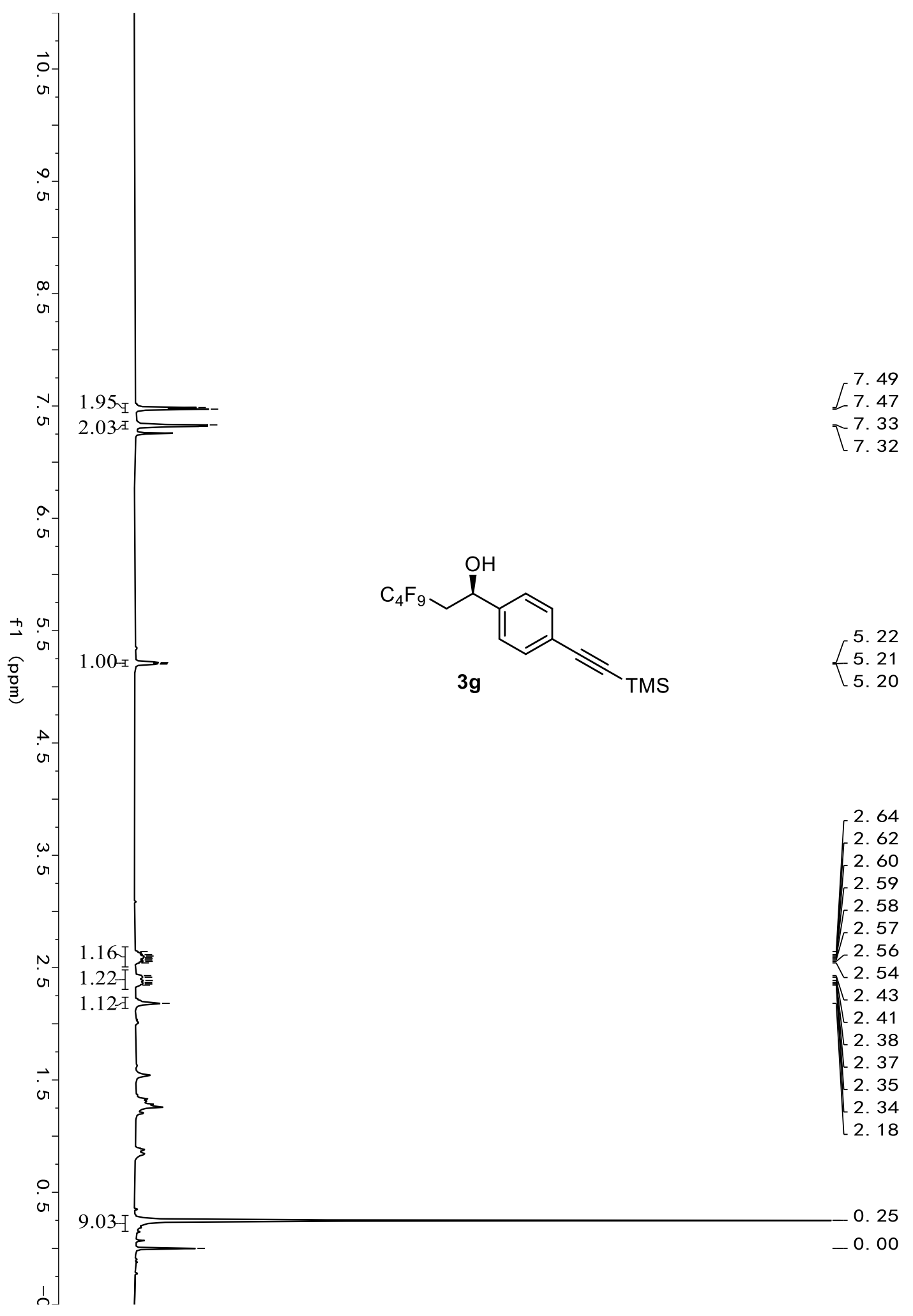




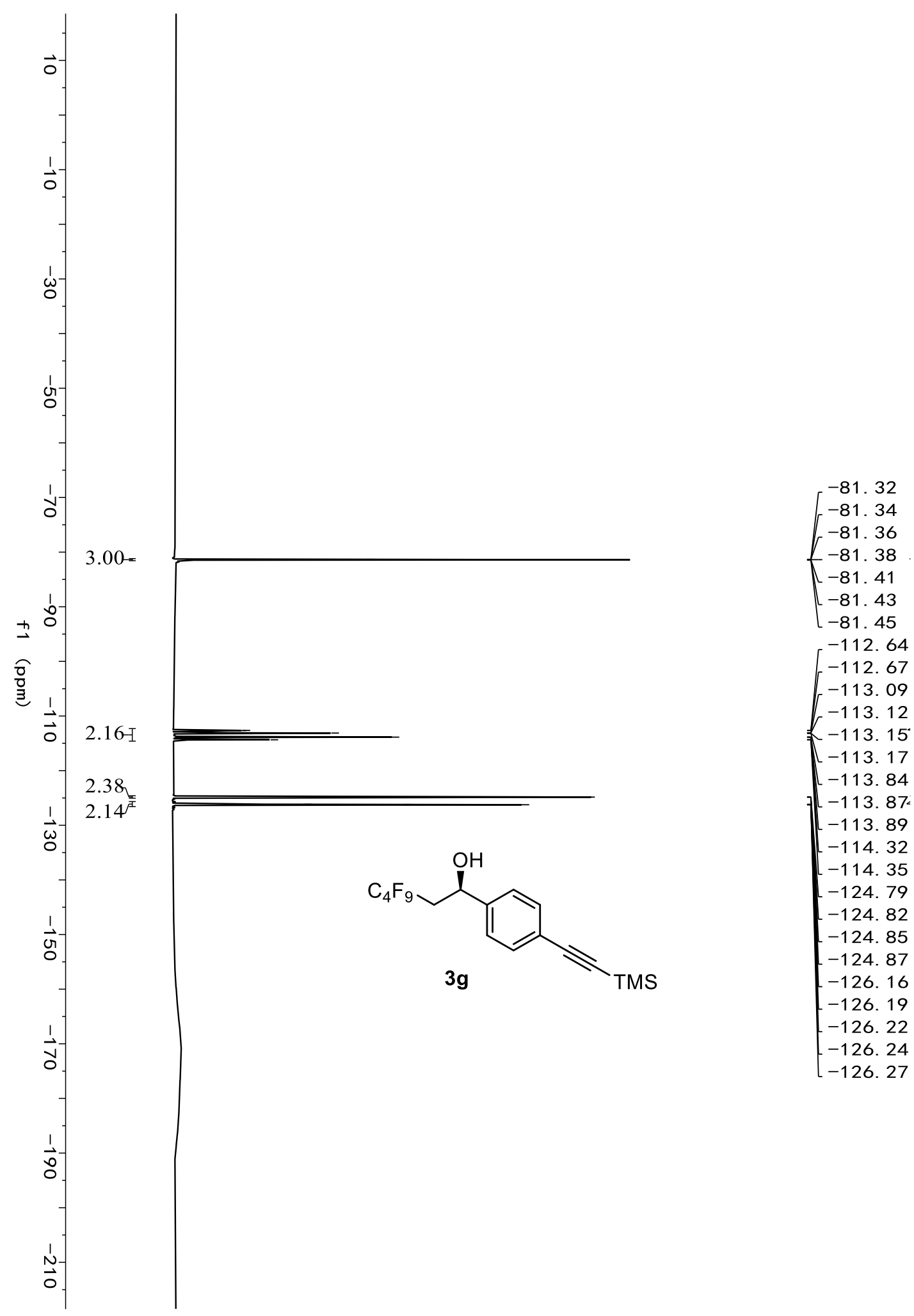




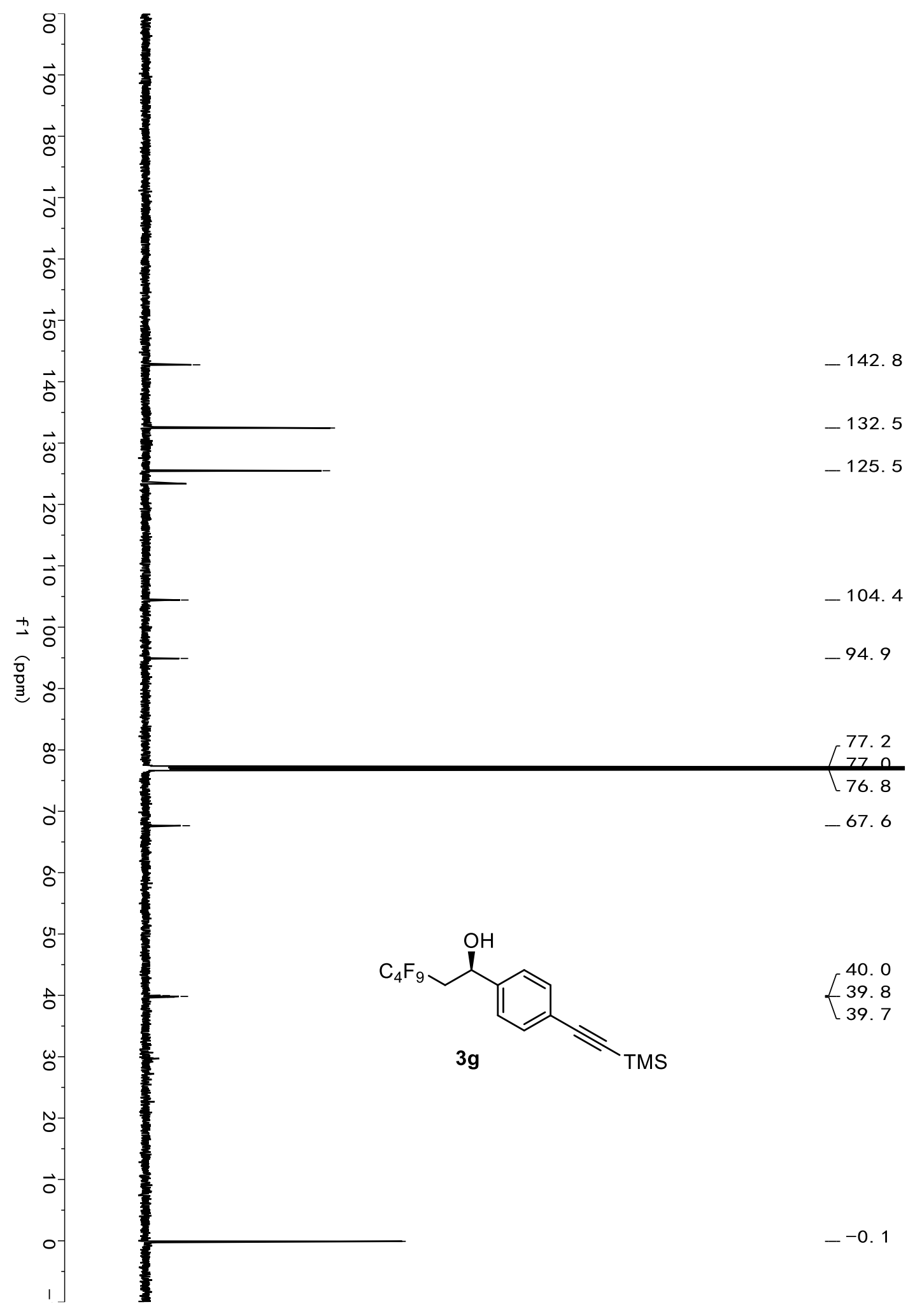




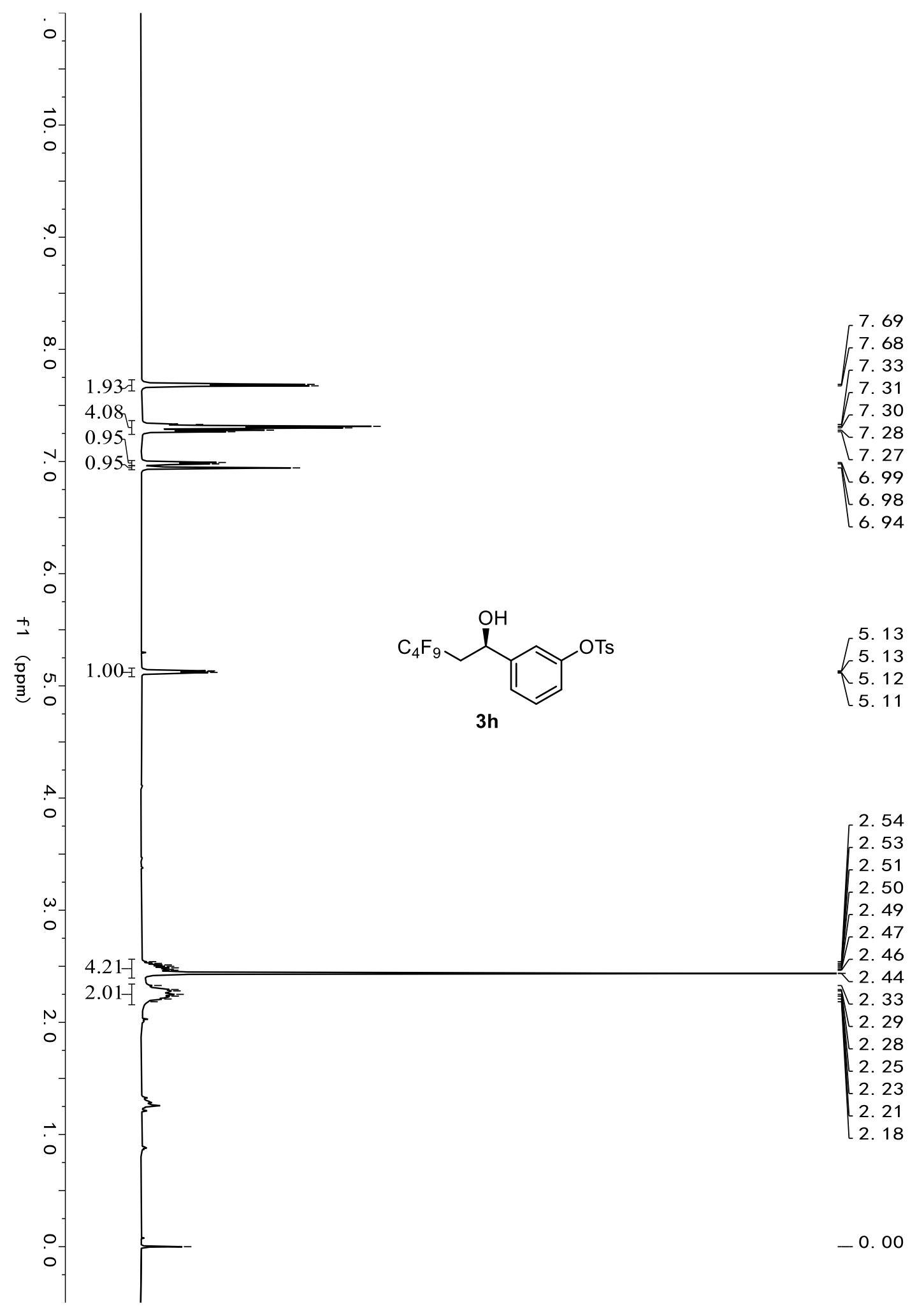




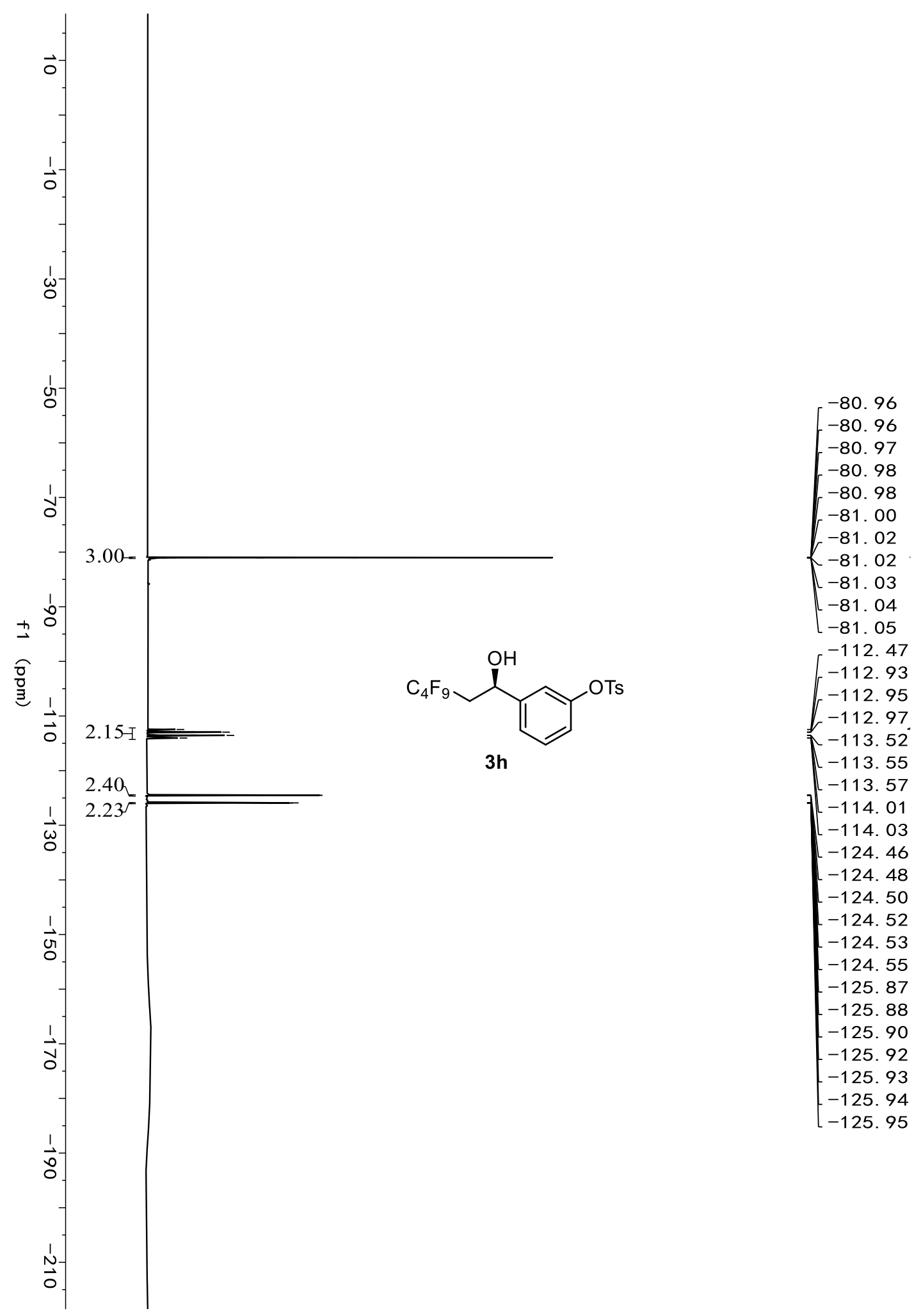




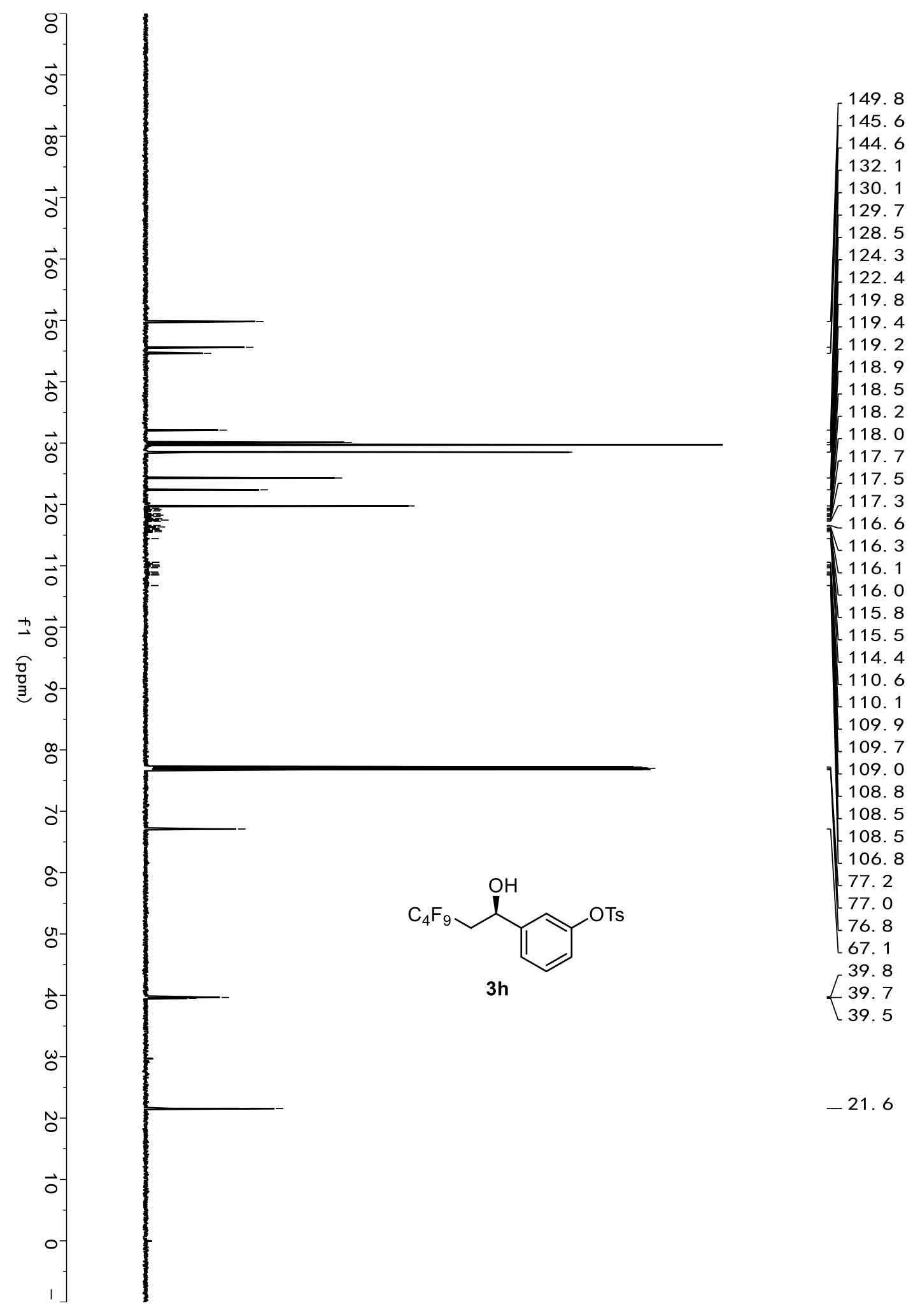




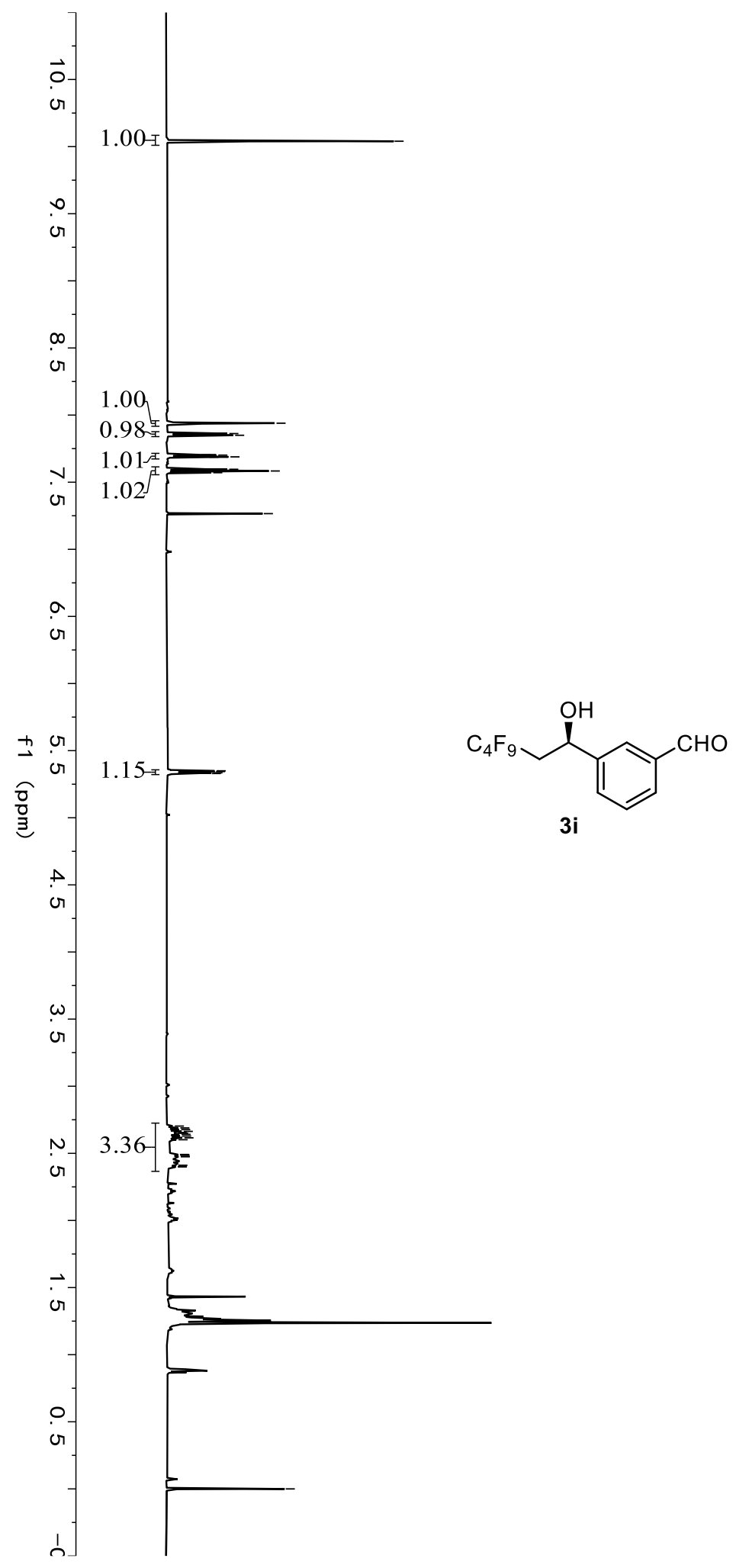

$-10.04$

7. 94

7. 86

7. 86

7.86

7. 85

7. 85

7.85

7.70

- 7.70

7. 70

7. 69

7. 69

7. 69

7. 60

7. 58

7. 57

7. 27

5. 35

$\begin{array}{r}5.34 \\ \hline\end{array}$

5. 34

5. 33

2. 70

2. 69

2. 68

2. 66

2. 66

2. 65

2. 64

2. 63

2. 62

2. 60

2. 49

2. 49

2. 49

2. 48

2. 47

2. 41

2. 40

2. 40

$--0.00$ 


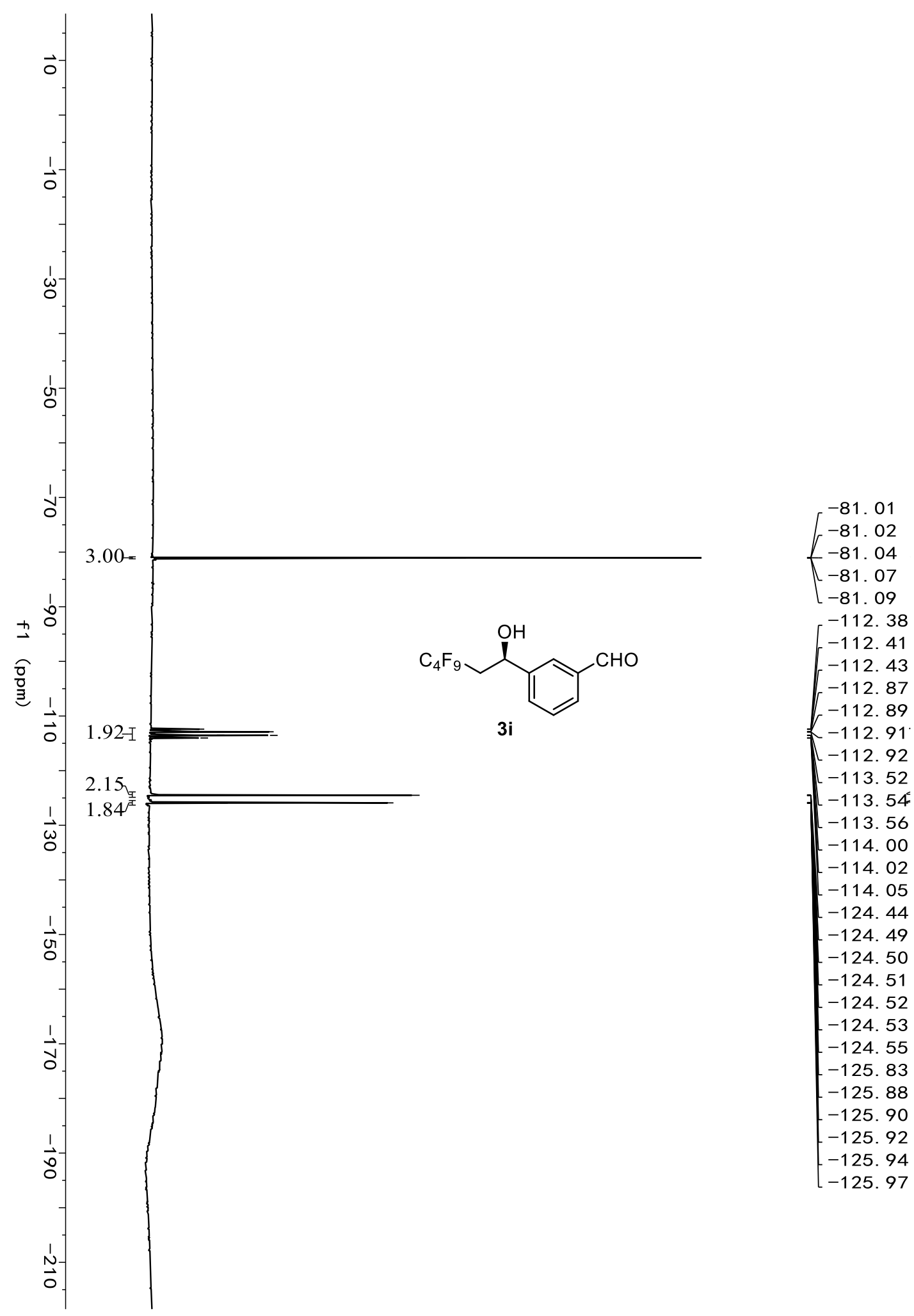




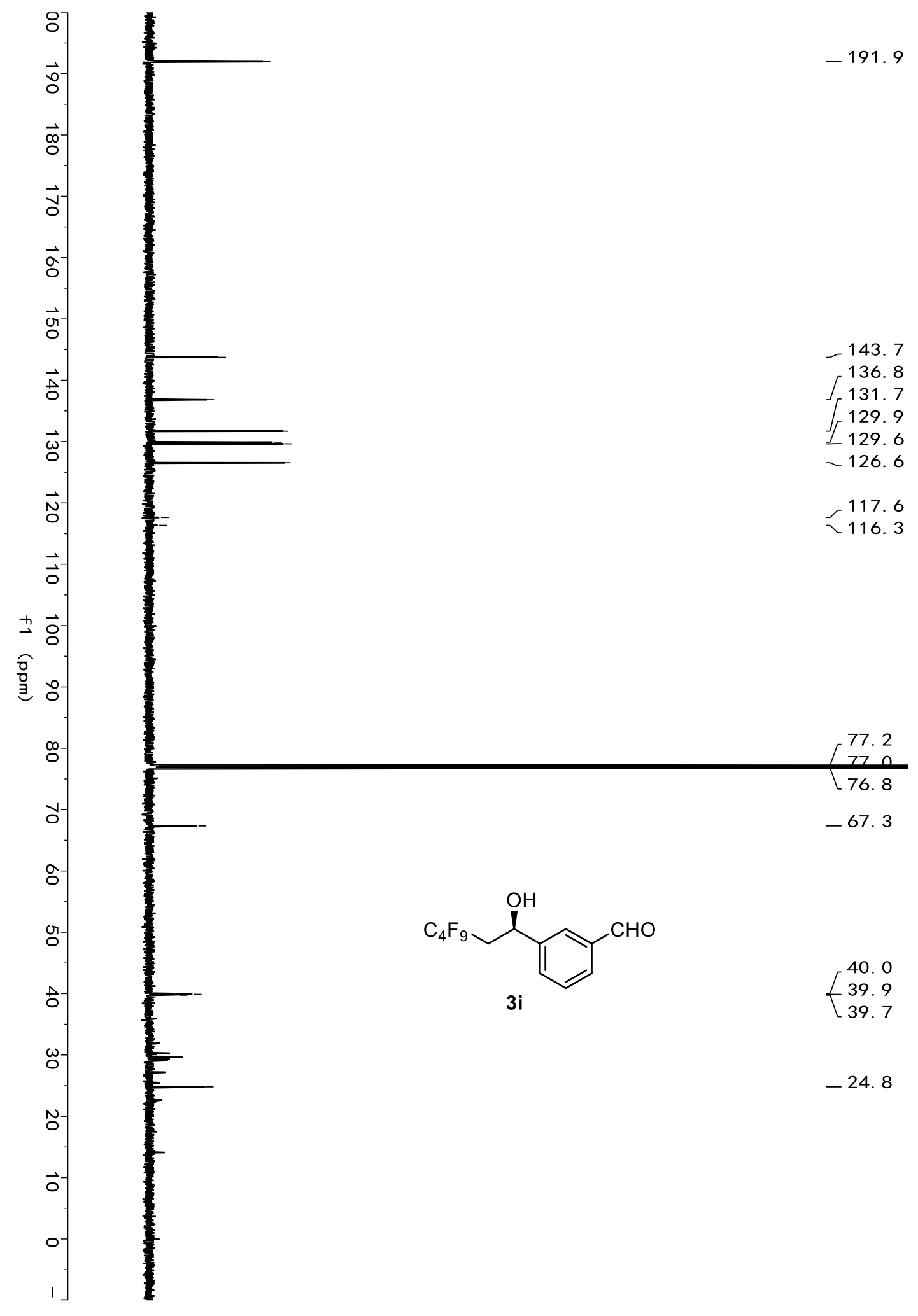




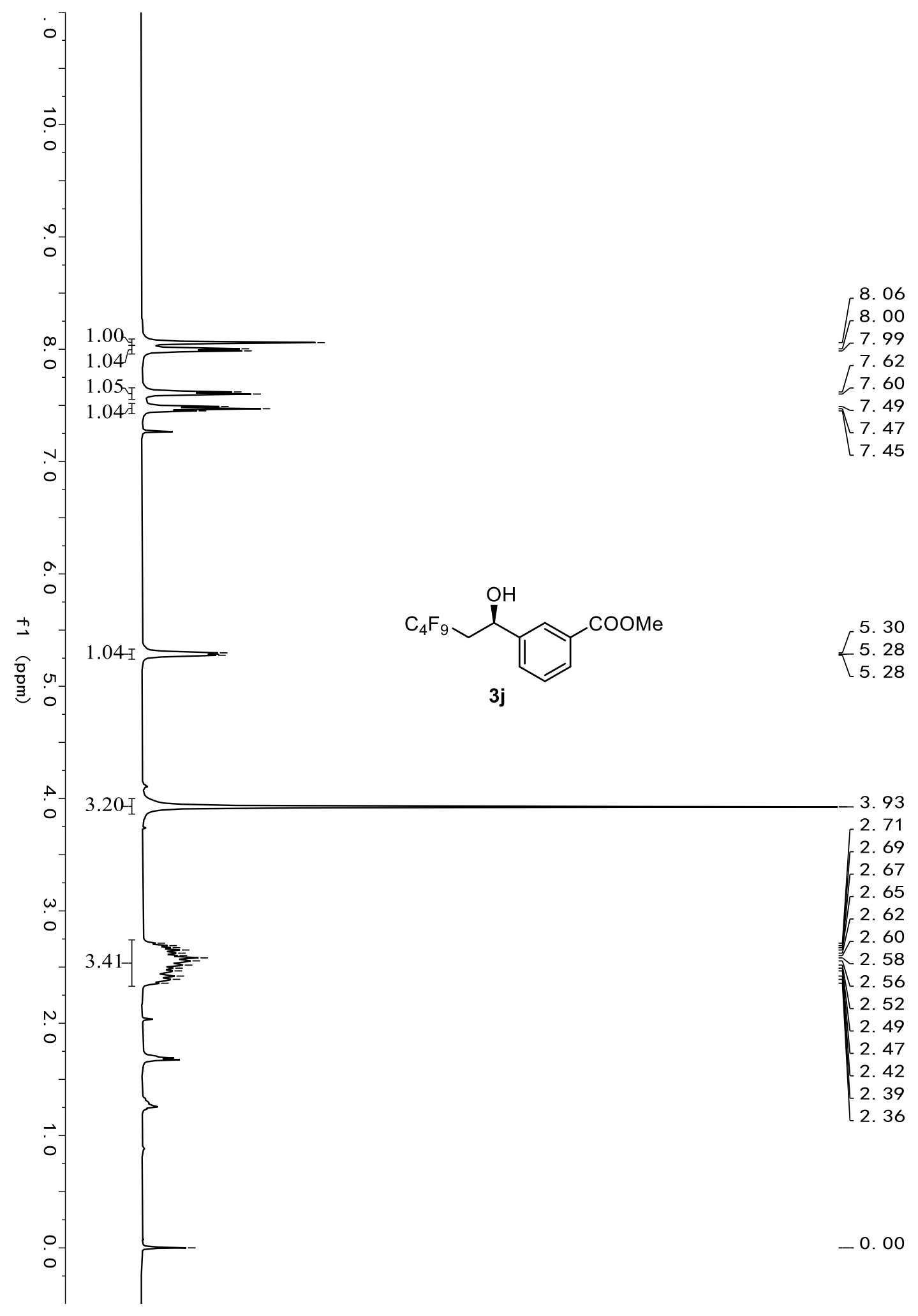




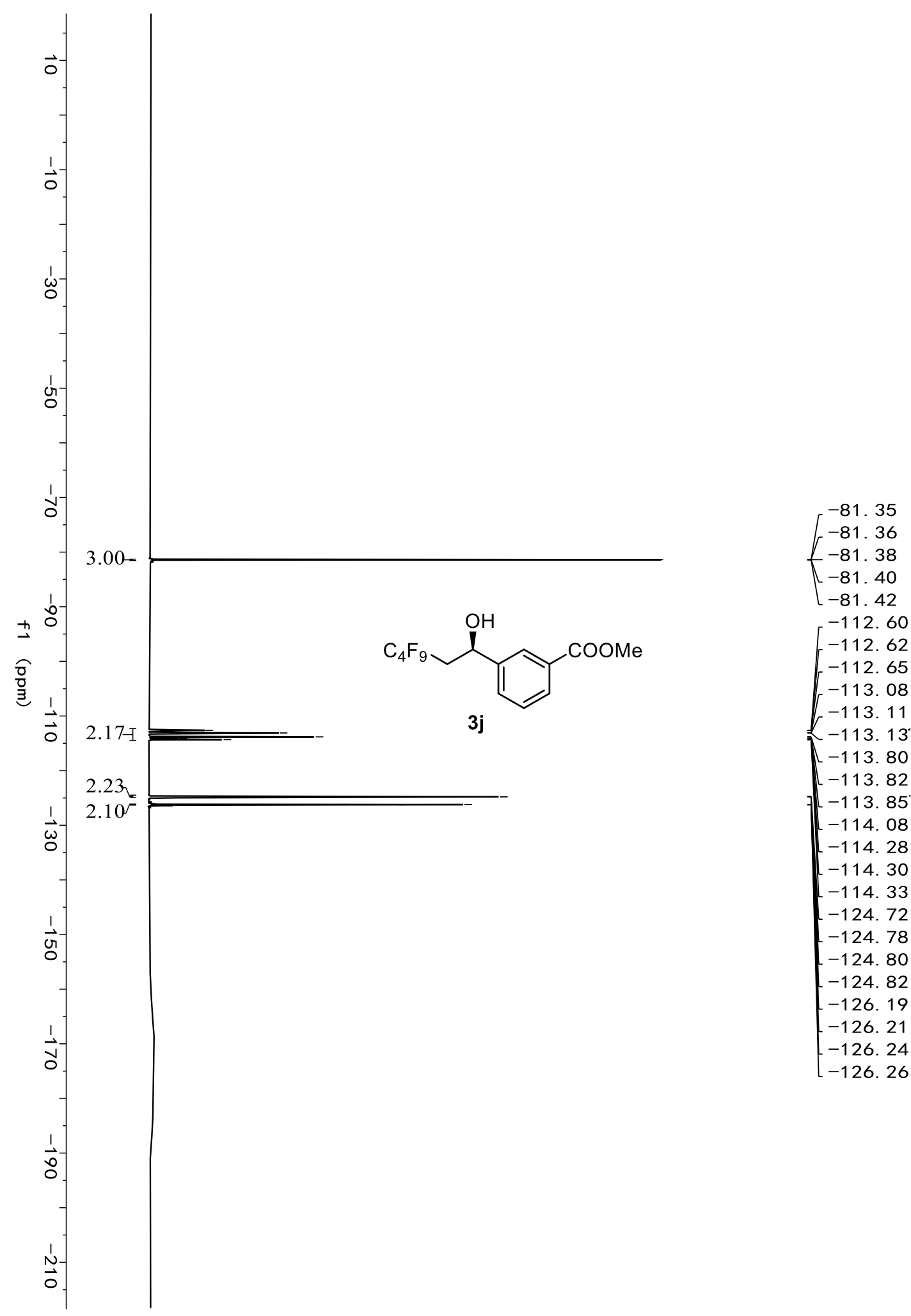




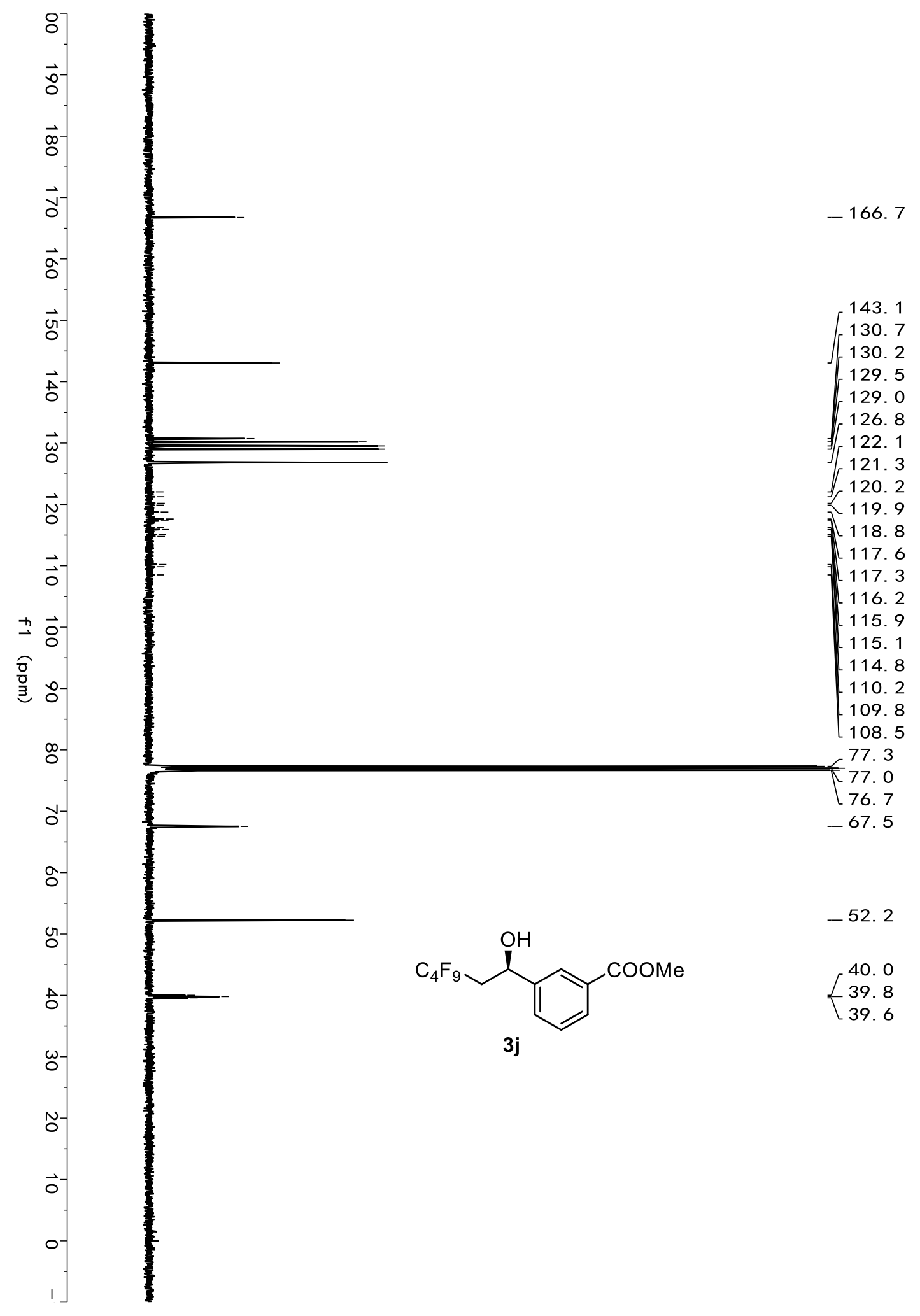




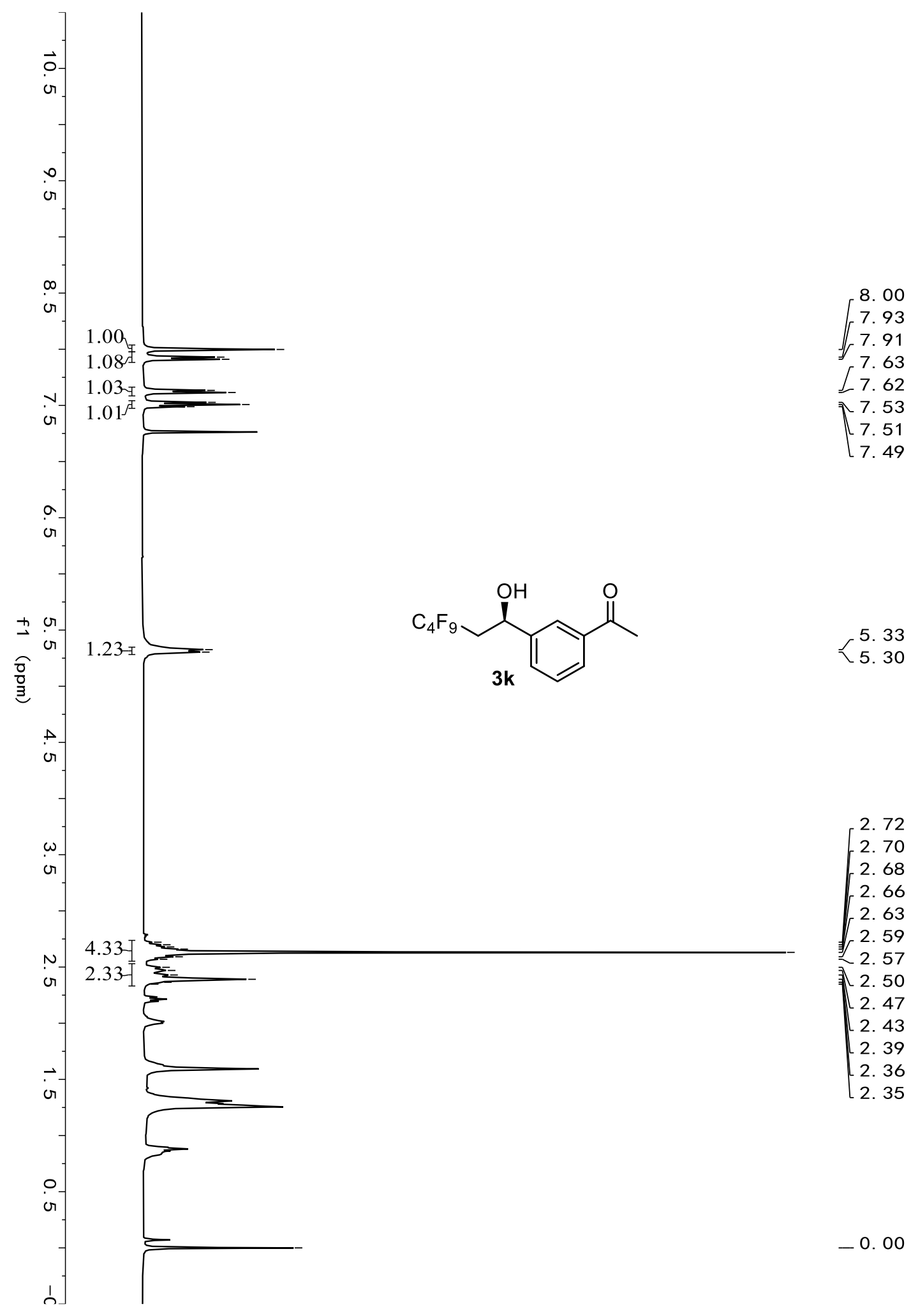




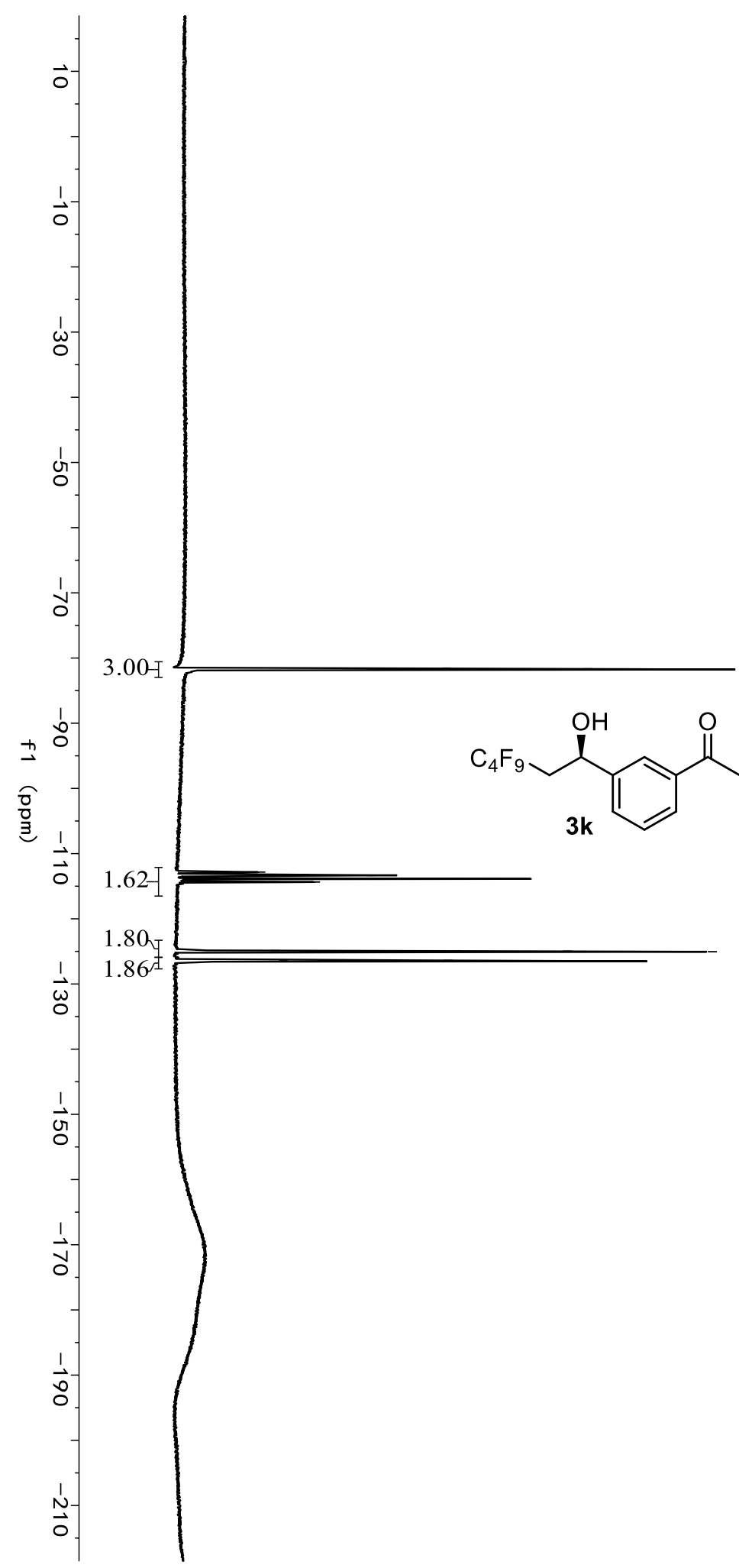

$-81.55$

$-81.66$

$-81.73$

$-81.77$

$-112.83$

$-112.87$

$-113.23$

$-113.31$

$-113.35$

$-113.82$

$-113.85$

$-113.87$

$-113.90$

$-114.31$

$-114.33$

$-114.36$

$-114.38$

$-124.88$

$-124.91$

$-124.97$

$-125.05$

$-126.32$

$-126.39$

$-126.51$

$-126.55$ 


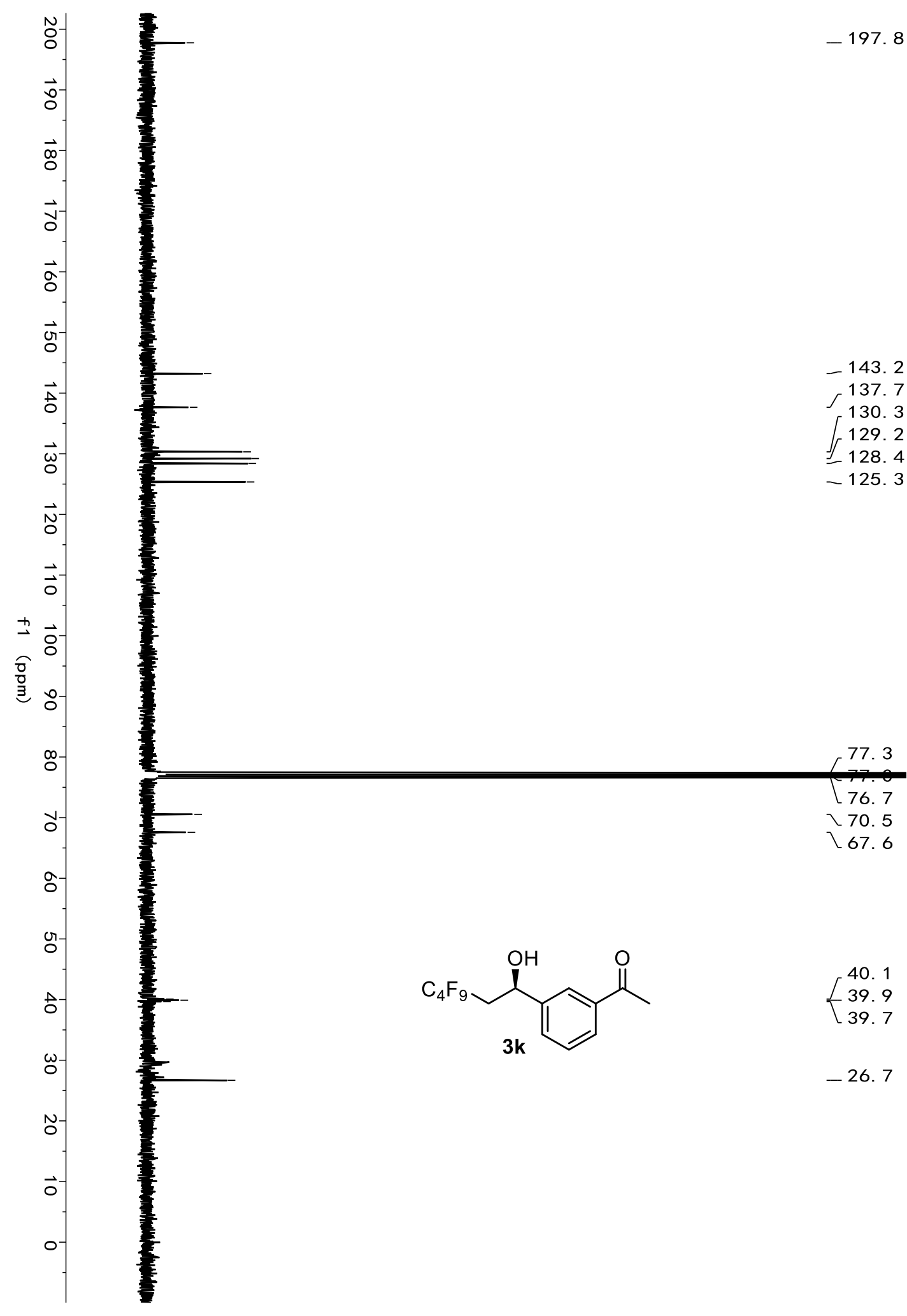




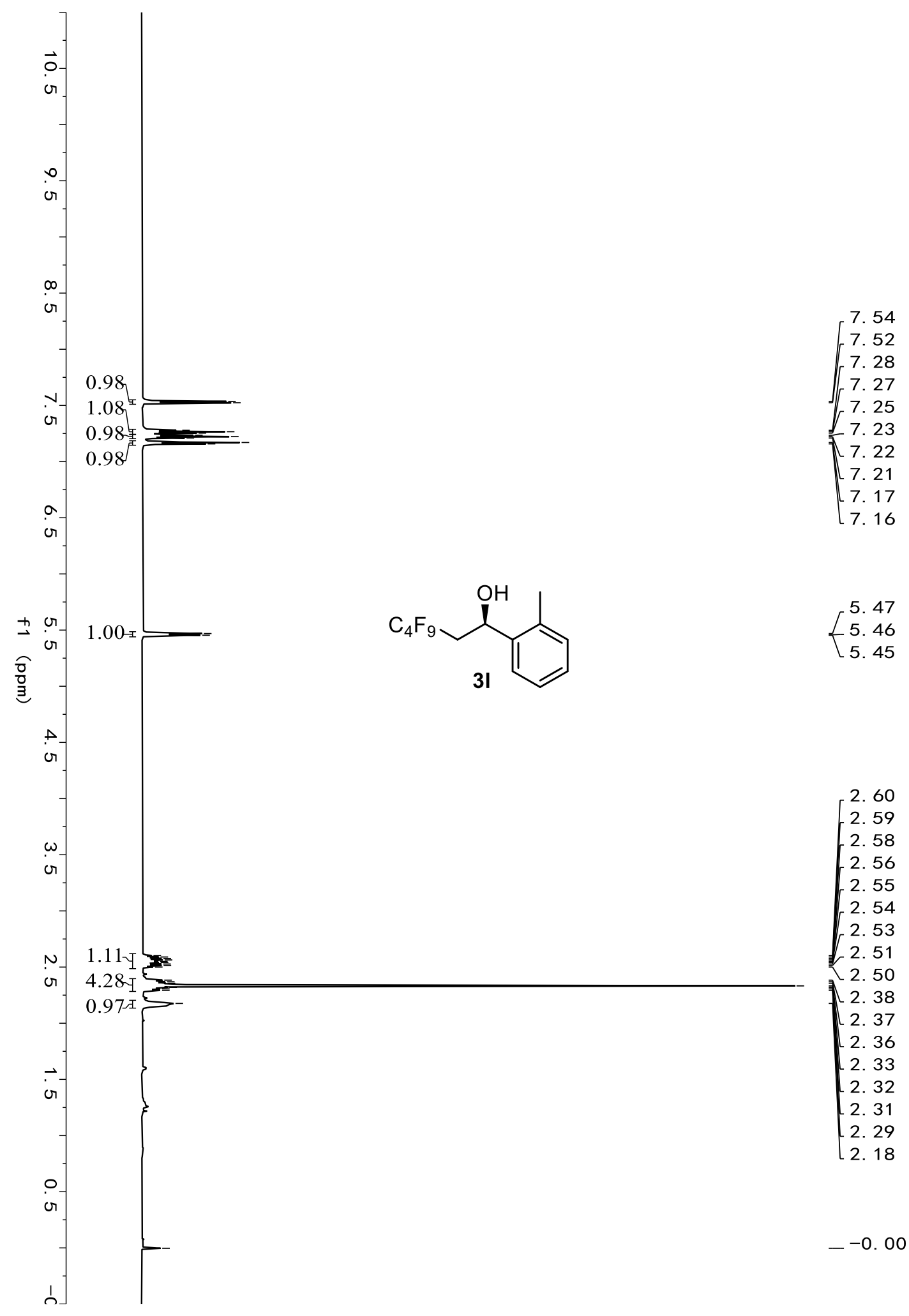




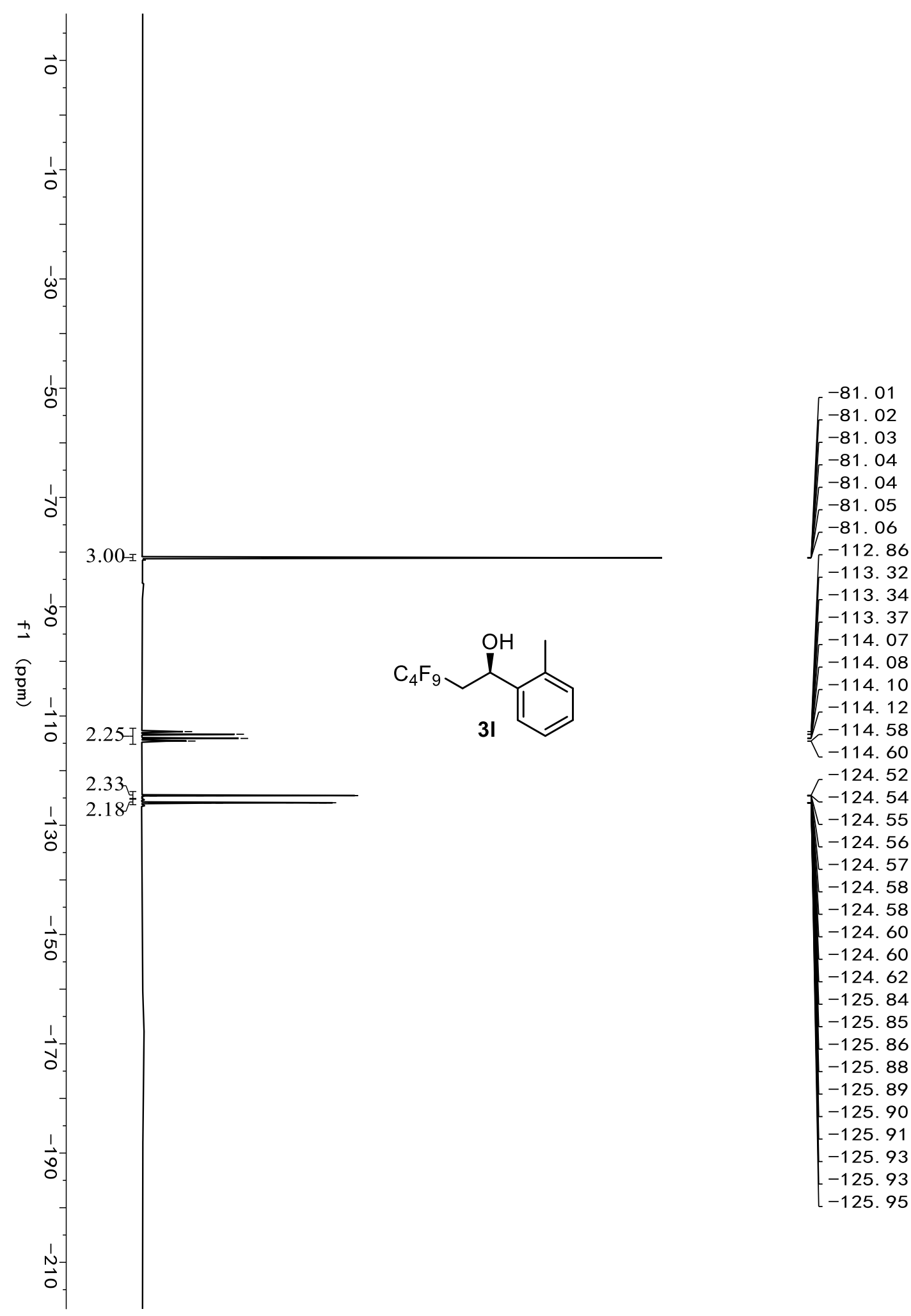




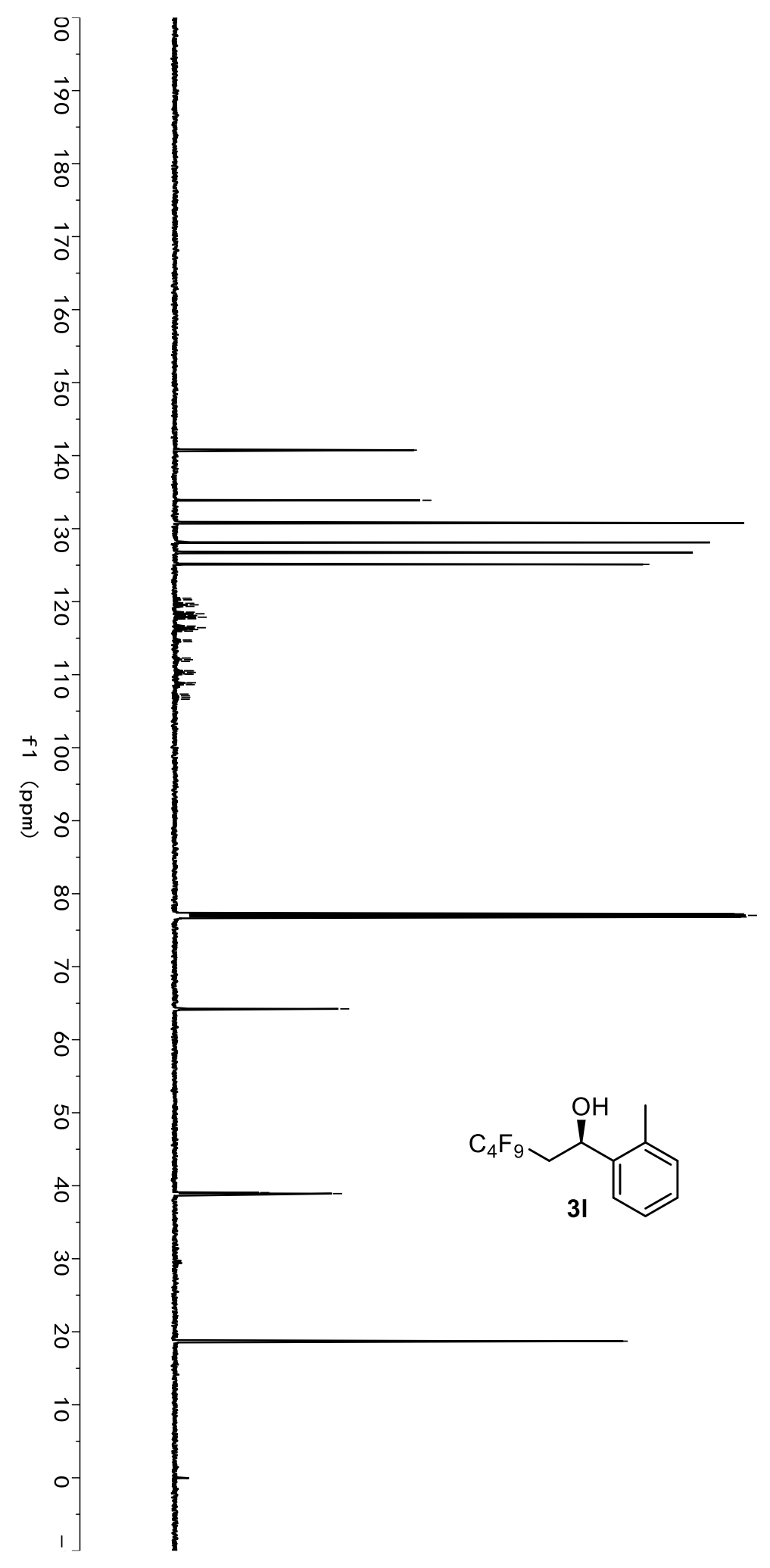

140. 8

133. 9

130. 8

128. 1

126. 7

125. 1

120. 3

$-119.8$

119.6

119. 4

118. 6

118. 3

118. 1

118. 1

117. 9

117. 7

116. 7

116. 4

116. 2

116. 2

116. 0

114. 7

114.5

$-112.1$

110. 5

110.5

110. 3

110. 1

110. 1

108. 9

108. 9

108,6

$-77.0$

76. 8

$-64.2$

39. 1

$-38.9$

38. 8

$-18.7$ 


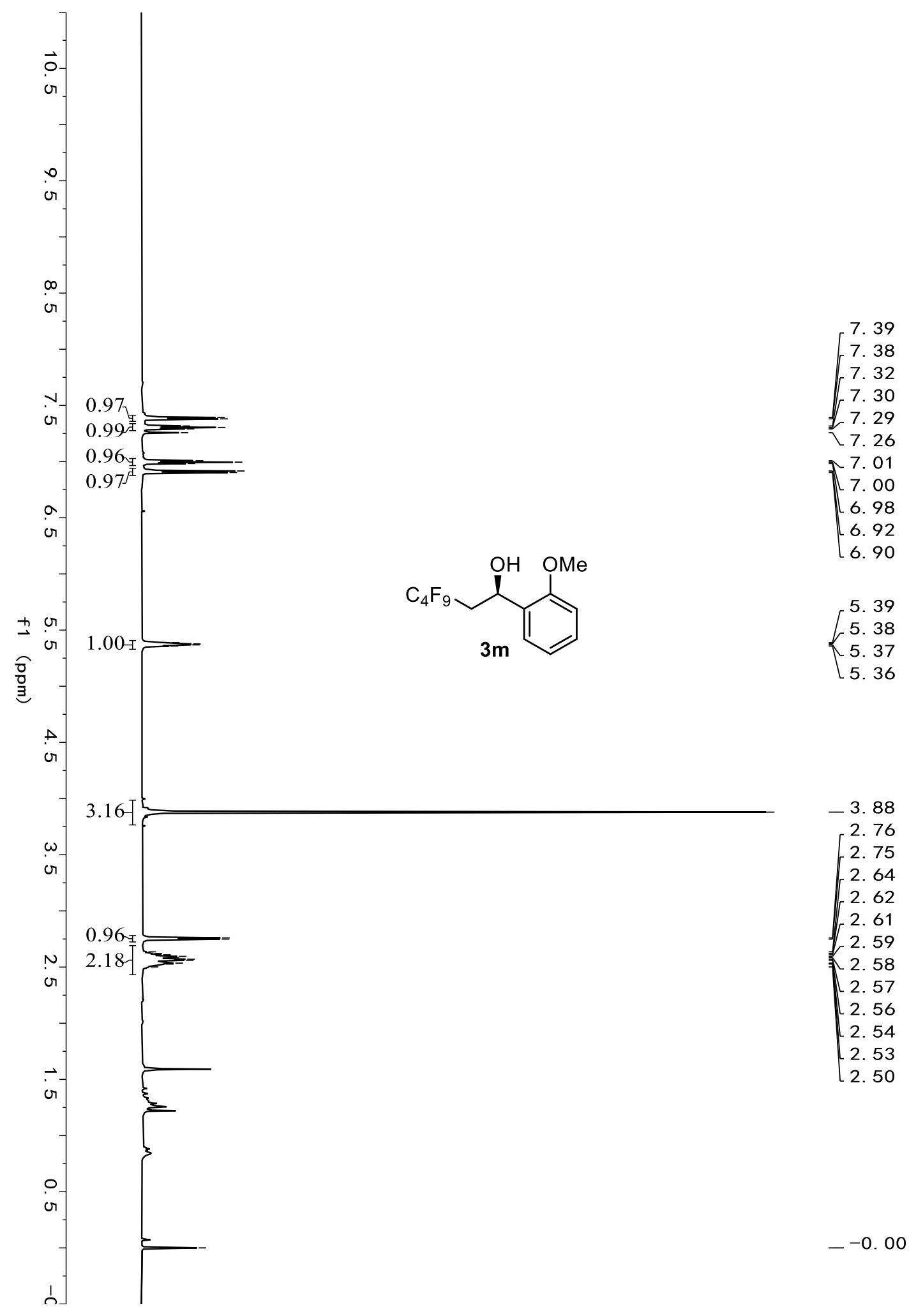




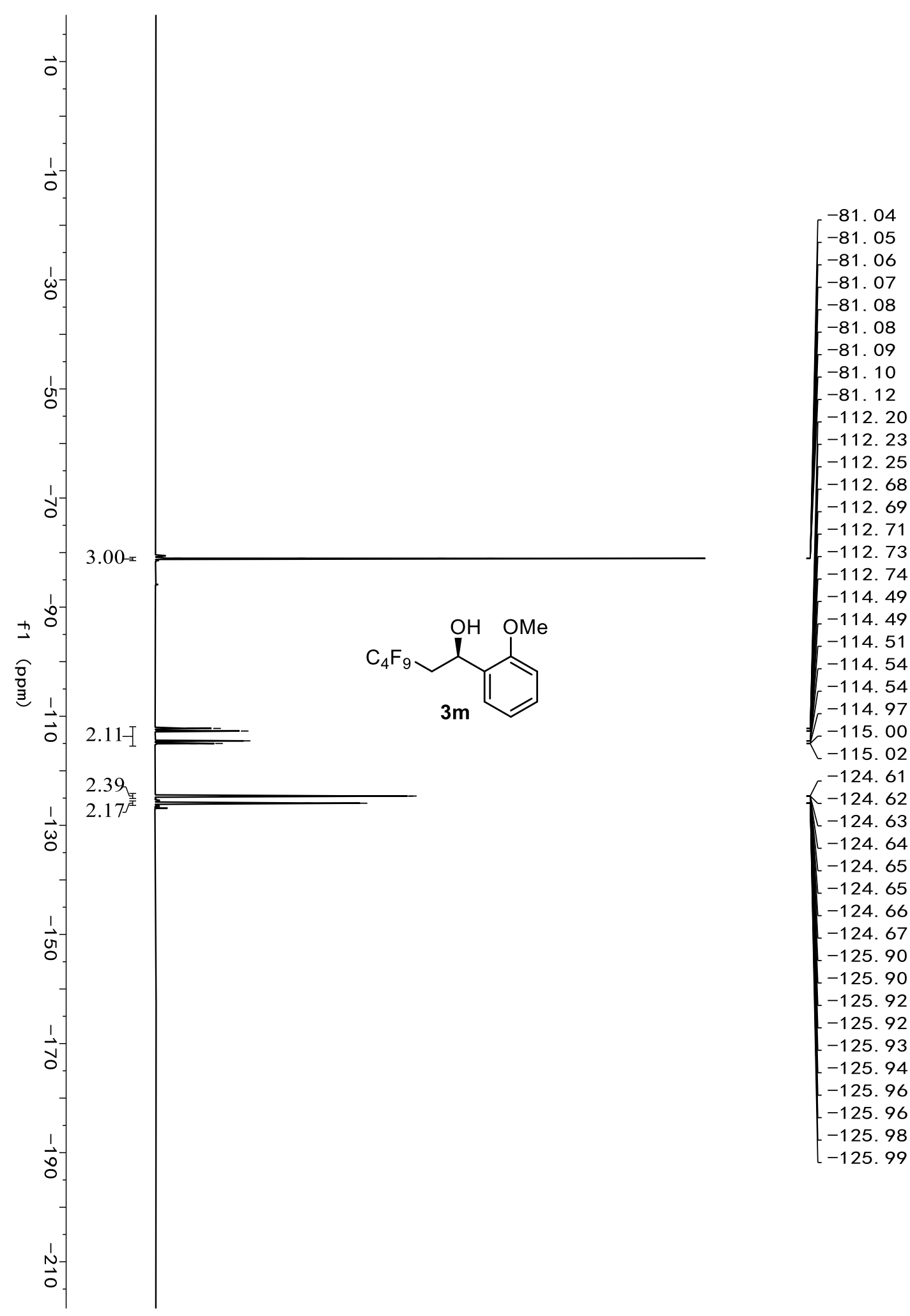




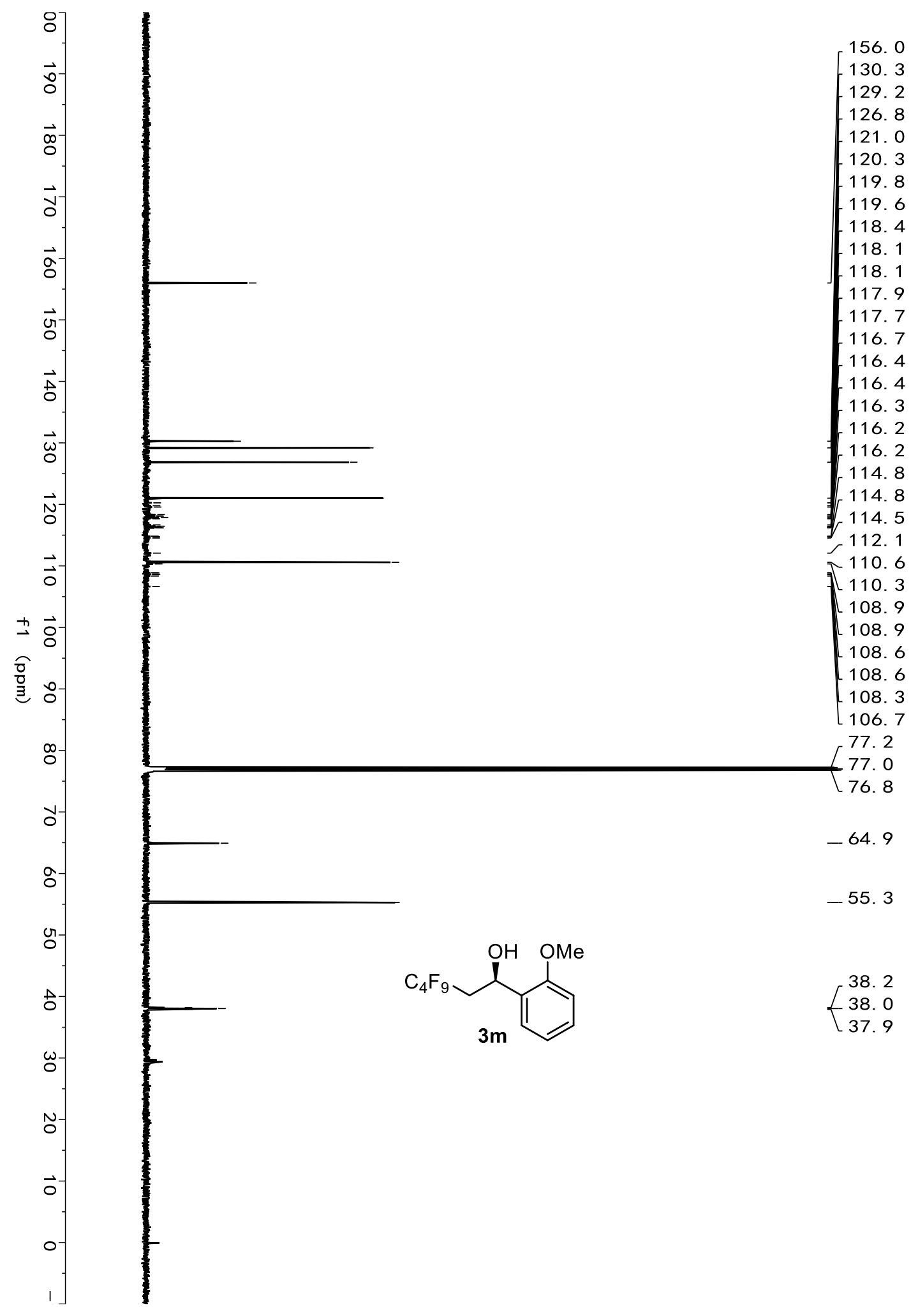




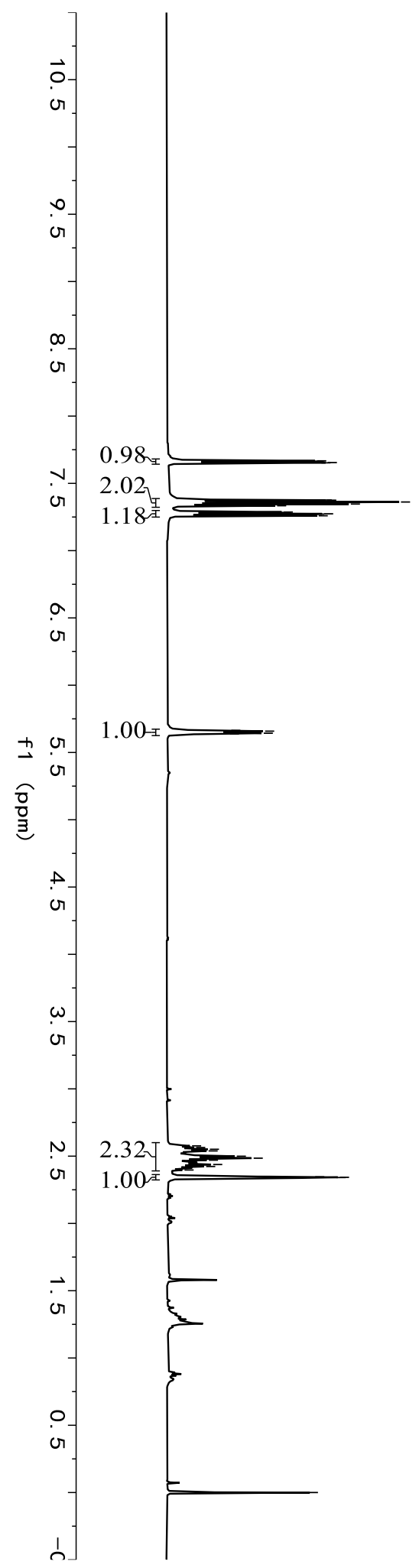

$\left[\begin{array}{l}7.67 \\ 7.67 \\ 7.65 \\ 7.65 \\ 7.38 \\ 7.37 \\ 7.36 \\ 7.35 \\ 7.33 \\ 7.29 \\ 7.28 \\ 7.27 \\ 7.27 \\ 7.26 \\ 5.66 \\ 5.66 \\ 5.65 \\ 5.65 \\ 5.64 \\ 5.64\end{array}\right.$

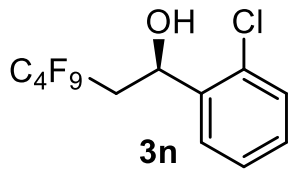

2. 58

2. 56

2. 55

2. 54

2. 50

2. 48

2. 47

2. 44

2. 42

2. 40

2. 35

2. 34

2. 34

$-0.00$ 

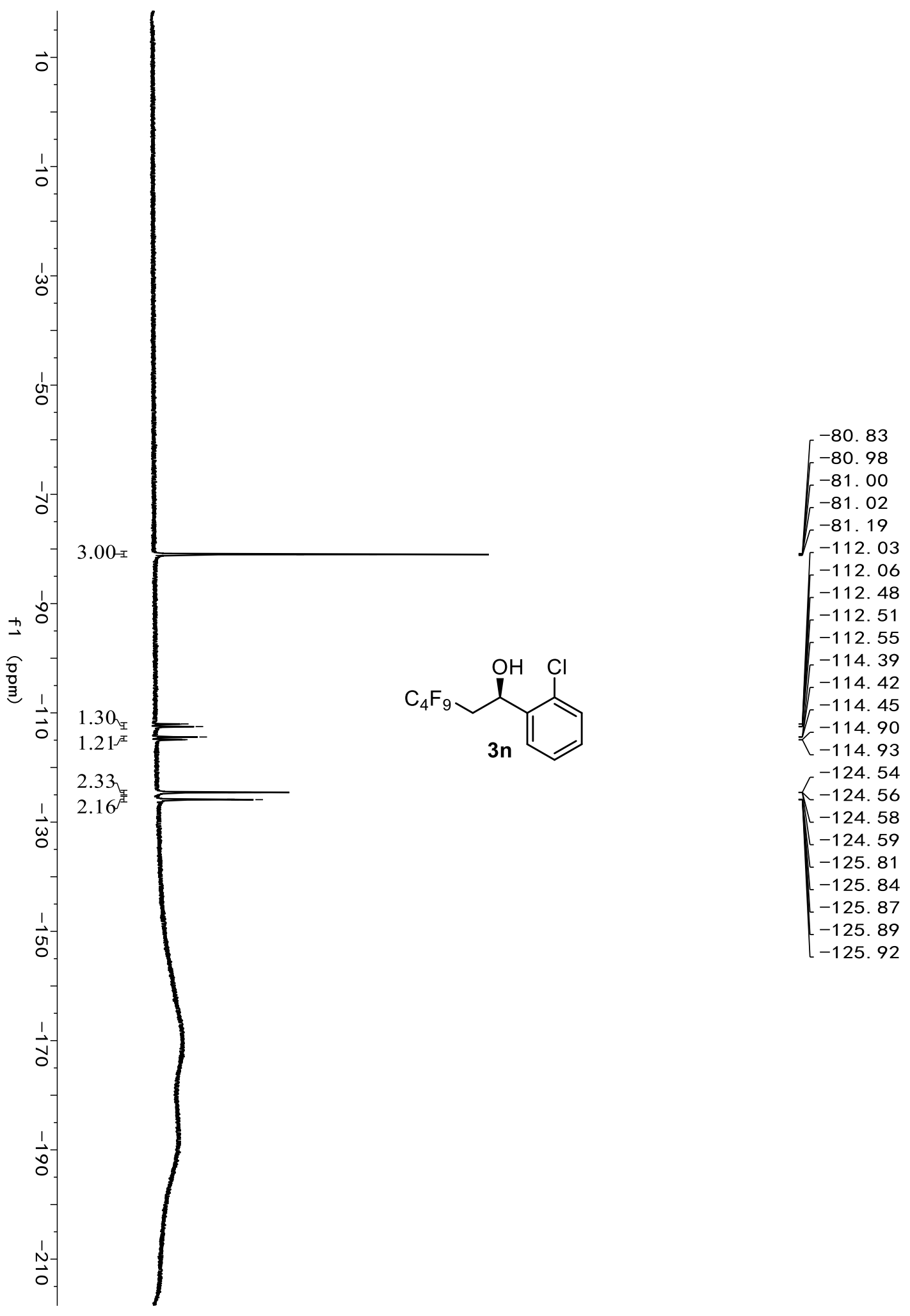

156 


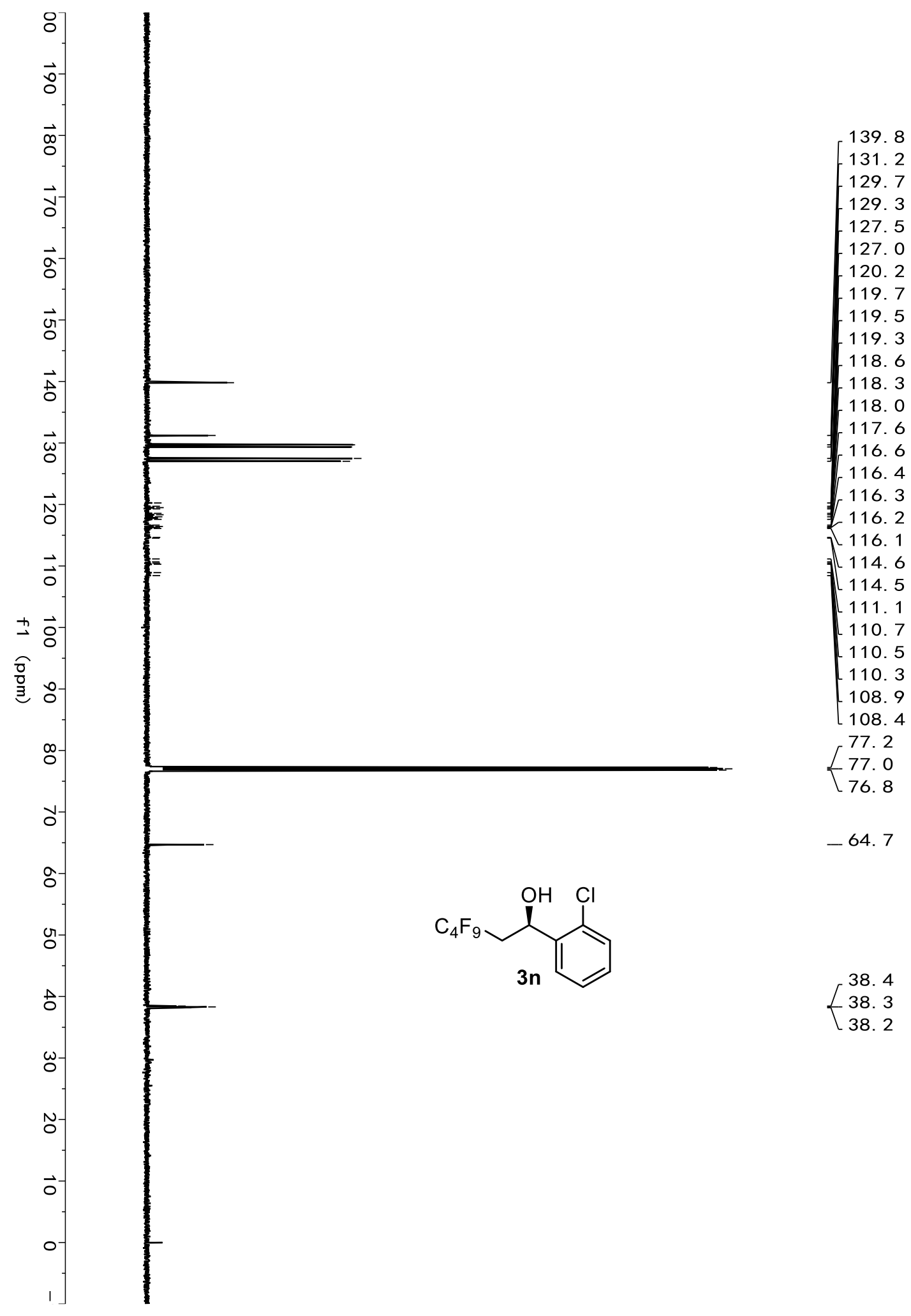




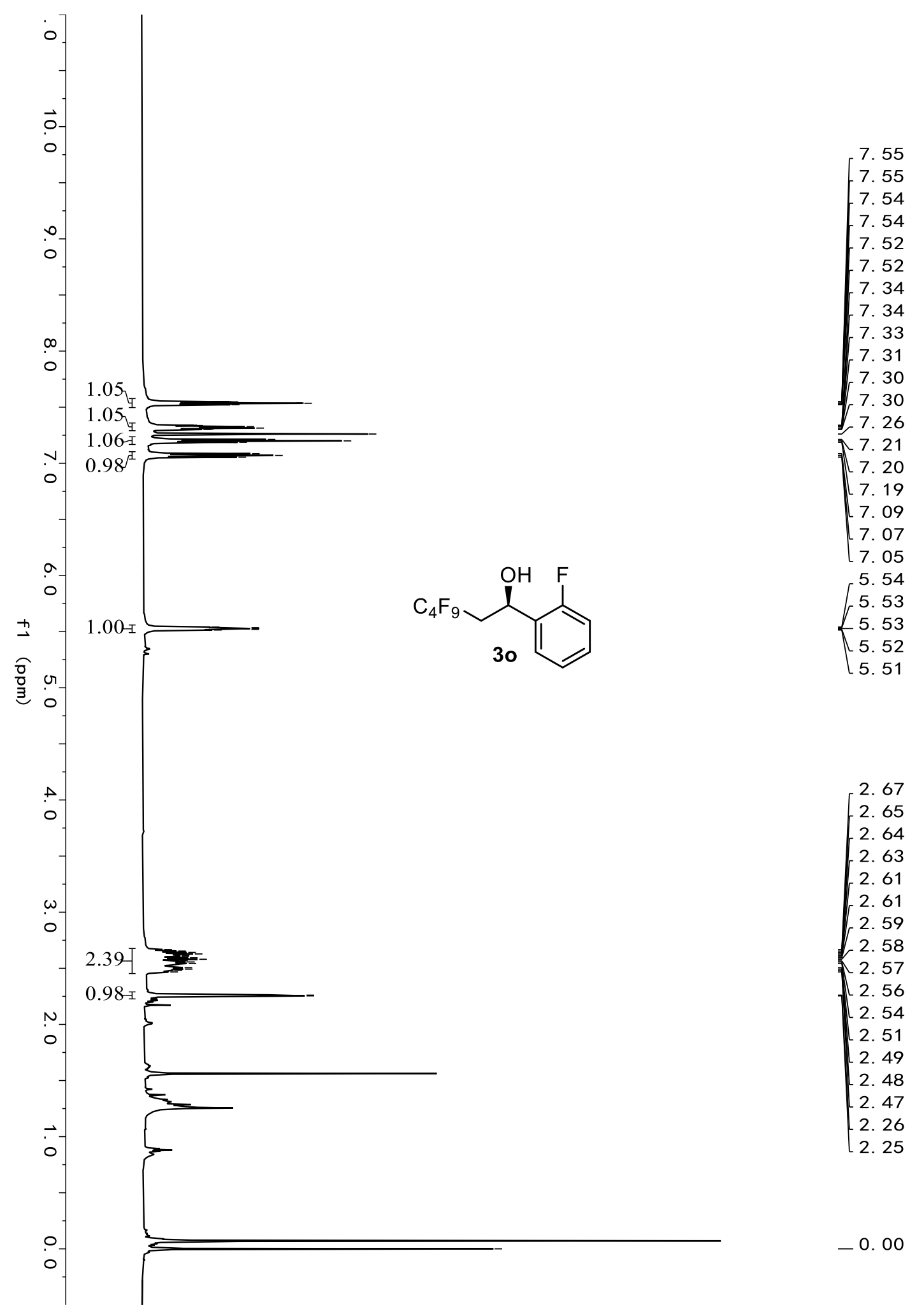




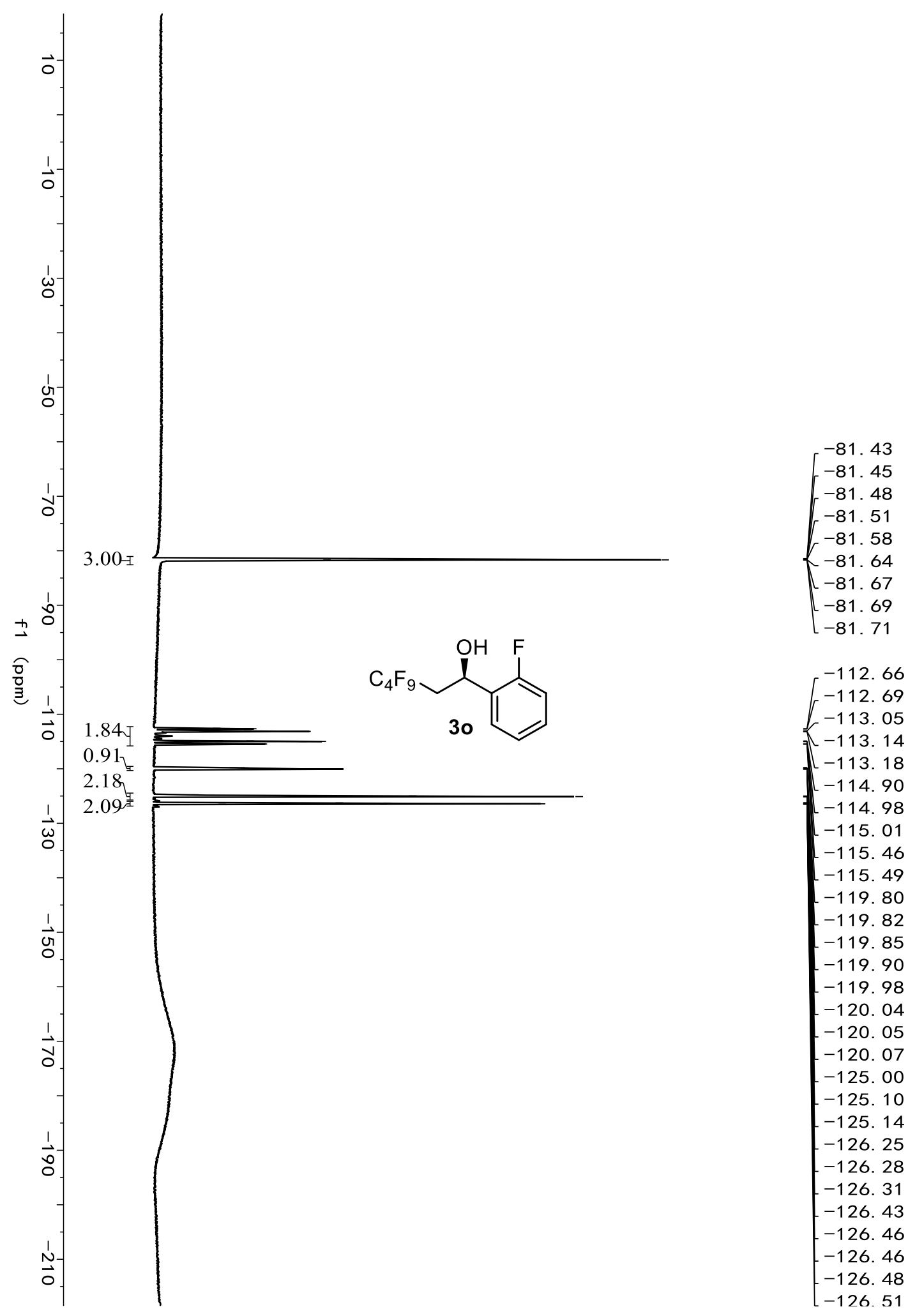




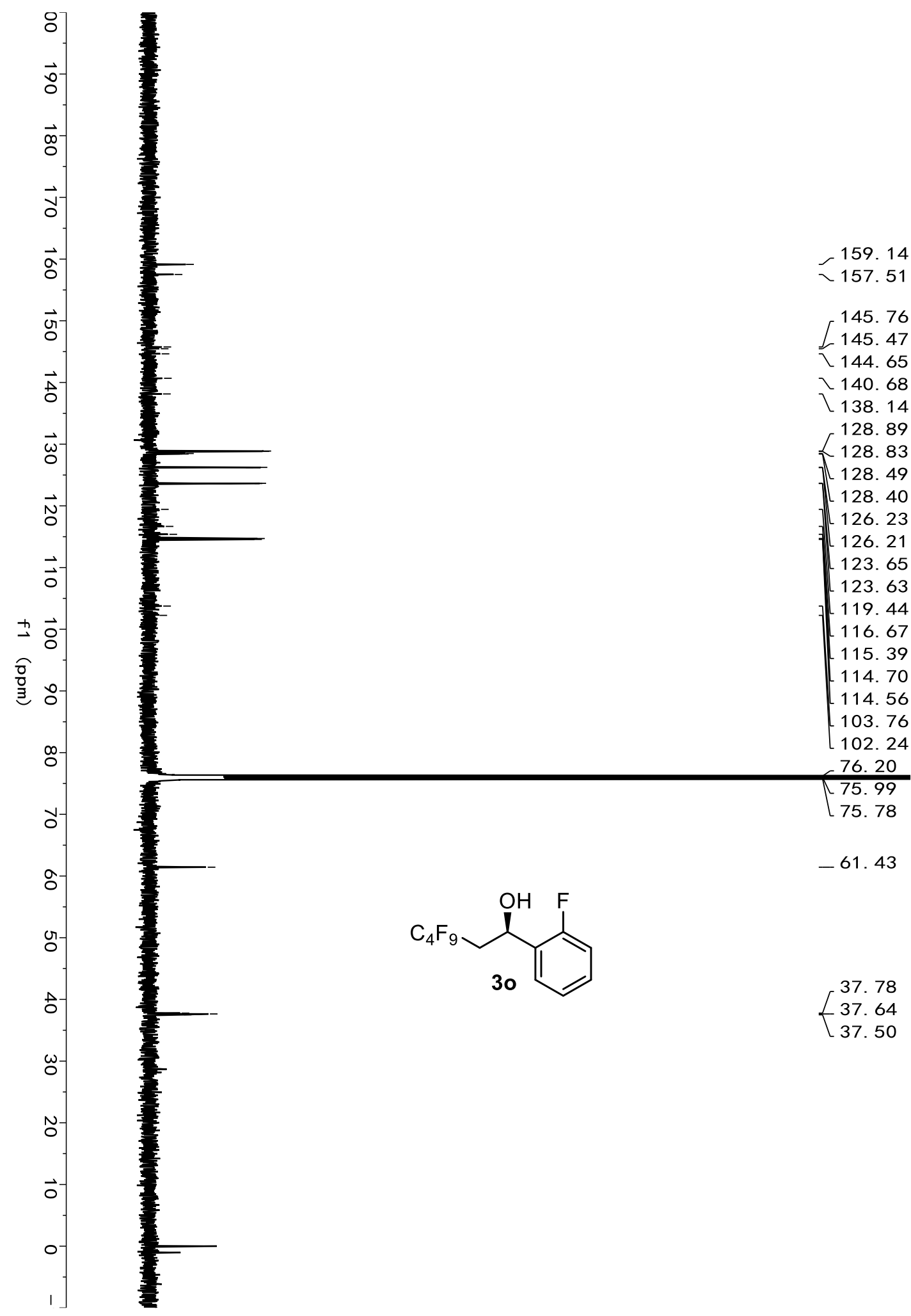




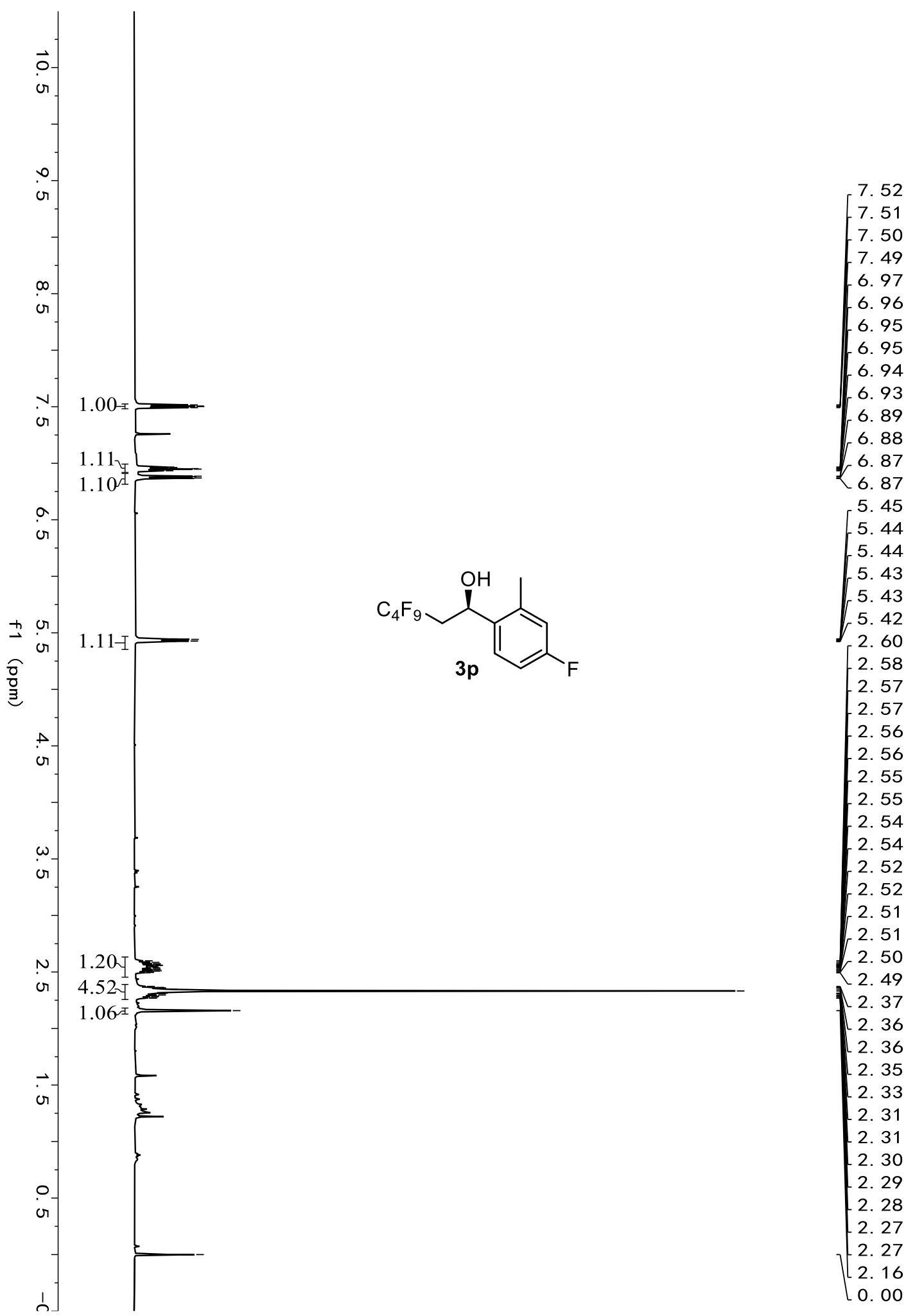

161 


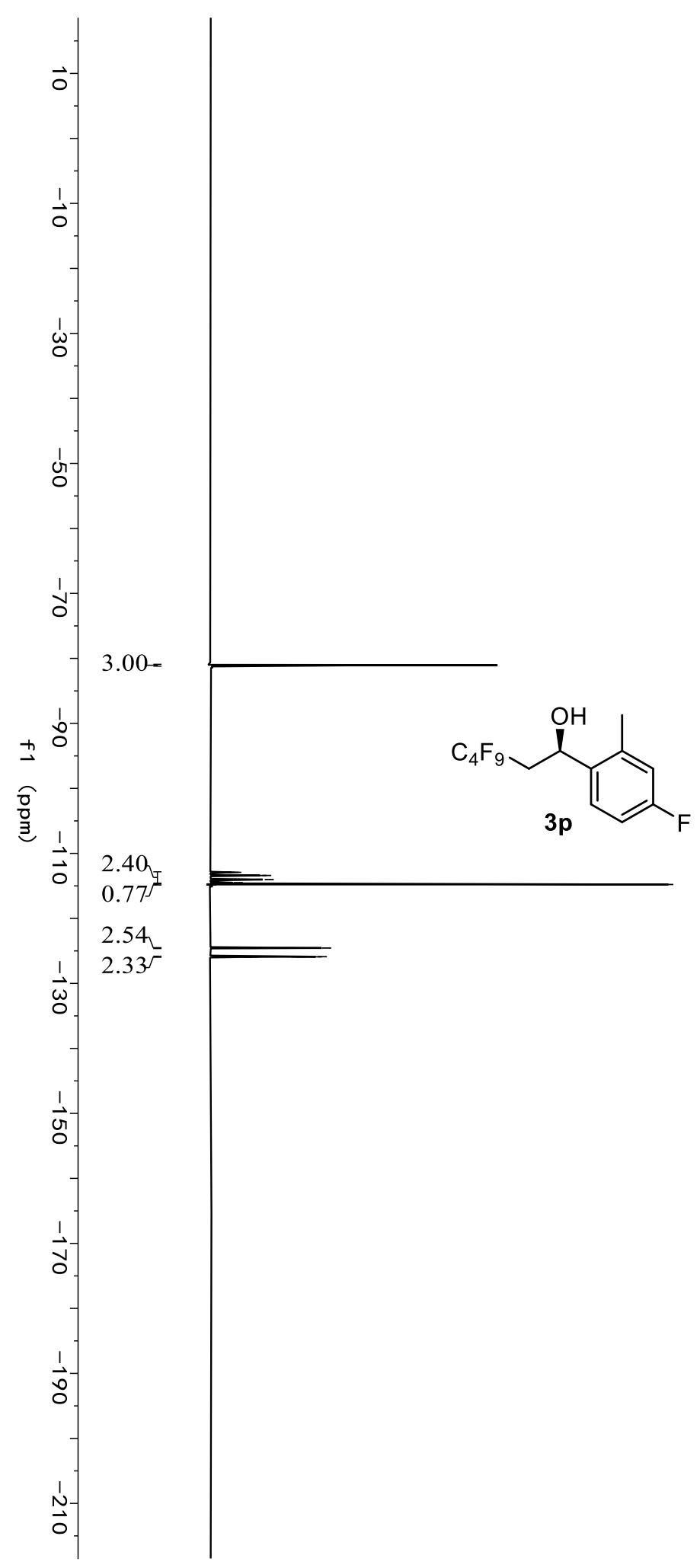

$-81.01$

$-81.02$

$-81.03$

$-81.03$

$-81.05$

$-81.06$

$-112.89$

$-112.89$

$-112.91$

$-112.91$

$-112.92$

$-112.94$

$-112.94$

$-113.37$

$-113.37$

$-113.39$

$-113.40$

$-113.40$

$-113.42$

$-113.42$

$-113.43$

$-113.99$

$-114.00$

$-114.01$

$-114.02$

$-114.04$

$-114.05$

$-114.05$

$-114.48$

$-114.50$

$-114.53$

$-114.78$

$-124.53$

$-124.53$

$-124.54$

$-124.55$

$-124.56$

$-124.57$

$-124.58$

$-124.59$

$-125.84$

$-125.86$

$-125.86$

$-125.87$

$-125.88$

$-125.89$

$-125.89$

$-125.90$

$-125.91$

$-125.92$

$-125.93$

$-125.93$

$-125.94$

$-125.96$ 


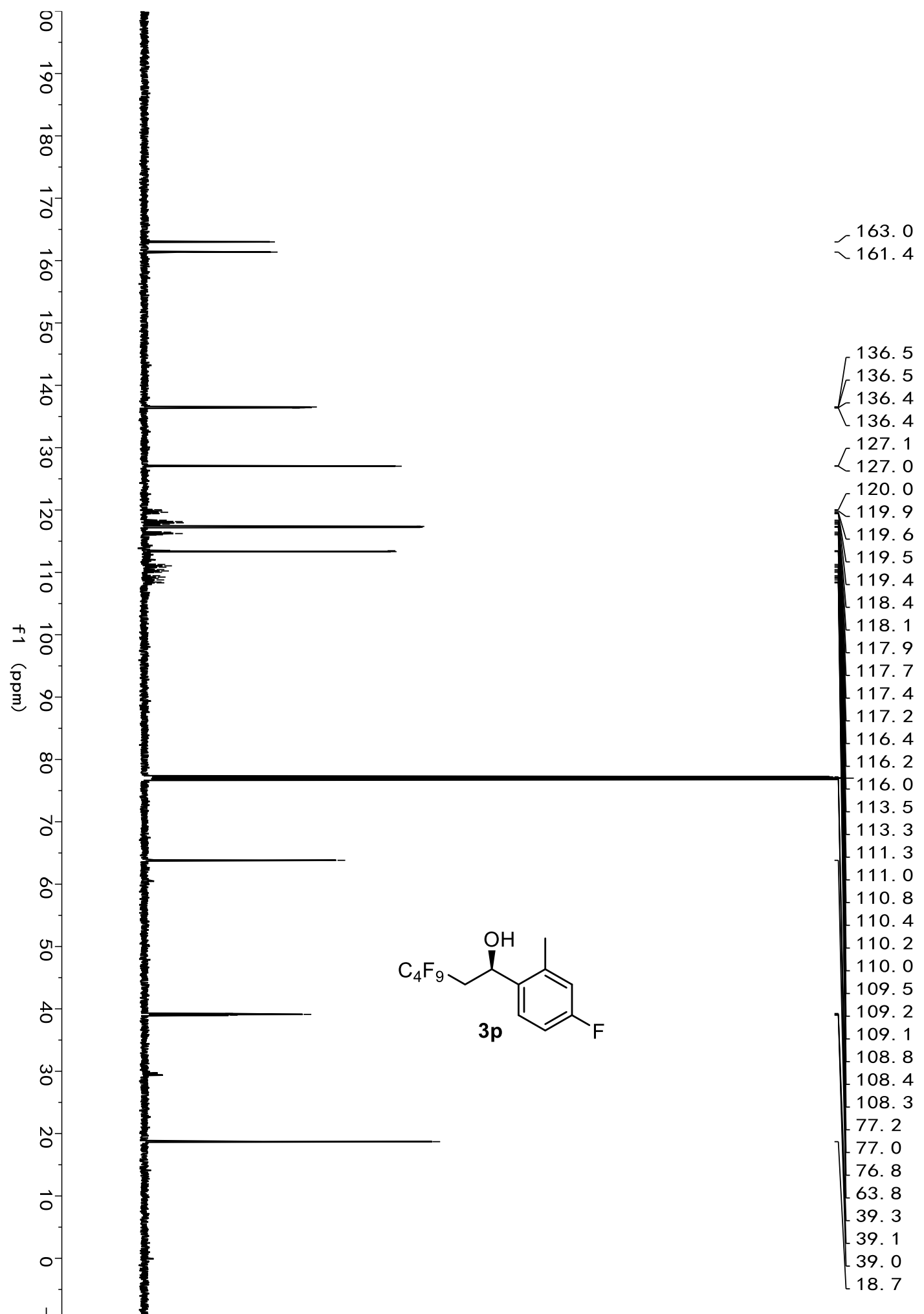

163 


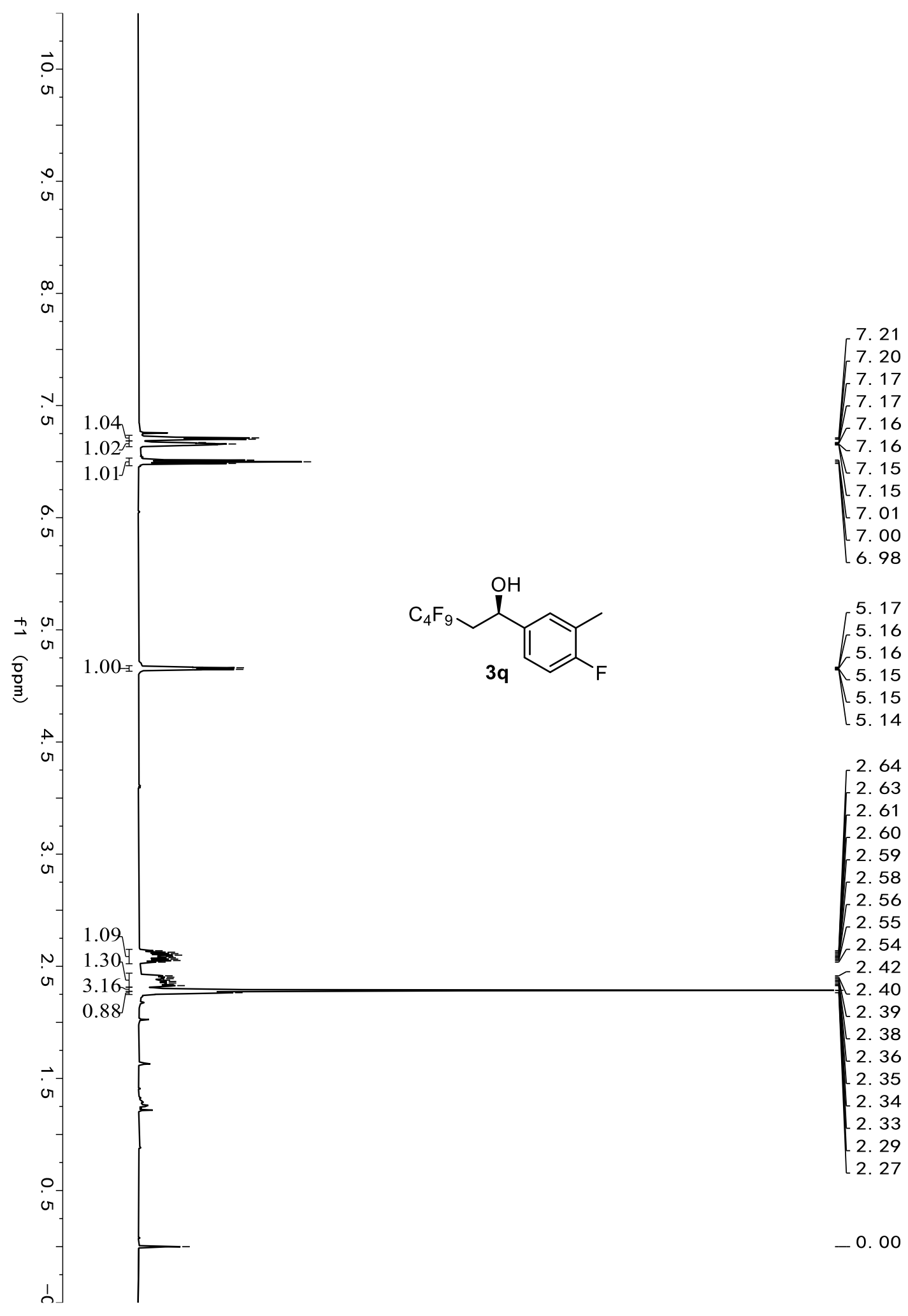




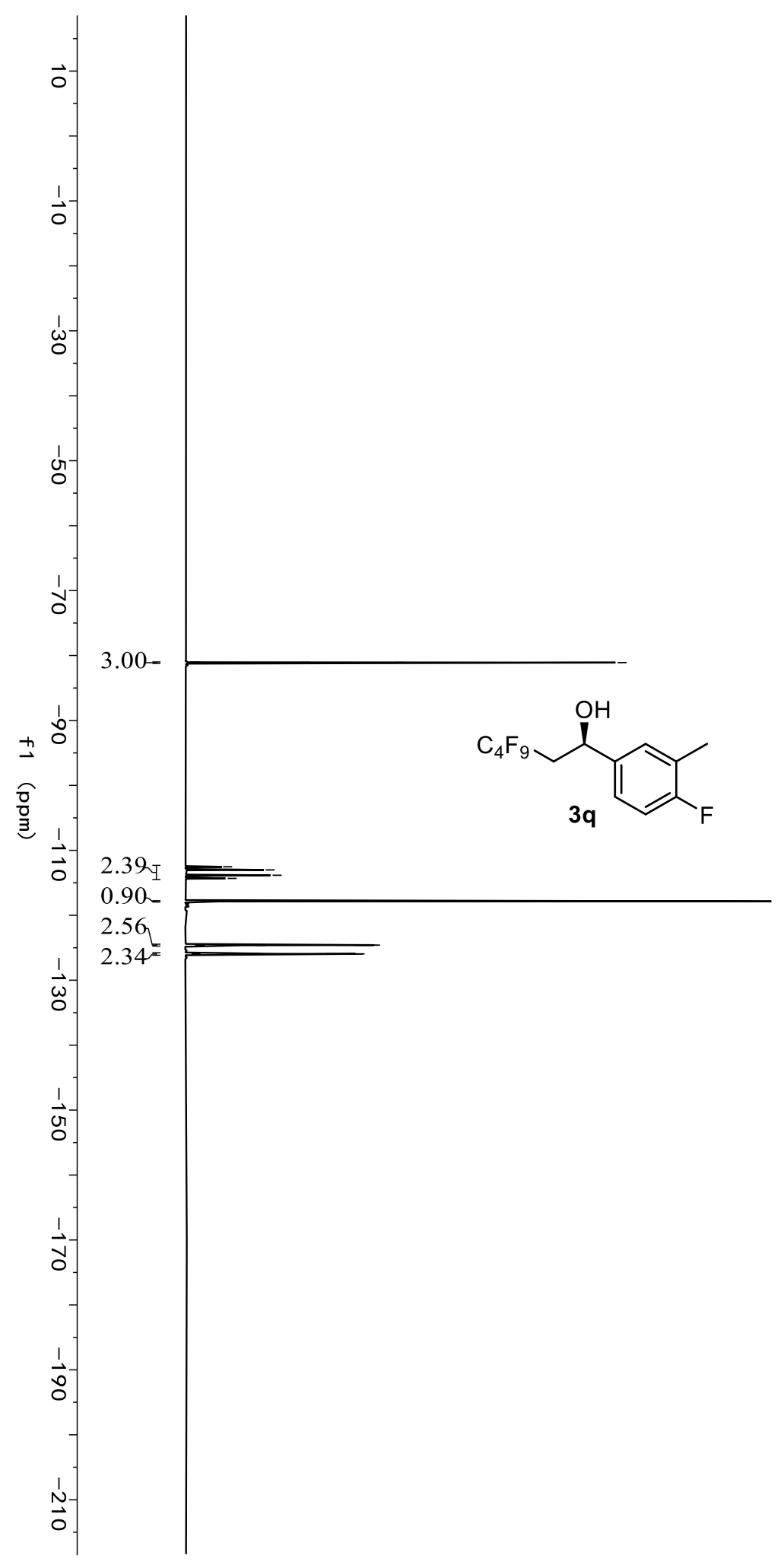

$\left[\begin{array}{l}-81.03 \\ -81.05 \\ -81.07 \\ -81.07 \\ -81.09 \\ -81.11 \\ -81.11 \\ -81.14\end{array}\right.$
$\left[\begin{array}{l}-112.53 \\ -112.56 \\ -112.99 \\ -113.02 \\ -113.04 \\ -113.82 \\ -113.84 \\ -113.86 \\ -114.30 \\ -114.32 \\ -114.34 \\ -117.87 \\ -124.56 \\ -124.57 \\ -124.58 \\ -124.59 \\ -124.59 \\ -124.60 \\ -124.60 \\ -124.61 \\ -124.62 \\ -125.92 \\ -125.93 \\ -125.94 \\ -125.95 \\ -125.96 \\ -125.97 \\ -125.98 \\ -125.99\end{array}\right.$ 


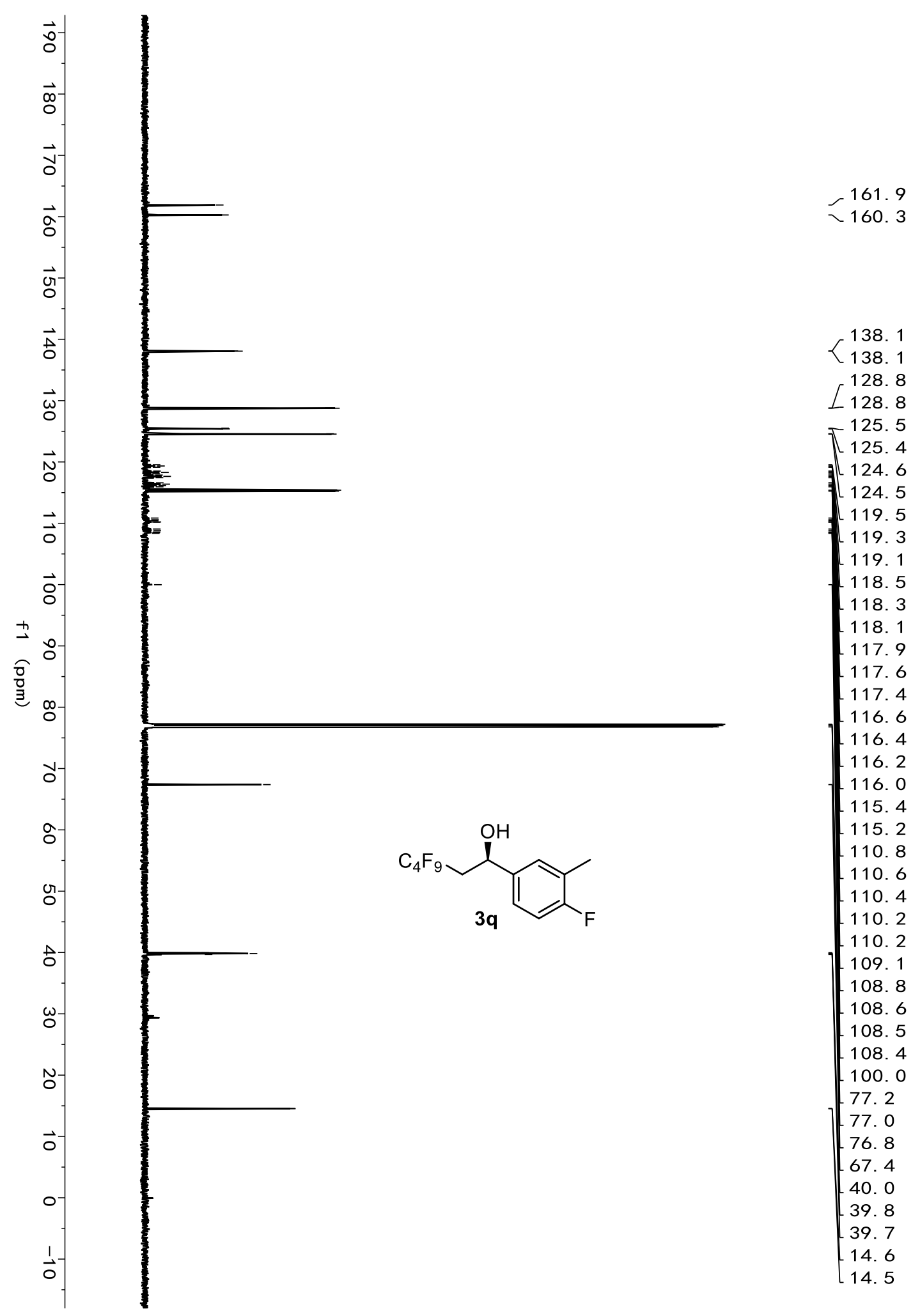




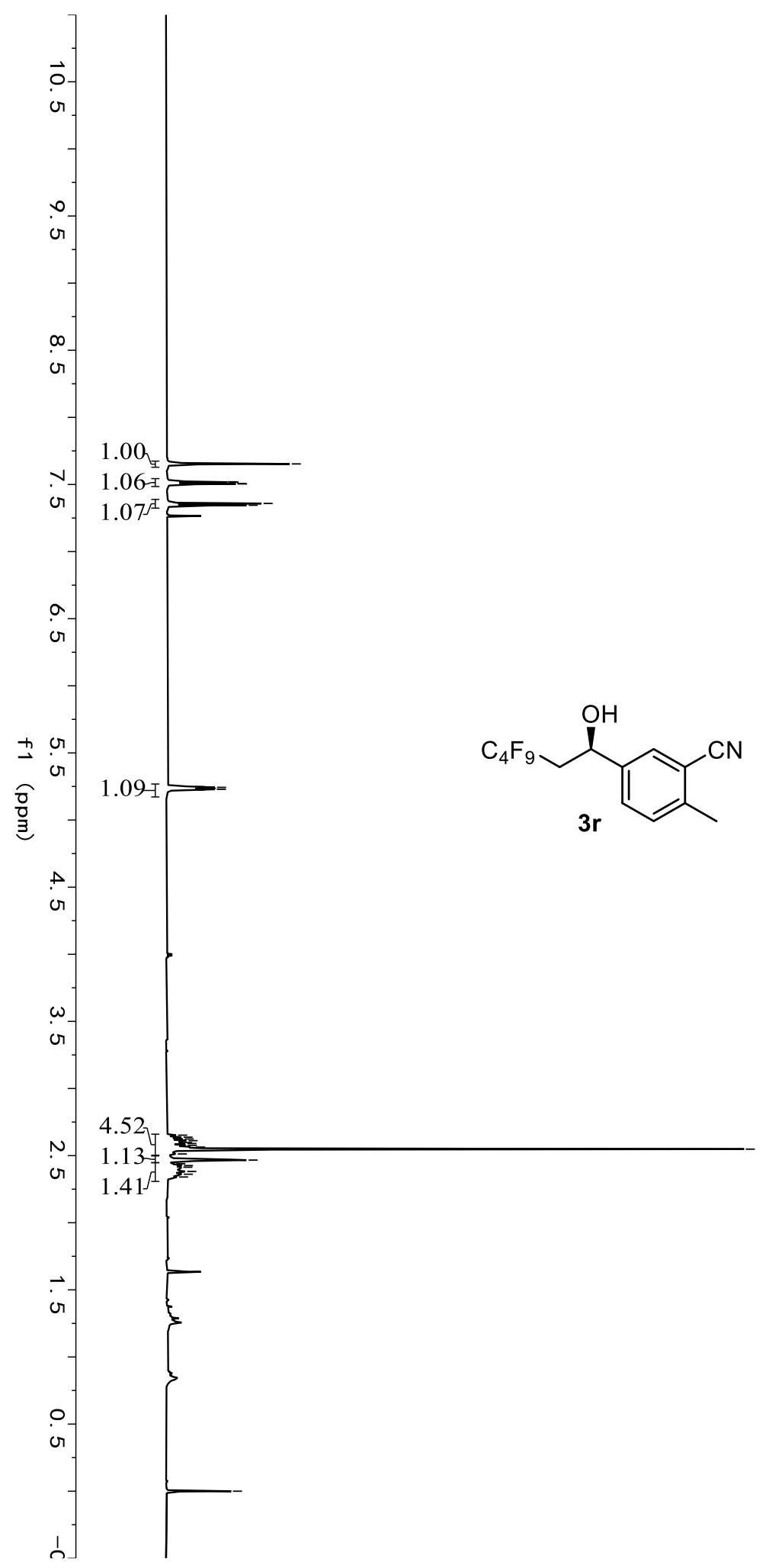

$\left\{\begin{array}{l}7.65 \\ 7.52 \\ 7.52 \\ 7.51 \\ 7.50 \\ 7.36 \\ 7.35\end{array}\right.$

5. 25

5. 24

$-5.24$

5. 23

5. 22

2. 65

2. 64

2. 62

2. 61

2. 60

2. 59

2. 58

2. 56

2. 55

2. 51

2. 47

2. 44

2. 43

2. 41

2. 38

2. 36

2. 34

$--0.00$ 


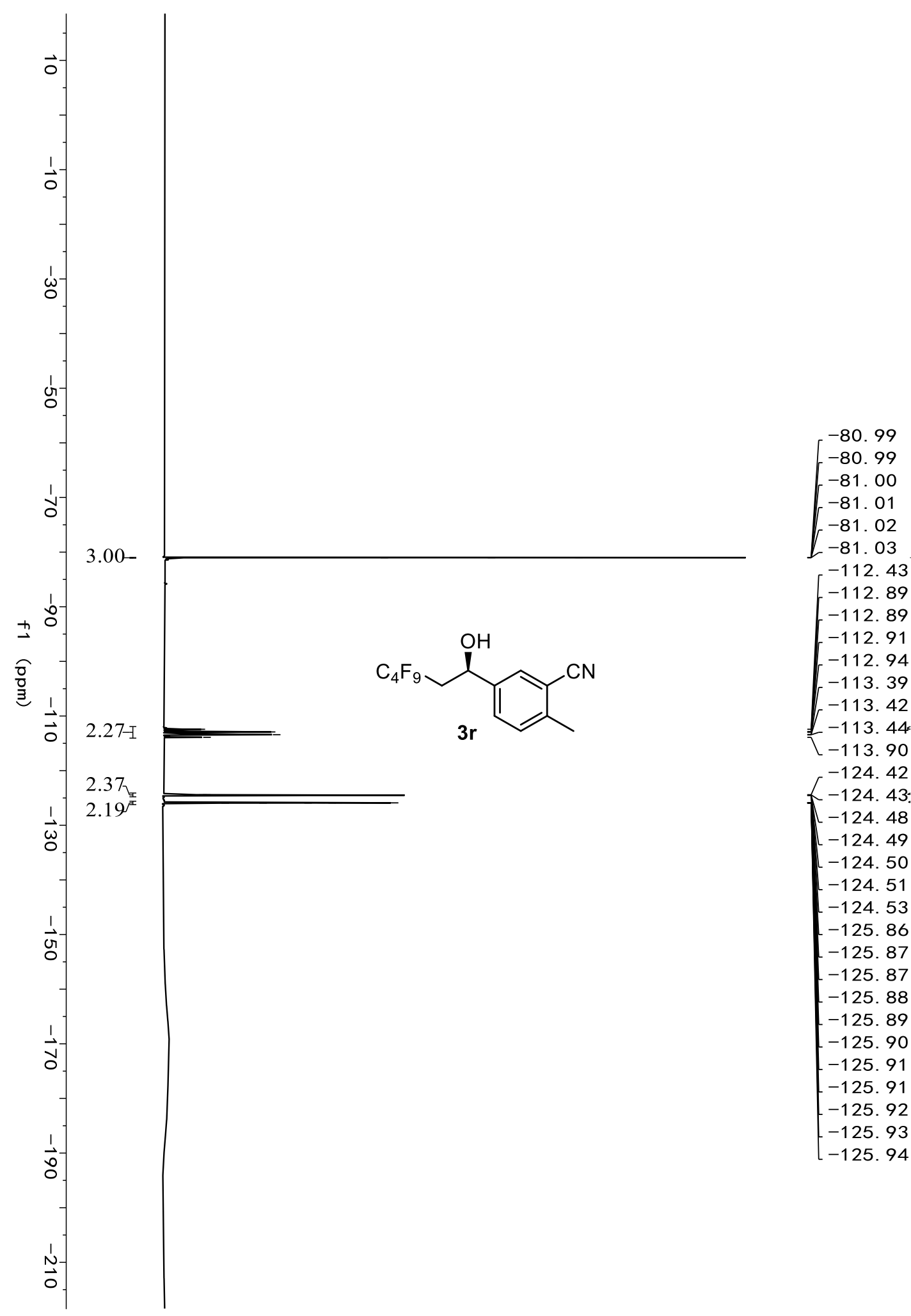



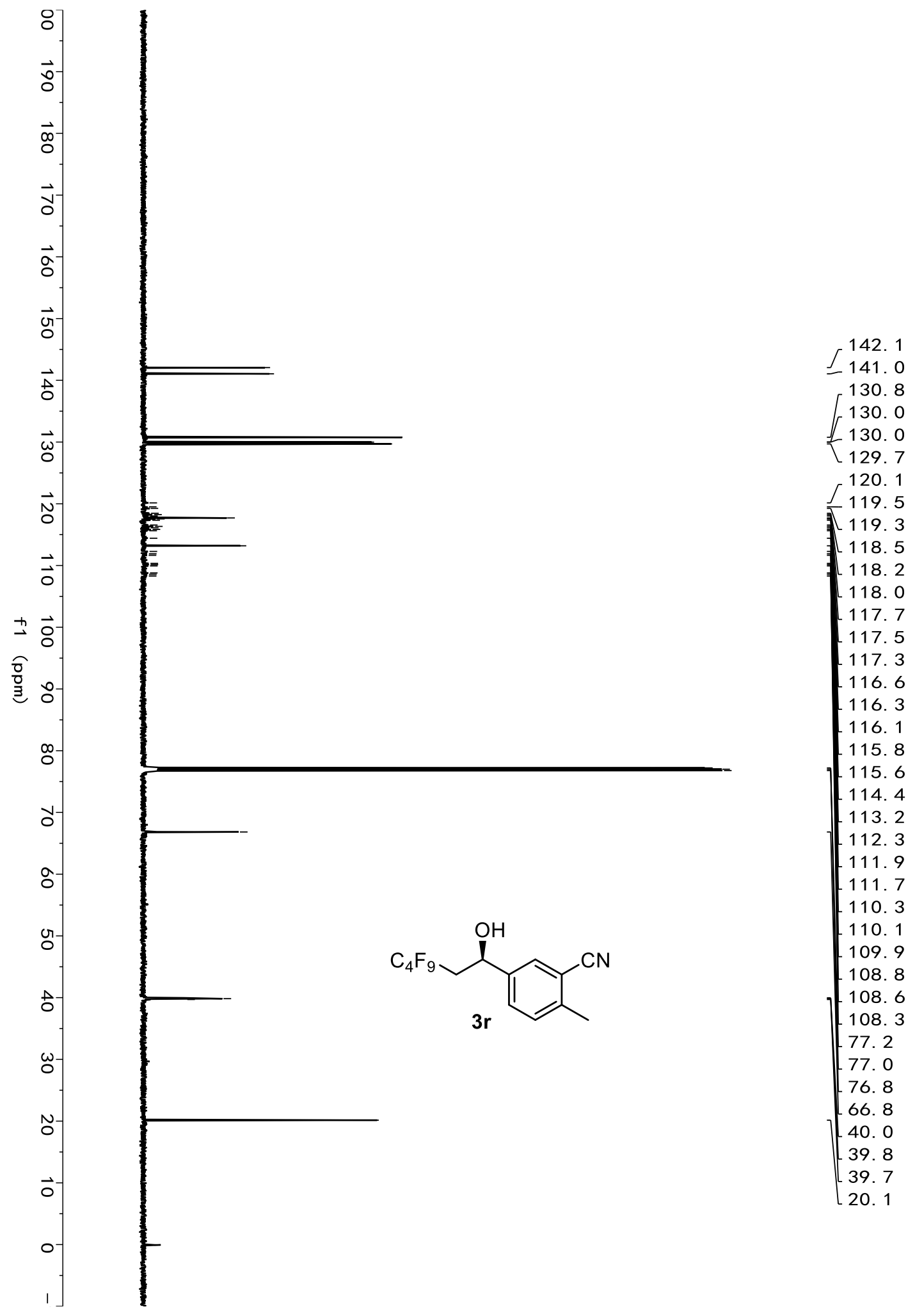

169 


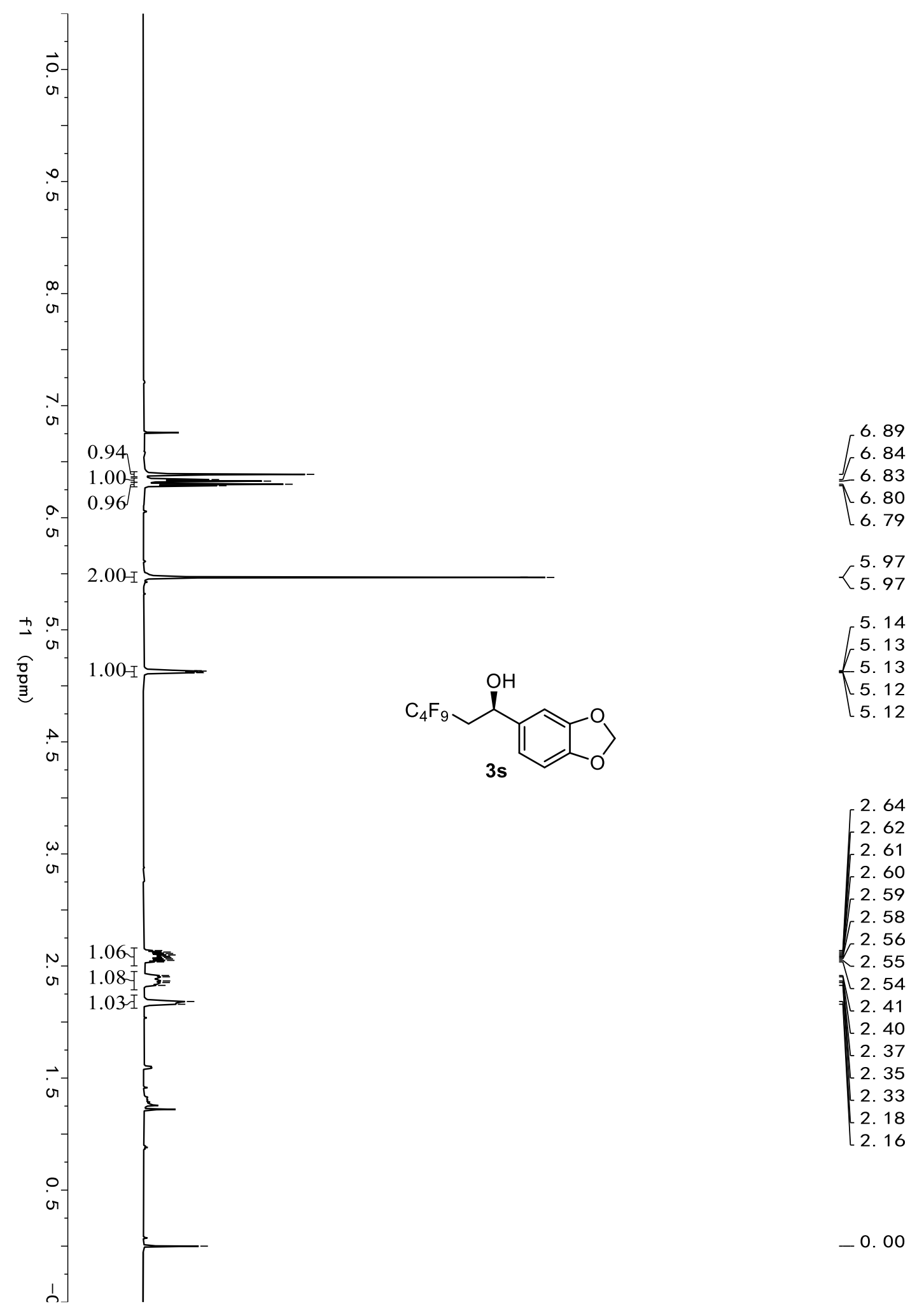




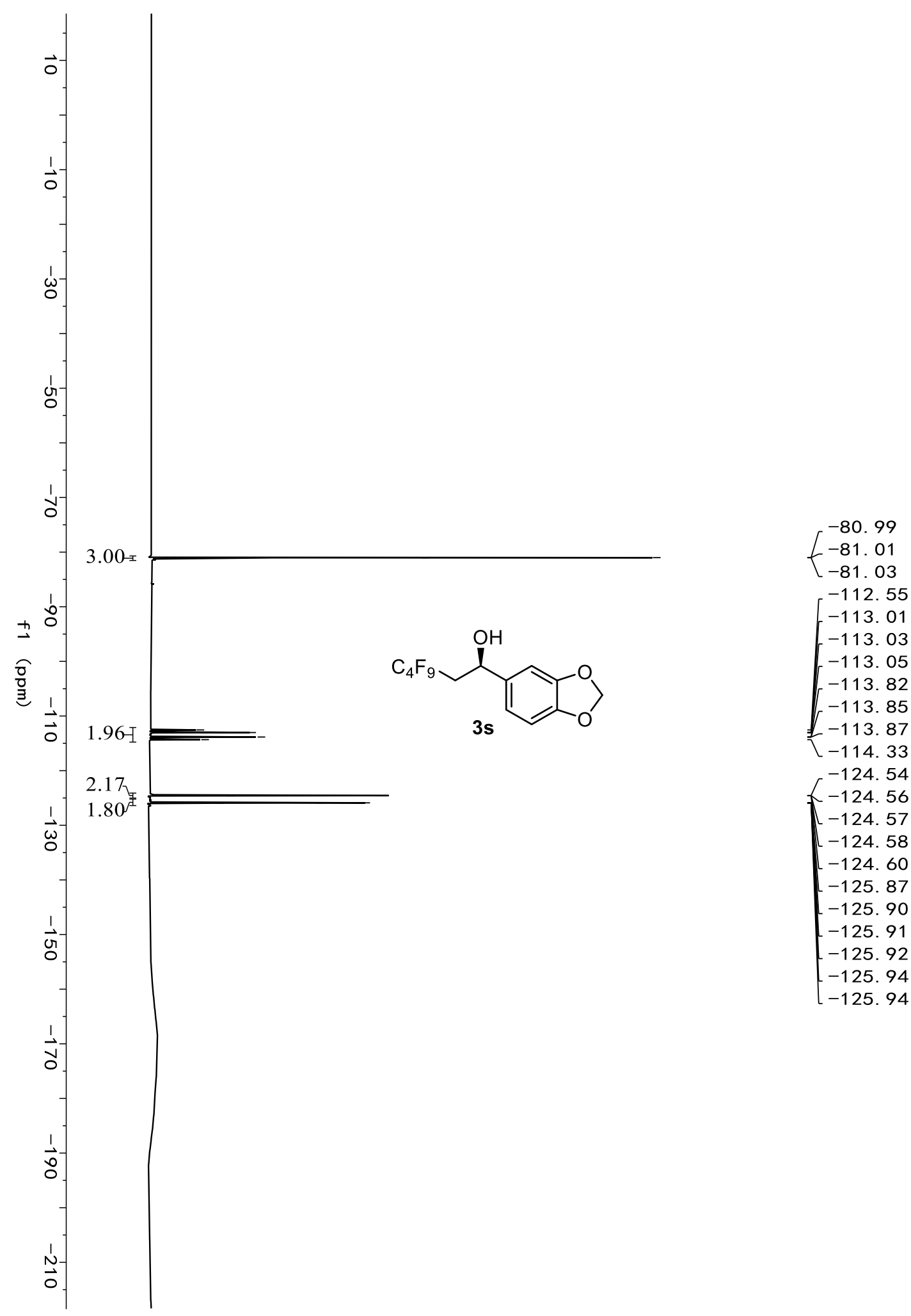




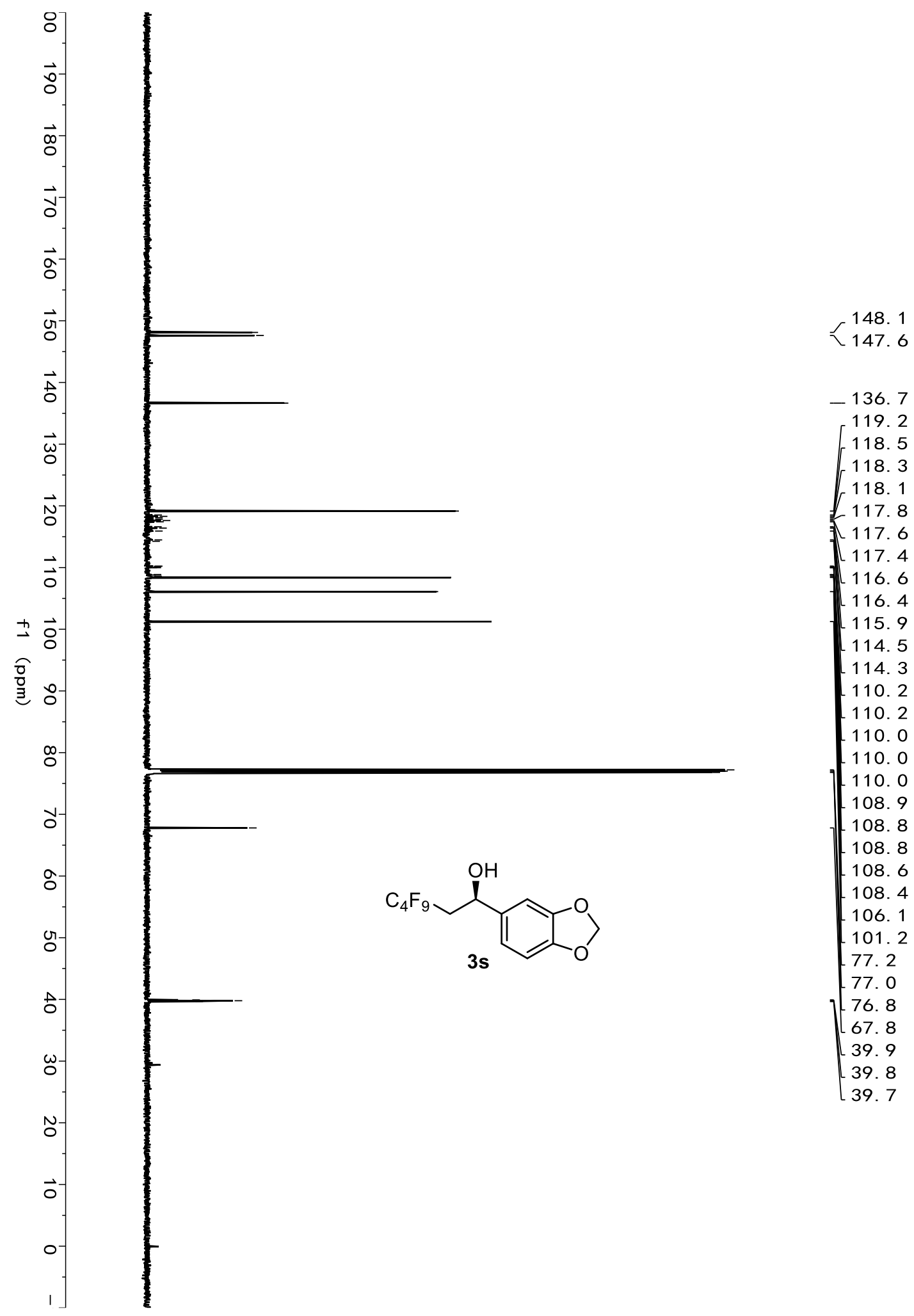




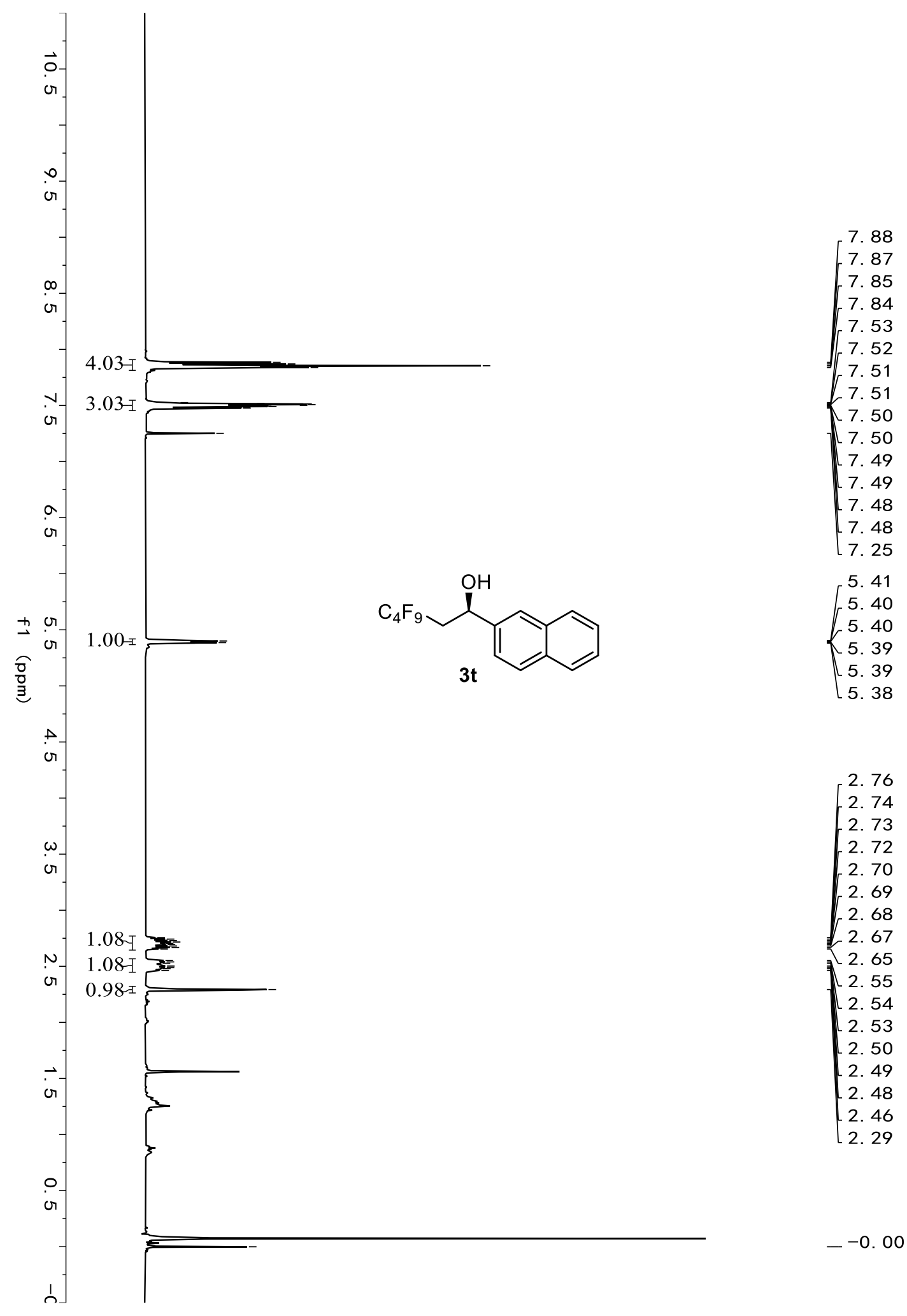




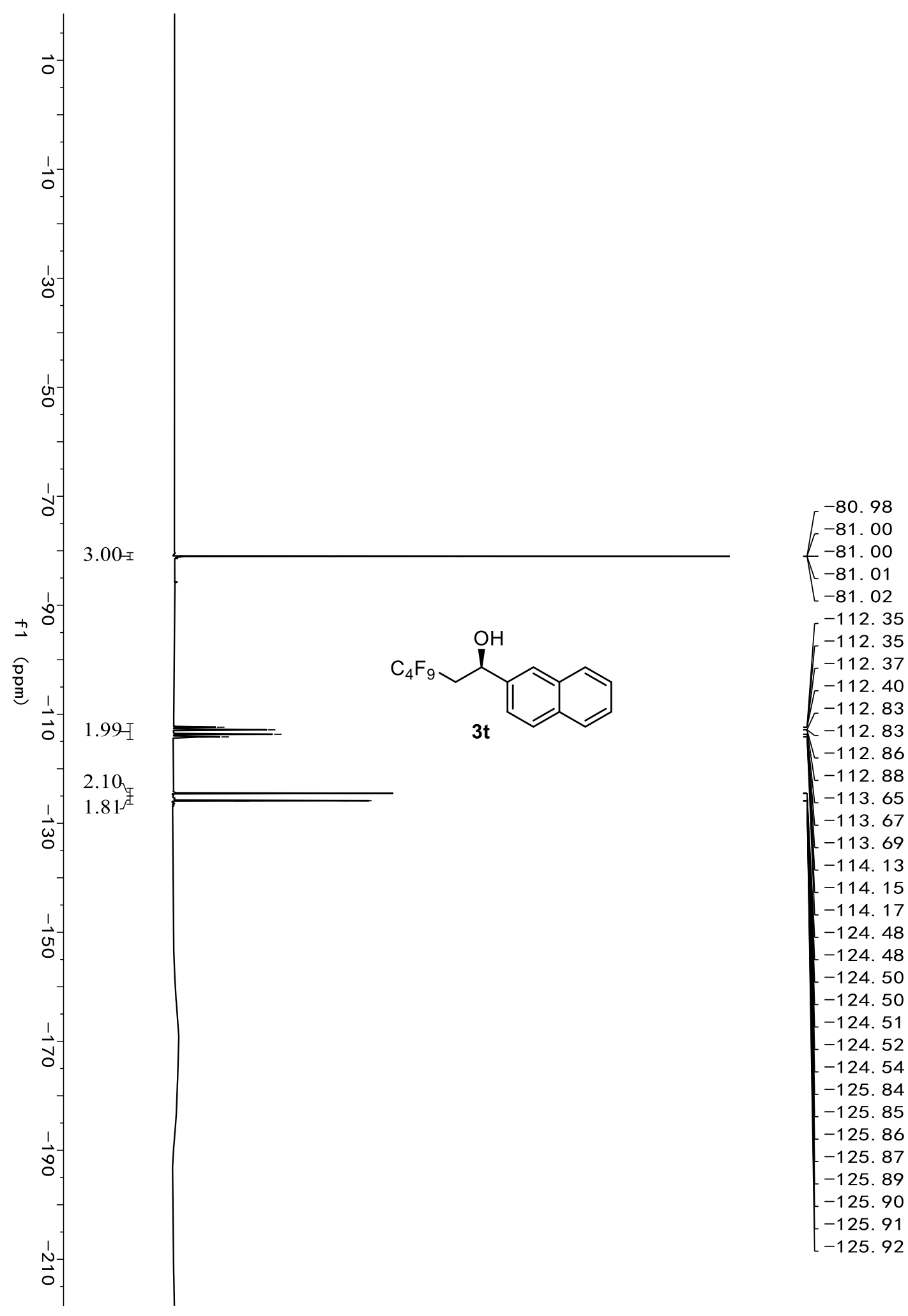




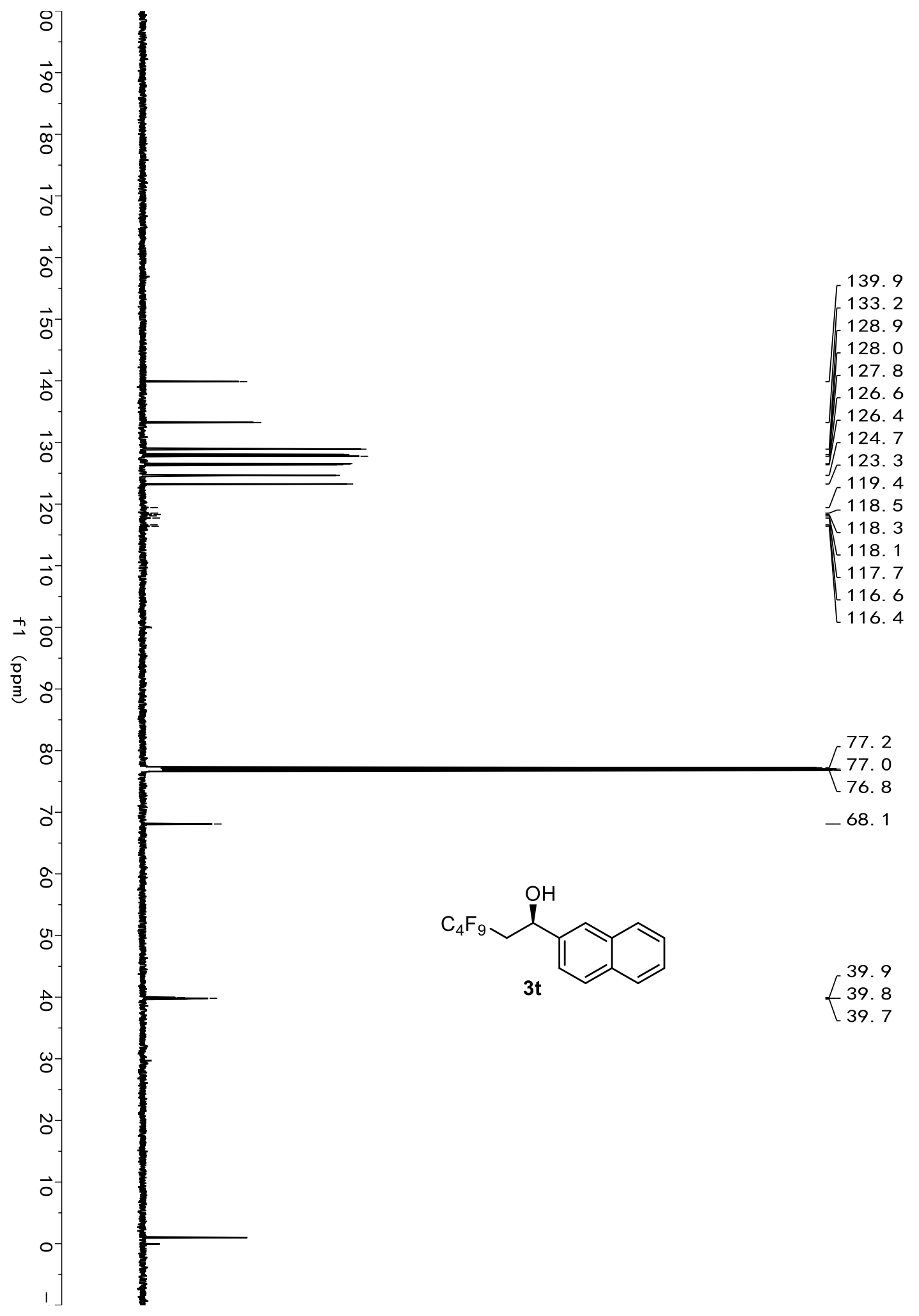

175 


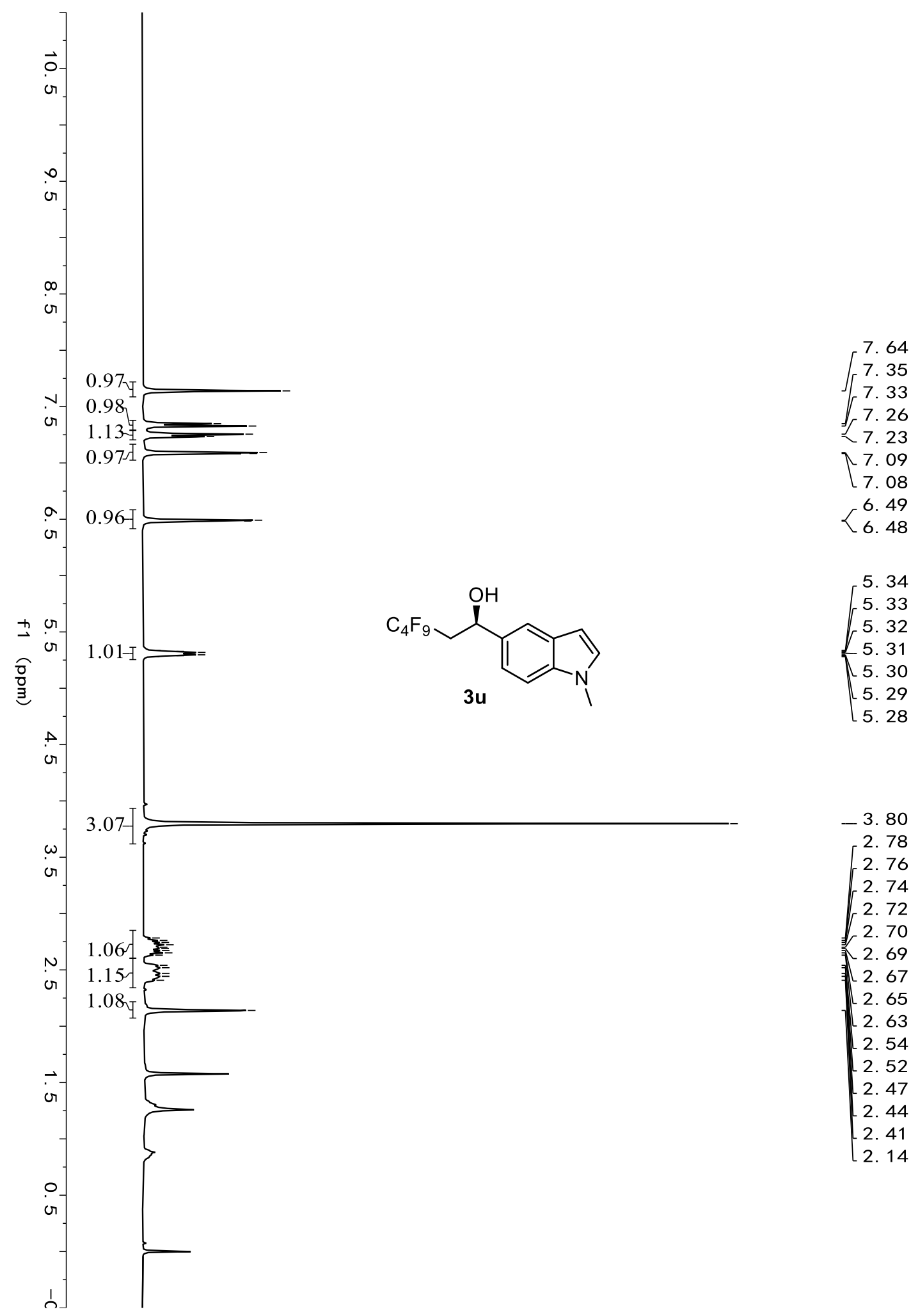




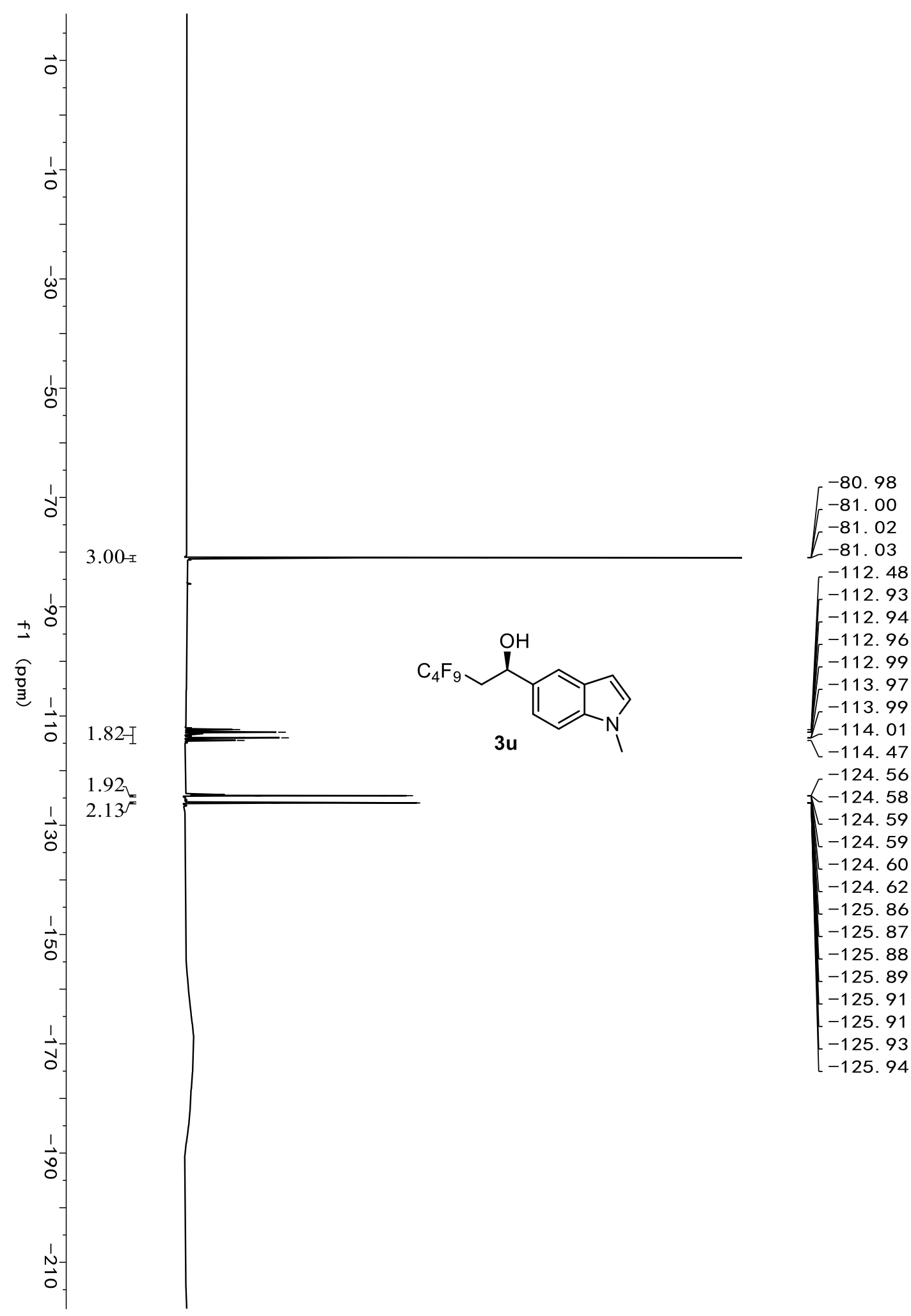




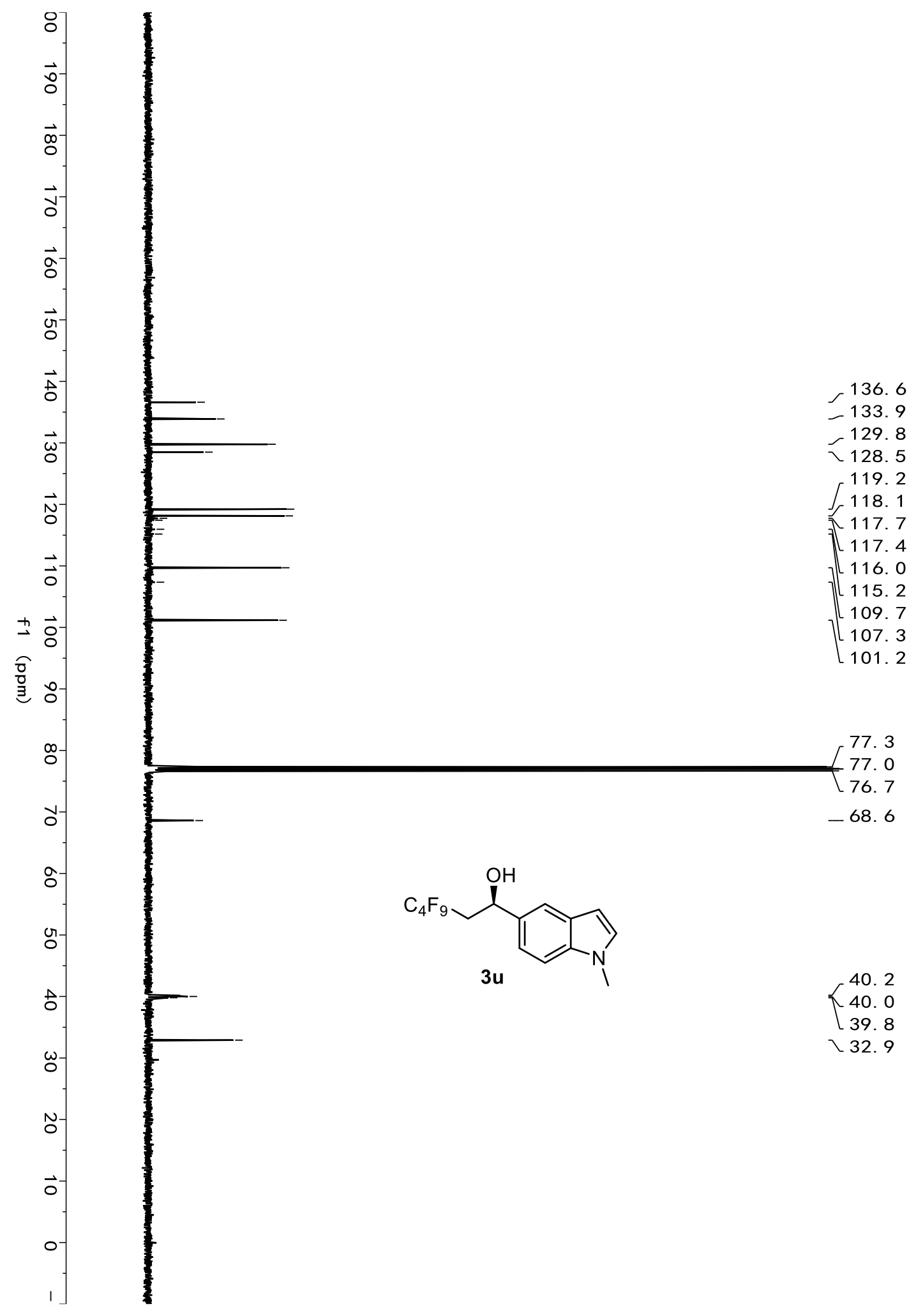




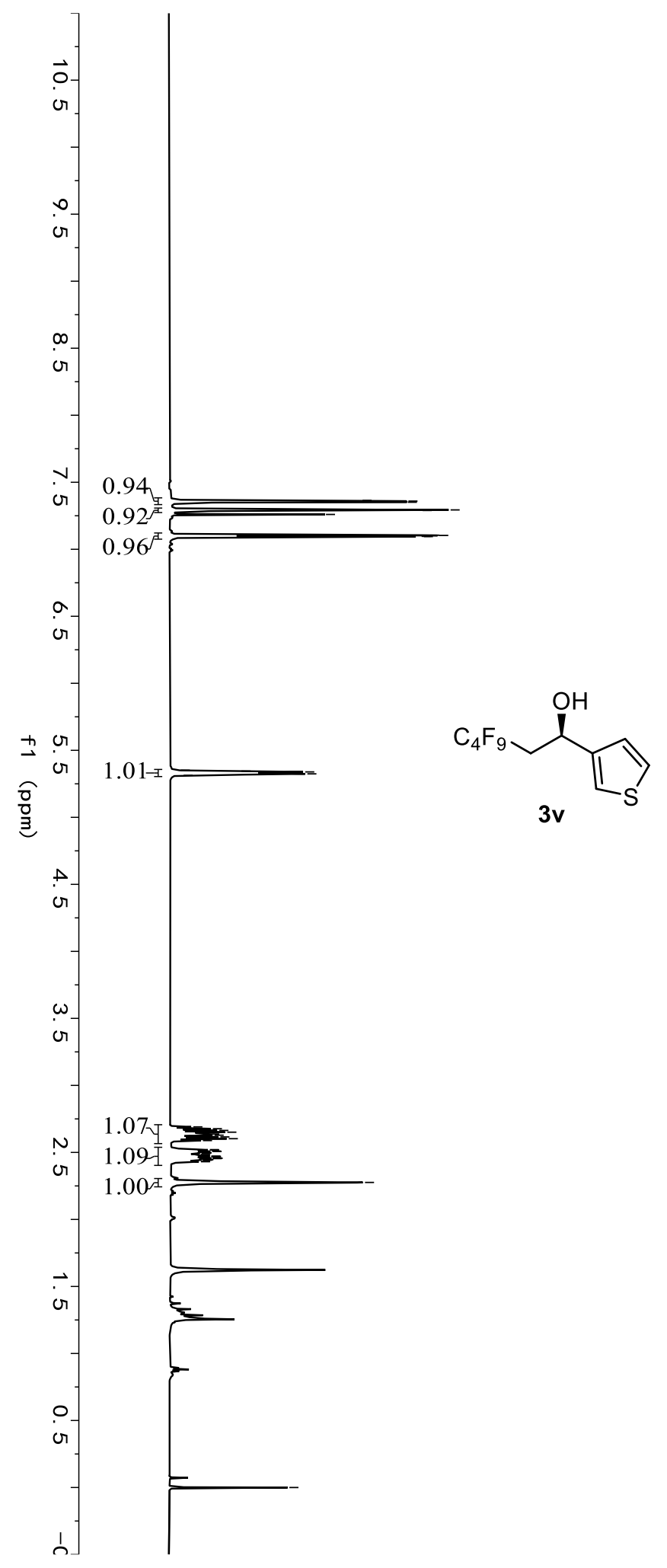

$\left[\begin{array}{l}7.36 \\ 7.36 \\ 7.36 \\ 7.35 \\ 7.29 \\ 7.29 \\ 7.26 \\ 7.10 \\ 7.10 \\ 7.10 \\ 7.09 \\ 5.35 \\ 5.34 \\ 5.34 \\ 5.33 \\ 5.32 \\ 5.32 \\ 5.31 \\ 2.69 \\ 2.68 \\ 2.66 \\ 2.65 \\ 2.64 \\ 2.63 \\ 2.62 \\ 2.60 \\ 2.59 \\ 2.52 \\ 2.52 \\ 2.52 \\ 2.51 \\ 2.51 \\ 2.51 \\ 2.50 \\ 2.50 \\ 2.49 \\ 2.49 \\ 2.48 \\ 2.48 \\ 2.48 \\ 2.47 \\ 2.47 \\ 2.47 \\ 2.46 \\ 2.46 \\ 2.45 \\ 2.45 \\ 2.44 \\ 2.44 \\ -00\end{array}\right.$ 


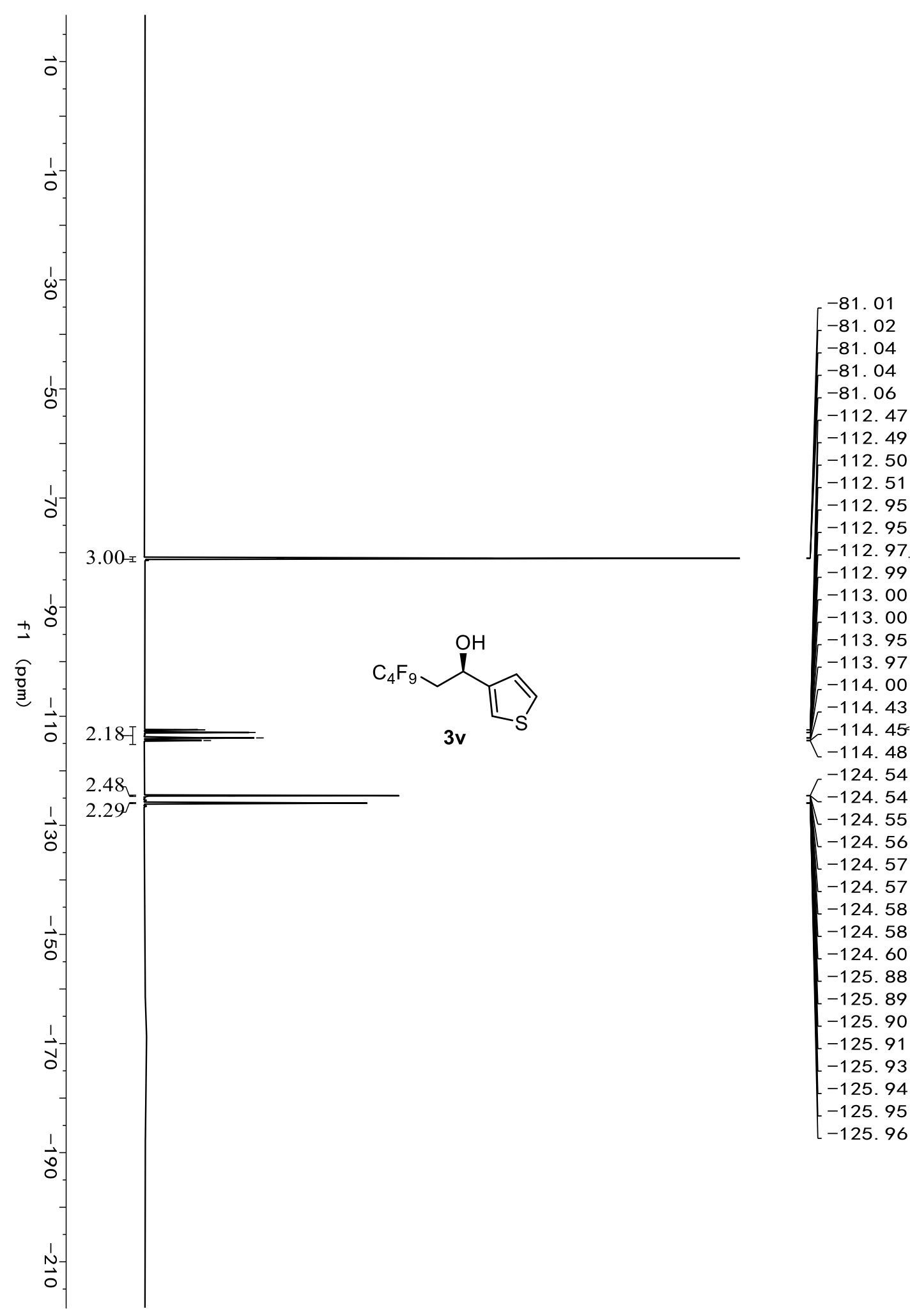




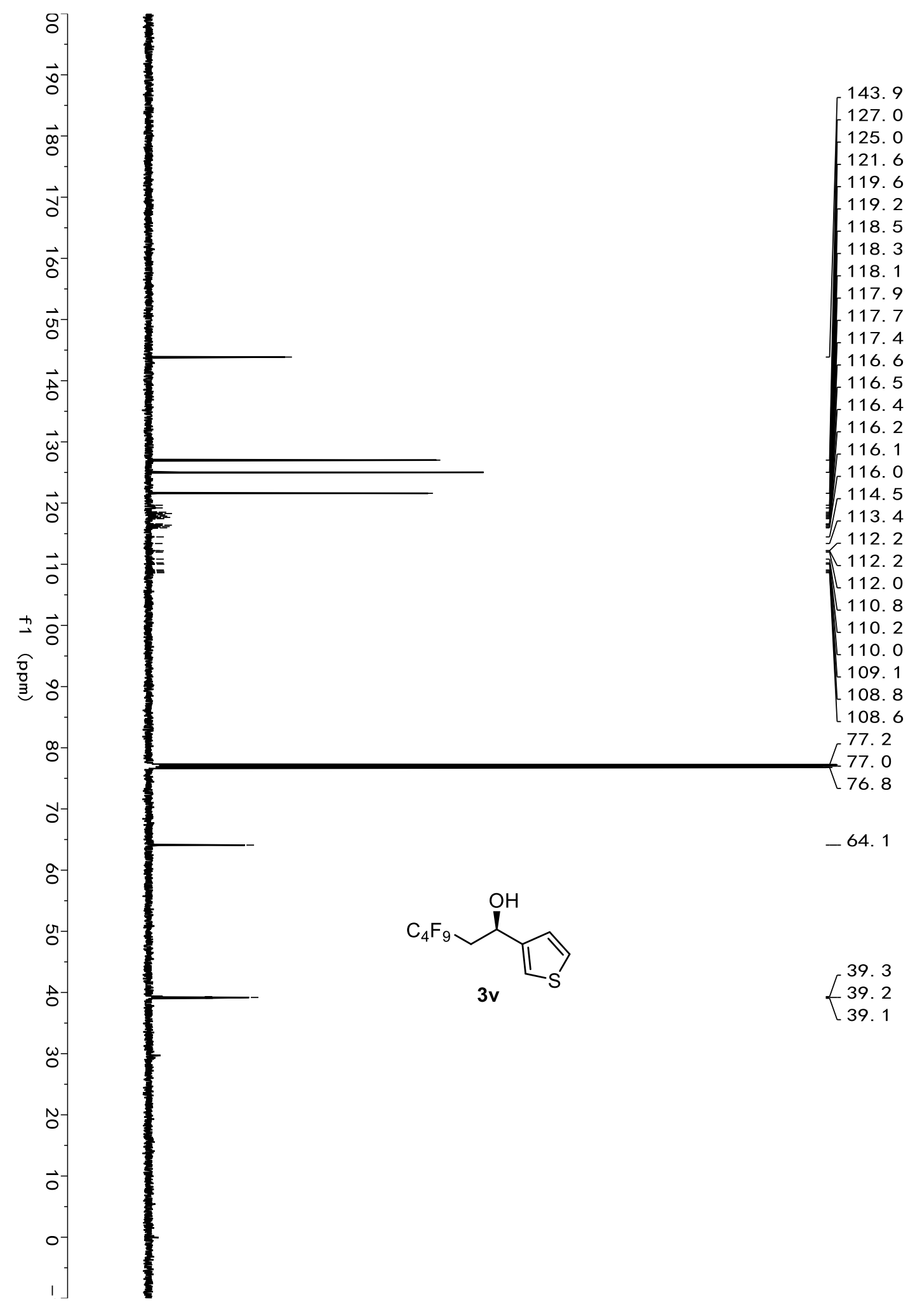




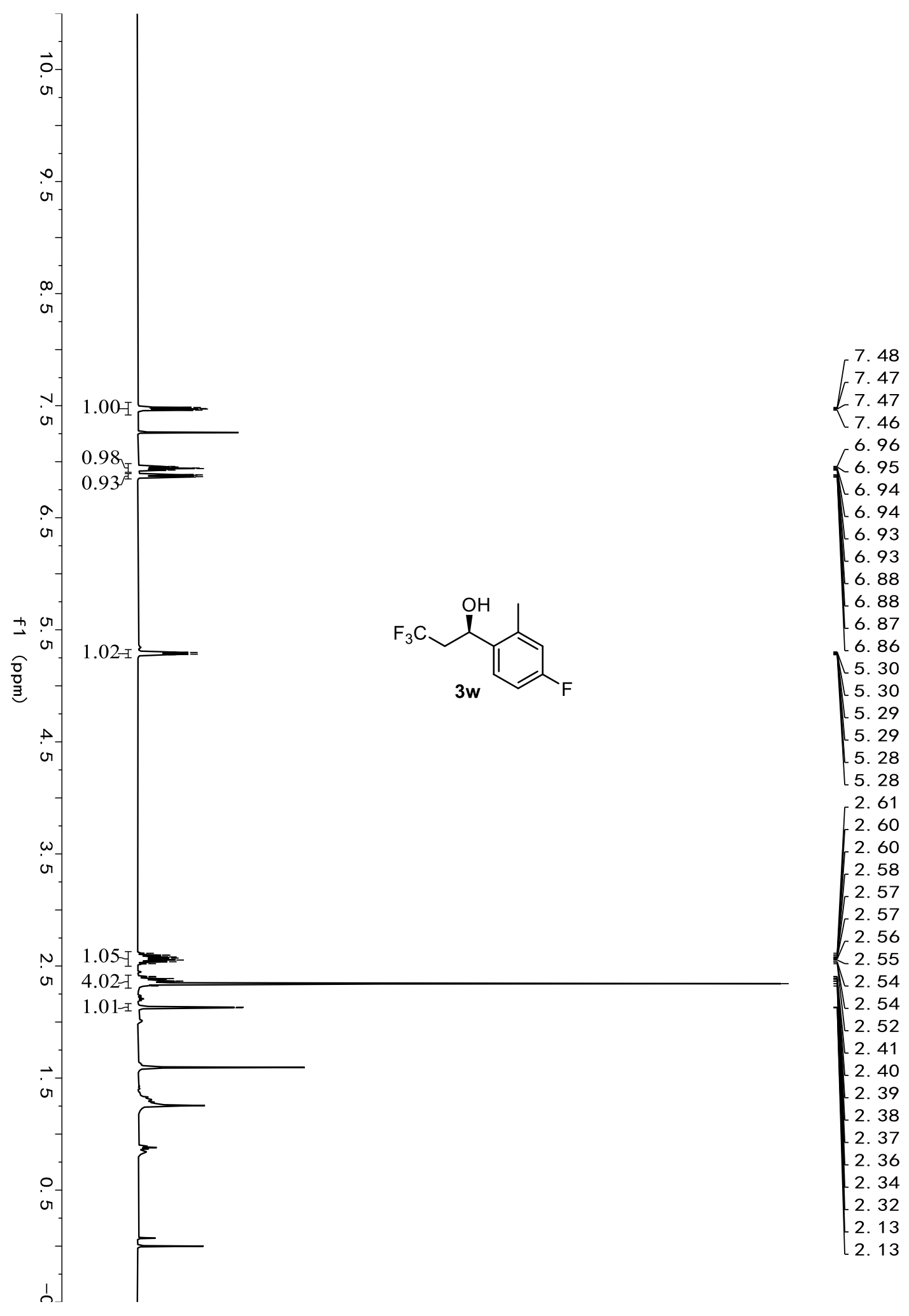




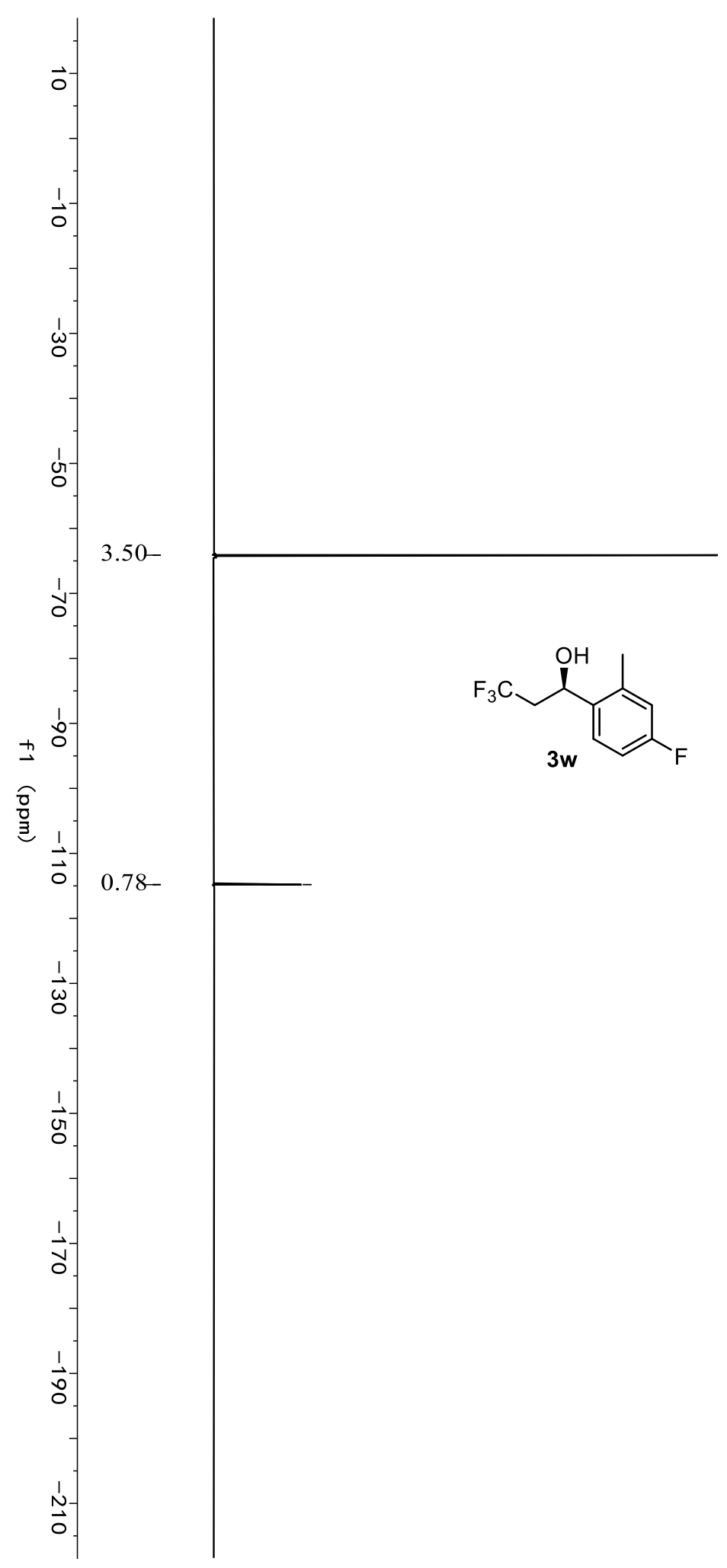

$--64.12$

$--114.81$ 


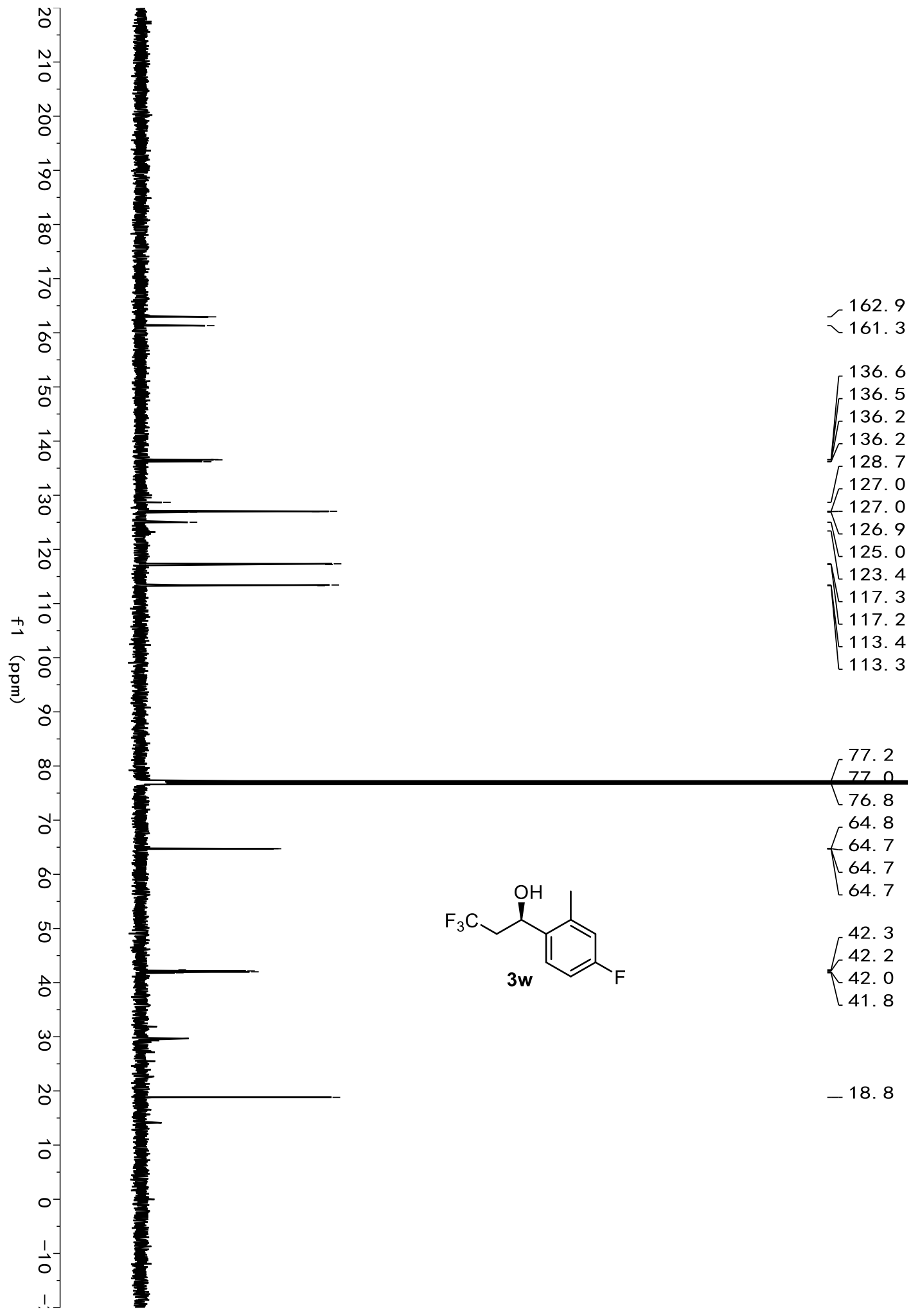

184 


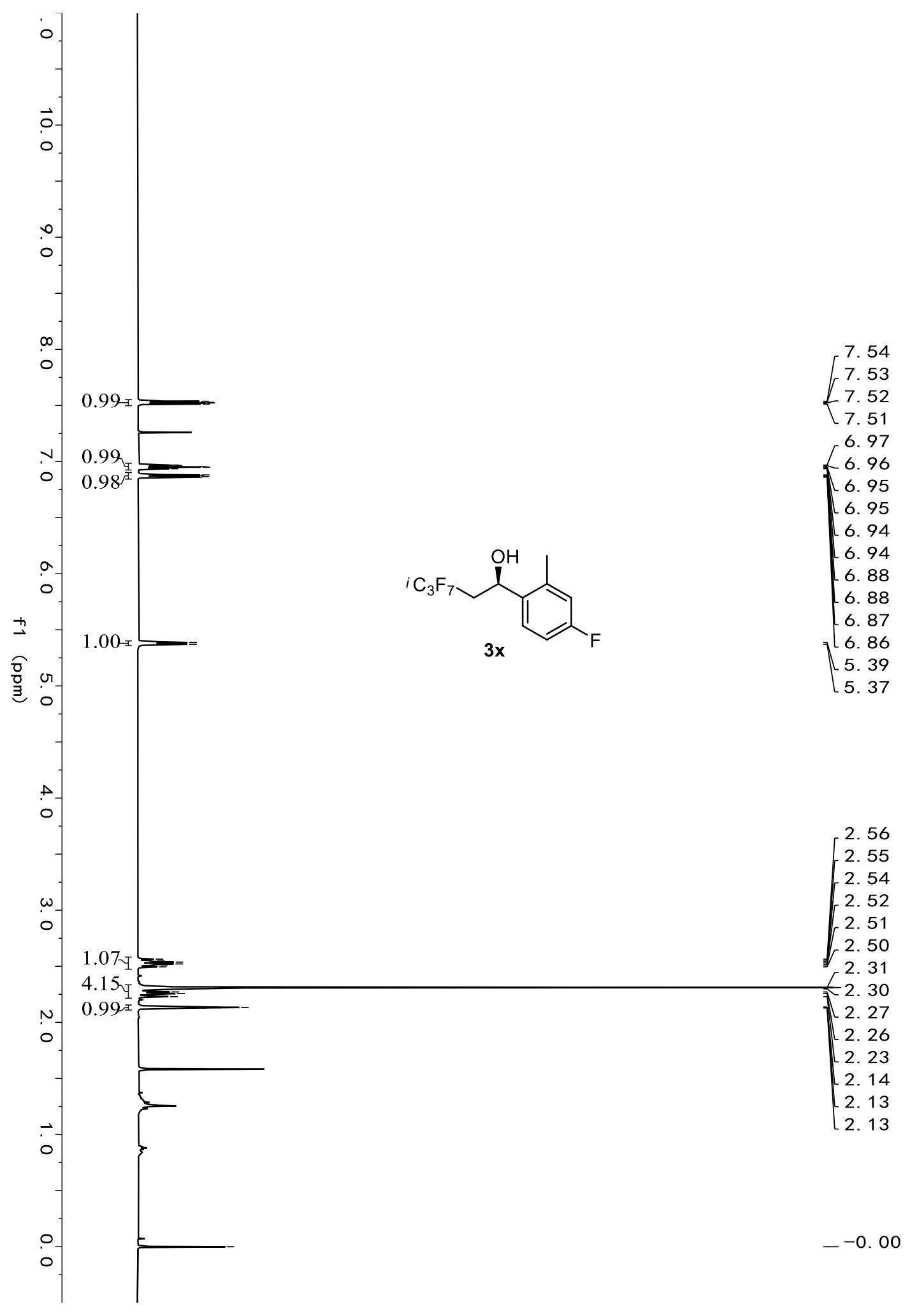




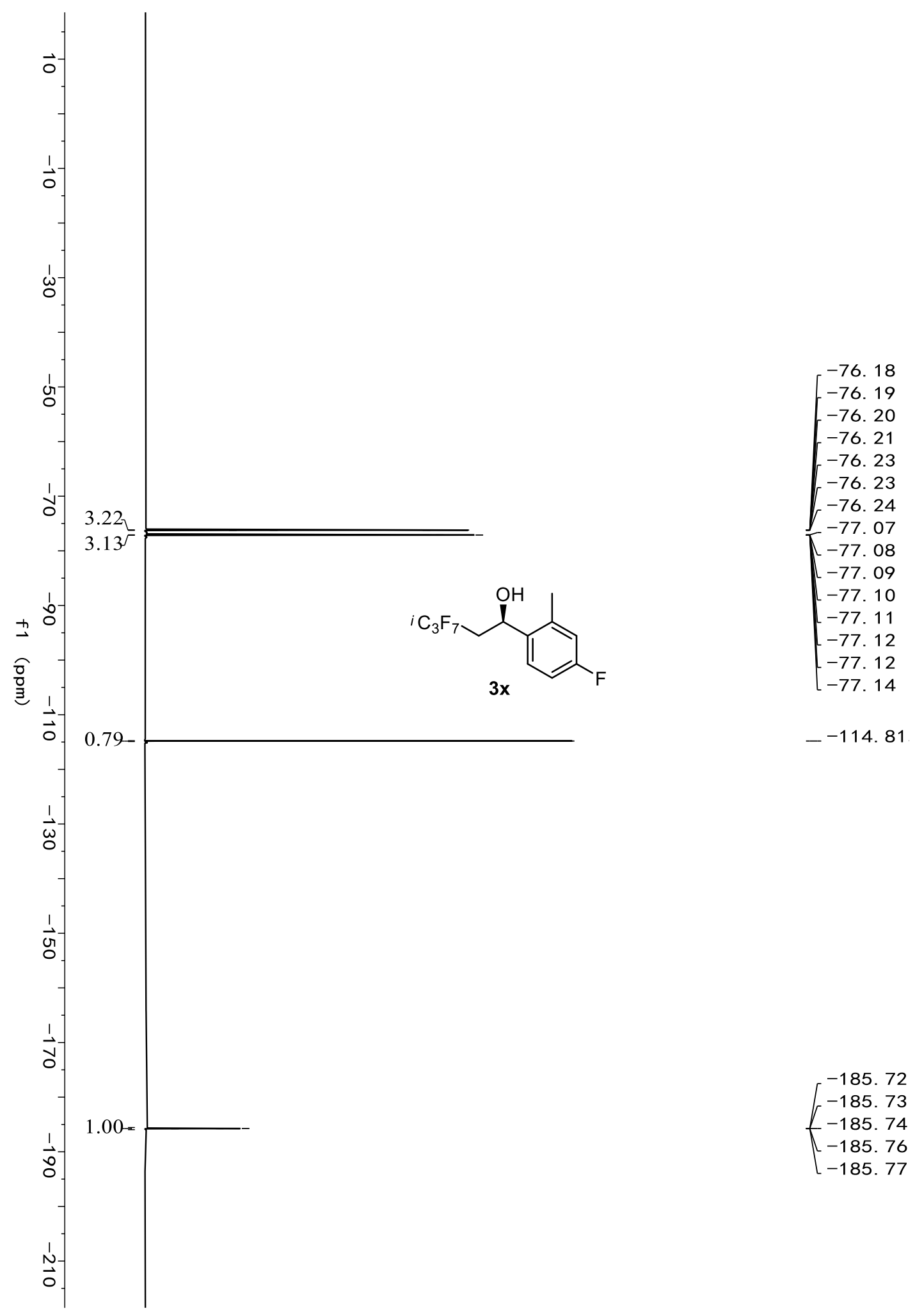




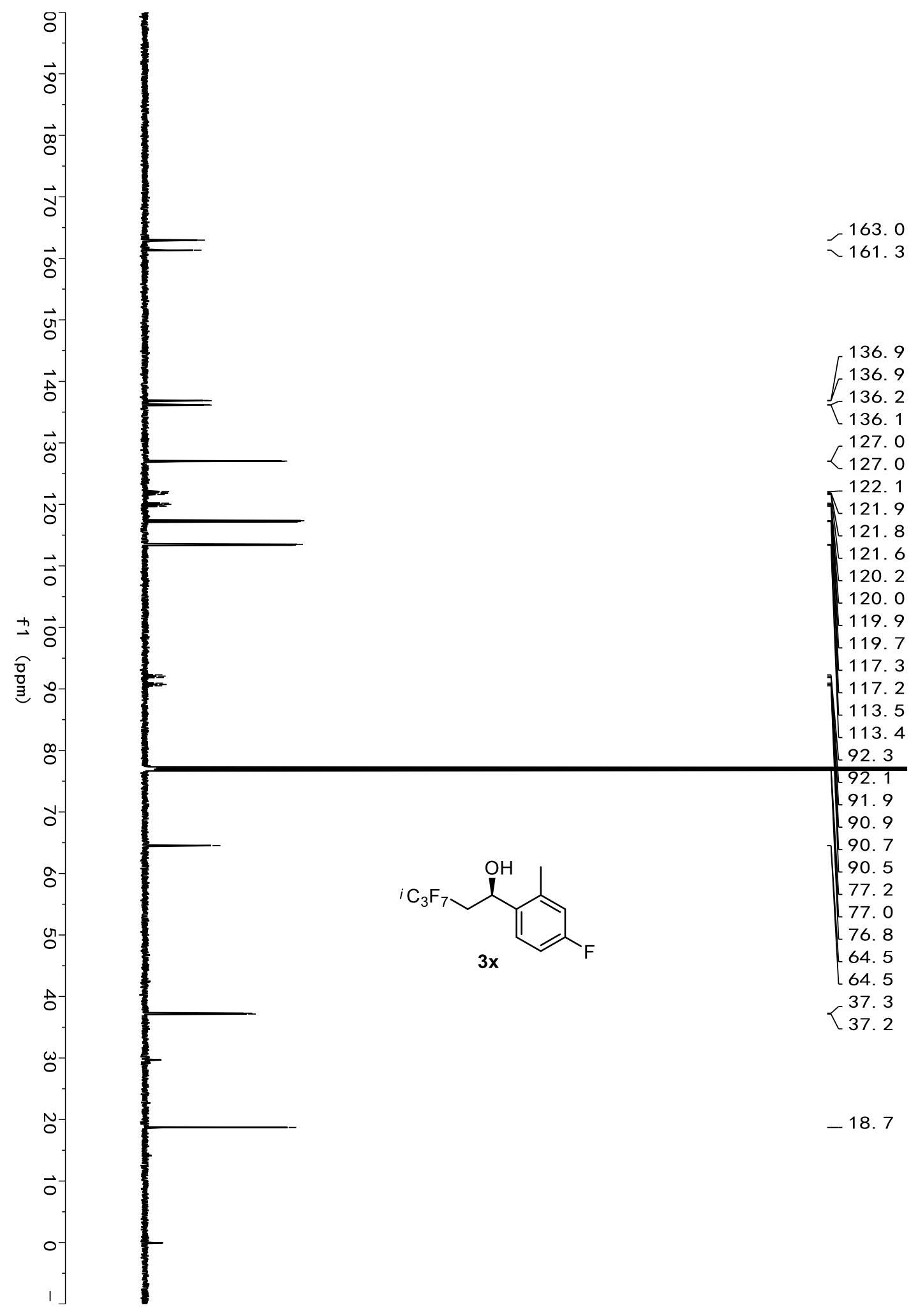




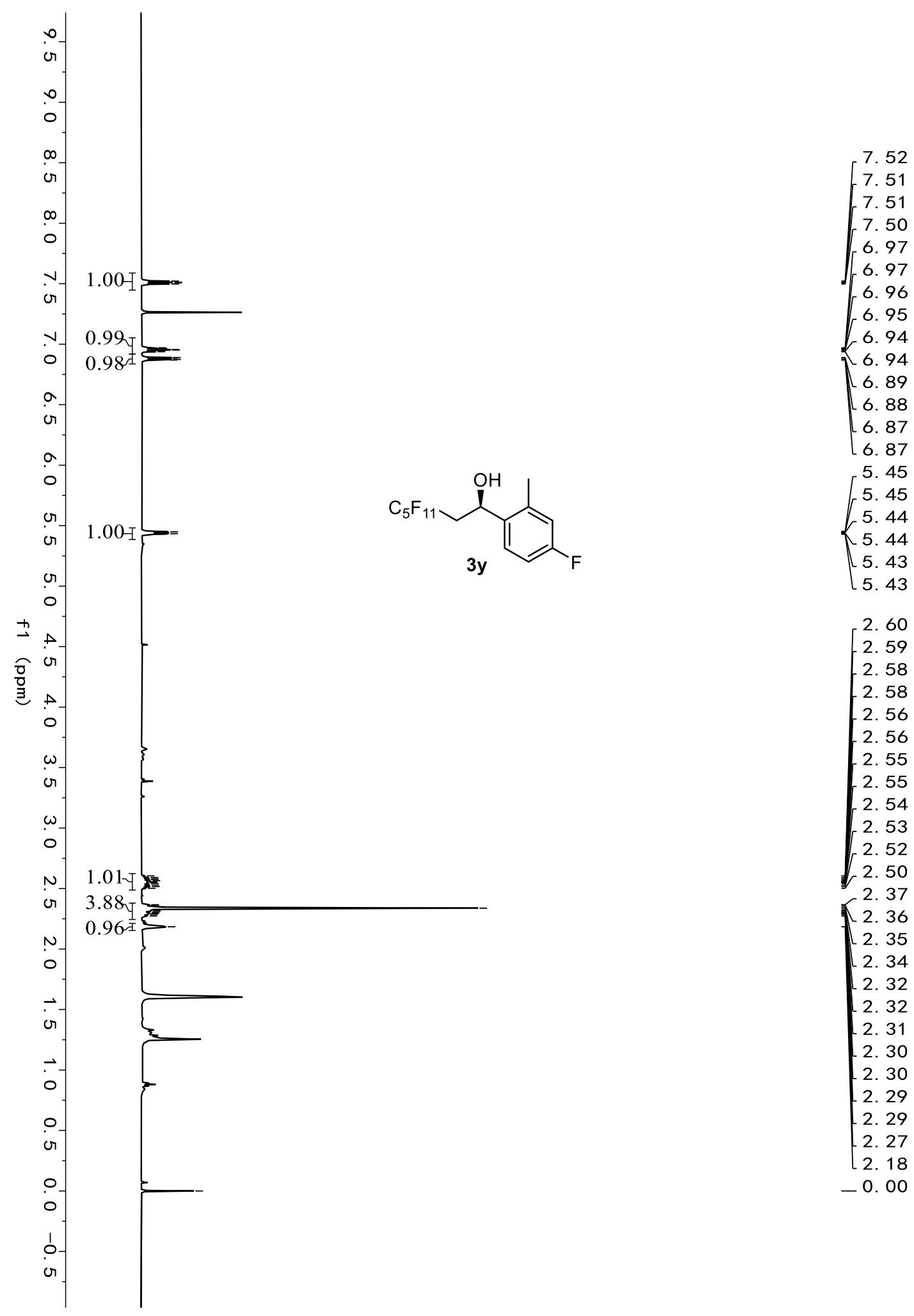




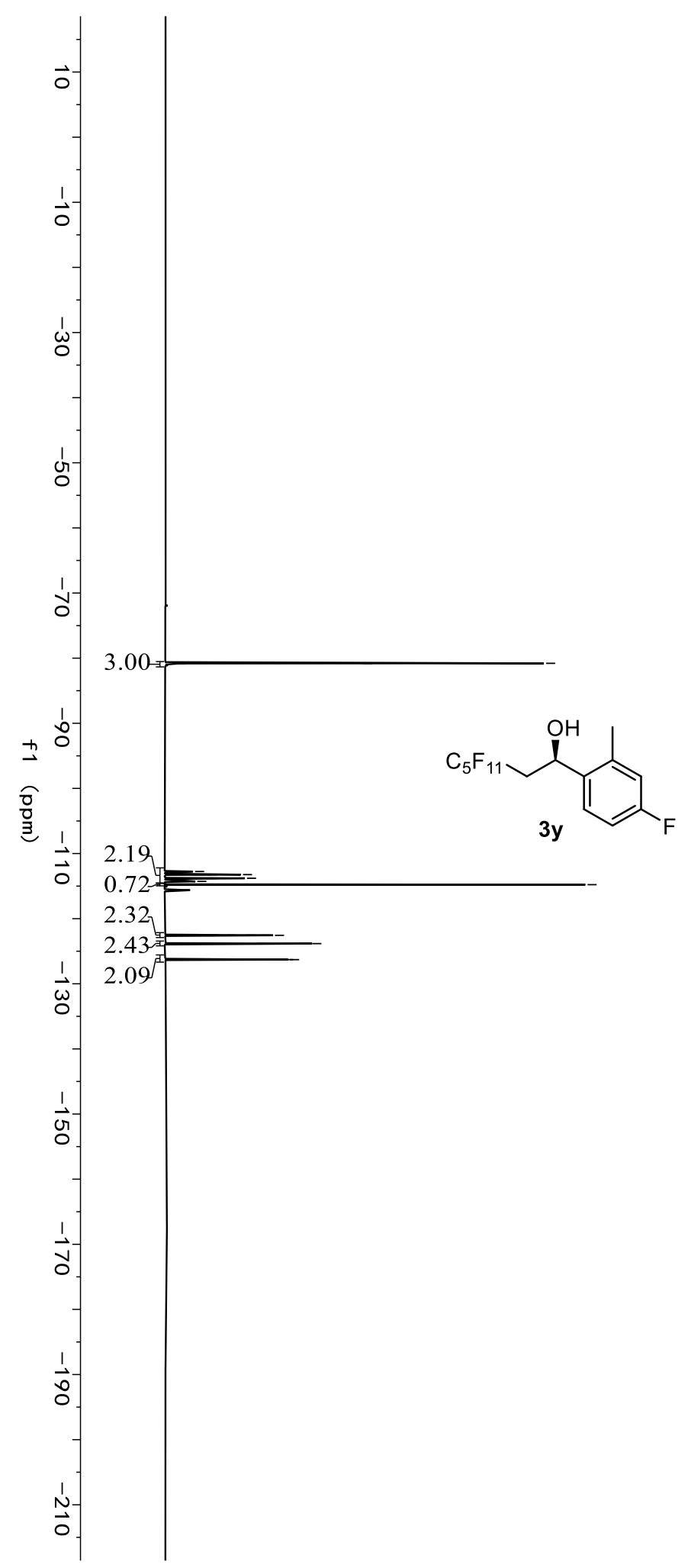

$\left\{\begin{array}{l}-80.75 \\ -80.77 \\ -80.79 \\ -80.81 \\ -80.82\end{array}\right.$

$-113.21$

$-113.21$

$-113.24$

$-113.26$

$-113.26$

$-113.77$

$-113.80$

$-113.82$

$-114.28$

$-114.78$

$-122.52$

$-122.53$

$-122.54$

$-122.54$

$-122.56$

$-122.57$

$-122.59$

$-122.60$

$-123.81$

$-123.83$

$-123.86$

$-126.26$

$-126.27$

$-126.28$

$-126.29$

$-126.29$

$-126.30$

$-126.31$

$-126.32$

-126. 33 


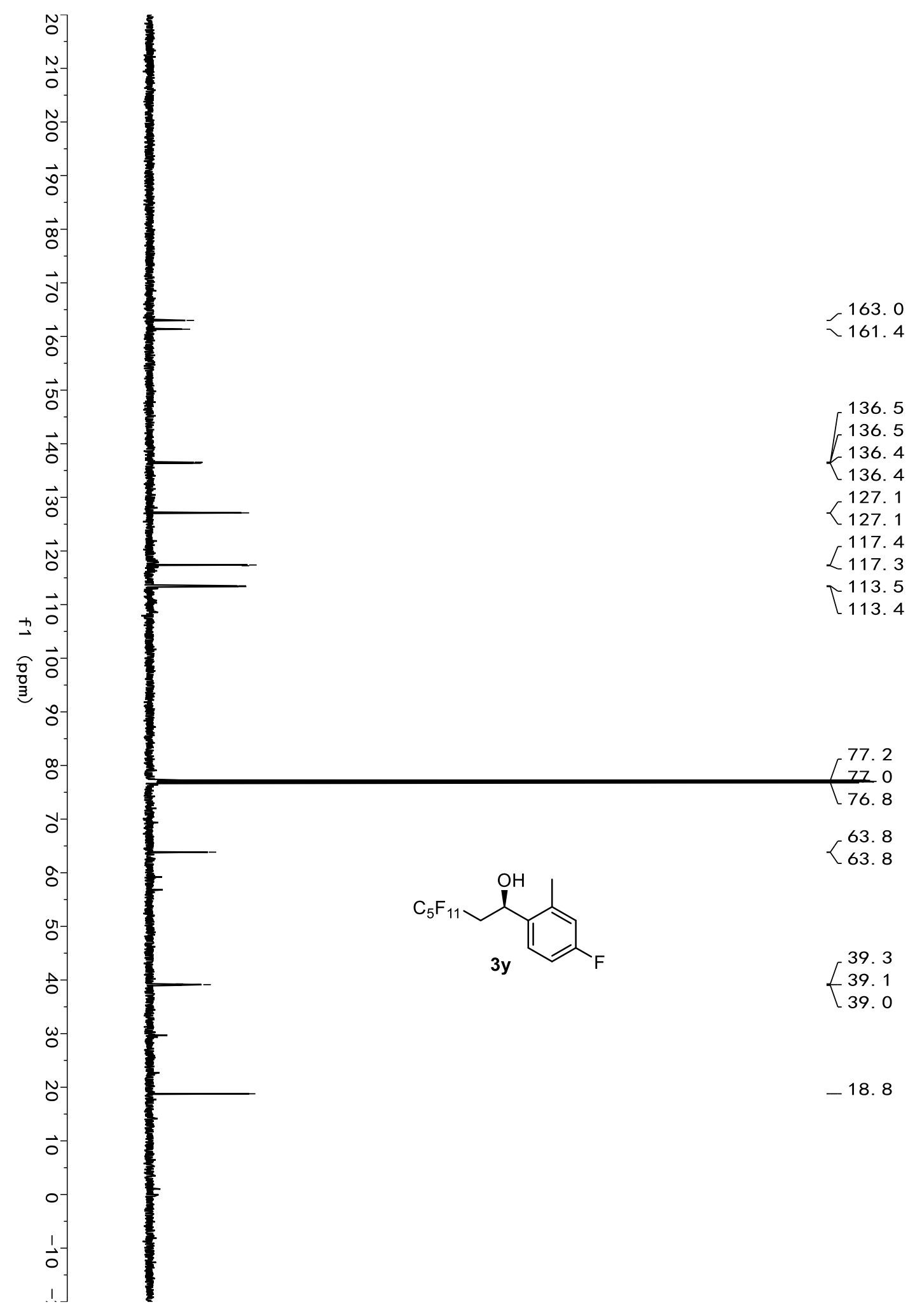




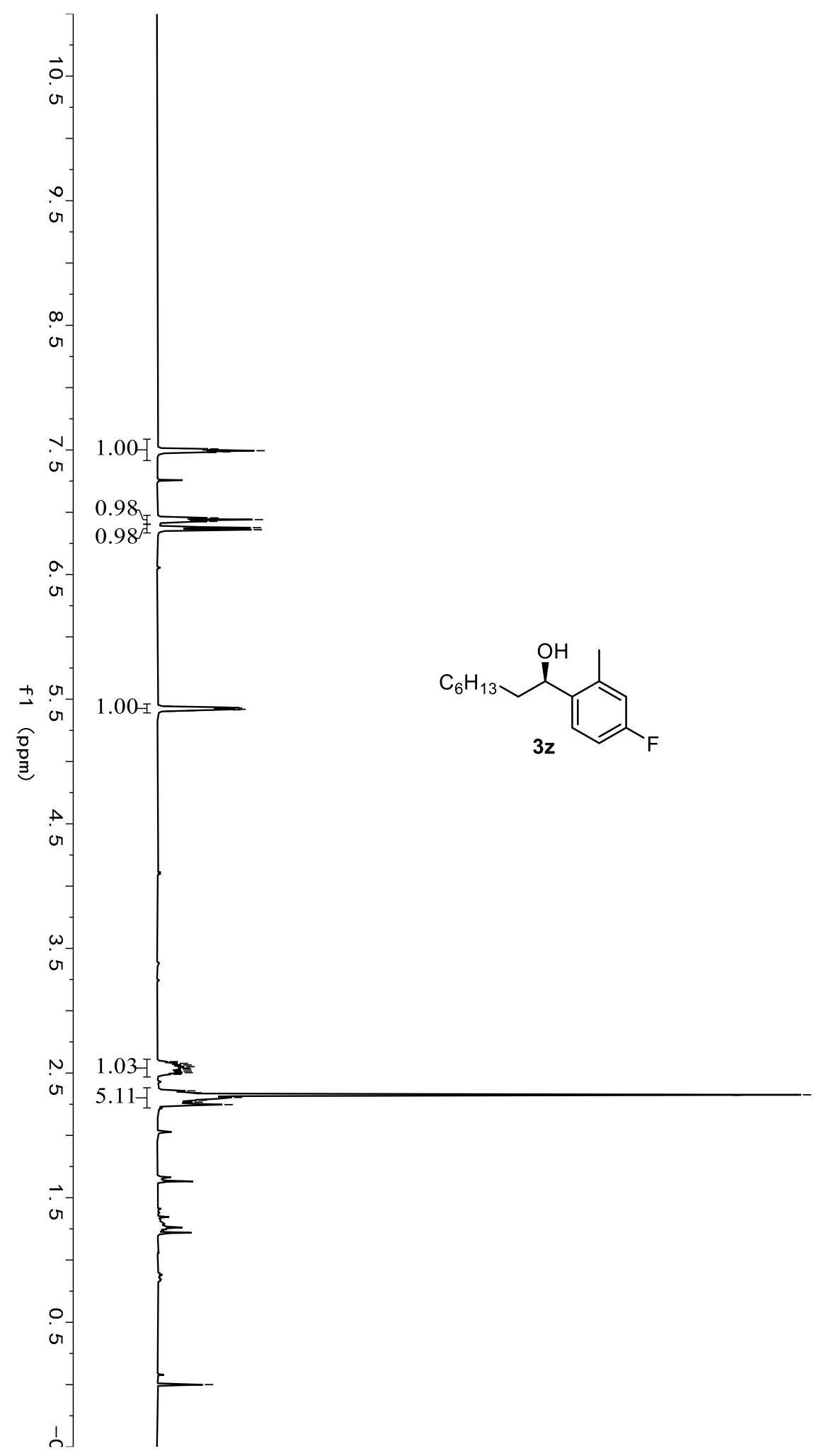

7.51
7.50

7. 50

7. 49

7.49

$-7.48$

7.48

6.96

6.94

6. 93

6. 88

6. 86

5. 44

5. 44

5. 43

5. 43

5.42

5. 42

5. 41

5. 41

5. 40

2. 59

2. 58

2. 57

2. 55

2. 54

2. 53

2. 52

2. 50

2. 49

氠 2.36

2. 34

2. 33

2. 32

2. 30

2. 28

2. 27

2. 25

$-0.00$ 


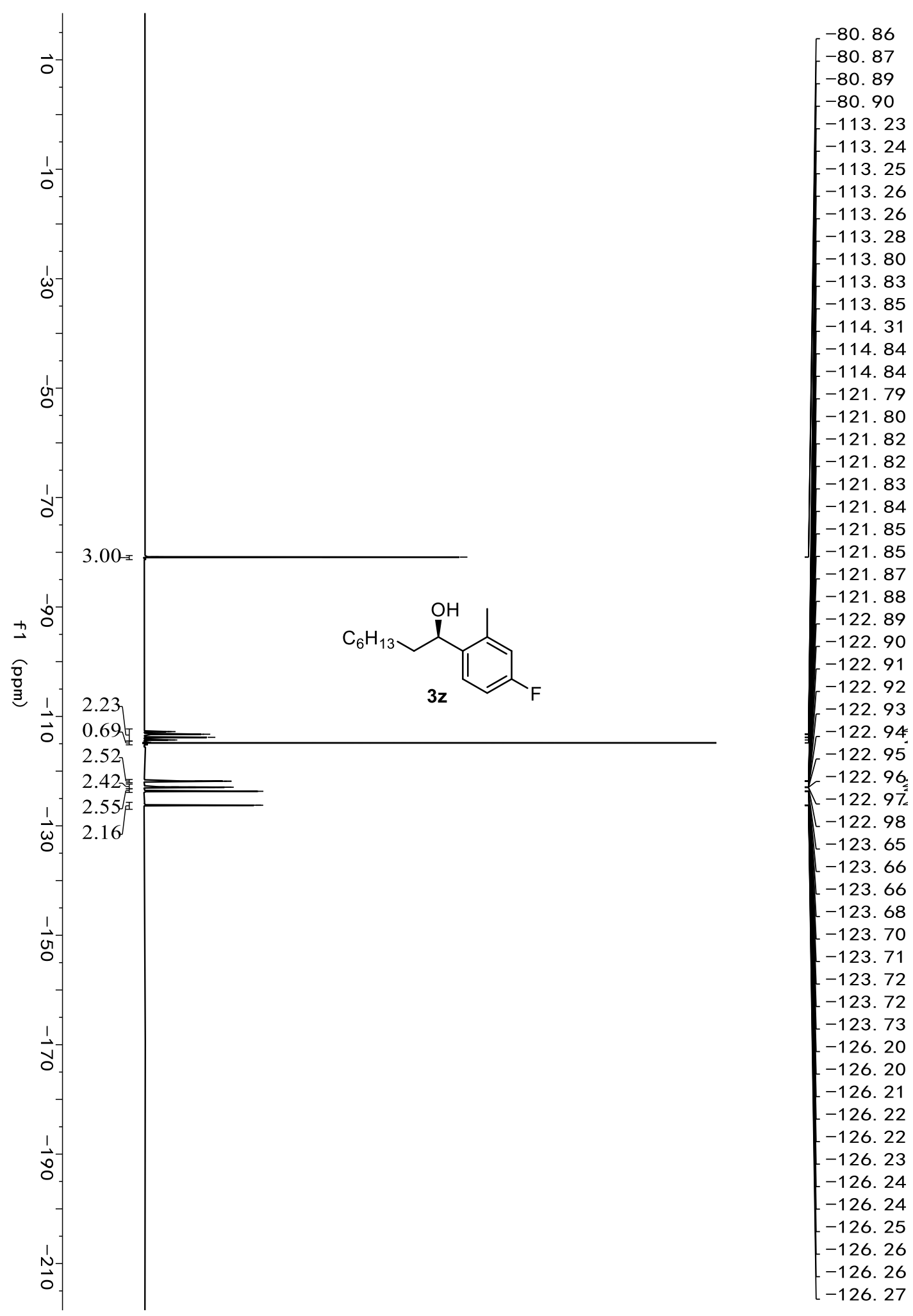




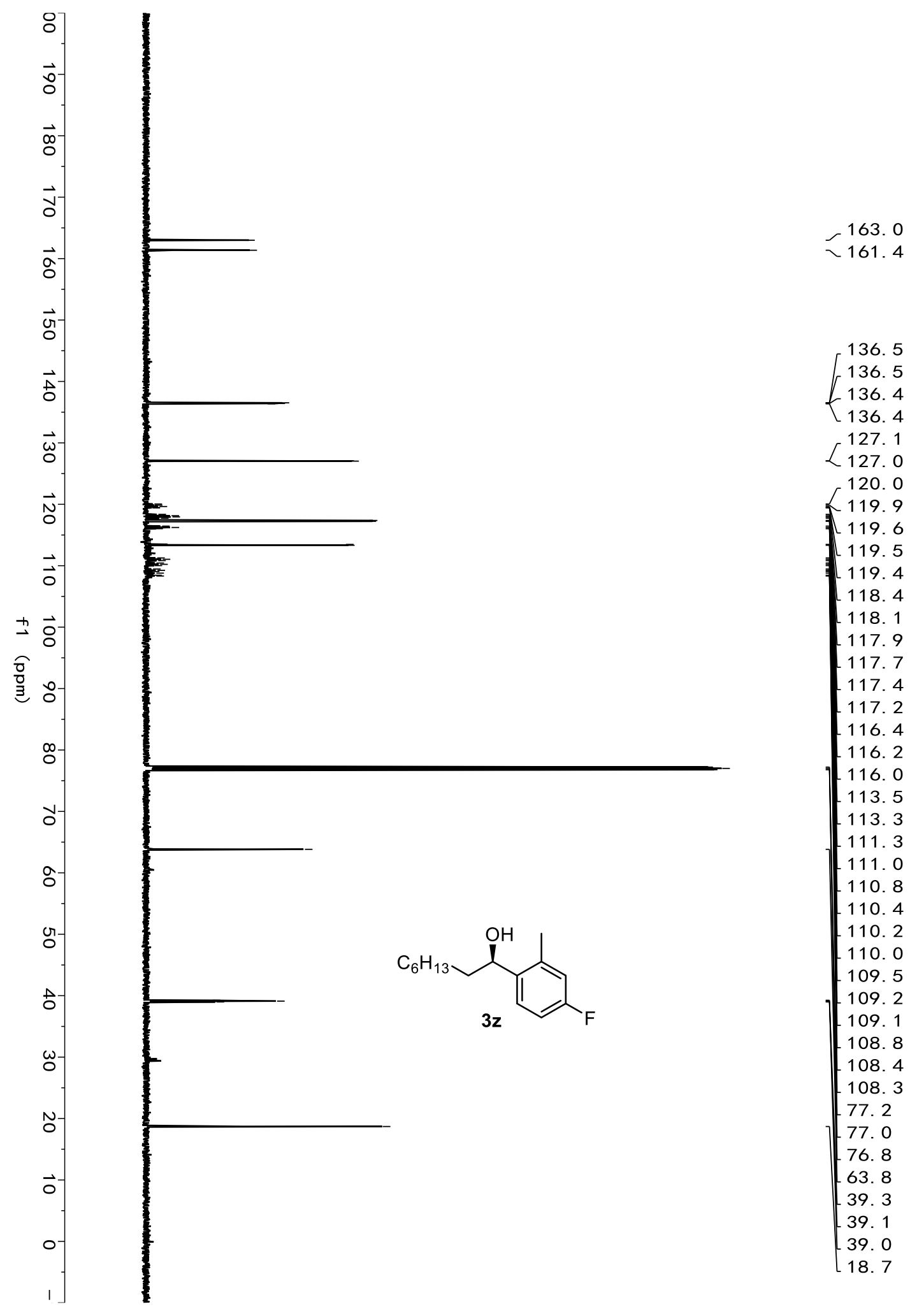




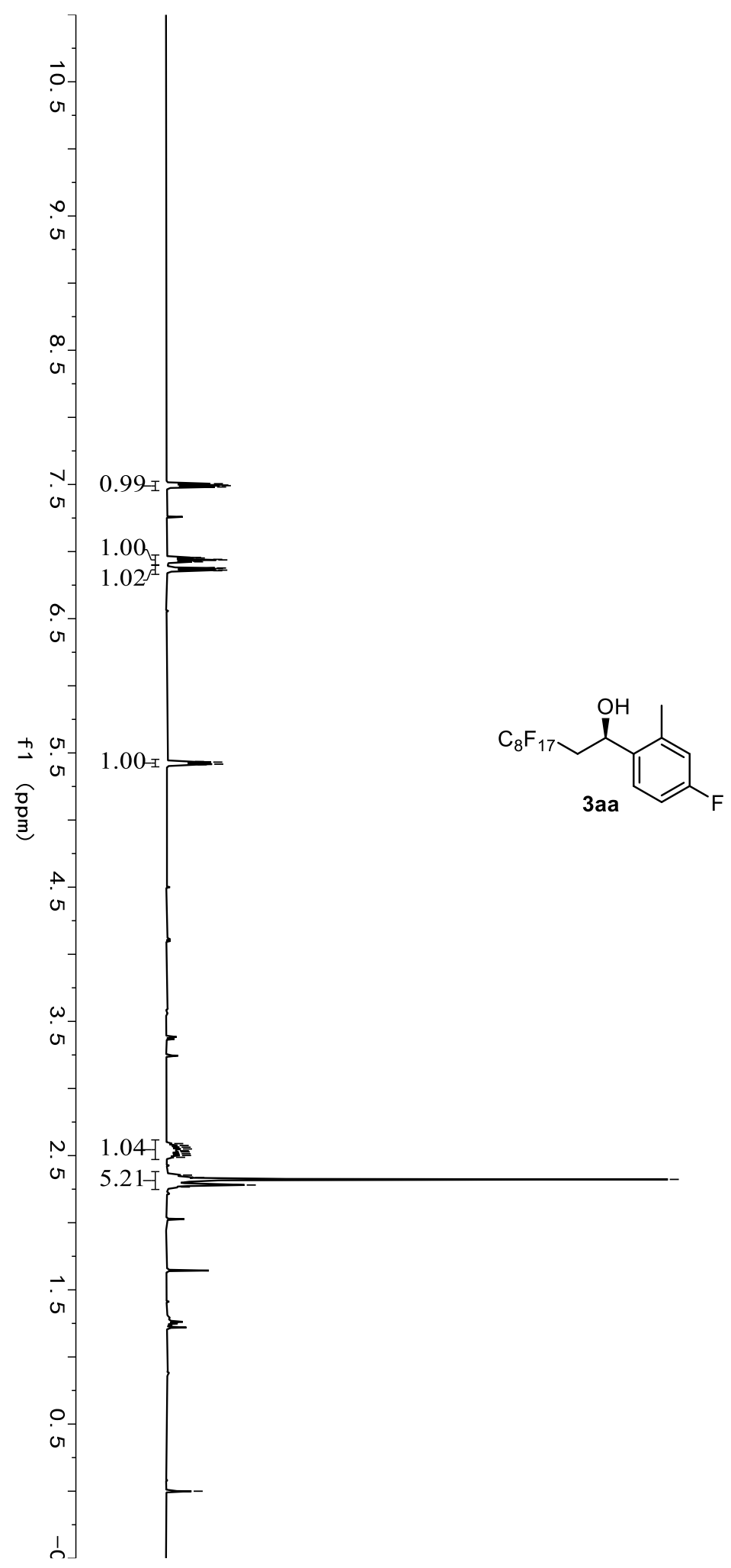

$\left[\begin{array}{r}7.51 \\ 7.50 \\ 7.49 \\ 7.48 \\ 6.96 \\ 6.95 \\ 6.94 \\ 6.94 \\ 6.93 \\ 6.92 \\ 6.88 \\ 6.87 \\ 6.86 \\ 6.86 \\ 5.44 \\ 5.43 \\ 5.43 \\ 5.42 \\ 5.42 \\ 5.41\end{array}\right.$

2. 59

2. 58

2. 56

2. 55

2. 54

2. 53

2. 51

2. 50

2. 49

2. 35

2. 34

2. 32

2. 28

2. 27

$-0.00$ 


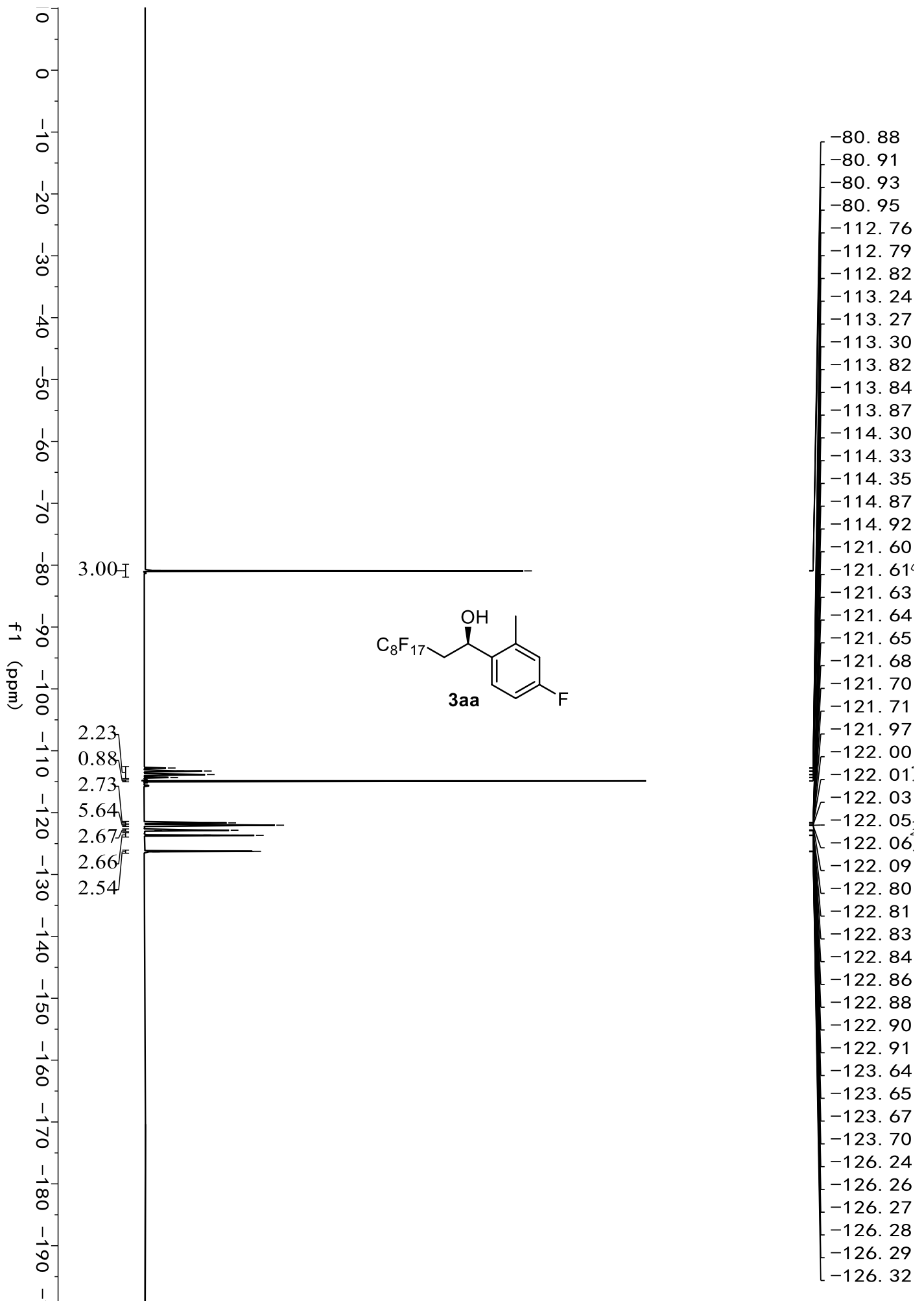

195 


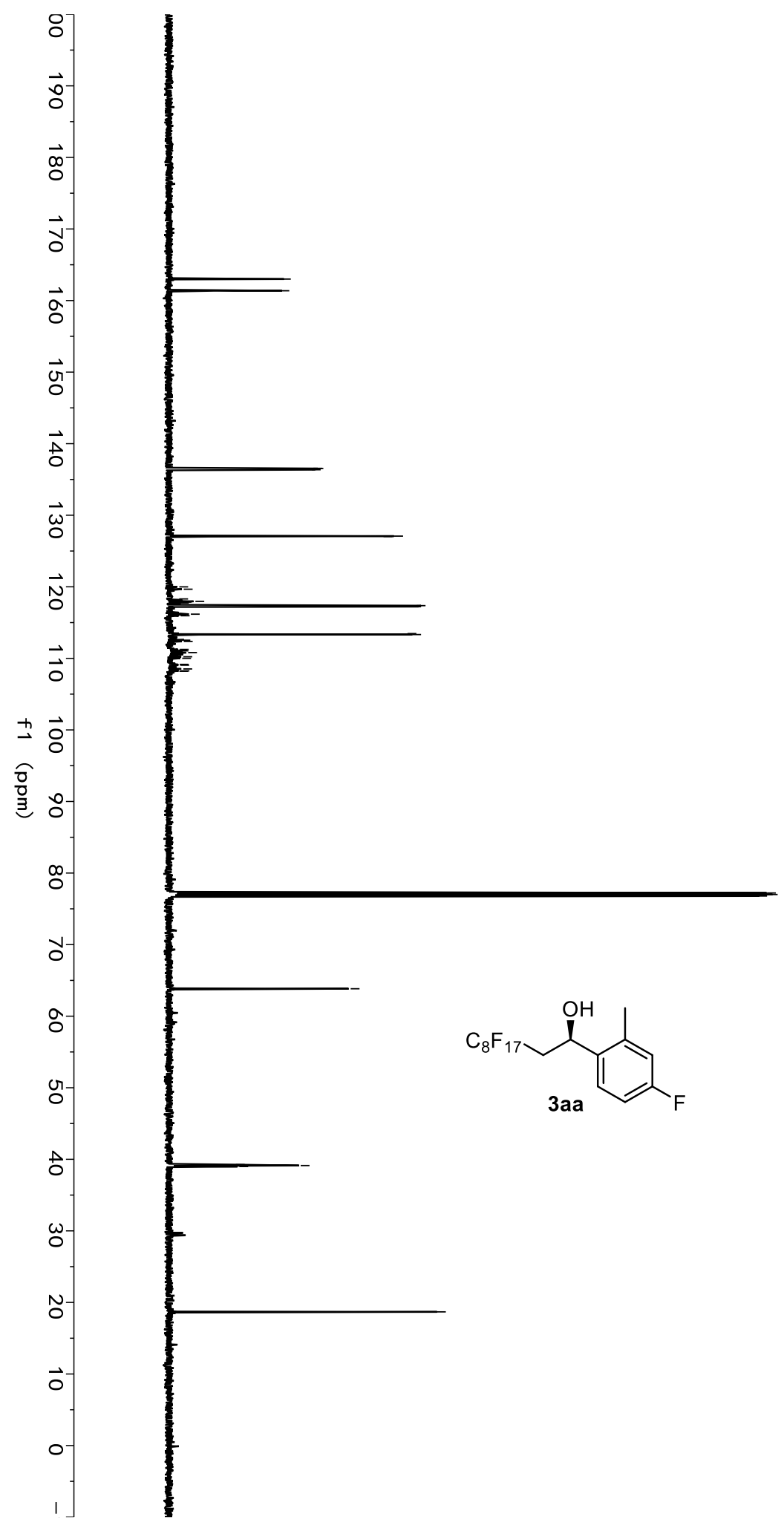

163.0
$\simeq 161.4$

136. 6

136. 5

136.4
-136.4

136. 4

127. 1

127.0

120.0
$\leftarrow 119.7$

119.7

118.3

118. 0

117. 9

117. 7

117. 4

117. 2

116. 2

116. 1

116. 0

113. 5

113. 3

112. 5

112. 4

111. 2

111. 1

111. 1

110. 8

110. 2

110. 0

109. 2

109. 0

108. 5

108. 3

108. 2

77. 2

77. 0

76. 8

63. 8

39. 3

39. 1

39. 0

18. 7 


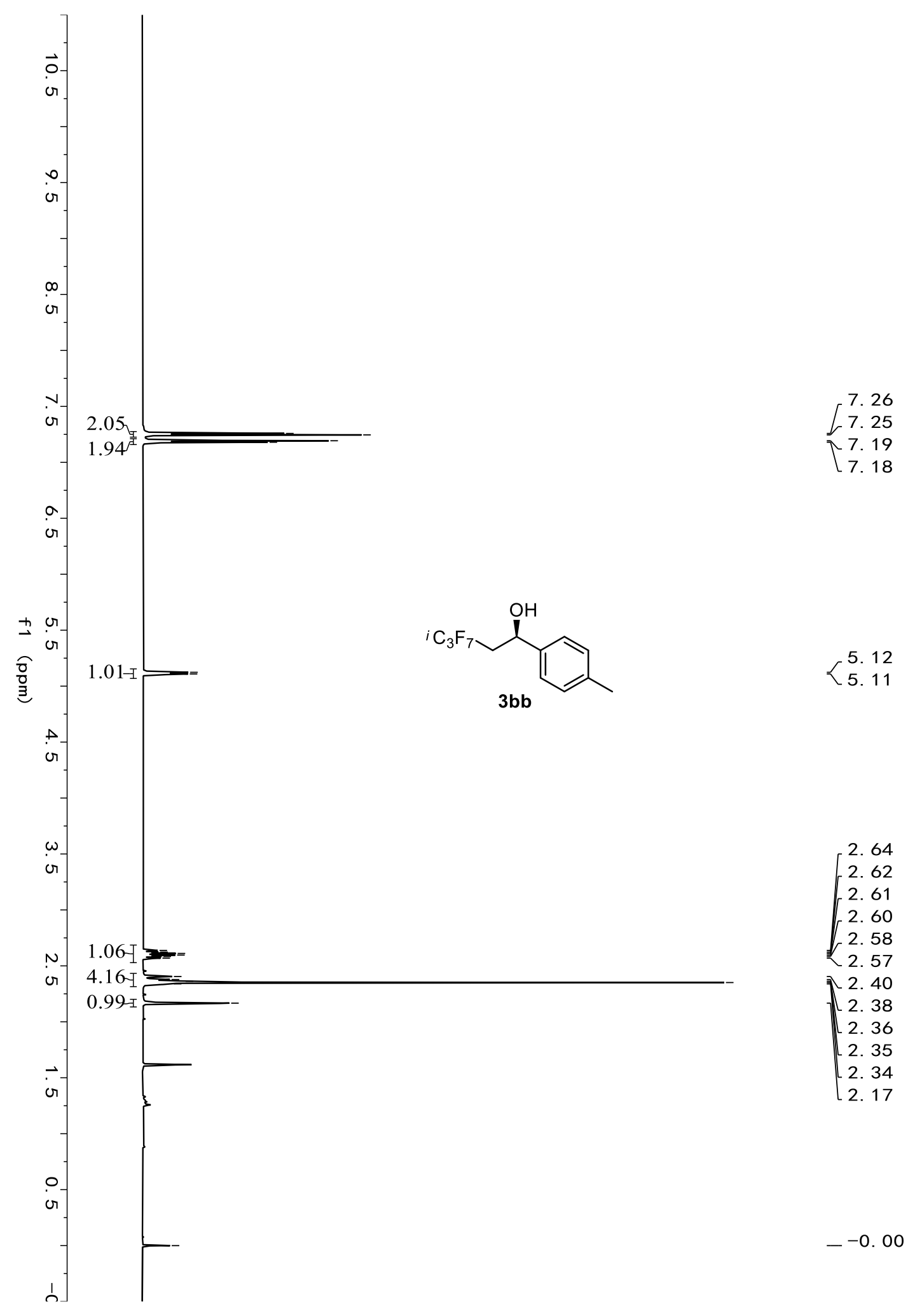



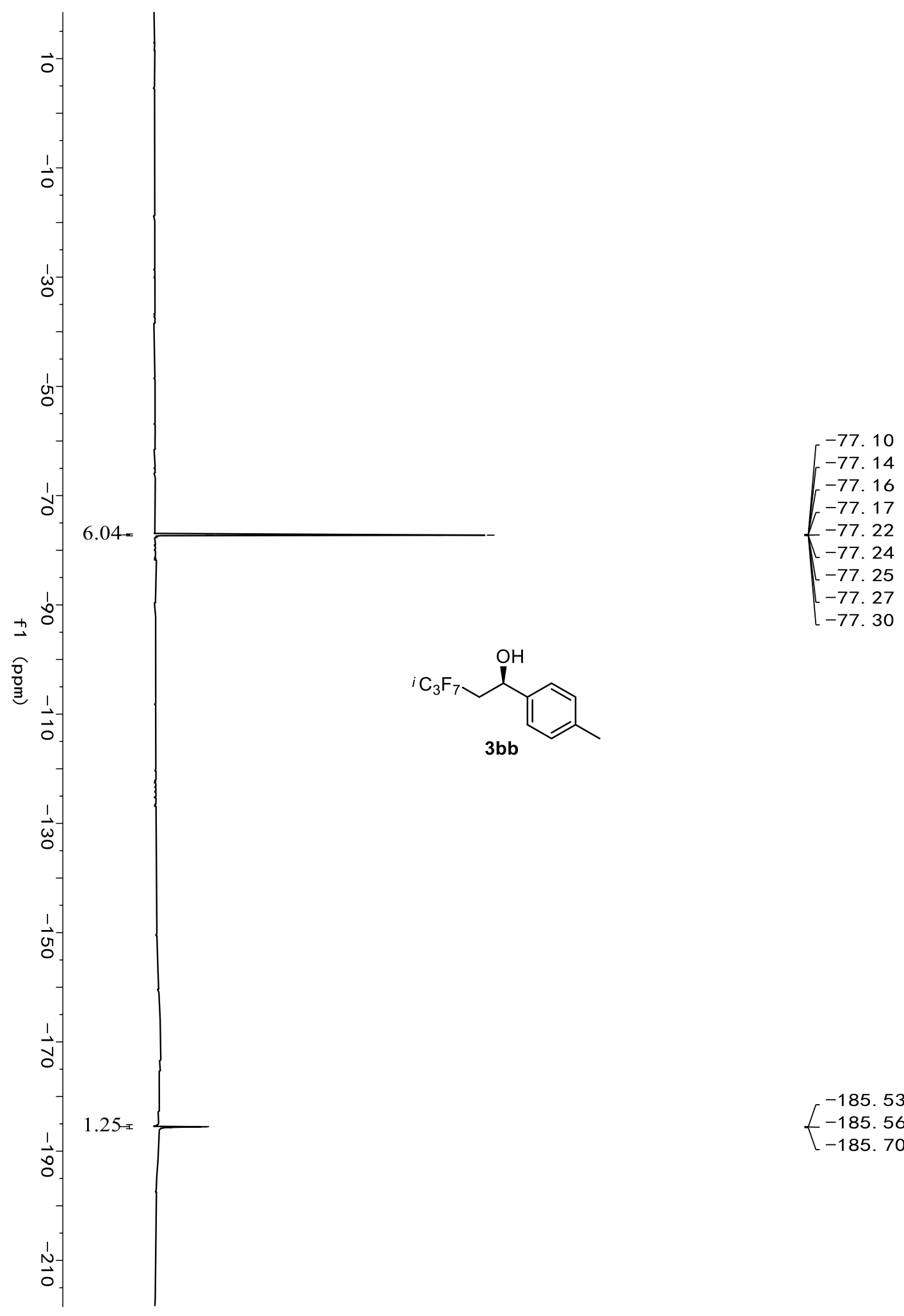

$\left\{\begin{array}{l}-185.53 \\ -185.56 \\ -185.70\end{array}\right.$

198 


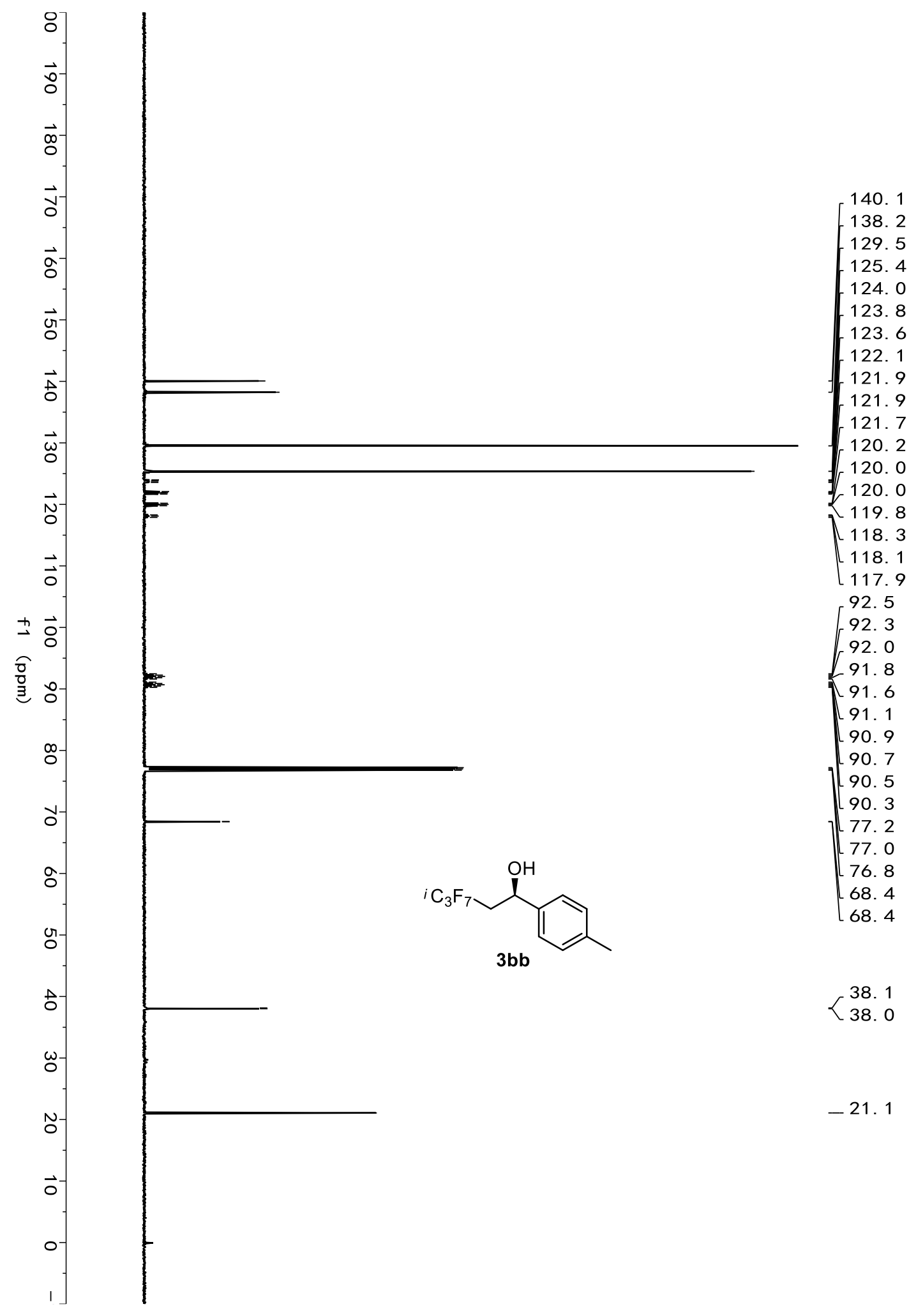




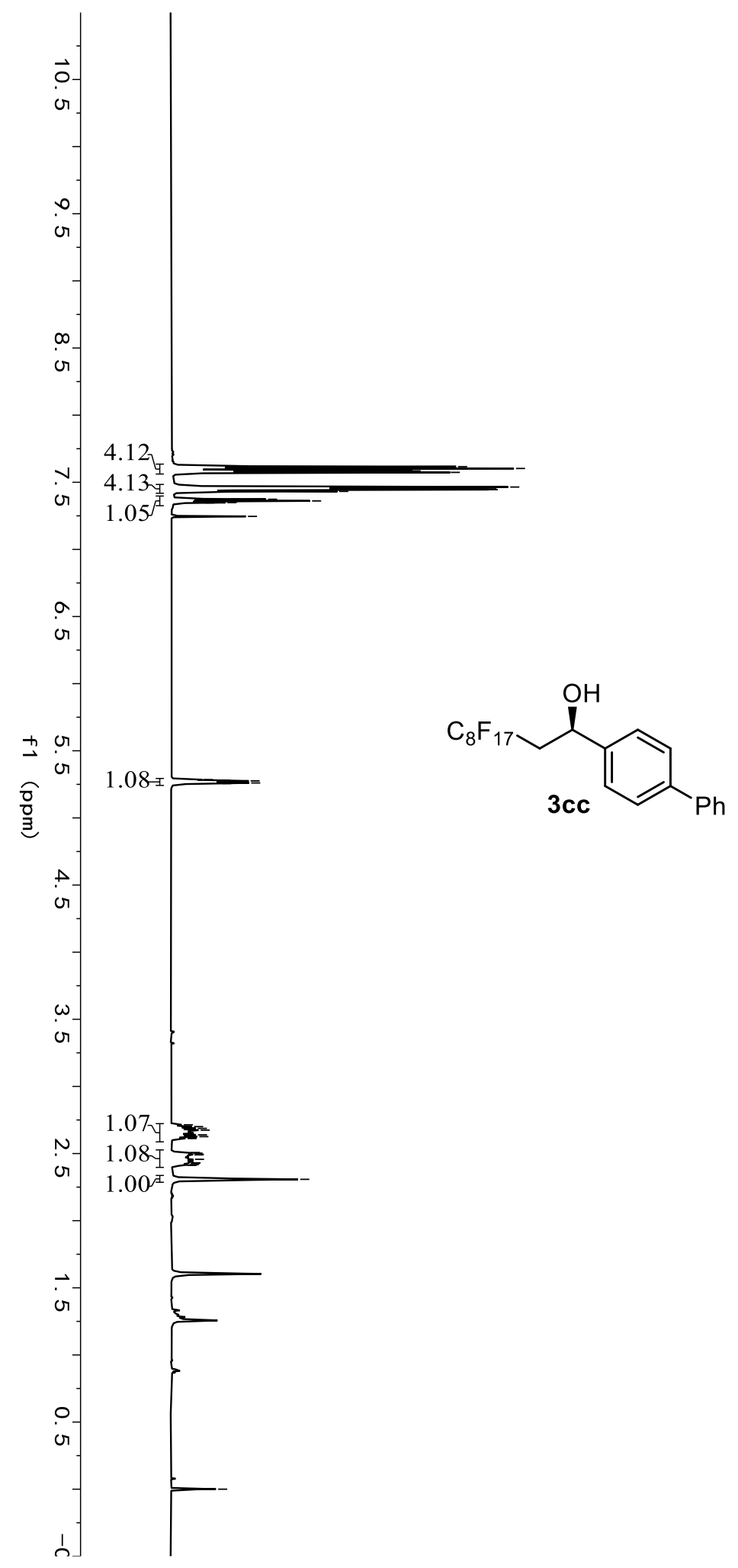

7. 62

7. 61

7. 61

7. 60

7. 59

7. 59

7. 58

7. 58

7. 57

7. 57

7. 46

7. 46

7. 45

7. 45

7. 45

7. 43

7. 43

7. 38

7. 37

7. 36

7. 36

7. 36

7. 35

7. 25

5. 28

5. 28

$-5.27$

5. 27

5. 26

5. 26

2. 71

2. 70

2. 69

2. 67

2. 64

2. 63

2. 61

2. 51

2.50

2. 49

2. 49

2. 46

2. 43

2. 43

2. 42

2. 41

2. 31

$-0.00$ 


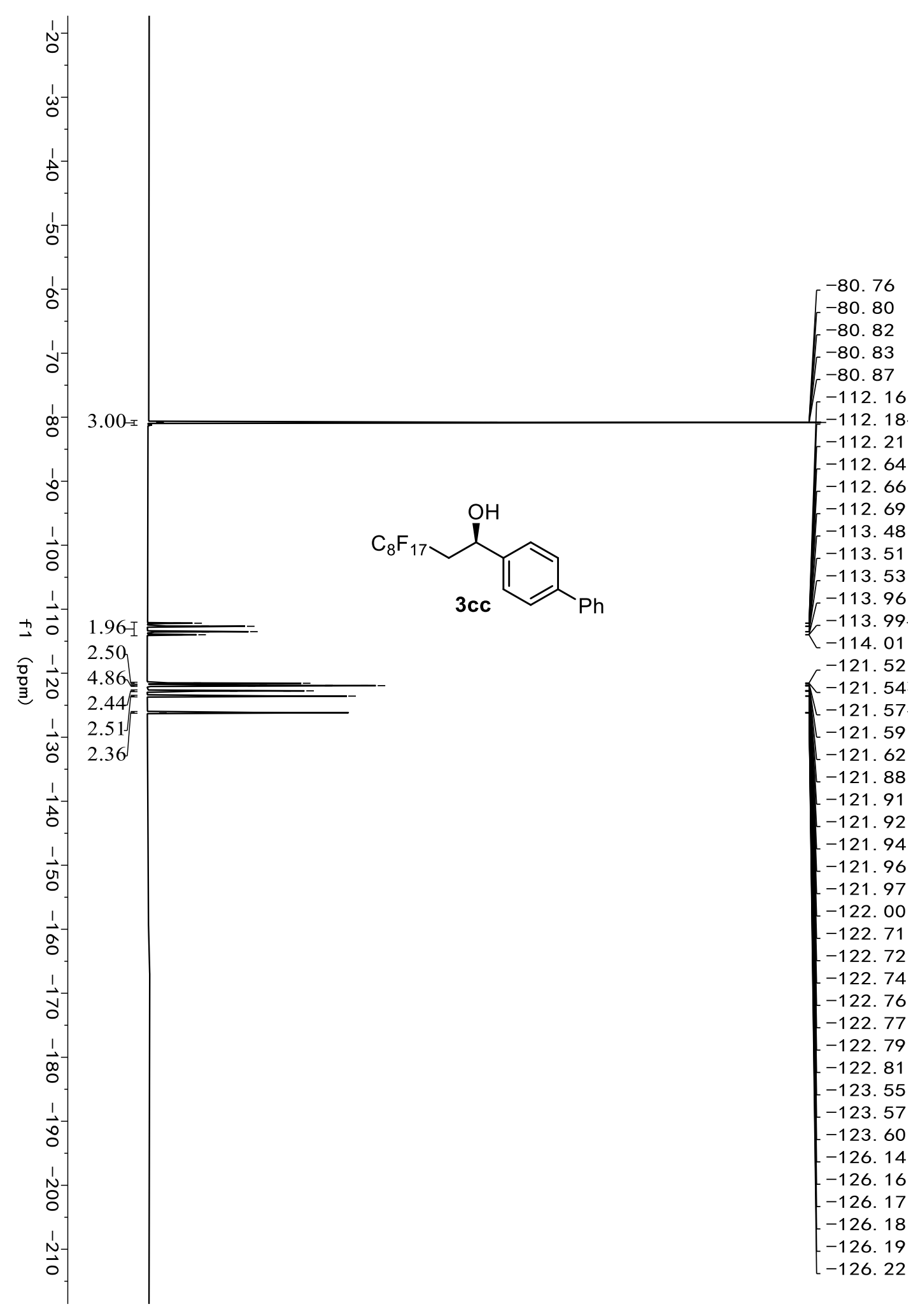




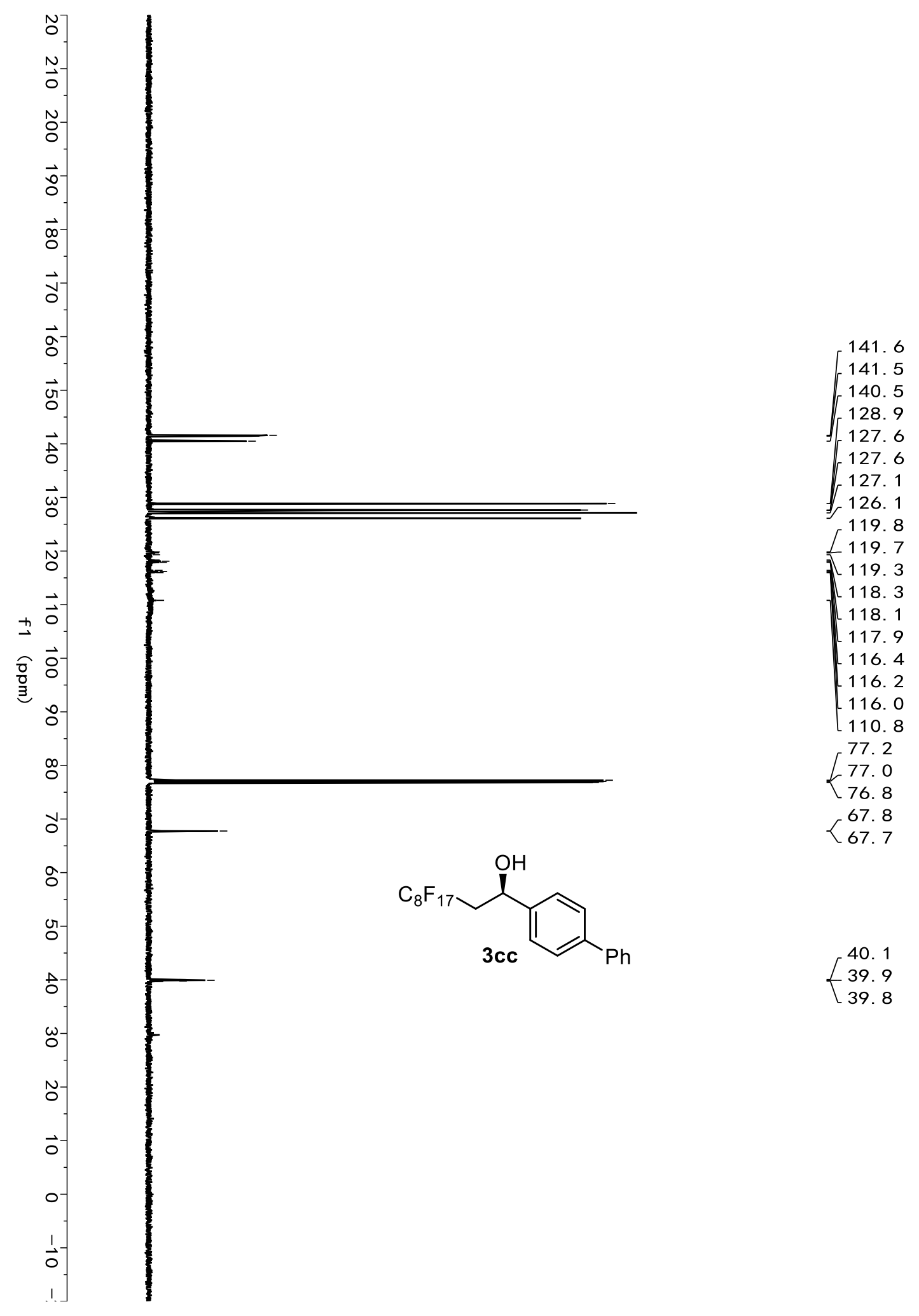




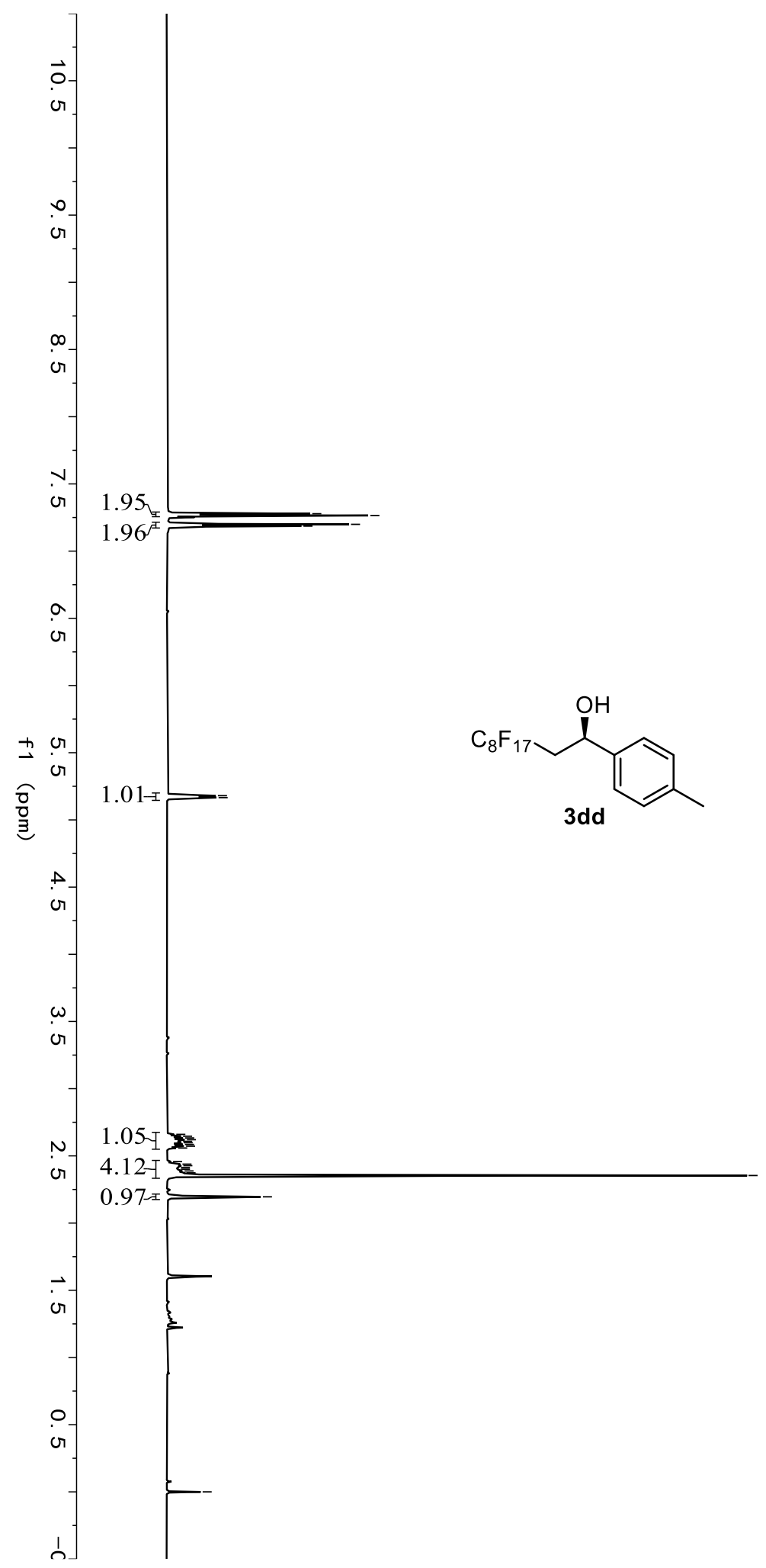




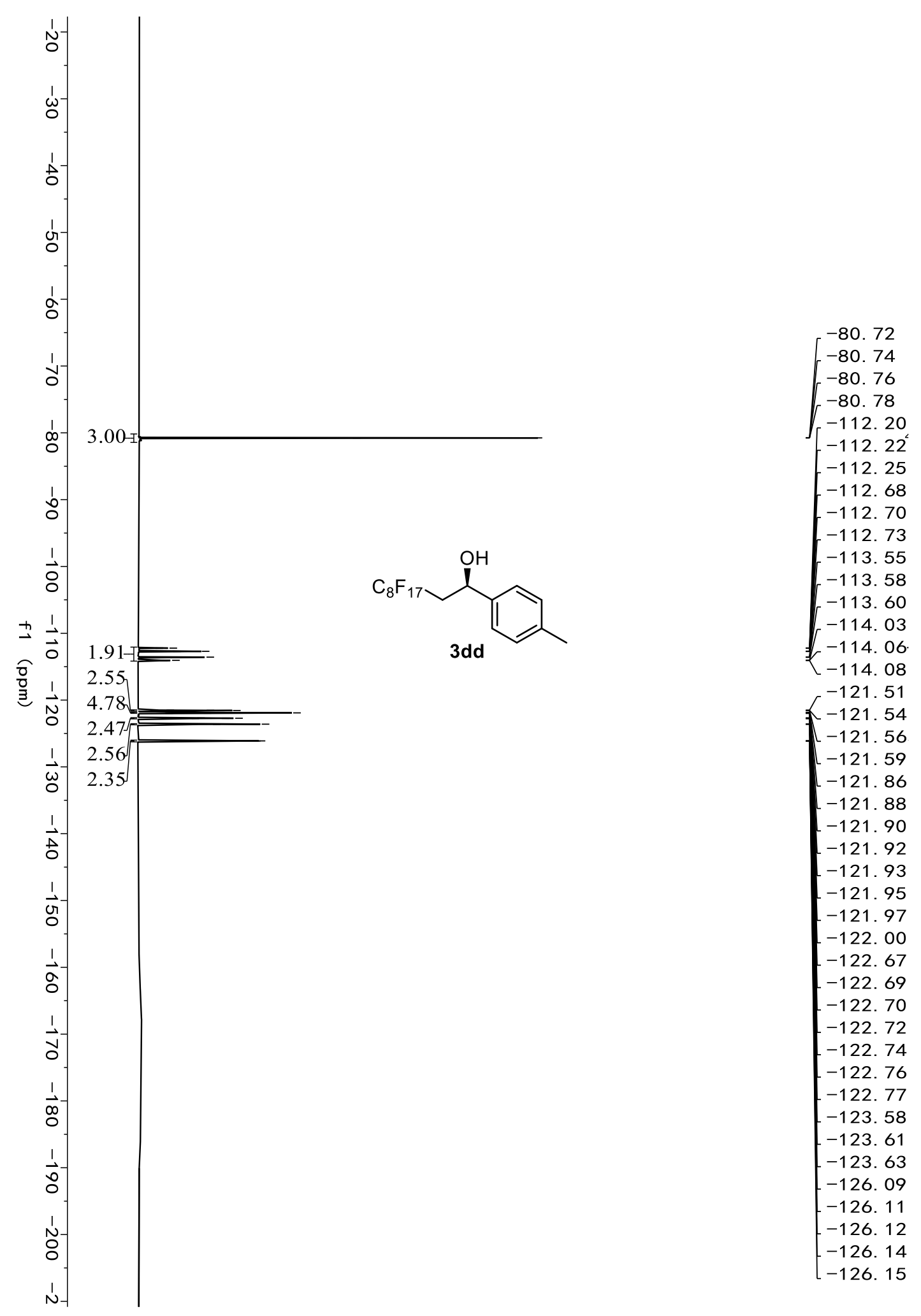




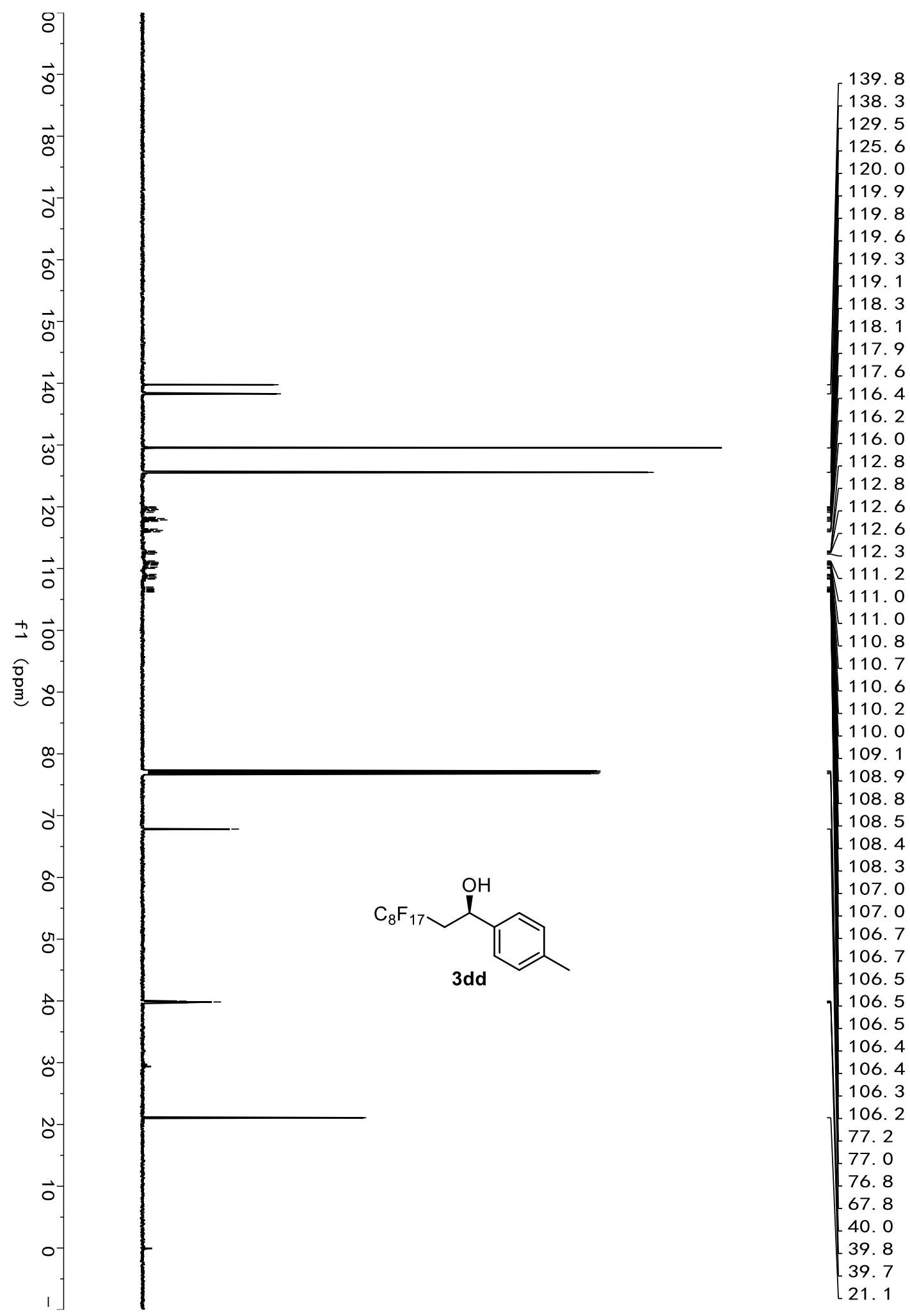




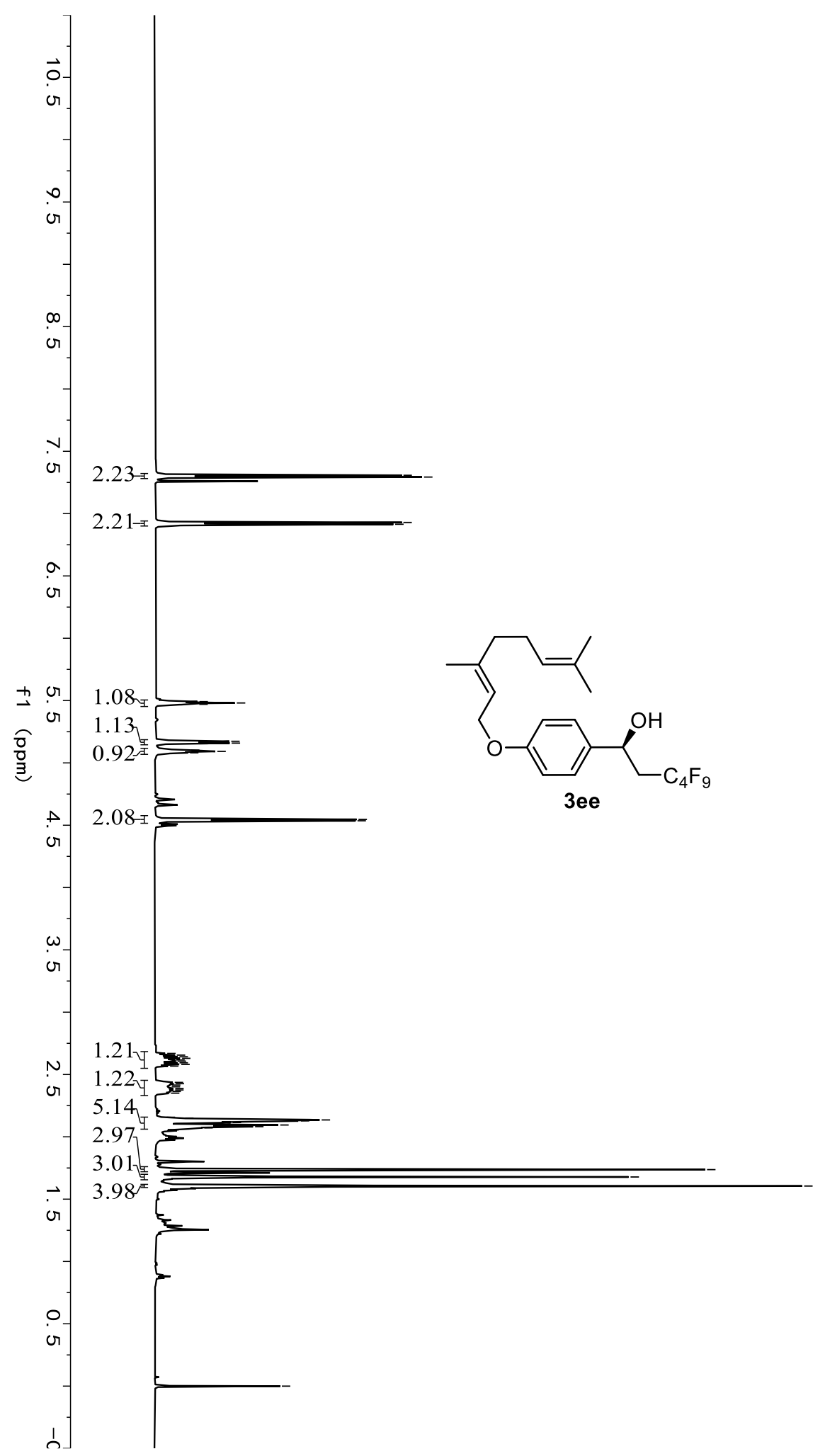

$\left[\begin{array}{l}7.31 \\ 7.29 \\ 6.93 \\ 6.91 \\ 5.49 \\ 5.48 \\ 5.47 \\ 5.18 \\ 5.17 \\ 5.17 \\ 5.16 \\ 5.16 \\ 5.15 \\ 5.10 \\ 5.09 \\ 5.08 \\ 4.55 \\ 4.54 \\ 2.67 \\ 2.66 \\ 2.64 \\ 2.63 \\ 2.62 \\ 2.61 \\ 2.59 \\ 2.58 \\ 2.57 \\ 2.44 \\ 2.43 \\ 2.42 \\ 2.40 \\ 2.39 \\ 2.38 \\ 2.36 \\ 2.35 \\ 2.16 \\ 2.15 \\ 2.14 \\ 2.13 \\ 2.11 \\ 2.09 \\ 2.08 \\ 2.07 \\ 2.06 \\ 1.74 \\ 1.68 \\ 1.61 \\ -0.00\end{array}\right.$




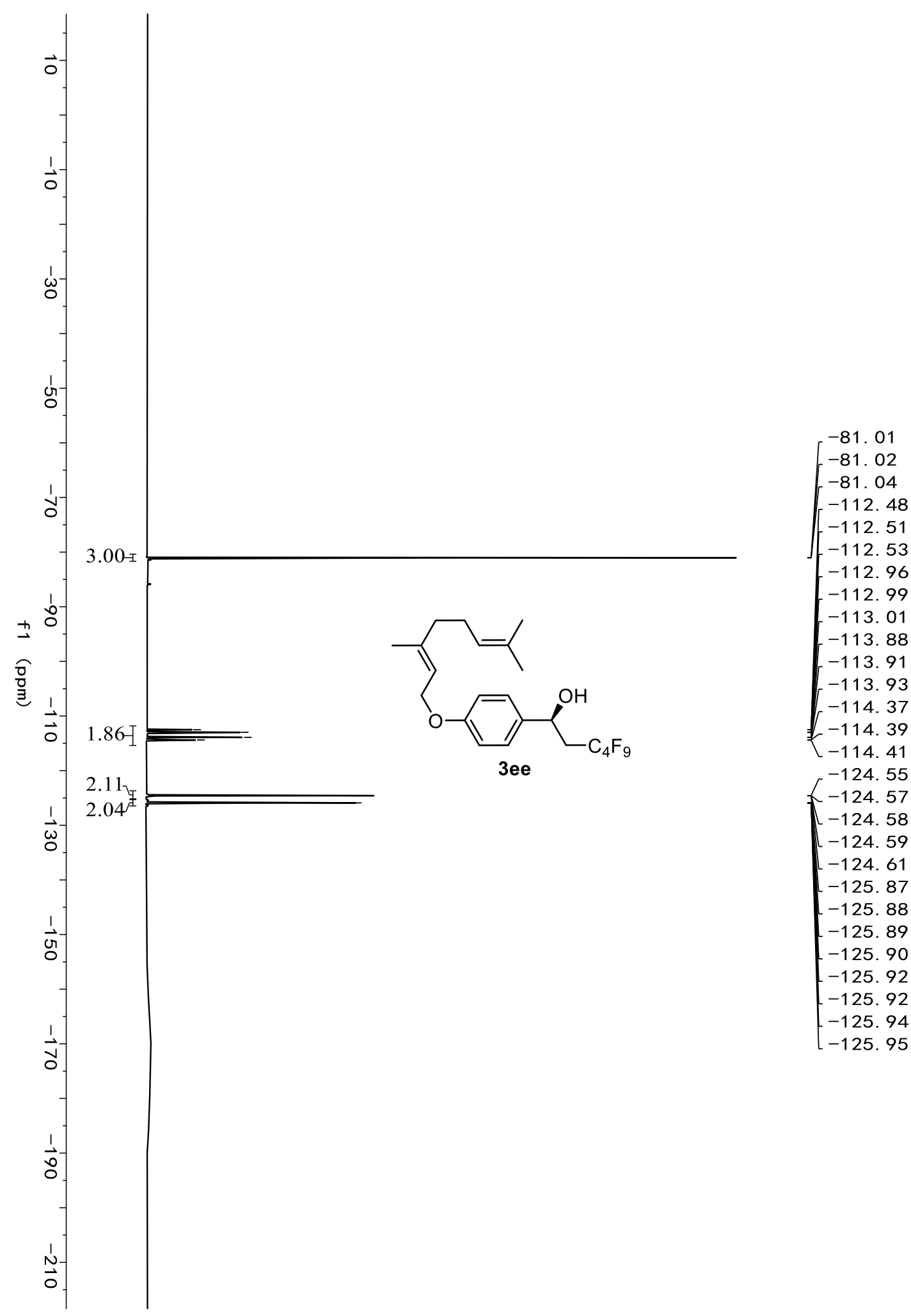




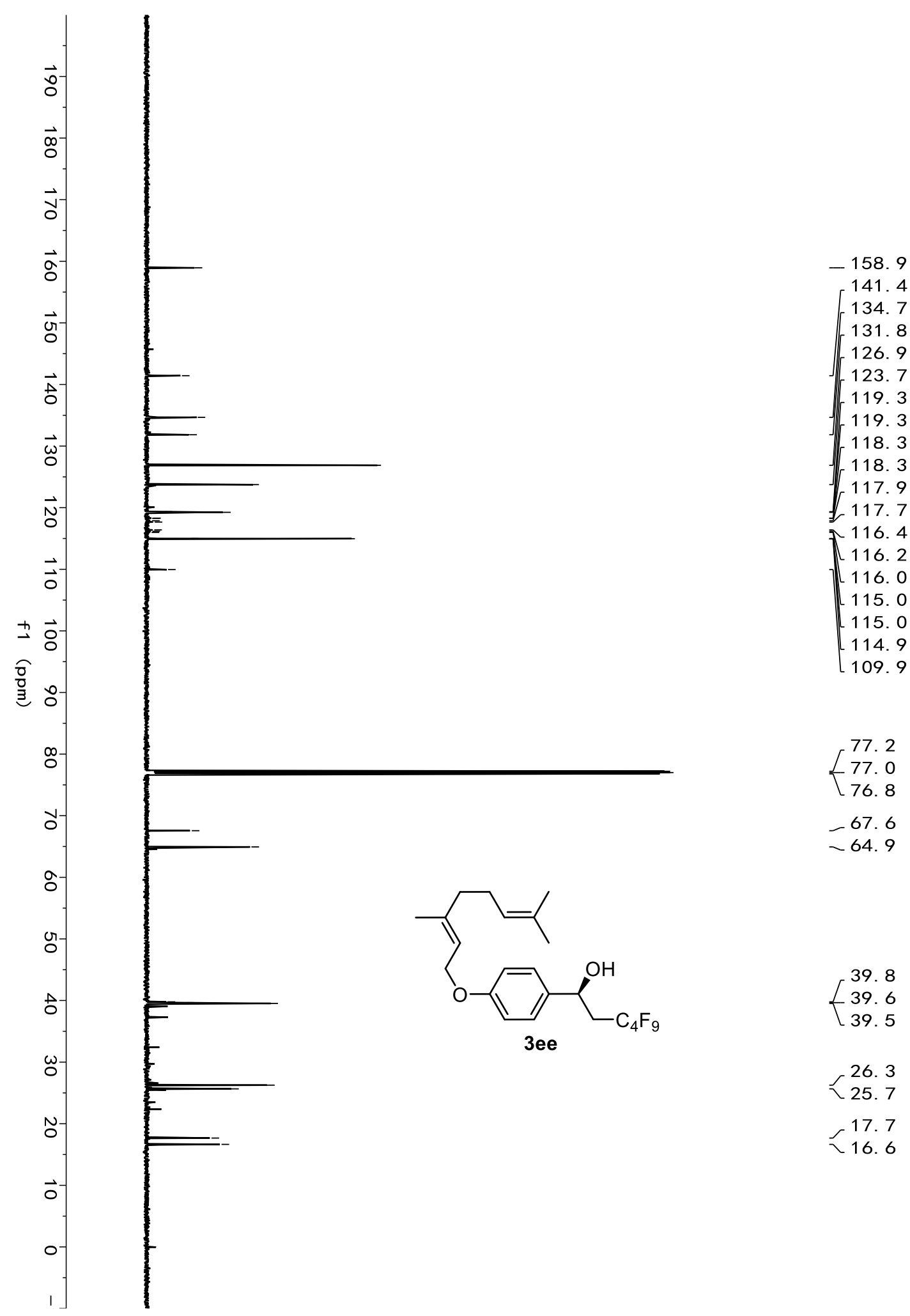




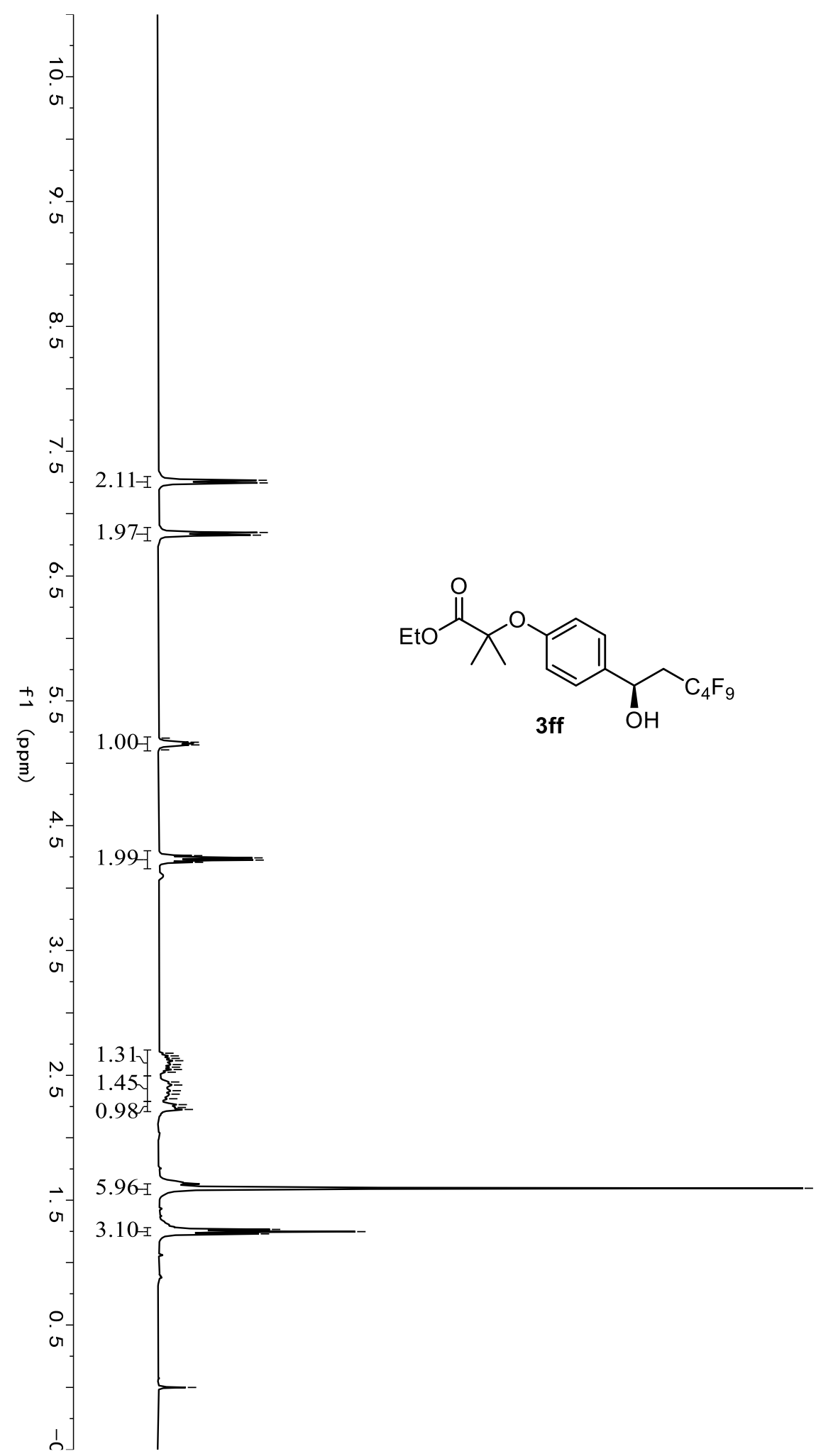




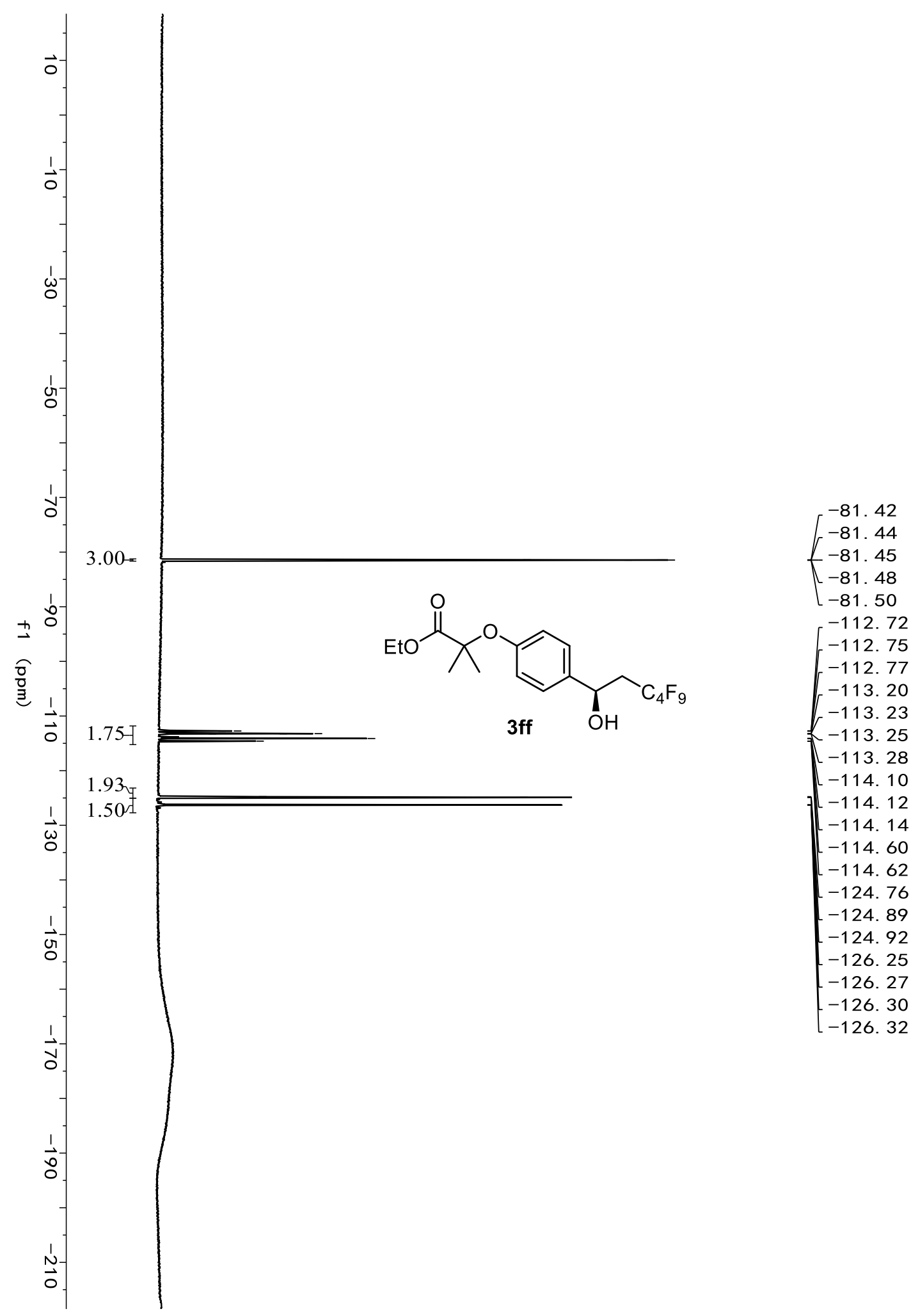




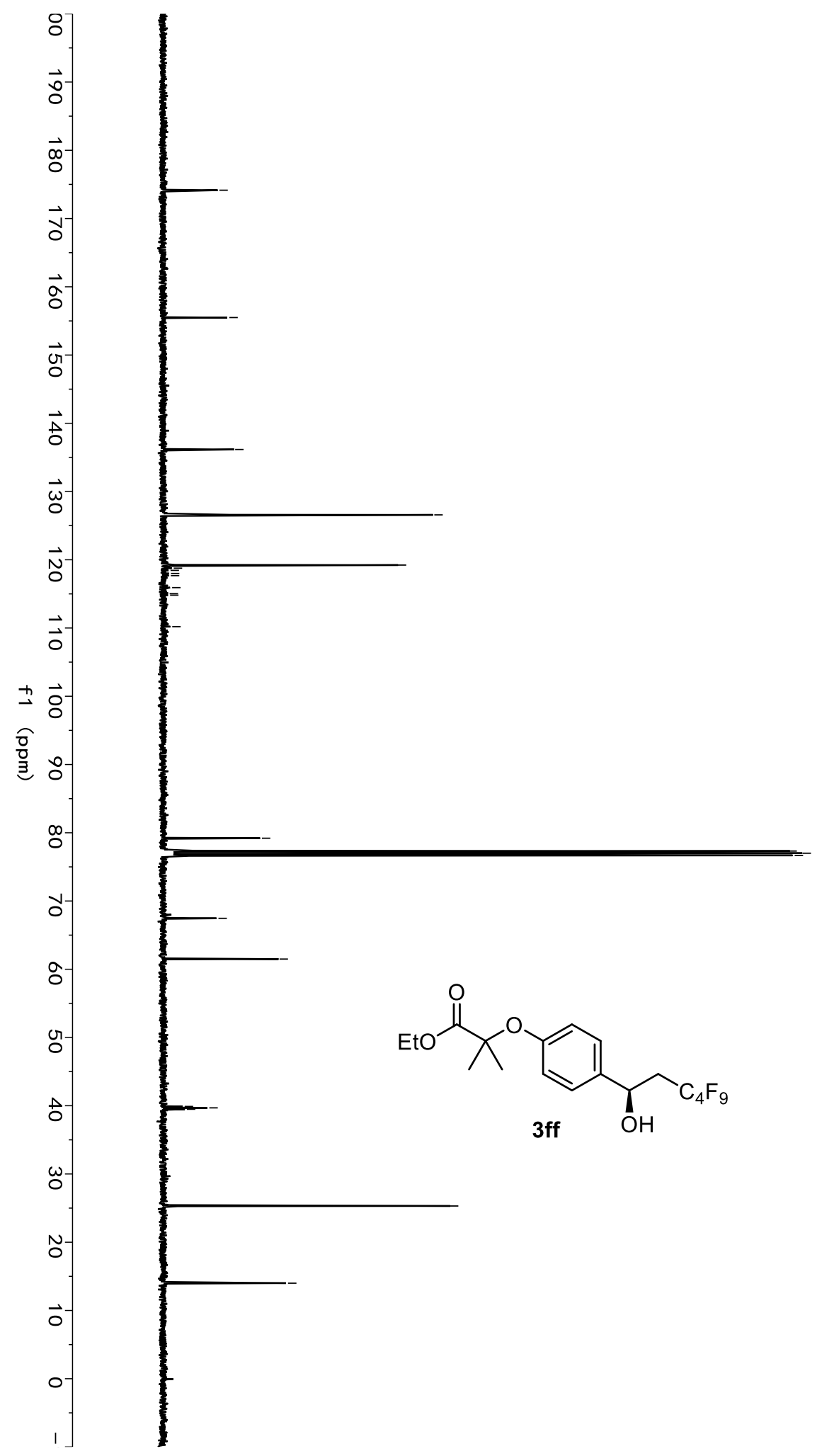

$-174.2$

$-155.5$

$-136.2$

$-126.6$

119. 2

118. 8

118. 4

118. 0

117.7

115. 9

115.0

114. 8

[110. 2

79. 2

$\int 77.3$

77.0
76.7

$-67.5$

$-61.5$

39. 9

$\left\{\begin{array}{r}39.9 \\ -39.7 \\ 39.5\end{array}\right.$

$-25.3$

$-14.0$ 


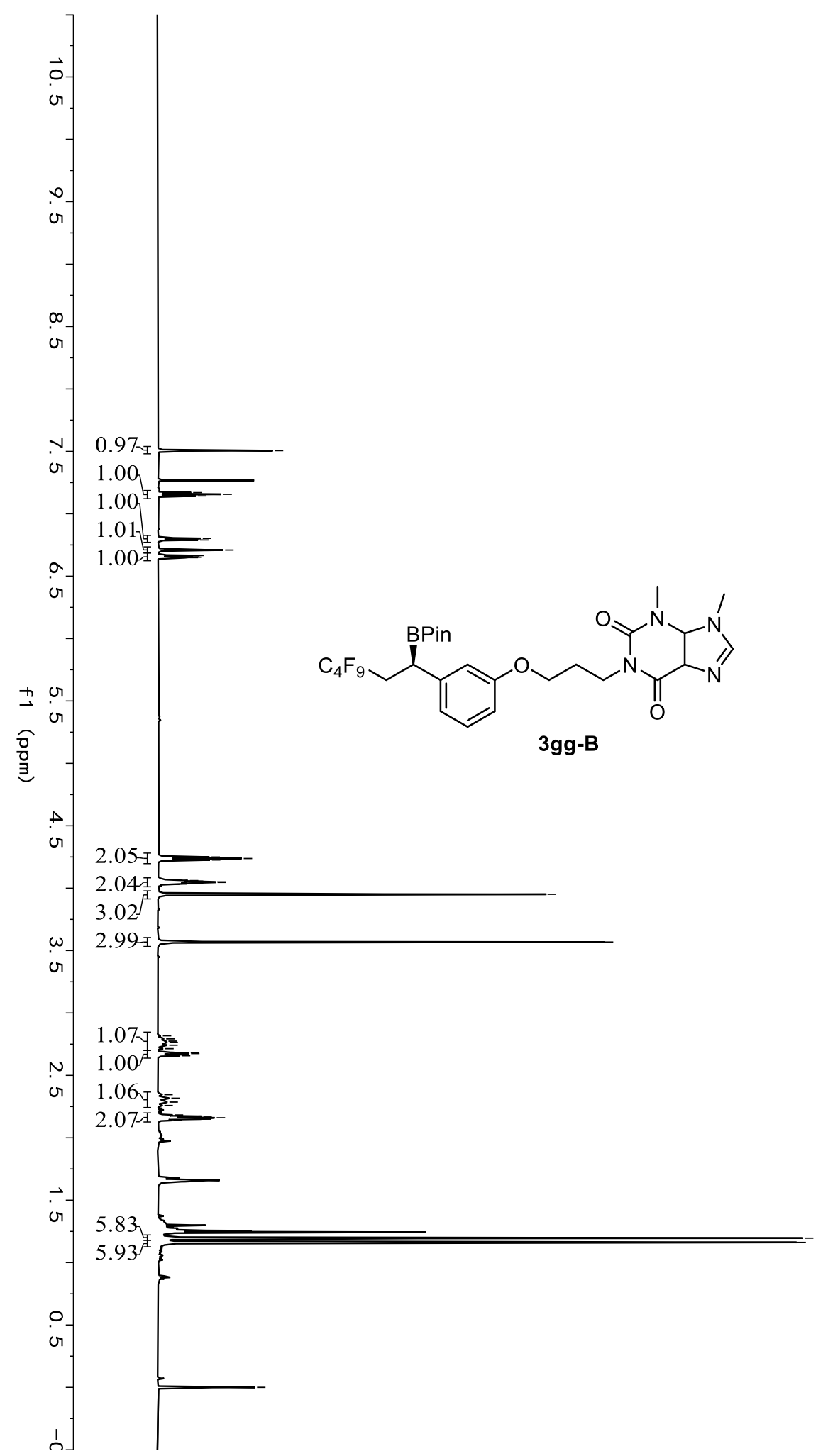

$\left[\begin{array}{l}7.51 \\ 7.17 \\ 7.16 \\ 7.14 \\ 6.80 \\ 6.79 \\ 6.72 \\ 6.71 \\ 6.71 \\ 6.71 \\ 6.70 \\ 6.67 \\ 6.66 \\ 6.65 \\ 6.65\end{array}\right.$

4. 25

4. 24

4. 23

4. 07

4. 06

4. 06

4. 05

4. 05

4. 04

4. 04

4. 03

3. 95

3.57

2. 82

2.79

2. 77

2. 76

2. 74

2.71

2. 68

2. 67

2. 66

2. 66

2. 35

2. 32

2. 29

2. 26

2. 18

2. 17

2. 16

2. 15

2. 14

1. 20

1. 16

-0.00 


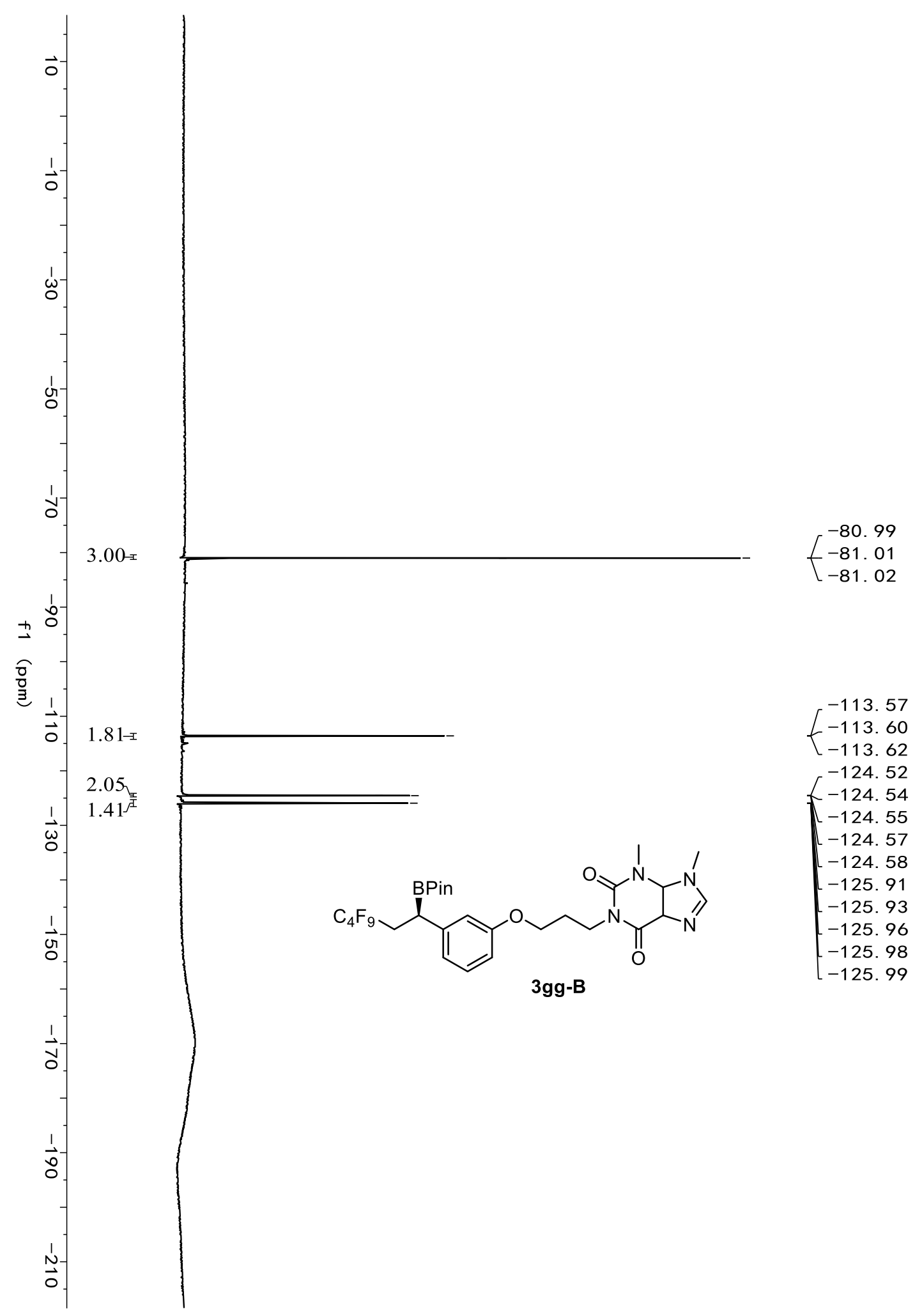




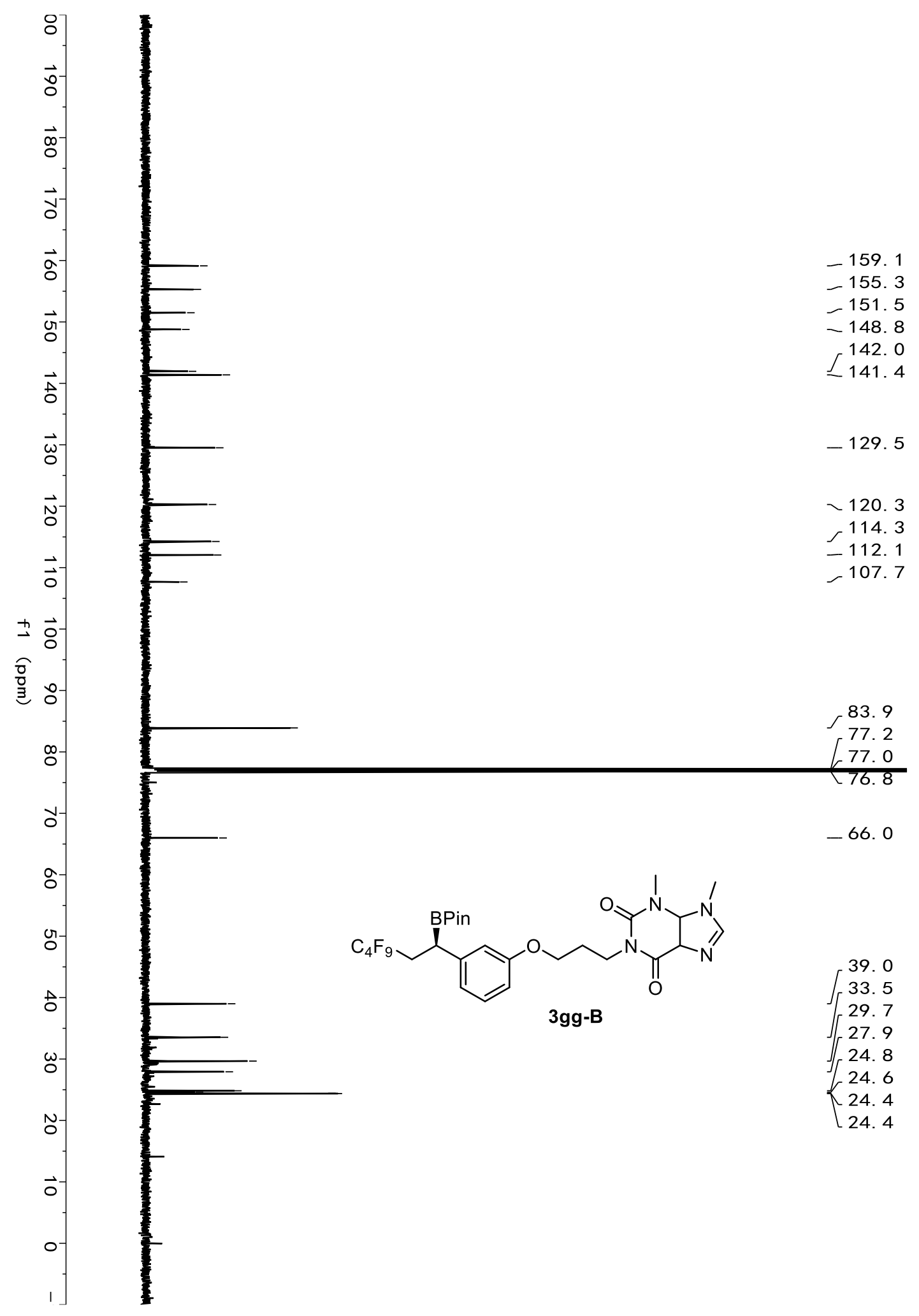




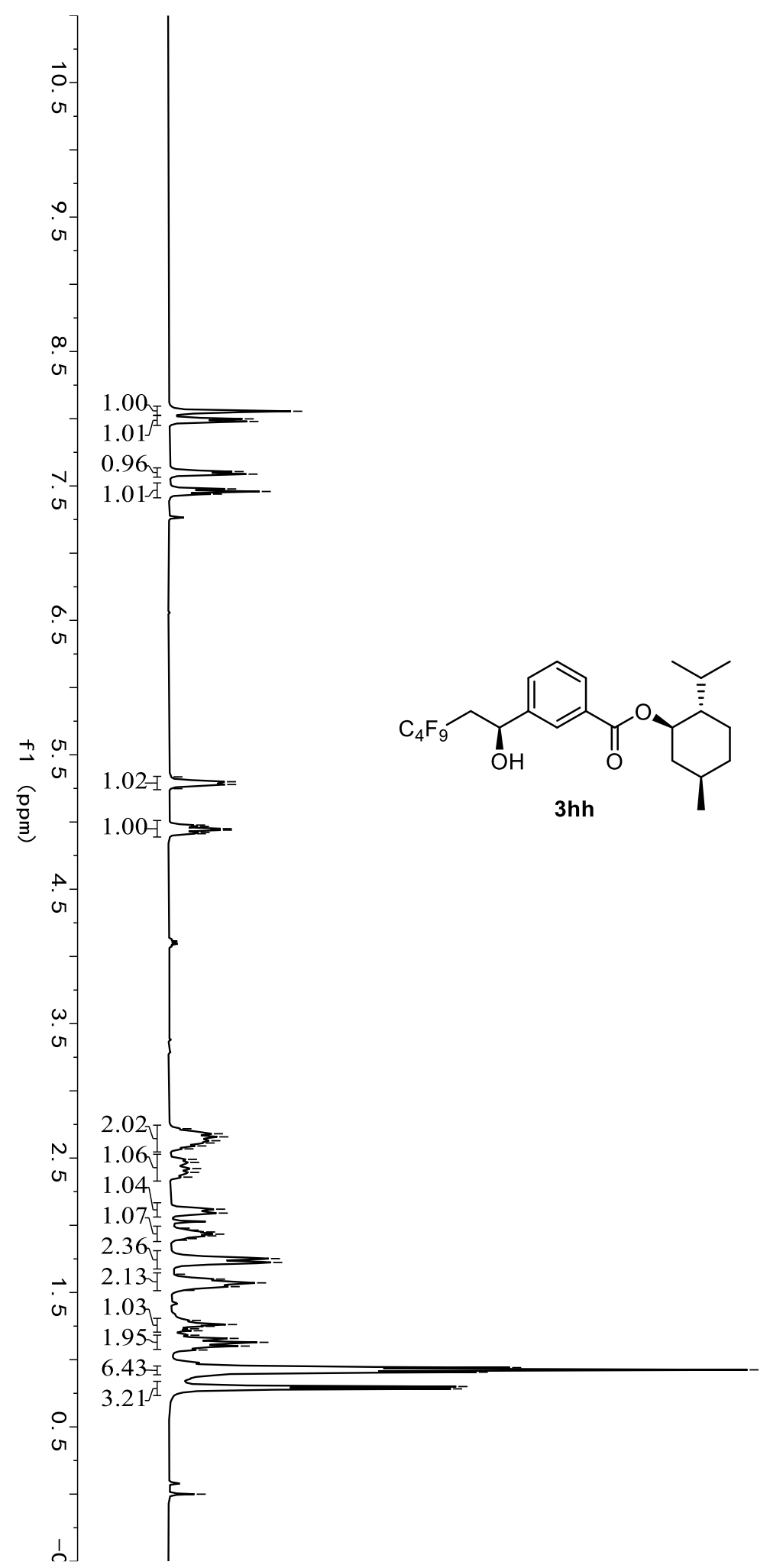

8. 06

8. 00

7. 98

7. 61

7. 59

7. 48

7. 46

7. 44

5. 30

5. 28

4. 98

4. 97

4. 95

4. 94

4. 92

4. 91

2. 72

2. 68

2. 66

2. 63

2. 61

2. 59

2. 57

2. 49

2. 47

2. 42

2. 39

2. 36

2. 12

2. 09

1. 96

1. 95

1. 93

1. 92

$-1.90$

1. 75

1. 72

$-1.60$

1. 57

1. 54

1. 52

1. 29

1. 26

1. 25

1. 23

1. 22

1. 18

1. 16

1.13

ก 1.10

[1.07

0.94

0.92

0.91

0.80

0.78

$-0.00$ 


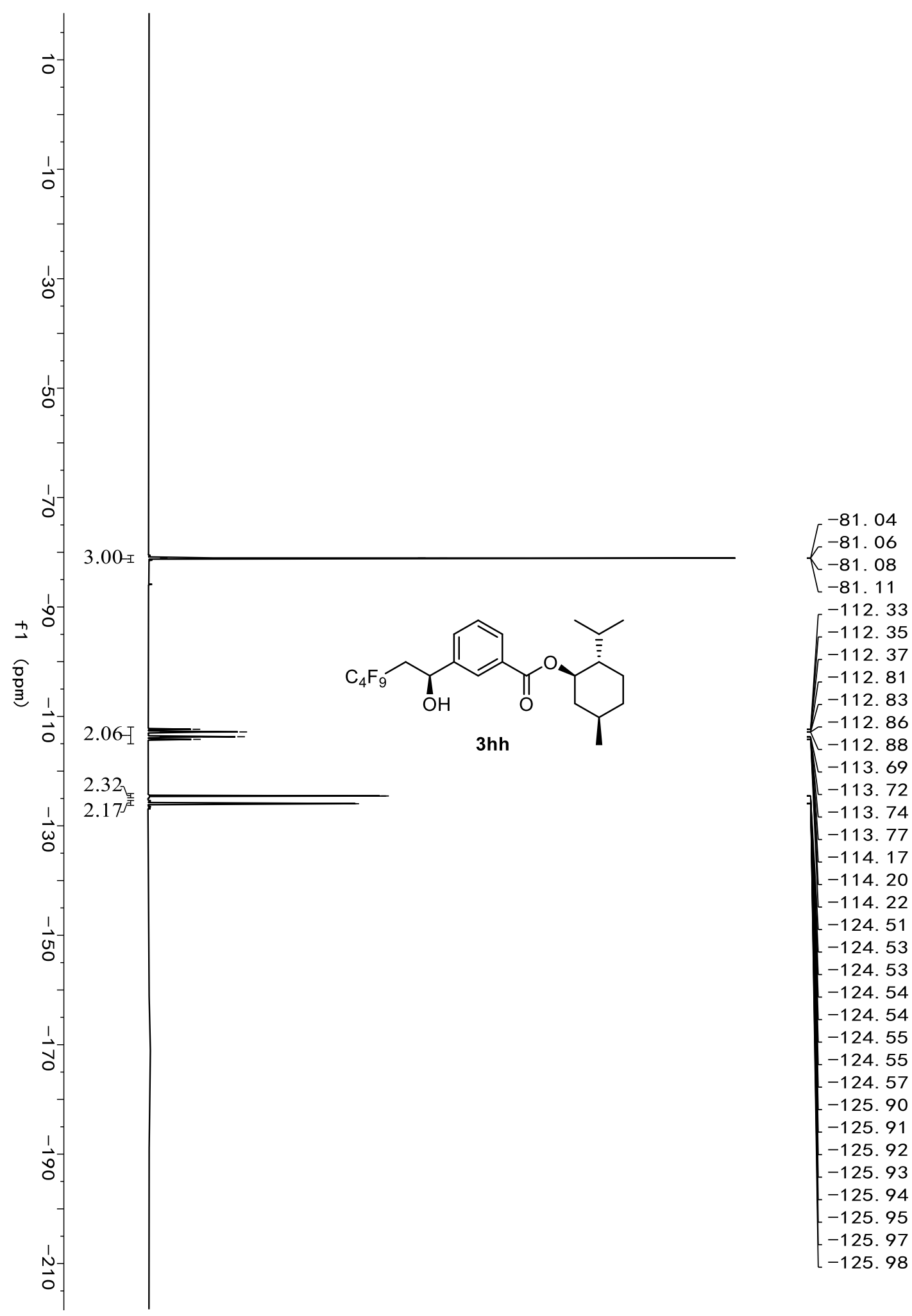




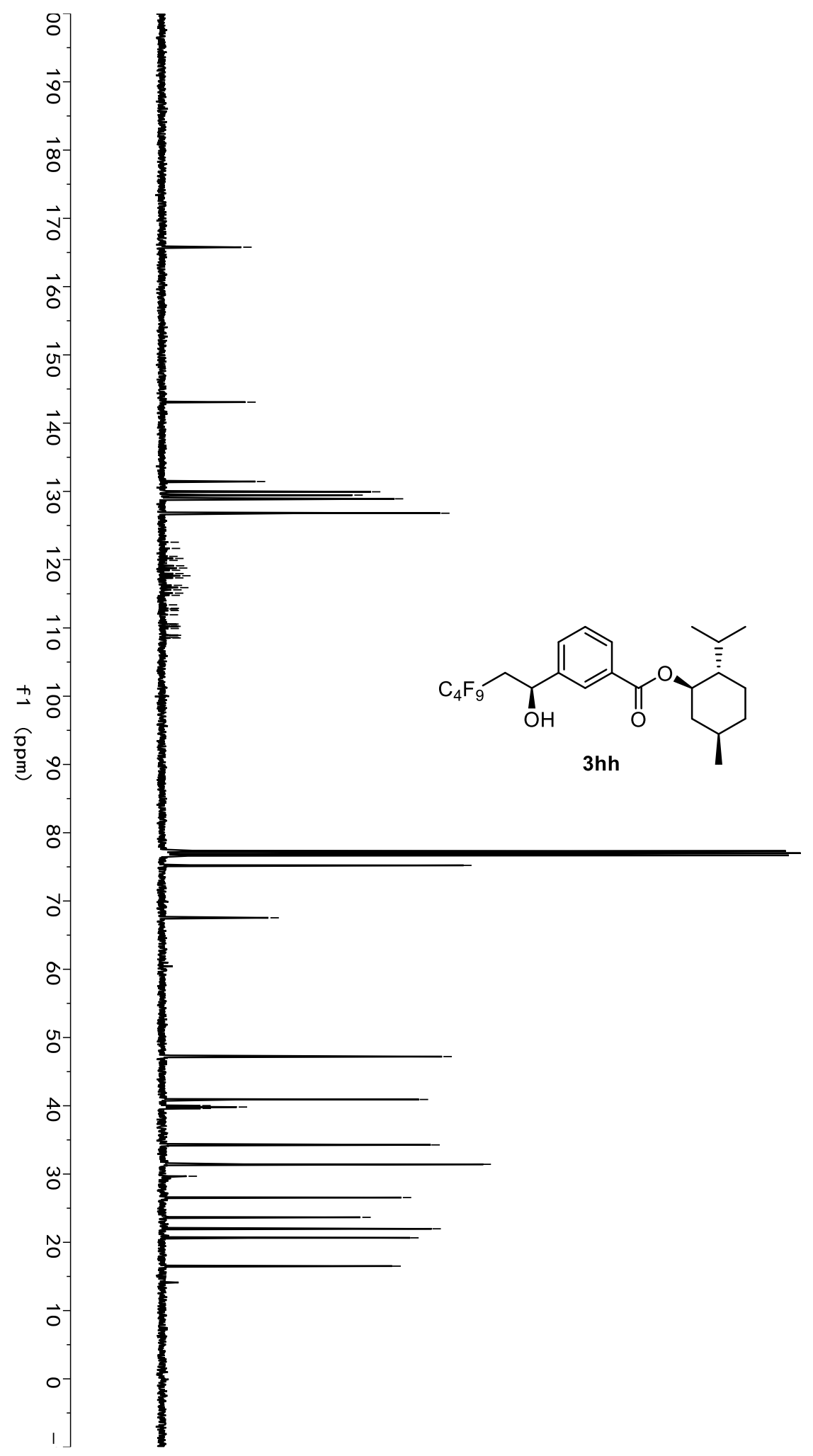

165. 8

143. 1

131.4

129. 9

129. 5

128. 9

126. 8

122. 6

121.6

120. 5

120. 2

119. 9

119. 1

118. 8

-118. 4

118. 0

117. 6

117. 3

116. 2

115.9

115.6

115. 1

114. 8

113. 4

112. 9

112. 8

112. 6

111.9

110.6

110. 6

110.5

110. 3

110. 2

110. 2

109. 9

109. 0

108. 9

108. 9

108. 6

108. 5

75. 2

67. 5

47. 2

40. 9

40. 0

39. 8

39.6

34. 3

31. 4

29. 7

26. 6

23. 7

22. 0

20. 7

16. 5 


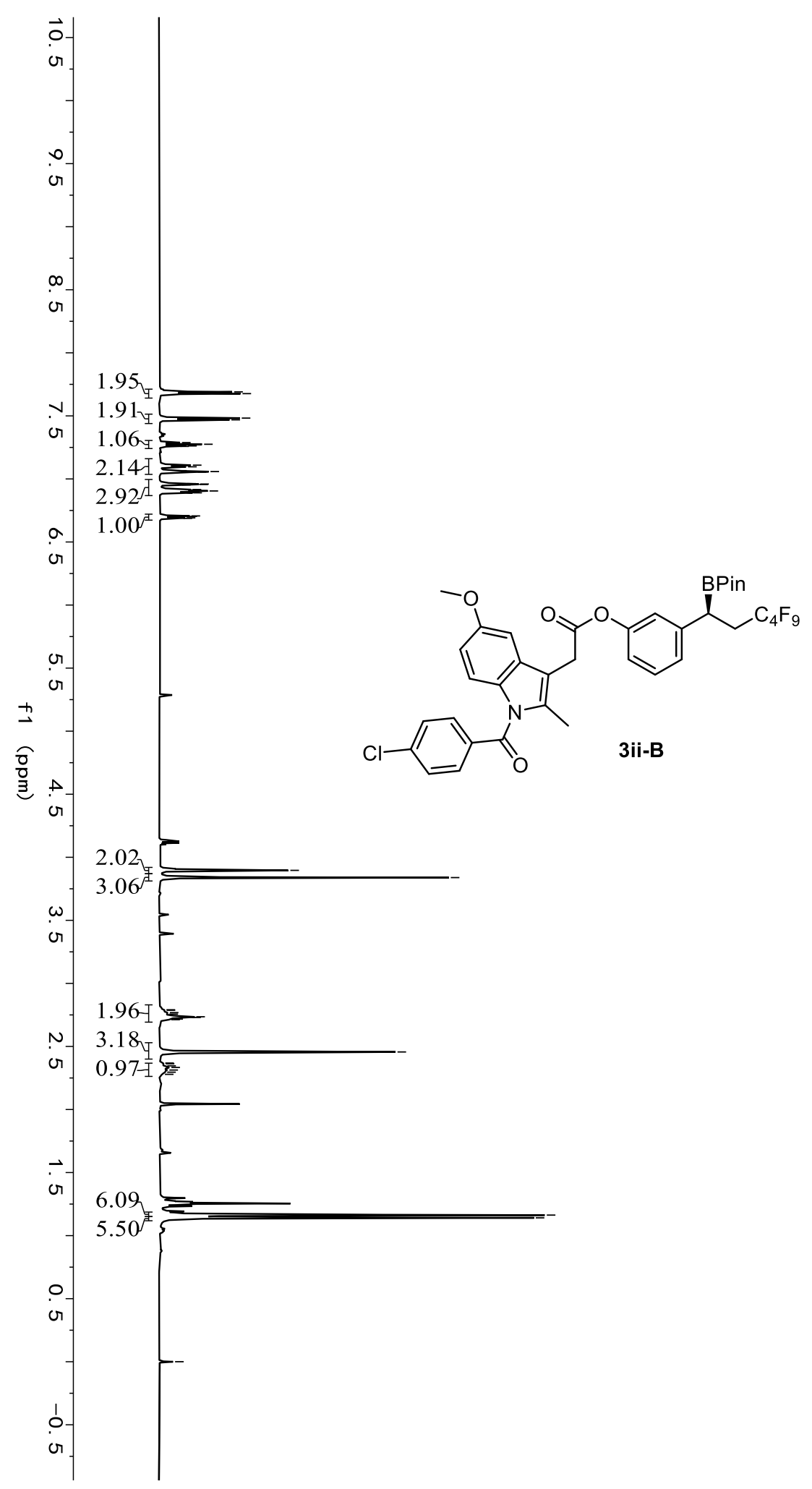

2. 72

2. 71

2. 46

2. 37

2. 36

2. 35

2. 33

2. 31

2. 30

2. 28

1. 16

1. 14

[0. 00 


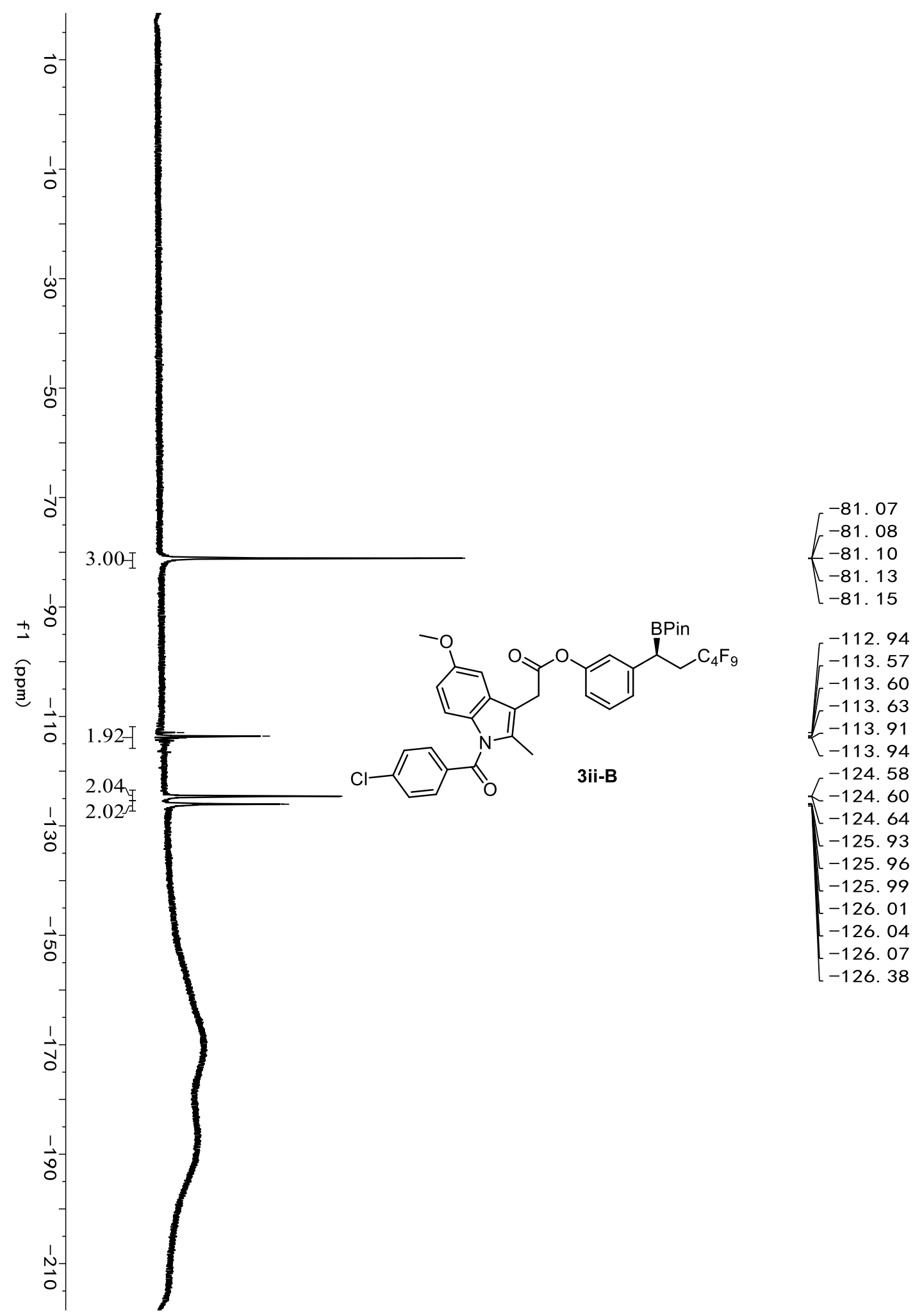




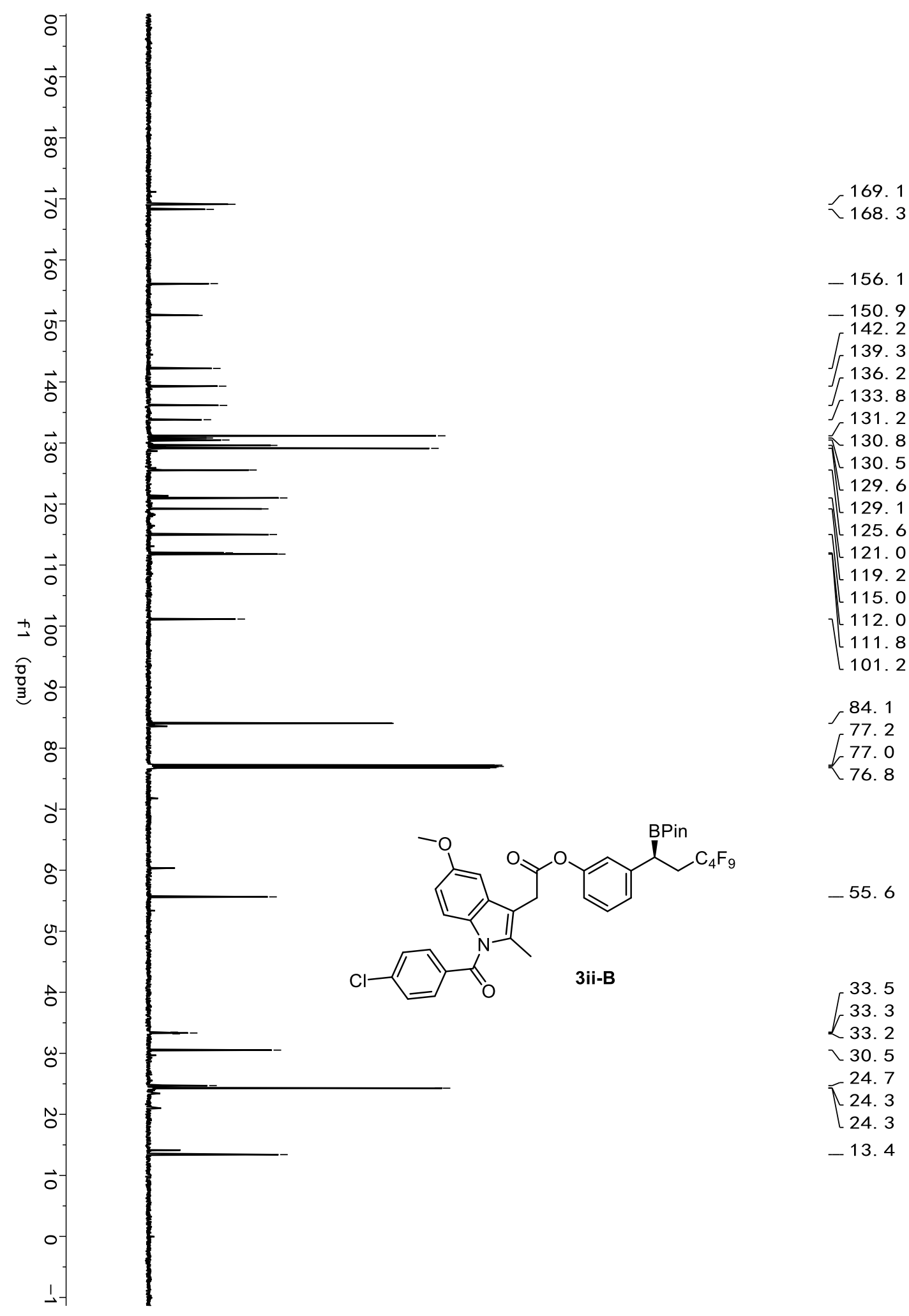



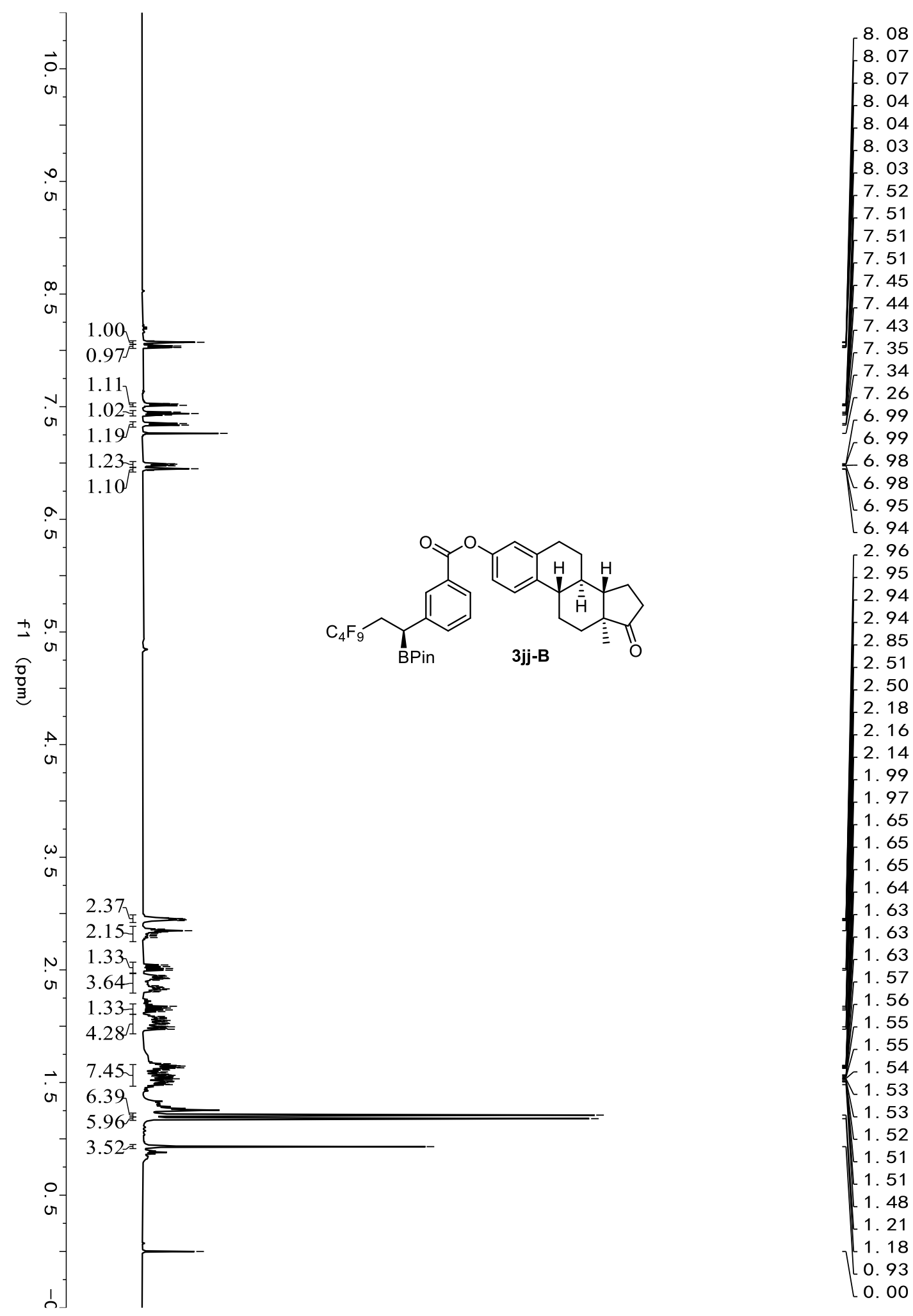


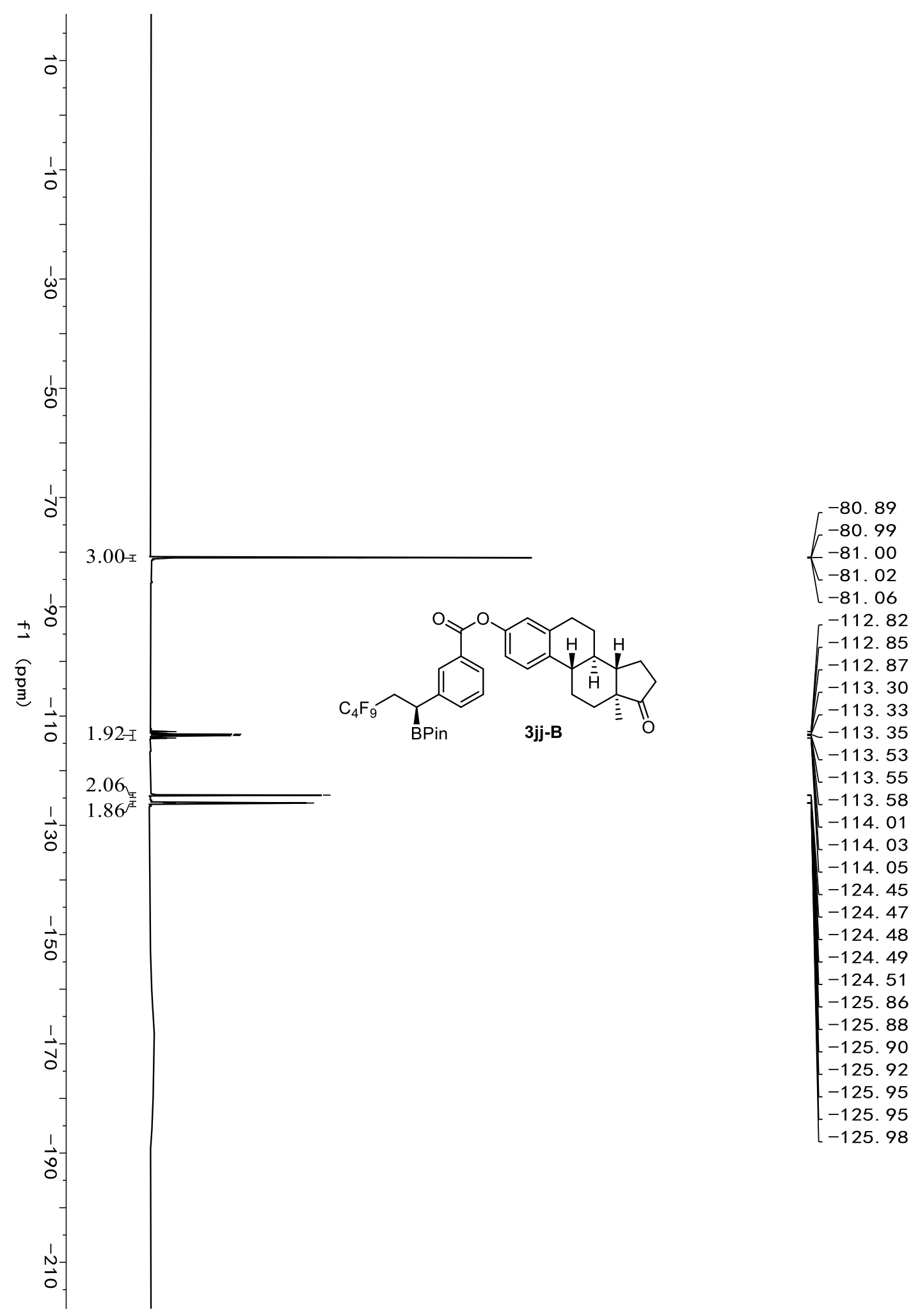




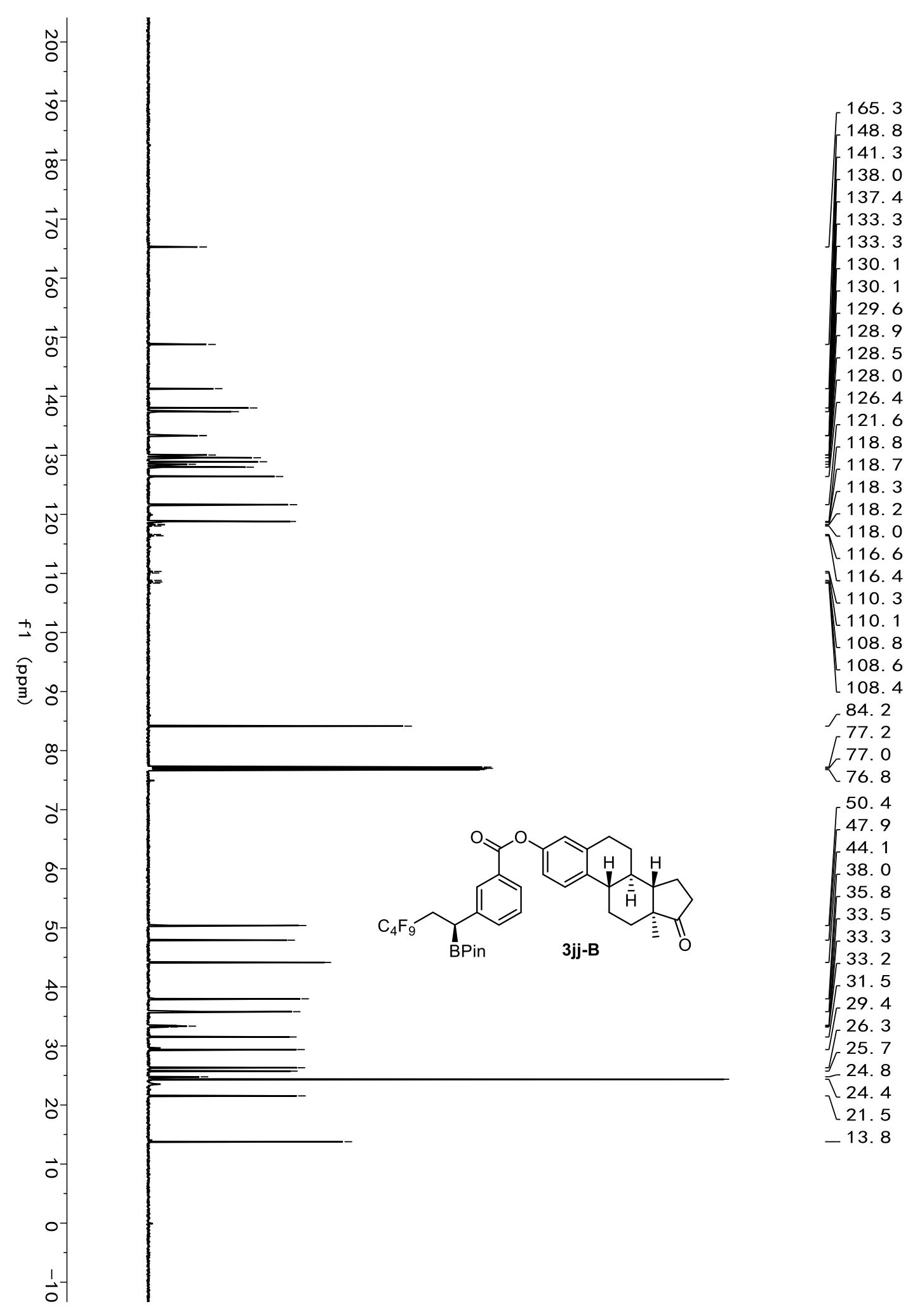




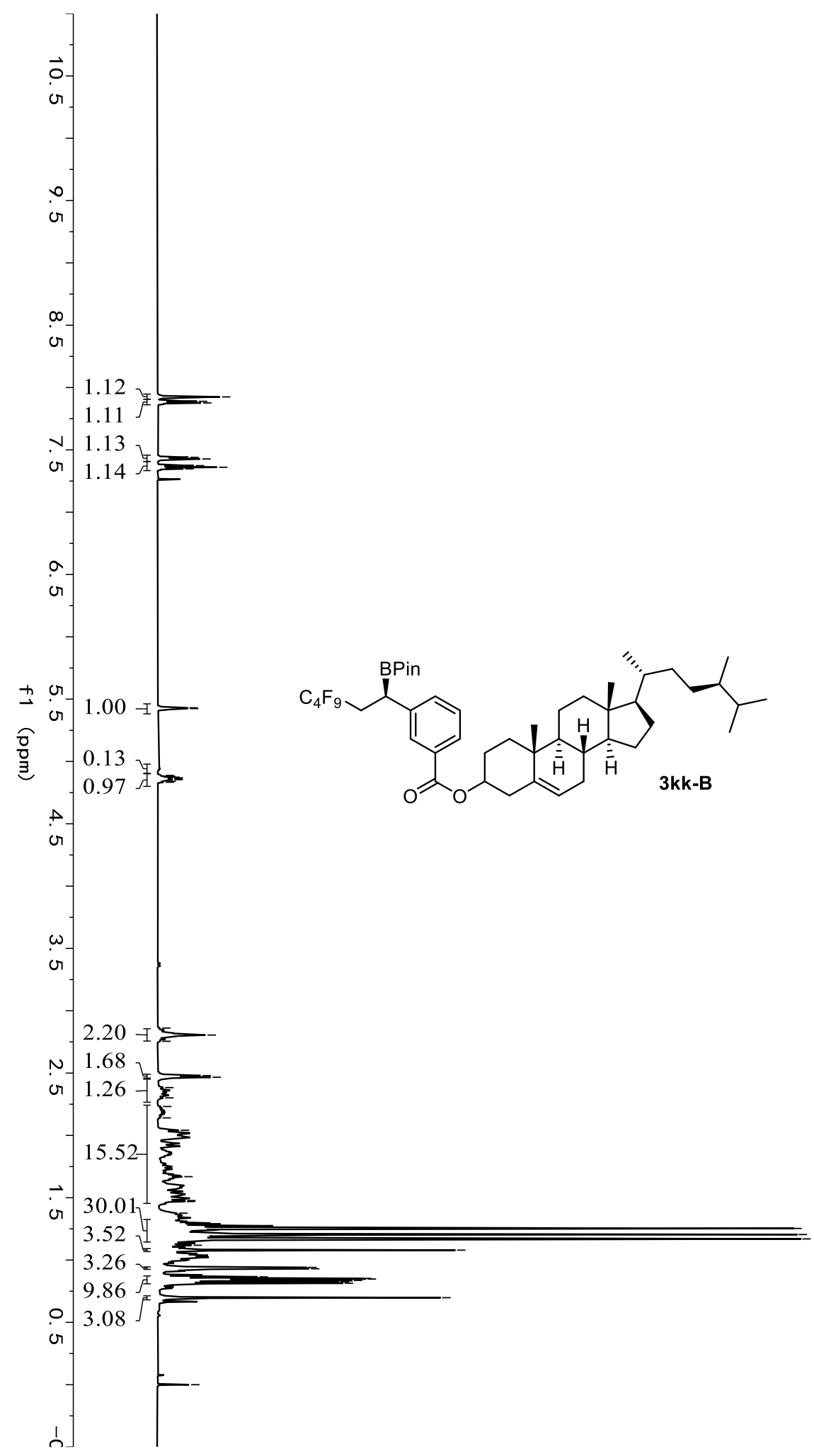

4. 87

4. 86

-4. 85

4. 84

4. 83

2. 86

2. 81

2. 76

2. 48

2. 47

2. 38

2. 30

2. 23

2. 14

2. 04

1. 67

1. 48

1. 47

1. 38

1. 25

1. 20

1. 17

1. 12

1. 08

0.94

0.93

0.86

0.85

0.85

0.84

0. 83

0.81

[0.70

0. 00 


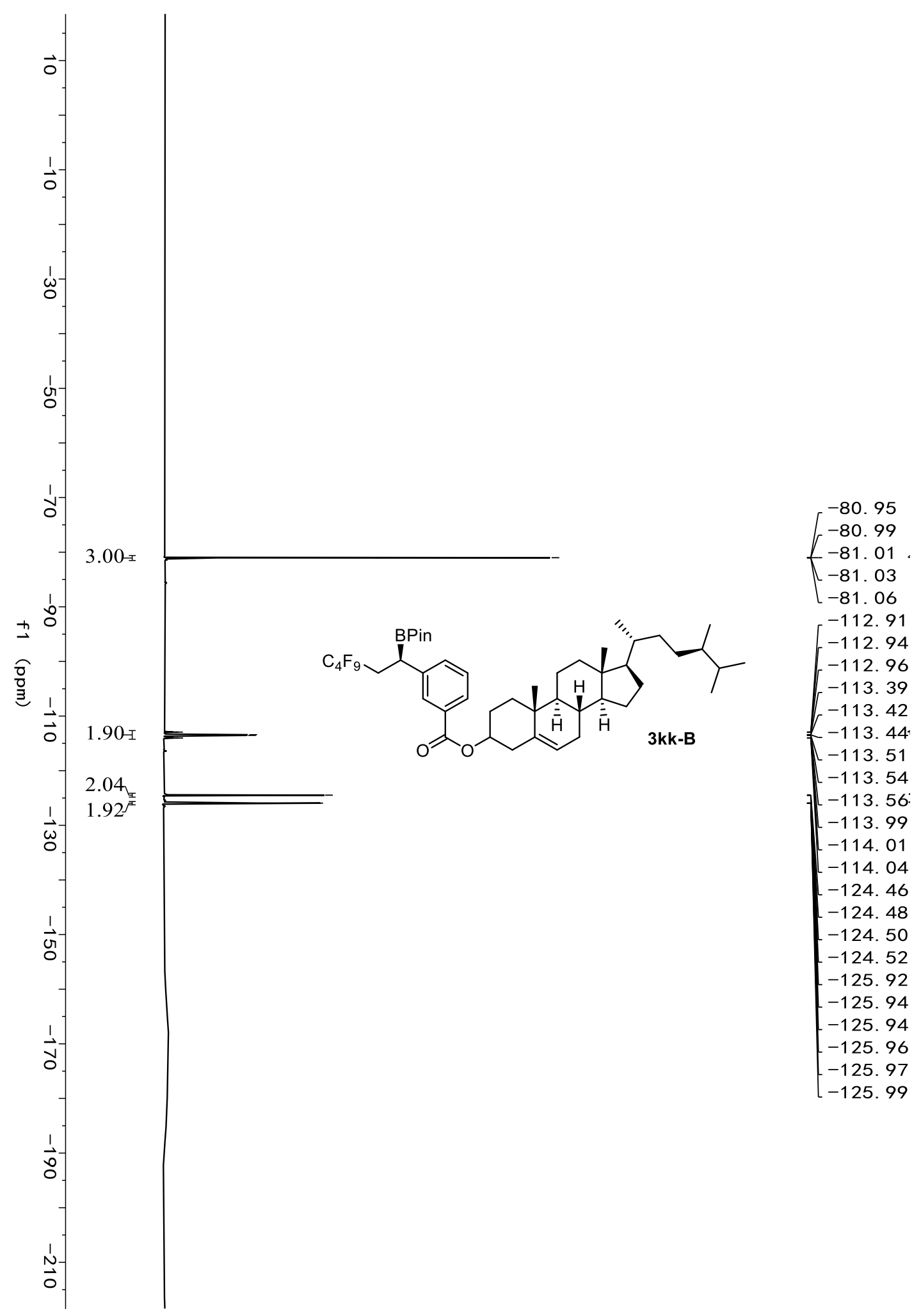




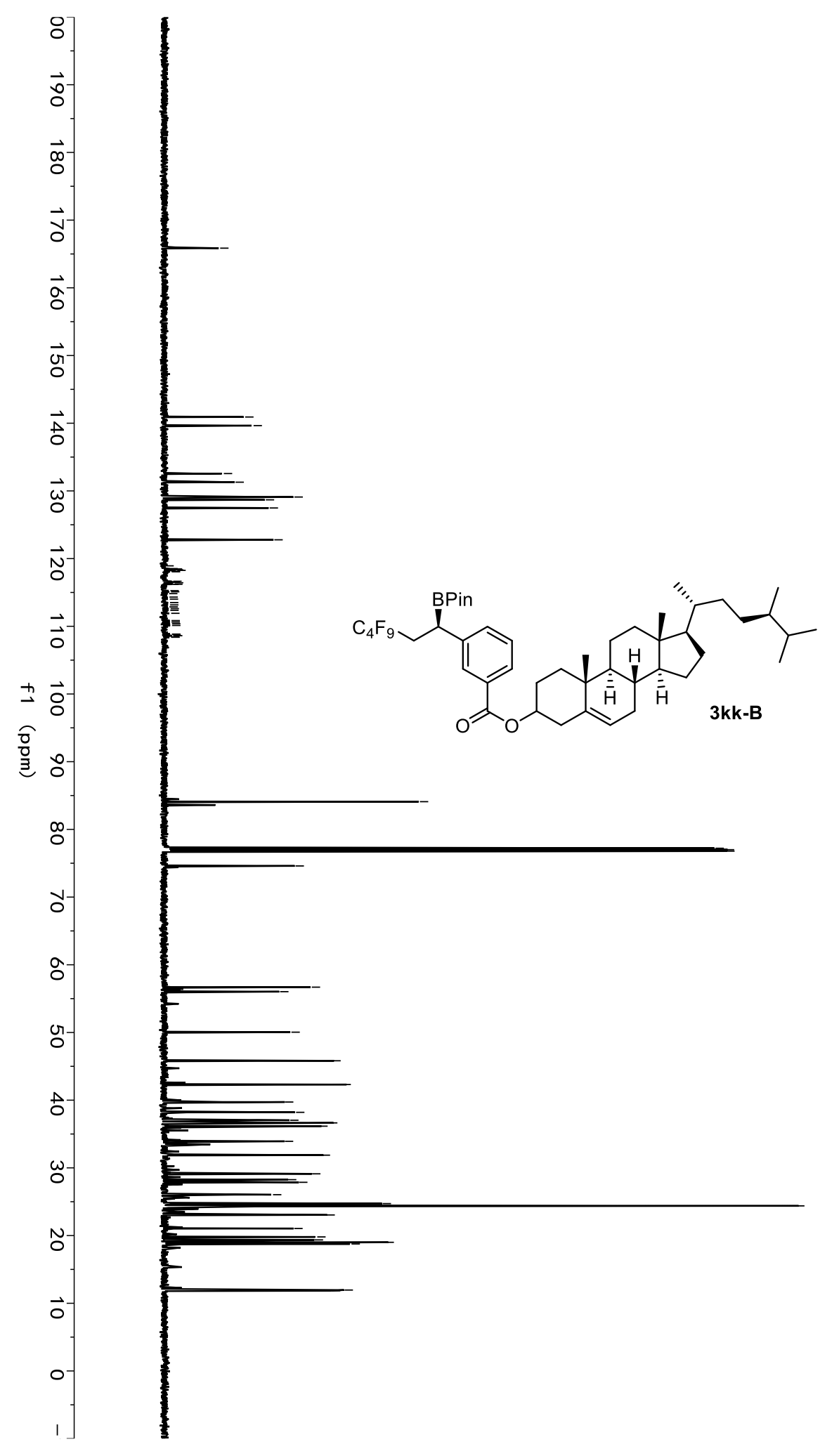

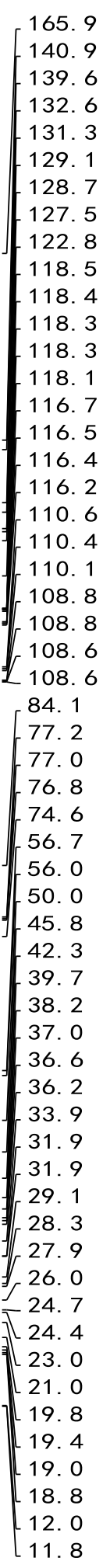




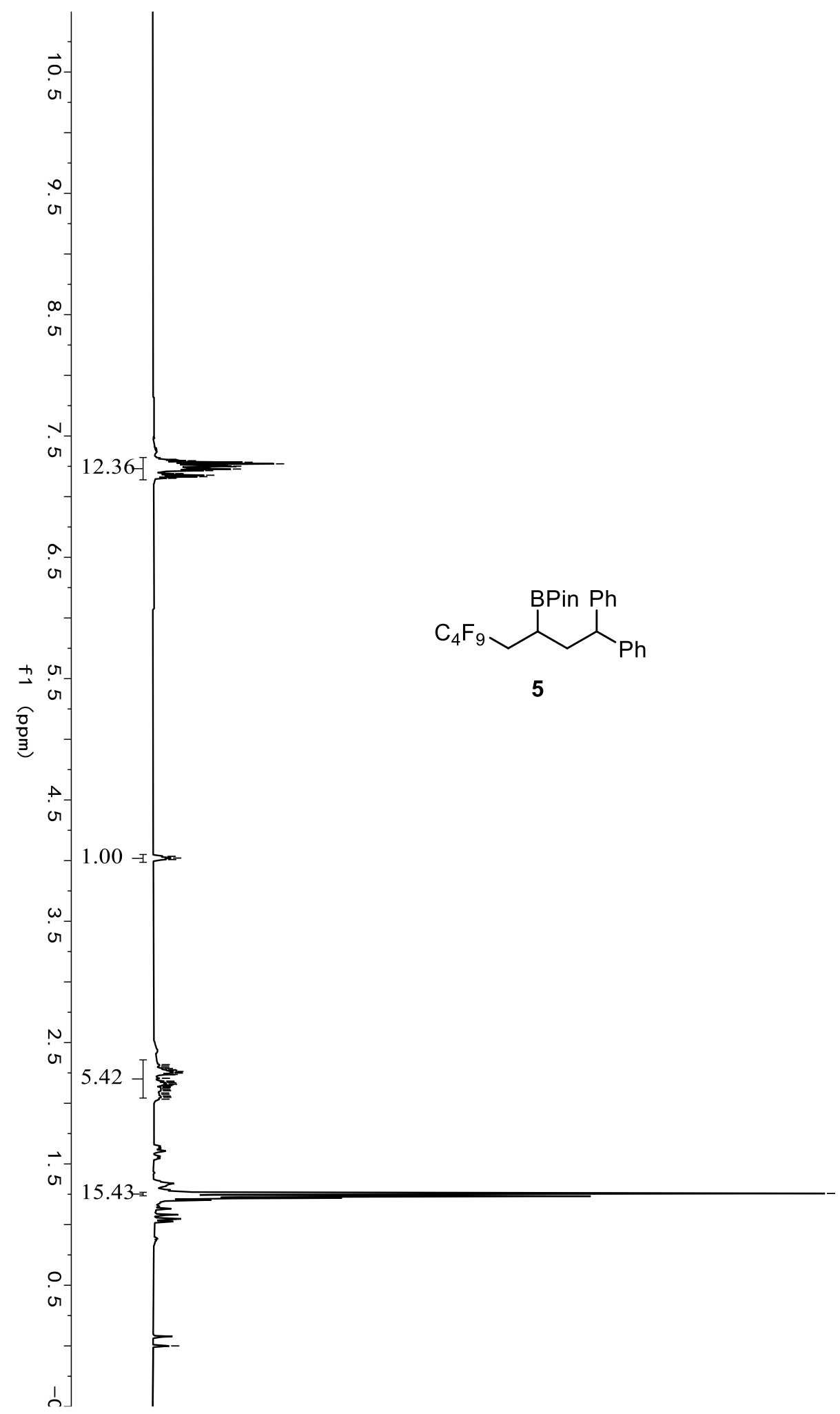

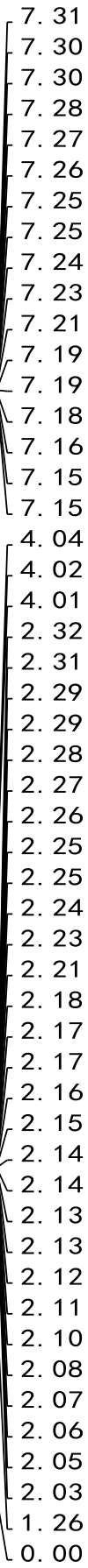




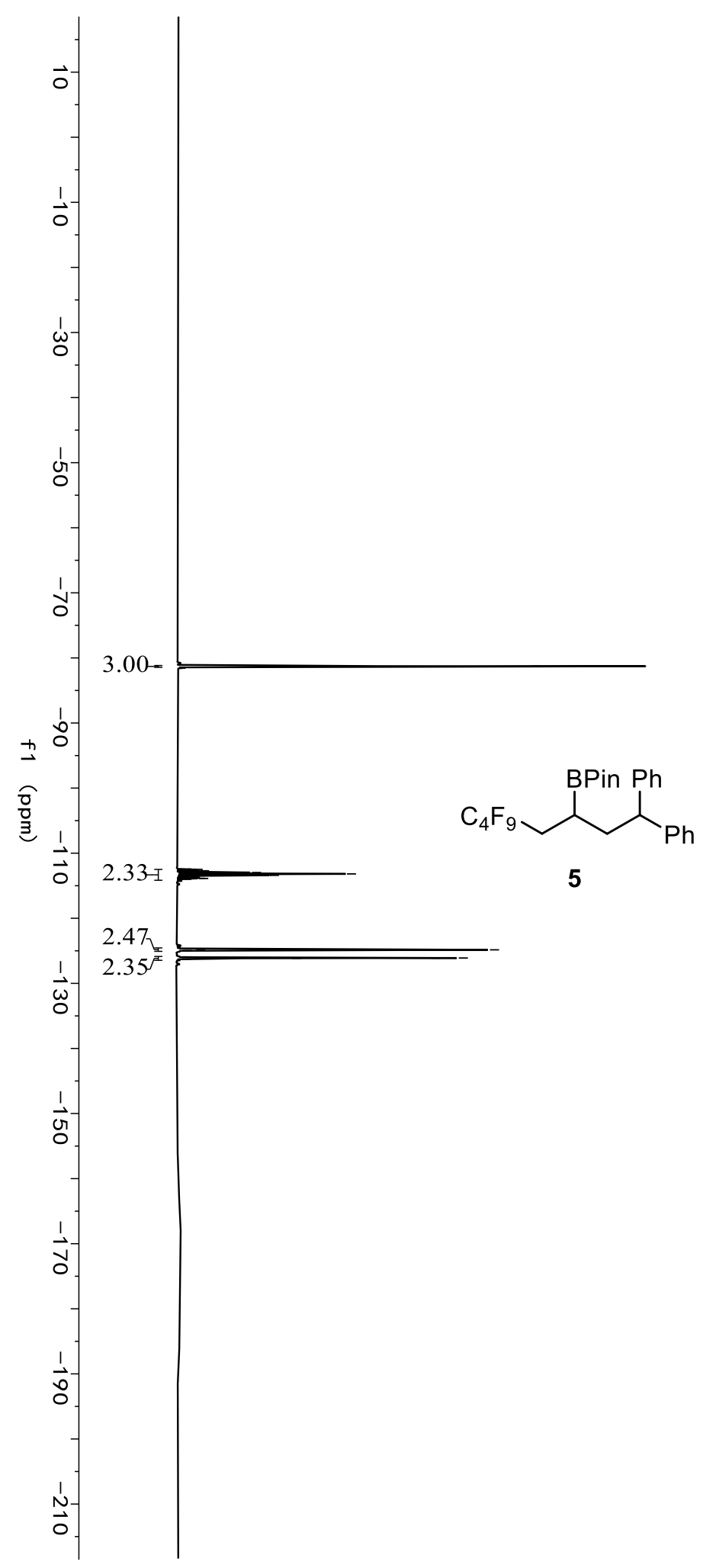

$-81.27$

$-81.28$

$-81.29$

$-81.31$

$-112.47$

$-112.49$

$-112.51$

$-112.68$

$-112.70$

$-112.73$

$-112.87$

$-112.89$

$-112.95$

$-112.97$

$-113.00$

$-113.16$

$-113.18$

-113. 21

$-113.32$

$-113.38$

$-113.40$

$-113.43$

$-113.66$

$-113.68$

$-113.71$

$-113.86$

$-113.88$

$-113.91$

$-113.97$

$-113.99$

$-124.78$

$-124.83$

$-124.85$

$-124.87$

$-124.97$

$-125.95$

$-126.08$

$-126.10$

$-126.12$

$-126.15$

-126. 27 


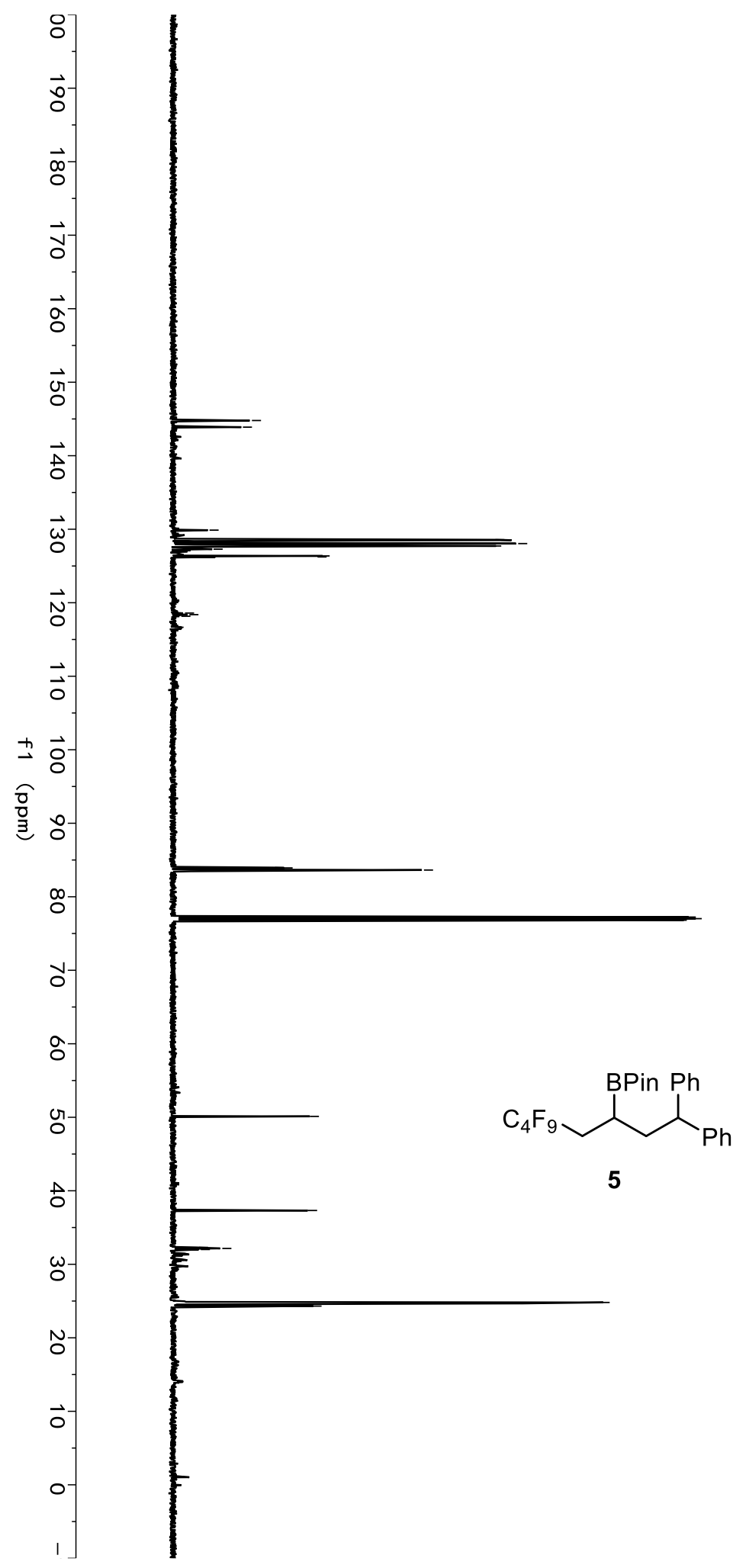

144. 8

143. 9

129.9

128. 6

128. 5

128. 3

128. 1

128. 0

127. 7

127. 3

126. 4

126. 2

118. 6

118. 4

118. 2

116. 7

116. 4

84. 0

83.9

83.6

77.2

77.0

76. 8

$-50.1$

37. 3

32.3

32.2

<2. 0

24.8

$\left\{\begin{array}{l}24.7 \\ 24.3\end{array}\right.$ 

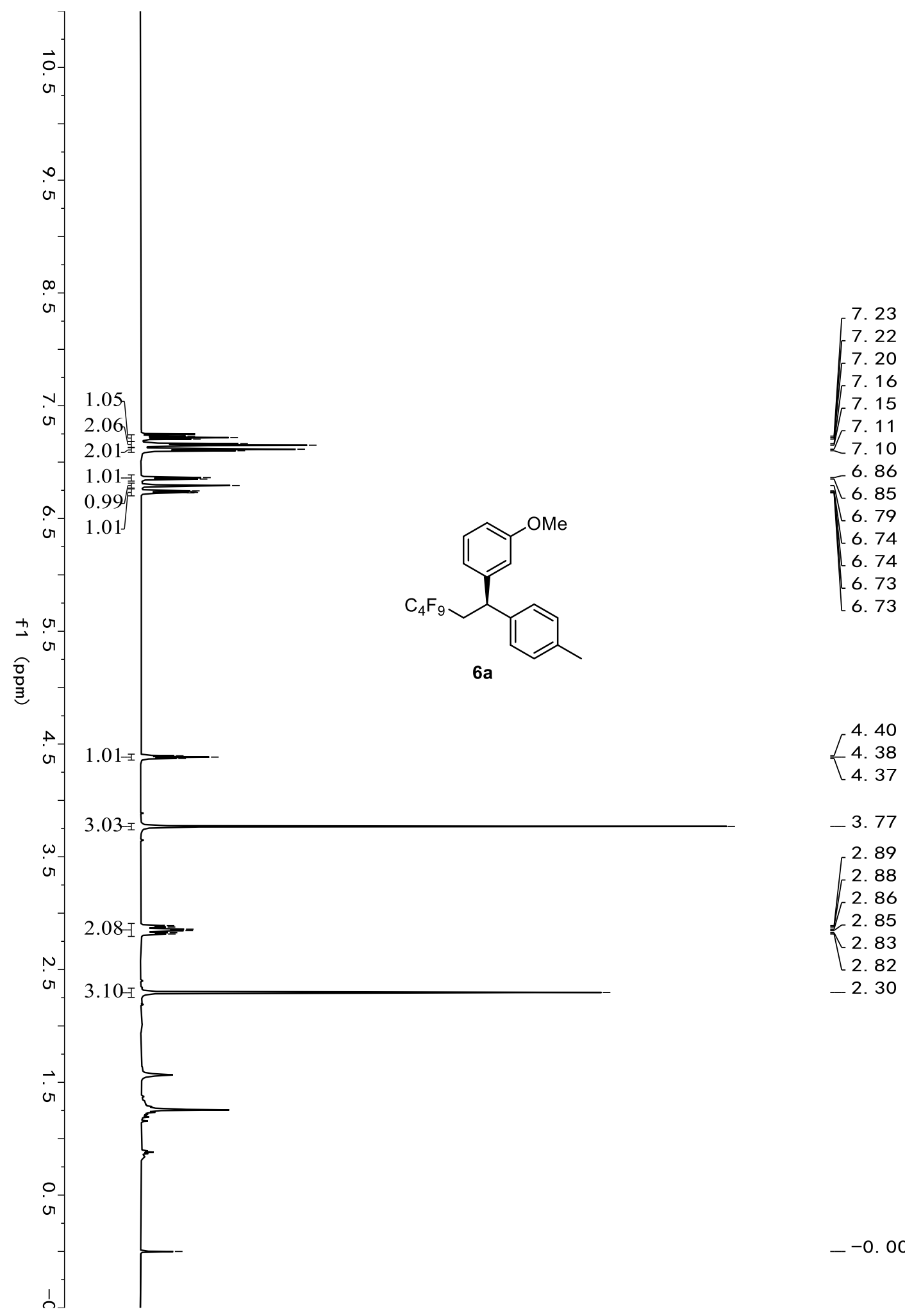

$\left\{\begin{array}{l}4.40 \\ 4.38 \\ 4.37\end{array}\right.$

$-3.77$

$\left[\begin{array}{l}2.89 \\ 2.88\end{array}\right.$

2.86
2.85

2.83

2. 82

$-2.30$

$--0.00$

230 


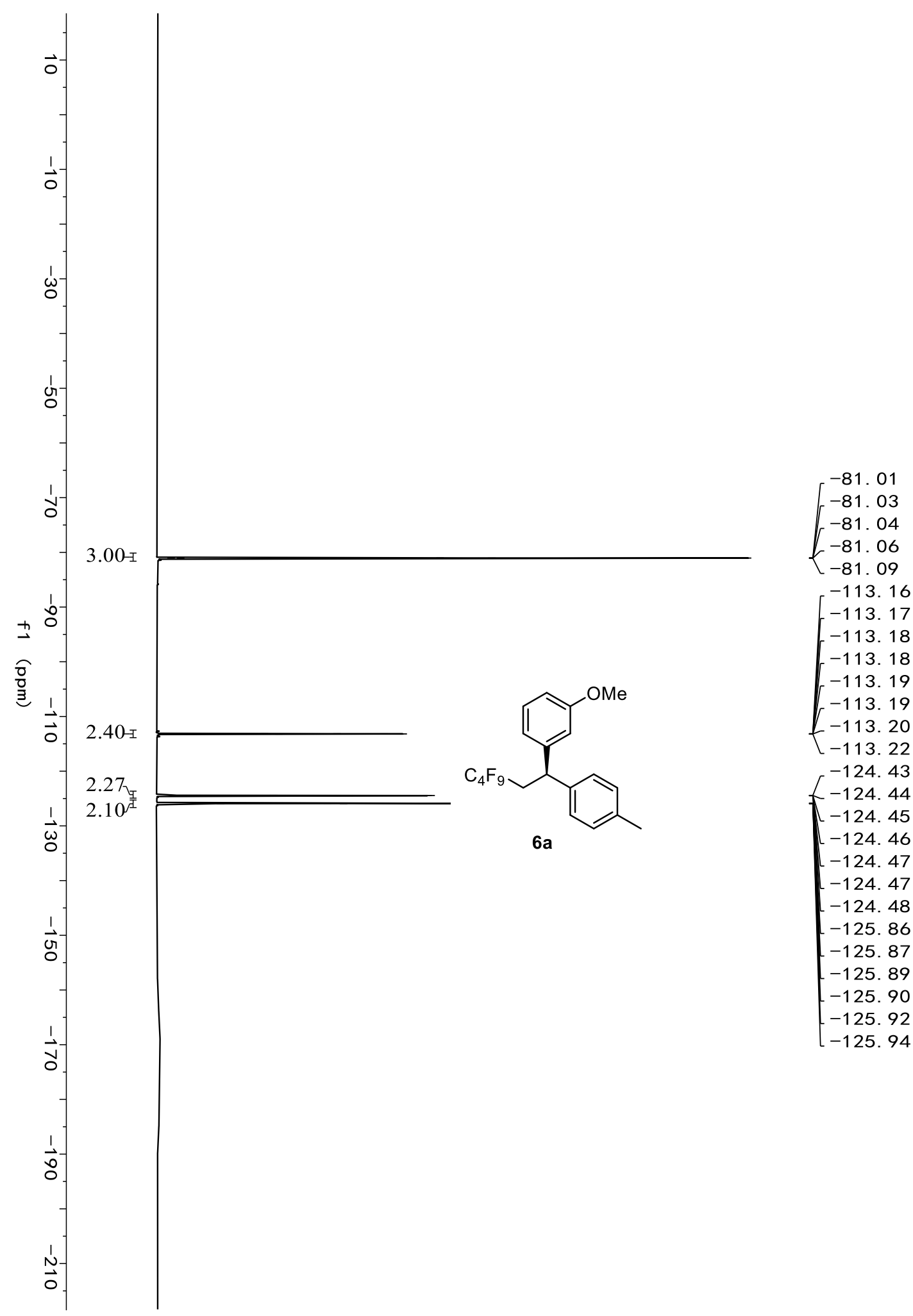




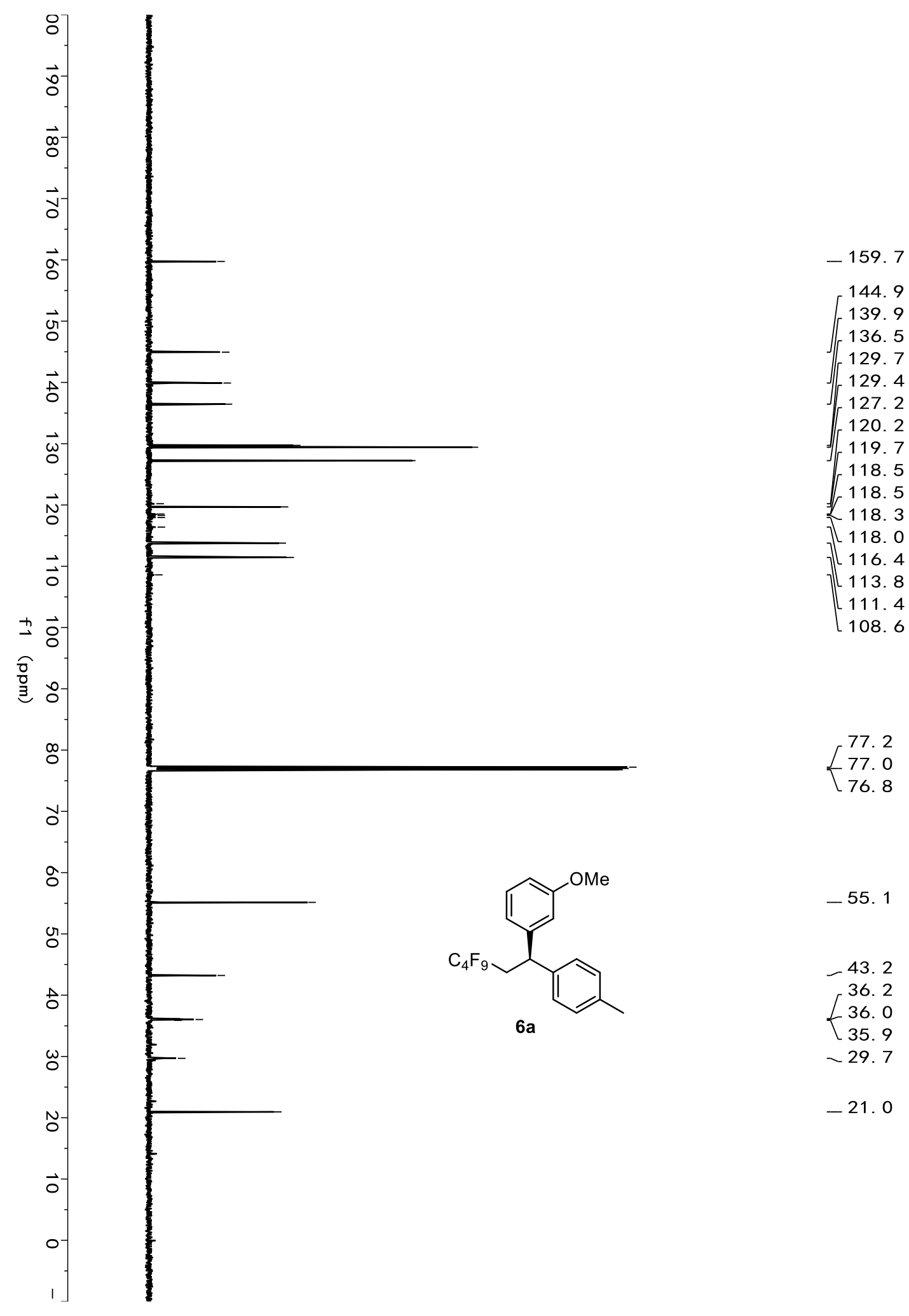




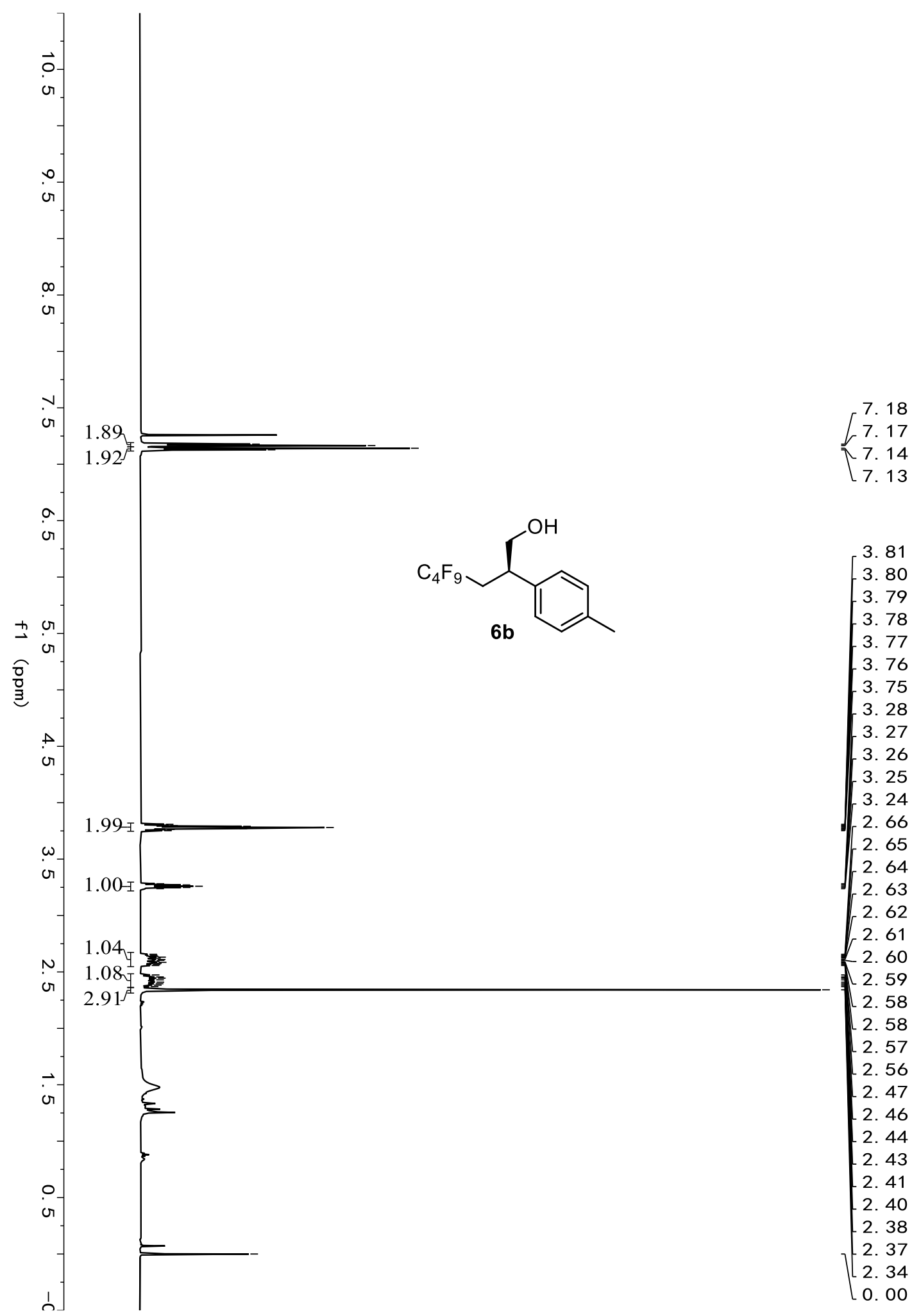




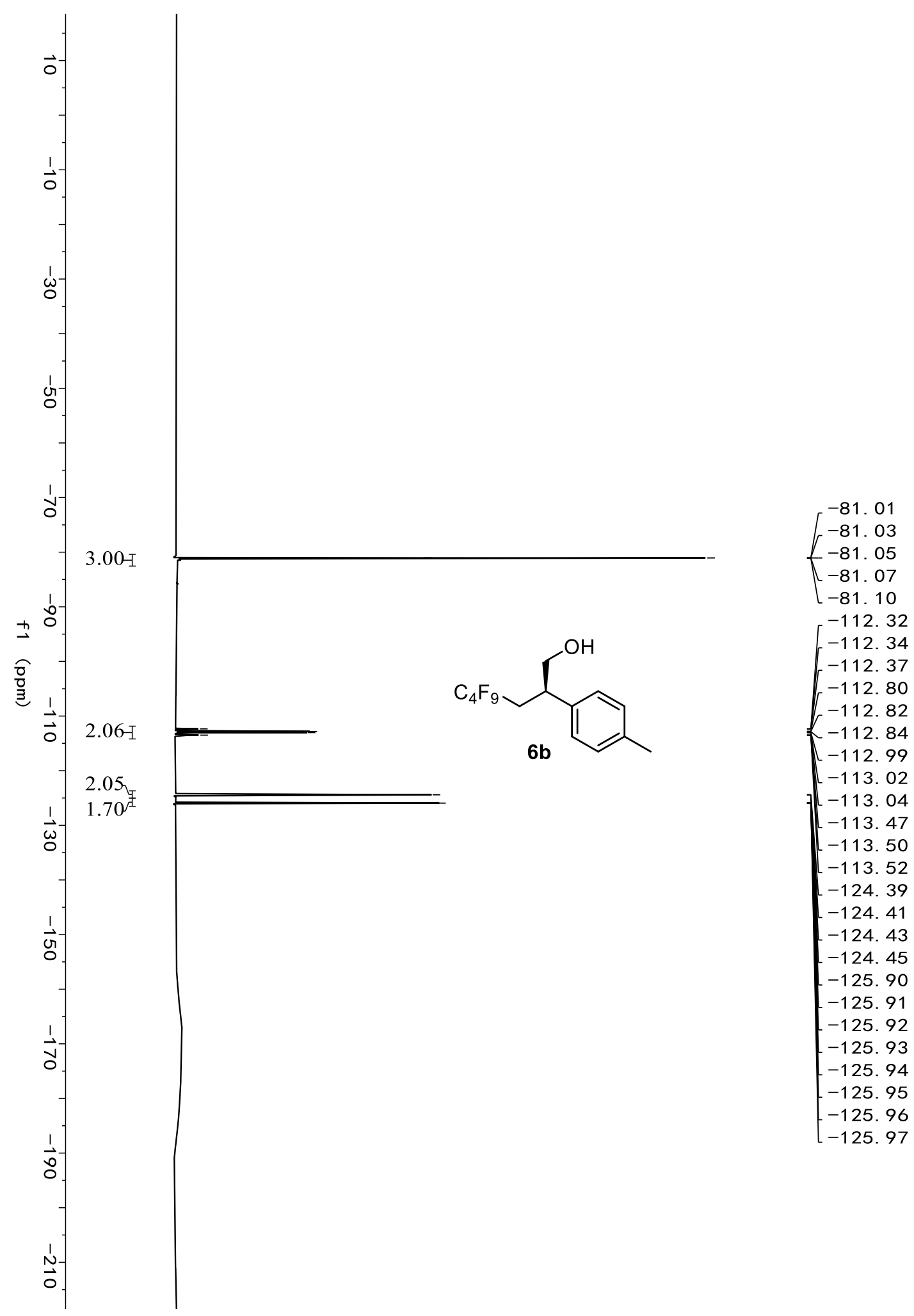




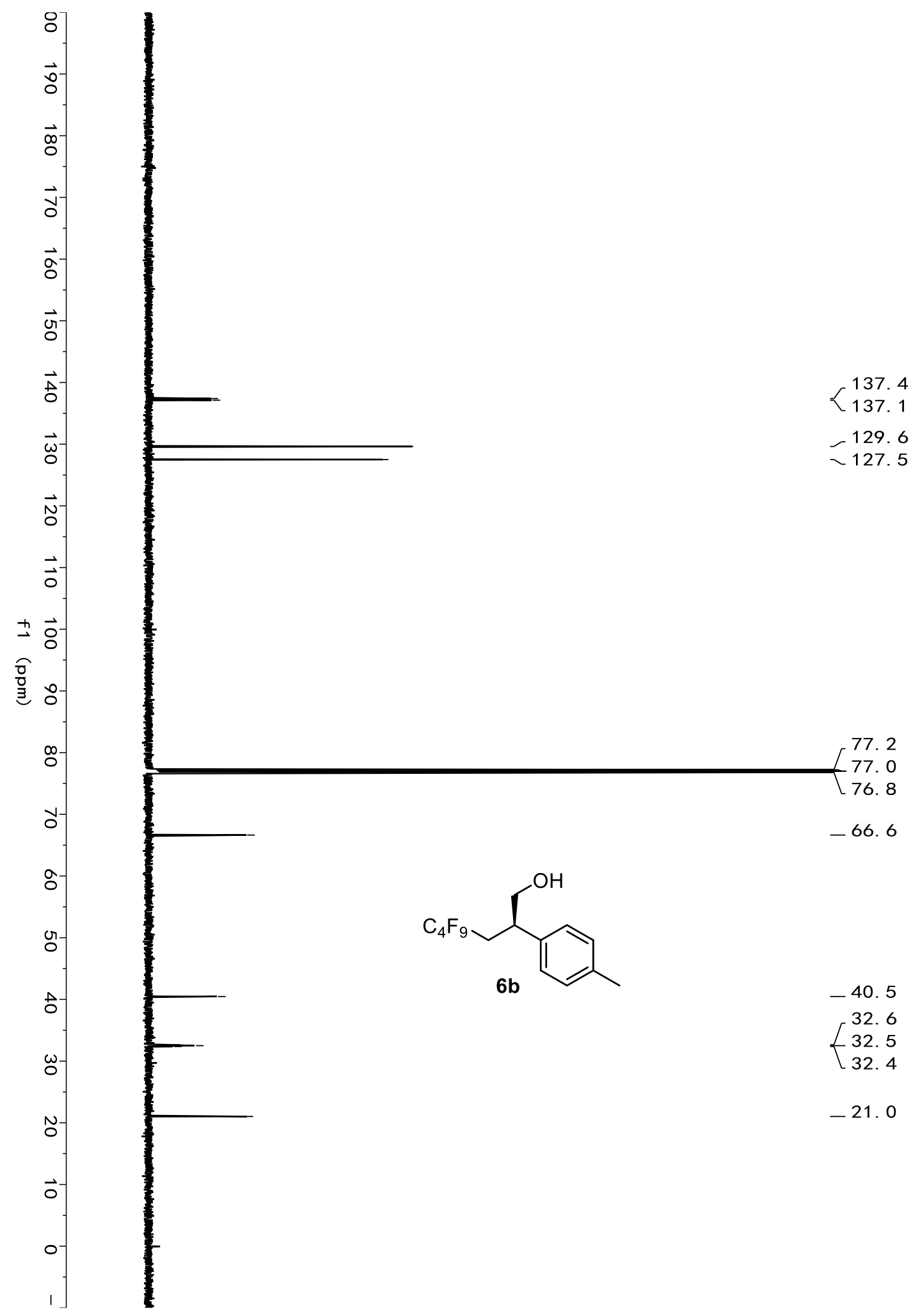




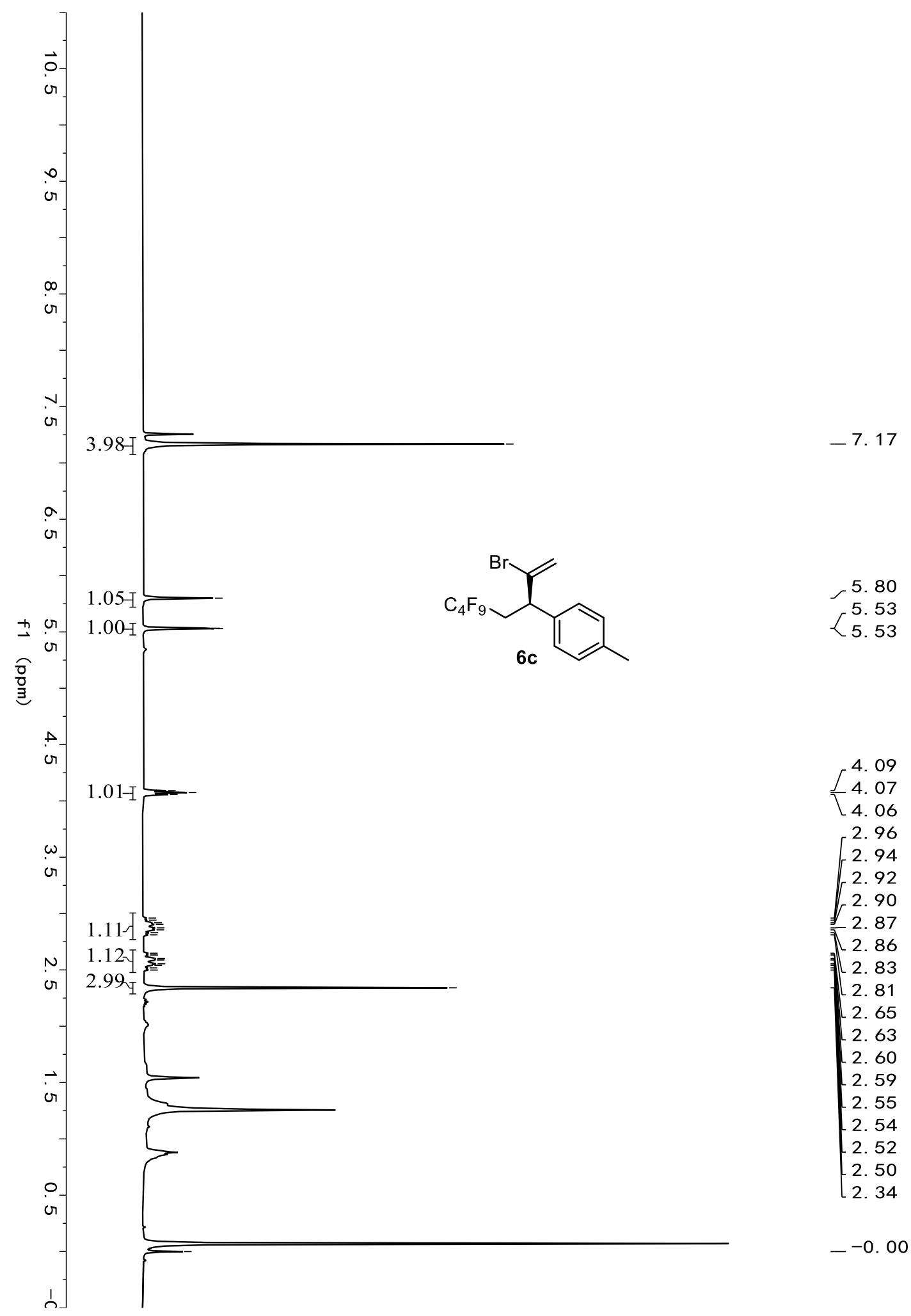




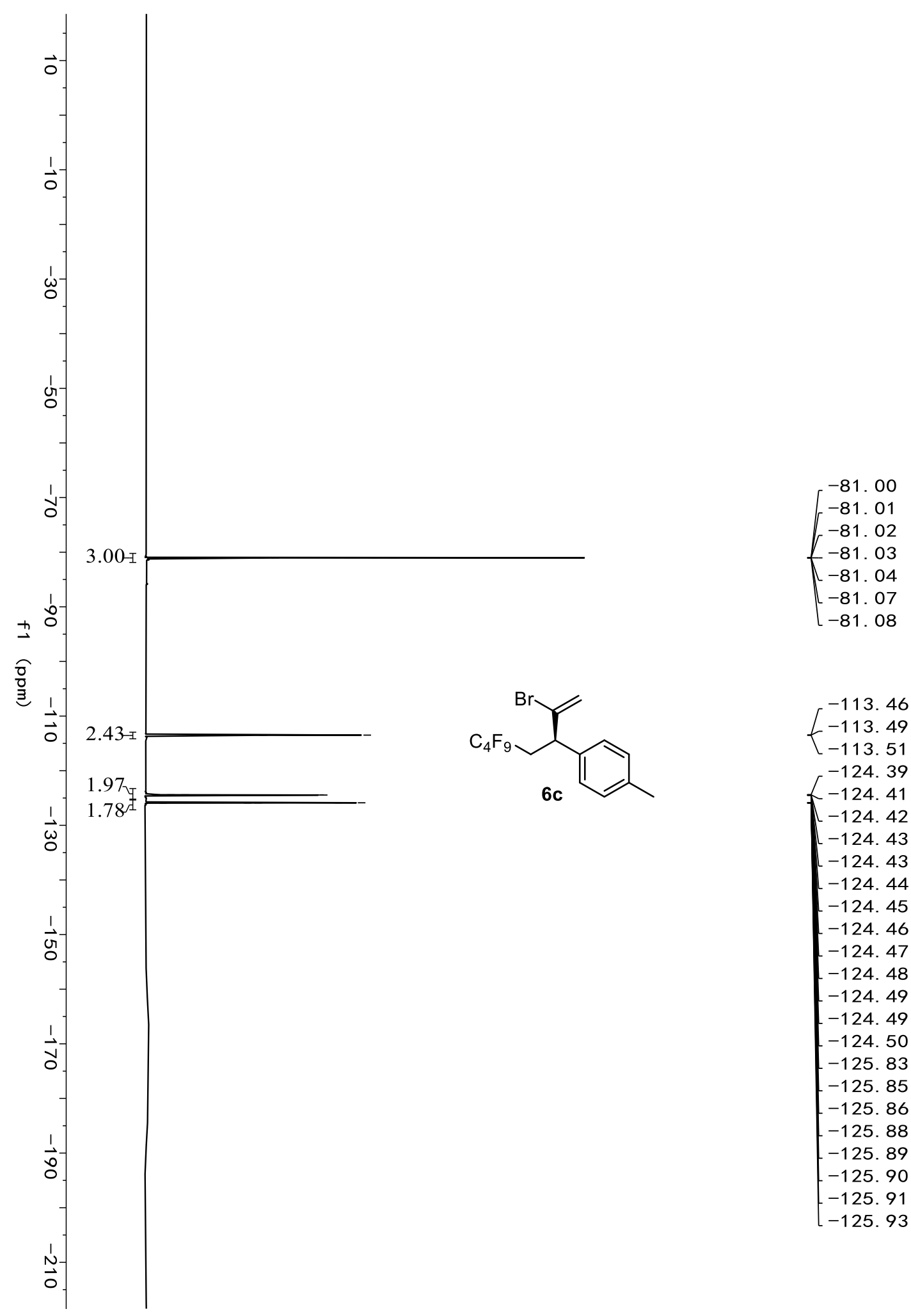




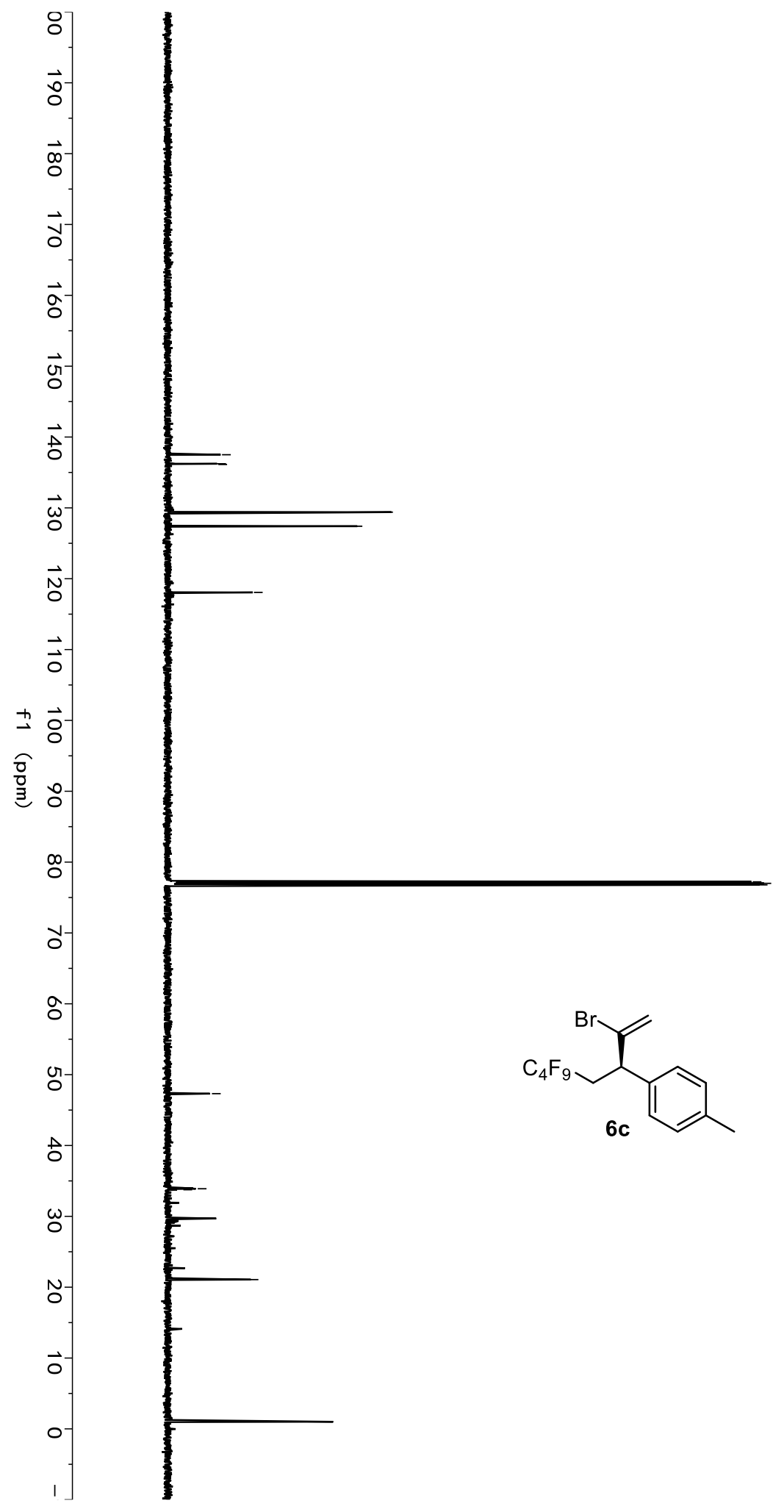

137.5
$\leftarrow 136.2$
136.1
-129.4
$\ulcorner 127.4$
-118.1

77.2
$\left\{\begin{array}{r}77.0 \\ 76.8\end{array}\right.$

$-47.3$

$\left\{\begin{array}{r}34.0 \\ 33.9 \\ 33.8\end{array}\right.$

$-21.1$

238 


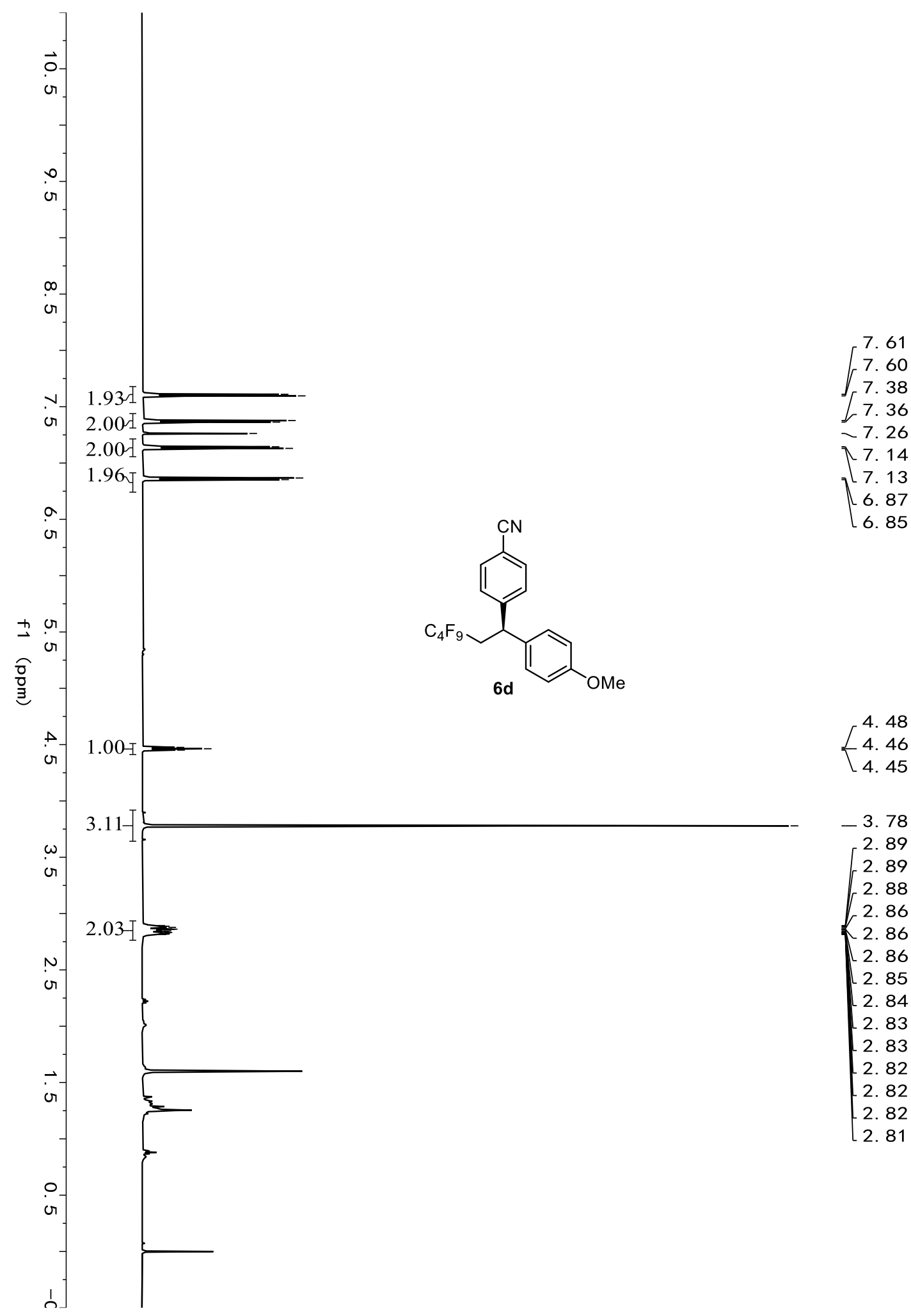




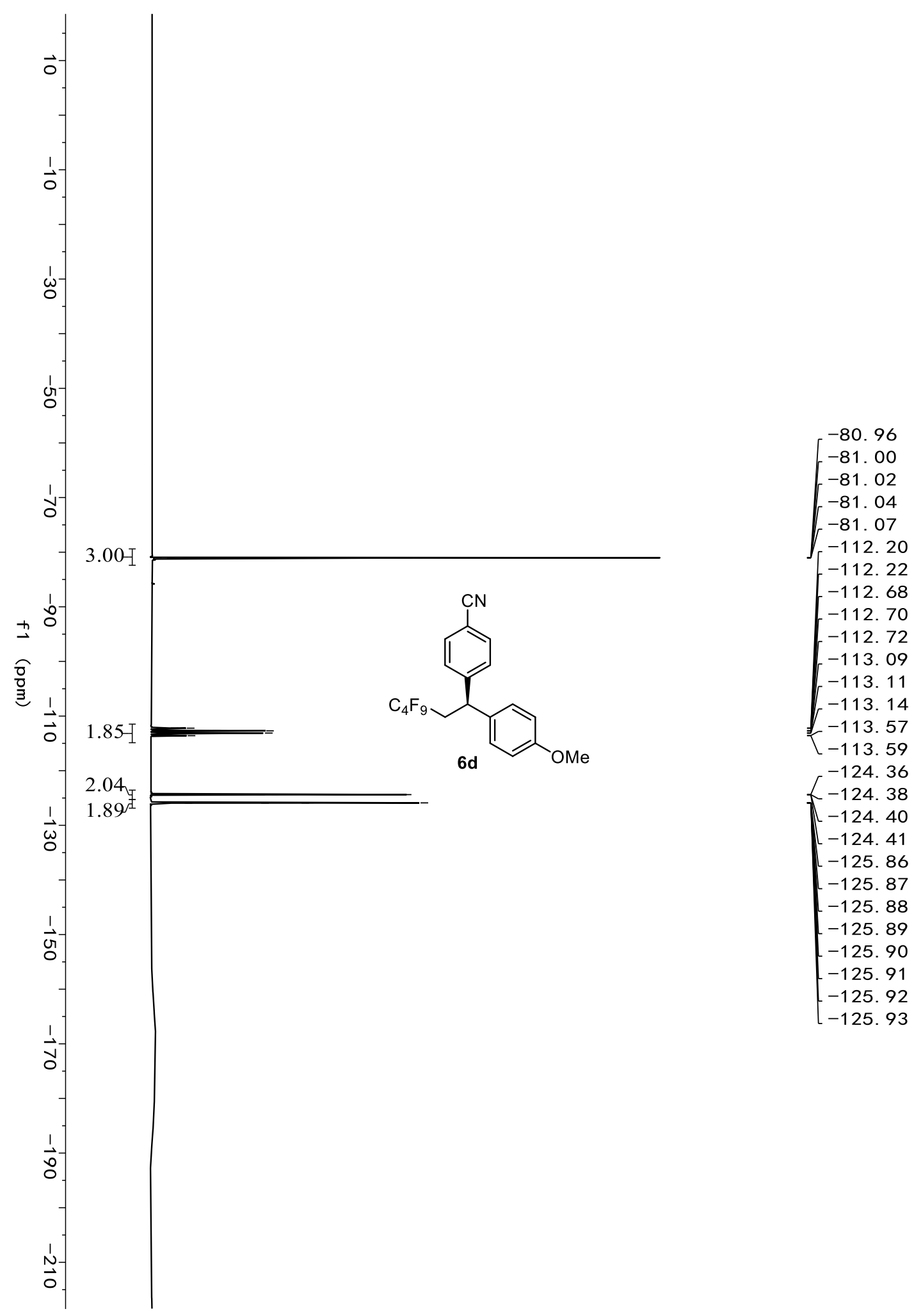




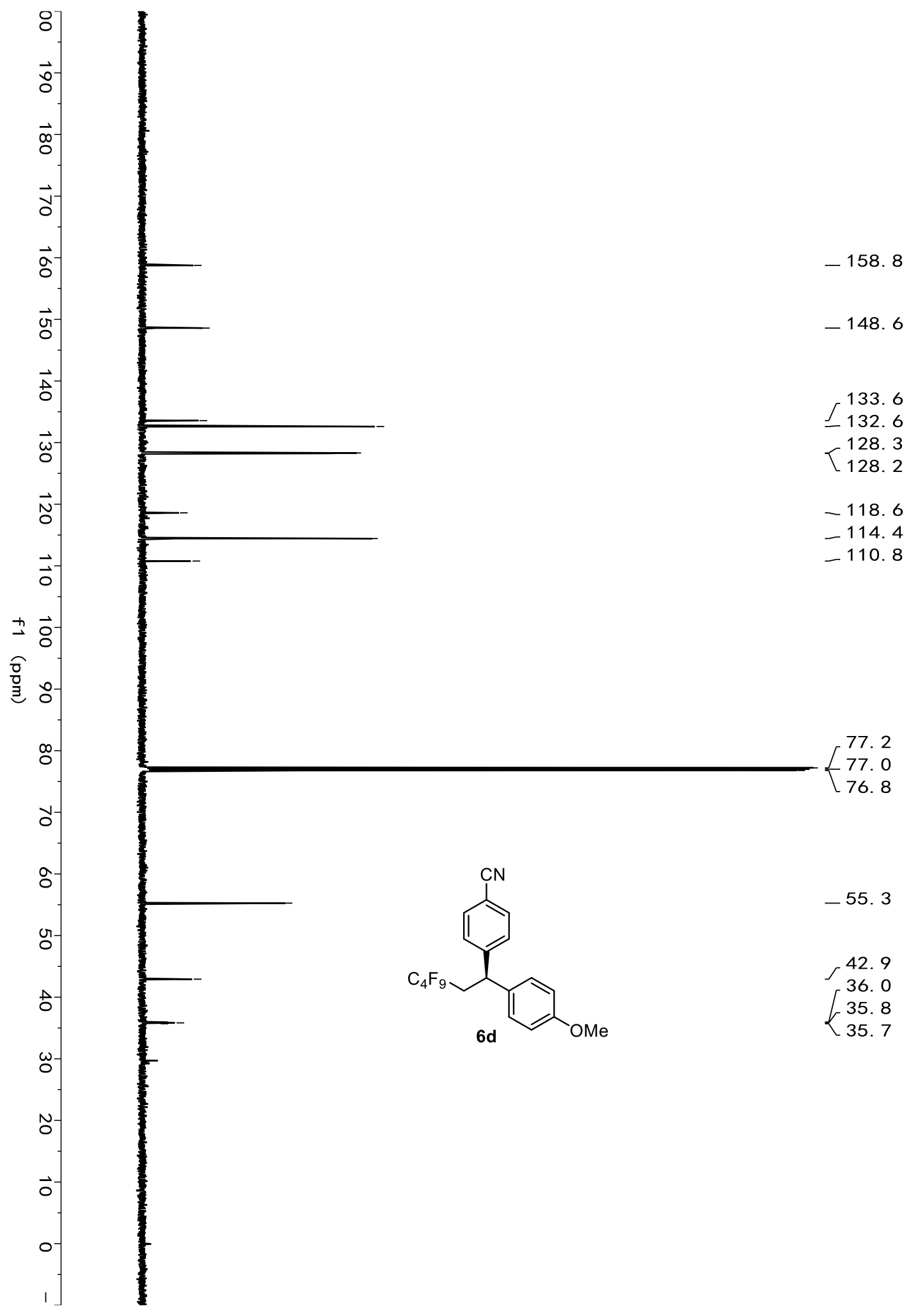

241 


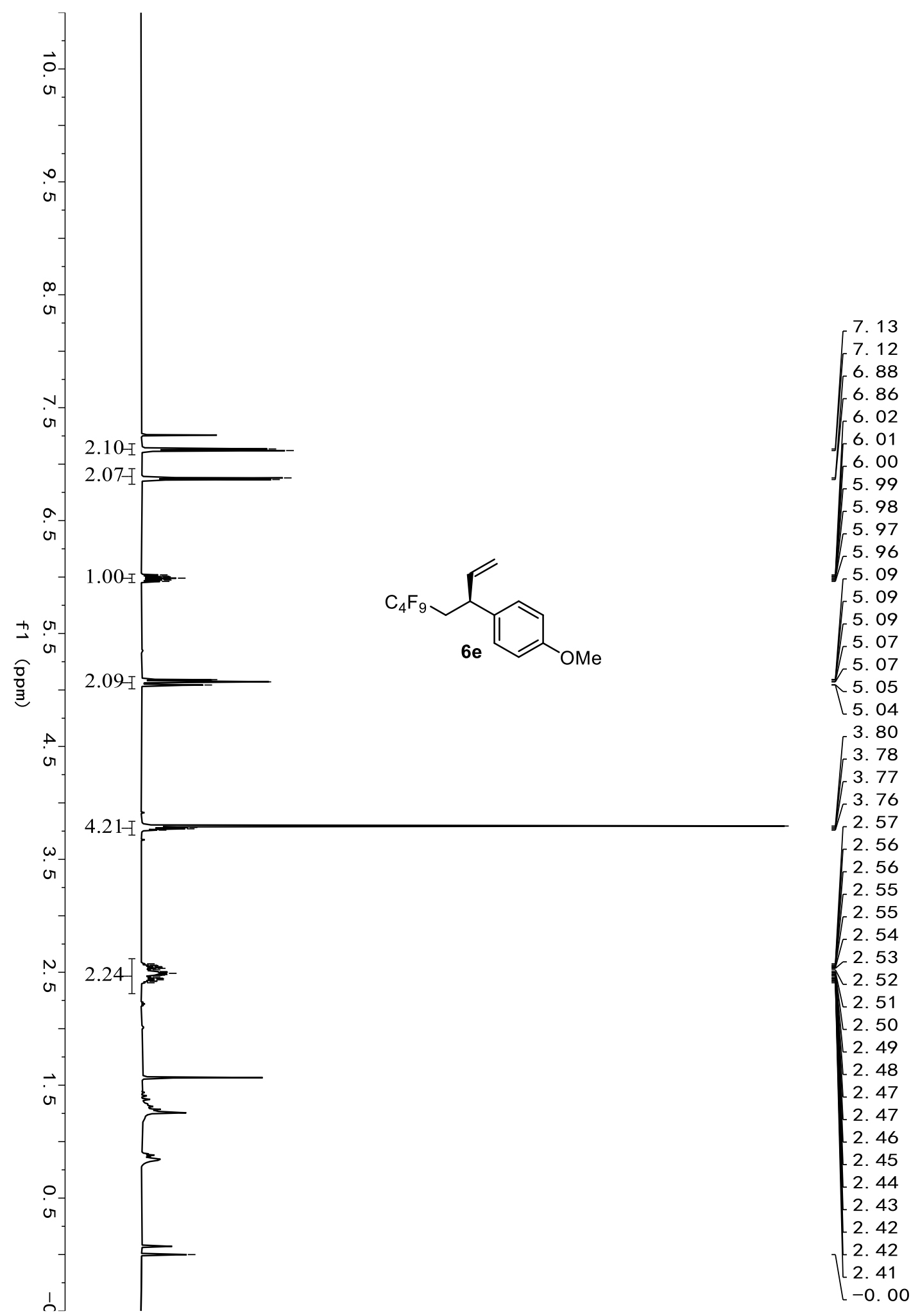




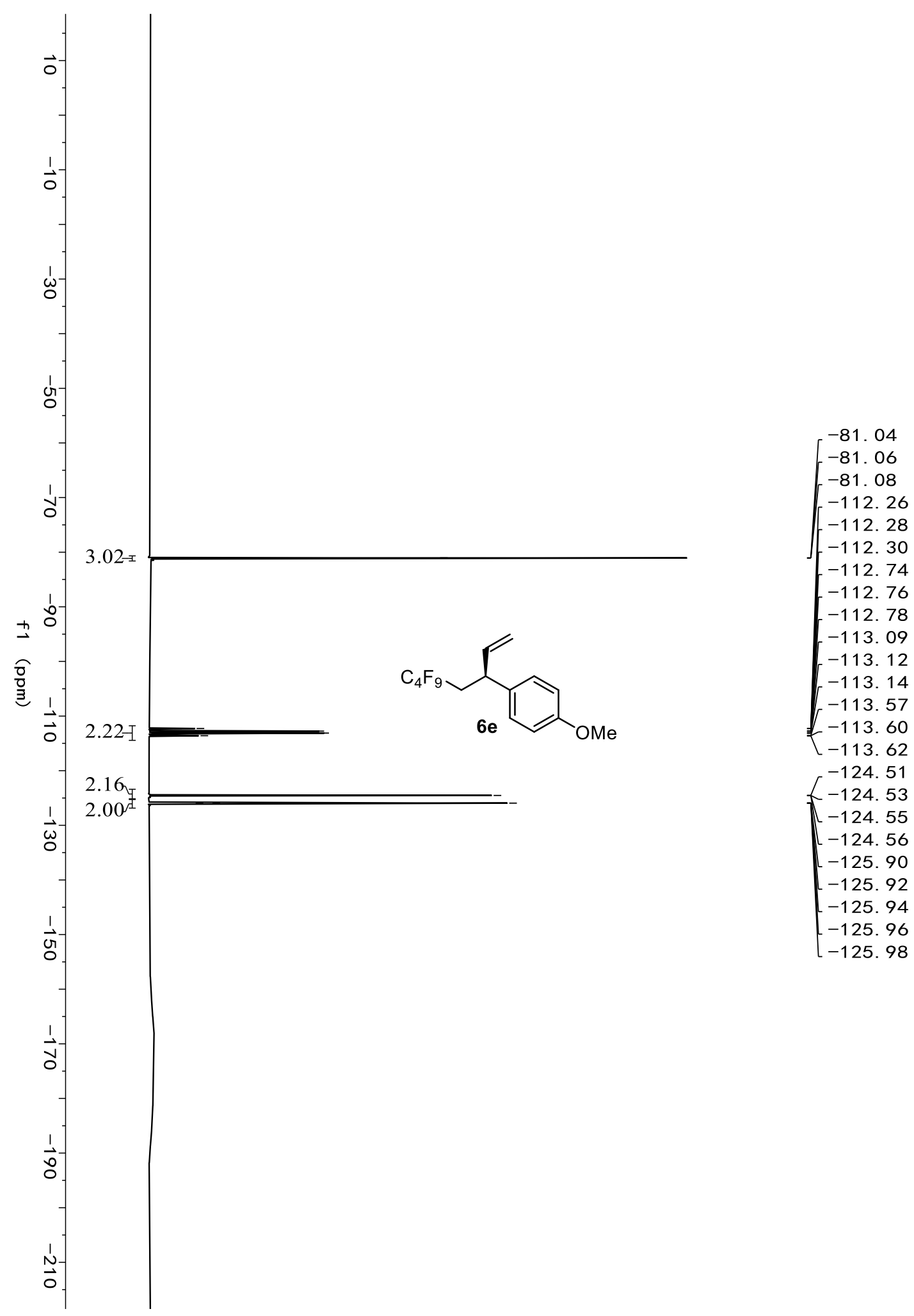




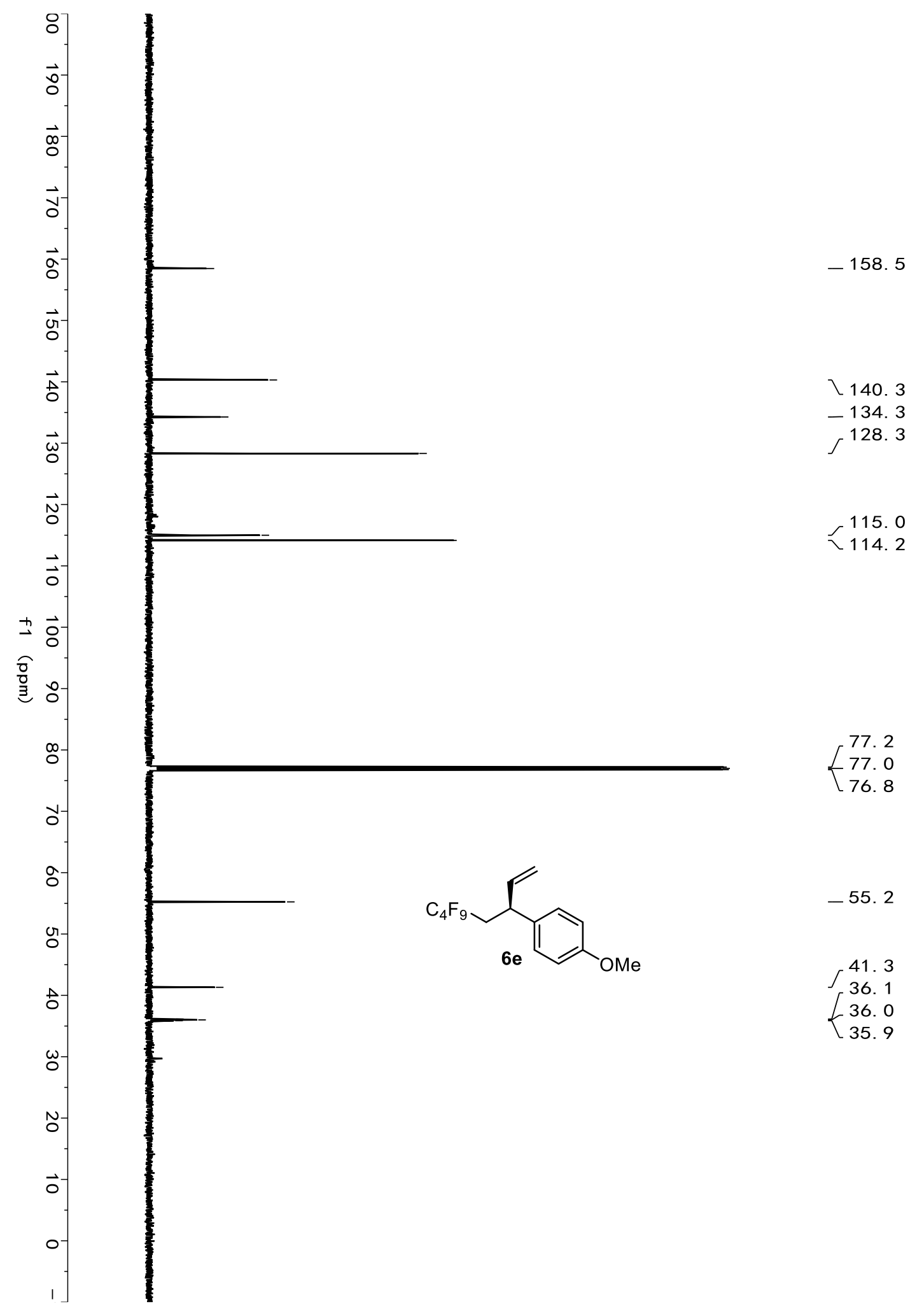




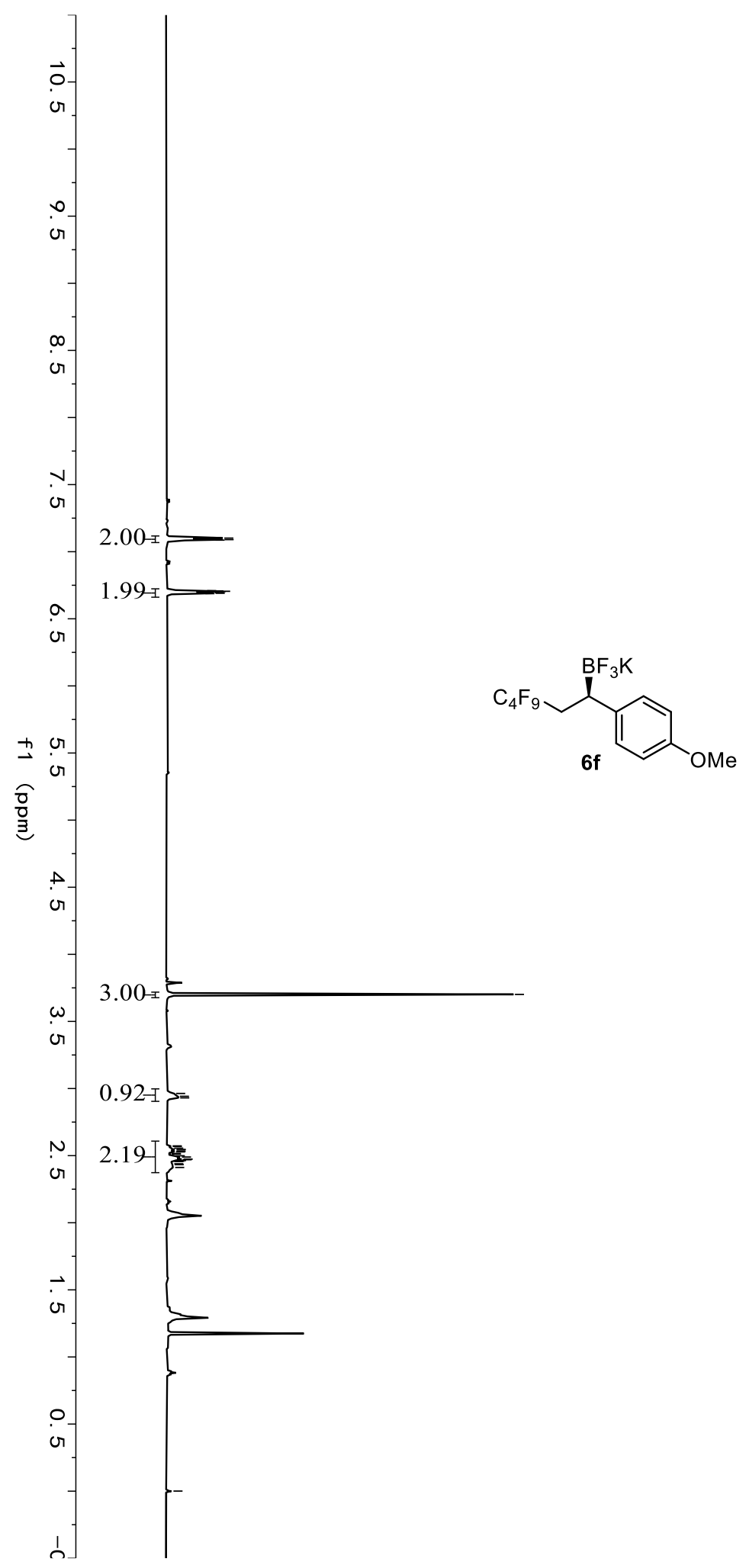

3. 70

2.96

2.94

2.93

2. 57

2. 57

2.56

2. 55

2. 54

2. 53

2. 53

2. 52

2. 51

2. 50

2. 49

2. 47

2. 47

2. 46

2. 46

-2. 46

2. 44

2. 43

2. 41

0.00 


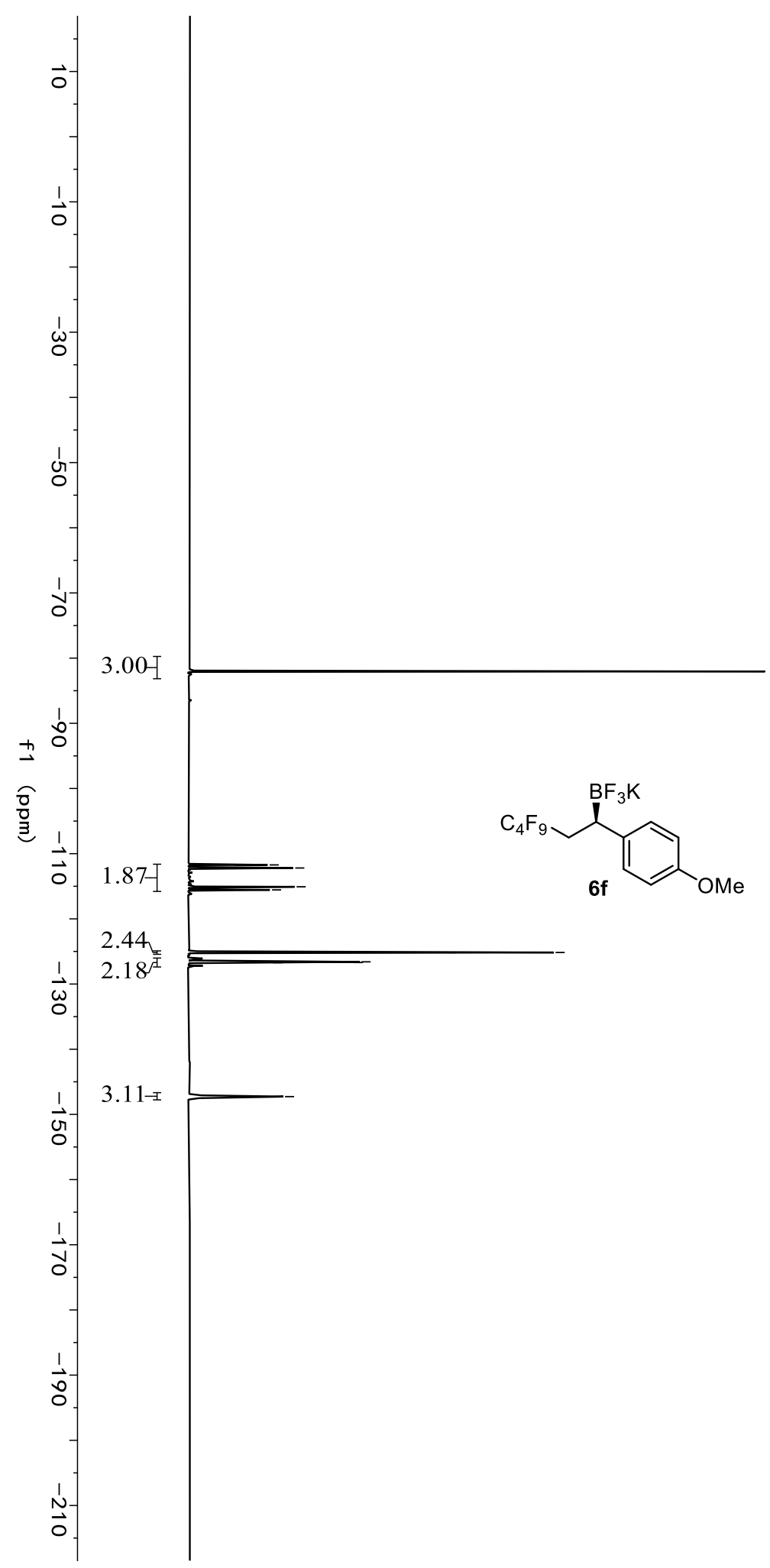

$-82.05$

$-111.70$

$-111.72$

$-111.75$

$-112.17$

$-112.20$

$-112.22$

$-115.06$

$-115.08$

$-115.10$

$-115.53$

$=-115.56$

$-115.58$

$-125.14$

$-125.16$

$-125.18$

$-125.20$

$-126.57$

$-126.59$

$-126.61$

$-126.67$

$-126.70$

-126. 72 


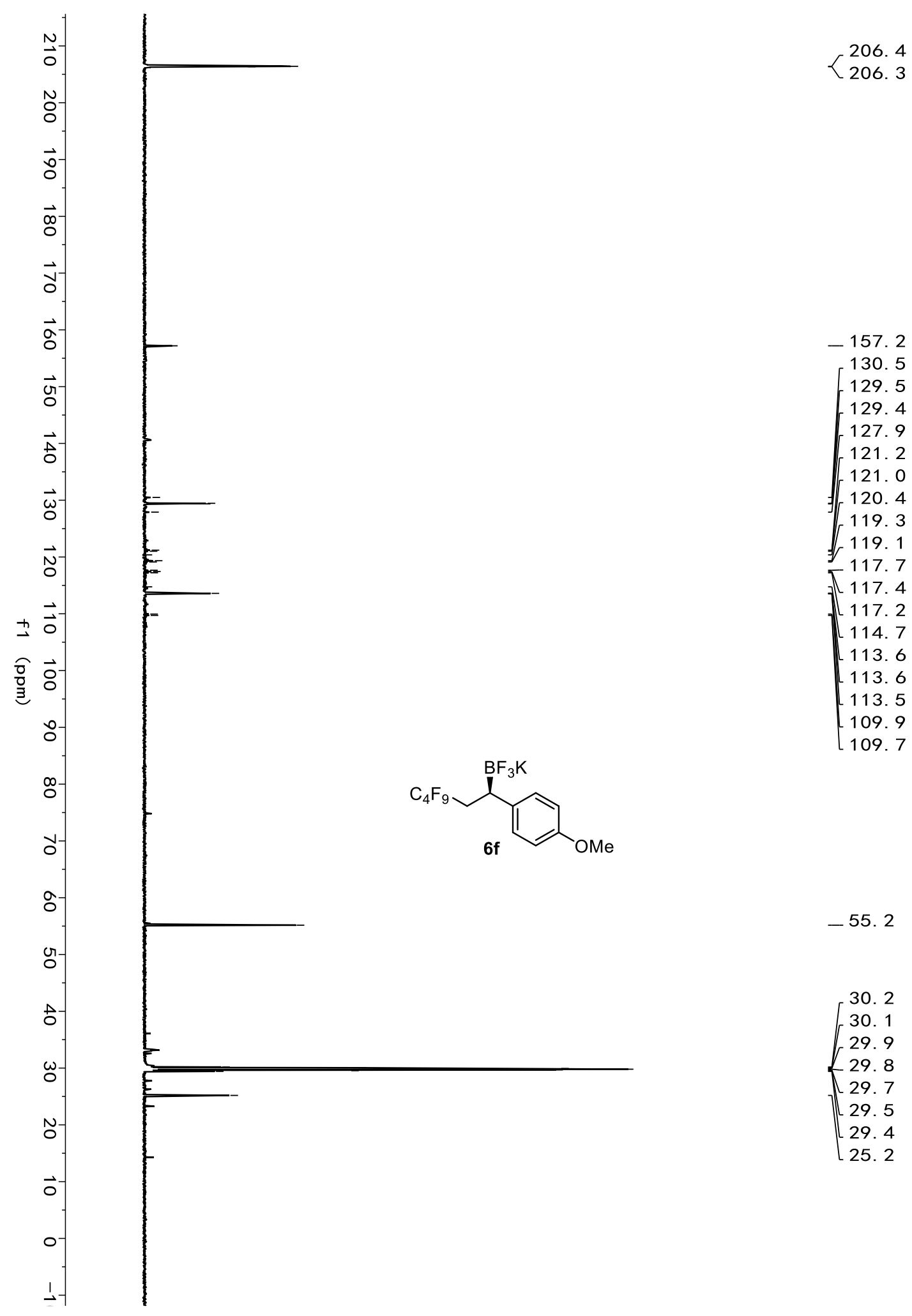

